Universidad deValladolid

PROGRAMA DE DOCTORADO EN INGENIERÍA INDUSTRIAL

TESIS DOCTORAL:

\title{
TÉCNICAS DE DETECCIÓN Y DIAGNÓSTICO DE FALLOS EN MOTORES DE INDUCCIÓN ALIMENTADOS POR INVERSOR EN ESTADO TRANSITORIO
}

Presentada por Vanessa Fernández

Cavero para optar al grado de

Doctora por la Universidad de Valladolid

Dirigida por:

Dr. Daniel Moríñigo Sotelo

Dr. Joan Pons Llinares

Dr. Oscar Duque Pérez 

"Soy de las que piensan que la ciencia tiene una gran belleza. Un científico en su laboratorio no es sólo un técnico: es también un niño colocado ante fenómenos naturales que le impresionan como un cuento de hadas."

-Marie Curie- 



\section{Agradecimientos:}

Cuando decides dar un cambio radical a tu trayectoria de vida es mucha la gente que no lo entiende e intenta disuadirte de tu objetivo, "por qué ahora... A estas alturas de la vida te vas a poner con esto... No es mejor seguir con lo que tienes... No es mejor conformarse con algo más tranquilo y estable..." Pero la palabra conformismo nunca me ha gustado, y la pasión y las ganas de aprender y seguir creciendo siempre están por encima de esto. Así que con y pese a todo, me lancé a esta aventura, que, si bien difícil y dura, la pasión por la ciencia y por el descubrimiento de algo nuevo hace que todo esfuerzo merezca la pena.

$\mathrm{Y}$ es en este momento donde le tengo que dar las gracias a toda la gente que me dijo adelante y, es más, me dieron la mano para hacerlo. Familia y amigos, eternamente os lo tengo que agradecer y la materialización de esta tesis es vuestra, un pedacito de cada uno de vosotros, porque todos, de algún modo u otro, habéis contribuido significativamente. Sería imposible enumerar a todos, así que me centraré en unos pocos. A todos los demás, aunque vuestro nombre no aparezca, gracias, de corazón, ¡gracias!

Al primero que se lo quiero agradecer, y a quien quiero dedicar esta tesis, es a Luis, mi padre, por inculcarme el amor por la ciencia desde mi más tierna infancia, concretamente por la Ingeniería Eléctrica, como te propusiste (y conseguiste) que mi primera palabra fuese "Luz" en lugar de mamá o papá como el resto de los niños, ya estabas marcando mi camino. Recuerdo perfectamente cómo me sentabas en tu mesa donde hacías tus "chapuzas" que yo admiraba con los ojos bien abiertos y me dabas un cable para que jugara, me llevabas a la térmica y me enseñabas las calderas, los motores, los paneles de control... que me parecían juguetes llenos de luces y color. Al final todo dio su fruto y mira, aquí estoy, intentando transmitir esa pasión que tú supiste transmitirme tan bien a mí.

De igual manera se lo tengo que agradecer a Adela, mi madre, por ser el mástil de mi vida, por estar siempre ahí y apoyarme en todas las cosas que emprendo, por muy absurdas que vea algunas siempre estás ahí tirando de mí y ayudándome a seguir creciendo cada día. Gracias por ser madre y, además, amiga. 
Para continuar, le tengo que dar las gracias a Daniel, Director y tutor de esta tesis, porque sin conocerme previamente, con tan solo una breve charla en su despacho, confió en mí y me abrió las puertas. A parte de haber descubierto en ti a una persona maravillosa, has sido el mejor tutor posible, un apoyo enorme en todo este tiempo, aguantando mis motivaciones y desmotivaciones, apoyándome y animándome a seguir adelante y a luchar por esto. Enseñándome cada día una cosa nueva, tengo mucho que aprender de ti, y espero seguir haciéndolo. Sin lugar a duda, sin ti, esto no habría sido posible.

A Joan, también director de esta tesis, por contagiarme su entusiasmo y sus ganas de trabajar y animarme en los momentos más bajos para seguir hacia adelante. Gracias por tus palabras de aliento, por esas llamadas cargadas de motivación, da ganas y sobre todo de vida, esas palabras que hacían que al colgar quisiera ponerme a trabajar en ese mismo instante. Gracias por esas largas horas de trabajo, por enseñarme y por seguir enseñándome tanto. Gracias por contagiar tus ganas de vivir, de trabajar y de aprender.

A otro de los directores de esta tesis, Óscar, por sus ánimos y sus palabras, por motivarme y hacerme ver que es posible y que hay que seguir adelante. Gracias por transmitirme que hay que seguir y que todo tiene su recompensa.

A Andrea, ejemplo de fuerza, coraje y tenacidad, una de las mujeres más fuertes y resueltas que jamás haya conocido. Y aunque ya no estás en este mundo de mortales, sé que desde allá donde estés me sigues mirando, animándome a seguir estudiando, siempre estudiando (como decías) y esforzándome en cada cosa que emprendo. Gracias por tus galletas con fanta de naranja, tus historias y tus ánimos para no conformarme y seguir siempre creciendo, siempre aprendiendo, siempre adelante.

A Xavi, por tu apoyo y comprensión, por motivarme cada día y confiar ciegamente en mí y en mis capacidades, aún y cuando yo misma dude de ellas. Gracias por tus palabras tan cargadas de cariño y por darme la mano para seguir adelante en todo y con todo, pase lo que pase.

Y al Dueño de la creación también gracias, por esta vida mortal, por la oportunidad de pasar por este mundo, por las personas que has puesto en mi camino, por todas las cosas que sé y por tantas que desconozco. 


\section{Índice}

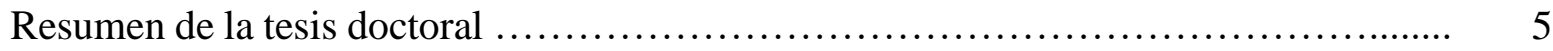

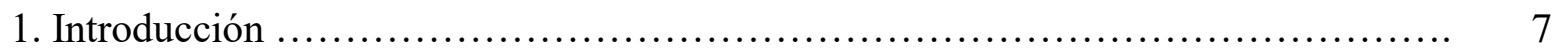

1.1. Antecedentes ..................................................... 9

1.2. Objetivos ................................................... 12

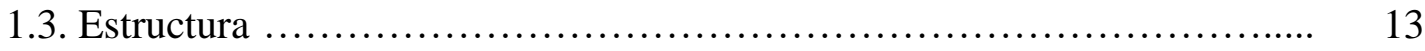

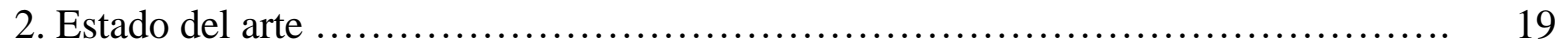

2.1. Introducción ................................................... 21

2.2. Motor Current Signature Analysis, MCSA .......................... 23

2.3. Régimen permanente .......................................... 27

2.4. Régimen transitorio .............................................. 32

2.4.1. Introducción .............................................. 32

2.4.2. Evolución temporal de los armónicos de fallo .................. 32

2.4.3. Transitorios arbitrarios .................................... 35

2.4.4. Transformada de Fourier ..................................... 37

2.4.5. Short Time Fourier Transform ............................ 38

2.4.6. Wavelets ................................................ 39

2.4.7. Distribución de Wigner-Ville ........................... 41

2.4.8. Transformada de Hilbert-Huang ............................ 42

2.4.9. Transformada de Pendiente Adaptable ........................ 43

2.4.10. Transformada Chirplet ..................................... 44

2.4.11. Multiple Signal Classification ............................ 45

2.4.12. Conclusiones parciales ................................. 45 
3. Aplicación de las técnicas de análisis tiempo-frecuencia presentes en la literatura técnica: alcance y limitaciones ............................................. 49

3.1. Introducción ..................................................... 51

3.2. Casos de estudio ................................................ 51

3.3. Aplicación y resultados de las técnicas presentes en la literatura técnica ..... 54

3.4. Conclusiones: comparativa, alcance y limitaciones de cada técnica ......... 83

4. Metodología Propuesta .................................................. 85

4.1. Introducción ..................................................... 87

4.2. Familia de átomos tiempo frecuencia ajustados a las evoluciones arbitrarias de los armónicos a seguir ................................................. 88

4.2.1. Introducción .................................................. 88

4.2.2. Definición, características principales y tipos de átomos tiempo-

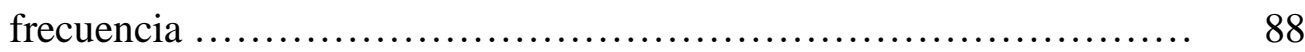

4.2.3. Átomos tipo Dragón ......................................... 93

4.3. Transformada Dragón .............................................. 100

4.3.1. Definición de la transformada Dragón ........................ 100

4.3.2. Ventajas e inconvenientes transformada Dragón ................ 108

4.3.3. Cuantificación ............................................ 109

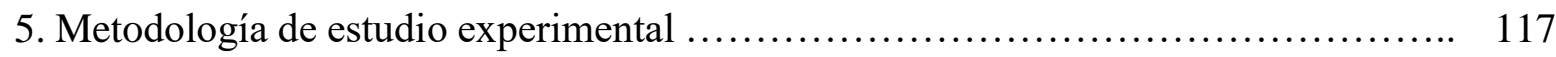

5.1. Introducción ...................................................... 119

5.2. Banco de Ensayos ................................................. 121

5.3. Ensayos experimentales .................................... 124

6. Resultados ........................................................ 133

6.1. Introducción .................................................. 135

6.2. Detección barras rotas durante el arranque ............................. 135

6.2.1. Análisis ensayos ....................................... 135

6.2.2. Conclusiones parciales ................................... 166

6.2.3. Ensayos especiales .................................. 167 
6.3. Detección de excentricidad mixta durante el arranque 178

6.4. Cuantificación del fallo de barras ..................................... 188

6.4.1. Introducción .............................................. 188

6.4.2. Curvas cuantificación ........................................ 190

6.4.3. Conclusiones parciales ................................ 212

6.5. Resultados relativos a la discriminación en motores con compensación por deslizamiento

7. Aportaciones y conclusiones (Contributions and conclusions) .................... 221

7.1. Conclusiones (Conclusions) ..................................... 223

7.2. Aportaciones (Contributions) .................................... 229

7.3. Posibles líneas de investigación a futuro (Possible future lines of research) 230

Abreviaturas

Bibliografía 237 


\section{Resumen de la tesis doctoral}

Analizando la competitividad de la industria actual es patente que una parte importante se basa en la reducción de costes, entre los cuales se encuentran los costes asociados al mantenimiento. El equipamiento industrial con el cual se trabaja se encuentra sujeto a una serie de esfuerzos, así, si se produce cierta combinación de estos o algún esfuerzo que se ejerza sobre la maquinaria superase el umbral fijado en el diseño, puede acabar produciéndose un fallo prematuro, con los consiguientes costes asociados a la parada de la producción, reparación o sustitución del equipo dañado.

Uno de los elementos más importantes en la industria actual es el motor de inducción o asíncrono. Este tipo de motor eléctrico es el principal convertidor de energía eléctrica en mecánica que se utiliza en la industria actual. Se puede alimentar directamente de red en aplicaciones de velocidad fija o bien a través de un inversor en aplicaciones de velocidad variable o que requieran un control del par, como es el caso en el que nos centramos en esta tesis. Se trata de una máquina muy robusta, así su tasa de fallos debería ser baja y su vida útil larga. Sin embargo, esta máquina puede sufrir fallos antes del final de su vida útil en algunos de sus componentes.

Para determinar la existencia o no de un fallo es necesario un sistema de detección y diagnóstico. Por esta razón es por la que existe una necesidad actual de técnicas adecuadas para identificar fallos en motores de inducción alimentados por inversor en estados transitorios, lo que supone todo un reto debido a la proximidad de las evoluciones de los armónicos de fallo con la componente fundamental y el cambio continuo de esta frecuencia durante los estados transitorios. Y es aquí donde surge la necesidad del desarrollo de la presente tesis.

En esta tesis se ha desarrollado una nueva técnica, la Transformada Dragón, basada en átomos tiempo-frecuencia, que permite solventar los problemas de resolución que aparecen en otras técnicas, consiguiendo una perfecta resolución de los armónicos de fallo, lo que permite poder visualizarlos con cualquier tipo de señal que se analice. La Transformada Dragón ha sido probada, de manera satisfactoria, tanto de manera teórica como experimental en el laboratorio de la Universidad de Valladolid, consiguiendo los resultados que en esta memoria se plasman. 


\section{Thesis summary}

The competitiveness of the industry is partly based on cost reduction, which includes costs associated with maintenance. The industrial equipment is subject to a series of efforts, thus, if there is some combination of these efforts or any effort that is exercised on the machinery exceeds the threshold set in the design, it can end up resulting in premature failure, with the consequent costs associated with the stop of production, repair or replacement of damaged equipment.

One of the most important elements in today's industry is the induction motor or asynchronous motor. This type of electric motor is today the main converter of electrical power into mechanical energy. It can be fed directly from the line in fixed speed applications or through an inverter in variable speed applications or that require a torque control. This machine is very robust, so its failure rate should be low, and it has a long life. However, induction motors can suffer failures before the end of its useful life in some of its components.

Detection and diagnosis systems are necessary to determine the existence or not of failures. For this reason, there is a current need to appropriate techniques to identify faults in inverter-fed induction motors in transient regimes, which is a challenge due to the proximity of the trajectories of the fault harmonics to the fundamental component and the continuous change of this frequency during the transient state. And this is where the need arises for the realization of this thesis, where a new technique called the Dragon Transform has been developed. This new tool is based on time-frequency atoms, which allow solving the resolution problems that appear in other methods, getting a perfect capture of the fault harmonics that permits to these harmonics with any signal.

The Dragon Transform has been satisfactorily tested, both theoretically and experimentally in the laboratory of the University of Valladolid and the results are shown in this dissertation. 
1. Introducción

\author{
1.1. Antecedentes \\ 1.2. Objetivos \\ 1.3. Estructura
}




\section{Introducción}

En este primer punto de la memoria se lleva a cabo una breve introducción en donde se analizan los antecedentes que dan lugar a la aparición de la presente tesis. Así mismo se van a ver los objetivos que se plantean conseguir con la misma y la estructura que se ha seguido para llevarla a cabo.

\subsection{Antecedentes}

Analizando la competitividad de la industria actual es patente que una parte importante se basa en la reducción de costes, entre los cuales se encuentran los costes asociados al mantenimiento. El equipamiento industrial con el cual se trabaja se encuentra sujeto a desgastes, fallos de montaje, tolerancias, entre otras. Así, si se produce cierta combinación de esfuerzos (como pueden ser térmicos, mecánicos, magnéticos, eléctricos, ambientales), o algún esfuerzo que se ejerza sobre la maquinaria superase el umbral fijado en el diseño, puede acabar produciéndose un fallo prematuro, con los consiguientes costes asociados a la parada de la producción, reparación o sustitución del equipo dañado.

Dentro del equipamiento con el que cuenta una industria, uno de los elementos más importantes es el motor de inducción o asíncrono. Este tipo de motor eléctrico es el principal convertidor de energía eléctrica en mecánica que se utiliza en la industria actual. Aproximadamente el $90 \%$ de los motores industriales son de este tipo. También es importante en tracción, sobre todo la ferroviaria, donde la mayoría de las cabezas tractoras de alta velocidad se equipan con este tipo de motor. El motor asíncrono se puede alimentar directamente de red en aplicaciones de velocidad fija o bien a través de un inversor en aplicaciones de velocidad variable o que requieran un control del par.

En general, el motor de inducción es una máquina muy robusta. Si el motor está bien seleccionado según los requerimientos de la aplicación, su tasa de fallos debería ser baja y su vida útil larga. Sin embargo, debido a defectos de fabricación, especificaciones incorrectas, ciclos de trabajo no adecuados, montaje incorrecto, etc., esta máquina puede 
sufrir fallos antes del final de su vida útil en algunos de sus componentes. Entre los elementos que pueden dar lugar a fallo se pueden citar el rotor, rodamientos, eje, bobinado del estator, etc. La importancia del fallo en la aplicación industrial depende de muchos factores:

- Importancia y/o criticidad del motor en el sistema de producción.

- Accesibilidad del motor para la reparación.

- Existencia de stocks.

- $\quad$ Tipo de mantenimiento.

Es cierto que, bajo ciertos tipos de fallo, prácticamente todos salvo el cortocircuito del estator y algunos fallos mecánicos asociados al eje, el motor puede seguir funcionando, aparentemente bien. Pero en algunos casos, la existencia del fallo afecta a la generación del par o produce oscilaciones de velocidad que, por ejemplo, pueden afectar al producto final en un ciclo de mecanizado o al confort en una aplicación de tracción.

Para determinar la existencia o no de un fallo es necesario un sistema de detección y diagnóstico. Las prestaciones y orientación del sistema de diagnóstico dependerán del tipo de mantenimiento y de los factores comentados anteriormente sobre la importancia del fallo. Es decir, en algunos casos será necesario diagnosticar el fallo incipiente, mientras que en otros basta con el diagnóstico de un fallo total, pero siempre antes de que el fallo derive en uno catastrófico, que podría suponer la destrucción total del motor, consecuencias para el resto del sistema productivo o elementos en la cadena cinemática, incluso, puede poner en riesgo la vida de las personas.

Los sistemas de diagnóstico requieren varias etapas, que a continuación se detallan adaptándolos a la tesis en cuestión:

- Conocimiento del sistema a diagnosticar. En este caso el sistema a diagnosticar será un motor alimentado por un inversor. El uso de un inversor para alimentar el motor de inducción está cada vez más generalizado en la industria por varias razones como son la mayor eficiencia energética del accionamiento, velocidad variable regulable, control de par producido, etc. Desde un punto de vista de la 
detección y diagnóstico de fallos, el uso del inversor supone una gran dificultad añadida por la presencia de ruido en la señal y otros interarmónicos y subarmónicos que pueden dificultar la observación de los indicadores de fallo. En algunos casos, también se modifica la firma del fallo con respecto a la alimentación directa de la red.

- Elección de las variables a monitorizar. En el caso de un motor de inducción las señales suelen ser la corriente eléctrica del estator, el flujo magnético, la temperatura, vibraciones mecánicas o el sonido. En esta tesis, se ha utilizado la corriente del estator como señal para monitorizar. Se ha elegido la corriente como magnitud medida, sobre otras variables, por las ventajas que presenta su análisis, como la de tratarse de una técnica no invasiva, es decir, se puede medir sin necesidad de actuar sobre el motor.

- Sistema de adquisición de datos, con los sensores adecuados. Los parámetros de adquisición, frecuencia y tiempo de muestreo, tienen que ser acordes con la capacidad del sistema de adquisición disponible, pero también con las técnicas de tratamiento de señales que se comentan a continuación.

- Procesamiento de las señales. Las señales normalmente se adquieren en el dominio del tiempo, pero para extraer información útil suele ser necesario transformar la señal al dominio de la frecuencia o del tiempo-frecuencia. La técnica de procesamiento de la señal dependerá de la naturaleza de la señal, estacionaria o transitoria, de la detectabilidad del fallo, etc. En este caso se analizarán las señales no estacionarias en el dominio tiempo-frecuencia.

- Obtención o cálculo de los indicadores de fallo. Después del procesamiento de la señal, es necesario extraer valores numéricos indicadores del estado del motor. Es importante que el resultado del procesamiento de la señal se pueda cuantificar si se quiere que el diagnóstico sea automático o realizado por un sistema experto.

- Construcción del sistema de diagnóstico. La mayoría de los sistemas de diagnóstico son de tipo supervisado. Es decir, necesitan de un entrenamiento con 
datos obtenidos previamente. El sistema de diagnóstico suele fundamentarse en el uso de un clasificador, entre los que se pueden citar las Redes Neuronales, Support Vector Machines, Decision Trees, etc. También puede haber sistemas no supervisados, pero son menos comunes.

Esta tesis se va a centrar en el desarrollo de una técnica de procesamiento de señales no estacionarias para la detección de fallos en motores alimentados por inversor.

En el apartado 2 se analizan las técnicas que se han usado históricamente para la detección de fallos. Para a continuación centrar la memoria en la técnica desarrollada durante el proyecto de tesis, con su análisis exhaustivo y posterior exposición de resultados.

\subsection{Objetivos}

Se plantea como objetivo principal el desarrollar una técnica de procesamiento de señales no estacionarias basada en la técnica de los átomos tiempo-frecuencia cuya pendiente puede adaptarse a la evolución de los armónicos de fallo en motores de inducción alimentados por inversor, obteniendo así toda la evolución temporal de la señal analizada.

Este objetivo principal se puede dividir en una serie de objetivos parciales que derivan del mismo.

- Diseñar un banco de pruebas que permita la captura de las señales de corriente del motor alimentado por diferentes inversores y red, y en diferentes condiciones de carga del motor.

- Aplicar diferentes técnicas de procesamiento de señales en el dominio tiempo-frecuencia para la detección de fallos en transitorios de arranque del motor. Realizar un estudio comparativo crítico de las diferentes técnicas. 
- Identificar qué técnicas permitirían adaptar la pendiente de las cajas de Heisenberg.

- Estudiar y proponer un algoritmo que permita adaptar de forma continua la pendiente de la caja de Heisenberg en función de las necesidades de resolución en tiempo y frecuencia.

- Aplicar la nueva técnica desarrollada aplicándola a la detección de fallos por barras rotas, excentricidad y evitar falsos positivos. Analizar los resultados obtenidos, para un motor de inducción alimentado por diferentes inversores.

\subsection{Estructura}

La presente memoria de esta tesis doctoral se ha estructurado en siete puntos, entre los que se incluye este primer apartado. Se detallan, a continuación, el contenido de cada uno de estos puntos.

\section{Introducción}

En este primer apartado se introducen las bases de la industria, así como el contexto industrial actual, para tener una primera visión del marco en el que se está trabajando y de la importancia del motor de inducción en el mismo y la necesidad del desarrollo de esta tesis.

Así mismo se explican los objetivos con los que se ha planteado la tesis y que se encuentran desarrollados a lo largo de esta memoria, así como la estructura de la misma.

Para desarrollar este punto, el apartado se divide, a su vez, en los siguientes subapartados:

1.1. Antecedentes

1.2. Objetivos

\subsection{Estructura}




\section{Estado del arte}

En este apartado se desarrolla el Estado del arte de las técnicas que aparecen en la literatura y que se han ido desarrollando en el ámbito del estudio de la detección de fallos en motores de inducción alimentados mediante inversor. Para ello se hará una breve introducción, se analiza el método con el que se va a trabajar durante el desarrollo de la transformada (objetivo principal de esta tesis), Motor Current Signature Analysis; Por otra parte, se analizan los tipos de fallos que se pueden encontrar en estos motores. Para, finalmente, analizar las técnicas que se han desarrollado en el estado permanente y el estado transitorio. El estado transitorio es en el cual se centra el desarrollo de esta tesis.

Así, este punto se encuentra dividido de la siguiente manera:

\subsection{Introducción}

2.2. Motor Current Signature Analysis, MCSA.

\subsection{Régimen Permanente}

\subsection{Régimen Transitorio}

\subsubsection{Introducción}

2.4.2. Evolución temporal de los armónicos de fallo

2.4.3. Transitorios arbitrarios

2.4.4. Transformada de Fourier

2.4.5. Short Time Fourier Transform

2.4.6. Wavelets

2.4.7. Distribución de Wigner-Ville

2.4.8. Transformada de Hilbert-Huang

2.4.9. Transformada de Pendiente Adaptable

2.4.10. Transformada Chirplet

2.4.11. Multiple Signal Classification.

2.4.12. Conclusiones parciales 
3. Régimen transitorio: aplicación de las técnicas de análisis tiempo-frecuencia presentes en la literatura técnica.

En este apartado se estudia la problemática que presenta las técnicas que se han desarrollado para el análisis en estado transitorio, que previamente se han analizado en el punto 2, concretamente en el epígrafe 2.4 .

Para analizar exhaustivamente cada una de estas técnicas se aplica cada método a dos señales experimentales que se han obtenido en el laboratorio. Por tanto, a lo largo de este apartado, se explica cómo se han obtenido las señales, se aplican las técnicas del apartado 2.4 y finalmente se sacan una serie de conclusiones, aludiendo a las ventajas e inconvenientes que presenta cada una de estas técnicas.

Por tanto, el punto 3 queda dividido de la siguiente manera:

\subsection{Introducción}

3.2. Casos de estudio

3.3. Técnicas de detección de fallos basadas en el análisis tiempo-frecuencia en motores de inducción alimentados mediante inversor

\subsection{Conclusiones}

\section{Metodología Propuesta}

El apartado 4 es el apartado central de esta tesis, ya que es en él donde se explica la técnica desarrollada y los resultados que se han obtenido con ella, que se van a analizar en profundidad en el siguiente punto (Punto 5). Para ello, en este apartado se introducen los átomos tiempo-frecuencia, su definición, características y cómo se llega al desarrollo de los átomos tipo "Dragón”. Una vez que se hecho este primer análisis en el siguiente bloque se analiza la transformada Dragón, para lo que se va a ver su definición, qué ventajas e inconvenientes presenta, así como su cuantificación. Finalmente aparece reflejado en una figura los pasos a seguir para analizar una señal mediante la transformada Dragón.

El punto 4 se encuentra dividido de la manera que sigue: 


\subsection{Introducción}

4.2. Familia de átomos tiempo frecuencia ajustados a las evoluciones arbitrarias de los armónicos a seguir

\subsubsection{Introducción}

4.2.2. Definición, características principales y tipos de átomos tiempofrecuencia

\subsubsection{Principio de Incertidumbre de Heisenberg}

\subsection{4. Átomos tipo Dragón}

\subsection{Transformada Dragón}

4.3.1. Definición de la transformada Dragón

4.3.2. Ventajas e inconvenientes de la Transformada Dragón

\subsubsection{Cuantificación}

\section{Metodología de estudio experimental}

En este apartado se concreta la validación experimental de todo lo que se ha desarrollado durante esta tesis. Se comienza por un análisis exhaustivo del banco de ensayos con el que se ha trabajado en el laboratorio, mediante el cual se ha llevado a cabo la realización de los ensayos, así se explicarán cada uno de los elementos que lo componen. Seguidamente se explica todo lo relativo a los ensayos que se han llevado a cabo.

Por tanto, el punto 5 queda dividido de la siguiente manera:

\subsection{Introducción}

5.2. Banco de ensayos

\subsection{Ensayos experimentales}

\section{Resultados}

Tras analizar los puntos anteriores, en este apartado se presentan los resultados experimentales que se han obtenido y que fundamentan el desarrollo de esta tesis, distinguiendo por tipo de fallo (fallo por barras rotas y excentricidad mixta) y relativos a falso positivo. Con lo que el punto se estructurará de la siguiente manera: 


\subsection{Introducción}

6.2. Detección barras rotas durante el arranque

6.2.1. Análisis ensayos

6.2.2. Conclusiones parciales

6.2.3. Ensayos especiales

6.3. Detección de excentricidad mixta durante el arranque

6.4. Cuantificación del fallo de barras.

6.4.1. Introducción.

6.4.2. Curvas cuantificación.

6.4.1. Conclusiones parciales.

6.5. Resultados relativos a la discriminación en motores con compensación por deslizamiento

\section{Aportaciones y conclusiones}

Con el desarrollo de los apartados 2 a 5 se puede llegar a una serie de conclusiones, que se pueden dividir en tres bloques; en el primero de ellos tendremos las conclusiones concernientes tanto de las técnicas desarrolladas que aparecen en la literatura para estado permanente y transitorio, que se ha desarrollado en el punto 2 de esta memoria, como de la aplicación experimental de las mismas, en estado transitorio, tal como se desarrolla en el apartado 3.

En el siguiente bloque aparecen las conclusiones concernientes al desarrollo de la transformada, objeto de estudio en esta tesis. Así se explicarán, a modo de resumen, las conclusiones a las que se han llegado en el desarrollo del apartado 4 de esta memoria.

Se extraerán una serie de conclusiones a raíz del apartado 5 de la memoria, que tratarán sobre lo que se ha obtenido de manera experimental durante el desarrollo de la tesis.

Finalmente, y en base a lo obtenido durante el desarrollo de los objetivos planteados en esta tesis, en este apartado se plantean una serie de líneas de investigación a futuro, con las que se ampliarán los resultados que se han obtenido en esta tesis. Algunas de estas líneas a futuro se han introducido, brevemente, en el apartado 5 de esta memoria, 
concretamente en el apartado 5.5., con el que se pueden obtener interesantes resultados tras su profundo análisis.

7. Aportaciones y conclusiones

7.1. Conclusiones

7.2. Aportaciones

7.3. Posibles líneas de investigación a futuro. 


\section{Estado del arte}

2.1. Introducción

2.2. Motor Current Signature Analysis, MCSA

2.3. Régimen permanente

2.4. Régimen transitorio

2.4.1. Introducción

2.4.2. Evolución temporal de los armónicos de fallo

2.4.4. Transformada de Fourier

2.4.5. Short Time Fourier Transform

2.4.6. Wavelets

2.4.7. Distribución de Wigner-Ville

2.4.8. Transformada de Hilbert-Huang

2.4.9. Transformada de Pendiente Adaptable

2.4.10. Transformada Chirplet

2.4.11. Multiple Signal Classification.

2.4.12. Conclusiones parciales 


\section{Estado del arte}

\subsection{Introducción.}

En este capítulo se realiza una revisión de las técnicas de análisis de señales que, hasta el momento, se han desarrollado y empleado para la detección de fallos en motores de inducción. Se va a diferenciar entre el régimen permanente y el transitorio.

Como se ha venido mencionando en la introducción, los motores de inducción (IM) son un elemento clave de la industria. Estos motores pueden funcionar alimentados directamente desde la red. En estas aplicaciones, la velocidad del motor no puede ser controlada y se prácticamente constante. Esto es habitual, por ejemplo, en el accionamiento de bombas y ventiladores que representan más del $50 \%$ de los electrodomésticos y usos industriales de los motores de inducción. Debido a que el funcionamiento a velocidad fija tiene un gran impacto en el consumo de energía y en los costos operacionales del motor durante su vida útil, estos usos son considerados muy ineficientes. Si el motor es alimentado por un inversor, su velocidad puede ser regulada y adaptada a las necesidades reales de la carga, y esto se traduce en un importante ahorro de energía [1]. Por ejemplo, un motor funcionando a la mitad de velocidad reduce su consumo en un $75 \%$ [2]. Las últimas directivas de la UE promueven el uso de motores energéticamente eficientes o el uso de motores alimentados mediante un inversor. Por esta razón, el número de motores de inducción alimentados por inversor ha aumentado significativamente en los últimos años. Hay otros campos donde el uso de un inversor para alimentar un motor es obligatorio como en tracción eléctrica [3] o en procesos de fabricación donde la flexibilidad es una necesidad [4]. Estas aplicaciones se caracterizan generalmente por un motor funcionando en condiciones de estado no estacionario.

Este cambio en el uso de los IM también ha tenido un impacto importante en el campo del mantenimiento, con una tendencia hacia el mantenimiento predictivo, el cual trata de detectar el fallo de antemano y evaluar su severidad, minimizando el impacto económico de dicho fallo a futuro [5]. La implantación de un programa de mantenimiento predictivo suele estar basada en el diseño e implementación de un sistema experto, como método que permite llevar a cabo este mantenimiento. 
Antes de comenzar con el análisis en profundidad, primeramente, es necesario distinguir qué es detección y qué parte corresponde a diagnóstico, dentro de un sistema experto; Se entiende por sistema experto "Programa de inteligencia artificial diseñado para resolver problemas o tomar decisiones en un ámbito determinado de modo análogo al razonamiento humano" [6]. Un sistema experto realiza tareas de monitorización, diseño, planificación, control, simulación, instrucción y recuperación de la información. En la Figura 1 se muestra de manera esquemática las partes de las que consta un sistema experto; resumidamente se puede decir que consta de una base de conocimientos, una base de hechos y un motor de inferencias. La base de conocimientos es el conjunto de reglas que permiten representar conocimientos del dominio de experto; la base de hechos contiene los datos de partida así como los criterios de parada; y el motor de inferencias es el programa de control. Además de contar con estas partes fundamentales es necesario que exista una interactuación.

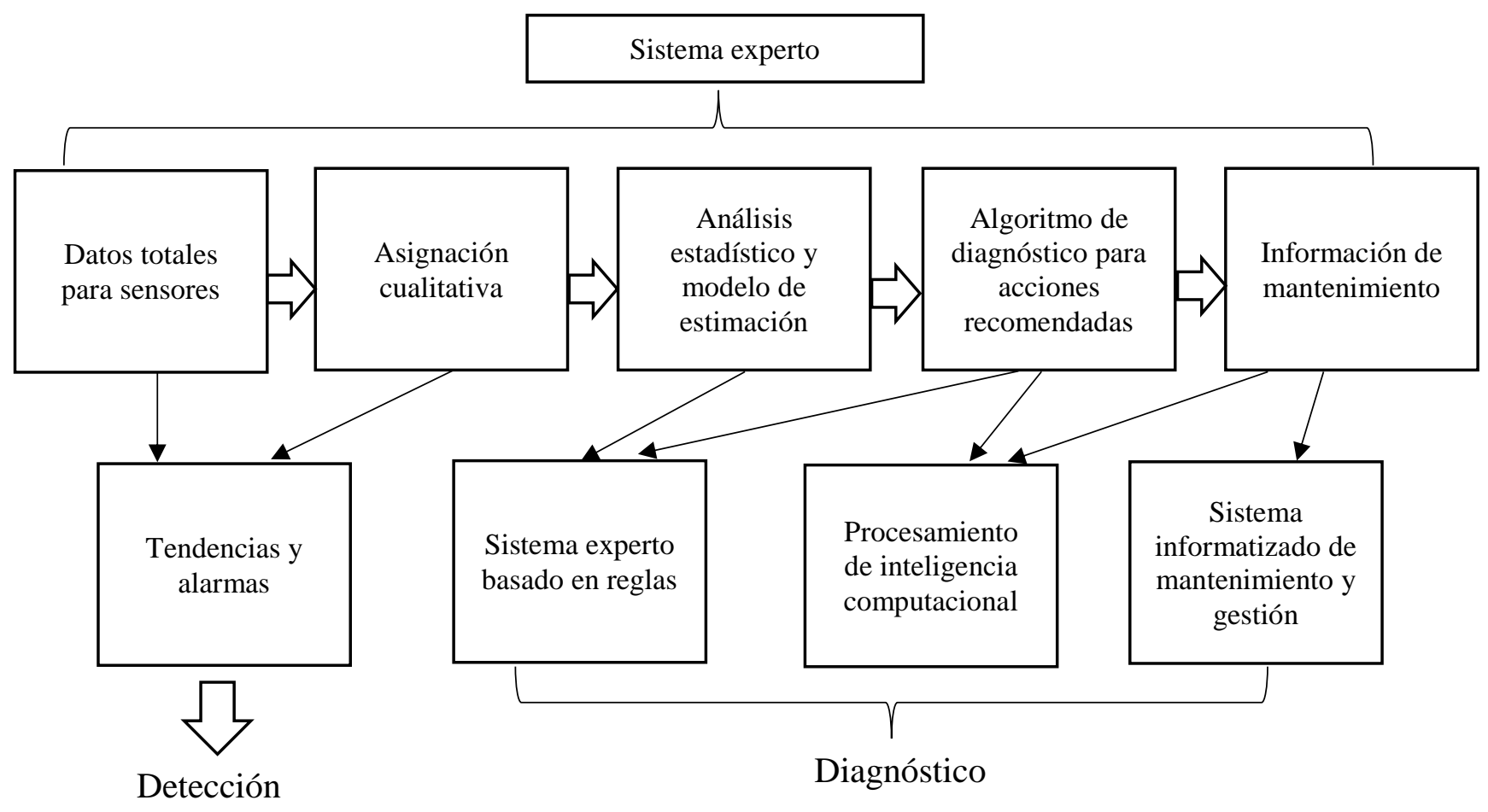

Figura 1. Etapas de un sistema experto. 
Es de vital importancia para conseguir un mantenimiento predictivo correcto que la detección del fallo sea adecuada. Dicha detección se basa en el análisis de una determinada variable física, pudiendo ser diversas las variables a analizar, tales como:

- Vibraciones: Se analiza la vibración de la máquina y su espectro en frecuencia, lo que dará información sobre la máquina así como sobre la posible presencia de fallos.

- Flujo axial de dispersión: Consiste en medir el flujo axial de dispersión, el cual es causado por asimetrías del estator y rotor.

- Termografía: en este caso se emplea termografía o termómetros de infrarrojos para localizar calentamientos que puedan ser debidos a defectos en la máquina.

- Análisis de la corriente: Motor Current Signature Analysis (MCSA). Se basa en el análisis de los componentes armónicos de la corriente. Se explicará en más profundidad en el apartado 2.2 pues es la base del presente estudio.

Por otro lado, también se puede atender al análisis del estado de la jaula. Este ensayo se utiliza para detectar daños y defectos de calidad en rotores de máquinas de inducción, pudiendo localizar el tipo y el lugar del fallo.

Para analizar estos aspectos, en el Apartado 2.2. se explica la técnica MCSA, así como una introducción a los tipos de fallo; para pasar posteriormente al Estado del arte, así se analiza en el Apartado 2.3. el Estado del arte en régimen permanente y en el Apartado 2.4. el Estado del arte para el régimen transitorio.

\subsection{Motor Current Signature Analysis, MCSA.}

Como se ha comentado en la introducción de este apartado, existen diversas técnicas que permiten la detección de fallos en un motor de inducción. Para la realización de esta tesis se va a centrar en la monitorización y análisis de la corriente del estator [7 10]. Este método, que se conoce por su nombre en inglés como Motor Current Signature Analysis, es una técnica no invasiva, lo que constituye su principal ventaja sobre otros esquemas de monitorización. 
MCSA es un método muy extendido que se basa en la monitorización de la corriente del estator y, posteriormente, su análisis espectral, mediante alguna técnica de detección de fallos, las cuales se explicarán en los apartados 2.3. y 2.4. Consiste en evaluar la amplitud relativa de los componentes armónicos que aparecen en la señal y que son provocados por la presencia de un fallo.

Sin embargo, podría no ser apropiado para aplicaciones donde la velocidad del motor está sujeta a continuos cambios [11]. La razón es que el análisis de la corriente del estator se basa en el uso de la transformada de Fourier, o su versión Fast Fourier Transform (FFT). Incluso, si los períodos de operación de estado estacionario son lo suficientemente largos, el espectro de la corriente es rico en contenido armónico originado por el mecanismo de la conmutación del inversor y la señal tiene generalmente un alto nivel de ruido.

Algunos armónicos producidos por el suministro pueden solaparse con armónicos relacionados con el fallo, haciendo más complicada su observación y posterior diagnóstico. Es decir, podrían dar lugar a un falso positivo, entendiendo por positivo la detección del fallo. Además, la amplitud de algunos armónicos de fallo depende también de la frecuencia de salida asignada o del tipo de control de inversor [12]-[14]. Todo esto ha originado un campo activo de investigación en diagnóstico de fallos en motores alimentados por inversor y detección de fallos en los regímenes estacionario y transitorio.

Así, resumidamente, se puede afirmar sobre la técnica $M C S A$, que:

- Mediante el análisis de la señal de la corriente se puede detectar si existe o no fallo en el motor analizado, analizando su espectro.

- Se basa en la monitorización de la corriente de estator del motor y su posterior análisis espectral.

- Consiste en evaluar la amplitud relativa de los diferentes armónicos que aparecen en la corriente debido al fallo.

- Este método es válido para la detección de distintos tipos de fallos, tales como barras rotas y excentricidad, que se van a comentar posteriormente en este apartado. 
Por otra parte, la medida de la corriente se puede realizar mediante diferentes métodos como son:

- Resistencia Shunt (Figura 2(a)): En este caso se usa el valor de la resistencia shunt para determinar la intensidad de corriente eléctrica que fluye a través de la carga; esto se hace mediante la medición de la diferencia de tensión a través de dicha resistencia, para lo que se aplica la ley de Ohm.

- Sensores de efecto Hall (Figura 2(b)): estos sensores se basan en el efecto Hall para la medición de la corriente.

- Transformadores de Corriente (Figura 2(c)): Estos transformadores son usados para suministrar la corriente adecuada a los instrumentos o equipos necesarios. El devanado primario del transformador de corriente se conecta en serie con el circuito donde circula la corriente que se desea medir, mientras que los instrumentos o equipos se conectan en serie a su devanado secundario.

- Bobina Rogowski (Figura 2(d)): se trata de un dispositivo electrónico que se usa para medir corriente alterna o pulsos rápidos de corriente. Consiste en una bobina de cable en forma de hélice, alrededor de una circunferencia, como un toroide, pero con núcleo de aire, y los dos terminales están cercanos entre sí; dicha bobina se cierra alrededor del cable conductor que transporta la corriente que se quiere medir.

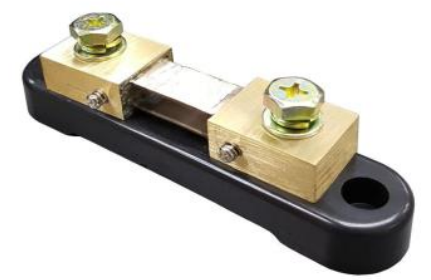

(a) Resistencia Shunt

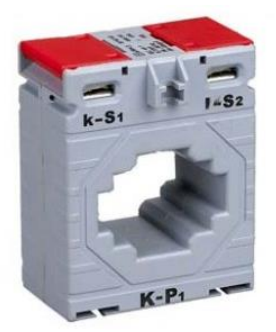

(c) Transformadores de Corriente

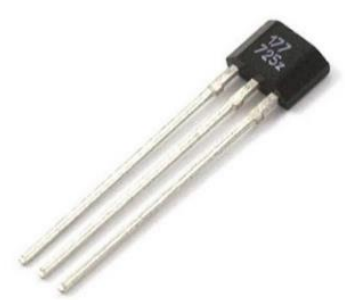

(b) Sensores de efecto Hall

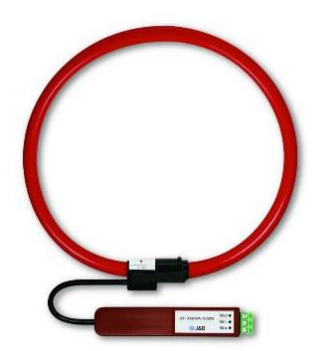

(d) Bobina Rogowski

Figura 2. Métodos medida de corriente. Fuentes: (a) DirectIndustry; (b) Techmake Electronics; (c) RadioSurtidora; (d) TransformerSeller 
$\mathrm{Al}$ analizar el contenido armónico de la corriente mediante el método MCSA, y trabajando en un caso ideal, es decir, considerando el hipotético caso de que el motor trabaje en condiciones ideales respecto a la alimentación y además el estator como el rotor no estén ranurados, y se considere que la distribución de los devanados en el espacio es una senoidal pura. Además el motor se encontraría directamente alimentado de red y operando en estado sano. En este caso ideal, la corriente consumida por el motor sólo tendría una frecuencia o componente, que sería la frecuencia impuesta por la red. Sin embargo, cualquier tipo de asimetría que ocurra en el mismo debida a un fallo, por ejemplo, provocará que aparezcan otras componentes de frecuencia en la corriente. Por lo tanto, este método es válido para aquellos fallos que se traducen en la aparición de componentes espectrales a unas frecuencias características del fallo, conocidas a priori. En la Tabla 1 se presentan los distintos tipos de fallo para los que esta técnica ha sido exitosamente aplicada, junto con la frecuencia o frecuencias características de fallo.

\begin{tabular}{|l|c|}
\hline \multicolumn{1}{|c|}{ Tipo de Falta } & Frecuencia característica de fallo \\
\hline \hline $\begin{array}{l}\text { Rotura de barras, agrietamiento de barras, } \\
\text { soldaduras débiles en el anillo de cortocircuito, } \\
\text { concentraciones de alta porosidad, etc. [14 - 15] }\end{array}$ & $f=f_{1}(1 \pm 2 n s)$ \\
$\mathrm{n}=1,2,3, \ldots$ \\
\hline Excentricidad estática [16] & $f=f_{1}\left[R \frac{1-s}{p} \pm 1\right]$ \\
\hline Excentricidad dinámica [17] & $f=f_{1}\left[\left(R \pm n_{d}\right) \frac{1-s}{p} \pm 1\right]$ \\
& $f=f_{1}\left[1 \pm n \frac{1-s}{p}\right]$ \\
\hline Fallos en rodamientos [18] & $f=\left|f_{1} \pm n f_{v}\right|$ \\
\hline
\end{tabular}

Tabla 1.- Tipo de fallo con su frecuencia característica.

En donde:

f: es la frecuencia característica de fallo.

$\mathrm{f}_{1}$ : es la frecuencia fundamental.

$\mathrm{n}$ : número natural que indica el orden del armónico; $n=1,2,3 \ldots$

$\mathrm{R}$ : número natural.

p: número de polos del motor.

s: deslizamiento.

$f_{v}$ : frecuencia característica de cada tipo de fallo provocado por rodamiento [18]. 
Estos fallos, por tanto, introducen nuevos armónicos en la corriente del motor. Así, al hacer un análisis de la misma, aparecerán una serie de armónicos que no aparecerían en el espectro de un motor sano.

Para detectar el fallo, se deberían emplear técnicas de procesamiento de señal que permitan conocer el contenido armónico de la corriente consumida por el IM. El procesamiento de esta señal se puede realizar en el dominio del tiempo, de la frecuencia o del tiempo-frecuencia.

Las técnicas basadas en el tiempo se basan en el análisis mediante medidas estadísticas. Las basadas en frecuencia se basan en la transformada de Fourier y se analizan en el apartado 2.3. Las técnicas en el dominio tiempo-frecuencia se usan para señales no estacionarias, y se estudiarán en el punto 2.4. de la memoria.

Esta tesis se centra en el estudio de las técnicas basadas en frecuencia y en tiempofrecuencia. Las técnicas basadas en el dominio del tiempo han sido las primeras en ser usadas, permitiendo obtener las características básicas de la señal, sin embargo, en la actualidad, aunque siguen en uso, se tiende a generalizar el uso de técnicas de mayor alcance.

\subsection{Régimen Permanente}

En esta sección se exponen los métodos de detección que aparecen en la literatura a lo largo de los años para el análisis del motor de inducción cuando éste se encuentra operando en estado estacionario o permanente. Atendiendo a la técnica MCSA que se ha introducido en el apartado 2.2. de este capítulo, el método que por excelencia se utiliza al trabajar en estado estacionario para calcular el espectro de la corriente estatórica estacionario del motor de inducción es la transformada rápida de Fourier $(F F T)$.

Mediante el espectro obtenido con la $F F T$ se tiene el contenido armónico que presenta la señal a estudio, lo cual da una respuesta a si el motor está sano o presenta algún tipo de fallo. Con la aplicación de la FFT en estado estacionario se obtiene que la 
resolución no representa un problema a la hora de detectar fallos, por lo que no es necesaria una técnica tan potente como cuando se está trabajando en estado transitorio, como se verá al pasar al punto 2.4 .

Se presenta, a continuación, un ejemplo práctico del espectro que se obtiene mediante el análisis de la señal al aplicar FFT. En la Figura 3 pueden observarse las corrientes estatóricas de dos motores de inducción, el motor 1 (IM1) está sano, mientras que el motor 2 (IM2) presenta barras rotas y excentricidad mixta. La componente fundamental se encuentra a $50 \mathrm{~Hz}$. Los espectros se han obtenido con una frecuencia de muestreo de $50.000 \mathrm{~Hz}$, con una duración total de la señal de $30 \mathrm{~s}$, por tanto, el número total de puntos, $N$, es de 1.500.000. (Para mayor información acerca de cómo se han generado los ensayos, se remite al capítulo 5 de esta memoria).

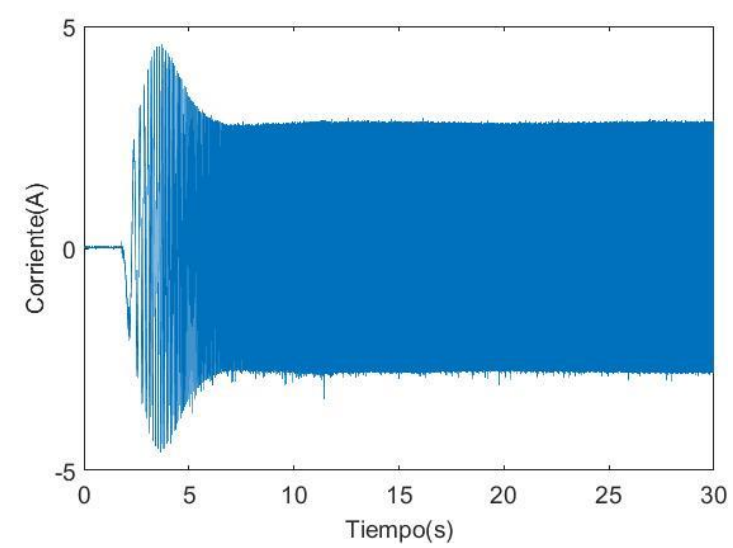

(a) Corriente IMI.

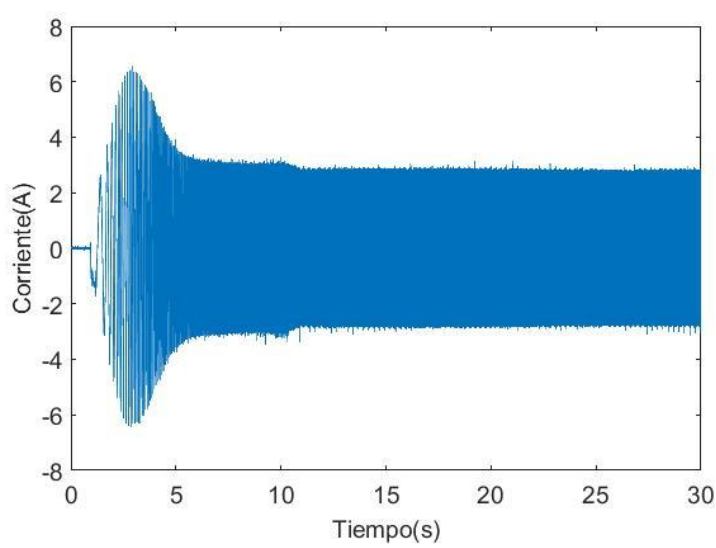

(b) Corriente $I M 2$.

Figura 3. Corrientes estatóricas IM1 y IM2.

$\mathrm{Al}$ analizar estas corrientes mediante la transformada rápida de Fourier, se tienen los espectrogramas resultantes de la Figura 4, en donde se puede observar, en ambos casos, la componente fundamental situada a $50 \mathrm{~Hz}$.

Para el análisis mediante $F F T$ se pueden utilizar una serie de funciones ventana que mejoran la claridad de la señal analizada, ya que reduce los efectos del análisis FFT sobre una señal con un número no entero de ciclos; estas ventanas reducen la discontinuidad de la forma de onda, dando así como resultado una forma de onda continua sin presentar transiciones bruscas. Existen numerosas ventanas que se pueden emplear (Hamming, Hann, Blackman-Harris...) dependiendo de la señal a analizar, sin embargo, 
no existe un único enfoque para la elección de esta ventana [19]; En este caso se ha optado por una ventana tipo Hann, ya que ésta consigue eliminar toda discontinuidad.

Se han analizado 10 segundos de ese periodo de funcionamiento estacionario, calculándose la densidad espectral de potencia (Power Spectral Density, PSD), aplicando una ventana de Hann, como se acaba de comentar, con lo que se obtiene la energía asociada a cada frecuencia.

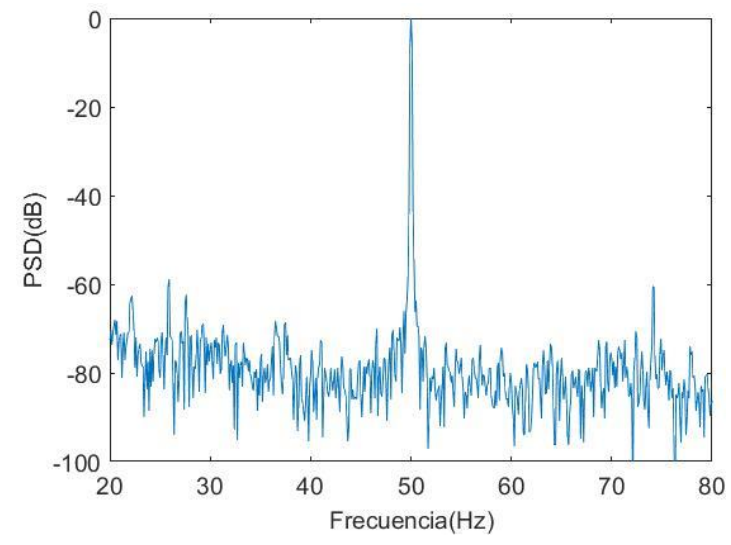

(a) Espectrograma resultante de aplicar FFT a Corriente $I M 1$.

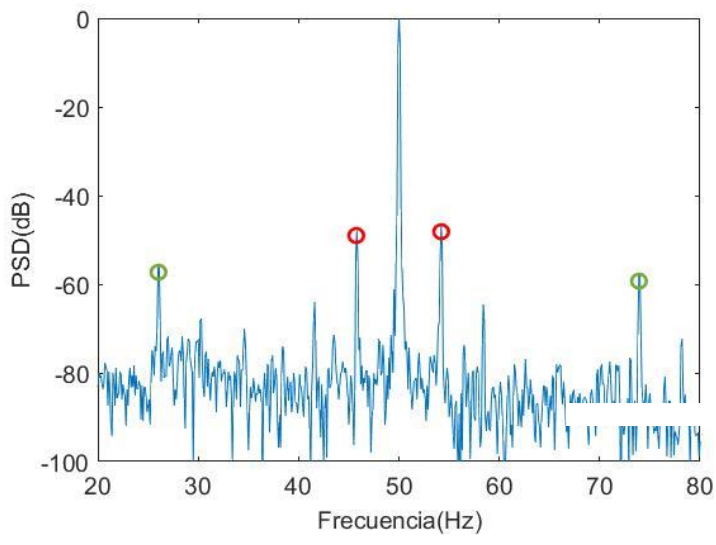

(b) Espectrograma resultante de aplicar FFT a Corriente $I M 2$.

Figura 4. Espectrogramas resultantes de aplicar FFT a corrientes de IMI y IM2.

Con la Figura 4 se puede corroborar que efectivamente con la Transformada Rápida de Fourier se pueden apreciar los fallos que el motor de inducción presentaba, cuando se está analizando el estado estacionario. Así en el caso de la Figura 4 (a), se puede corroborar que se trata de un motor sano, ya que no presenta contenido armónico; Mientras que en la Figura 4 (b), se puede distinguir actividad armónica, que indica la presencia de fallos debidos a barra rota (marcado con círculos rojos) y de excentricidad mixta (marcado con círculos verdes).

Por otra parte, algunos autores han elaborado Tablas con valores de amplitud de los armónicos que indican si existe fallo y en qué nivel de severidad se encuentra el mismo. Un ejemplo de estas Tablas aparece en la Tabla 2 [20]. El problema que estas Tablas presentan es que no tienen en cuenta otras condiciones de entorno que influyen en la amplitud de los armónicos de fallo. 


\begin{tabular}{|c|c|c|c|}
\hline Límite Superior & ABB & Nicholas & EASA \\
\hline$(\mathrm{dB})$ & $>70 \%$ carga & $>75 \%$ carga & $>50 \%$ carga \\
\hline 60 & Motor sano & Excelente & Bueno \\
\hline 54 & Motor sano & Bueno & $\begin{array}{l}\text { Condición } \\
\text { Marginal }\end{array}$ \\
\hline 49 & Algún punto de alta resistencia & Bueno & $\begin{array}{l}\text { Condición } \\
\text { Marginal }\end{array}$ \\
\hline 48 & Algún punto de alta resistencia & Moderado & $\begin{array}{l}\text { Condición } \\
\text { Marginal }\end{array}$ \\
\hline 46 & Varios puntos de alta resistencia & Moderado & $\begin{array}{l}\text { Condición } \\
\text { Marginal }\end{array}$ \\
\hline 45 & Varios puntos de alta resistencia & Moderado & $\begin{array}{l}\text { Condición } \\
\text { Marginal }\end{array}$ \\
\hline 44 & $\begin{array}{l}\text { Muchos puntos de alta } \\
\text { resistencia o barra rota }\end{array}$ & Moderado & $\begin{array}{l}\text { Al menos una barra } \\
\text { rota }\end{array}$ \\
\hline 42 & $\begin{array}{l}\text { Muchos puntos de alta } \\
\text { resistencia o barra rota }\end{array}$ & $\begin{array}{lrr}\text { Barras } & \text { rotas } & \text { o } \\
\text { superficies } & \text { de } & \text { alta } \\
\text { resistencia } & & \end{array}$ & $\begin{array}{l}\text { Al menos una barra } \\
\text { rota }\end{array}$ \\
\hline 40 & $\begin{array}{l}\text { Muchos puntos de alta } \\
\text { resistencia o barra rota }\end{array}$ & $\begin{array}{lrr}\text { Barras } & \text { rotas } & \text { o } \\
\text { superficies } & \text { de } & \text { alta } \\
\text { resistencia } & & \end{array}$ & $\begin{array}{l}\text { Al menos una barra } \\
\text { rota }\end{array}$ \\
\hline 39 & $\mathrm{Al}$ menos una barra rota & $\begin{array}{lrr}\text { Barras } & \text { rotas } & \text { o } \\
\text { superficies } & \text { de } & \text { alta } \\
\text { resistencia } & & \end{array}$ & $\begin{array}{l}\text { Más de una barra } \\
\text { rota }\end{array}$ \\
\hline 36 & $\mathrm{Al}$ menos una barra rota & $\begin{array}{l}\text { Múltiples superficies } \\
\text { de alta resistencia }\end{array}$ & $\begin{array}{l}\text { Más de una barra } \\
\text { rota }\end{array}$ \\
\hline 35 & Varias barras rotas & $\begin{array}{l}\text { Múltiples superficies } \\
\text { de alta resistencia }\end{array}$ & $\begin{array}{l}\text { Más de una barra } \\
\text { rota }\end{array}$ \\
\hline 30 & & Múltiples barras rotas & Daño severo \\
\hline
\end{tabular}

Tabla 2. Nivel de severidad de fallo provocado por barra rota.

Así, atendiendo a estas Tablas, si se observan los valores de amplitud obtenidos en el espectrograma resultante al analizar la corriente del IM2, se obtiene que efectivamente, corroboran que existe un fallo debido a barra rota. 


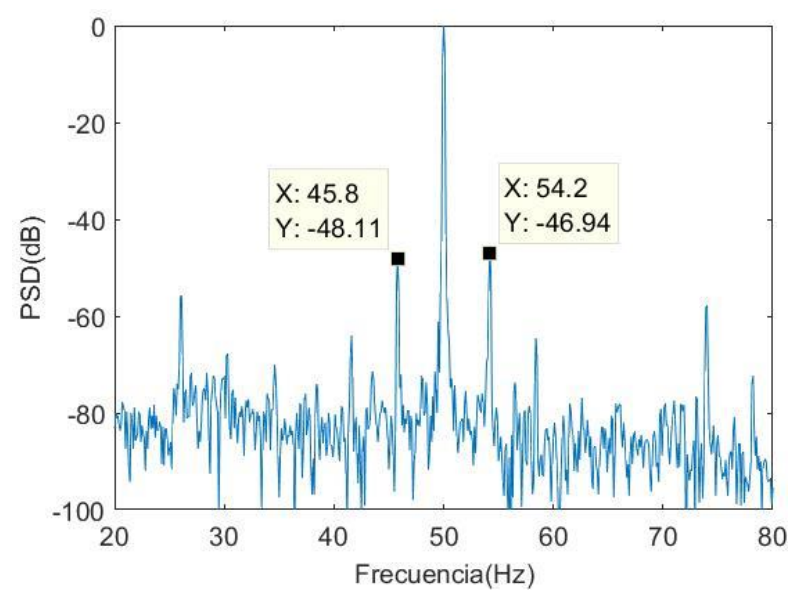

Figura 5. Espectrograma FFT Corriente IM2.

Si bien la Transformada Rápida de Fourier se utiliza para esta tarea, tiene limitaciones intrínsecas [21] como sensibilidad al cociente signal-to-noise bajo (es decir la relación que existe entre la potencia de la señal y el ruido presenta un valor bajo); superposición de componentes espectrales cercanas, dificultad de analizar señales no estacionarias (tal como se verá en el apartado 2.4 de este capítulo) y la dispersión espectral.

Uno de los inconvenientes que presenta esta técnica es la dispersión espectral, que puede dar lugar a que se enmascaren los armónicos. Para resolverlo se han venido desarrollando diversas técnicas, como el Espectro Promediado (Averaged Spectrum) capaz de capturar la corriente en sucesivos intervalos de tiempo, calcular el espectro de cada fragmento de corriente capturada y obtener el espectro final como su media.

Otro método para mejorar los resultados de los métodos basados en FFT, reduciendo así la dispersión espectral, es la utilización del remuestreo fraccional [21].

Por otra parte, para mejorar la resolución de frecuencia, es decir, obtener la máxima resolución en la franja de interés, se han propuesto una serie de técnicas basadas en la FFT, como son la Transformada Zoom-FFT [22], Transformada Chirp Z [22] y el método de máxima Covarianza [22].

También sería posible el uso de modelos autorregresivos, como es MUSIC (Multiple Signal Classification Method) [22], teniendo en cuanta que MUSIC es conocido 
más como una técnica de subespacios; que disminuye el ruido, detecta componentes de baja amplitud en comparación con la amplitud de la relación señal-ruido, aunque tiene el inconveniente de tener un elevado tiempo computacional, que se puede reducir empleando técnicas como Zoom-MUSIC [22], o bien el uso de poliespectros [22].

\subsection{Régimen Transitorio.}

\subsubsection{Introducción}

Para la detección de los fallos en los $I M$ funcionando en régimen transitorio son necesarias otras herramientas matemáticas, distintas de la transformada de Fourier. Algunas de estas técnicas todavía están en desarrollo o proceden de otros campos de la ciencia y la ingeniería. Son utilizadas principalmente para analizar la corriente del estator y en algunos casos vibraciones. Estas transformadas matemáticas pertenecen al campo de las herramientas de descomposición tiempo-frecuencia (TFD).

Transformadas como la Wavelet y la distribución de Wigner-Ville aparecen en la literatura para detectar fallos en el arranque directo del motor de inducción [23,24], pero, como se mostrará más adelante, su aplicación en $I M$ alimentados por inversor no es sencilla. La principal diferencia entre un arranque directo y otro alimentado por inversor es el bajo deslizamiento del motor durante todo el transitorio y la evolución temporal de la frecuencia fundamental. Como resultado, las trayectorias de algunos armónicos de fallo evolucionan muy cerca de la trayectoria de la componente principal. Como se explica a continuación, es ésta la característica que hace que la detección de fallos en motores alimentados mediante inversor sea tan diferente en comparación con los alimentados directamente de red y también más complicada.

\subsubsection{Evolución temporal de los armónicos de fallo}

En el caso de motores alimentados directamente de la red, la frecuencia fundamental es constante y se establece desde el mismo instante de la conexión del motor a la línea. En un arranque directo, algunos armónicos de fallo evolucionan de una manera muy conveniente ya que sus trayectorias se alejan del primer armónico, que es el componente espectral de mayor energía. Por ejemplo, el armónico conocido como banda 
lateral izquierda (Left Sideband Harmonic, LSH), relacionado con el fallo de barras en el rotor de jaula, presenta una trayectoria en el plano tiempo-frecuencia con un patrón característico en forma de V [25].

La Figura 6 muestra las trayectorias teóricas, tanto del fallo de excentricidad mixta (Eccentricity Harmonic Negative, ERH(-) y Eccentricity Harmonic Positive, ERH(+)) como los armónicos relativos a asimetrías del rotor de jaula (Left Sideband Harmonic, LSH y Upper Sideband Harmonic, USH) durante un arranque directo. Al trabajar con un IM alimentado directamente de red, la frecuencia fundamental permanece fija y establecida desde el primer instante que el motor se pone en marcha, por tanto la velocidad de sincronismo también es constante; por esta razón la velocidad de giro del rotor aumenta linealmente desde cero hasta casi llegar a la velocidad de sincronismo, a su vez el deslizamiento disminuye, de igual manera, desde un valor de 1 hasta llegar a un valor muy pequeño, próximo a cero. En ambos fallos, estos armónicos evolucionan lejos del componente principal. Por lo tanto, al menos durante el arranque, se pueden observar fácilmente.

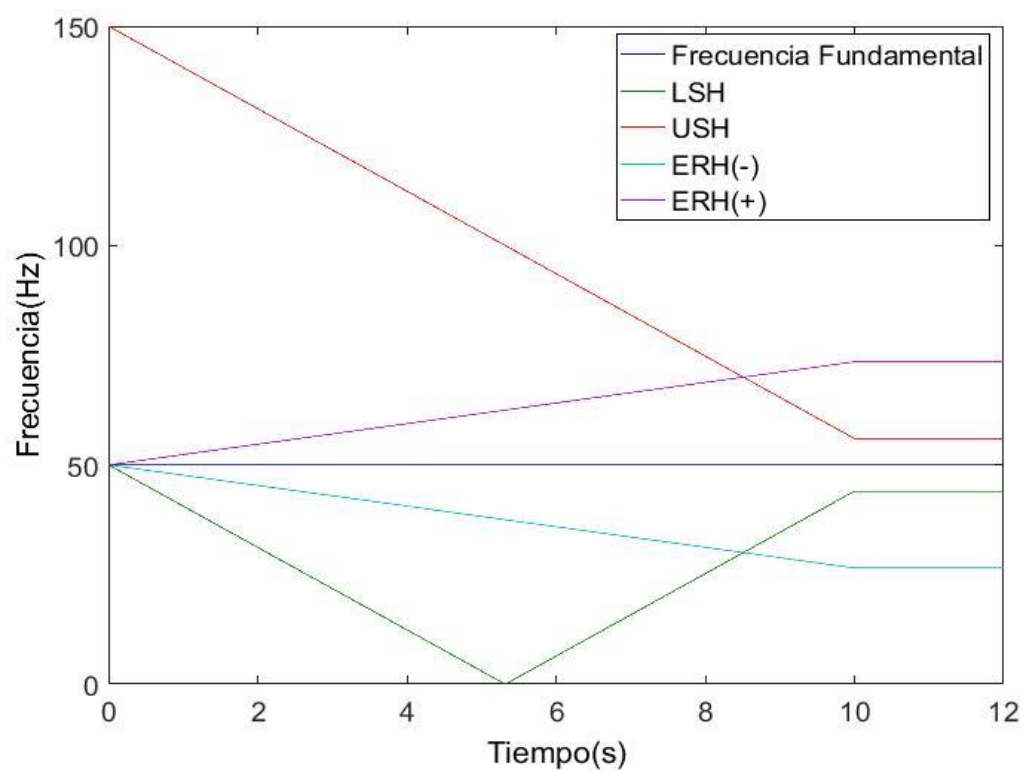

Figura 6. Trayectorias teóricas de los componentes armónicos en un arranque directo de 10 s de duración.

Los armónicos LSH y USH son los armónicos relativos a las asimetrías que presenta el rotor de jaula. ERH(-) y ERH(+) son los armónicos relativos a excentricidad mixta. 
La diferencia principal entre el arranque directo y mediante inversor, con respecto a la detección de fallos, es que la evolución temporal que siguen los armónicos durante este transitorio es totalmente distinta $[3,11]$.

Cuando el motor de inducción está alimentado por inversor, las trayectorias son muy diferentes, como puede verse en la Figura 7. El inversor permite evitar la sobrecorriente típica del arranque directo y controla la duración de este transitorio. Como resultado, la puesta en marcha del motor de inducción es, en principio, suave y libre de pares elevados. Independientemente del tipo de control del motor de inducción, en estos casos la componente principal de la tensión de salida del inversor se incrementa desde 0 $\mathrm{Hz}$ a la frecuencia de funcionamiento final asignada.

Por otra parte, la velocidad del motor va aumentando hasta hacerse cercana a la velocidad de sincronismo, por lo que el deslizamiento del motor es muy bajo. En consecuencia, los armónicos de fallo evolucionan durante la puesta en marcha de manera paralela a la frecuencia principal, al menos desde el punto de vista teórico. Los armónicos del rotor presentan baja energía, estando especialmente cerca del componente principal durante la duración del transitorio. Esto hace especialmente difícil su detección. La trayectoria de los componentes de fallos relativos a excentricidad mixta es más ventajosa y similar a la puesta en marcha con alimentación directa de red, sobre todo al final del período transitorio de arranque, si bien, durante el inicio, también están muy próximos al armónico fundamental. Por lo tanto, la detección de fallos en este transitorio dependerá de la capacidad que tenga la transformada tiempo-frecuencia empleada para "rastrear" armónicos de baja energía con respecto a la componente fundamental y otros armónicos como los de bobinado y los de ranura. Es decir, el éxito de la detección descansará en la resolución en tiempo y en frecuencia que pueda proporcionar la técnica utilizada.

Además de las técnicas que se han visto en el apartado 2.3, que se utilizan para analizar el estado estacionario, en el transitorio se han venido usando herramientas numéricas, tanto en el dominio de la frecuencia como en el dominio del tiempofrecuencia, para el régimen transitorio. A continuación, se analizan los fundamentos matemáticos de algunas de ellas, concretamente, la transformada de Fourier, la transformada Corta de Fourier, la transformada Wavelet, la distribución de Wigner- 
Ville, la transformada de Hilbert-Huang, la transformada de Pendiente Adaptable, MUSIC y la técnica Bi-Frequency Sliding.

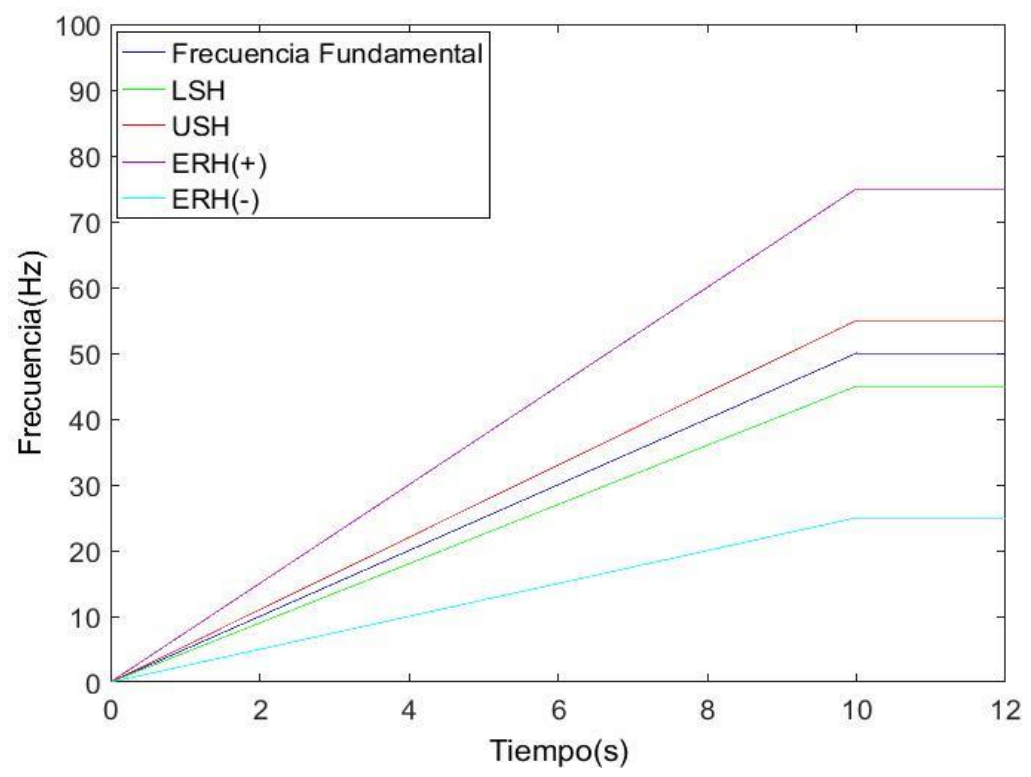

Figura 7. Trayectorias teóricas de los componentes armónicos durante 10s con arranque con inversor con una evolución lineal de la frecuencia fundamental de 0 a $50 \mathrm{~Hz}$.

\subsubsection{Transitorios arbitrarios}

Aunque no es objetivo de esta tesis el desarrollo y prueba de transitorios arbitrarios, la técnica desarrollada es susceptible de aplicarse a los mismos, de la misma forma que se encuentra aplicada en transitorios de arranque especiales y en estacionarios con carga y frecuencia de consigna oscilantes (subapartado 6.2.3). Por tanto, cabe mencionar este tipo de transitorio y algunos de los trabajos que en la literatura técnica aparecen registrados. Así esta condición de transitorios arbitrarios se encuentra añadida como una línea de investigación a futuro, apareciendo reflejado en el apartado 7.3. de este manuscrito.

Se entiende que una máquina operan en condición de transitorio arbitrario cuando se encuentra trabajando en régimen no estacionario. Por lo que, concierne a todas aquellas aplicaciones en las que el funcionamiento supone un cambio de las magnitudes de la máquina, lo que viene impuesto por el proceso que impulsa a la misma. Ejemplo de este tipo de funcionamiento puede verse en los generadores eólicos, que se encuentran sometidos a una velocidad del viento cambiante. 
Así, la dificultad de este tipo de funcionamiento aparece en que los componentes asociados al giro del motor poseen una frecuencia cambiante, por lo que las herramientas para su análisis deben de ser más complejas. Sin embargo, es posible su análisis mediante el seguimiento de la evolución de la energía de los componentes armónicos de fallo, para lo que es necesario el desarrollo de técnicas más potentes de las estudiadas para el régimen estacionario.

Este tema se encuentra ampliamente estudiado en [26], en donde el autor desarrolla una metodología para diagnosticar motores de inducción, tanto de rotor de jaula como de rotor bobinado, que se encuentran operando en régimen no estacionario. Esta metodología se basa en el análisis de los componentes de fallo que aparecen la corriente. El análisis se lleva a cabo en el plano deslizamiento-frecuencia. La técnica desarrollada se ha aplicado para el diagnóstico de asimetrías estatóricas y rotóricas y para excentricidad mixta, con alimentación tanto de red como mediante convertidor.

Uno de los trabajos en el que se abordan este tipo de transitorios [27] los autores analizan los métodos de diagnóstico de fallo que son adecuados para los casos en los que el motor de inducción se encuentra operando bajo condiciones de cargas y velocidades fluctuantes durante su ciclo de trabajo, es decir la carga y la velocidad se encuentran fluctuando continuamente y de manera aleatoria, lo que dificulta el diagnóstico de manera considerable; Se analiza las técnicas que existen y buscan la mejor implementación posible para hacer posible este diagnóstico.

Avanzando en este diagnóstico se encuentra el siguiente trabajo [28] en el que se trata de extraer los componentes de fallo presentes en la señal mediante el desarrollo de un filtro basado en el Teorema de muestreo y las propiedades de la Transformada discreta de Fourier, con lo que se consigue extraer tanto los fallos en estado estacionario o como durante el régimen transitorio.

El trabajo [29] se centra en generadores que se usan para molinos de viento, ya que en estos casos, como se ha dicho al comienzo de esta sección, las condiciones de funcionamiento no son estacionarias, ya que la velocidad del viento es variable, por lo que es necesario un diagnóstico de acuerdo a estas condiciones. Los autores proponen el 
uso de una transformada continua de frecuencia, obteniendo una correcta resolución de tiempo y frecuencia en todo el plano, validando de manera experimental la metodología propuesta.

Por último cabe mencionar otro trabajo [30], en el que se presenta un método para detectar asimetrías en el rotor de motores de inducción de rotor bobinado que operan en transitorio. Este método se va a basar en el análisis de la corriente del rotor y una posterior transformada. Con lo que, mediante pocos parámetros y mediante un gráfico simple, permite evaluar el estado de la máquina. Se obtiene un buen funcionamiento en generadores eólicos, permitiendo la transmisión de datos al centro de control remoto, facilitando el análisis y diagnóstico.

\subsubsection{Transformada de Fourier}

La transformada de Fourier, es la base del análisis del estator del motor de inducción en regímenes en estado estacionario, como se ha comentado anteriormente. La FT permite obtener el contenido armónico de una señal periódica y la energía asociada a cada frecuencia. La detección de fallos se realiza mediante la identificación de ciertas frecuencias en el espectro de la corriente del estator. Si la señal de la corriente es no periódica o es el resultado de un régimen transitorio del motor de inducción, la transformada de Fourier no resulta adecuada para esta tarea. En estos casos, es importante conocer el contenido de armónicos de la señal, pero también la componente de frecuencia en cada instante de tiempo. Desafortunadamente, esta información no es suministrada por la $F T$.

Para superar esta limitación de la transformada de Fourier con señales no estacionarias en motores alimentados mediante inversor, una expansión de Fourier-Bessel fue propuesta en [31]. Se trata de una serie de Fourier basada en las funciones de Bessel cuyos coeficientes son únicos, lo que las hace adecuadas para el análisis de los regímenes transitorios del motor de inducción. En este trabajo, esta técnica fue utilizada para detectar excentricidad y fallos de rodamientos. 
Otro enfoque para superar las limitaciones de la transformada de Fourier fue presentado en [32] donde la Transformada de Fourier fraccional, Fractional Fourier Transform (FrFT), se utiliza para detectar barras rotas. La FrFT de una señal puede interpretarse como una descomposición de la señal en términos de chirps. Esto puede usarse para detectar señales tipo chirp como las generadas por armónicos de fallo de barras rotas durante la puesta en marcha de un motor de inducción alimentado directamente de línea. En este trabajo, la corriente del estator es previamente filtrada con una Transformada Wavelet Discreta $(D W T)$ y luego se procesa con la FrFT. La técnica está optimizada para generar un espectro donde se muestran los diferentes armónicos de fallo a frecuencia variable como las líneas espectrales individuales que facilitan el diagnóstico de fallos. No hay referencias en la literatura donde se haya utilizado esta técnica para la detección de fallos en motores de inducción alimentados mediante inversor.

\subsubsection{Short Time Fourier Transform}

Otra técnica para hacer frente a las señales no estacionarias es la Transformada corta de Fourier, Short Time Fourier Transform (STFT) [33]. De forma muy simple, esta transformada funciona primero dividiendo la señal en ventanas muy cortas, que pueden ser consecutivas o superpuestas, y luego se calcula la Transformada de Fourier en cada ventana. Esta transformada también es conocida como FT ventaneada porque se ve la señal a través de una ventana sobre la cual la señal es aproximadamente estacionaria, es posible usar diferentes tipos de ventanas como pueden ser las ventanas Bartlett, Hamming, Hanning o Blackman [34], entre otras. El resultado es una representación tridimensional de la señal sobre el plano tiempo-frecuencia. El resultado es una representación tridimensional de la señal sobre el plano tiempo-frecuencia. La longitud en tiempo de la ventana fija la resolución de la transformada en tiempo y en frecuencia. Por lo tanto, la resolución de tiempo y la de frecuencia no son independientes. Una longitud larga de ventana significa una buena resolución en frecuencia, pero se disminuye la resolución en tiempo. Por otro lado, una longitud corta de ventana mejora la resolución en tiempo a costa del empeoramiento de la resolución en frecuencia. Esta es la principal limitación de esta técnica. 
La STFT es la transformada estándar en el dominio tiempo-frecuencia, y el resto de transformadas se comparan generalmente con ella. Debido a sus limitaciones en cuanto a resolución, son pocos los trabajos que reportan su uso. Por ejemplo, en [35], los autores utilizaron la STFT en combinación con wavelets y un factor de mérito basado en la densidad espectral de potencia $(P S D)$ para detectar cortocircuitos en el estator y barras rotas en el rotor.

\subsubsection{Wavelets}

La transformada Wavelet, Wavelet Transform (WT), se desarrolló para resolver los problemas de resolución en tiempo-frecuencia que aparecen en los métodos que se basan en Fourier, y que se derivan del uso de una función cuya ventana presenta una longitud fija. Esta transformada se basa en una función, que se fija como función Wavelet madre, en base a la cual se va a descomponer la señal. Las funciones Wavelet se van a distribuir en familias, dicha familia de funciones Wavelet se va a escoger en función de la señal a estudio; así los tipos de Wavelet madre más usadas son Daubechies, coiflet, simlet, biorthogonal o discrete Meyer [36].

La Transformada Wavelet Continua, Continous Wavelet Transform (CWT), es similar a la $S T F T$, donde la señal a analizar es convolucionada con una función wavelet cuya forma depende del punto del plano tiempo-frecuencia analizado.

La $C W T$ permite realizar el análisis a cualquier frecuencia. La principal diferencia entre $C W T$ y $S T F T$ es que la primera se considera una transformada de resolución múltiple porque la longitud de tiempo de la función wavelet se establece según su frecuencia. Como resultado, esta transformada ofrece una resolución pobre en frecuencia y buena en tiempo a altas frecuencias. Por otro lado, a bajas frecuencias, la resolución de la frecuencia es mucho mejor y empeora la resolución temporal.

La versión discreta de la CWT es conocida como la Transformada de Wavelet Discreta, Discret Wavelet Transform (DWT). La DWT proporciona resultados que están asociados con bandas de frecuencias siguiendo una escala diádica que es fijada por la 
frecuencia de muestreo y el número de niveles de descomposición previamente seleccionado.

La identificación del fallo puede llevarse a cabo mediante un análisis de los coeficientes resultantes de la descomposición o el estudio de las señales wavelet de alto nivel, que son las señales de aproximación y detalle del fallo. Como se afirma en [37], el uso de las señales wavelet en lugar de los coeficientes también permite una interpretación física del fenómeno de fallo.

El uso de transformadas basadas en Wavelet para detectar fallos en IM está ampliamente divulgado en la literatura. La $C W T$ fue utilizada en [38] para detectar excentricidad mixta en estados transitorios de un motor de inducción alimentado mediante inversor. En este caso, $C W T$ se aplica utilizando una transformada Wavelet analítica, Analytic Wavelet Transform (AWT), para obtener un gráfico en tiempofrecuencia detallado de la corriente del estator. La buena resolución obtenida permite seguir la evolución de los armónicos asociados a la excentricidad mixta durante periodos transitorios tales como un arranque, donde la frecuencia principal aumenta linealmente de 0 a $50 \mathrm{~Hz}$, u otros transitorios donde se cambió la frecuencia asignada de salida del inversor. En [39], se mejoró la resolución de la $C W T$, principalmente alrededor de la frecuencia fundamental, con Frequency B-Spline (FBS) usada como wavelet madre. La metodología propuesta se probó con la corriente de estator de un arranque de línea sin carga donde logra trazar de manera precisa los armónicos producidos por una rotura de barra del rotor.

Otra variante de la $C W T$ es la Transformada Wavelet Compleja Continua, Complex Continuous Wavelet Transform (CCWT), que utiliza valores complejos de las funciones Wavelet, en vez de los reales. La CCWT fue aplicada en [40] a la corriente del estator para rastrear el armónico LSH. Una descomposición de alta resolución de la corriente del estator logra representar el módulo de la $C C W T$, y la característica en V del LSH fue claramente identificada.

El uso de la $D W T$ también está ampliamente documentado en la literatura. Por ejemplo, en [41] se detectaron algunos fallos electromecánicos de un motor de inducción en regímenes transitorios. En este caso, las funciones de alto nivel proporcionadas por 
DWT después de procesar la corriente del estator de un motor de inducción alimentado directamente de red fueron utilizadas para visualizar los patrones de fallo característicos relativos a barras rotas y excentricidad mixta. Incluso se cuantificó la severidad de fallo mediante el cálculo de la energía de las señales wavelet resultantes del análisis corriente del estator.

Otras publicaciones recientes usan la potencia reactiva instantánea como señal monitorizada para establecer la condición del motor de inducción en transitorios provocados por el par, la velocidad o las oscilaciones de tensión [42,43]. Los fallos considerados fueron barras rotas y excentricidad mixta. Los fallos se detectan a través de funciones de alto nivel y los coeficientes resultantes de aplicar DWT para la potencia reactiva instantánea, cuyo cálculo requiere la medición de las tensiones y corrientes trifásicas. Los autores también definen un factor de severidad de fallo evaluando la energía en algunas señales wavelet. Otra metodología basada en la Transformada Wavelet Discreta Múltiple fue presentada en [44], donde se utiliza para detectar fallos de barras rotas en las unidades alimentadas directamente de línea con velocidad variable, operando a diferentes velocidades.

También es muy interesante el trabajo presentado en [45]. Una variante de $D W T$ conocida como Descomposición de Paquetes Wavelet, Wavelet Packets Decomposition $(W P D)$, fue utilizada para la detección temprana de fallos de rodamientos en motores de inducción alimentados mediante inversor.

\subsubsection{Distribución de Wigner-Ville}

Como se ha mencionado antes, la resolución en tiempo y frecuencia no puede ser mejorada al mismo tiempo en la $S T F T$ y $C W T$. La STFT tiene una resolución fija mientras que en la $C W T$ la resolución varía con la frecuencia analizada. $W V D$ pertenece a la familia de representaciones tiempo-frecuencia cuadráticas y es adecuada para el análisis de señales no estacionarias; permite obtener una distribución de la energía en el plano tiempo-frecuencia sin pérdida de resolución. Es decir, muestra las evoluciones de los componentes de fallo como perfectas líneas finas. 
El inconveniente más importante que presenta esta técnica es la presencia de términos cruzados, que crean interferencias en la distribución de la energía tiempofrecuencia en el análisis de las señales de varios componentes; es decir, aparecen términos extra que no deberían de estar ahí, ya que por cada par de componentes se introduce un término ficticio nuevo. En la literatura también se puede encontrar otra posible solución, basada en el uso de kernels, que permiten reducir al mínimo la interferencia de términos cruzados, aunque conlleva una pérdida de resolución. También se ha propuesto el uso de filtros anteriores al cálculo de la distribución tiempo-frecuencia [46-48].

$W D$ fue utilizado en [49-51] para detectar asimetrías de rotor y excentricidad en motores alimentados directamente de línea durante el arranque. La señal monitorizada es la corriente del estator. En [49] se utilizan filtros para eliminar algunos componentes de frecuencia constante y evitar los inconvenientes de los términos cruzados. La buena resolución permite el seguimiento de los armónicos relativos al fallo en el plano tiempofrecuencia. Los autores también propusieron un método de cuantificación para evaluar la severidad de fallo calculando la energía de los puntos de interés. En [50], se utilizan filtros de partículas como un procedimiento de extracción previo al cálculo de la WVD. Estos autores proponen utilizar este procedimiento cuando se trabaja con señales multicomponentes en $I M$ que se encuentran sometidos a transitorios complejos.

\subsubsection{Transformada de Hilbert-Huang}

La transformada de Hilbert-Huang, o Hilbert Huang Transform (HHT), es una combinación de análisis espectral de Hilbert o transformada de Hilbert, Hilbert Transform (HT), y la descomposición de modo empírico, Empirical Mode Decomposition (EMD). La HHT puede aplicarse a señales no estacionarias y no lineales. La EMD realiza una descomposición adaptativa basada en características de la señal en un número finito de funciones de modo intrínseco, Intrinsic Mode Functions (IMF). Estas IMF son individuales y se puede decir que casi señales monocomponentes, por lo que resultan más adecuadas para el uso de la $H T$, que aplicada a las $I M F$ conduce a la identificación de frecuencias instantáneas y amplitudes con un significado físico. Si la $H T$ se aplica a la señal original multicomponente y ruidosa, el resultado genera falsas amplitudes sin significado físico. 
El procedimiento explicado fue utilizado con éxito en [51] para detectar barras rotas y se comparaba con el $D W T$. La señal monitorizada es la corriente del estator de un arranque de un motor de inducción alimentado directamente de línea. La HHT presenta algunas ventajas en comparación con $D W T$. Por ejemplo, no es necesario elegir una función wavelet madre adecuada. Sin embargo, la detección de fallos con el espectro de Hilbert-Huang parece no ser superior al conseguido con DWT. Otros dos trabajos interesantes [52,53] aplican la $H H T$ y EMD para el diagnóstico de motores de inducción alimentados mediante inversor. La $H H T$ fue aplicada a la corriente durante el arranque del estator. La cuantificación de fallos se realiza computando la energía instantánea de la transformada Hilbert de IMFs específicas. La viabilidad del procedimiento se evalúa considerando diferentes condiciones de operación del motor de inducción y diferentes tensiones de alimentación.

El algoritmo EMD presenta algunos inconvenientes como efectos de borde, modo mixto o criterio de parada inciertos. Se han desarrollado algunos métodos asistidos para superar estos problemas, como el de Descomposición de Modo Empírico Completo, Complete Ensemble Empirical Mode Decomposition (CEEMD) o el de Descomposición Empírica en modos por conjuntos, Ensemble Empirical Mode Decomposition (EEMD). Esta última implementación EEMD se aplicó en [54] a las señales de vibración para extraer patrones de fallo para diagnóstico de fallos en rodamientos.

\subsubsection{Transformada de Pendiente Adaptable}

La Transformada de Pendiente Adaptable, Adaptive Slope Transform (AST), recientemente desarrollada [55], pertenece a la familia de transformadas lineales, como la STFT y la $C W T$, donde la señal a analizar se correlaciona con un átomo de tiempofrecuencia (TFA). Un TFA es una función cuya energía se concentra alrededor de un punto en el plano tiempo-frecuencia. La distribución de energía del átomo tiempo-frecuencia se caracteriza por su caja de Heisenberg, Heisenberg Box (HB). Los átomos en STFT y CWT son diferentes a los utilizados en la AST. Estos átomos son inclinados (en el plano tiempofrecuencia) y definidos por dos pendientes. Matemáticamente se conocen como "chirplets". La transformada lineal con estos átomos es también conocida como transformada Chirplet, Chirplet Transform (CT) [3] (ver punto 2.4.10. de esta memoria). 
La pendiente y el espesor del átomo tiempo-frecuencia pueden ser adaptados a la pendiente de la trayectoria del armónico de fallo. Esto permite una observación precisa de los componentes de fallo en regímenes transitorios, incluso si están cerca de la componente principal, pero requiere un conocimiento previo de la evolución temporal de los armónicos de interés.

Esta técnica fue utilizada con éxito para detectar barras rotas y excentricidad mixta en motores de inducción alimentados por inversor [3,11]. La resolución en tiempo y frecuencia alcanzada permite observar la trayectoria del $L S H$ y $U S H$ durante todo el transitorio (incluso en las inmediaciones de la frecuencia fundamental) y calcular la energía durante la trayectoria para propósitos de diagnóstico.

\subsubsection{Transformada Chirplet}

Al igual que la $A S T, S T F T$ y $C W T$, la transformada Chirplet es una transformada lineal. Lo que diferencia a esta transformada de las anteriores es que ésta no presenta una compensación entre las resoluciones de tiempo y frecuencia cuando se captura una evolución siguiendo una línea recta, es decir, en el caso de la $C T$ se consigue que el espesor sea mucho menor ya que la dispersión de la energía del componente aparece a lo largo de la evolución del componente en sí mismo cuando sigue una línea recta; mientras que en las otras transformadas el espesor viene dado por la dispersión de la energía del componente en torno a su evolución real a lo largo de los ejes de tiempo y frecuencia, así si el átomo utilizado es largo en el tiempo, este espesor será grande a lo largo del eje del tiempo, y al contrario, si el átomo utilizado es largo en frecuencia este grosor será significativo a lo largo del eje de frecuencia. La transformada Chirplet logra esto mediante el uso de átomos t-f cuya energía se distribuye desde su centro a lo largo de una cierta dirección, por lo hay que elegir en qué dirección del plano t-f se dispersa la energía.

Una vez que se ha elegido esta dirección, a lo largo de esta se va a dispersar la energía del átomo, siendo igual para cada átomo utilizado, sin importar en qué punto del plano t-f se encuentren, por lo que si la trayectoria de la componente cambia en algún punto del transitorio capturado, más allá de ese punto no se tendrá una captura óptima de esa trayectoria. Por otra parte, si los armónicos evolucionan con diferentes pendientes, entonces tampoco se capturarán de una manera correcta. 
Esta técnica se ha utilizado con éxito para detectar por primera vez las barras rotas durante el arranque de un motor de inducción alimentado por inversor [3].

\subsubsection{Multiple Signal Classification.}

Este algoritmo, también conocido como MUSIC, proporciona un pseudo-espectro de alta resolución de la señal analizada, mitigando los efectos del ruido. Se basa en el análisis de autovalores de la matriz de autocorrelación de la señal. La matriz de autocorrelación $R$ es la suma de las matrices de autocorrelación de la señal y del ruido. Los vectores modo frecuencia son ortogonales a los vectores propios del subespacio de la matriz de ruido. El pseudo-espectro MUSIC se calcula teniendo en cuenta la ortogonalidad de los vectores frecuencia a los vectores propios del subespacio ruido. Este procedimiento fue utilizado en [56] para la detección de barras rotas durante el arranque de motor de inducción alimentado mediante inversor utilizando la corriente del estator. Es notable la alta resolución de la representación tiempo-frecuencia de la corriente del estator. Los armónicos relativos de fallo fueron igualmente observables durante los regímenes transitorios y estacionarios.

Otro modo de aplicación de esta técnica es usado en [57] en donde junto con la técnica Zero-Sequence Current (ZCS) se analiza el arranque directo de un motor; al aplicarlo junto con esta técnica se consigue obtener una mejora con respecto al análisis de una sola fase de la forma de onda de la corriente, obteniéndose así resultados satisfactorios en la detección de barras rotas tanto en el estado transitorio como en el estacionario.

\subsubsection{Conclusiones parciales}

En el apartado 2.4, el más importante del presente capítulo, se han resumido las características principales relativas a las distintas transformadas utilizadas en la literatura técnica para resolver el problema de la detección de averías en motores de inducción alimentados mediante inversor y operando en régimen transitorios de arranque. Los comentarios relativos a los resultados que se pueden obtener con estas transformadas, se han basado en su análisis teórico, así como en la observación y análisis de los resultados 
plasmados en los artículos citados. Para poder realizar un análisis más preciso, además de una comparativa justa, en el siguiente capítulo se han implementado dichas técnicas, mostrando los resultados obtenidos al aplicarlas a dos corrientes provenientes de dos motores con dos tipos de averías distintos (barras rotas y excentricidad mixta). A continuación se resumen las técnicas presentes en Estado del arte, añadiendo comentarios que son también conclusión abreviada del análisis realizado en el siguiente capítulo.

Las transformadas basadas en $F F T$, que permiten una buena identificación del fallo en estados estacionarios, no son apropiadas cuando se trabaja con regímenes transitorios.

La STFT tiene un compromiso de resolución fijo en todo el plano tiempofrecuencia, que, si bien es suficiente para obtener la evolución de los componentes de fallo por excentricidad mixta, falla al diagnosticar fallos debidos a rotura de barras.

Para solucionar este inconveniente, se propusieron otras técnicas tales como la transformada Wavelet con una resolución mejorada. Más concretamente, la CWT permite una resolución variable con la frecuencia analizada. Sin embargo, esta variación no es la adecuada para los tipos de evoluciones de los componentes de fallo en las corrientes de estator de motor de inducción, y, por tanto, se debe evitar. Por otra parte, la DWT descompone la corriente en subseñales relacionadas con diversas bandas de frecuencia, pero no permite obtener las evoluciones de los armónicos de fallo, ya que la presencia de la componente fundamental en las subseñales enmascara los componentes.

La WVD elimina el problema de resolución t-f que existe en las anteriores técnicas, pero introduce términos cruzados (una componente ficticia por cada par de componentes reales), los cuales, al superponerse con las evoluciones de las componentes reales presentes en la corriente, dificultan su detección. Al aplicar esta técnica en motores alimentados directamente desde la red, es común el filtrado de la componente fundamental y los armónicos de devanado, para disminuir el número de términos cruzados. No obstante, al alimentar mediante un inversor, no resulta posible, pues las frecuencias de dichas componentes son variables. En definitiva, aparecen muchos términos cruzados, impidiendo ver los armónicos de fallo durante el transitorio de 
arranque. Sólo resulta útil para detectar las evoluciones de las componentes de excentricidad mixta.

HHT trata de dividir la corriente en sus componentes, para luego obtener su frecuencia instantánea. Sin embargo, sólo tiene éxito para separar la componente de excentricidad mixta inferior de la corriente.

MUSIC muestra una resolución uniforme en todo el plano tiempo-frecuencia.

La $A S T$ ofrece una resolución t-f completamente adaptable y esto permite una gran mejora con respecto a la STFT. Sin embargo, esta mejora no es suficiente para observar la evolución de los armónicos de rotura de barra durante el arranque.

La $C T$ elimina los problemas de resolución de las transformadas anteriores sin introducir términos cruzados. Como consecuencia, se pueden detectar perfectamente las evoluciones de las componentes de barra rota durante el transitorio de arranque. No obstante, la detección de componentes de excentricidad mixta no mejora con respecto a la $A S T$.

A la hora de elegir una técnica, la $S T F T$ es una buena opción para detectar componentes de excentricidad mixta, mientras que la $A S T$ consigue optimizar el resultado de la STFT. Para obtener las evoluciones de rotura de barra durante el arranque, hay que recurrir a la $C T$. No obstante, no existe una técnica capaz de identificar fallos en motores de inducción alimentados por inversor en estados transitorios arbitrarios y de forma completa y precisa. La dificultad estriba en la proximidad de las evoluciones de los armónicos de fallo con la frecuencia fundamental (en especial los de barra rota) y el cambio continuo de la frecuencia fundamental durante los estados transitorios. En el capítulo 4 se presenta una técnica capaz de abordar y resolver el mencionado problema. 

3. Aplicación de las técnicas de análisis tiempo-frecuencia presentes en la literatura técnica: alcance y limitaciones

\subsection{Introducción}

3.2. Casos de estudio

3.3. Aplicación y resultados de las técnicas presentes en la literatura técnica

3.4. Conclusiones: comparativa, alcance y limitaciones de cada técnica 
Capítulo 3. Aplicación de las técnicas de análisis tiempo-frecuencia presentes en la literatura técnica: alcance y limitaciones 


\section{Aplicación de las técnicas de análisis tiempo-frecuencia presentes en la literatura técnica: alcance y limitaciones}

\section{1.- Introducción}

En este capítulo se trata de analizar en profundidad la problemática relativa al diagnóstico de un motor de inducción alimentado por inversor, operando en régimen transitorio (expuesta en el punto 2.4. de esta memoria), a través del uso de las transformadas tiempo-frecuencia presentes en la literatura técnica.

Para ello se han analizado dos señales diferentes obtenidas experimentalmente en el laboratorio. Estas señales han sido obtenidas tal como se presenta en el epígrafe 3.2 de este apartado.

A dichas señales se les han aplicado las técnicas presentadas teóricamente en el punto 2.3 y 2.4 de esta memoria: FFT, STFT, Wavelet, Wigner-Ville, HHT, AST, Chirplet y MUSIC. De esta forma se ha comprobado, de manera experimental, cómo responde cada una de ellas, y se puede corroborar lo explicado de manera teórica acerca de cada técnica.

A partir de estos resultados experimentales obtenidos, se puede llegar a una serie de conclusiones sobre las mismas, que se exponen en el epígrafe 3.4. de este apartado, mostrando su potencial, pero también sus limitaciones.

Son estas últimas las que llevan a la necesidad de definir una nueva transformada, presentada en el capítulo 4, y testeada experimentalmente de forma extensiva en el capítulo 6.

\subsection{Casos de estudio:}

Para estudiar en profundidad las distintas técnicas que se utilizan en el estado transitorio, se han obtenido experimentalmente dos señales en el laboratorio, 
correspondientes a dos motores de inducción trabajando en diferentes condiciones. Ambos motores presentan las siguientes características:

- $\quad 0,75 \mathrm{~kW}$ de potencia

- conexión estrella

- $\quad 400 \mathrm{~V}$ de tensión

- $\quad 1410 \mathrm{rpm}$ de velocidad nominal

- $\quad 2,6$ A de corriente nominal.

Las características del motor empleado, así como los detalles referentes al banco de ensayos utilizado y cómo se han ejecutado los ensayos y los diversos inversores utilizados, se pueden consultar en el capítulo 5 de esta memoria de tesis.

Volviendo al apartado en cuestión, en cuanto al primer motor de inducción, éste presenta una barra de rotor agujereada (en el capítulo 6 de esta memoria aparece cómo se han provocado los distintos fallos) y es alimentado mediante un inversor AB PowerFlex 40. Por otra parte, este motor de inducción también presenta un bajo nivel de excentricidad mixta debido al montaje del motor en el banco de ensayos.

En este primer caso se programa un arranque largo de 10 segundos, con una frecuencia de salida que aumenta linealmente desde 0 a $50 \mathrm{~Hz}$. La corriente de fase (señal 1), incluye el arranque transitorio y una operación en estado estacionario largo. En la Figura 8 se muestra la señal obtenida y la trayectoria del primer armónico en el plano tiempo-frecuencia durante esta prueba de laboratorio. Para obtener dicha evolución, se ha aplicado un método basado en calcular en primer lugar la STFT, para seguidamente obtener el máximo del resultado de la transformada para cada instante de tiempo [58]. Como el primer armónico es la componente de mayor amplitud, dicho máximo se sitúa siempre a su frecuencia. Este motor consume 2,4 A en el final de estado estacionario.

El segundo motor presenta un alto nivel de excentricidad mixta, que ha sido provocada, y es alimentado mediante un variador Siemens Micromaster 420. El arranque está programado para que pasados 2,2 segundos la frecuencia de salida haya aumentado 
linealmente desde 0 a $50 \mathrm{~Hz}$. La segunda señal (señal 2) es una de las corrientes capturadas, que incluye al transitorio de arranque seguido por un periodo de estado estacionario. La señal es de 6 segundos en total. La Figura 9 muestra la señal 2 así como la trayectoria de su primer armónico en el plano tiempo-frecuencia. La corriente consumida en el final del estado estacionario fue de 2,55 A.

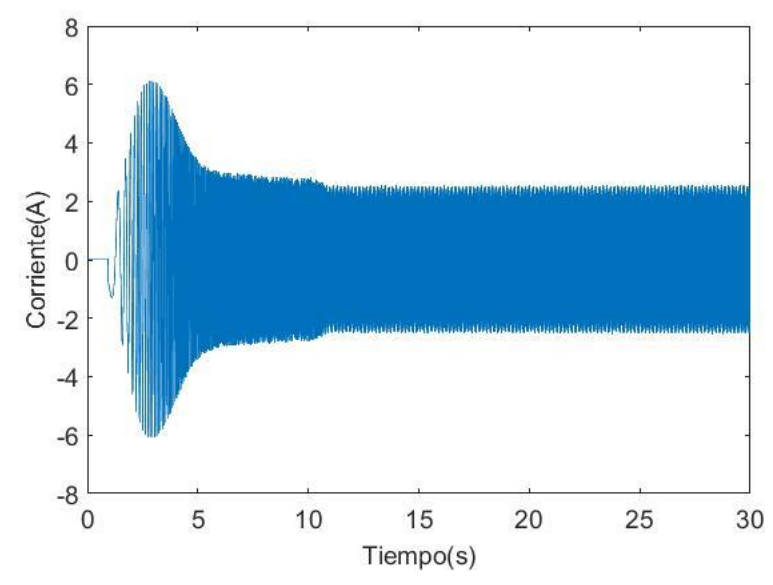

(a) Corriente del motor de inducción con una barra rota (señal 1).

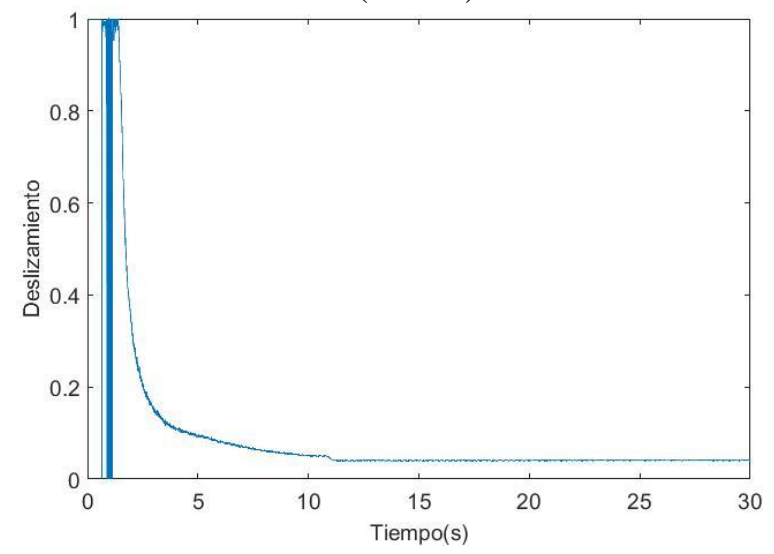

(c) Evolución temporal del deslizamiento de la señal 1.

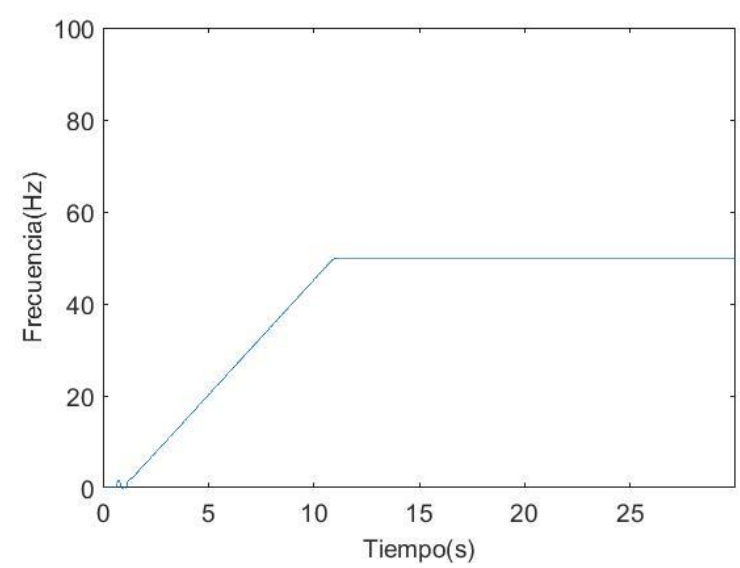

(b) Trayectoria en el plano tiempo-frecuencia del

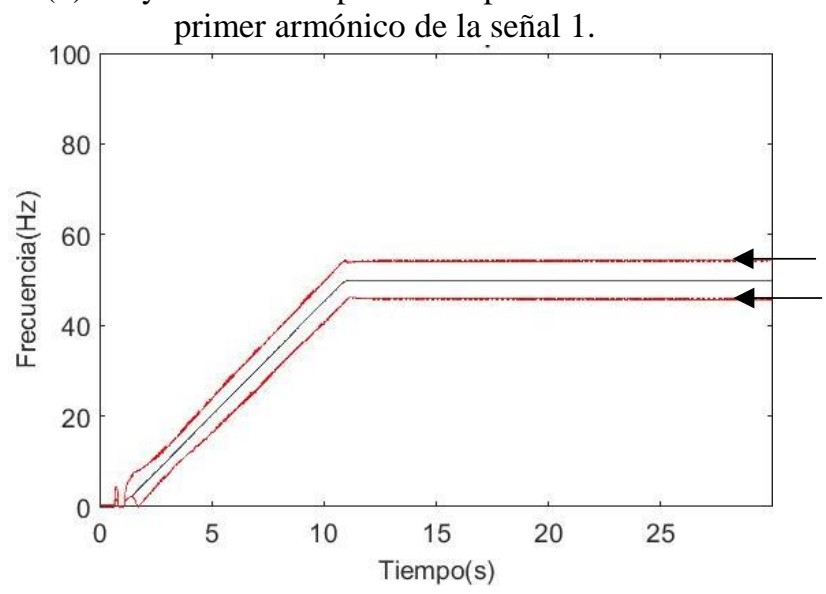

(d) Trayectoria en el plano tiempo-frecuencia del primer armónico, del $L S H$ y del $U S H$ de la señal 1 (señalado con flechas negras en la imagen).

Figura 8. Captura y análisis preliminar de la señal 1, búsqueda de la trayectoria de fallo debido a $B R B$.

Una vez introducidas las dos señales sobre las que se va a trabajar, se pasa a continuación, en el apartado 3.3., a analizar estas dos señales con las diferentes técnicas tiempo-frecuencia previamente vistas. 
Capítulo 3. Aplicación de las técnicas de análisis tiempo-frecuencia presentes en la literatura técnica: alcance y limitaciones

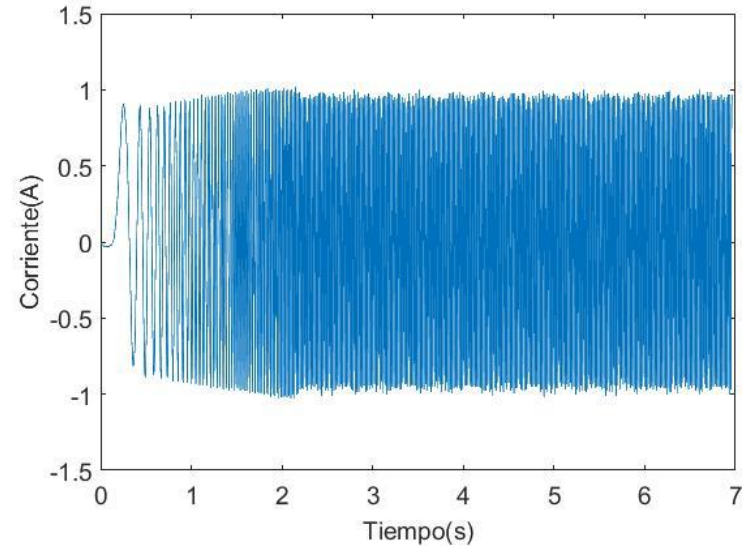

(a) Corriente del segundo motor de inducción con un alto nivel de excentricidad mixta (señal 2).

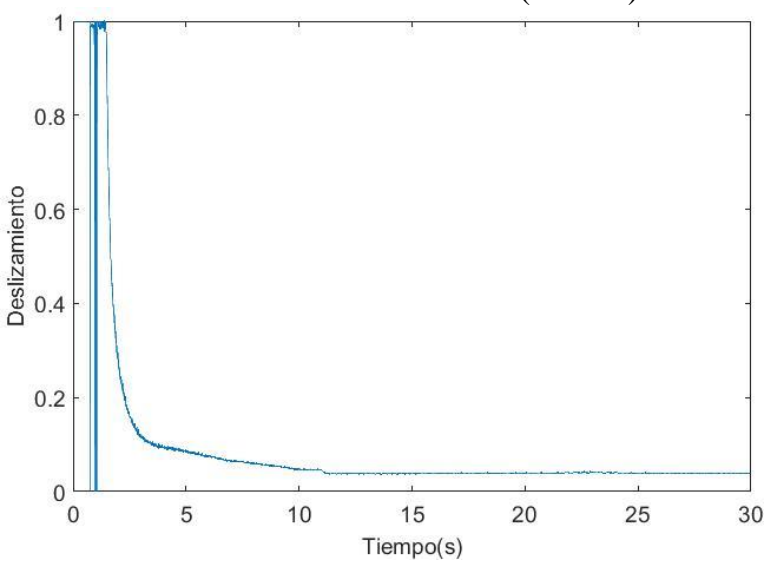

(c) Evolución temporal del deslizamiento de la señal 2.

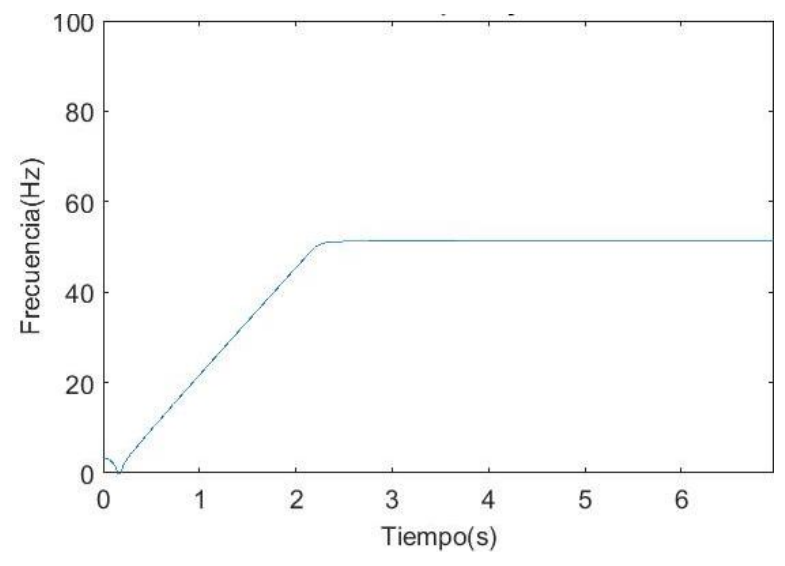

(b) Trayectoria en el plano tiempo-frecuencia del

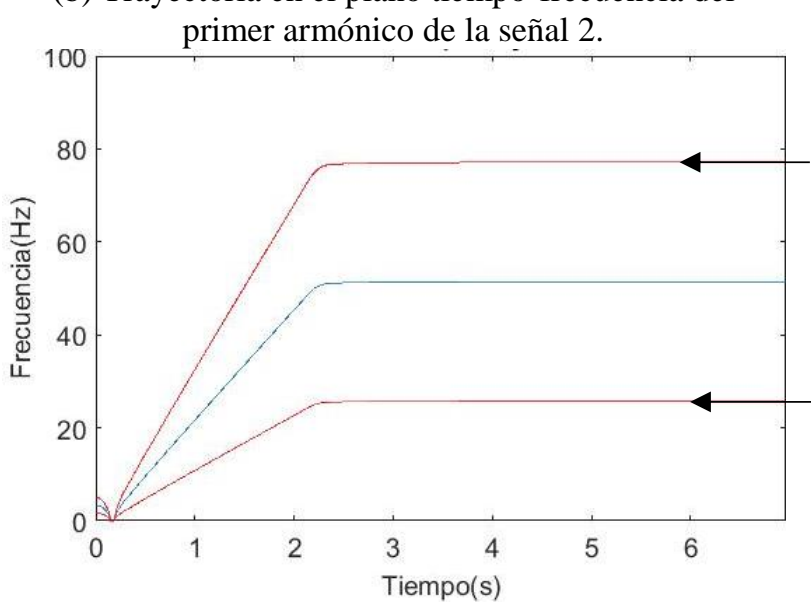

(d) Trayectoria en el plano tiempo-frecuencia del primer armónico, del $E R H(+)$ y del $E R H(-)$ de la señal

2 (señalado con flechas negras en la imagen).

Figura 9. Captura y análisis preliminar de la señal 2, búsqueda de trayectoria de fallo debido a Excentricidad Mixta.

\subsection{Aplicación y resultados de las técnicas presentes en la literatura técnica.}

En esta sección se analizan las herramientas más significativas utilizadas para detectar fallos en motores de inducción en su estado transitorio, las cuales han sido introducidas en el estado del arte presentado en el apartado 2.4.

Se ha empezado con la técnica clásica en régimen permanente $(F F T)$, observando sus limitaciones y la necesidad de utilizar transformadas tiempo-frecuencia, para, a continuación, aplicar las principales técnicas de este tipo presentes en la literatura técnica. 
Las dos señales experimentales (Señal 1 y 2) explicadas en el punto 3.2., se van a utilizar para probar estas técnicas, con motores alimentados mediante inversor, y así poder comparar sus ventajas y puntos débiles. Con esto se presenta una verdadera comparativa, teniendo en cuenta que cada técnica es aplicada al mismo par de señales, pudiendo comparar la calidad de los resultados obtenidos con cada una de ellas. Así, se permite a los ingenieros de campo elegir la técnica más adecuada de entre las presentes en la literatura técnica. Los problemas no resueltos son revelados, mostrando la necesidad de definir nuevas técnicas que los resuelvan (ver el capítulo 6 para ampliar información acerca de los ensayos).

Las herramientas que finalmente se van a comparar son: FFT, STFT, Wavelet, Wigner-Ville, HHT, AST, Chirplet y MUSIC. Para cada una de las técnicas (introducidas en el capítulo 2) se van a describir sus características, mostrar ventajas e inconvenientes inherentes y finalmente presentar los resultados y analizarlos de acuerdo con las características de la transformada previamente descritas.

\section{Fast Fourier Transform}

La transformada de Fourier, Fourier Transform (FT), como se expuso en el apartado 2.3. de esta memoria, es la base del análisis de la corriente del estator del motor de inducción en estado estacionario. Proporciona información sobre el contenido de armónicos de una señal y la energía asociada a cada armónico; la identificación de armónicos vinculados a averías permite el diagnóstico de motores. No obstante, no se obtiene una buena respuesta cuando la señal se captura durante un régimen transitorio: las frecuencias de los harmónicos pueden ser variables con el tiempo, y dicha variación no queda adecuadamente representada en el resultado de la transformada. Por lo tanto, es necesario buscar otras técnicas que permitan superar estas limitaciones, tal y como se expondrá en las siguientes subsecciones.

La transformada de Fourier de una función $f$ se define como:

$$
\hat{f}(\varepsilon)=\frac{1}{\sqrt{2 \pi}} \int_{-\infty}^{+\infty} f(x) e^{-i \varepsilon x} d x
$$


Capítulo 3. Aplicación de las técnicas de análisis tiempo-frecuencia presentes en la literatura técnica: alcance y limitaciones

Aplicando esta transformada a las señales de estudio, señal 1 y señal 2, se obtienen las imágenes de la Figura 10. Analizando primeramente las Figuras 10 (a) y (b), obtenidas al aplicar la transformada rápida de Fourier ( $F F T$ en sus siglas en inglés) a la corriente completa (incluyendo el transitorio de arranque), estas Figuras muestran los espectros, que son consistentes con la capacidad técnica de la transformada y el contenido armónico de las dos señales transitorias.

Los espectros muestran un contenido armónico elevado entre 0 y $50 \mathrm{~Hz}$. Esta energía está vinculada a la componente fundamental de la corriente, generada por el primer armónico de la tensión de salida del inversor, cuya frecuencia incrementa linealmente con el tiempo de 0 a $50 \mathrm{~Hz}$ durante el arranque. Teniendo en cuenta que dicho armónico cubre la banda de 0 a $50 \mathrm{~Hz}$ durante su evolución, la FFT muestra como resultado energías elevadas en dicha banda. Por otra parte, la energía de este armónico es mucho mayor que el resto de armónicos y domina el espectro en este rango de frecuencias. Es por esta razón que resulta complicado observar otros componentes armónicos, como los armónicos relativos de fallo de barras para la señal 1 o de excentricidad para la señal 2; sin embargo, en la segunda señal, sí se ve una componente entre 0 y $50 \mathrm{~Hz}$, representada por un pico muy ligero que sobresale respecto al fundamental: el armónico de excentricidad mixta inferior, a $26,58 \mathrm{~Hz}$.

Una vez se ha pasado la banda de $50 \mathrm{~Hz}$, en donde aparece la componente fundamental, sí es posible observar los armónicos que aparecen en esta parte del espectrograma, como puede verse en la Figura 10 (a), apareciendo un pico representativo de barra rota en 54,2 Hz, y en la Figura 10 (b), donde se puede observar también el pico debido a la componente armónica de excentricidad superior situado en 76,43 Hz.

Se habrá observado que la energía de la componente fundamental es mayor a 50 $\mathrm{Hz}$ que en el resto de frecuencias por las que pasa durante su evolución en el arranque. Esto no significa que su amplitud sea necesariamente mayor cuando llega a esta frecuencia. Para obtener la $F F T$, cuando se correlaciona la señal a analizar con una exponencial compleja a una determinada frecuencia, si dicha frecuencia se encuentra por debajo de los $50 \mathrm{~Hz}$ que la fundamental alcanza en permanente, la exponencial que se utiliza para correlacionar se cruza con la fundamental tan solo en un instante: aquel en el que la fundamental pasa por la frecuencia de la exponencial. Pero cuando se correlaciona 
con una exponencial a la frecuencia de permanente (50 Hz en este caso), la coincidencia entre la fundamental y la frecuencia de la exponencial se produce no en un instante, sino en todo el intervalo en el que el motor se encuentra en régimen permanente. Es por ello que la energía capturada es mayor a dicha frecuencia.

En definitiva, al aplicar la $F F T$ a señales con una parte en transitorio no es posible localizar las frecuencias en el tiempo durante su evolución temporal, pero sí se pueden llegar a observar las frecuencias en su parte de régimen permanente de componentes vinculadas a fallos si ningún armónico de energía elevada tiene una evolución que se cruce con su frecuencia.

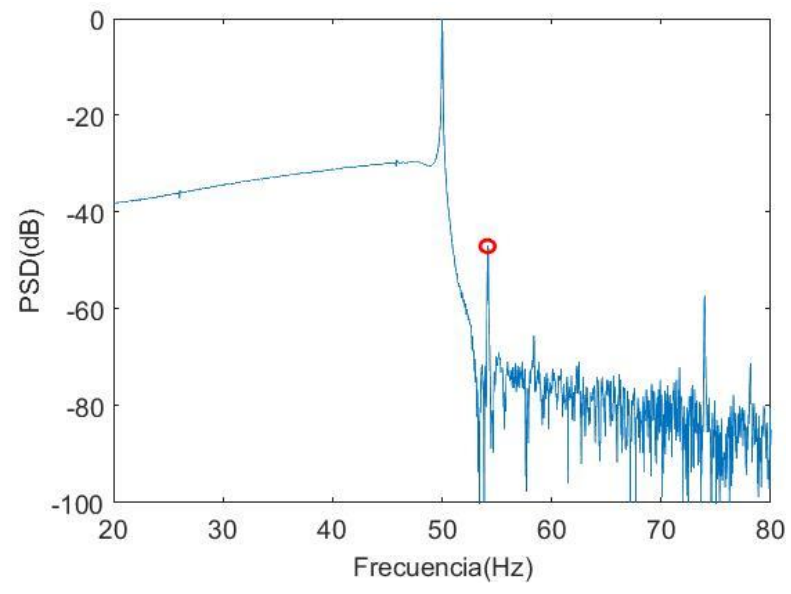

(a) Espectro de la señal 1 completa (transitorio y estacionario) calculado con FFT.

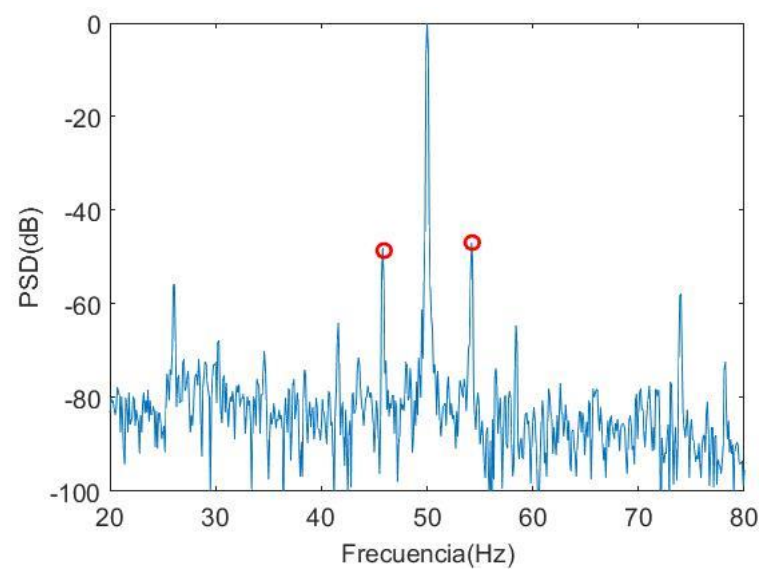

(c) Espectro del estacionario de la señal 1 calculado con $F F T$. Señalado en rojo armónicos de fallo por $B R B$.

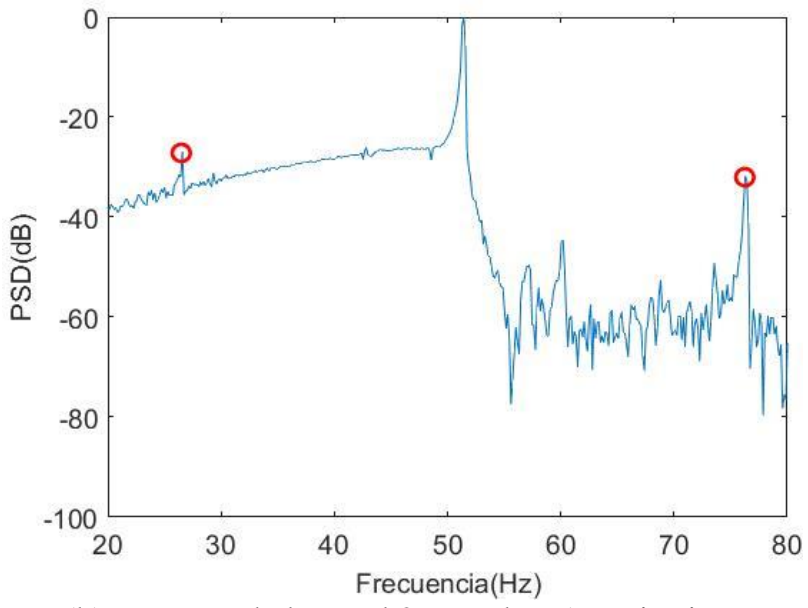

(b) Espectro de la señal 2 completa (transitorio y estacionario) calculado con FFT.

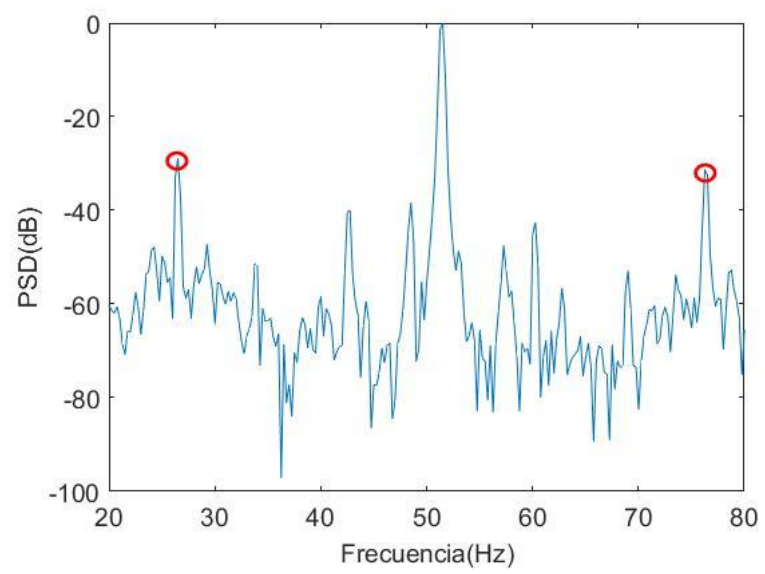

(d) Espectro del estacionario de la señal 2 calculado con $F F T$. Señalado en rojo armónicos de fallo por Excentricidad Mixta.

Figura 10. Análisis mediante $F F T$ de las señales 1 y 2. 
Sin embargo, si se analiza sólo la parte del estacionario de cada una de las señales, se tiene que la frecuencia fundamental no varía en el estado estacionario, identificando perfectamente su posición en una única frecuencia, como puede verse en las Figuras 10 (c) y (d). Esto facilita la detección de otras componentes, como son las vinculadas a averías. En particular, se pueden observar los armónicos debidos a fallo de barras en la Figura 10 (c), así como los armónicos debidos a excentricidad en la Figura 10 (d). Estos armónicos se observan debido a que el estado estacionario capturado es largo con respecto al transitorio, siendo el doble de tiempo de éste.

En este caso de analizar únicamente el estado estacionario, se pueden aplicar las ecuaciones para el cálculo teórico de las frecuencias de fallo, en caso de barras rotas y excentricidad mixta, que se explicaron en el apartado 2 de esta memoria, con lo que se obtendría, teniendo en cuenta que los deslizamientos durante el estado estacionario son de 0,042 para el motor de la señal 1 y 0,045 para el motor de la señal 2:

- Señal 1 (Figura 10c): LSH: 45,8 Hz y USH: $54,2 \mathrm{~Hz}$.

- Señal 2 (Figura 10d): ERHn: 26,58Hz y ERHp: 76,43Hz.

Este resultado demuestra que esta técnica no es adecuada para la detección de fallos en estados transitorios porque no proporciona información sobre la trayectoria temporal de los armónicos y no es posible identificar sus trayectorias características. Sin embargo, por una parte, incluso al analizar corrientes no estacionarias, si estas tienen un período estacionario, durante dicho período se pueden observar la presencia de componentes si sus frecuencias no se ven intersectadas por las de componentes de muy elevada energía, como es el caso de la componente fundamental. Por otra parte, es la técnica ideal para aplicar al estado estacionario, en donde la componente fundamental se encuentra en una posición de frecuencia única, haciendo así posible la identificación del resto de los componentes armónicos con la máxima precisión posible.

\section{Short Time Fourier Transform}

Como se ha explicado en el punto 2.4., la primera técnica para hacer frente a las señales no estacionarias es la denominada Short Time Fourier Transform (STFT) [31]. La 
STFT es una transformada tiempo-frecuencia, y como tal permite una representación tridimensional de la señal: la distribución de su energía en el plano tiempo-frecuencia. Esta técnica es la más simple, la que más limitada está respecto a la resolución del resultado obtenido, pero que resulta suficiente en determinadas condiciones. En definitiva, la $S T F T$ se calcula como:

$$
\operatorname{STFT}_{x}^{g}(t, f)=\int_{-\infty}^{+\infty} x\left(t^{\prime}\right) g^{*}\left(t^{\prime}-t\right) e^{-j 2 \pi f t^{\prime}} d t^{\prime}
$$

donde $g$ es la ventana seleccionada, $g^{*}$ su conjugado, $x$ es la señal a analizar y $(t, f)$ el punto del plano donde se quiere calcular la transformada.

Para calcular la STFT, la ventana se centra en el instante de tiempo y se modula a la frecuencia donde la transformada necesita calcularse. El objetivo de la transformada es correlacionar la señal de estudio $x$ con la ventana, centrada en un instante $t$ y modulada a una frecuencia $f$. La ventana centrada y modulada, se llama también átomo tiempofrecuencia, pues su energía está bien concentrada alrededor del punto t-f. Como ejemplo, la Figura 11 (a) muestra una familia de este tipo de funciones; concretamente, se muestra la distribución de la energía de 30 de estas funciones, centradas en distintos puntos del plano, formando una matriz (la distribución de la energía de los átomos en el plano se ha obtenido utilizando la distribución de Wigner-Ville). Tal y como se detallará más adelante, esta familia de funciones (Figura 11 (a)) es la utilizada para analizar la primera señal descrita en el apartado anterior, obteniendo como resultado de la STFT la Figura 11 (b); análogamente, la familia de funciones utilizada para analizar la segunda señal se muestra en la Figura 12 (a), obteniendo como resultado la Figura 12 (b).

Normalmente, la dispersión de la energía del átomo se describe mediante una caja, la caja de Heisenberg, de tal forma que la caja contiene prácticamente toda la energía del átomo (se introducirá este concepto con mayor detalle en el capítulo 4, donde se analizarán además con detalle los átomos tiempo-frecuencia, su construcción, características y resultados que obtienen). Al correlacionar la señal $x$ con el átomo, medimos la energía de $x$ en la zona del plano donde el átomo tiene concentrada su energía. Más concretamente, se obtiene una medida de la densidad de energía de la señal en el punto del plano t-f donde el átomo está centrado. Si se repite esta operación en una 
extensión de puntos del plano t-f, centrando el átomo en diferentes puntos, se obtiene la distribución en el plano de la densidad de energía de la señal analizada. La densidad de energía es elevada en las zonas del plano por las que evolucionan armónicos de la señal. De esta forma, la representación de la densidad de energía muestra las evoluciones de los armónicos (Figuras 11 (b) y 12 (b)). Estas, aunque físicamente son líneas perfectamente finas, en el resultado aparecen con un cierto grosor, el cual es función del compromiso entre resolución temporal y frecuencial que tiene todo resultado de transformada lineal. Así, una longitud de ventana larga significa una resolución buena en frecuencia, pero se reduce la resolución temporal; al contrario, una longitud corta de ventana mejora la resolución temporal a costa del empeoramiento de la resolución en frecuencia.

Todas las transformadas lineales están basadas en la correlación de la señal a analizar con una familia de átomos tiempo-frecuencia. La única diferencia entre las distintas transformadas son las características de la familia de átomos utilizada por cada transformada. Concretamente, la STFT se basa en el uso de una ventana igual para todo el plano tiempo-frecuencia; esta ventana elegida va a fijar la resolución que se tendrá en tiempo y frecuencia a lo largo de todo el plano.

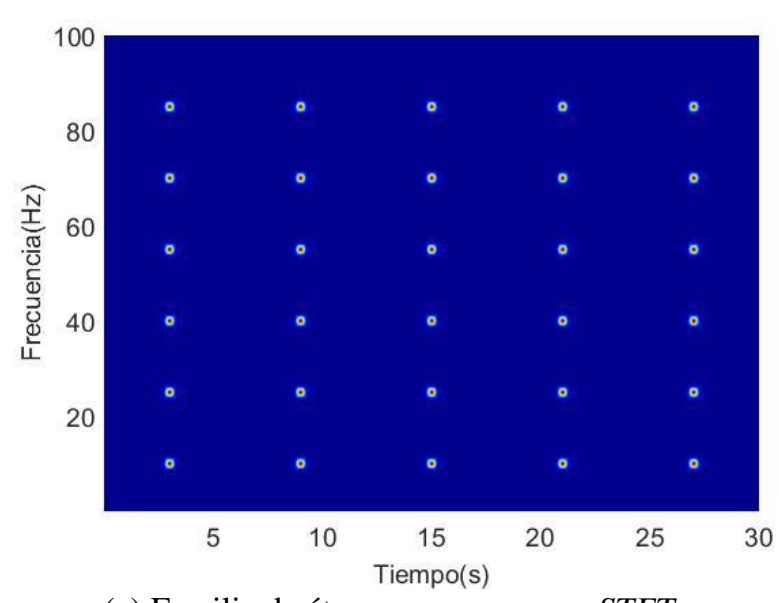

(a) Familia de átomos para generar STFT.

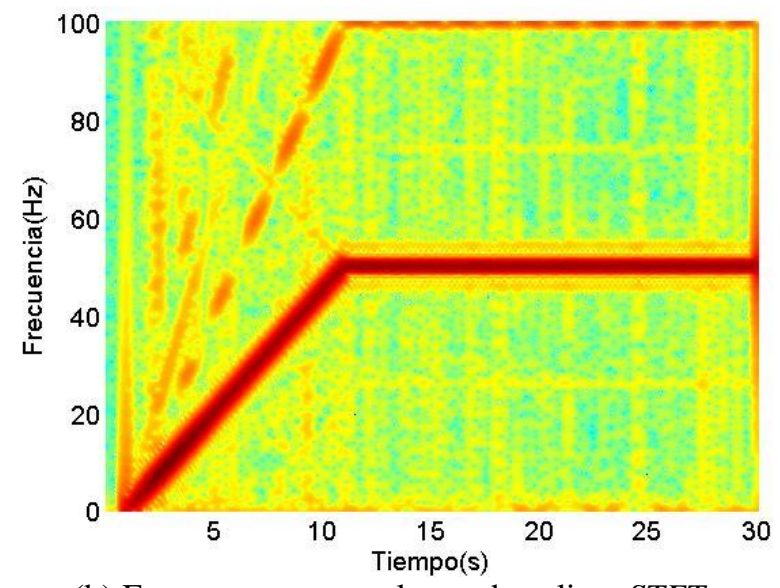

(b) Espectrograma resultante de aplicar STFT.

Figura 11. Espectrograma de la STFT de señal 1 (IM con BRB). 


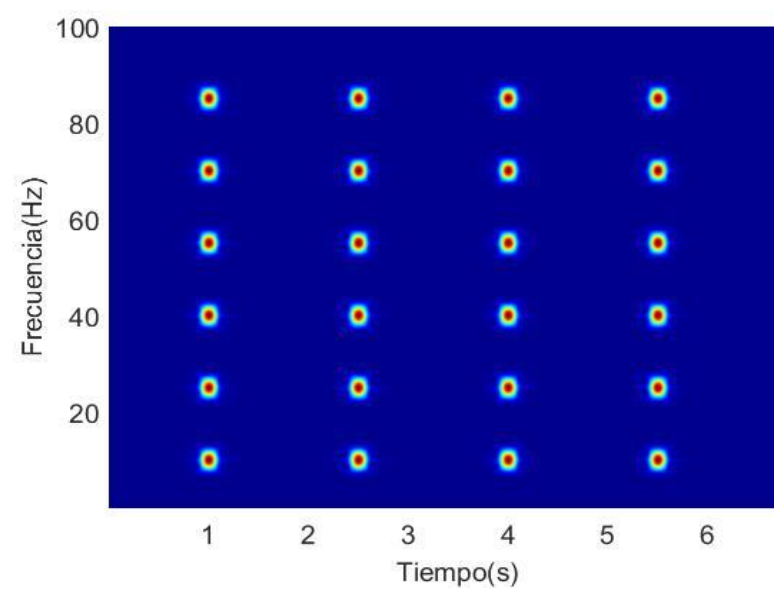

(a) Familia de átomos para generar STFT.

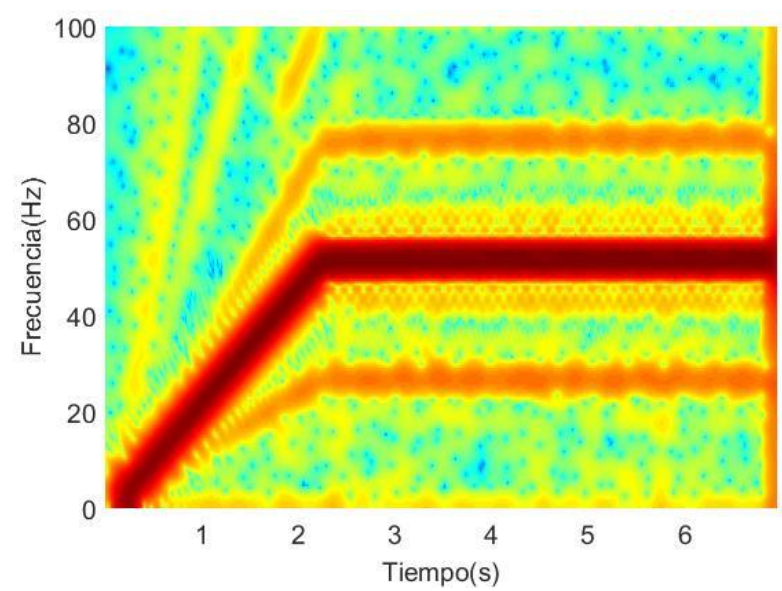

(b) Espectrograma resultante de aplicar STFT.

Figura 12. Espectrograma de la STFT de señal 2 (IM con excentricidad mixta).

En las Figuras 11 (a) y 12 (a) se pueden observar las familias de átomos que han sido usadas para generar los espectrogramas resultantes de aplicar la STFT. Es decir, en estas Figuras se ve la distribución de energía de algunos de los átomos t-f usados. Como se ha mencionado antes, estos átomos capturan la energía de la señal en la zona del plano t-f en donde se concentra la energía del átomo. Se puede decir que el átomo actúa como un filtro t-f, capturando la energía de la señal alrededor del punto analizado. Si el átomo es largo en tiempo, al centrarlo en un punto y correlacionarlo con la señal, se obtiene, asociado a ese punto, información de la señal en ese punto de muchos instantes de tiempo, por lo que la resolución en tiempo es mala. El inconveniente aparece cuando se trata de acortar este átomo en tiempo, ya que inevitablemente éste crece en frecuencia, al correlacionar con la señal se obtiene información de la señal a muchas frecuencias, por lo que la resolución en frecuencia será mala. Por tanto, la resolución que se obtiene no puede ser buena simultáneamente en tiempo y en frecuencia. En el caso de la STFT, el tipo de átomo es el mismo en todos los puntos del plano tiempo-frecuencia, como puede verse en las Figuras 11 (a) y 12 (a). Por tanto, en el caso de la STFT, la resolución tiempofrecuencia es la misma en todos los puntos del plano.

Si se centra un átomo en un punto de la evolución de la componente fundamental, cuanto más largo en el tiempo sea el átomo, más se debe desplazar a otros instantes de tiempo para que deje de capturar energía de la componente fundamental. Por tanto, cuanto más alargado en el tiempo sea el átomo mayor es el grosor medido en el sentido del eje 
de tiempo que se obtiene de la evolución de la componente fundamental. Por otra parte, cuanto más alargado sea el átomo en el sentido del eje de frecuencias, más se debe separar de la frecuencia de la componente fundamental a otras frecuencias para que deje de capturar la energía de dicha componente, y por tanto mayor será el grosor con el que se obtiene la evolución de la componente fundamental, medido en la dirección del eje de frecuencias. En definitiva, el grosor con el que se obtiene la evolución de la componente fundamental, el cual se puede medir en la dirección del eje temporal o en la dirección del eje de frecuencias, es función de la forma en la que el átomo se dispersa en el plano t-f. Como no se puede disminuir el tamaño del átomo simultáneamente en tiempo y en frecuencia, no es posible disminuir el grosor de la evolución por debajo de un determinado mínimo.

Si la evolución de la componente a detectar es horizontal, entonces se debe de utilizar un átomo largo en el tiempo, para que la resolución frecuencial sea elevada y se pueda establecer bien la frecuencia de dicha componente con evolución horizontal. Si por el contrario, la evolución de la componente es vertical, se debe utilizar un átomo largo en frecuencia, para determinar bien el instante en el que la frecuencia de dicha componente cambia abruptamente. Finalmente, si la evolución es genérica, la proporción entre la longitud en frecuencia y en tiempo del átomo debe ser igual a la proporción entre el cambio de frecuencia de la componente y el tiempo que tarda en producirse dicho cambio (esto es, la derivada de la frecuencia de la componente respecto al tiempo). Al aplicar este criterio, se consigue minimizar el grosor de la evolución medida en sentido perpendicular a la propia evolución.

Para las dos señales analizadas se ha aplicado el criterio de la pendiente (la tangente a la evolución de la componente en un punto coincide con la diagonal del átomo utilizado en dicho punto [58]). Dicho criterio se ha aplicado (ajustando adecuadamente la longitud de la ventana), para optimizar la captura de la evolución de la componente fundamental durante el arranque, obteniendo la familia de átomos a usar (Figuras 11 (a) y 12 (a)) y determinando la resolución tiempo-frecuencia a obtener. Consecuentemente, se ha minimizado la finura de la línea de la componente fundamental durante el arranque, consiguiendo el resultado más ideal posible al respecto. 
Concretamente, observando la Figura 11 (señal 1) se ha llegado a un compromiso de resolución entre tiempo y frecuencia, de tal manera que la longitud de ventana elegida permite la observación de los armónicos de fallos de barras, Broken Rotor Bars (BRB), durante el final del estado estacionario. Sin embargo, esto no es suficiente para poder observar las trayectorias de estos armónicos durante el periodo de arranque del motor de inducción, ya que en este caso la línea de la trayectoria de la componente fundamental no es lo suficientemente fina como para separarse de los armónicos debidos a fallo de barras. En otras palabras, el grosor con el que se obtiene la componente fundamental no es suficientemente pequeño, y las trayectorias de las componentes de avería, que se encuentran muy cerca, quedan enmascaradas.

Por otra parte, en la Figura 12 (señal 2), se ha procedido de manera análoga a la anterior. La longitud de ventana ajustada permite observar las trayectorias de los armónicos debidos a excentricidad mixta, que son visibles durante el arranque, ya que en este caso los armónicos se encuentran más lejos de la $F C$ que en el de barra rota. No obstante, no se llegan a observar por debajo de 1 segundo pues en esta zona sus trayectorias sí son demasiado próximas. En el régimen de estado estacionario se observan trayectorias adicionales alrededor del componente principal. Estos armónicos son producidos por el mecanismo de la conmutación del inversor, pero la mejor resolución alcanzable con la STFT no permite observarlos correctamente y pueden ser confundidos con los armónicos $B R B$.

La principal ventaja de la STFT es el bajo esfuerzo computacional. Con respecto a los resultados, esta técnica permite la observación de armónicos de baja energía que distan de la alta energía del primer armónico, como ocurre con el fallo de excentricidad mixta. Sin embargo, si estos armónicos están muy cerca de la componente principal, como ocurre en el caso de fallo de barras, la mejor resolución posible en el plano tiempofrecuencia no es suficiente para poder distinguirlos.

\section{Wavelets}

La transformada wavelet se desarrolló para resolver los problemas de resolución de tiempo y frecuencia que aparecen con la STFT. La transformada wavelet continua, 
Continuous Wavelet Transform (CWT) es similar a la STFT: la señal a analizar se correlaciona con una función llamada wavelet. Dicha función tiene su energía concentrada alrededor de un punto del plano tiempo-frecuencia, por lo que puede ser categorizada como átomo tiempo-frecuencia (tiene además algunas propiedades matemáticas particulares). Los problemas de la STFT se derivan del uso de funciones de longitud fija a lo largo del plano, lo cual implica un compromiso de resolución t-f fija. Para resolver este problema, la longitud de la wavelet depende de la frecuencia a la que se encuentra centrada.

La familia de wavelets se define como una traslación y escalado de una wavelet original, centrada en el origen, llamada wavelet madre. Esto es un procedimiento similar al de traslación y modulación de una ventana, realizado para calcular la STFT. La diferencia es que la modulación no produce cambios en las características de la ventana original, más allá de la frecuencia a la que se encuentra centrada, mientras que el escalado sí cambia sus características, provocando un cambio en la resolución tiempo-frecuencia obtenida al correlacionarla con la señal a analizar. Por lo tanto, la resolución obtenida con la $C W T$ cambia con la frecuencia analizada, a diferencia de la STFT, que presenta una resolución fija en todos los puntos del plano. Además, dicha variación de la resolución sigue una ley prestablecida, tal y como se comenta más adelante.

La transformada wavelet discreta, Discrete Wavelet Transform (DWT), es la versión discreta de la $C W T$. La $D W T$ realiza una descomposición de la señal en un número previamente seleccionado de subseñales asociada a distintas bandas de frecuencia, tal como se explica en el apartado 2.4. Las bandas de frecuencia asociadas a las subseñales siguen una escala diádica, la cual depende de la frecuencia de muestreo y del número de niveles de descomposición elegidos.

La función wavelet se puede expresar como:

$$
\Psi_{t, a}\left(t^{\prime}\right)=\frac{1}{\sqrt{a}} \Psi\left(\frac{t^{\prime}-t}{a}\right)
$$

donde $t^{\prime}$ representa el tiempo, $t$ una traslación en el tiempo y $a$ es un factor de escala. 
Matemáticamente, la $C W T$ se define como:

$$
\operatorname{CWT}_{x}^{\Psi}(t, a)=\int_{-\infty}^{\infty} x\left(t^{\prime}\right) \frac{1}{\sqrt{a}} \Psi^{*}\left(\frac{t^{\prime}-t}{a}\right) d t^{\prime}
$$

donde $x$ es la señal a descomponer.

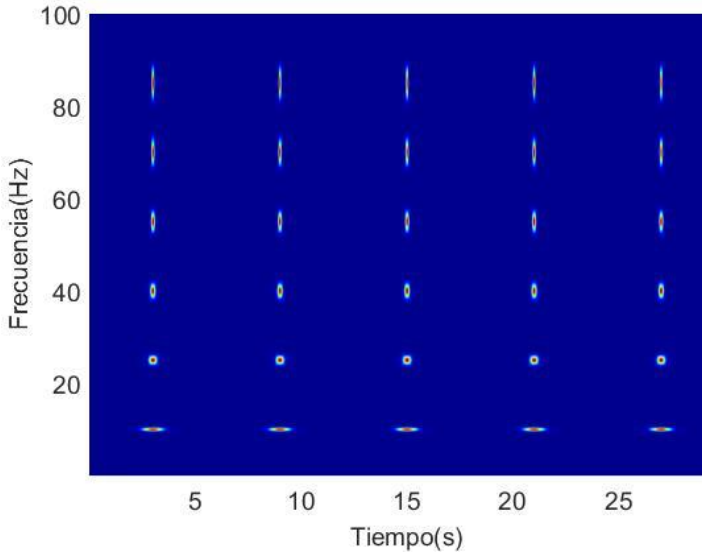

(a) Familia de átomos para generar $C W T$.

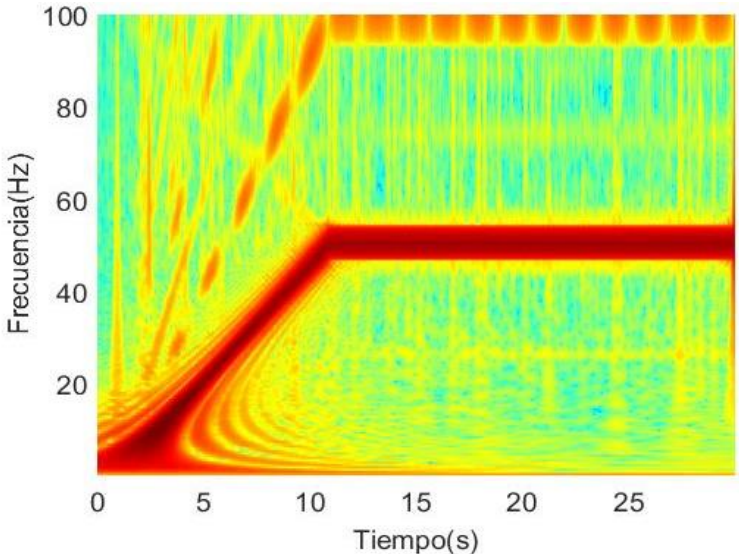

(b) Espectrograma resultante de aplicar $C W T$.

Figura 13. Espectrograma de la $C W T$ de la señal $1(I M \operatorname{con} B R B)$.

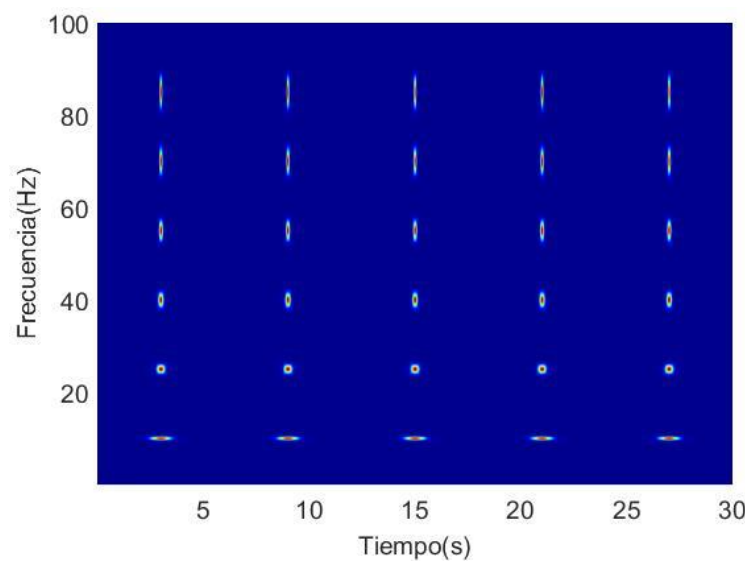

(a) Familia de átomos para generar CWT.

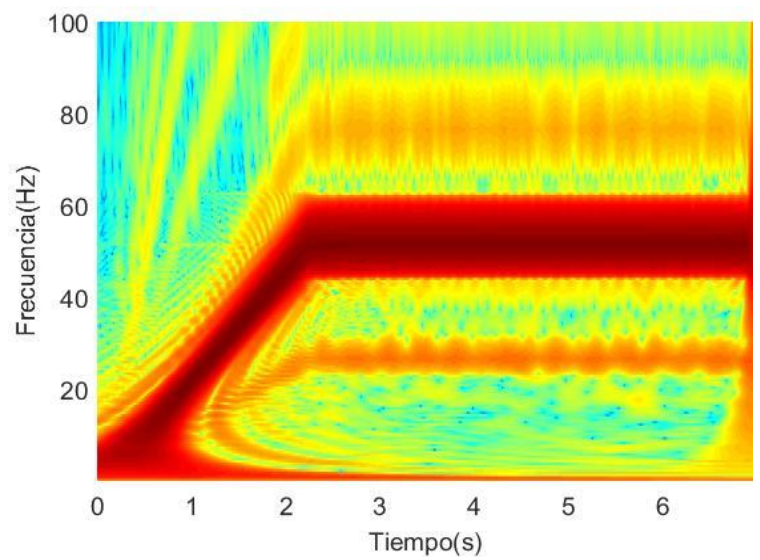

(b) Espectrograma resultante de aplicar $C W T$.

Figura 14. Espectrograma de la $C W T$ de la señal 2 (IM con excentricidad mixta).

Las dos señales propuestas han sido analizadas usando la $C W T$. Los resultados, que se muestran en las Figuras 13 y 14 ilustran las características de esta transformada. Para calcular los espectrogramas resultantes se utiliza una familia de átomos tiempofrecuencia, atendiendo al criterio de la pendiente [58], de tal manera que se optimice la captura de la componente fundamental en el punto donde su frecuencia alcanza los $25 \mathrm{~Hz}$, 
tal y como se ha procedido con la STFT. De esta forma, el compromiso entre la resolución en tiempo y en frecuencia es el mismo que el obtenido con la STFT a $25 \mathrm{~Hz}$ (aunque distinto en el resto). Al aplicar dicho criterio se consigue minimizar el grosor de la componente fundamental a dicha frecuencia.

Las Figuras 13 (a) y 14 (a) representan las familias de átomos que han sido utilizadas para generar los espectrogramas $C W T$ de las señales 1 y 2 . Si se compara esta familia de átomos con los usados para generar los espectrogramas de la STFT (Figuras 11 (a) y 12 (a)), solo coinciden los átomos que se encuentran a la frecuencia de $25 \mathrm{~Hz}$, siendo diferentes para el resto de frecuencias. En el caso de la $C W T$ a bajas frecuencias estos átomos presentan una mayor base, es decir, son largos en tiempo y cortos en frecuencia. A medida que esta frecuencia se va incrementando, se modifican estos átomos haciéndose más altos y con menor base, es decir se van reduciendo en tiempo y aumentando en frecuencia. Esto explica que la resolución tiempo-frecuencia no sea uniforme a lo largo de todo el plano tiempo-frecuencia. Es por esto que con esta transformada se obtiene peor resolución en tiempo a bajas frecuencias y una mayor resolución temporal a altas frecuencias. Con la resolución frecuencial ocurre lo inverso.

En cuanto a la resolución obtenida con la aplicación de la CWT es claro que la resolución en tiempo es peor a frecuencias inferiores a $25 \mathrm{~Hz}$, mientras que la resolución frecuencial mejora. Esto provoca una mayor dispersión en el tiempo de la componente fundamental, ocultando en mayor medida las evoluciones de las componentes de fallo. Por otra parte, se incrementa la resolución temporal a frecuencias superiores a $25 \mathrm{~Hz}$, a costa de empeorar la resolución en frecuencia. No obstante, la tasa de cambio no es lo suficientemente rápida como para ver claramente el efecto al llegar a los $100 \mathrm{~Hz}$ analizados (podría observarse mejor a $1000 \mathrm{~Hz}$ ). Aun así, este cambio en la familia de átomos para calcular la $C W T$ no hace que estos átomos se adapten mejor a las evoluciones a capturar, y por tanto no mejoran el resultado obtenido: en ambas señales, a frecuencias superiores a $25 \mathrm{~Hz}$, se observan evoluciones horizontales que están relacionadas con el régimen estacionario, para las que sería deseable contar con átomos largos en tiempo, cosa que no sucede al trabajar con la $C W T$, cuyos átomos van justamente en tendencia contraria. 
Con respecto al caso de barras rotas, al observar la Figura 13 (b) se aprecia que el primer armónico presenta una alta energía con una larga extensión en tiempo por debajo de $25 \mathrm{~Hz}$, y aumentando su espesor en frecuencia por encima de $25 \mathrm{~Hz}$, lo que impide que se observen los armónicos relativos a fallo de barras. Sin embargo, las trayectorias de los armónicos provocados por excentricidad mixta se desarrollan lejos de la alta energía del primer armónico, por lo que estos sí son observables, sobre todo en la parte de estado estacionario, a pesar de los problemas de resolución que se encuentran, tal como se aprecia en la Figura 14 (b).

Se puede concluir que esta técnica no mejora los resultados que se obtienen mediante la aplicación de la STFT (las componentes de rotura de barra siguen sin observarse, y las de excentricidad mixta se observan peor), por lo que es conveniente evitarla para el análisis de este tipo de señales.

Por otro lado, la formulación matemática de la $D W T$ es:

$$
x(t)=\sum_{i} \alpha_{i}^{n} \varphi_{i}^{n}(t)+\sum_{j=1}^{n} \sum_{i} \beta_{i}^{j} \Psi_{i}^{j}(t)=a_{n}+\sum_{k=1}^{n} d_{k}
$$

donde $x$ es la señal a descomponer, $\alpha_{i}^{n}$ y $\beta_{i}^{j}$ son la escala y coeficiente de la wavelet, $\varphi_{i}^{n}$ es la función de escalamiento en el nivel $n, \Psi_{i}^{j}$ es la función wavelet en el nivel $j, a_{n}$ es la señal de aproximación en el nivel $n$ y $d_{k}$ es la señal de detalle en el nivel $k$. El nivel de descomposición viene dado por $n$.

Ambas señales también se han descompuesto con la $D W T$, pero no se observan las características de las evoluciones de los fallos consideradas en los diferentes detalles, debido a que la componente fundamental se encuentra presente en todos ellos enmascarando al resto de armónicos debido a su elevada amplitud. Es por ello que dichos resultados se han omitido en el presente documento. 
Capítulo 3. Aplicación de las técnicas de análisis tiempo-frecuencia presentes en la literatura técnica: alcance y limitaciones

\section{Adaptive Slope Transform}

La transformada de pendiente adaptable, Adaptive Slope Transform (AST) [55] pertenece a la familia de transformadas lineales, tal como se ha explicado en el punto 2.4. de esta memoria. Su formulación matemática es la siguiente [59]:

$$
\langle h, \emptyset\rangle=\int_{-}^{+\infty} h(t) \emptyset^{*}(t) d t
$$

Es decir, se trata de la correlación de la señal $h$ con un átomo $\emptyset$. La ventana se va adaptando para capturar la energía de la señal según su pendiente, esta ventana es una caja de Heisenberg cuya altura y base se ajustan en cada punto del plano para capturar de manera correcta el armónico de interés, es decir, se aplica el criterio de la pendiente [58].

El mismo enfoque usado con las anteriores transformadas se ha tomado con la $A S T$ [10], con lo que se obtienen las Figuras 15 y 16, que presentan la familia de átomos y espectrogramas resultantes de aplicar esta transformada. Tal y como se ha visto, las dos transformadas lineales previamente analizadas, presentan un compromiso entre la resolución obtenida en tiempo y la obtenida en frecuencia, el cual fija el grosor con el que se obtiene la evolución de la componente, distinto al de la una línea perfectamente fina que represente la evolución física real. En el caso de la STFT, este compromiso es el mismo a lo largo de todo el plano, mientras que en el caso de la $C W T$, el compromiso cambia con la frecuencia analizada de una determinada forma que hace el análisis apto para evoluciones parabólicas. En el caso de la $A S T$, esta transformada permite que exista un compromiso diferente en cada punto del plano, con lo que se consigue optimizar el resultado a lo largo de todo el plano, reduciéndose así el grosor de las evoluciones capturadas en todos sus puntos, mejorando la detección. En motores alimentados directamente desde la red, esto permite obtener la evolución del $L S H$ durante un arranque y las posteriores oscilaciones de carga. En motores alimentados con inversor, su uso es menos crítico, aportando mejoras de menor relevancia.

En el caso del motor con una barra rota, es decir, el correspondiente a la señal 1 (Figura 15), se aplica el criterio de la pendiente a la evolución de la componente 
fundamental durante el arranque, por lo que se trata de reducir al mínimo el espesor con el que se obtiene la evolución de dicha componente durante este intervalo de tiempo. Por otra parte, la $A S T$ permite que estos átomos se vayan adaptando a la evolución de la componente fundamental, haciéndose más largos en tiempo según se va entrando en el estado estacionario, para lograr un aumento en la resolución frecuencial, que es lo necesario cuando las evoluciones son horizontales (Figura 15 (a)). Más concretamente, a partir del instante $11 \mathrm{~s}$, se aumenta la resolución frecuencial gracias a la adaptabilidad de la $A S T$, disminuyendo el grosor de la componente fundamental en el sentido del eje de frecuencias (Figura 15 (b)). Como se ha visto en el apartado 2 (concretamente en la Figura 6 del epígrafe 2.4. de esta memoria), las evoluciones de los armónicos de fallo debidos a rotura de barra son casi paralelas a la evolución de esta componente fundamental. Por lo tanto, los átomos utilizados para las componentes de fallo y la componente fundamental son los mismos, como resultado de aplicar el criterio de la pendiente (Figura 15 (a)). Es por ello que, los átomos sólo cambian para esta corriente en función del tiempo, siendo idénticos si comparamos átomos a distintas frecuencias, pero centrados en un mismo instante. Las componentes de fallo de barras y la componente fundamental están demasiado cercanas, lo cual impide el poder observar la trayectoria de estos armónicos de fallo durante el arranque, tal como se aprecia en la Figura 15 (b), a pesar de haber minimizado el espesor de la componente fundamental con el criterio de la pendiente. No obstante, los armónicos debidos a fallo de barras son fácilmente observables durante el estado estacionario, tal como se muestra en la Figura 15 (b), mejorando la distinción con respecto al resultado de la $S T F T$.

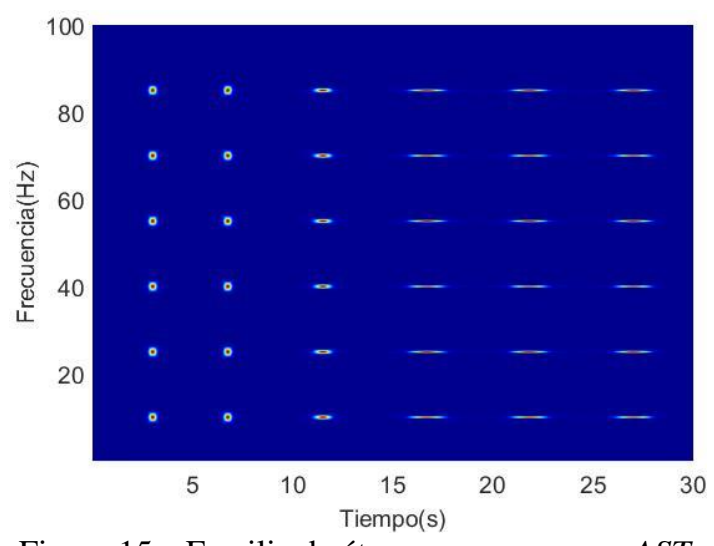

Figura 15a. Familia de átomos para generar $A S T$.

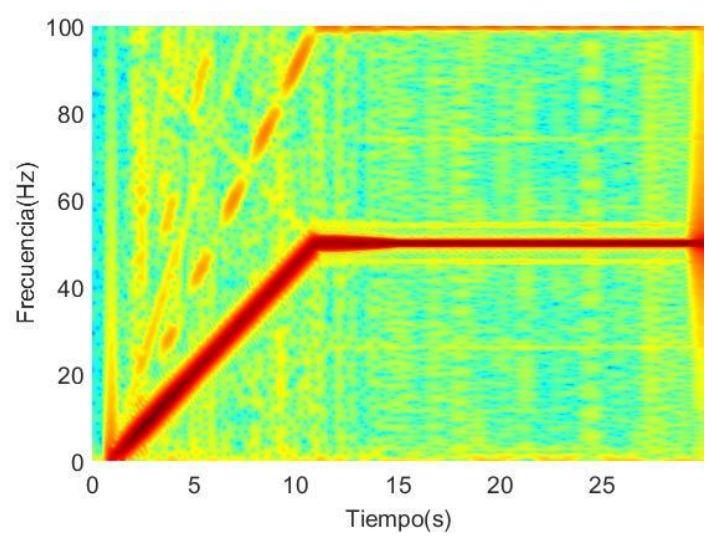

Figura 15b. Espectrograma resultante de aplicar AST.

Figura 15. Espectrograma $A S T$ de la señal 1 (IM con $B R B)$. 
Capítulo 3. Aplicación de las técnicas de análisis tiempo-frecuencia presentes en la literatura técnica: alcance y limitaciones

Análogamente a lo anteriormente expuesto, ocurre cuando analizamos el motor que presenta excentricidad mixta (señal 2, Figura 16). En la Figura 16 (a) se muestra cómo, mediante la aplicación del criterio de la pendiente, se adaptan las pendientes de los átomos tiempo-frecuencia a la evolución de la componente fundamental durante el transitorio de arranque y el posterior permanente, para minimizar el grosor con el que se obtiene dicha evolución (esta adaptación es análoga al caso anterior con barras rotas). Aunque las evoluciones de las componentes de excentricidad mixta ya no son estrictamente paralelas a la de la componente fundamental, como en el caso de barras rotas, las pendientes de estas componentes no son excesivamente diferentes. Es por ello que se aplica el mismo compromiso de resolución tanto en la componente fundamental como en las evoluciones de excentricidad mixta. Como consecuencia, los átomos a las distintas frecuencias de un mismo instante de tiempo son los mismos. Los átomos se van afinando (Figura 16 (a)), empezando a aumentar su base en instantes posteriores a 2,2 segundos, adquiriendo una resolución en frecuencia óptima al llegar a 3 segundos (Figura 16 (b)), lo que permite disminuir el grosor de las evoluciones medida paralelamente al eje de frecuencias, y por lo tanto poder observar de manera más precisa la frecuencia de los armónicos durante el estado estacionario, con lo que se pueden separar y distinguir mejor las frecuencias.

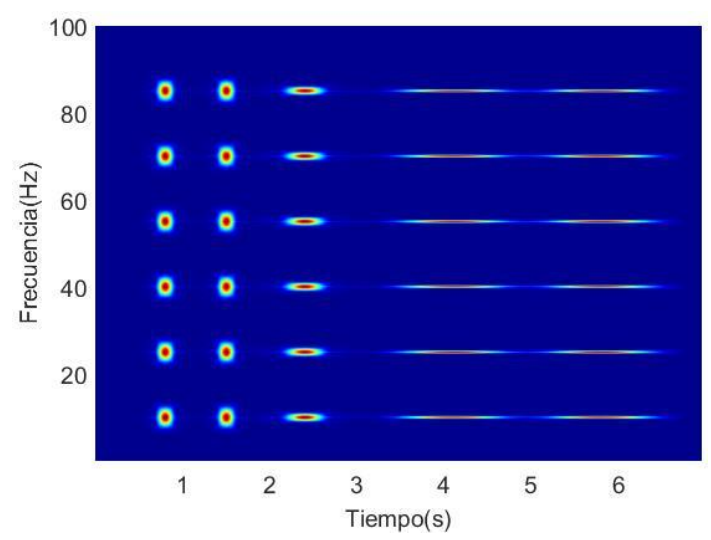

(a) Familia de átomos para generar AST.

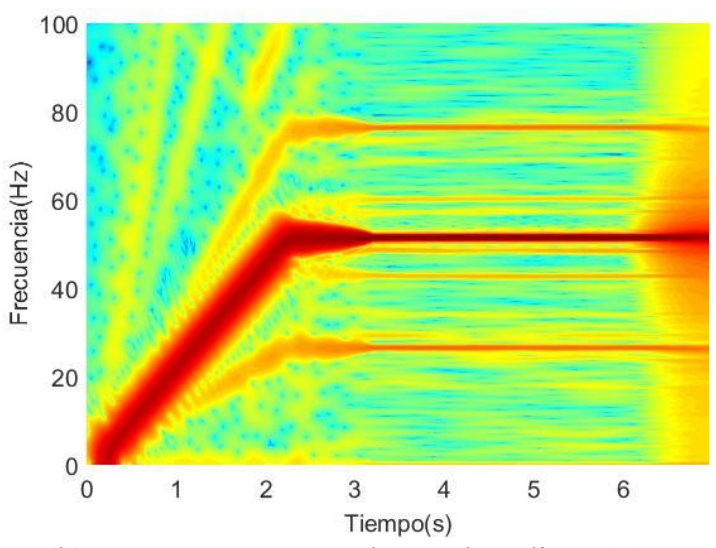

(b) Espectrograma resultante de aplicar $A S T$.

Figura 16. Espectrograma AST de la señal 2 (IM con excentricidad mixto).

Por lo tanto, con la $A S T$, la forma del átomo se adapta dinámicamente a la pendiente de las trayectorias de los armónicos de fallo en el plano tiempo-frecuencia. En el caso de motores alimentados con inversor, esto se traduce en una adaptación a la evolución de la componente fundamental, a medida que esta cambia con el tiempo, pero 
manteniendo las características del átomo a las otras frecuencias, pues las otras evoluciones son paralelas (barras rotas) o con pendientes parecidas (excentricidad mixta). Esto permite obtener una buena resolución tanto durante el arranque como en el régimen permanente. No obstante, esto no resulta suficiente para observar las evoluciones de los armónicos de barras rotas durante el arranque, o para ver dichas evoluciones completas en el caso de excentricidad mixta, debido a que incluso capturándola de forma óptima, la componente fundamental aparece representada con una cierta dispersión en el arranque, enmascarando a las componentes de fallo. Esta transformada marca más la diferencia en motores alimentados desde la red, debido a que la componente fundamental y los armónicos vinculados a fallos tienen en este caso trayectorias muy distintas.

\section{Chirplet Transform}

Si el átomo tiempo-frecuencia, que se ha venido mencionando, se define por una función Chirplet, la transformada lineal con estos átomos se conoce como Transformada Chirplet (Chirplet Transform, CT) [3], tal y como se explica en el punto 2.4.

En el resultado de una transformada lineal existe la dispersión de la energía de una componente alrededor de su evolución real. Esto, que se traduce en la representación de dicha evolución con un determinado grosor, se puede medir en la dirección del eje del tiempo, o en la dirección del eje de frecuencia. Si se usa un átomo largo en tiempo, es decir, con una gran base, entonces tratamos de disminuir el grosor de la línea en el eje de frecuencias; sin embargo, el grosor medido en la dirección del eje del tiempo aumentará. Ocurre lo opuesto si el átomo es largo en frecuencia, es decir, alto y con poca base: se trata de disminuir el grosor medido paralelamente al eje de tiempo, pero se aumenta dicho grosor medido paralelamente al eje de frecuencias. Todos estos átomos utilizados en apartados anteriores tienen una dispersión de la energía alrededor de su centro que se puede representar esquemáticamente mediante un rectángulo. Las funciones chirplet distribuyen su energía alrededor de su centro de forma muy distinta: en vez de formar un cuadrado, forman un rectángulo muy fino que se encuentra inclinado (Figura 17 (a)); la inclinación de dicho rectángulo viene determinada por el usuario. Así pues, se puede decir que las funciones chirplet son átomos tiempo-frecuencia cuya energía se distribuye desde su centro a lo largo de una dirección determinada. Como consecuencia, al trabajar con la 
$C T$, la dispersión de la energía de la componente aparece a lo largo de su evolución cuando esta sigue una línea recta, si el átomo escogido tiene una inclinación igual a la de dicha recta, con lo que se logra un menor espesor de la línea que traza la evolución de la componente. En definitiva, la $C T$, al contrario que las transformadas lineales anteriores, no tiene un compromiso de resolución en el tiempo y en la frecuencia, cuando se trata de capturar evoluciones de componentes que trazan trayectorias en línea recta.

El principal problema que presenta esta transformada es que se debe elegir a lo largo de qué dirección del plano tiempo-frecuencia se va a dispersar la energía, o en otras palabras, se debe elegir la inclinación del átomo, siendo esta la misma en cualquier punto del plano donde centremos un átomo para analizarlo. La $C T$ puede ser aplicada para optimizar la captura de un armónico con una evolución rectilínea, eligiendo un átomo tal que disperse energía en la dirección en la que evoluciona la componente, para así tener una evolución del armónico lo más delgada posible en el resultado de la transformada. El problema aparece cuando se encuentran otros armónicos que, si bien están evolucionando en líneas rectas, presentan diferentes pendientes: la captura no sería óptima para éstos. De la misma forma, tampoco será óptima la captura de la evolución de la componente, si en algún momento deja de ser rectilínea. Así, en los casos que se están analizando se ha escogido que los átomos utilizados se inclinen en la dirección de la componente fundamental durante el transitorio de arranque, ajustándose, a lo largo de este estado, perfectamente a la misma (Figuras 17 y 19).

Atendiendo ahora a la señal 1 (Figura 17), como se acaba de comentar, se aplica la $C T$ para la captura de la $F C$ durante el arranque (Figura 17 (a)). Como consecuencia, su evolución durante el arranque aparece (Figura 17 (b)) como una línea muy delgada. Como se aprecia en la Figura 6 del apartado 2.4., las trayectorias de los armónicos debidos a fallo de barra son casi paralelas a la componente fundamental. Por lo tanto, el análisis de $C T$, que optimiza la captura de la $F C$ durante el arranque, también optimiza la captura de estos armónicos durante el arranque. Como resultado, la resolución durante el arranque es óptima para seguir la evolución de los $L S H$ y $U S H$ (ver zoom, Figura 18), pudiendo distinguirlas a pesar de encontrarse muy próximas. Esta ha sido la primera técnica en conseguir detectar componentes de barra rota durante el arranque de un motor alimentado por inversor. No obstante, los resultados durante el estado estacionario son mucho peores. 
Aquí se puede notar la pendiente y la forma del átomo porque la energía de la componente fundamental se extiende sobre una amplia gama de frecuencias en la dirección de inclinación del átomo, no permitiendo la observación, durante el régimen peramente, de los armónicos debidos a fallo de barras.

Las Figuras 19 y 20 muestran la aplicación de la $C T$ para el motor con excentricidad mixta (señal 2). En este caso se han aplicado dos pendientes distintas: ajustando a la evolución de la componente fundamental (Figura 19) y ajustando a la evolución del armónico de excentricidad mixta inferior (Figura 20).

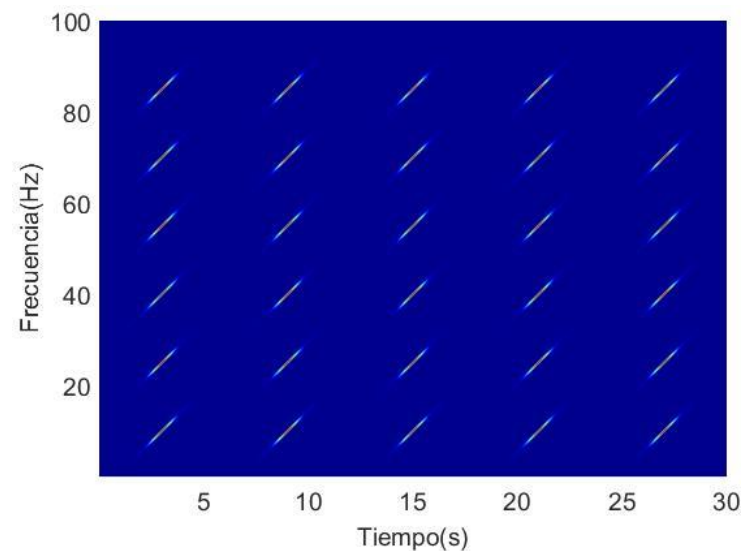

(a) Familia de átomos para generar la transformada Chirplet.

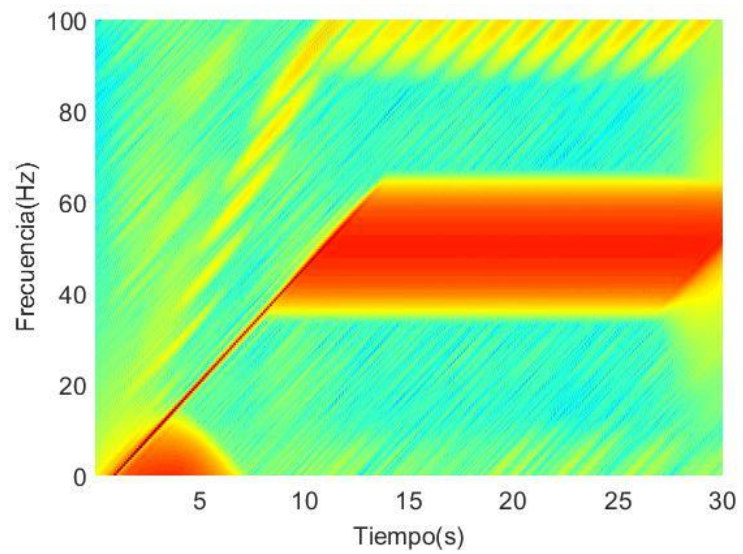

(b) Espectrograma resultante de aplicar la transformada Chirplet.

Figura 17. Resultado de la aplicación de la transformada Chirplet a la señal 1.

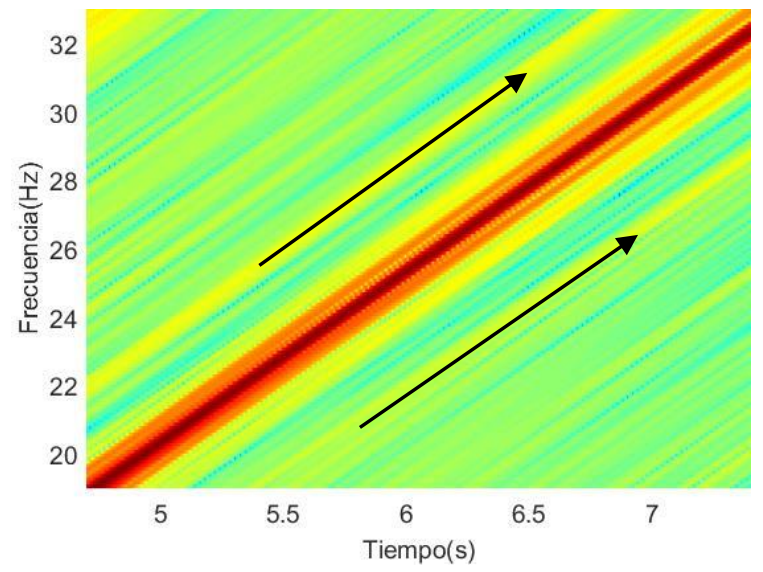

Figura 18. Zoom de la Figura 17 (b), entre los instantes 4,7 y 7,4s.

En el caso de la Figura 19, se ha aplicado la $C T$ para capturar de manera óptima la evolución de la componente fundamental durante el transitorio de arranque. Para ello, se han utilizado átomos cuya energía se dispersa a lo largo de la dirección de la evolución 
de la componente fundamental durante este arranque, tal como se muestra en la Figura 19 (a). No obstante, las trayectorias de los armónicos de excentricidad mixta presentan una pendiente distinta a la de la componente fundamental (tal como se puede comprobar gráficamente viendo la Figura 6 del apartado 2.4. de la memoria). Así pues, los átomos escogidos se adaptan a la evolución de la componente fundamental, pero no a la de los armónicos de excentricidad mixta; por esta razón, sus evoluciones aparecen menos nítidas. Aun así, se observan las evoluciones durante un intervalo de tiempo mayor, pues el fundamental tiene menos dispersa su energía. Finalmente, la pendiente elegida hace que la energía del primer armónico y de los armónicos de excentricidad mixta se extienda sobre un amplio rango de frecuencias al capturar el estado estacionario, tal como se ha explicado que ocurre en la Figura 17 (b), dispersando su energía en la dirección del átomo escogido: la del fundamental durante el arranque.

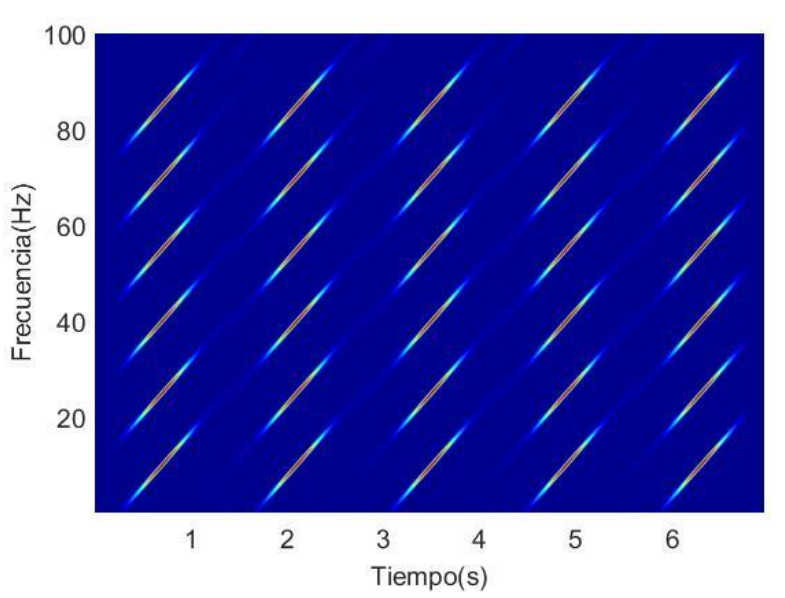

(a) Familia de átomos para generar la transformada Chirplet.

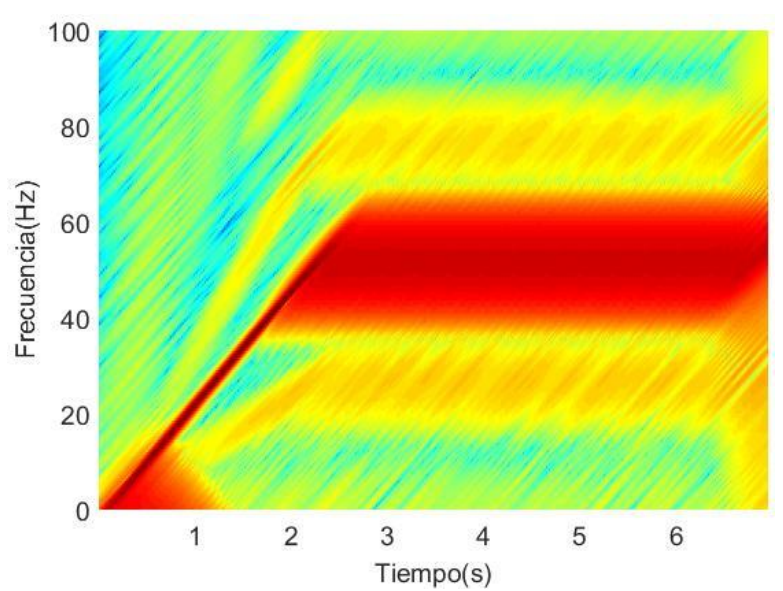

(b) Espectrograma resultante de aplicar la transformada Chirplet.

Figura 19. Resultado de la aplicación de la transformada Chirplet a señal 2.

Para obtener el espectrograma de la Figura 20 (b), la pendiente del átomo tiempofrecuencia, tal como se aprecia en la Figura 20 (a), fue adaptada a la pendiente de la componente $E R H(-)$ durante el arranque, es decir, la energía de los átomos usados dispersa a lo largo de la dirección de este armónico. Por tanto, la evolución de este armónico durante el arranque aparece representada con una línea delgada, en contra de lo que ocurre con el $E R H(+)$ y con la componente fundamental, cuya evolución aparece con una línea más gruesa, pues en este caso el átomo no se adapta de forma óptima a estas dos componentes. Como ésta pendiente es menor que la utilizada en la Figura 19, asemejándose más a los átomos horizontales necesarios para analizar un régimen 
permanente, se mejoran los resultados relativos a dicho período, en donde, pese a no tener una resolución muy fina, pueden distinguirse los armónicos debidos a excentricidad, así como el primer armónico.

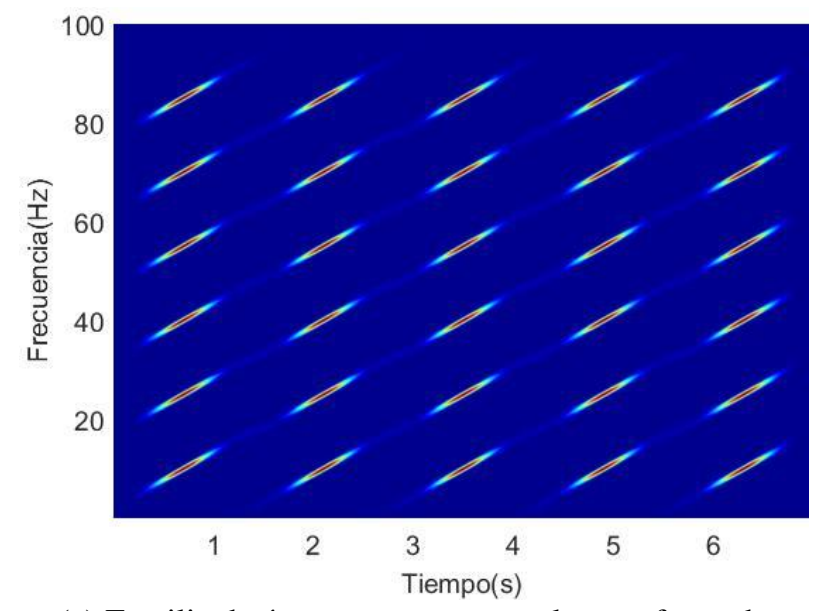

(a) Familia de átomos para generar la transformada Chirplet.

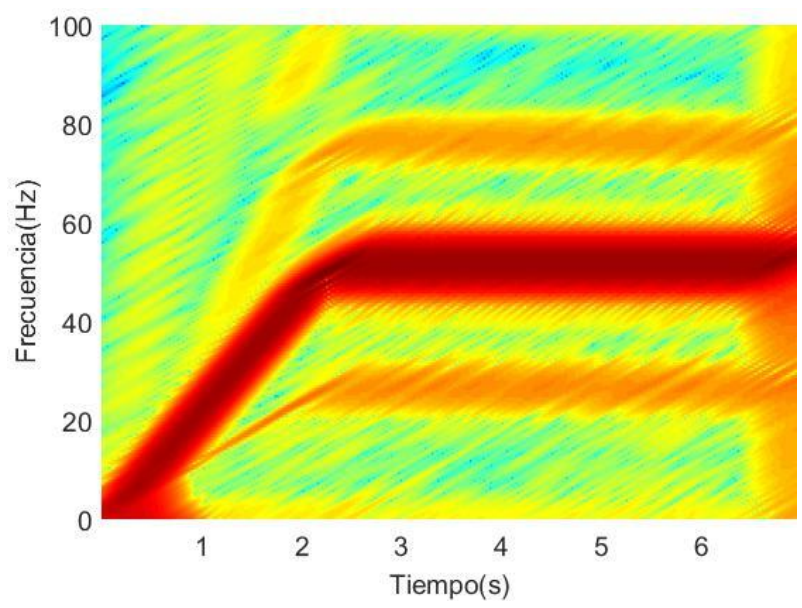

(b) Espectrograma resultante de aplicar la transformada Chirplet.

Figura 20. Resultado de la aplicación de la transformada Chirplet a la señal 2 con diferente pendiente.

\section{Wigner Ville Distribution}

La Wigner Ville Distribution (WVD) fue definida para obtener una distribución de la energía en el plano tiempo-frecuencia sin pérdida de resolución, tal como se explica en el apartado 2.4. Se trata de una técnica, que, a diferencia de las anteriores, no se basa en átomos tiempo-frecuencia. Esto evita el compromiso entre resolución en tiempo y en frecuencia que los átomos introducen (fijo para la STFT, variable en frecuencia para la $C W T$, adaptable en todo el plano para la $A S T$ y óptimo en una dirección del plano para la $C T)$. Así pues, la $W V D$ trata de obtener las evoluciones como líneas perfectamente finas, evitando el grosor típico de las transformadas lineales (salvo la $C T$, que consigue optimizar en una dirección).

La distribución de Wigner de una señal $x(t)$ se expresa de la siguiente manera [60]:

$$
W(t, \omega)=\frac{1}{2 \pi} \int_{-\infty}^{+\infty} x\left(t+\frac{\tau}{2}\right) x^{*}\left(t-\frac{\tau}{2}\right) e^{-j \omega \tau} d \tau
$$


La versión discreta de la distribución de Wigner Ville se puede expresar de la siguiente manera [61]:

$$
W_{x}[f, n]=\sum_{m=0}^{N-1} x[n+m] x \cdot[n-m] e^{-j \pi f m}
$$

Siendo $n$ el tiempo discreto y $m$ el desfase de tiempo discreto. Por lo tanto, la distribución de Wigner es la FT de la autocorrelación de la señal de entrada, no siendo una operación lineal, causando así la aparición de términos cruzados.

Por tanto, esta distribución funciona bien en señales unitarias, siendo el principal problema de esta transformada la aparición, en señales multicomponentes, de los denominados términos cruzados. Más concretamente, en el resultado de la transformada aparece un término ficticio, que físicamente no está presente en la señal analizada, por cada par de componentes reales de la señal. En motores alimentados directamente desde la red, este problema se ha reducido filtrando antes la componente fundamental, así como sus múltiplos. También se ha propuesto el uso de WVD modificadas, en las que se aplica una función llamada núcleo, que suaviza el resultado, eliminando términos cruzados, pero introduciendo el compromiso entre resolución tiempo y frecuencia, que precisamente se pretendía evitar con esta transformada. Con respecto a motores alimentados por inversor, no existen trabajos al respecto, probablemente debido a que al presentar la componente fundamental una frecuencia variable, no es posible ni aplicar un filtro ni definir con facilidad una función núcleo que elimine los términos cruzados.

Las distribuciones de Wigner Ville de las señales analizadas se muestran en las Figuras 21 y 23. Estas señales contienen muchas componentes, lo cual implica la aparición de muchos términos cruzados. La presencia de términos cruzados impide la observación de las trayectorias de los armónicos de fallo, creando además confusión al interpretar la distribución de energía de la señal, debido a su carácter ficticio.

En la Figura 21, donde se analiza la señal 1, en el estado estacionario se pueden distinguir los armónicos de barras rotas (ver zoom, Figura 22); sin embargo, durante el régimen transitorio se hace imposible seguir la trayectoria de los componentes de fallo, ya que los términos cruzados impiden poder visualizarlos. 
Capítulo 3. Aplicación de las técnicas de análisis tiempo-frecuencia presentes en la literatura técnica: alcance y limitaciones

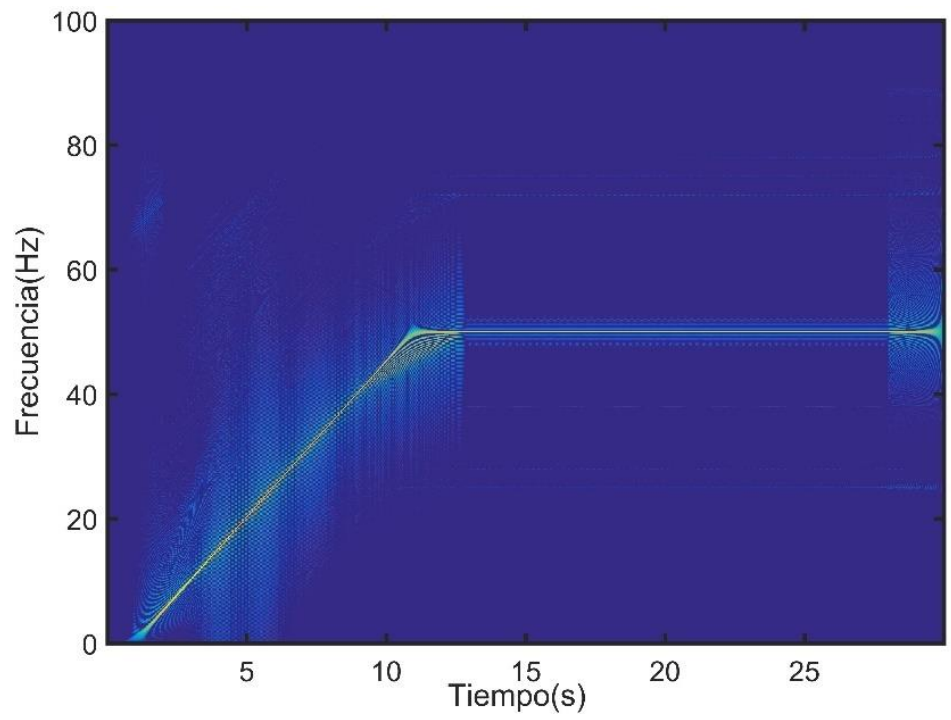

Figura 21. Resultado de la aplicación de la transformada $W V D$ señal 1.

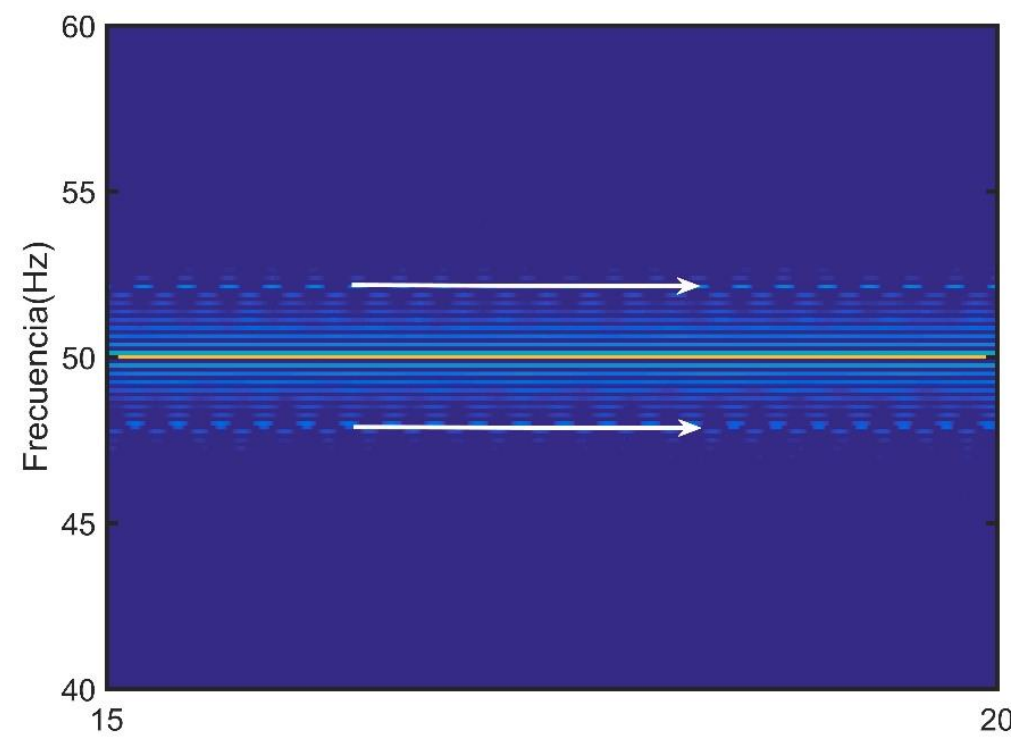

Figura 22. Zoom entre los segundos 15 y 20 del espectrograma de la Figura 21.

Con respecto al resultado de analizar la señal 2 (Figura 23), se intuyen las evoluciones de las componentes de excentricidad mixta, pero de forma débil, y confundidas por la presencia de numerosos términos cruzados. 
Capítulo 3. Aplicación de las técnicas de análisis tiempo-frecuencia presentes en la literatura técnica: alcance y limitaciones

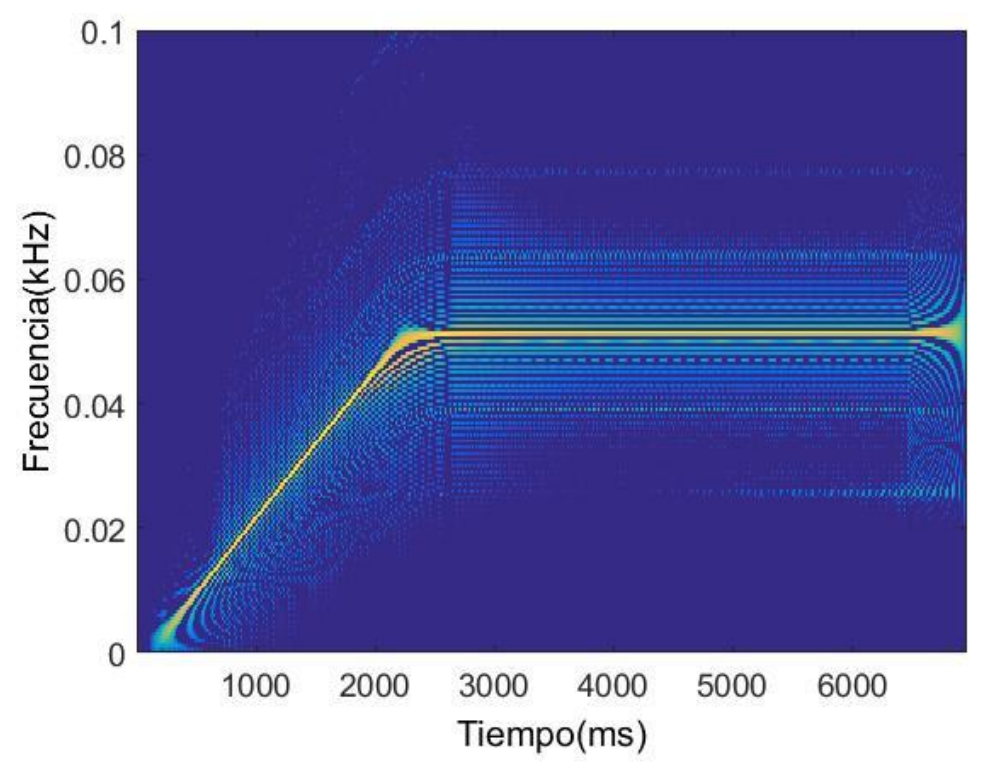

Figura 23. Resultado de la aplicación de la transformada WVD señal 2.

\section{Hilbert-Huang Transform}

La transformada de Hilbert-Huang (Hilbert-Huang Transform, HHT), es una técnica empírica, que, como se ha visto en el apartado 2.3., supone una combinación del análisis espectral de Hilbert o transformada de Hilbert (Hilbert Transform, HT) y la descomposición de modo empírico (Empirical Mode Decomposition, EMD). Esta transformada puede trabajar con señales no estacionarias y no lineales.

La $E M D$ es una descomposición adaptativa, basada en características locales de la señal, que descompone dicha señal en un número finito de funciones de modo intrínseco (Intrinsic Mode Funcions, IMF). Estas funciones son señales casi monocomponentes, y por tanto adecuadas para ser analizadas con la $H T$.

Sea la señal $x(t)$ una de las $I M F$, se considera como la parte real de una $z$ compleja, cuya parte imaginaria se obtiene aplicando la $H T$ a la señal $x(t)$, dada por:

$$
y(t)=\frac{1}{\pi} \int_{-\infty}^{+\infty} \frac{x(\tau)}{t-\tau} d \tau
$$


La parte compleja o señal analítica se define como sigue:

$$
\mathrm{z}(\mathrm{t})=\mathrm{x}(\mathrm{t})+\mathrm{i} \cdot \mathrm{y}(\mathrm{t})
$$

La amplitud y la fase de $z$ se definen como funciones analíticas de la variable $t$. La frecuencia instantánea se calcula como sigue:

$$
\omega(t)=\frac{d \theta(t)}{d t}
$$

Donde $\theta(t)$ es la fase de $z$. El espectro de Hilbert-Huang (Hilbert Huang Spectrum, $H H S$ ) es la representación tiempo-frecuencia de la amplitud de z.

La transformada de Hilbert permite identificar amplitudes y frecuencias instantáneas de las señales monocomponentes $(I M F)$. Si se aplica directamente a la señal original, la cual es multicomponente, los resultados serían deficientes.

Los inconvenientes de la $E M D$ son la presencia de efectos de borde, el mezclado de modos, o un criterio de parada incierto. Estos problemas se han tratado de resolver definiendo la EEMD (Ensemble EMD) o la CEEMD (Complete Ensemble EMD).

Las dos señales propuestas en este trabajo han sido analizadas con esta técnica. Ambas señales se descomponen en $I M F$ con la versión EMD propuesta en [62] y con los siguientes parámetros: desviación estándar de ruido: 0.1; 100 etapas; 200 iteraciones y mismo nivel de ruido en todas las etapas. En el caso del motor con una barra de rotor rota, el EMD descompone en $16 I M F$, pero falla en la separación de los modos de oscilación correspondiente al LSH y USH del modo correspondiente a la componente fundamental. Sus frecuencias y modos de oscilación están demasiado cercanos, y el algoritmo EMD no es capaz de separarlos. 
IMF 1

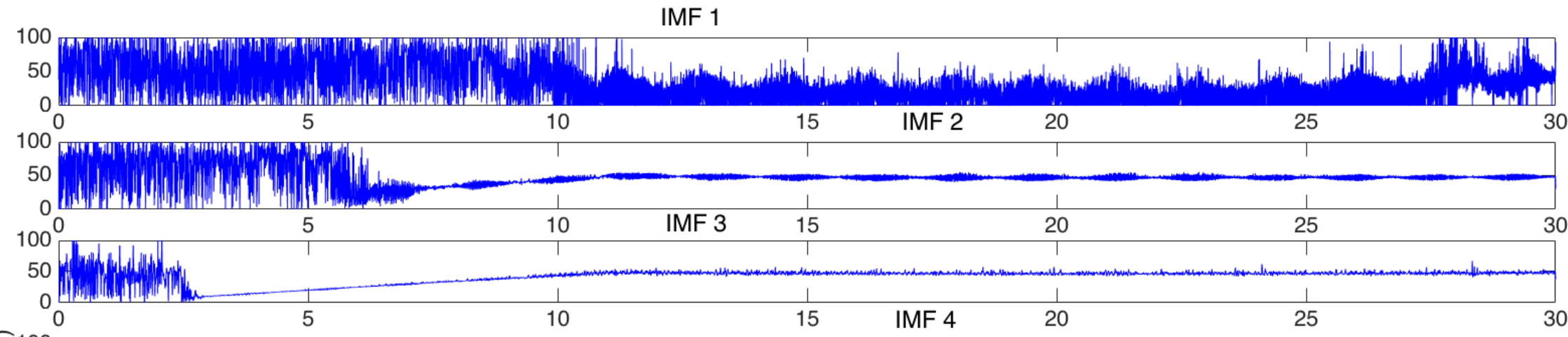

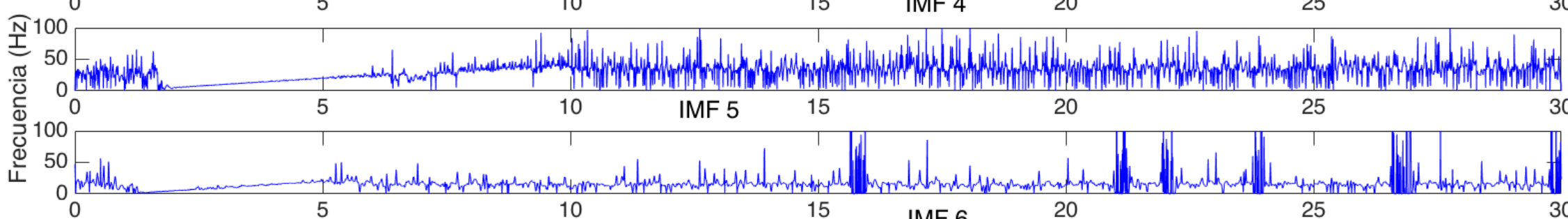
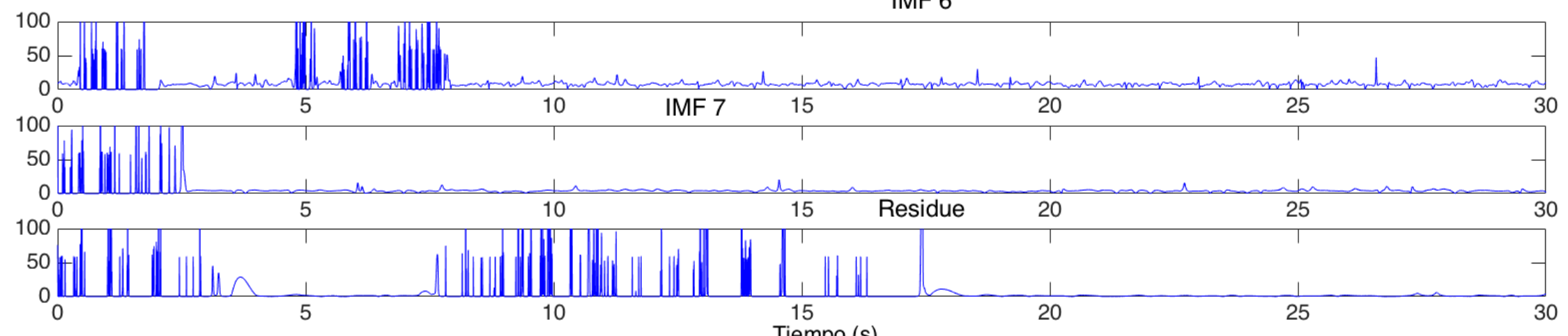

(a) Frecuencia instantánea calculada para cada $I M F$.
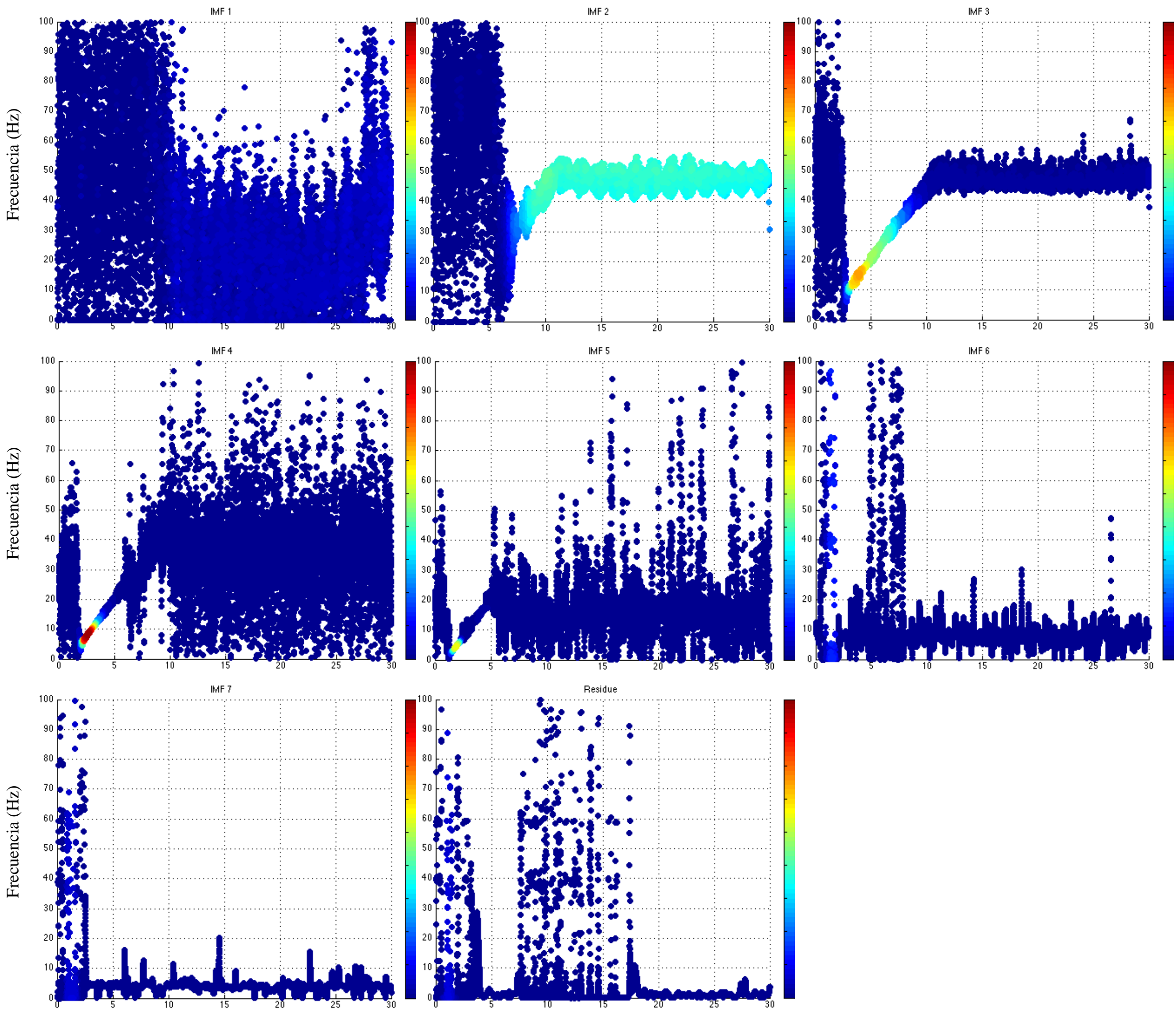

Tiempo (s)

(b) Espectros resultantes de aplicar $H H$ a cada IMF

Figura 24. Resultado de aplicar $H H T$ a la Señal 1. 
En la Figura 24 se puede apreciar cómo esta técnica no es capaz de extraer todos los modos, con lo que no proporciona información suficiente para determinar fallo o no en el motor, para lo que se ilustran 8 de las $I M F$ en las que se ha descompuesto la señal.

La señal del motor con excentricidad mixta, señal 2, se descompone en 12 IMF. La IMF número 4 contiene el modo de oscilación correspondiente a la $E R H p$, pero el ERHn no está separado de la componente principal. El IMF 4 y su espectro de HilbertHuang, mostrando el ERHp, se representan en las Figuras 25 (a) y (b) respectivamente.

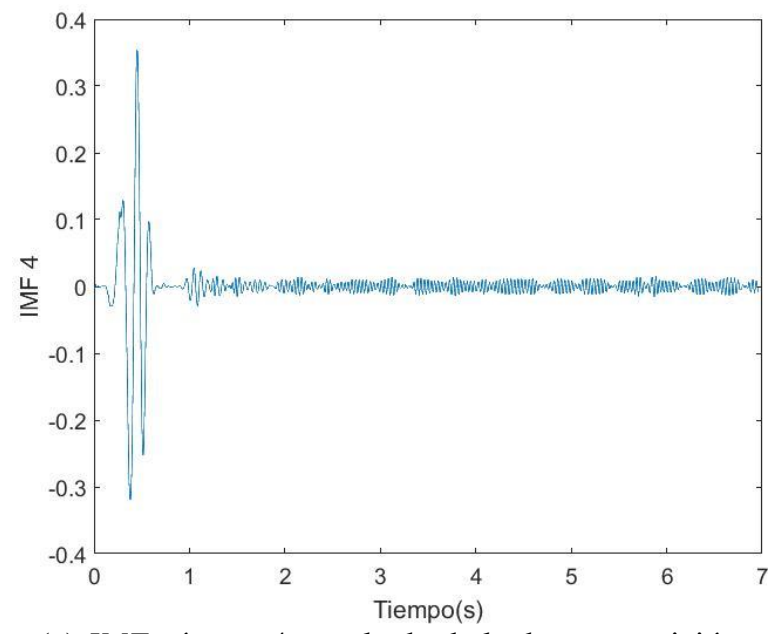

(a) IMF número 4, resultado de la descomposición con CEEMD de señal 2.

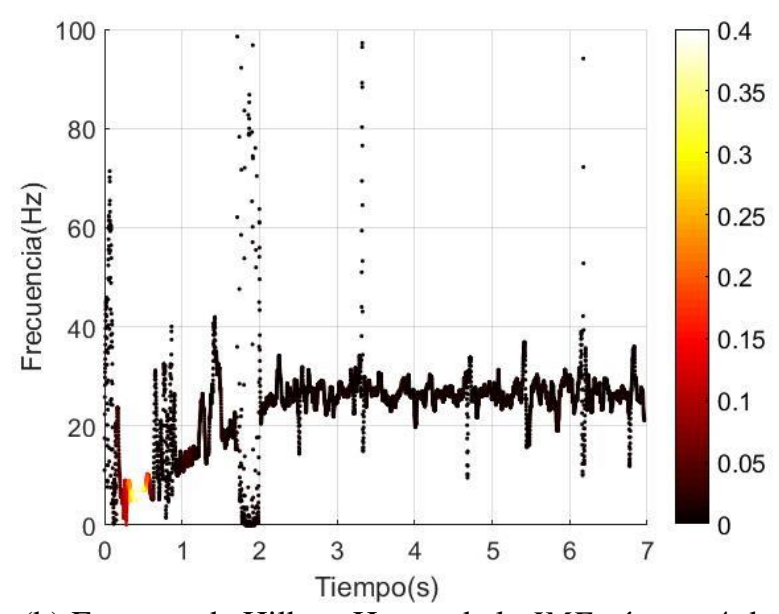

(b) Espectro de Hilbert-Huang de la $I M F$ número 4 de la señal 2, mostrando la componente armónica $E R H p$ relativa a la excentricidad mixta.

Figura 25. Resultado de aplicar la transformada $H H T$ a la señal 2.

\section{Multiple Signal Classification}

Este algoritmo, también conocido como MUSIC, proporciona un pseudo-espectro de la señal analizada con alta resolución. Tal como se detalla en el apartado 2.4. El pseudo-espectro MUSIC se calcula teniendo en cuenta la ortogonalidad de los vectores de modo de frecuencia a los eigenvectores de subespacio ruido, dado por:

$$
R=\frac{1}{N} \sum_{n=0}^{N-1} x[n] x[n]^{\prime}
$$


Capítulo 3. Aplicación de las técnicas de análisis tiempo-frecuencia presentes en la literatura técnica: alcance y limitaciones

donde $x[n]$ es la señal muestreada. La matriz de autocorrelación $R$ es la suma de matrices de autocorrelación de señal y ruido. Los vectores de modo frecuencia son ortogonales a los vectores propios de la matriz de subespacio de ruido.

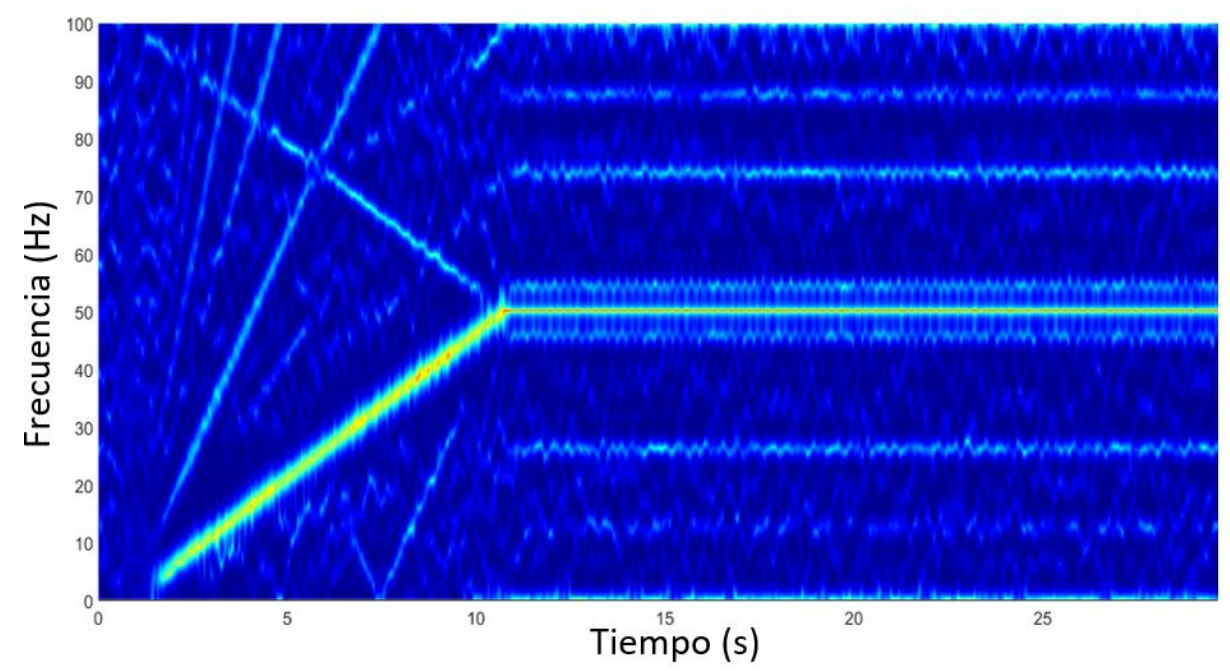

Figura 26. Espectrograma $M U S I C$ de la señal $1(I M \operatorname{con} B R B)$.

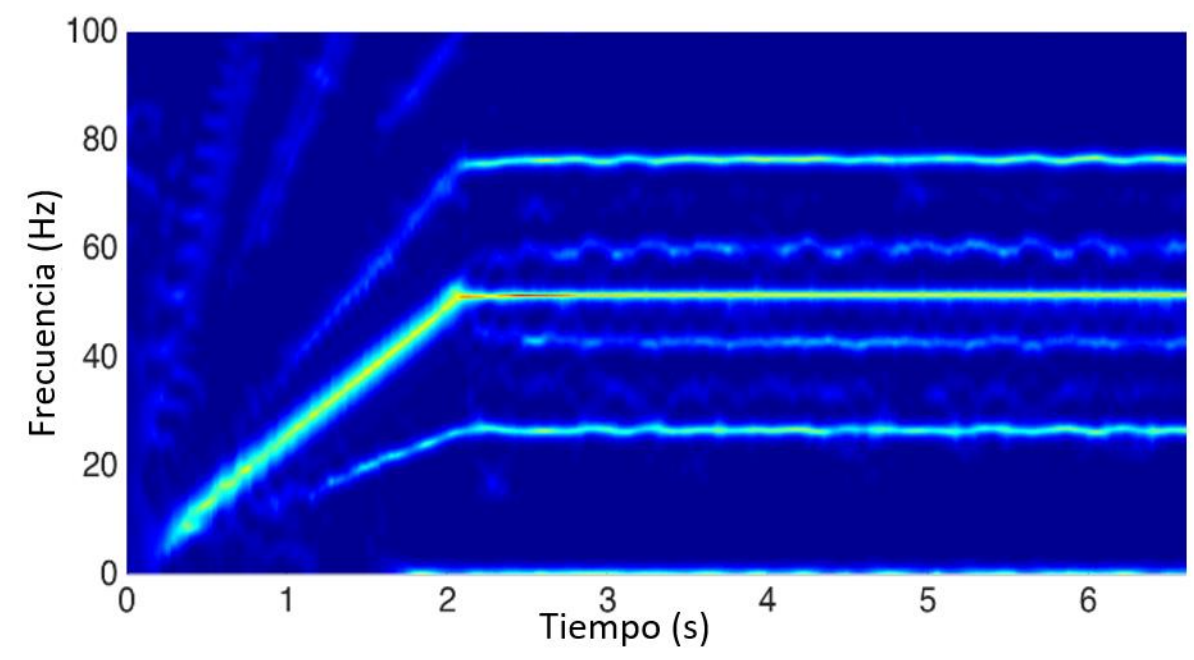

Figura 27. Espectrograma MUSIC de la señal 2 (IM con excentricidad mixta).

En la Figura 26 se ilustra el espectrograma resultante de aplicar MUSIC a la señal 1: se pueden apreciar los armónicos de fallo debidos a barras rotas de manera clara en el estado estacionario; sin embargo, no se tiene suficiente resolución para apreciar el estado transitorio, por lo que, en este caso, la técnica no es lo suficientemente potente para poder diagnosticar el fallo en el IMI. 
Capítulo 3. Aplicación de las técnicas de análisis tiempo-frecuencia presentes en la literatura técnica: alcance y limitaciones

e igual manera, se ha aplicado esta técnica a la señal 2, cuyo resultado se tiene en la Figura 27, donde se pueden observar con una buena resolución los armónicos debidos a la excentricidad mixta, tanto en el estado estacionario como en el transitorio.

\subsection{Conclusiones: comparativa, alcance y limitaciones de cada técnica.}

Una vez se han aplicado las distintas transformadas presentes en la literatura técnica a la detección de barras rotas y excentricidad mixta en dos motores de inducción alimentados por convertidor, se pueden extraer las siguientes conclusiones.

En primer lugar, las transformadas lineales introducen un compromiso entre la resolución en tiempo y en frecuencia que impide observar las evoluciones de las componentes como líneas perfectamente finas, de acuerdo con su evolución física real, sino que presentan un determinado grosor al aparecer representadas en el resultado de la transformada. Dicho compromiso es constante en la STFT, variable en frecuencia, pero con una variación inadecuada para la señal a analizar, en la $C W T$ y completamente adaptable en la $A S T$. Incluso al utilizar una adaptabilidad absoluta del compromiso a lo largo del plano t-f, la optimización del resultado no es suficiente para obtener las evoluciones de las componentes cuando estas se encuentran especialmente cerca (como es el caso de los armónicos de barras rotas durante el arranque).

Si se emplea la transformada $C T$, esta sí permite distinguir evoluciones muy cercanas, pero solo en el caso de que estas sean rectilíneas y que evolucionen siempre de forma paralela.

Las opciones por fuera de las transformadas lineales no aportan mejores resultados: la $W V D$ enmaraña el plano con la aparición de los términos cruzados, lo que imposibilita en muchos casos la detección de evoluciones, la HHT no consigue separar las componentes cuando estas tienen evoluciones muy cercanas y MUSIC tampoco alcanza la resolución suficiente para distinguirlas en este mismo caso.

Por tanto, y después de analizar ambas señales con cada una de las técnicas, se ve que existe una necesidad de seguir investigando hacia una técnica apropiada para la 
Capítulo 3. Aplicación de las técnicas de análisis tiempo-frecuencia presentes en la literatura técnica: alcance y limitaciones

identificación de fallos en motores de inducción alimentados mediante inversor en estados transitorios, que sea capaz de obtener las evoluciones completas de las componentes, tanto en arranques, como en permanentes o en transitorios arbitrarios, con una resolución suficientemente elevada como para poder distinguirlas incluso cuando se encuentran especialmente próximas. 


\section{Metodología Propuesta}

4.1. Introducción

4.2. Familia de átomos tiempo frecuencia ajustados a las evoluciones arbitrarias de los armónicos a seguir

4.2.1. Introducción

4.2.2. Definición, características principales y tipos de átomos tiempofrecuencia

4.2.3. Átomos tipo Dragón

4.3. Transformada Dragón

4.3.1. Definición de la transformada Dragón

4.3.2. Ventajas e inconvenientes de la Transformada Dragón

4.3.3. Cuantificación 


\section{Metodología Propuesta}

\subsection{Introducción}

Este capítulo dedicado a la metodología de diagnóstico propuesta, es el punto central en torno al cual se articula toda la tesis. El objetivo de la misma es proponer una técnica de procesamiento de señales no estacionarias, basada en el uso de los átomos tiempo-frecuencia, cuya forma se adapte perfectamente a la evolución de los armónicos de fallo, para así conseguir una representación de sus evoluciones con la máxima precisión posible, y poder detectarlas de forma completa incluso en el caso de motores de inducción alimentados por inversor, cuando estos se encuentran especialmente próximos.

El capítulo se divide en dos apartados. En el primer apartado se va a profundizar en el estudio de los átomos tiempo-frecuencia. El estudio de sus características permitirá determinar cómo se pueden definir átomos con una distribución de la energía completamente arbitraria, consiguiendo que estos se adapten, afinen y doblen cuando sea necesario. Eso permitirá definir átomos que sigan las evoluciones contenidas en la señal a analizar, independientemente de sus características.

En el segundo apartado, una vez construidos los átomos necesarios, se definirá la Transformada Dragón (Dragon Transform, DT). Dicha transformada estará caracterizada por el uso de átomos que realizan un seguimiento perfecto de las evoluciones a detectar. Una vez definida la transformada, se analizarán sus ventajas e inconvenientes. Finalmente, se presentará un método para cuantificar la presencia de una componente, y por tanto de su avería asociada. 


\subsection{Familia de átomos tiempo frecuencia ajustados a las evoluciones arbitrarias de los armónicos a seguir.}

\subsubsection{Introducción.}

En el presente apartado se retoman en primer lugar algunas de las explicaciones relativas a la definición y características de los átomos tiempo-frecuencia, que ya se avanzaron en el capítulo anterior (en especial en el apartado vinculado a la STFT), abordándolas desde un punto de vista más técnico, a través de la definición de las cajas de Heisenberg. Esto se complementa con ejemplos de los tipos de átomos tiempofrecuencia más utilizados comúnmente. Una vez dado este paso, se introduce una metodología para generar los átomos más idóneos para capturar las evoluciones de los armónicos en el plano tiempo frecuencia, minimizando la dispersión de energía, sea cual sea la trayectoria de dichas evoluciones. Como se ha venido mencionando, lo que va a condicionar el átomo tiempo-frecuencia a utilizar es el conocimiento previo de la estructura de la corriente a analizar, tal como aparece especificado en el apartado 2 de esta memoria. Lo que se debe de tener en cuenta en cada punto es el contenido energético de la señal, o más concretamente, las características de la evolución de la componente en el punto donde se va a centrar el átomo, para poder escogerlo.

\subsubsection{Definición, características principales y tipos de átomos tiempo-frecuencia.}

En primer lugar, se recuerda que un átomo tiempo-frecuencia es una función cuya energía se encuentra bien concentrada alrededor de un punto del plano tiempo-frecuencia. La energía de un átomo se distribuye normalmente alrededor de su centro en una forma aproximadamente elíptica (circular en el caso de la Figura 28).

Para caracterizar mejor la distribución de la energía de un átomo alrededor de su centro, se define la caja de Heisenberg. Esta caja aparece en la Figura 28 representada por un rectángulo negro. Presenta dos dimensiones: una temporal y una frecuencial, que se definen como $\sigma_{t}$ y $\sigma_{f}$ respectivamente. Estas dos dimensiones de la caja se definen matemáticamente como la desviación típica de la distribución de la energía del átomo paralelamente al eje temporal y al eje de frecuencias, respectivamente. Es decir, si se 
entiende que la energía del átomo dispersa desde su centro paralelamente al eje del tiempo siguiendo la forma de una campana, $\sigma_{t}$ indica lo estrecha o alargada que es dicha campana. Análogamente con $\sigma_{f}$ para la dispersión paralela al eje de frecuencias. Mediante estas dos dimensiones se puede definir la pendiente del átomo como:

$$
\mathrm{S}=\frac{\sigma_{\mathrm{f}}}{\sigma_{\mathrm{t}}}
$$

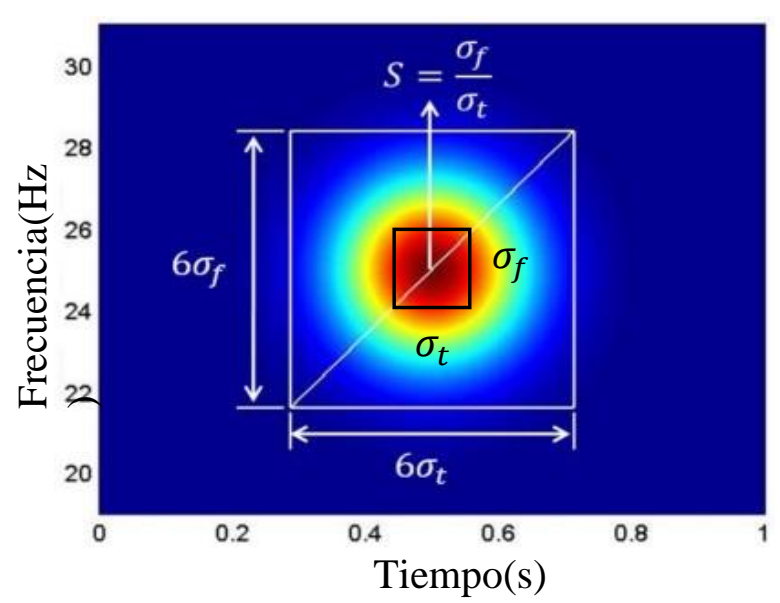

Figura 28. Átomo tiempo-frecuencia centrado en un punto del plano y sus parámetros.

En esta misma Figura 28 se pueden ver las dimensiones de la caja de Heisenberg ampliada, definida como la caja de Heisenberg multiplicada por 6 (representada de color blanco); esta caja ampliada, al contrario que la caja tradicional, contiene en su interior prácticamente toda la energía del átomo. Esta caja de Heisenberg ampliada da información acerca de cómo se distribuye la energía desde el centro del átomo y en los ejes del plano tiempo-frecuencia, y además permite afirmar que la energía del átomo se encuentra concentrada completamente dentro de su interior.

La definición de la caja de Heisenberg nos lleva a exponer el principio de incertidumbre, el cual fue formulado por W. Heisenberg en 1927: "El hecho de que cada partícula lleva asociada consigo una onda, impone restricciones en la capacidad para determinar al mismo tiempo su posición y su velocidad". Es decir, el principio de incertidumbre o principio de indeterminación viene a decir que es imposible medir simultáneamente, y con precisión absoluta, el valor de la posición y la cantidad de movimiento de una partícula. Por tanto, la precisión con que se pueden medir estas dos magnitudes es limitada, y el límite viene fijado por la constante de Planck: 


$$
\Delta \mathrm{x} \cdot \Delta \mathrm{p}_{\mathrm{x}} \geq \frac{\mathrm{h}}{4 \pi}
$$

donde $\Delta x$ representa la indeterminación en el espacio, $\Delta p_{x}$ la indeterminación en la cantidad de movimiento y $h$ la constante de Planck con un valor de h=6,626·10-34 J·s.

Esta propiedad se relaciona directamente con las transformadas tiempofrecuencia. En su caso, existe un compromiso entre la resolución en tiempo y la resolución en frecuencia, como ocurre en el caso de la STFT, tal como se explica en el capítulo anterior. Más concretamente, en mecánica cuántica, las funciones que determinan la posición y la velocidad de una partícula vienen dadas por sendas funciones de distribución de probabilidad. Se demuestra matemáticamente que, las desviaciones estándar de ambas funciones están relacionadas, de tal manera que, si se disminuye la relativa a la posición para obtenerla con precisión, la relativa a la velocidad aumenta, y a la inversa. La distribución de la energía de un átomo en el tiempo y en frecuencia, viene descrita por dos funciones, que desde un punto de vista matemático tienen una estructura idéntica a las distribuciones de probabilidad de la posición y la velocidad de una partícula en mecánica cuántica. Es por ello que, si se intenta concentrar la energía del átomo en el tiempo (disminuir la desviación típica de su función de distribución), la energía se dispersa en frecuencia (aumenta esta segunda desviación típica), y a la inversa. Como estas dos desviaciones típicas son las que caracterizan la caja de Heisenberg del átomo, este principio se puede entender como sigue: el área de la caja es constante, y si se disminuye su base, aumenta su altura, y a la inversa.

Como se ha mencionado en la introducción de este apartado, existen numerosos tipos de átomos tiempo-frecuencia, que se definirán mediante sus respectivas expresiones matemáticas. Esta tesis se va a centrar en dos tipos de funciones: funciones de Gabor [63] y Frequency B-Splines [64]. Se hace uso concretamente de estas funciones puesto que las funciones Gabor presentan las cajas de Heisenberg de área mínima, con lo que se consigue que el conjunto de resolución tiempo-frecuencia es el mejor posible. Por otra parte, en el caso de las funciones Frequency B-Splines el área de la caja de Heisenberg es mayor, sin embargo, cortándola apropiadamente en su primer paso por cero, permite obtener un resultado de buena calidad con una considerable reducción del tiempo de cálculo. 
Las funciones de Gabor se pueden definir como:

$$
\psi(t)=C_{\sigma} e^{-\frac{t^{2}}{2 \sigma^{2}}} e^{i 2 \pi f_{c} t}
$$

en donde $\sigma \in \mathbb{R}$ representa el parámetro de dispersión o desviación; $f_{c} \in \mathrm{R}$ es la frecuencia central de la función y $C_{\sigma}$ es la constante de normalización, que viene definida como:

$$
C_{\sigma}=\frac{1}{\sqrt[4]{\pi} \sqrt{\sigma}}
$$

Gabor [63] introdujo estas ventanas para hacer posible el observar una parte determinada de la señal, en el punto que por el estudio en cuestión sea de interés. Es decir, en el instante analizado las componentes frecuenciales de la señal dependen exclusivamente de las proximidades del punto, sin influir en aquel instante las partes alejadas de la señal, concentrando en ese punto la máxima energía posible. Esto no es más que el principio rector de los átomos tiempo-frecuencia, avanzado en el capítulo anterior, y que se recuerda más adelante.

La pendiente de estos átomos depende del parámetro de dispersión o desviación $\sigma:$

$$
P=\frac{1}{2 \pi \sigma^{2}}
$$

Las Frequency B-Splines son funciones complejas de variable real, que se pueden definir como:

$$
\psi(t)=C_{m, f_{b}}\left[\operatorname{sinc}\left(\frac{f_{b} t}{m}\right)\right]^{m} e^{i 2 \pi f_{c} t}
$$

en donde $m$ es un número natural, $f_{b}$ es el ancho de banda, $f_{c}$ es la frecuencia central de la función y $C_{m, f_{b}}$ es la constante de normalización que vendrá definida como: 


$$
C_{m, f_{b}}=\sqrt{\frac{f_{b}}{m \int_{-\infty}^{+\infty}[\operatorname{sinc}(x)]^{2 m} d x}}
$$

La pendiente de estos átomos depende del parámetro de ancho de banda $f_{b}$ :

$$
P=\frac{\pi f_{b}^{2}}{2 \sqrt{30}}
$$

El espectrograma que se obtiene como resultado de aplicar una transformada lineal basada en el uso de átomos tiempo-frecuencia, no es más que la correlación de los átomos, centrados en todos los puntos del plano tiempo-frecuencia a analizar, con la señal cuyas componentes y sus evoluciones se quieren obtener.

Tal y como ya se avanzó en el capítulo anterior, la correlación de un átomo con la señal de estudio da como resultado una medida de la densidad de energía de la señal en la zona del plano definida por la caja de Heisenberg ampliada. La correlación con el átomo no es más que un filtro de la señal, que permite quedarse con la energía contenida dentro de dicha caja ampliada. Si se repite en muchos puntos del plano, obteniendo la distribución de la densidad de energía a lo largo del plano. La densidad de energía es elevada en los puntos por los que evoluciona una componente de la señal, siendo baja en el resto. Por lo tanto, el espectrograma muestra la evolución temporal de las frecuencias de las componentes presentes en la señal.

Tal y como ya se explicó con detalle al aplicar la STFT en el capítulo anterior, las evoluciones de las componentes vienen representadas en este tipo de transformadas con un determinado grosor, en vez de por una línea ideal perfectamente fina, que es lo que físicamente contiene la señal. Dicho grosor se puede medir paralelamente al eje del tiempo o paralelamente al eje de frecuencias. Estos dos grosores son proporcionales a las dimensiones temporal y en frecuencia de la caja de Heisenberg del átomo. Se razonó ya que, cuanto más base de la caja tiene el átomo, más tenemos que alejar en tiempo el centro del átomo de la evolución real de la componente para que deje de capturar su energía, lo cual implica un mayor grosor con el que se obtiene la evolución, medido paralelamente 
al eje del tiempo. De la misma forma, cuanta mayor altura tiene la caja de Heisenberg del átomo, más se tiene que trasladar en frecuencias su centro alejándola de la evolución a detectar, para que deje de capturar energía, y mayor es el grosor con el que se obtiene dicha evolución, medido paralelamente al eje de frecuencias. Como ambas dimensiones no se pueden reducir de forma simultánea, debido al principio de incertidumbre de Heisenberg, existe un grosor mínimo. La AST, basada en la definición del criterio de la pendiente, permite minimizar dicho grosor, al garantizar que la pendiente del átomo (definida en (14)) sea igual a la derivada de la frecuencia de la componente.

Se ha analizado también en el capítulo anterior, que se pueden generar átomos cuya distribución de energía no sea esencialmente un rectángulo, sino que además dicho rectángulo tenga una base muy elevada y una determinada inclinación. El uso de estos átomos da lugar a la transformada Chirplet. Este tipo de átomo se consigue en primer lugar mediante una caja de Heisenberg de base alargada y poca altura, para seguidamente "inclinar" dicha caja. En el apartado siguiente se describe con mayor detalle esta operación. En la presente tesis se propone ampliar la versatilidad de los átomos, consiguiendo que tracen evoluciones arbitrarias. A este tipo de átomos se les ha llamado átomos tipo dragón, y la transformada a la que dan lugar, transformada Dragón (Dragon Transform, DT).

\subsection{3. Átomos tipo Dragón.}

Aunque la evolución temporal de la frecuencia de una componente sea una línea, al correlacionar con átomos esta línea queda representada en el espectrograma con un cierto grosor que es función de la forma de la caja de Heisenberg del átomo que se haya elegido. Cuanto más fino sea el átomo y más adaptado esté a la señal, más fina será la línea resultante que representa la evolución de esa componente. Hasta ahora, el mejor resultado posible obtenido con átomos convencionales es el que produce la $A S T$, que se ha presentado en el capítulo 2, y analizado con detalle en el 3, y se ilustra además con un ejemplo en el punto 4.3.1 de este capítulo. La AST optimiza la captura de las componentes de la señal a analizar, pero no en términos de evitar que la línea tenga un cierto grosor, tan sólo lo minimiza. La $C T$ sí consigue disminuir arbitrariamente el grosor, siempre y cuando la evolución sea rectilínea. Esto se consigue provocando que la dispersión de la 
energía de la componente se produzca a lo largo de la propia evolución de la componente. Para evitar que aparezca un grosor en la línea, se propone que la dispersión de la energía del átomo utilizado se produzca a lo largo de la evolución de la componente. En otras palabras, la dispersión de la energía del átomo debe describir la evolución de la componente, tal y como se muestra a continuación. Además, cuanto más fino sea el átomo, más fina será la evolución obtenida.

Como se ha mencionado, el área de la caja de Heisenberg del átomo es siempre constante, teniendo además un mínimo del que no se puede bajar. Por tanto, se puede modificar la forma de la caja de Heisenberg, pero manteniendo siempre esa misma área. Como ejemplo, partimos del átomo mostrado en la Figura 29 (a), cuya caja muestra una forma aproximadamente cuadrada. Concretamente, este átomo tiene una pendiente de 1 $\mathrm{Hz} / \mathrm{s}$. Si disminuimos la pendiente del átomo sucesivamente, pero inevitablemente se mantiene el área, pasando por las Figuras (b) y (c), con unas pendientes de 0.5 y 0.25 $\mathrm{Hz} / \mathrm{s}$, hasta llegar a la (d), con una pendiente de $0.1 \mathrm{~Hz} / \mathrm{s}$. Para ello, basta con escoger el parámetro adecuado, aplicando las ecuaciones (18) y (21) para las funciones de Gabor y Frequency B-Splines respectivamente.

Para obtener los átomos que aparecen en la Figura 30, se parte del átomo más fino obtenido en la Figura 29 (d) (con $\mathrm{P}=0.1 \mathrm{~Hz} / \mathrm{s}$ ). Seguidamente, se debe aumentar progresivamente la inclinación del átomo. Dicha inclinación se define, asumiendo que el átomo tiene forma de segmento (rectángulo idealmente fino), como el intervalo de frecuencia entre los dos extremos del segmento, dividido por el intervalo de tiempo entre los mismos extremos (el cual, en los átomos representados en la Figura 30, es constante). Como ejemplo, en la Figura 30 (c) se muestra un átomo que cubre un intervalo de tiempo de 4 segundos (entre 2 y 6 segundos), y una banda de frecuencia de $4 \mathrm{~Hz}$ (entre 5.5 y 9.5 $\mathrm{Hz}$ ). Por tanto, dicho átomo tiene una inclinación de $1 \mathrm{~Hz} / \mathrm{s}$. El resto de átomos presentan inclinaciones de $0.1,0.5$ y $2 \mathrm{~Hz} / \mathrm{s}$. Para conseguir este efecto, basta con, en las ecuaciones (14) y (19) para funciones de Gabor y Frequency B-Splines respectivamente, no utilizar como argumento de la exponencial compleja una frecuencia constante, sino una frecuencia que varíe linealmente con el tiempo. Este tipo de átomos se llaman chirplets, y son los utilizados por la $C T$. 


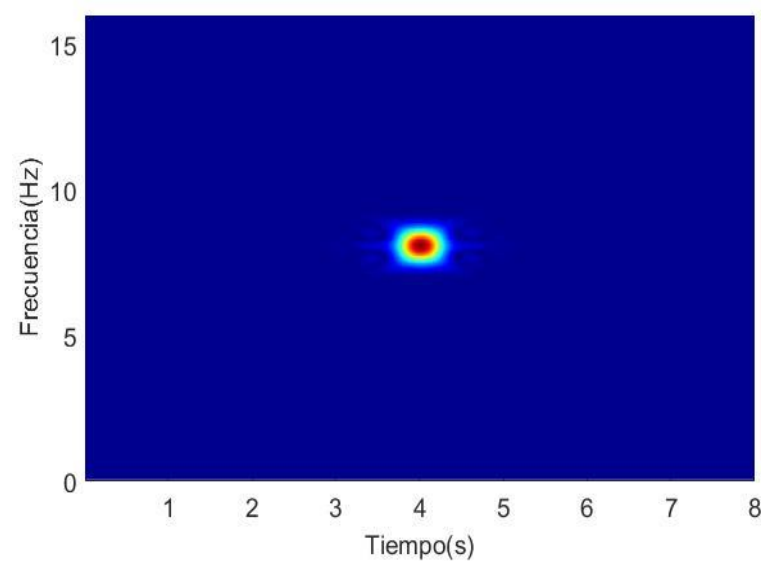

(a) Átomo horizontal $\mathrm{p}=1$.

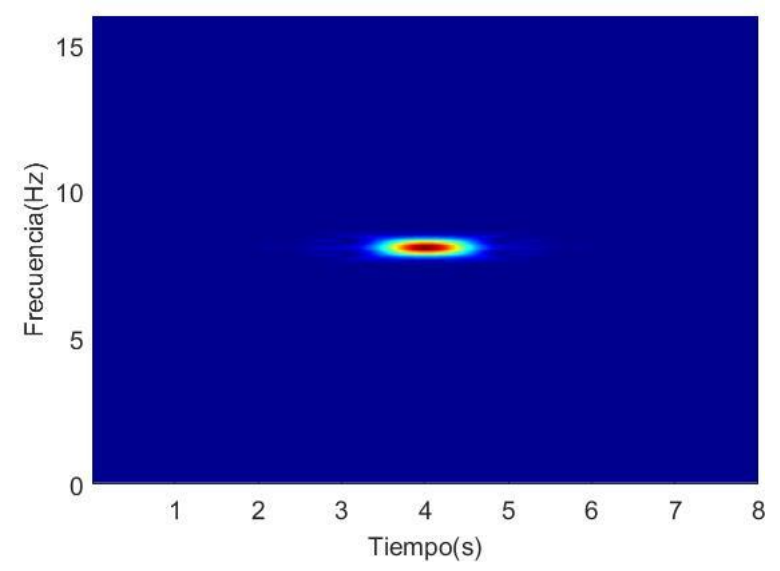

(c) Átomo horizontal $\mathrm{p}=0.25$.

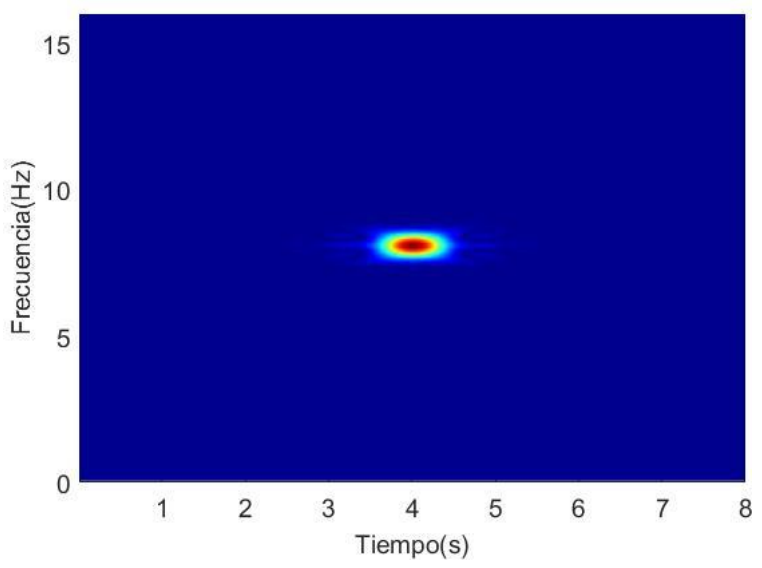

(b) Átomo horizontal $\mathrm{p}=0.5$.

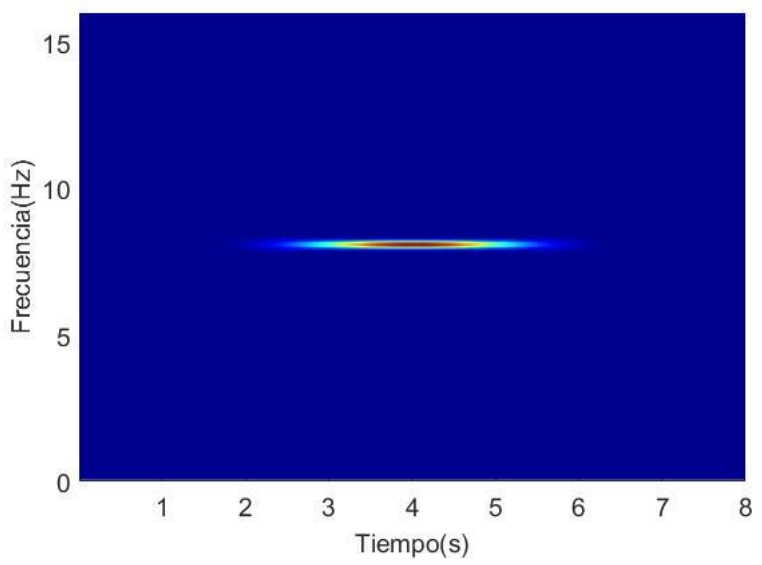

(d) Átomo horizontal $\mathrm{p}=0.1$.

Figura 29. Átomos horizontales de distintos grosores.

Una vez que se sabe cómo definir un átomo con una determinada pendiente (para el caso anterior, una pendiente baja), y seguidamente darle inclinación, el siguiente paso es conseguir que se adapte, de tal forma que siga la evolución de una determinada componente. En el ejemplo que aparece en la Figura 31, se puede apreciar una familia de 3 átomos que siguen la evolución temporal de la frecuencia mostrada en la parte izquierda de la Figura. Esta evolución es la propia de la componente fundamental al arrancar con inversor, con un arranque lineal, fijando la frecuencia final en $50 \mathrm{~Hz}$, y el tiempo de arranque ligeramente superior a 10 segundos. Se han definido tres átomos: el primero se encuentra en la zona del arranque, el tercero en la zona de permanente y el segundo justo en el codo en el que se termina el arranque y empieza el permanente. Como se observa, los átomos son capaces de "seguir" la evolución de la componente. En otras palabras, la dispersión de la energía de cada uno de los tres átomos se produce en la dirección de la evolución de la componente. Para ello, otra vez recurrimos a las ecuaciones (14) y (19), 
pero esta vez debemos definir un ángulo de la exponencial compleja, tal que su derivada sea igual a la frecuencia de la componente a detectar.

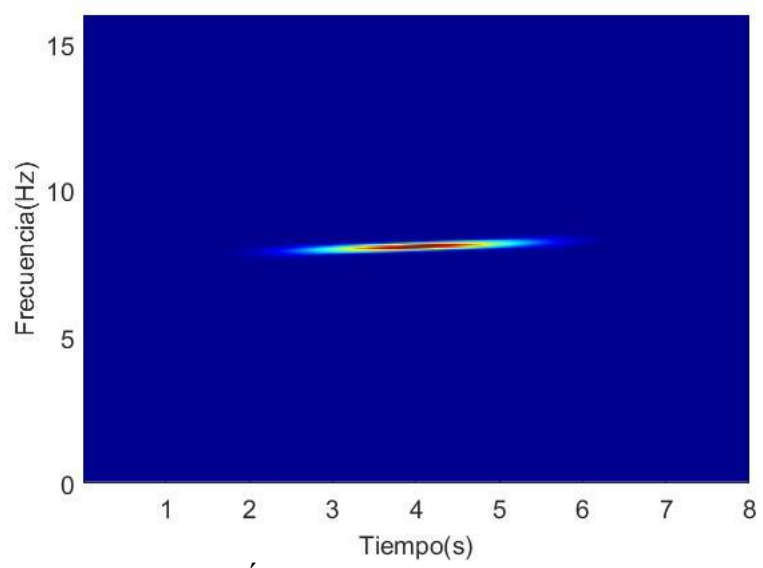

(a) Átomo inclinado $\beta=0.1$.

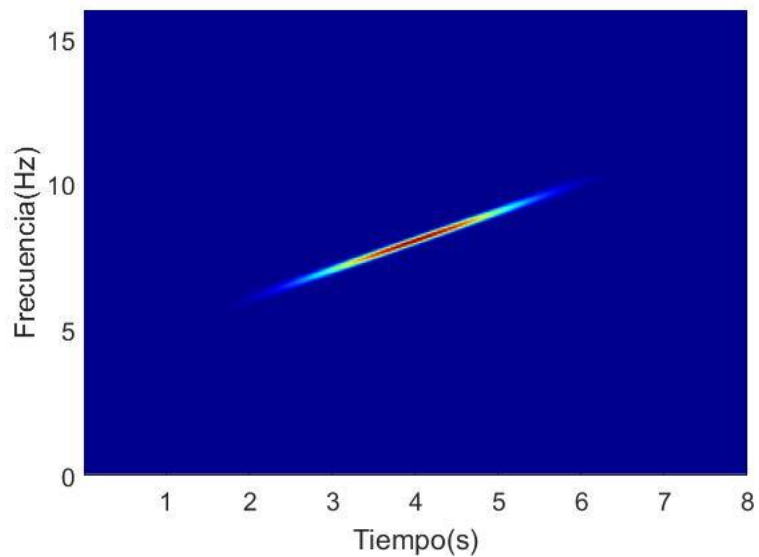

(c) Átomo inclinado $\beta=1$.

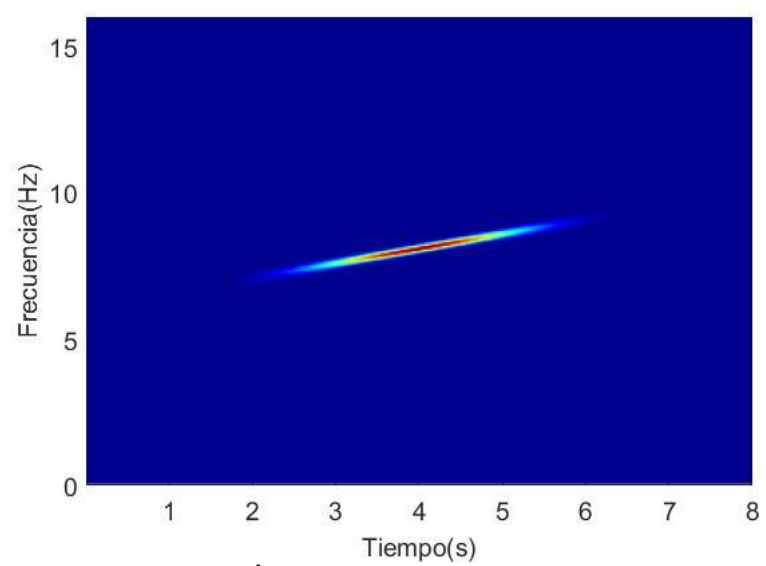

(b) Átomo inclinado $\beta=0.5$.

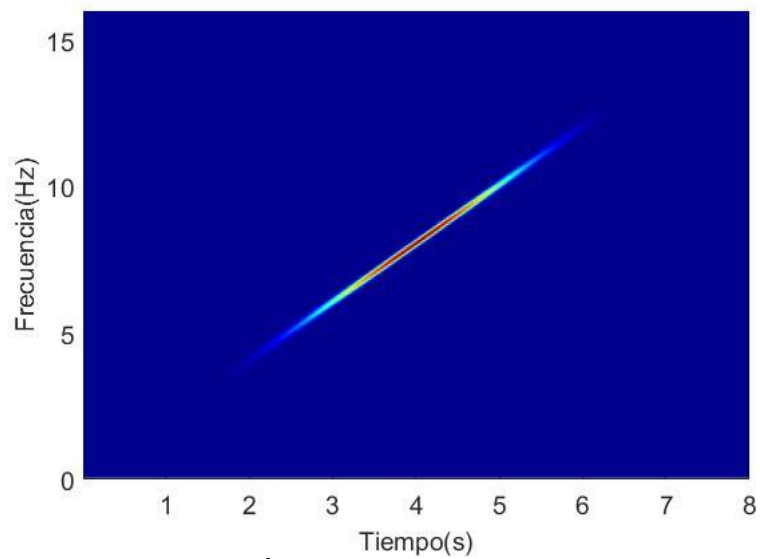

(d) Átomo inclinado $\beta=2$.

Figura 30. Átomos del mismo grosor con distintas inclinaciones
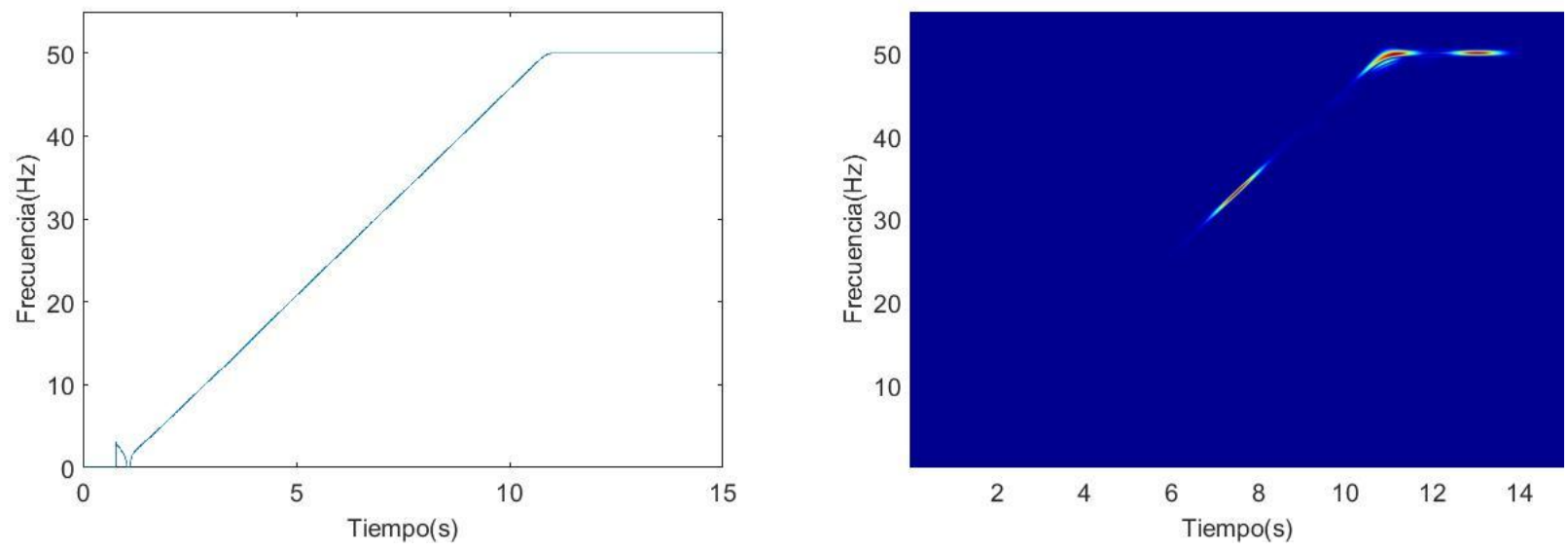

Figura 31. Familia de átomos para seguir una señal. 
En la parte izquierda de la Figura 32 se han trazado evoluciones arbitrarias de tres componentes distintas, aunque no sean representativas de las que se encuentran en las corrientes de motores de inducción alimentados por inversor. Con estos otros ejemplos se pretende terminar de constatar que los átomos dragón son capaces de seguir la evolución de una componente, sin importar sus características.

Finalmente, se obtienen los átomos a utilizar al analizar las evoluciones de los componentes armónicos de una señal real, en la que el motor presenta fallo de barras. Concretamente, en la parte derecha de la Figura 33 se observan las evoluciones de las componentes a detectar (de arriba abajo): componente fundamental, lateral inferior, lateral superior de barra rota y las tres evoluciones juntas. En la parte de la izquierda de la Figura, se observan ejemplos de átomos centrados en distintos puntos de estas evoluciones. Si por cada punto del plano se escoge un átomo siguiendo este criterio, se obtiene la familia de átomos a utilizar para correlacionarlos con la señal. Este será el resultado de la $D T$, definida en el siguiente apartado.

Si los átomos tienen formas rectangulares, la dispersión de la energía con la que se muestra la evolución de una componente reproduce la dispersión de la energía del propio átomo: dispersión a lo largo del tiempo al utilizar átomos con una base elevada, y dispersión a lo largo del eje de frecuencias al utilizar átomos con una altura elevada. Si el átomo tiene una forma tal que sigue la evolución de la componente, al correlacionarlo con la señal, la energía de la componente se dispersa a lo largo de su propia evolución, pudiendo obtenerla como una línea fina (tan fina como el átomo utilizado), tal y como se verá en el siguiente apartado. 


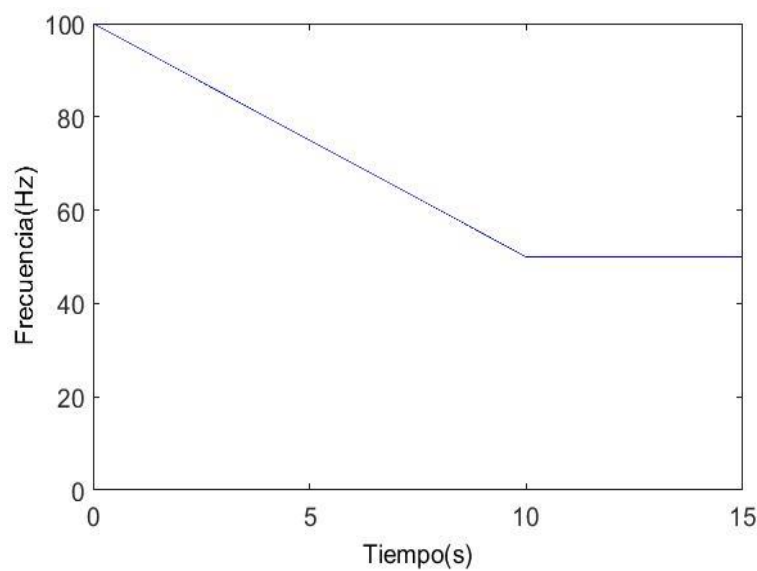

(a) Señal descendente.

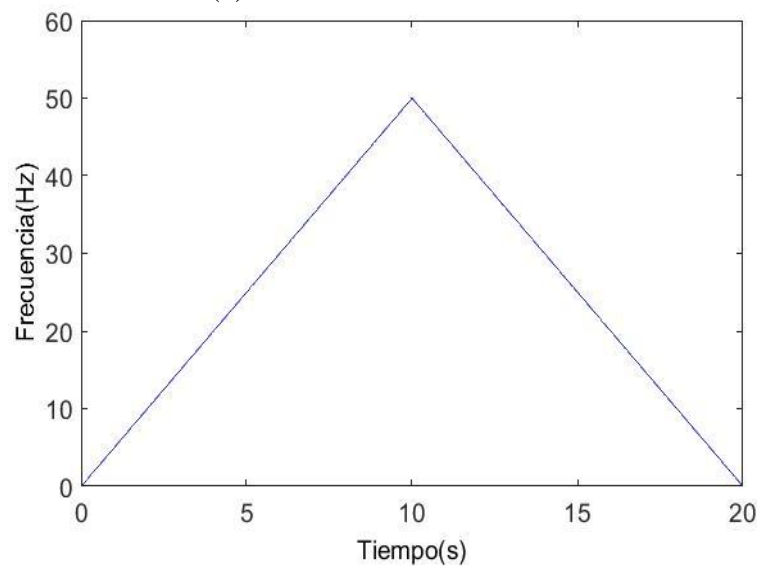

(c) Señal triangular.

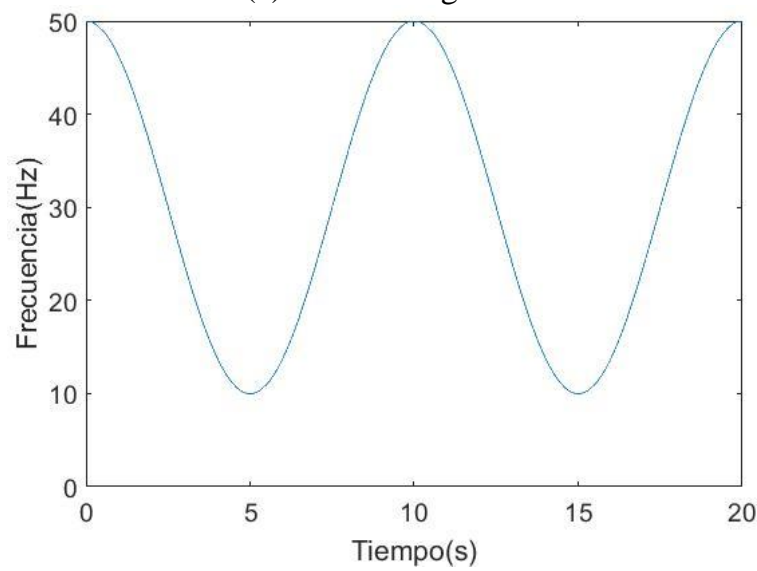

(e) Señal senoidal.

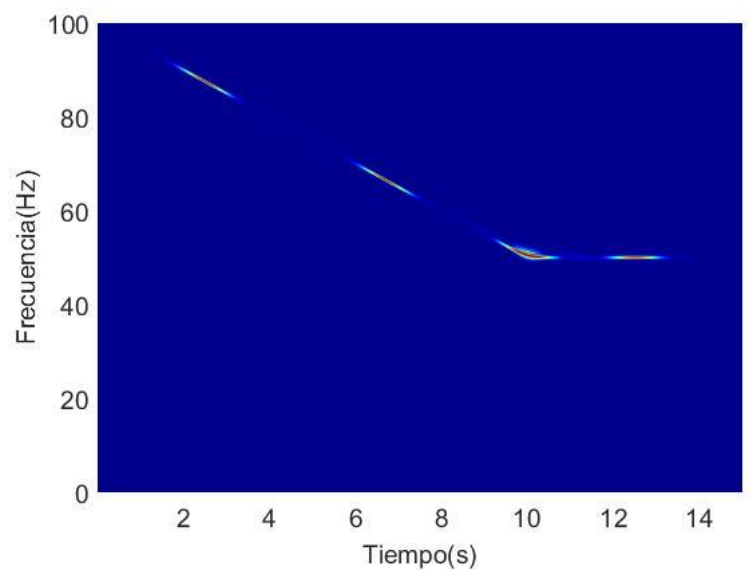

(b) Familia de átomos señal descendente.

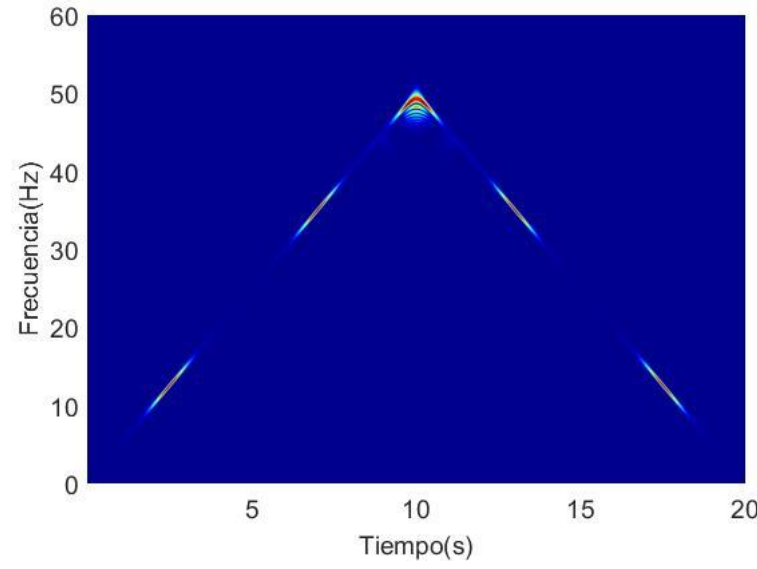

(d) Familia de átomos señal triangular.

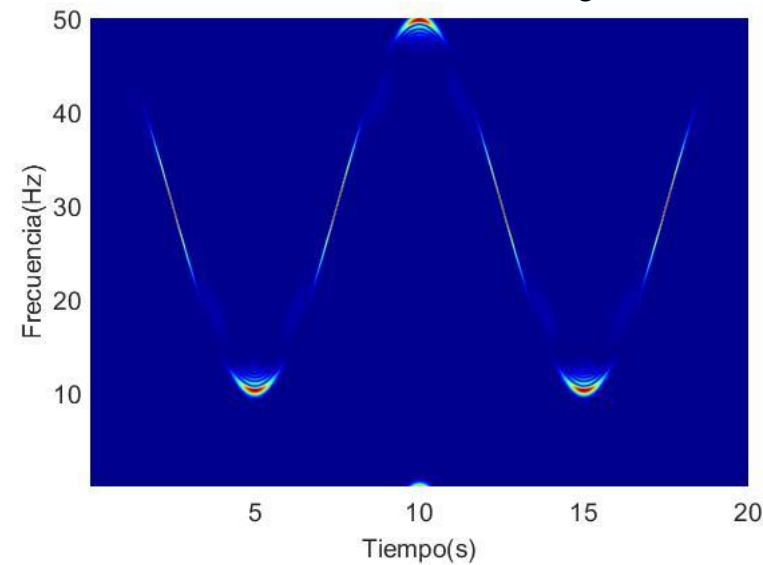

(f) Familia de átomos señal senoidal.

Figura 32. Átomos tiempo frecuencia siguiendo distintas señales sintéticas. 


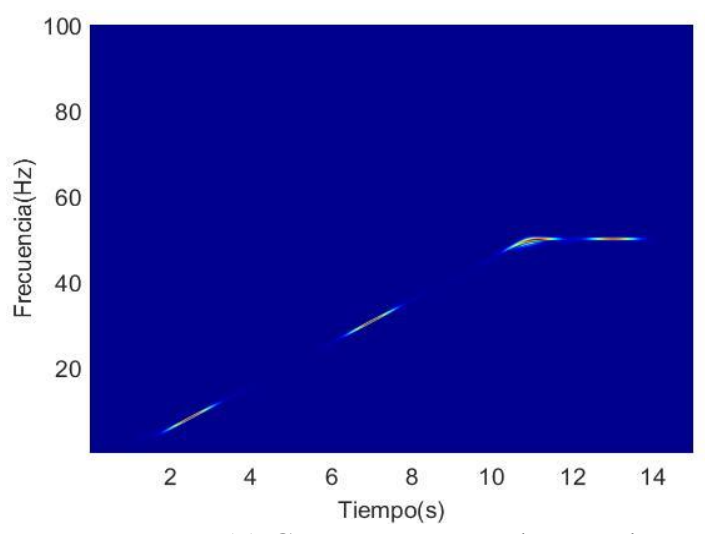

(a) Componente Fundamental

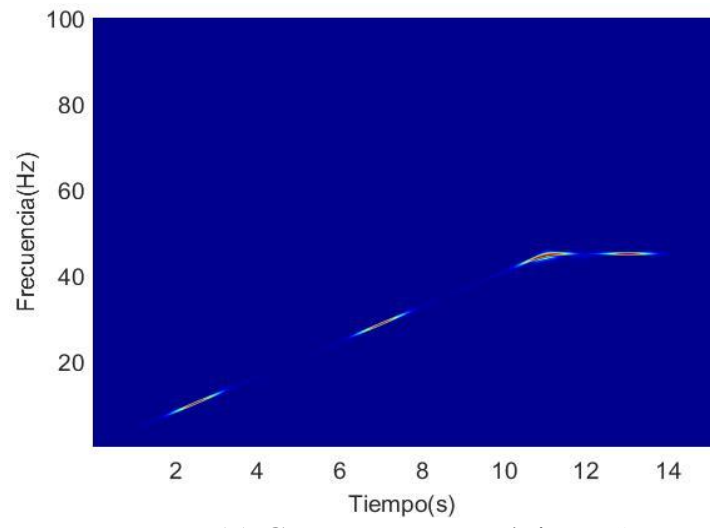

(c) Componente armónico $\mathrm{LSH}$

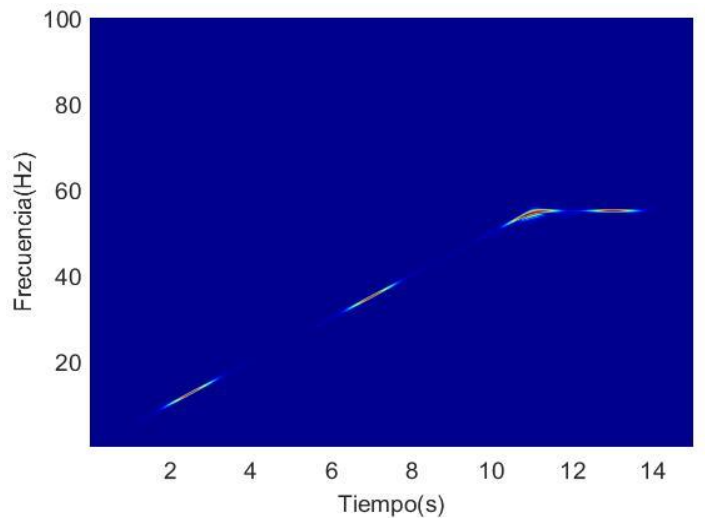

(e) Componente armónico USH

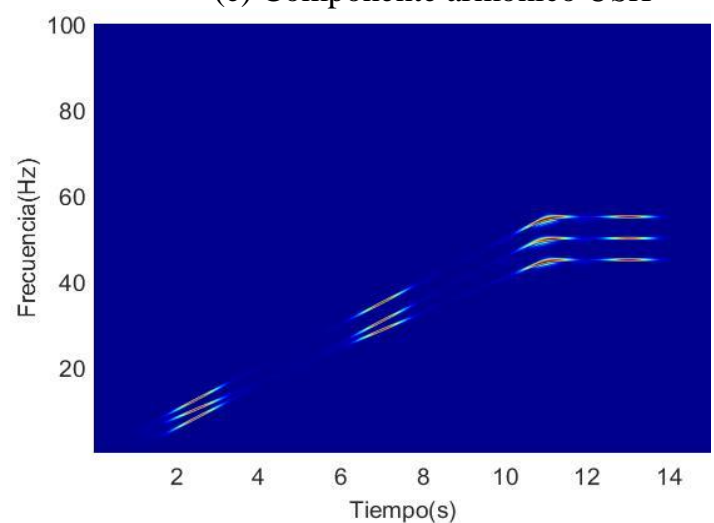

(g) Representación conjunta $F C, L S H$ y $U S H$.

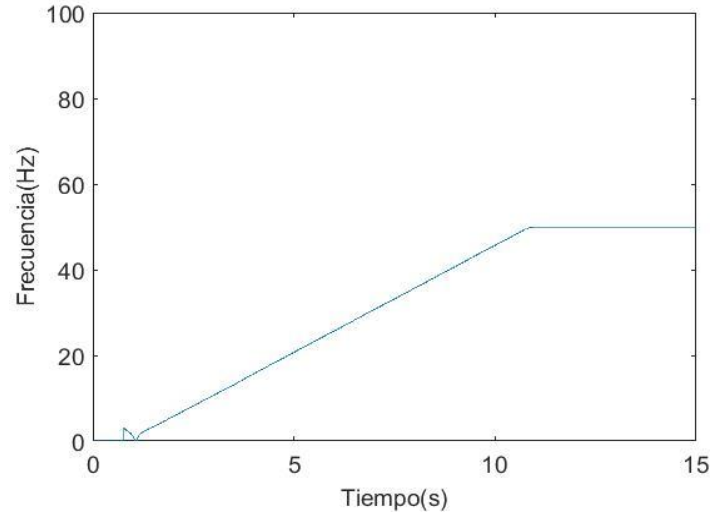

(b) Trayectoria Teórica Componente Fundamental

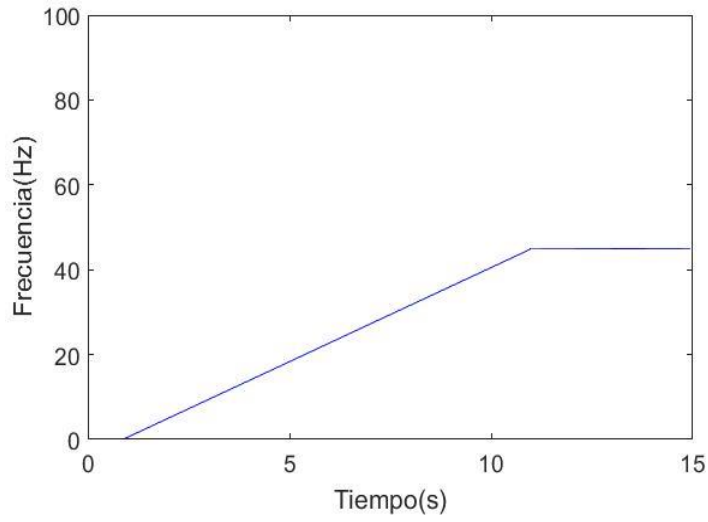

(d) Trayectoria Teórica $L S H$

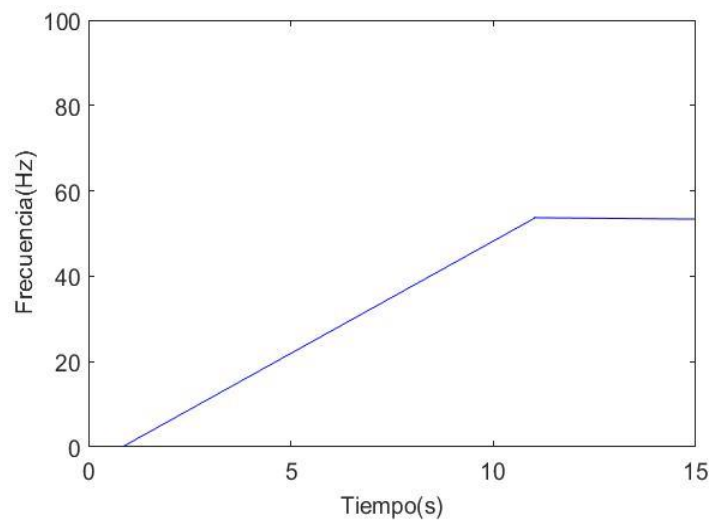

(f) Trayectoria Teórica $U S H$

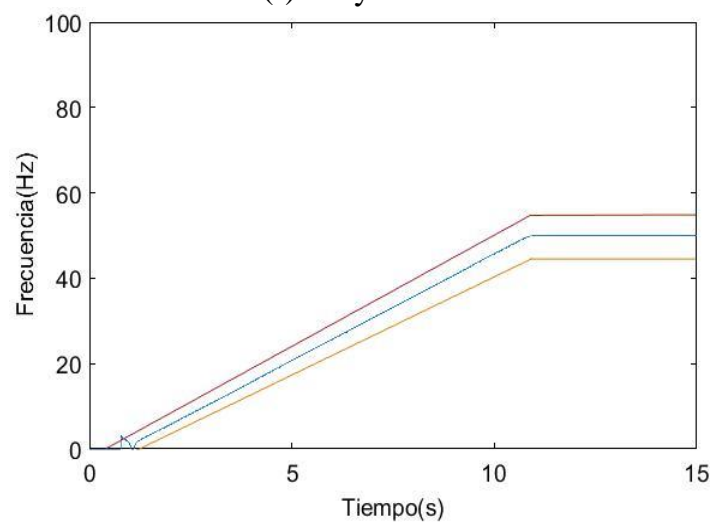

(h) Trayectorias teóricas juntas $F C, U S H$ y $L S H$.

Figura 33. Átomos ajustados a los componentes armónicos de una señal real. 


\subsection{Transformada Dragón.}

Este apartado constituye el punto central de la tesis, sobre el que se articula todo el desarrollo de la misma, ya que es en éste donde se va a presentar la transformada en la que se basa la metodología propuesta: la "Transformada Dragón”.

\subsubsection{Definición de la transformada Dragón.}

La transformada desarrollada se define en un punto como la correlación entre la señal a analizar y el átomo dragón centrado en ese punto del plano tiempo-frecuencia. La particularidad consiste en que se ha conseguido definir átomos cuya "forma" reproduce la evolución de la componente a detectar. De esta manera se consigue obtener una representación de la densidad de energía de la señal en la que las evoluciones de las componentes vienen representadas por líneas muy finas.

Para presentar la transformada desarrollada se muestra un ejemplo práctico. En este ejemplo se analiza una señal correspondiente a un motor de inducción que presenta barras rotas, cuya corriente en el estator es la que aparece en la Figura 34 (a) y su componente fundamental en la Figura 34 (b). Este motor se encuentra alimentado mediante un inversor que está programado para un arranque de 10 segundos durante el cual la frecuencia aumentará linealmente de 0 a $50 \mathrm{~Hz}$, presentando la señal, una duración total de 30 segundos.

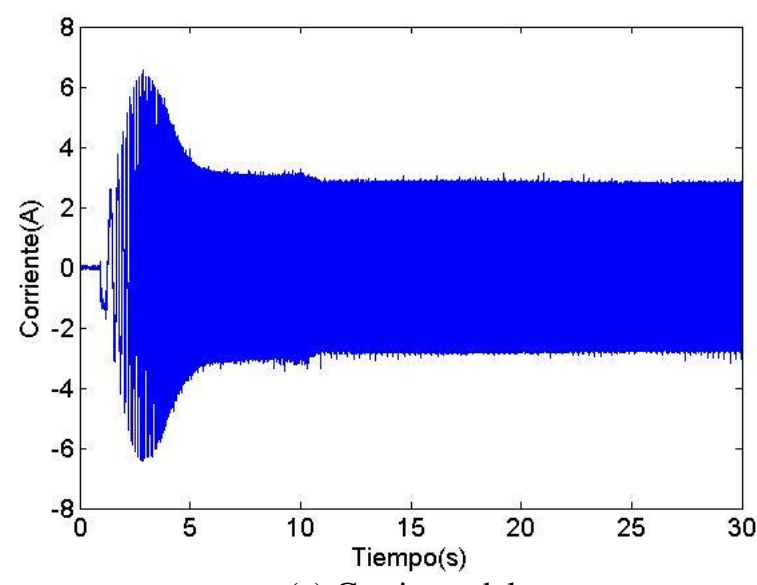

(a) Corriente del estator.

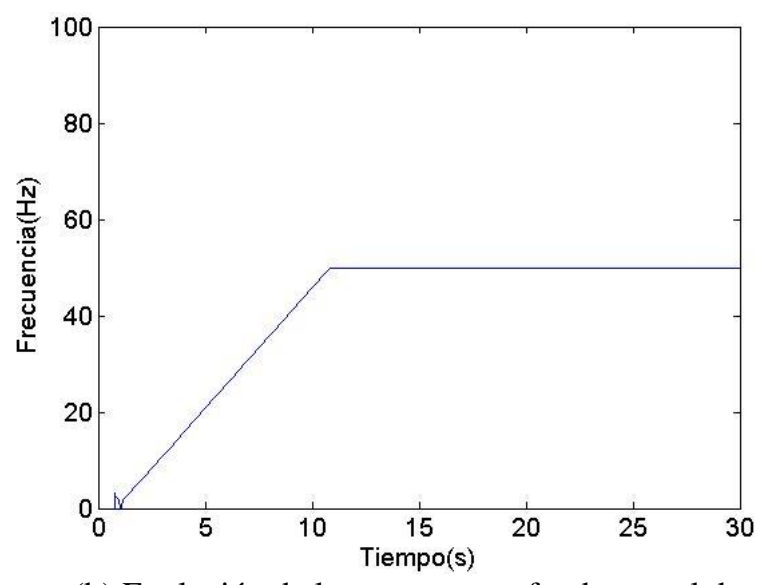

(b) Evolución de la componente fundamental de frecuencia para la señal de corriente de la figura 35 .a

Figura 34. Parámetros señal a analizar. 
Presentada la señal a estudiar, se pasan a analizar las componentes armónicas de la misma, para lo cual hay que fijarse en la Figura 35. En la Figura 35 (a) se encuentran las trayectorias teóricas de las componentes armónicas que deseamos detectar. Como se ha dicho que se trata de un motor con barras rotas, las componentes son la fundamental, así como los laterales de barra rota. En la Figura 35 (b) aparecen las familias de átomos que siguen las trayectorias de estos componentes armónicos. Por otra parte, en la Figura 35 (c) aparece la familia de átomos a lo largo de todo el plano tiempo-frecuencia. Los átomos centrados en los puntos donde hay una evolución a detectar deben seguir dicha evolución, pero ¿cómo se definen los átomos donde no hay ninguna evolución? Simplemente se debe intentar que la familia de átomos forme un conjunto en armonía, sin cambios bruscos que produzcan cambios bruscos en la resolución tiempo-frecuencia con la que se obtiene el resultado. Finalmente, en la Figura 35 (d) se tiene el espectrograma resultante de aplicar la $D T$ a la señal: correlación de la señal con la familia de átomos de la Figura 35 (c).

Para visualizar mejor el espectrograma resultante de la transformada Dragón que se tiene en la Figura 35 (d), se obtienen las Figuras 36 (a) y 36 (b), en donde se presenta un zoom en la parte del arranque (Figura 36 (a)) concretamente entre los segundos 3,5 y 6,4, y otra en el codo de la señal (Figura 36 (b)), entre los segundos 9,8 y 12, para visualizar sin lugar a dudas, que efectivamente se están siguiendo las evoluciones de los armónicos de fallo, incluso en el paso del transitorio al permanente.

Así, en las Figuras 36 (a) y 36 (b), se ve como, en el resultado de la transformada Dragón, se detectan de forma nítida las evoluciones de las componentes, cuya dirección se señala con flechas negras. Por lo que se puede concluir que con los átomos adaptables desarrollados, "átomos Dragón”, se consigue, por primera vez en la literatura técnica, poder detectar en el plano tiempo-frecuencia la evolución completa de las componentes de avería rotórica de un motor de inducción operado por inversor, además de su pertinente cuantificación, tanto en el estado estacionario como en el transitorio de arranque, a pesar de la dificultad debida a la proximidad entre dichas evoluciones y la componente fundamental. 


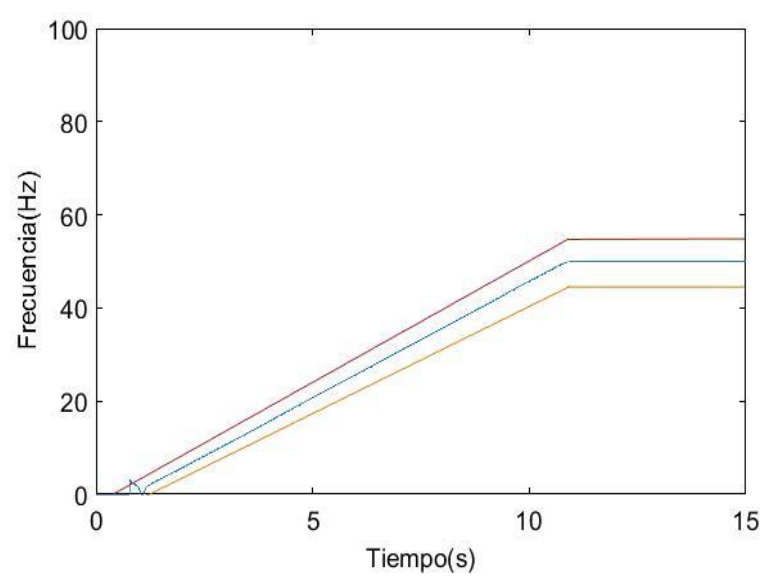

(a) Trayectorias teóricas de las componentes armónicas de falta para un motor de inducción.

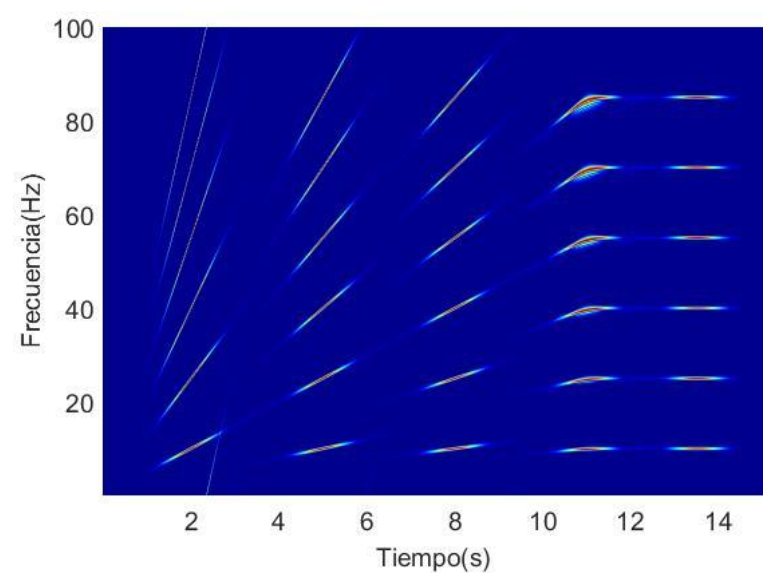

(c) Familias de átomos a lo largo de todo el plano tiempo frecuencia.

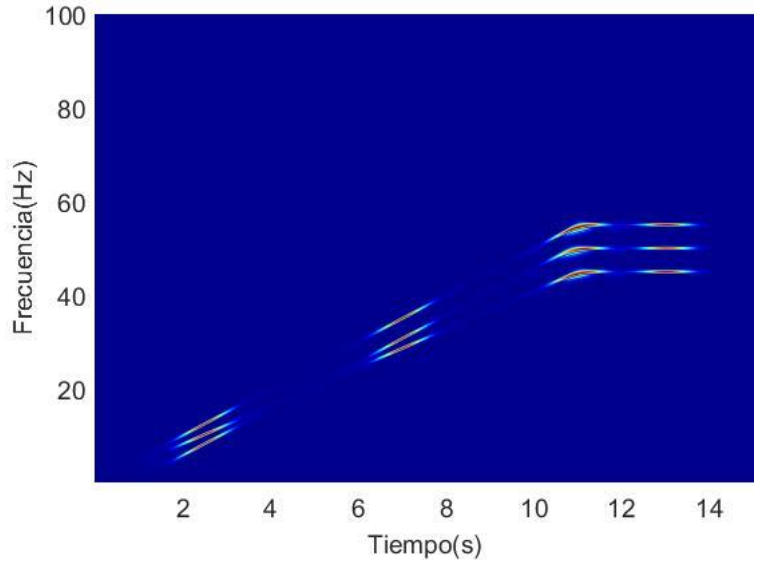

(b) Familias de átomos que siguen las evoluciones de las componentes armónicas.

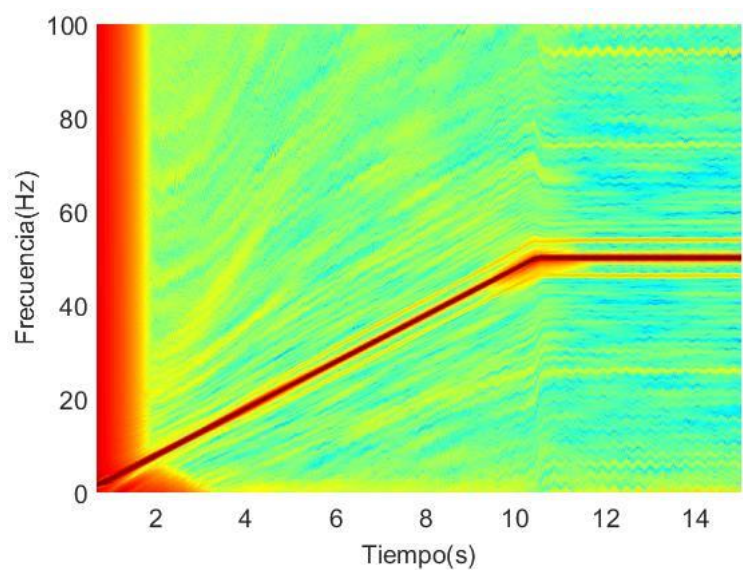

(d) Espectro resultante de aplicar la transformada Dragón.

Figura 35. Pasos para el cálculo de la transformada Dragón.

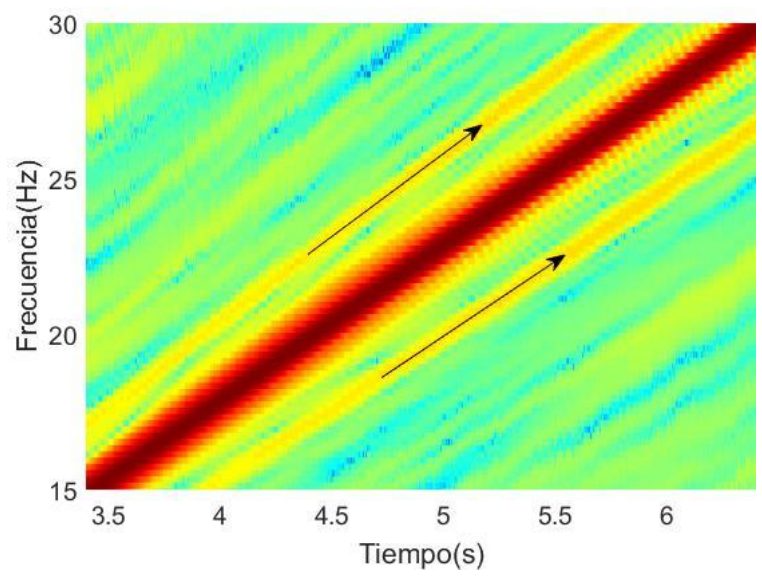

(a) Zoom entre los seguidos 3,4 y 6,4.

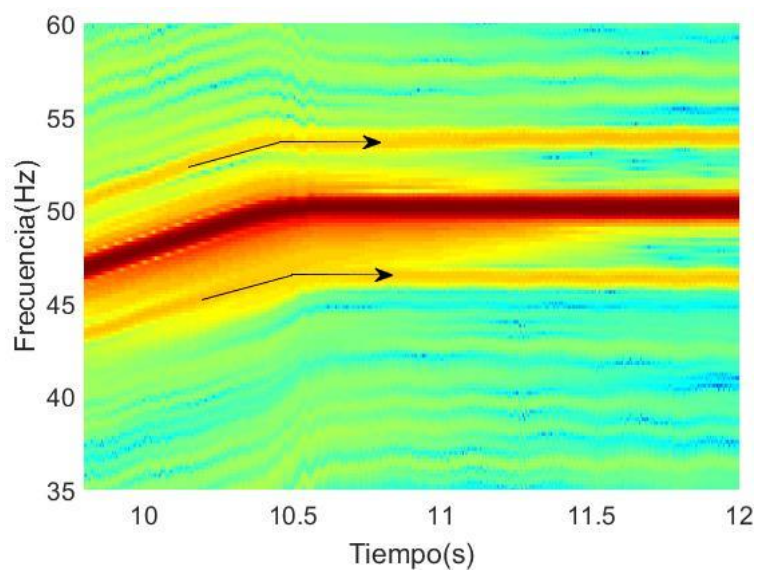

(b) Zoom entre los segundos 9 y 12 .

Figura 36. Zoom en régimen transitorio y codo de la Figura 36 (d). 
A continuación, para destacar la calidad del resultado obtenido, se procede a comparar dicho resultado con los obtenidos por las transformadas presentes en la literatura técnica, que se introducen a lo largo del capítulo 2 de esta tesis, y cuyos resultados ya han sido avanzados en el capítulo 3. Como ya se ha constatado, y ahora se vuelve a remarcar, estas transformadas no son capaces de detectar la evolución a lo largo de todo el intervalo de tiempo capturado, pudiendo a lo sumo observar las componentes, o bien en el permanente (STFT, AST) o bien en el arranque (CT).

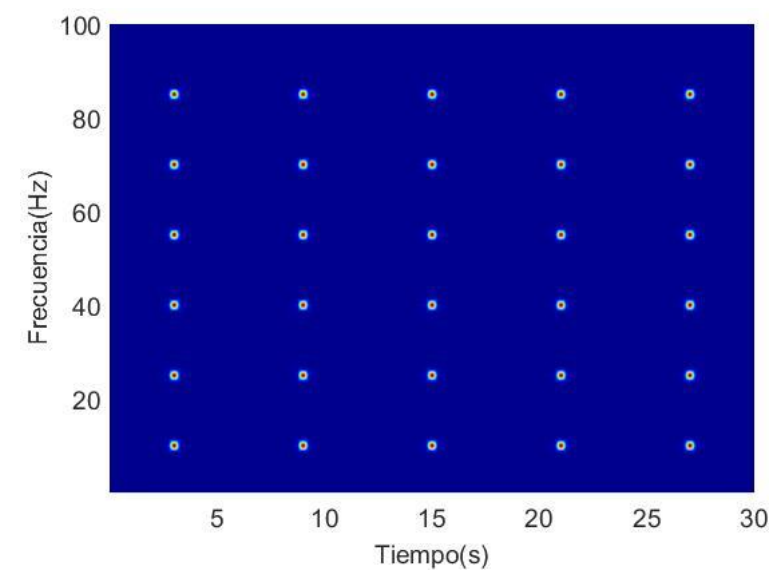

(a) Familia de átomos para generar STFT.

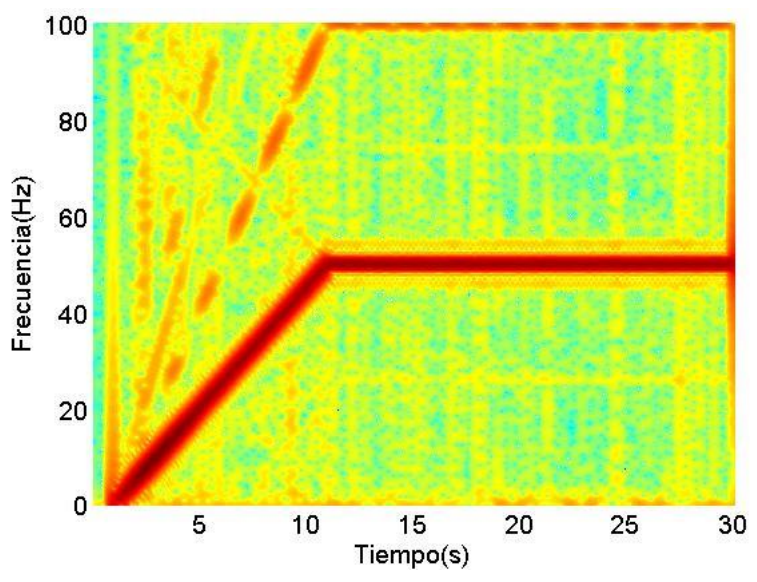

(b) Espectrograma resultante de aplicar STFT.

Figura 37. Resultado de aplicar STFT

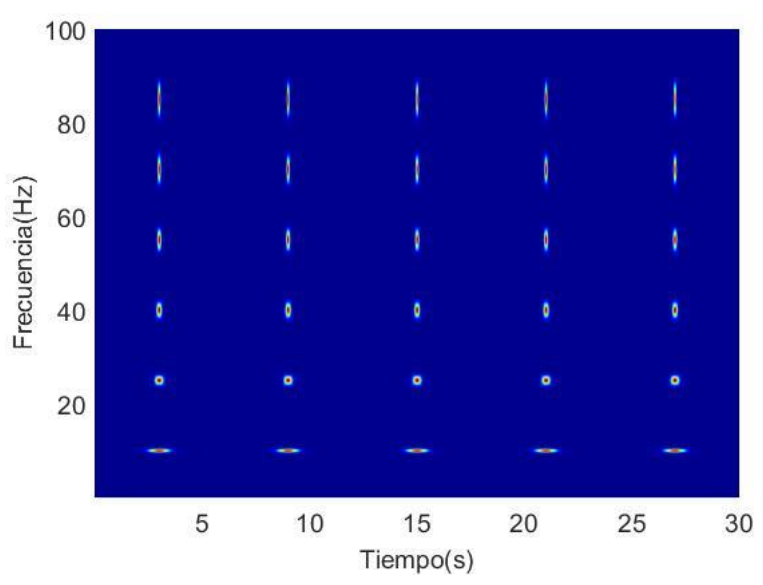

(a) Familia de átomos para generar $C W T$.

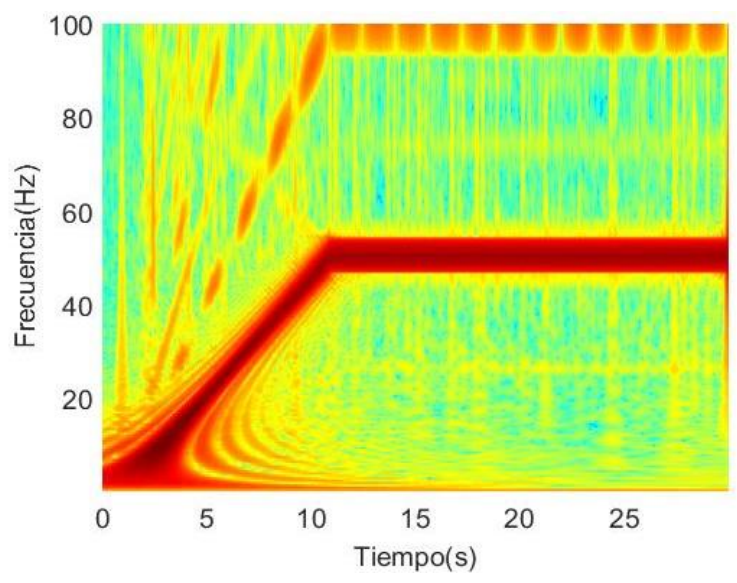

(b) Espectrograma resultante de aplicar $C W T$.

Figura 38. Resultado de aplicar CWT. 


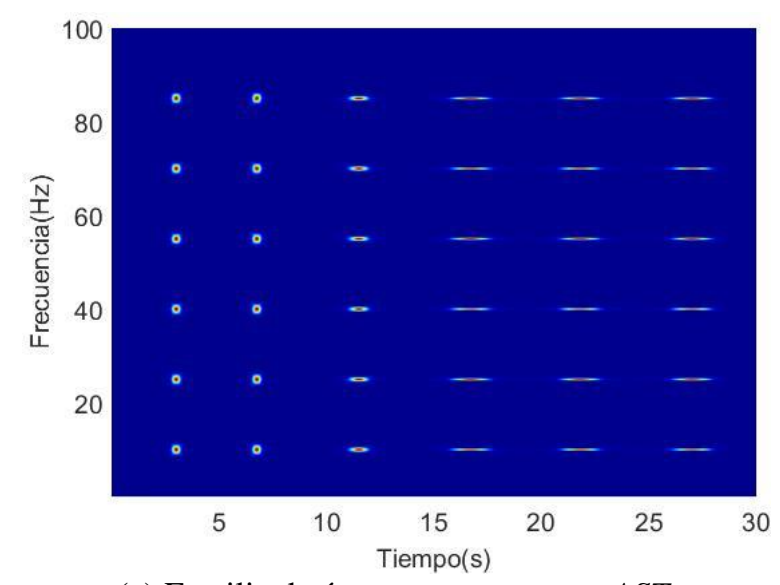

(a) Familia de átomos para generar AST.

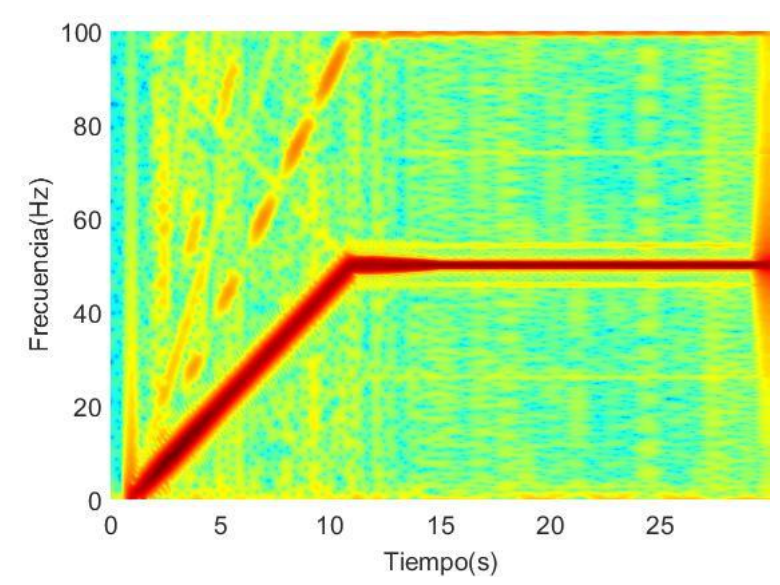

(b) Espectrograma resultante de aplicar AST.

Figura 39. Resultado de aplicar AST.

La Figura 37 establece el resultado de la STFT: en la Figura 37 (a) se puede observar la familia de átomos definida para el cálculo de la transformada con la mejor resolución posible. Como se ha explicado, en las transformadas lineales se busca un equilibrio entre las resoluciones tiempo y frecuencia, siendo el mismo para todos los puntos en el plano t-f en el caso de la STFT. En este caso se ha aplicado el criterio de la pendiente para fijar este equilibrio, es decir, se ha ajustado la longitud de la ventana para optimizar la captura de la evolución de la $F C$ durante el arranque, de tal manera que la pendiente de los átomos utilizados sea igual a la pendiente de la evolución a capturar. Como consecuencia, el espesor de la $F C$ durante el arranque se reduce al mínimo.

No obstante, el espesor mínimo para el que se ha obtenido la mejor definición de la componente fundamental no es suficiente para que se puedan observar las trayectorias de los componentes de fallo debidos a barras rotas, tal y como se observa en el Figura 37 (b), pero sí se obtiene la suficiente resolución para observar dichos armónicos a lo largo del estado estacionario, ya que en este caso el grosor de la trayectoria de la componente fundamental es lo suficientemente fino para poder separarse de los componentes debidos al fallo de barras.

En la Figura 38 se observa el resultado de la $C W T$. Para el caso que nos ocupa, las características de la wavelet han sido elegidas para optimizar la captura de la frecuencia fundamental en el punto donde su frecuencia llega a alcanzar los $25 \mathrm{~Hz}$, usando el criterio de la pendiente, al igual que se ha llevado a cabo con la STFT. La familia de átomos 
finalmente utilizada, teniendo en cuenta la variación de la forma de los átomos propia de la $C W T$, se puede observar en la Figura 38 (a).

Si se atiende a la imagen de la Figura 38 (b), se puede observar que la resolución en frecuencia aumenta mientras que la resolución en tiempo obtiene peores valores en frecuencias inferiores a $25 \mathrm{~Hz}$, aumentando la dispersión en tiempo de la energía de la componente fundamental con respecto a la $S T F T$. Para frecuencias superiores a $25 \mathrm{~Hz}$, el compromiso tiene una evolución inversa (mejora resolución temporal y empeora en frecuencia), aumentando la dispersión en frecuencia. Tal y como se explicó en el capítulo 3 , esto no mejora la detección de las componentes. Como resultado, se puede ver que no se detectan las componentes de fallo de barras.

El resultado de la $A S T$ se puede observar en la Figura 39. En este caso se han ajustado los átomos para tener una perfecta resolución al capturar la componente fundamental, tanto durante el arranque como en el estado estacionario. A diferencia de la STFT, en el estacionario se han utilizado átomos finos y horizontales, como aparecen en la Figura 39 (a). Al observar el espectrograma resultante, Figura 39 (b), en el estado estacionario la resolución de la frecuencia se incrementa gracias a la adaptabilidad de la $A S T$, lo que disminuye el espesor de la frecuencia fundamental medida en la dirección del eje de frecuencia, obteniéndose una línea perfectamente fina que permite detectar de manera precisa los armónicos de fallo de barras, mejorando el resultado de la $S T F T$.

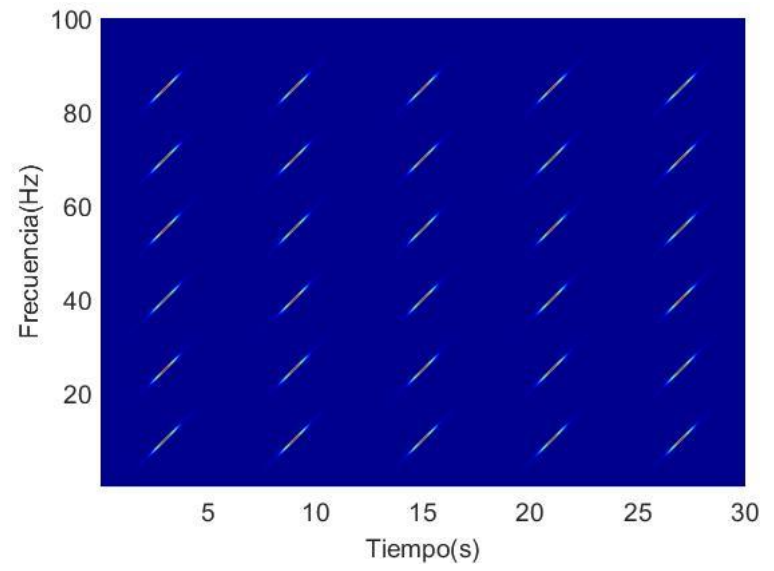

(a) Familia de átomos para generar la transformada Chirplet.

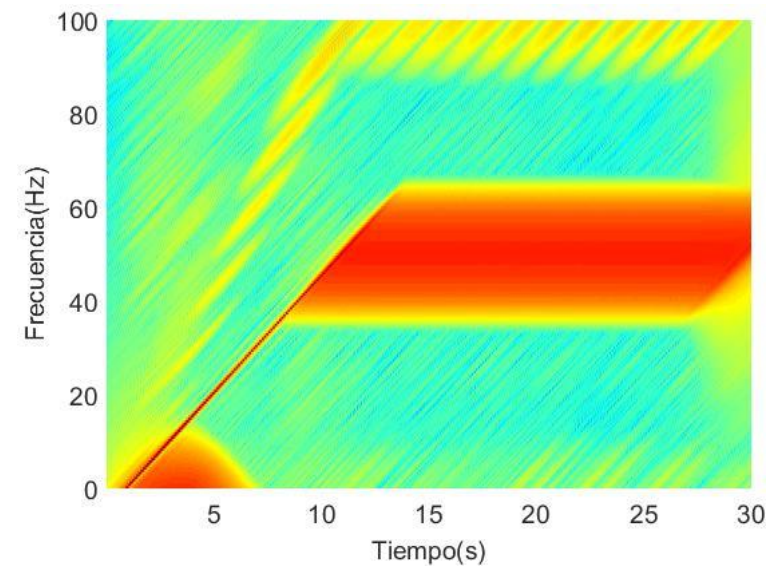

(b) Espectrograma resultante de aplicar la transformada Chirplet.

Figura 40. Resultado de aplicar la transformada Chirplet. 


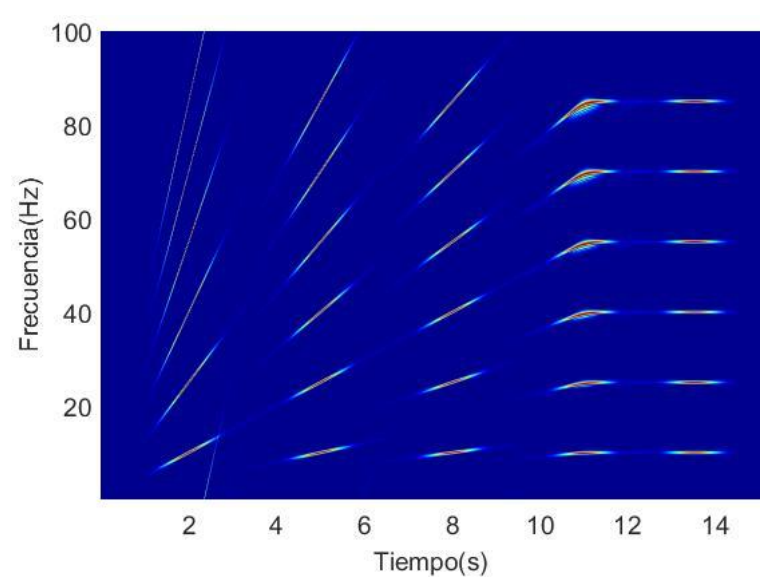

(a) Familia de átomos para generar la transformada Dragón.

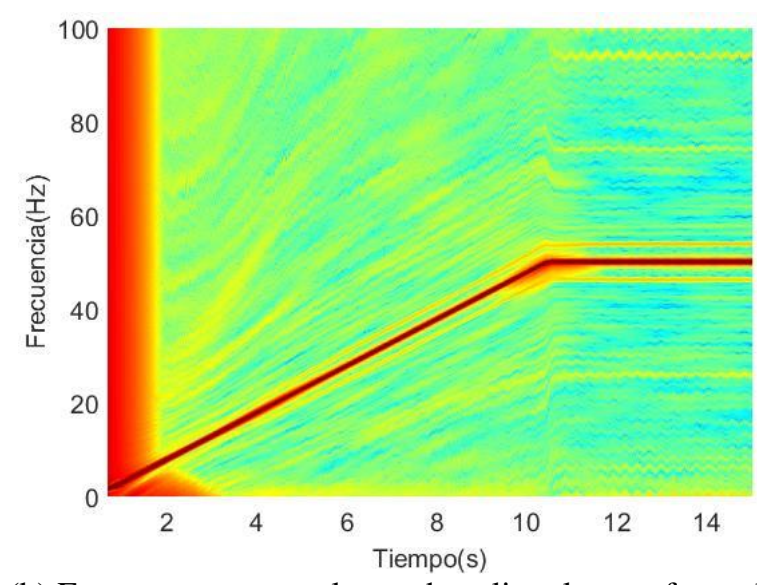

(b) Espectrograma resultante de aplicar la transformada Dragón.

Figura 41. Resultado de aplicar la transformada Dragón.

En la Figura 40 se tiene el resultado de la $C T$, que se ha utilizado para optimizar la captura de la frecuencia fundamental durante el arranque, por lo que se han utilizado átomos finos e inclinados, como aparece en la Figura 40 (a). Con estos átomos se obtiene una mejor resolución si lo que se busca es visualizar de manera perfecta el régimen transitorio: la evolución de la frecuencia fundamental aparece como una línea muy fina (Figura 40 (b)), de la misma manera que se optimiza la captura de los armónicos de barras rotas. Con el fin de observar estas evoluciones con un mayor detalle, se muestra un zoom del resultado, entre los segundos 4,7 y 7,4, en la Figura 42.

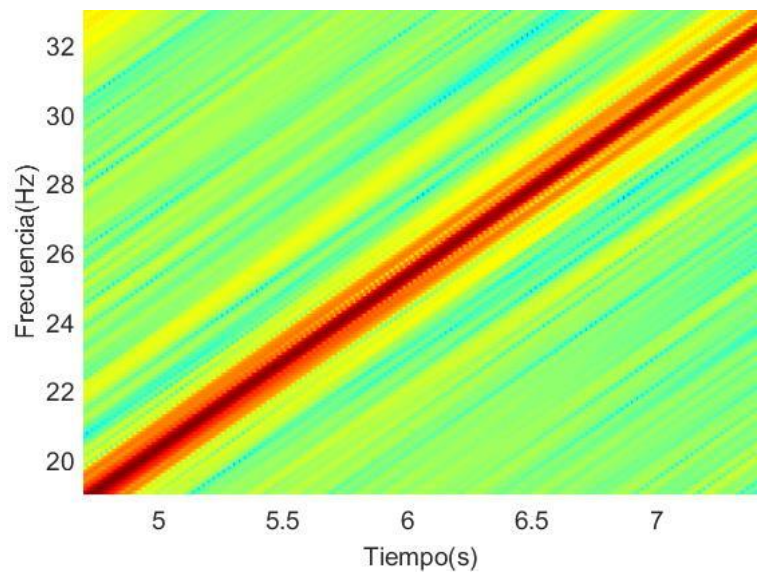

Figura 42. Zoom de la Figura 40 (b), entre los segundos 4,7 y 7,4.

Finalmente, en la Figura 41 se puede observar el resultado de aplicar la transformada Dragón a la señal, tal como se ha explicado previamente en este mismo apartado. En la Figura 41 (a) aparecen los átomos, a lo largo del plano tiempo-frecuencia, que se han usado para esta transformada. En la Figura 41 (b) se muestra el resultado de la 
transformada, donde se puede apreciar la diferencia que existe con las transformadas anteriores, en las que, debido a los problemas de resolución y a los átomos hasta ese momento definidos, no se pueden observar simultáneamente las evoluciones de los armónicos en el estado estacionario y transitorio. En el caso de la DT, gracias a los átomos dragón que se han desarrollado, se obtiene, por primera vez en la literatura técnica, la evolución completa en el plano tiempo-frecuencia de las componentes de avería del rotor de un motor de inducción alimentado por inversor, tanto durante el arranque como en el régimen permanente.

Por último, en la Figura 43 se presenta el resultado de la $W V D$, que, como se ha mencionado ya, fue definida para obtener una distribución de la energía en el plano t-f sin pérdida de resolución. La ventaja de utilizar $W V D$ es que representa las evoluciones con líneas perfectamente finas, ya que no tiene pérdida de resolución. No obstante, presenta un gran inconveniente: la presencia de términos cruzados en la distribución de energía tiempo-frecuencia cuando se analizan señales multi-componentes (como es el caso); esta desventaja se puede apreciar de manera muy clara en la Figura 43 (a), viendo como dichos términos cruzados, que aparecen sobre todo alrededor de la fundamental, emborronan el resultado. Sin embargo, como se aprecia en la Figura 43 (b), en donde aparece un zoom durante los segundos 15 y 20, durante el estado estacionario sí que es posible distinguir levemente los armónicos de fallo, así como la componente fundamental, que aparece claramente definida por una fina línea.

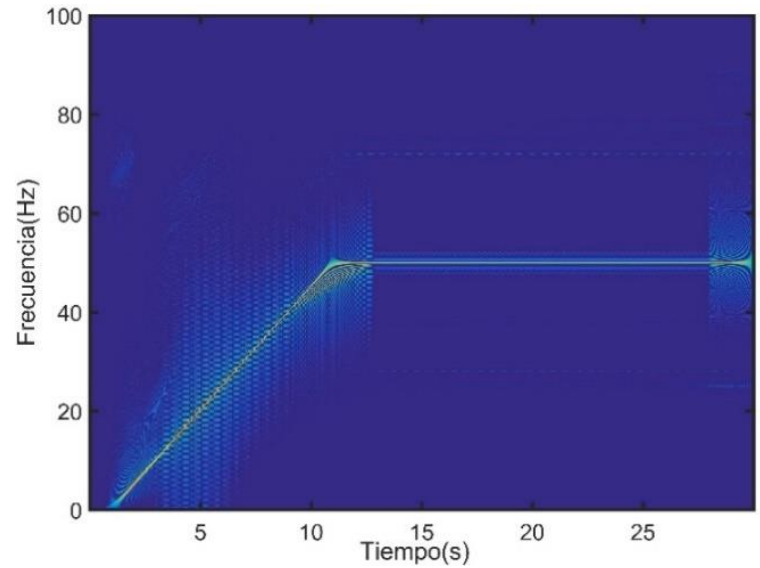

(a) Espectrograma resultante de aplicar la transformada $W V D$.

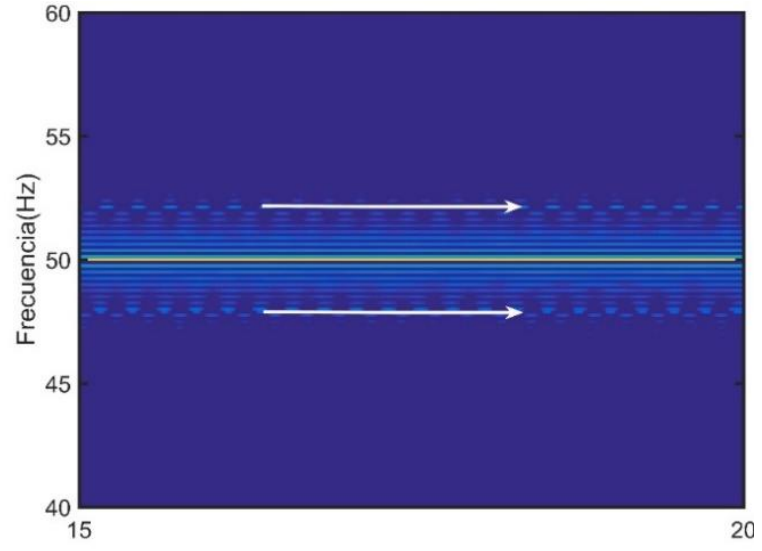

(b) Zoom entre los segundos 15 y 20 del espectrograma de la Figura 44 (a).

Figura 43. Análisis mediante la transformada $W V D$ de la señal analizada. 


\subsubsection{Ventajas e inconvenientes de la Transformada Dragón.}

Como todas las técnicas de detección de fallos, la transformada Dragón presenta ventajas, pero también una serie de inconvenientes o limitaciones a la hora de calcularla. Estas se exponen a continuación.

La principal ventaja de la $D T$ es que se adecúa a las necesidades de diagnóstico, es decir, se adapta a las evoluciones de las componentes de fallo que la señal a analizar presente. Así, en comparación con otras transformadas analizadas a lo largo de esta memoria, la mejora que incluye la $D T$ es la de generar átomos que se adaptan por completo a las evoluciones de las componentes que se desea detectar, a lo largo de toda la captura, con independencia de la complejidad del transitorio capturado, consiguiendo detectar dichas evoluciones con gran nitidez, tanto en tramos estacionarios como durante transitorios, con una pérdida de resolución extremadamente baja.

La primera de las limitaciones con la que se encuentra esta transformada es la necesidad de conocer a priori la estructura de la señal, ya que es necesario conocer la evolución teórica de la componente fundamental y de los armónicos de fallo para poder generar los átomos Dragón adecuados.

En segundo lugar, la transformada dragón presenta problemas cuando se quieren detectar evoluciones de componentes que se cruzan en determinados puntos del plano con pendientes distintas: en el punto de intersección, la transformada determina dos átomos a utilizar, siendo cada uno de ellos óptimo para una componente, pero desaconsejable para la otra, no pudiendo obtener adecuadamente ambas a la vez.

Finalmente, teniendo en cuenta que la energía del átomo se dispersa a lo largo de la evolución a detectar, al correlacionar con la señal no detectamos correctamente la amplitud de la componente en un punto, pues la densidad de energía que obtenemos en dicho punto depende de la amplitud a lo largo del tramo de evolución abarcada por el átomo: la amplitud en cada punto es una media de las que tiene alrededor a lo largo de esta evolución. 


\subsubsection{Cuantificación}

En este apartado se ilustra el método de cuantificación propuesto, siguiendo con el ejemplo del apartado anterior (en el capítulo 5 se explica en profundidad cómo se han realizado estos experimentos). Se trata de un motor de inducción de $0,75 \mathrm{~kW}$ de potencia nominal, con una barra rota (causada taladrando un agujero en la jaula del rotor) y alimentado con un inversor de ABB PowerFlex 40. Este inversor está programado para proporcionar un arranque de 10 segundos con la frecuencia de salida aumentando linealmente desde 0 a $50 \mathrm{~Hz}$. La corriente del estator ha sido capturada a $50 \mathrm{kHz}$ como frecuencia de muestreo, utilizando un sensor de efecto Hall, incluyendo el arranque y un estacionario largo. El motor consume $2.4 \mathrm{~A}$ al final del estado estacionario. En la Figura 44 (a) se puede observar la corriente del estator, en la Figura 44 (b) la velocidad medida y en la Figura 45 la evolución de la componente fundamental.

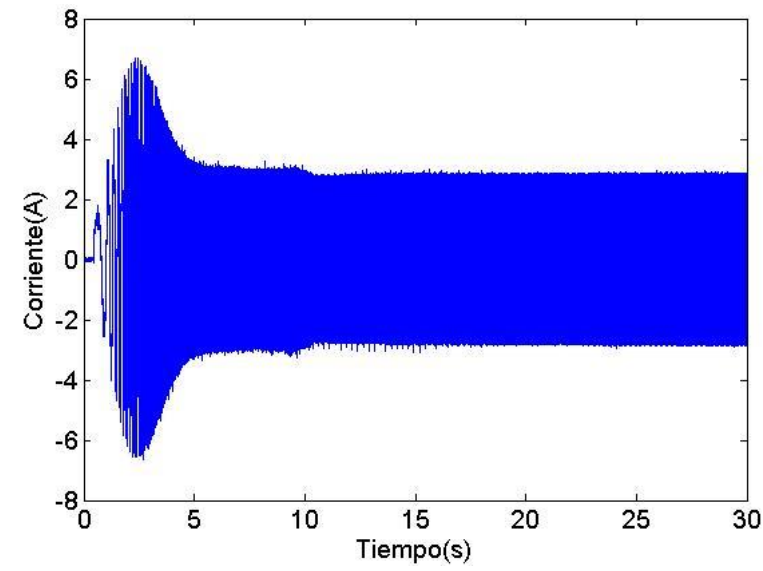

(a) Corriente de la señal analizada.

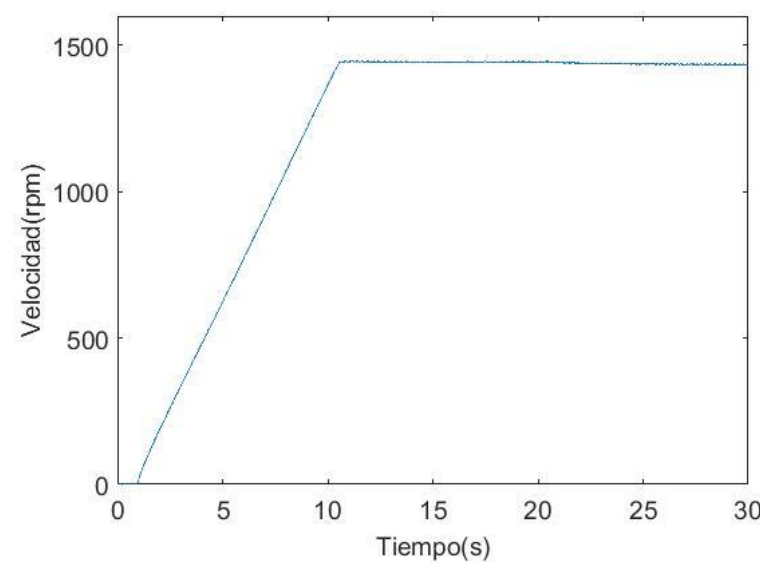

(b) Velocidad de la señal analizada.

Figura 44. Componentes señal a analizar.

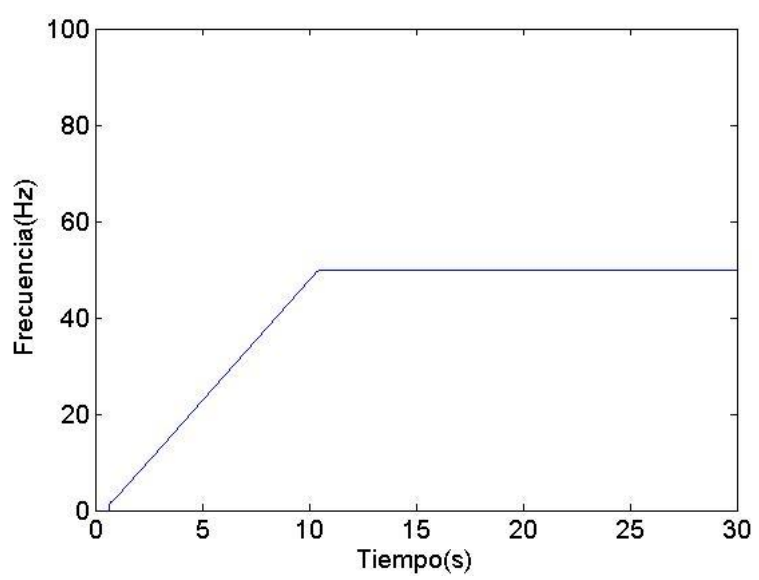

Figura 45. Evolución de la componente de frecuencia fundamental. 
Una vez que se tiene la corriente a analizar, la velocidad del motor y la componente fundamental, se pueden saber las trayectorias de los armónicos de fallo (Figura 46, LSH en amarillo y USH en marrón).

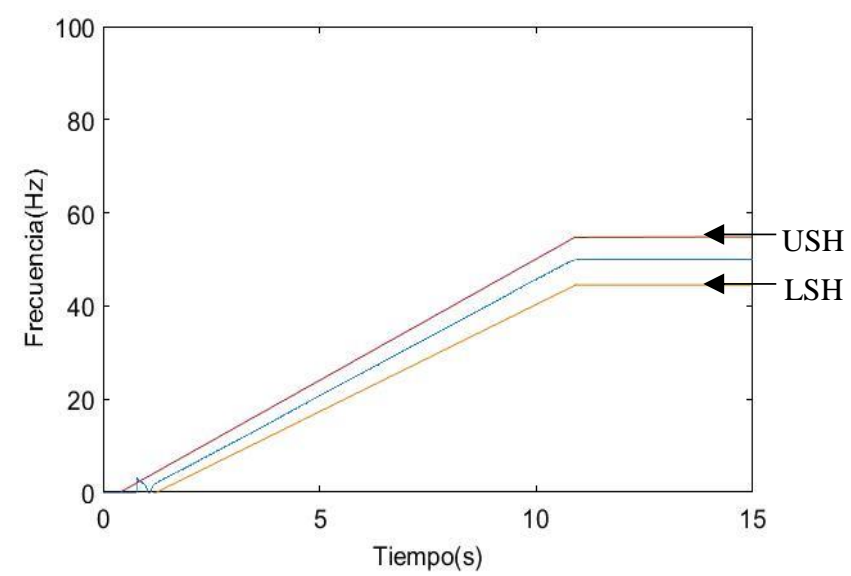

Figura 46. Evolución trayectorias componente fundamental, LSH y USH.

Tal y como se ha explicado anteriormente, con estos datos y la familia de átomos ajustados a estas trayectorias, se obtiene el espectrograma resultante de aplicar la $D T$, que aparece en la Figura 47.

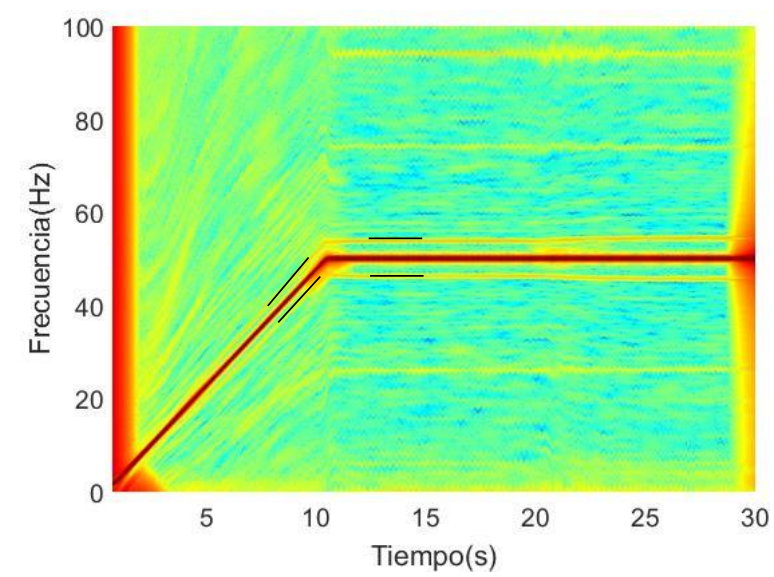

Figura 47. Análisis mediante la transformada Dragón de la señal a estudio.

En la Figura 48 se presenta un zoom entre los segundos 3,4 y 6,4 de la Figura 47, en donde se puede apreciar dos segundos del estado transitorio de la señal, viendo cómo la transformada dragón es capaz de seguir los armónicos de fallo tanto en el estado estacionario como en el estado transitorio, tal como se ha introducido anteriormente. 


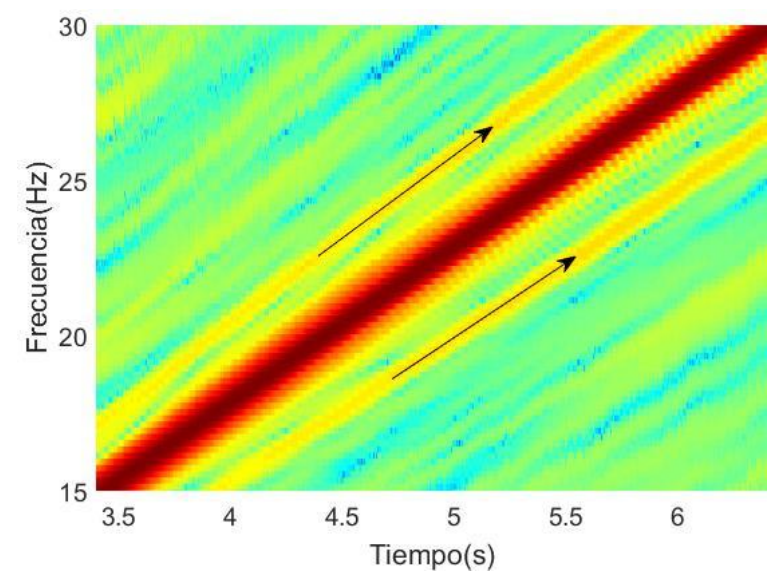

Figura 48. Zoom entre los segundos 3.4 y 6.4 de la Figura 47.

En la Figura 49 (a) y 49 (b) se puede observar una vista en tres dimensiones del espectrograma resultante de la Figura 47. En la Figura 49 (b) se pueden apreciar en 3D las trayectorias teóricas de las componentes armónicas, representadas en la Figura 48, superpuestas al espectrograma resultante, que permiten corroborar que lo que se está obteniendo es correcto, pues las evoluciones teóricas y las reales coinciden.

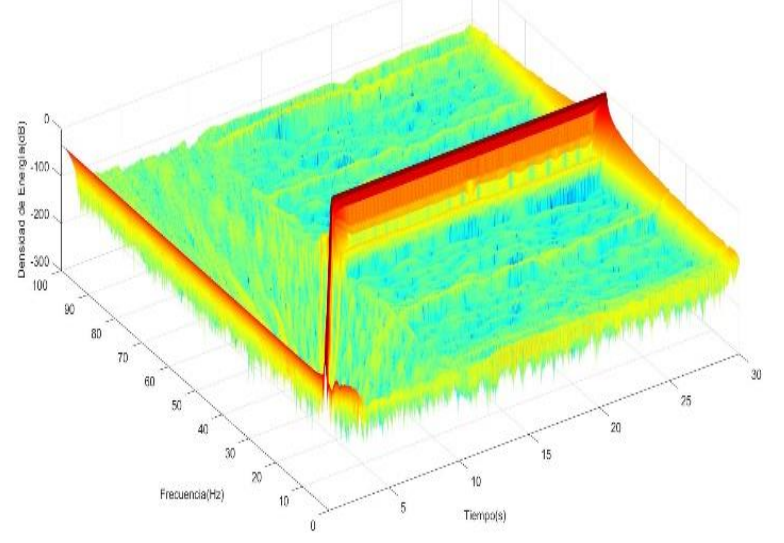

(a) Vista en 3D del espectro resultante en la Figura 47.

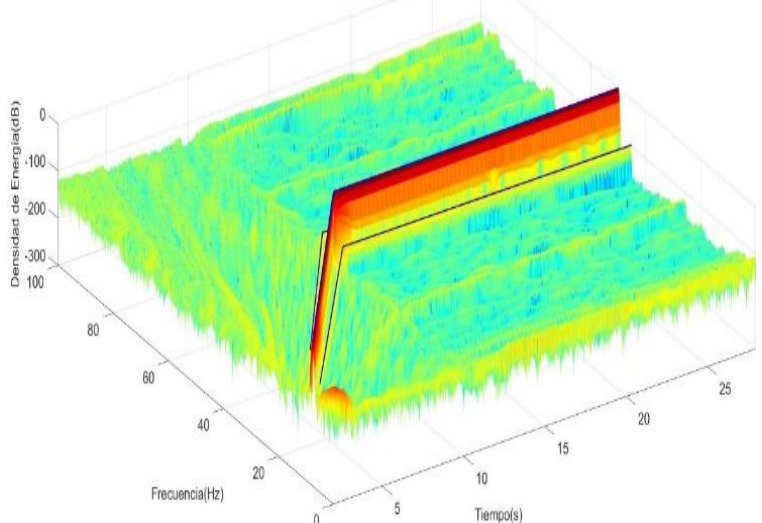

(b) Vista en 3D del espectro resultante en la Figura 47 con las trayectorias teóricas superpuestas.

Figura 49. Vistas 3D de la Figura 48.

Para poder cuantificar se deben definir unas bandas de búsqueda alrededor de las evoluciones teóricas, tal como se ve en la Figura 50 (apenas apreciables al ser muy estrechas). La amplitud de estas bandas se establece de manera experimental, de forma que se obtenga la mejor captura de los armónicos de fallo: ni demasiado amplia para 
interferir con el fundamental, ni demasiado estrecha para que la evolución real quede fuera de la banda alrededor de la teórica considerada.

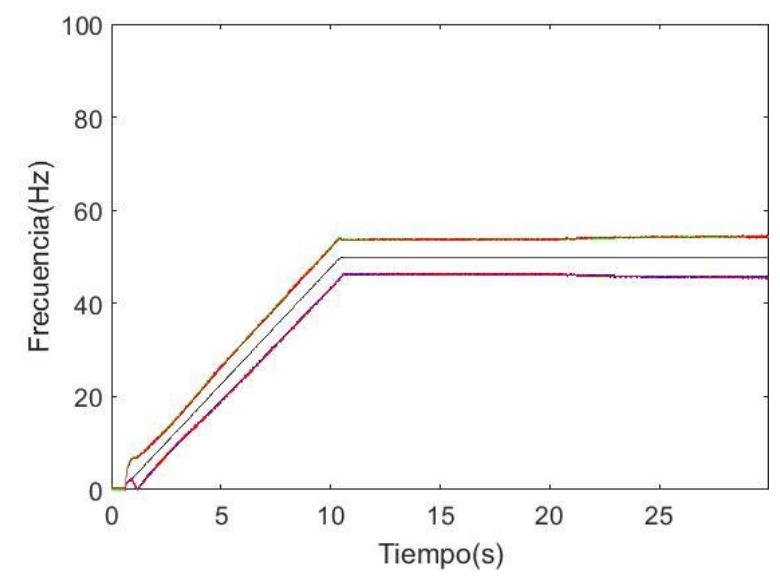

Figura 50. Evolución componente fundamental y armónicos por fallo de barra.

Dentro de cada banda, definida alrededor de la evolución teórica, se busca el máximo en cada instante de tiempo, lo que permite detectar la posición real de la componente de fallo con la mejor exactitud posible.

En la Figura 51 se han superpuesto las evoluciones finalmente obtenidas de los armónicos de fallo, sobre el resultado de la transformada Dragón, viendo así que, efectivamente, las evoluciones obtenidas de manera experimental coinciden con las que se tendrían de manera teórica, por lo que el resultado del espectrograma que se obtiene se puede concluir que es correcto.

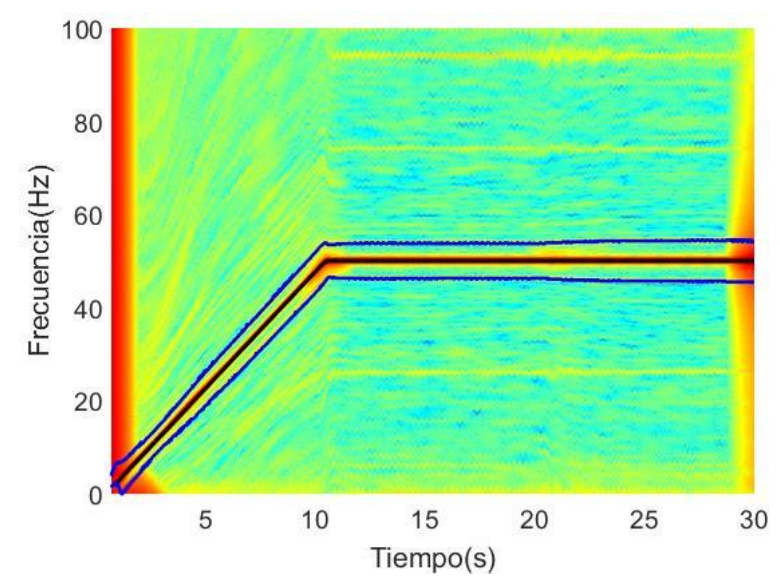

Figura 51. Espectrograma resultante con trayectorias armónicas superpuestas. 


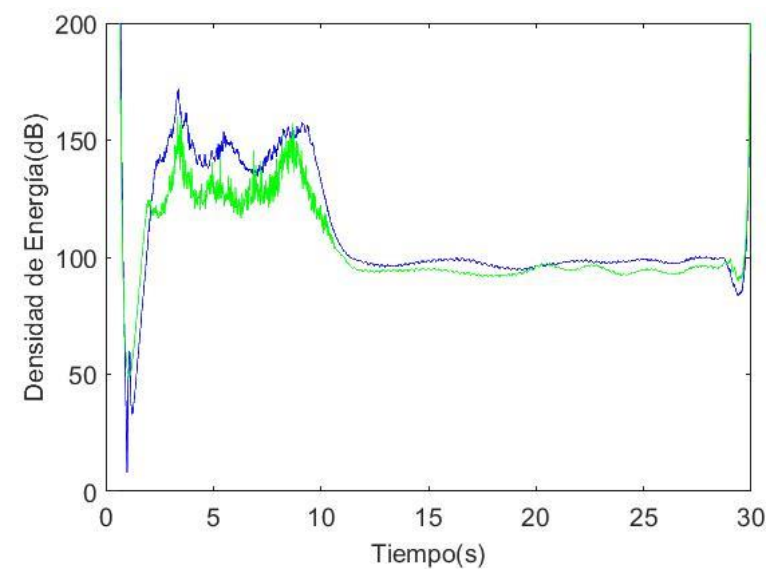

Figura 52. Densidades de Energía, en verde $L S H$ y en azul $U S H$.

Finalmente, en la Figura 52 se pueden observar las evoluciones temporales de las densidades de energía de los componentes de fallo de barras, Lower Sideband Harmonic y Upper Sideband Harmonic, que se representan en verde y azul, respectivamente. Esta Figura (Figura 52) funciona como un indicador de la amplitud instantánea de los armónicos de fallo a lo largo del transitorio. El crecimiento en su amplitud es un indicador de la presencia del fallo. Estas evoluciones se obtienen "midiendo" la densidad de energía del espectrograma de la Figura 51, a lo largo de las evoluciones superpuestas en dicha Figura, representadas de color azul.

Gracias a la técnica de cuantificación propuesta, se consigue obtener la evolución temporal de la densidad de energía de los armónicos de fallo, durante toda la captura realizada, con independencia de la complejidad del transitorio. La técnica aquí presentada ha sido comprobada con multitud de corrientes, a diversos niveles de carga y en diferentes situaciones, tal como se explica a continuación en el capítulo 5. Los pasos para calcularla aparecen a modo de diagrama en la Figura 53. 


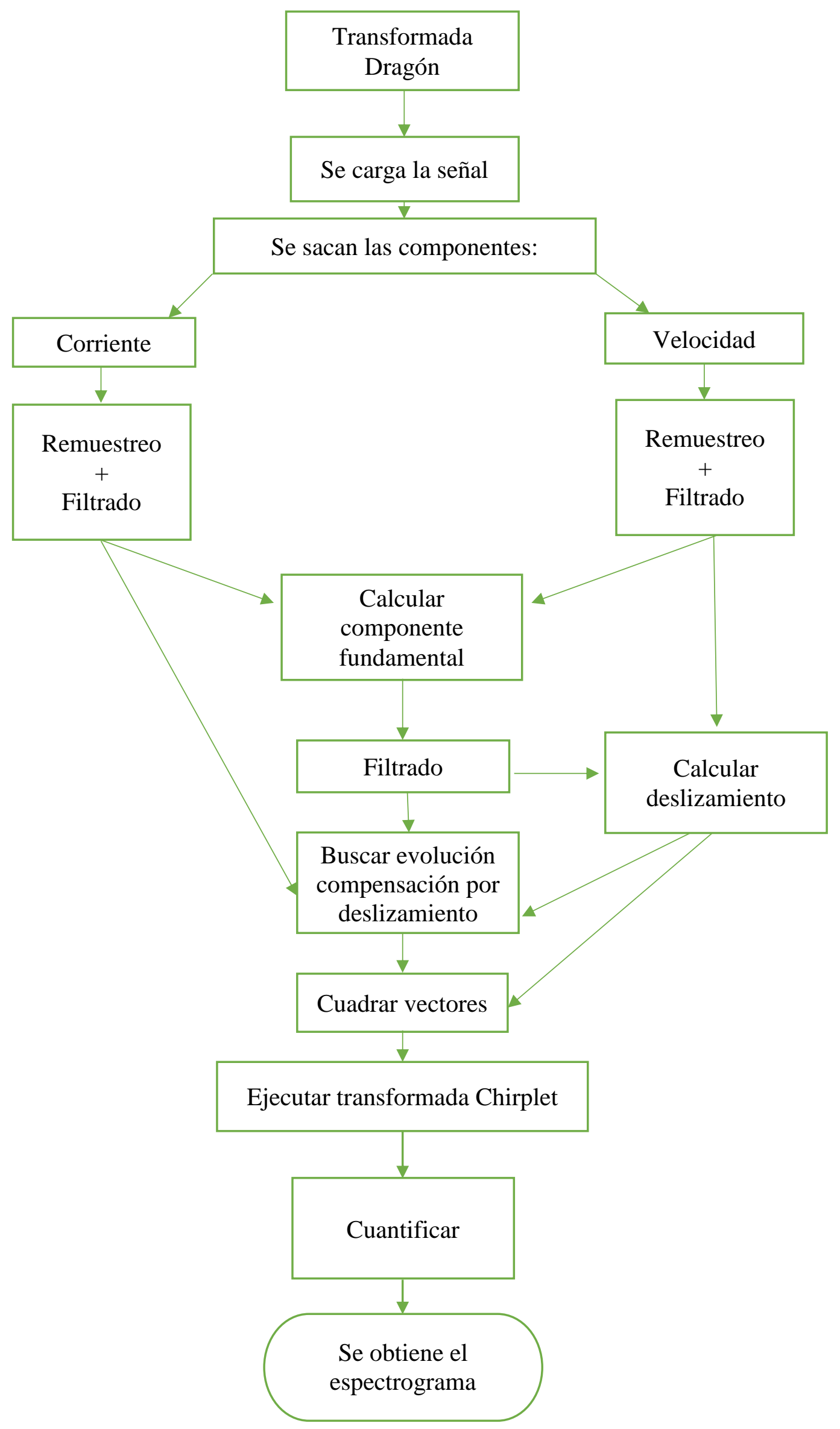

Figura 53. Diagrama de bloques resumen de los pasos para calcular la transformada Dragón. 
Es decir, los pasos para el cálculo de la transformada Dragón, que se exponen en la Figura 53, se pueden resumir en los siguientes:

1. El primer paso es cargar la señal que previamente ha sido medida en el laboratorio, esta señal presenta un tiempo total de 30 segundos, con 10 segundos de transitorio de arranque y se ha medido a una frecuencia de muestreo de $50 \mathrm{kHz}$.

2. De todas las variables que componen esta señal se sacaran los componentes que interesan para el cálculo de la transformada; corriente y velocidad.

3. Se procede a remuestrear ambas variables, pasando de una frecuencia inicial de muestreo de $50 \mathrm{kHz}$ a una frecuencia final de $256 \mathrm{~Hz}$. Del mismo modo, se realiza un filtro paso-bajo, para eliminar ruido a las variables.

4. Con la corriente y la velocidad filtradas y remuestreadas se calcula la componente fundamental.

5. Se realiza un segundo filtrado paso-bajo a la componente fundamental, para obtenerla libre de ruido.

6. Con la componente fundamental ya filtrada y la velocidad se obtiene el deslizamiento.

7. En este momento se calcula la componente compensación por deslizamiento.

8. Se cuadran vectores para que todas las variables presenten la misma longitud y poder operar con ellas.

9. Se ejecuta la transformada Chirplet, para conseguir que las trayectorias resultantes presenten el menor espesor posible.

10. Se cuantifica.

11. Finalmente se obtiene el espectrograma. 

5. Metodología de estudio experimental

\author{
5.1. Introducción
}

5.2. Banco de ensayos

5.3. Ensayos experimentales 


\section{Metodología de estudio experimental}

\subsection{Introducción}

En este capítulo se muestra la metodología experimental empleada para probar la capacidad de la transformada tiempo-frecuencia propuesta para la detección del fallo de barras durante el transitorio de arranque del motor, alimentado por un inversor. Se explicará a continuación el banco de ensayos empleado, incluyendo las diferentes fuentes de alimentación utilizadas junto con sus características más importantes. Así mismo, se hará una descripción detallada de todos los ensayos realizados. En la Figura 54 se muestra un diagrama explicativo de la metodología experimental empleada. 


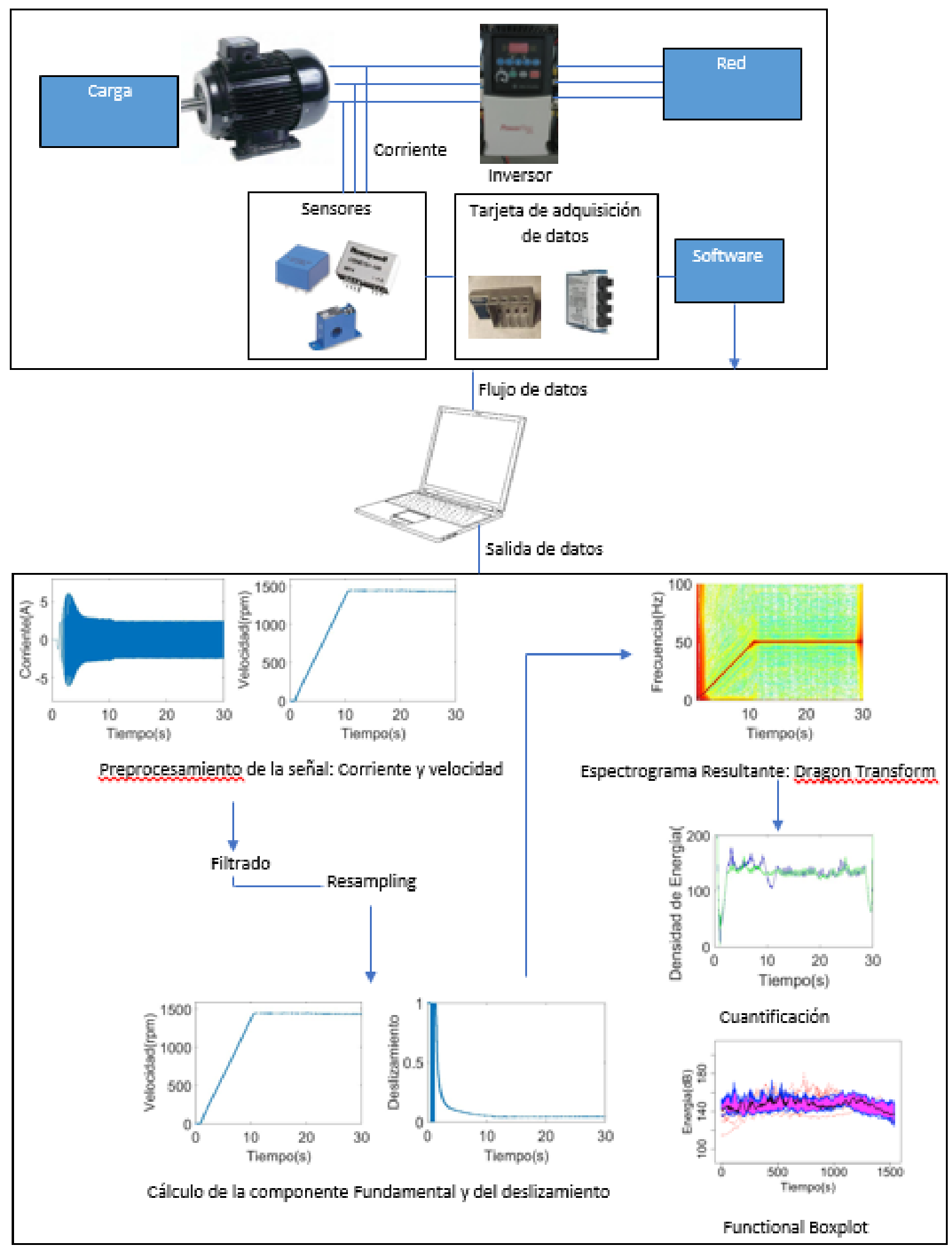

Figura 54. Esquema de la metodología empleada. 


\subsection{Banco de ensayos}

Los elementos que componen el banco de ensayos son los que se muestran en la Figura 55 y se describen en la Tabla 3.

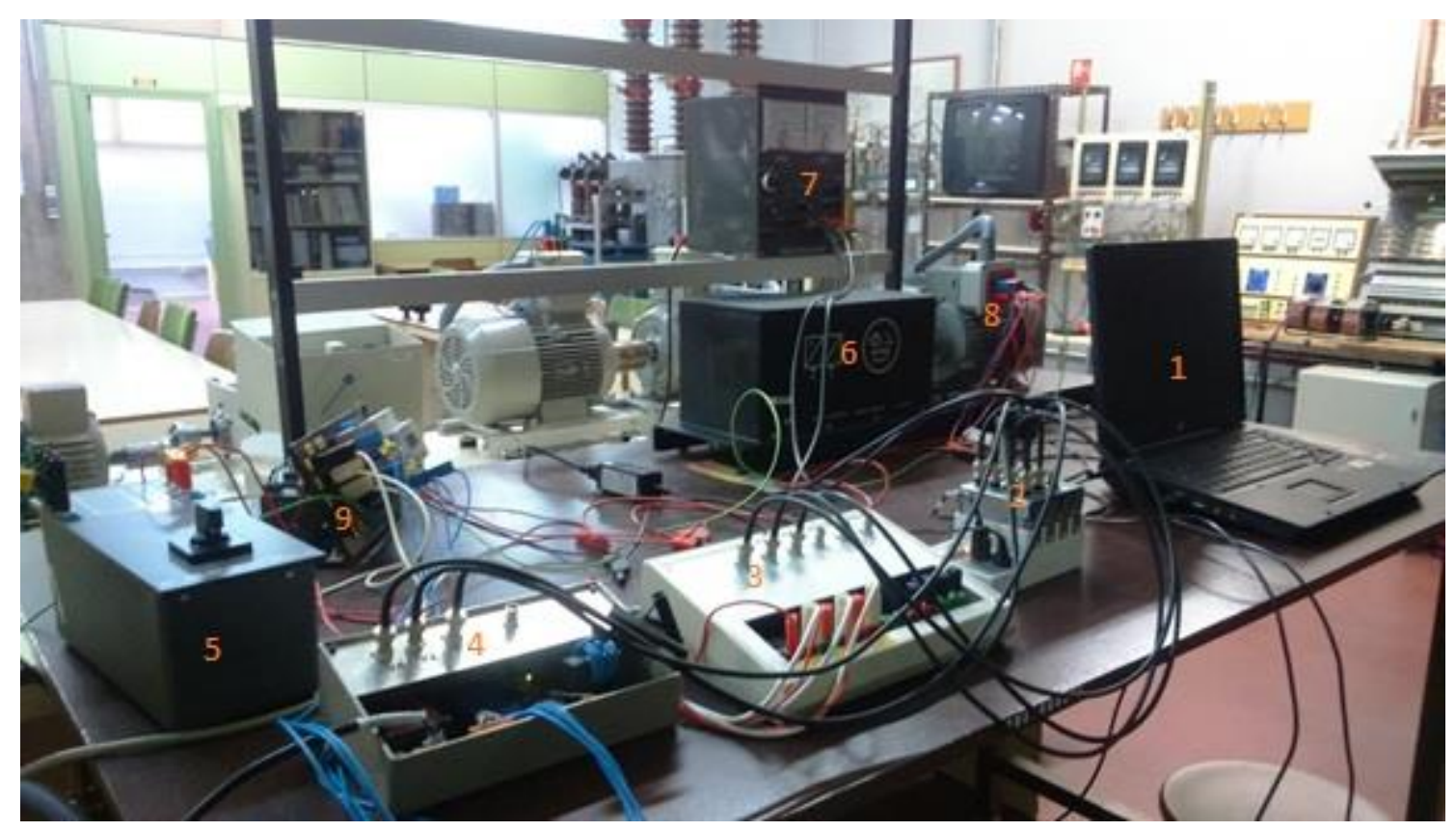

Figura 55.- Vista general del banco de ensayos.

Se ha utilizado un motor de Siemens, cuyas características físicas se pueden ver en la Figura 56, junto con la placa de características. En la Tabla 4 se muestra un resumen de los valores nominales de este motor.

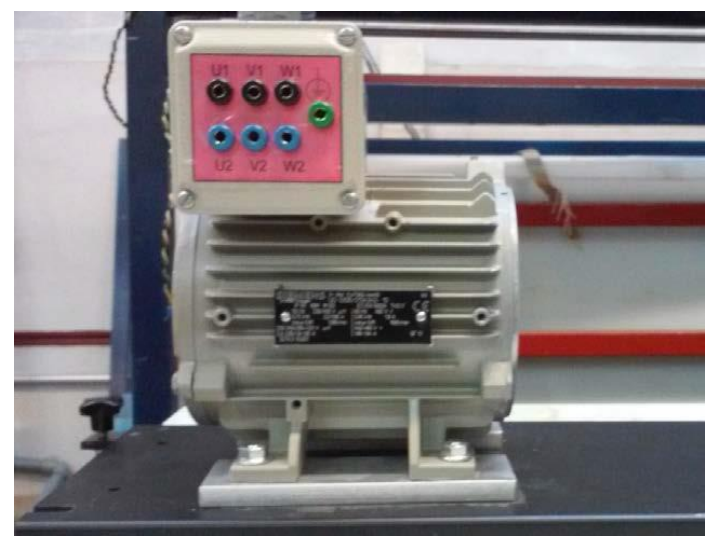

(a) Motor de inducción

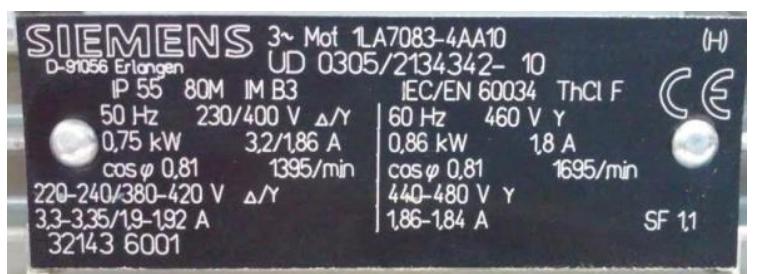

(b) Placa de características del motor de inducción

Figura 56. Motor de inducción con el que han sido realizados los ensayos y su placa de características 


\begin{tabular}{|c|c|c|}
\hline Elemento & Marca & Características \\
\hline 1. PC & HP Compaq 6715s & $\begin{array}{l}\text { Procesador AMD Turion } 64 \text { X2 TL-56 } \\
\text { Memoria Ram: } 2048 \text { MB } \\
\text { Disco Duro: 120GB SATA } \\
\text { Sistema Operativo: Windows } 7 \text { Pro } 64 \text { bits }\end{array}$ \\
\hline 2. Software & & $\begin{array}{l}\text { Librería NI-DAQmx } 15.5 \\
\text { Matlab, versión 2016a } \\
\text { Matlab Toolbox: Data Acquisition (Version 9.0), Signal } \\
\text { Processing (Version 9.0) }\end{array}$ \\
\hline \multirow[t]{2}{*}{$\begin{array}{l}\text { 3.Tarjeta de } \\
\text { adquisición de } \\
\text { datos }\end{array}$} & Chasis NI cDAQ-9174 & $\begin{array}{l}4 \text { ranuras } \\
\text { Rango entrada de voltaje: } 9 \mathrm{~V}-30 \mathrm{~V} \\
4 \text { Contadores/Temporizadores de } 32 \text { bits }\end{array}$ \\
\hline & 3 Módulos NI 9215 & $\begin{array}{l}4 \text { entradas diferenciales de } \pm 10 \mathrm{~V} \text {, aisladas respecto a tierra } \\
\text { Conector tipo BNC } \\
\text { Precisión: } 0,003456 \mathrm{~V} \\
\text { Canales simultáneos, no multiplexados } \\
\text { Frecuencia de muestreo máxima: } 100 \mathrm{kS} / \mathrm{s} \text { y canal } \\
\text { Resolución de entrada analógica: } 16 \mathrm{bits}\end{array}$ \\
\hline \multirow[t]{2}{*}{$\begin{array}{l}\text { 3. Placa de } \\
\text { sensores }\end{array}$} & & $\begin{array}{l}\text { Placa electrónica, hecha a medida, con fuente de } \\
\text { alimentación TRACO, con sensores de tensión }\end{array}$ \\
\hline & $\begin{array}{l}3 \text { Transductores de } \\
\text { tensión LEM LV 25-P }\end{array}$ & $\begin{array}{l}\text { Tipo: Efecto Hall } \\
\text { Tensiones de entrada: } 10 \ldots 500 \mathrm{~V} \\
\text { Tensión de alimentación: } \pm 12 \ldots 15 \mathrm{~V} \\
\text { Precisión a } 25^{\circ} \mathrm{C}: 0,9 \% \\
\text { Ancho de banda: } 0-10 \mathrm{kHz} \\
\text { Montaje: PCB }\end{array}$ \\
\hline \multirow[t]{3}{*}{$\begin{array}{l}\text { 4. Placas } \\
\text { sensores }\end{array}$} & & $\begin{array}{l}\text { Placa electrónica, hecha a medida, con fuente de } \\
\text { alimentación TRACO, con sensores de corriente }\end{array}$ \\
\hline & $\begin{array}{l}2 \text { Transductores de } \\
\text { corriente LEM LA } \\
25-\mathrm{NP}\end{array}$ & $\begin{array}{l}\text { Tipo: Efecto Hall } \\
\text { Corrientes de entrada: } 25 \mathrm{~A} \\
\text { Tensión de alimentación: } \pm 15 \mathrm{~V} \\
\text { Precisión a } 25^{\circ} \mathrm{C}: \pm 0,5 \% \\
\text { Ancho de banda: } 0-150 \mathrm{kHz} \\
\text { Montaje: PCB }\end{array}$ \\
\hline & $\begin{array}{l}1 \text { Transductor de } \\
\text { corriente Honeywell } \\
\text { CSNE151 }\end{array}$ & $\begin{array}{l}\text { Tipo: Efecto Hall } \\
\text { Corrientes de entrada: } 36 \mathrm{~A} \\
\text { Tensión de alimentación: } \pm 15 \mathrm{~V} \\
\text { Precisión a } 25^{\circ} \mathrm{C}: \pm 0,5 \% \\
\text { Ancho de banda: } 0-150 \mathrm{kHz} \\
\text { Montaje: PCB }\end{array}$ \\
\hline 5. Interruptor & & Interruptor de 4 polos \\
\hline $\begin{array}{l}\text { 6. Freno } \\
\text { Electromagnético }\end{array}$ & Lucas-Nüelle & $\begin{array}{l}\text { Modelo: SE2662-5R } \\
\text { Tipo: De polvo electromagnético con una potencia de } 1,1 \mathrm{~kW}\end{array}$ \\
\hline $\begin{array}{l}\text { 7. Unidad de } \\
\text { control del Freno }\end{array}$ & Lucas-Nüielle & $\begin{array}{l}\text { Modelo: SO3213-7S } \\
\text { Dos señales de salida en forma de tensión en el rango 0-10 V } \\
\text { proporcional al par motor y a la velocidad de giro }\end{array}$ \\
\hline
\end{tabular}

Tabla 3. Elementos banco ensayo de la Figura 55.

\begin{tabular}{|l|l|}
\hline \multicolumn{1}{|c|}{ Marca } & \\
\hline \hline Potencia & $0,75 \mathrm{~kW}$ \\
\hline Conexión: & En estrella \\
\hline Tensión nominal: & $400 \mathrm{~V}$ \\
\hline Corriente nominal en estrella & $1,86 \mathrm{~A}$ \\
\hline Velocidad de plena carga & $1395 \mathrm{rpm}$ \\
\hline Deslizamiento de plena carga & 0,07 \\
\hline Factor de potencia & 0,81 \\
\hline Par nominal & $5,13 \mathrm{Nm}$ \\
\hline
\end{tabular}

Tabla 4. Datos del motor de inducción de la Figura 56. 
Como fuentes de alimentación del motor se ha empleado un conjunto de inversores de distintos fabricantes. En la Figura 57 se muestra una fotografía de cada inversor y en la Tabla 5 se describen las características de cada uno ellos. Todos los inversores empleados generan la tensión de salida según un esquema de modulación por ancho de pulso, conocido por sus siglas en inglés PWM (Pulse Width Modulation). Posteriormente, en la Tabla 6, donde se explican los ensayos realizados, se muestran las características diferenciales de los inversores en cuanto a opciones de programación, control, etc.

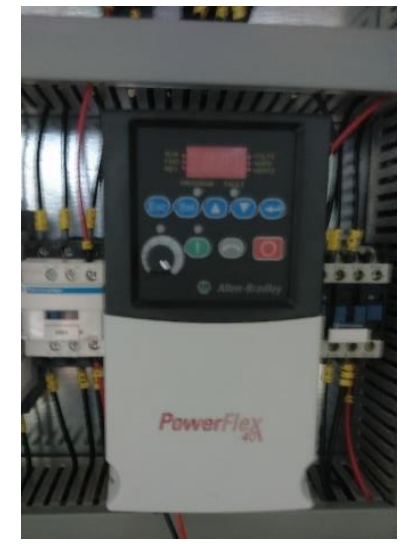

(a) Inversor PowerFlex 40 de Allen Bradley

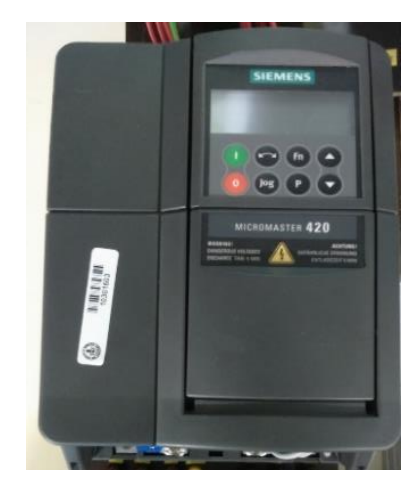

(d) Inversor Micromaster 420 de Siemens

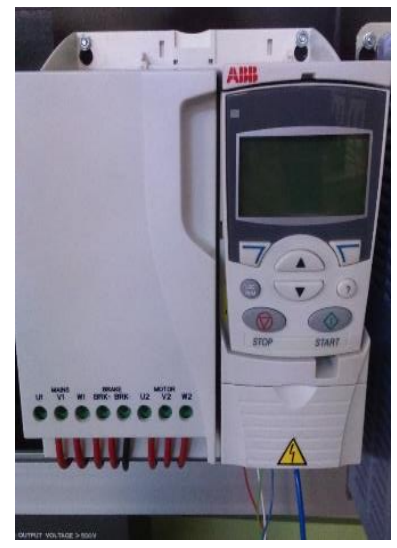

(b) Inversor ACS355 de ABB

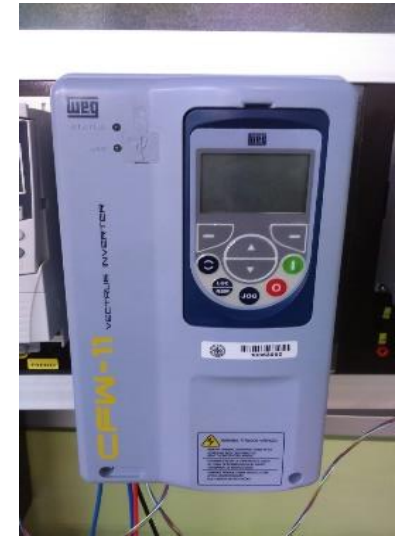

(c) Inversor CFW 11 de WEG

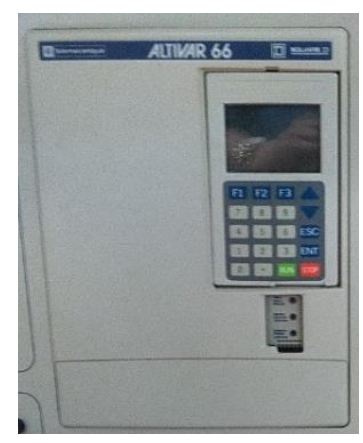

(d) Inversor Altivar 66 de Telemecánica

Figura 57. Inversores empleados en la experimentación. 


\begin{tabular}{|l|l|l|l|l|l|}
\hline $\begin{array}{c}\text { Fabricante y } \\
\text { acrónimo }\end{array}$ & $\begin{array}{c}\text { Allen Bradley } \\
\text { (AB) }\end{array}$ & \multicolumn{1}{|c|}{ ABB } & Siemens (SM) & $\begin{array}{c}\text { Telemecánica } \\
\text { (TM) }\end{array}$ & WEG \\
\hline Modelo & PowerFlex 40 & $\begin{array}{l}\text { ACS355-03E } \\
15 \mathrm{~A} 6-4\end{array}$ & $\begin{array}{l}\text { Micromaster } \\
420\end{array}$ & Altivar 66 & CFW-11 \\
\hline $\begin{array}{l}\text { Potencia } \\
\text { Nominal }\end{array}$ & $2,2 \mathrm{~kW}$ & $7,5 \mathrm{~kW}$ & $4 \mathrm{~kW}$ & $2,2 \mathrm{~kW}$ & $1,5 \mathrm{~kW}$ \\
\hline $\begin{array}{l}\text { Frecuencia de } \\
\text { conmutación por } \\
\text { defecto }\end{array}$ & $4 \mathrm{kHz}$ & $4 \mathrm{kHz}$ & $4 \mathrm{kHz}$ & $4 \mathrm{kHz}$ & $5 \mathrm{kHz}$ \\
\hline $\begin{array}{l}\text { Rango } \\
\text { frecuencias } \\
\text { PWM }\end{array}$ & $2-16 \mathrm{kHz}$ & $4-16 \mathrm{kHz}$ & $2-16 \mathrm{kHz}$ & - & $5-10 \mathrm{kHz}$ \\
\hline $\begin{array}{l}\text { Intensidad } \\
\text { Nominal }\end{array}$ & $7,5 \mathrm{~A}$ & $15,6 \mathrm{~A}$ & $10,2 \mathrm{~A}$ & $5,8 \mathrm{~A}$ & $3,6 \mathrm{~A}$ \\
\hline $\begin{array}{l}\text { Tensión } \\
\text { salida }\end{array}$ & $380-400 \mathrm{~V}$ & $380-400 \mathrm{~V}$ & $380-480 \mathrm{~V}$ & $400-460 \mathrm{~V}$ & $380-480 \mathrm{~V}$ \\
\hline $\begin{array}{l}\text { Tiempo de } \\
\text { aceleración } \\
\text { programable }\end{array}$ & $0-600 \mathrm{~s}$ & $0-1800 \mathrm{~s}$ & $0-999 \mathrm{~s}$ & $0,1-999,9 \mathrm{~s}$ & $0-999 \mathrm{~s}$ \\
\hline
\end{tabular}

Tabla 5. Características de los inversores utilizados.

\subsection{Ensayos experimentales}

En la validación experimental, gracias a disponer de una gran variedad de inversores, se busca probar la técnica de análisis en diferentes condiciones de funcionamiento. Se ha planteado un conjunto de ensayos donde se han ido modificando los siguientes parámetros:

- $\quad$ Nivel de carga del motor. Se han considerado dos niveles de carga distintos. El motor se ha ensayado a un nivel de carga bajo y un nivel de carga alto. Como nivel medio de carga se ha considerado un par resistente entre 2,2 y 3,0 $\mathrm{Nm}$, mientras que para nivel de carga alto el par resistente se fijaba entre 3,6 y 4,0 Nm. Lo que supone, en porcentaje, con respecto al par nominal del motor, que se está cargando al $I M$ al operar en nivel de carga bajo con un par resistente de entre el $42,88 \%$ al 58,48\%, siendo para el nivel de carga alto el par resistente fijado entre el 70,17\% y el $77,97 \%$.

- Forma o modalidad del arranque: Como se puede ver en la Tabla 6, algunos variadores permiten programar la relación entre la frecuencia de salida y el tiempo durante el transitorio de arranque. Por esta razón, se han programado arranques lineales, pero también en forma de $S$. 
- $\quad$ Tiempo de arranque: Aunque este parámetro también es ajustable, todos los transitorios de arranque se han programado en 10 segundos, ya que se considera que la variación de este parámetro no añade información útil al estudio.

- Frecuencia de conmutación del inversor: Se ha observado que las estrategias empleadas por cada fabricante para generar la onda de tensión tipo PWM son diferentes y por esta razón, considerando que este parámetro podría tener alguna influencia en los resultados, en los inversores que tenían esta opción, es posible que se modifique la frecuencia de conmutación o de la onda portadora. Aunque se ha experimentado a distintos valores de PWM, para los resultados que en esta tesis se muestran se han llevado a cabo con las frecuencias de conmutación por defecto de cada inversor, es decir, se han fijado en $4 \mathrm{kHz}$, salvo para WEG, cuya frecuencia de conmutación se fija por defecto en $5 \mathrm{kHz}$.

- $\quad$ Tipo de control (V/f lineal, SVC(Vel), SVC(Par)): todos los inversores empleados operan con el tipo de control V/f lineal, sin embargo, algunos variadores pueden funcionar con otro tipo de control (por ejemplo, el SVC: Sensorless Vector Control de WEG). En algunos casos, se ha decidido incluir en el estudio estos tipos de control.

- Grado de compensación por deslizamiento: El tipo de control empleado es en lazo abierto. Es decir, no se mide la velocidad para calcular su error y modificar de esta forma, la frecuencia fundamental de salida del inversor. El inversor solamente fija la frecuencia fundamental de la tensión de salida y la mantiene constante. De esta forma, la velocidad real del motor despenderá del nivel de carga, es decir, del deslizamiento. Algunos de los inversores empleados incorporan un sistema que se denomina de compensación por deslizamiento. Estiman este parámetro a partir de la corriente del estator y luego modifican la frecuencia de salida del inversor para aumentar la velocidad real de motor y que se acerque a la frecuencia de consigna. El grado de compensación se tiene que ajustar experimentalmente según un procedimiento que explica cada fabricante. En este caso, en los inversores que lo permiten, se ha modificado de forma arbitraria, que no aleatoria, escogiendo entre las opciones y valores disponibles para cada fabricante. Los 
niveles de compensación por deslizamiento que se han considerado se presentan en la Tabla 6. Según el inversor, el nivel de compensación se fija en Hz, en porcentaje, o según el tipo de control, como el Flux Current Control $(F C C)$ disponible en el inversor de Siemens.

- Frecuencia de consigna: se mantiene constante, en todos los ensayos, a $50 \mathrm{~Hz}$, aunque es otro de los parámetros ajustables de los inversores.

En la Tabla 6, se exponen de forma detallada todos los parámetros que se han modificado durante los ensayos según el inversor empleado en cada caso.

El estudio se ha completado con el simulacro de diferentes severidades del fallo. La rotura de una barra del rotor se ha simulado mediante un taladro en la unión de la barra con el anillo de cortocircuito. Se han considerado 5 estados de fallo distintos:

- $\quad$ Estado R1: Motor sin excentricidad y sin barra rota. Se puede considerar el caso sano.

- $\quad$ Estado R2: Motor con excentricidad mixta por desalineamiento del eje del motor con el freno. La profundidad del taladro ejecutado es de $4,2 \mathrm{~mm}$ y el diámetro del agujero es de $2,5 \mathrm{~mm}$.

- Estado R3: Fallo de barra rota con excentricidad mixta por la misma causa que en el estado R2. La profundidad del taladro ejecutado es de 9,4 $\mathrm{mm}$ y el diámetro del agujero es de $2,5 \mathrm{~mm}$.

- Estado R4: Fallo de barra rota sin excentricidad. La profundidad del taladro ejecutado es de $17 \mathrm{~mm}$ y el diámetro del agujero es de $2,5 \mathrm{~mm}$.

- Estado R5: Fallo de barra completamente rota sin excentricidad. La profundidad del taladro ejecutado es de $17 \mathrm{~mm}$ y el diámetro del agujero es de $3,5 \mathrm{~mm}$.

En la Figura 58 se muestran varias imágenes donde se puede apreciar la posición del taladro realizado para simular la rotura de la barra. Posteriormente, se cortó una jaula para ver la forma y longitud real de las barras del rotor. 


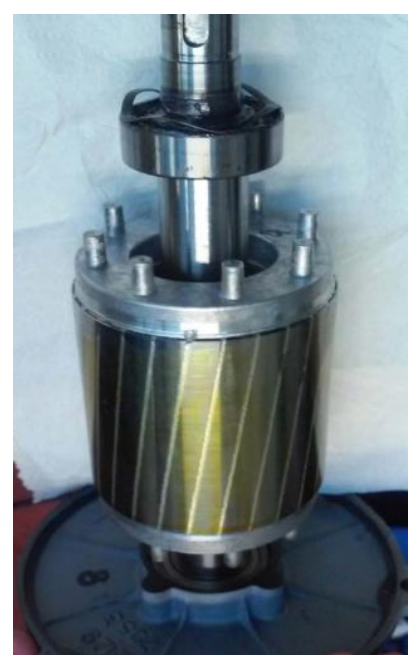

(a) Perforación en una de las barras del rotor para la severidad R2 de $4.2 \mathrm{~mm}$ de profundidad

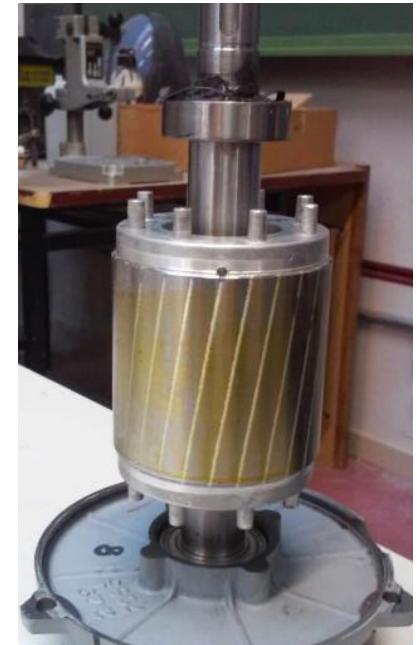

(b) Perforación en una de las barras del rotor para la severidad $\mathrm{R} 5$ de $17 \mathrm{~mm}$ de profundidad.

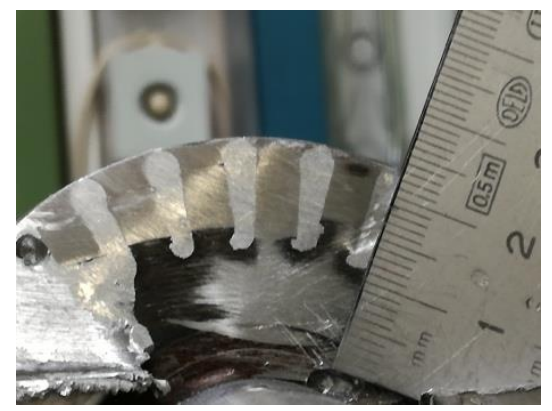

(c) Detalle de un corte de la jaula donde se observa la forma de la barra

Figura 58. Perforaciones de la jaula del rotor.

Se ha realizado una batería de ensayos muy extensa considerando las diferentes combinaciones de parámetros de la Tabla 6 y las diferentes severidades de fallo. En el caso del motor sano (Estado R1) se han realizado 30 ensayos por nivel de carga. Para el resto de estados considerados, se han realizado 15 pruebas por nivel de carga. En algunos casos, por ejemplo, el arranque en $\mathrm{S}$, sólo se han realizado 5 ensayos por nivel de carga para la severidad de sano (R1) y tres para el resto de casos. Así en total se han llevado a cabo un total de 2765 ensayos.

Todos los ensayos realizados han seguido el mismo procedimiento y se han capturado en las mismas condiciones. En cada ensayo se han capturado las siguientes variables físicas:

- Corriente de línea de las tres fases del motor.

- Tensión fase-neutro de las tres fases. 
- Velocidad de giro del motor, proporcionada por la unidad de control del freno electromagnético.

- Par motor, proporcionado por la unidad de control del freno electromagnético.

\begin{tabular}{|c|c|c|c|c|c|}
\hline Parámetros & ABB & $\mathbf{A B}$ & TM & WEG & Siemens \\
\hline Frecuencia de consigna & $50 \mathrm{~Hz}$ & $50 \mathrm{~Hz}$ & $50 \mathrm{~Hz}$ & $50 \mathrm{~Hz}$ & $50 \mathrm{~Hz}$ \\
\hline \multicolumn{6}{|l|}{ Nivel de carga: } \\
\hline Vacío & $\mathrm{X}$ & $\mathrm{X}$ & $\mathrm{X}$ & $\mathrm{X}$ & $\mathrm{X}$ \\
\hline Nivel bajo & $\mathrm{X}$ & $\mathrm{X}$ & $\mathrm{X}$ & $\mathrm{X}$ & $\mathrm{X}$ \\
\hline Nivel alto & $\mathrm{X}$ & $\mathrm{X}$ & $\mathrm{X}$ & $\mathrm{X}$ & $\mathrm{X}$ \\
\hline \multicolumn{6}{|l|}{ Tipo de control: } \\
\hline V/f Lineal & $\mathrm{X}$ & $\mathrm{X}$ & $\mathrm{X}$ & $\mathrm{X}$ & $\mathrm{X}$ \\
\hline SVC/velocidad & $\mathrm{X}$ & $\mathrm{X}$ & & $\mathrm{X}$ & \\
\hline SVC/Par & $\mathrm{X}$ & & & & \\
\hline \multicolumn{6}{|l|}{ Tipo de arranque V/f: } \\
\hline Lineal & $\mathrm{X}$ & $\mathrm{X}$ & $\mathrm{X}$ & $\mathrm{X}$ & $\mathrm{X}$ \\
\hline S & $\mathrm{X}$ & & & & \\
\hline $\mathrm{U}$ & & $\mathrm{X}$ & $\mathrm{X}$ & & \\
\hline Tiempo arranque & $10 \mathrm{~s}$ & $10 \mathrm{~s}$ & $10 \mathrm{~s}$ & $10 \mathrm{~s}$ & $10 \mathrm{~s}$ \\
\hline Frecuencia de Conmutación & $4 \mathrm{kHz}$ & $4 \mathrm{kHz}$ & $4 \mathrm{kHz}$ & $5 \mathrm{kHz}$ & $4 \mathrm{kHz}$ \\
\hline \multicolumn{6}{|l|}{ Comp. por deslizamiento: } \\
\hline $0 \%$ & $\mathrm{X}$ & $\mathrm{X}$ & $\mathrm{X}$ & $\mathrm{X}$ & $\mathrm{X}$ \\
\hline $50 \%$ & & & & & $\mathrm{X}$ \\
\hline $2 \mathrm{~Hz}$ & & $\mathrm{X}$ & & & \\
\hline $3 \mathrm{~Hz}$ & & & $\mathrm{X}$ & & \\
\hline $4 \mathrm{~Hz}$ & & $\mathrm{X}$ & & & \\
\hline FCC & & & & & $\mathrm{X}$ \\
\hline
\end{tabular}

Tabla 6. Parámetros de los inversores modificados en los ensayos del motor.

La frecuencia de muestreo se fijó en $50 \mathrm{kHz}$ y el tiempo total del ensayo fue 30 segundos. En todos los ensayos capturados se distinguen tres períodos de tiempo diferenciados:

- Período inicial donde el motor está parado hasta que se ordena al inversor que inicie la maniobra de arranque del motor. Esta fase es muy corta en el tiempo. 
- Período de arranque del motor. Su duración está controlada por el inversor y siempre se fijó en 10 segundos.

- Período final de funcionamiento en estado estacionario. La duración de esta fase es variable pero nunca inferior a 10 segundos, hasta completar los 30 segundos totales del ensayo.

La captura de los ensayos se realiza con Matlab desde el ordenador portátil que se muestra en la Figura 55. Se emplean funciones de Matlab pertenecientes a la "Data Acquisition Toolbox" que permiten acceder directamente a la API de National Instruments que controla la tarjeta de adquisición de datos. De esta forma, los datos se graban directamente en el formato “*.mat" de Matlab. En la Figura 59 se representan, a modo de ejemplo, las variables medidas en un ensayo del motor alimentado con el inversor $\mathrm{AB}$, con arranque V/f lineal y control lineal, con severidad de fallo R1 y nivel de carga alto.

La frecuencia de muestreo es muy elevada, por esta razón para procesar los ensayos se han empleado técnicas de resampleado fraccional ([65], [66]). Esta técnica permite cambiar la frecuencia de muestreo a un valor adecuado al análisis que se va a realizar posteriormente. Para aplicar este método se parte de la muestra inicial con una frecuencia de muestreo elevada y se aplica primero interpolación y posteriormente un diezmado a la misma, en función de la frecuencia final que se quiera conseguir, a razón del cociente $R / D$, siendo $R$ la razón de interpolación y $D$ la razón de diezmado. Así al realizar un remuestreo de la señal, el número de muestras de la señal también cambia. Por ejemplo, si el rango de frecuencias de interés de la señal es 0 a $100 \mathrm{~Hz}$, se puede realizar un remuestreo con una frecuencia final de $256 \mathrm{~Hz}$. Esto reduce el número de muestras de la señal y, por consiguiente, el esfuerzo computacional al aplicar la técnica de análisis tiempo-frecuencia, sin perder calidad o precisión en el resultado, tal como se muestra en las Figuras 60 y 61. 


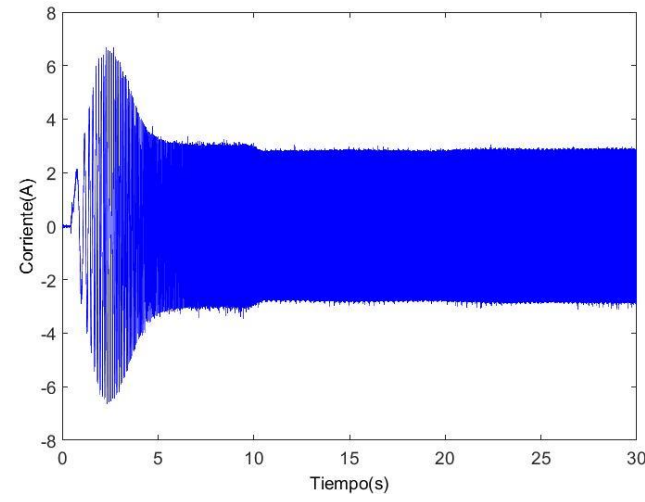

(a) Corriente Fase $\mathrm{R}$

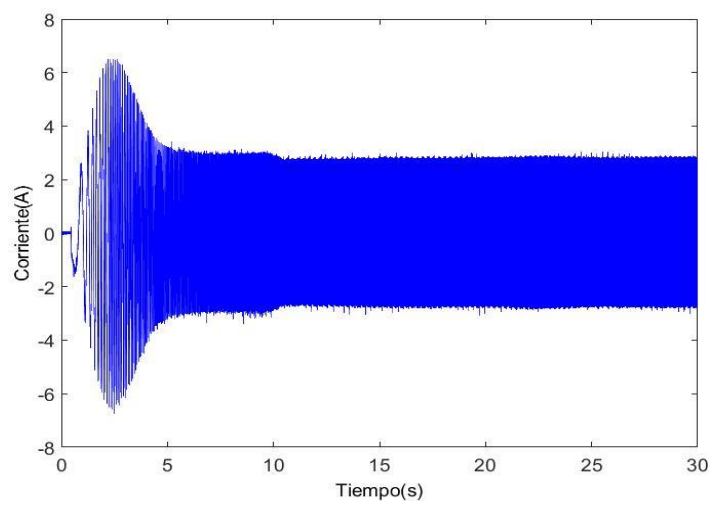

(c) Corriente Fase $\mathrm{S}$

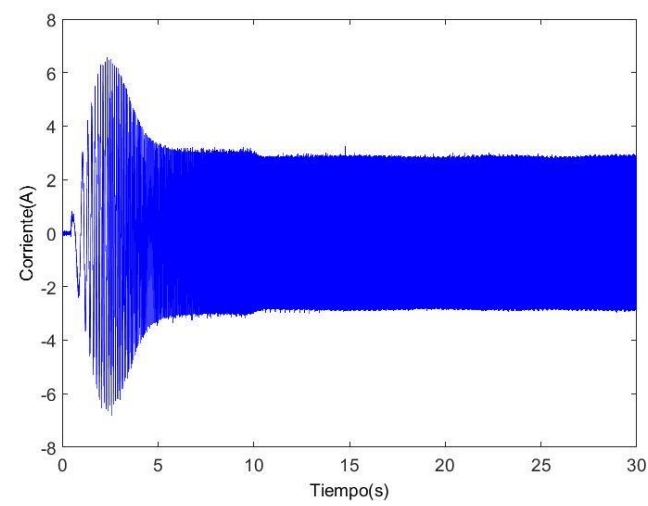

(e) Corriente Fase $\mathrm{T}$

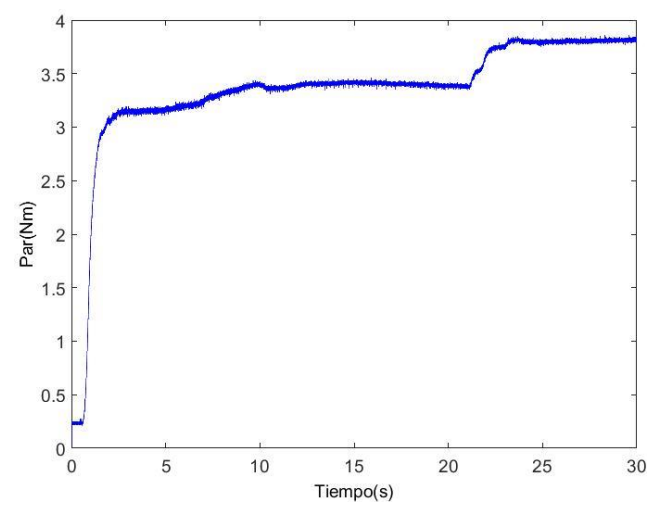

(g) Par resistente

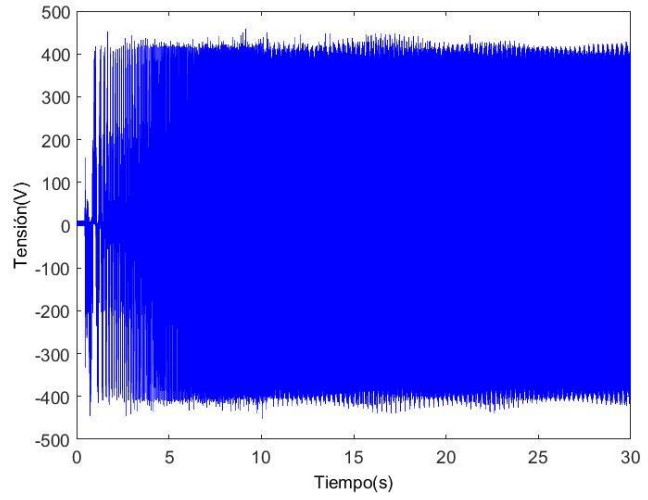

(b) Tensión Fase R

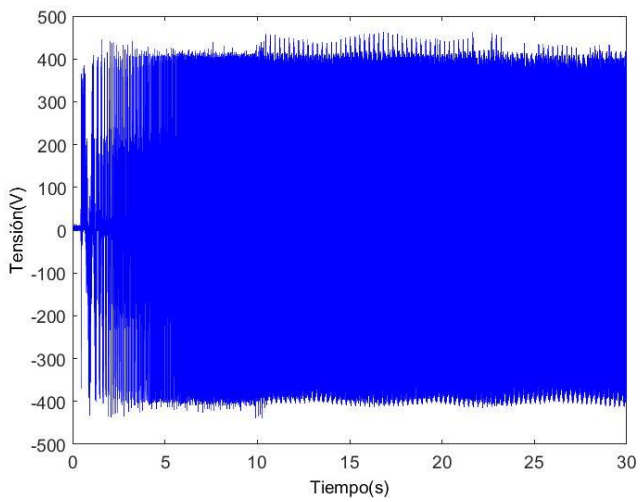

(d) Tensión Fase S

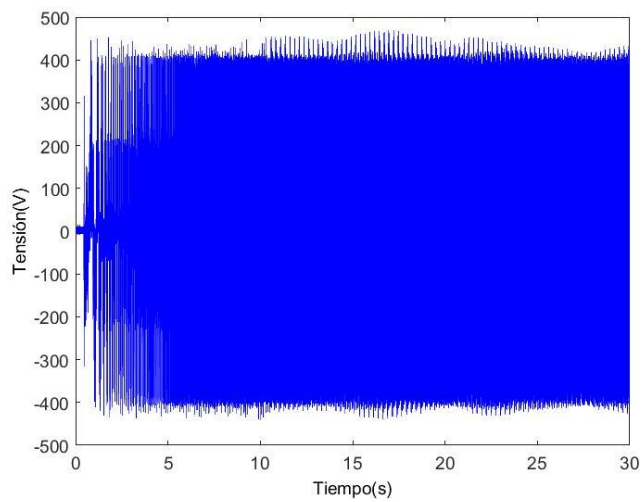

(f) Tensión Fase T

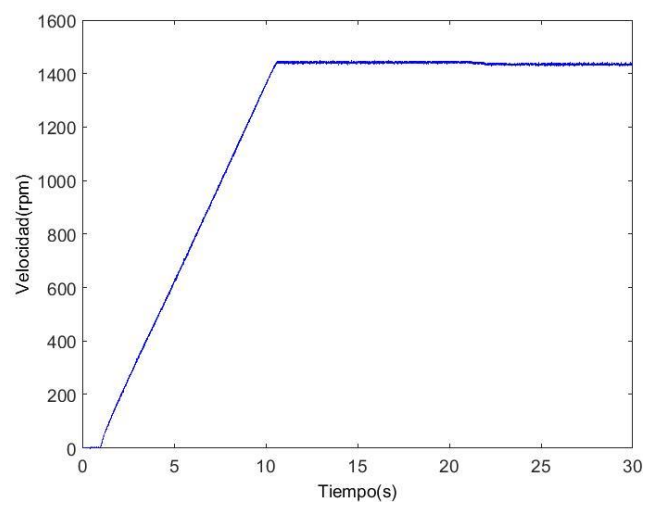

(h) Velocidad de giro

Figura 59. Variables registradas en el banco de ensayos en un arranque del motor alimentado por el inversor $\mathrm{AB}$. 
En estas Figuras que a continuación aparecen (donde se avanzan resultados), se muestra el resultado de aplicar la transformada Dragón a una señal de corriente durante el arranque del motor. La Figura 61 se obtuvo para una frecuencia de muestreo de $50 \mathrm{kHz}$ y el tiempo de computación aproximado fue de 2 horas. Para obtener la Figura 60 con la misma técnica, se remuestreó la señal a una frecuencia de $256 \mathrm{~Hz}$. En este caso, no se observa pérdida de resolución en tiempo o en frecuencia y el tiempo de computación se redujo a 10 minutos. Resulta notable la disminución de tiempo computacional obtenida aplicando la técnica de resampleado fraccional sin pérdida de resolución.

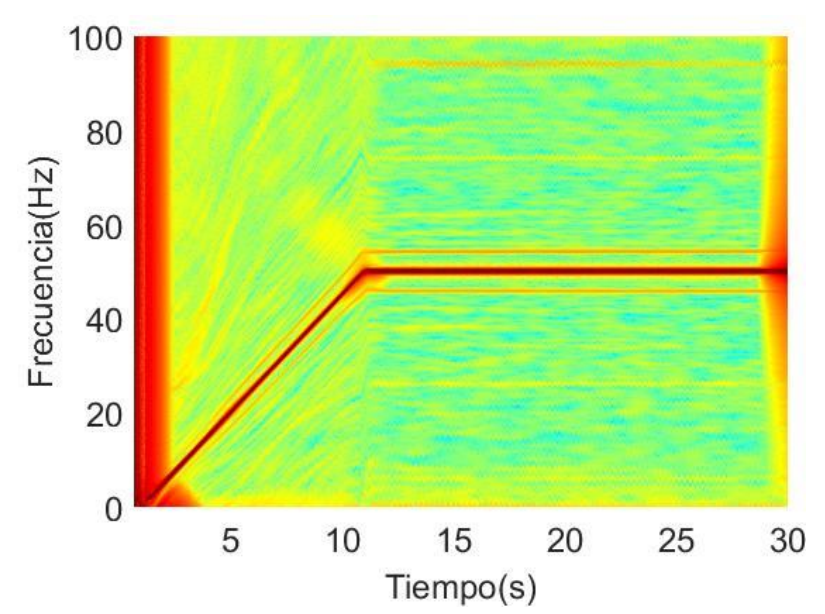

Figura 60. Análisis de la señal de la corriente de la Figura 59, empleando una frecuencia de muestreo de $256 \mathrm{~Hz}$.

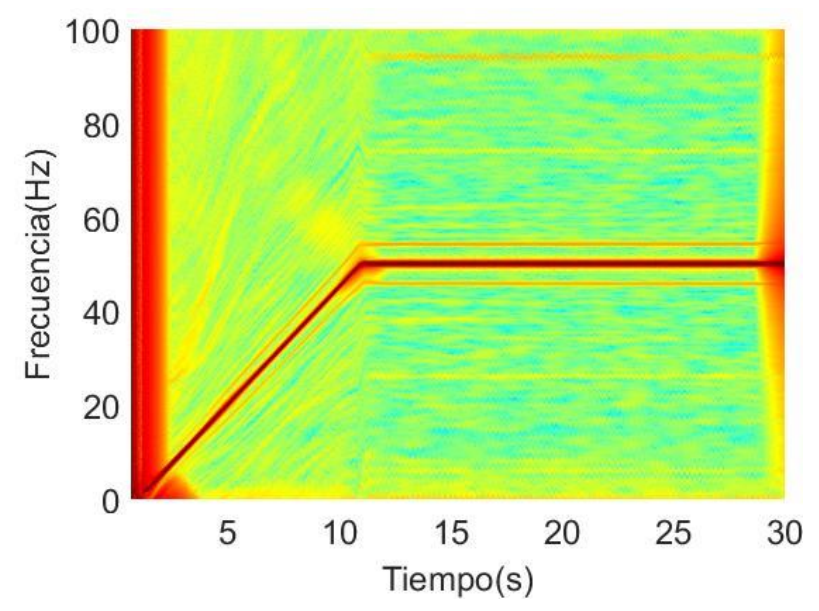

Figura 61. Análisis de la señal de la corriente de la Figura 59, empleando una frecuencia de muestreo de $50 \mathrm{kHz}$. 



\section{Resultados}

\subsection{Introducción}

6.2. Detección de barras rotas durante el arranque

6.2.1. Análisis de los ensayos

6.2.2. Conclusiones parciales

6.2.3. Ensayos especiales

6.3. Detección de excentricidad mixta durante el arranque

6.4. Cuantificación del fallo de barras.

6.4.1. Introducción.

6.4.2. Curvas cuantificación.

6.4.3. Conclusiones parciales.

6.5. Resultados relativos a la discriminación en motores con compensación por deslizamiento. 


\section{Resultados}

\subsection{Introducción}

En el capítulo 5 se ha descrito la metodología experimental para probar el método propuesto en el capítulo 4 para detectar fallos durante el arranque por inversor de un motor de inducción, así en este capítulo se muestran los resultados obtenidos.

\subsection{Detección barras rotas durante el arranque}

En este apartado se tratan, concretamente, los fallos debidos a barras rotas $(B R B)$, para lo cual se hizo un gran número de experimentos, que se detallan en el punto 5 de esta memoria. En este caso, se van a presentar los resultados correspondientes a dos severidades de fallo: motor sano (R1) y motor con una barra completamente rota (R5). Para analizar las prestaciones de la transformada propuesta se van a presentar los siguientes resultados: distribución tiempo-frecuencia, distribución tiempo-frecuencia con la trayectoria teórica de los armónicos de fallo, espectro del estado estacionario y cuantificación del fallo. En este último resultado, se entiende por cuantificación del fallo la amplitud del armónico durante la trayectoria real en el plano tiempo-frecuencia. Es decir, es la energía del armónico de fallo en función del tiempo.

\subsubsection{Análisis de los ensayos}

A continuación, y para ilustrar el uso de la técnica, se muestran los resultados del análisis de dos ensayos. Uno corresponde al motor sano y otro al del motor con una barra rota. Los datos de estos ensayos son los siguientes:

- Inversor: Allen-Bradley

- $\quad$ Nivel de carga y deslizamiento: Alto

- Tipo de control: V/f lineal

- Tipo de arranque: lineal 
En la Figura 62 se muestran los resultados de análisis de estos dos ensayos. La trayectoria del armónico fundamental (Figura 62 (a)) se calcula a partir del espectrograma, detectando los puntos de máxima energía. La velocidad se mide durante el ensayo. Esto permite determinar el deslizamiento del motor (Figura 62 (b)) y, por tanto, también la trayectoria teórica de los armónicos de fallo (Figura 62 (a)).

En la Figura 62 (c) se muestra el espectro de los dos ensayos en el estado estacionario posterior al arranque. El deslizamiento del motor en el ensayo con una barra rota es 0,042 . Los valores de las frecuencias de fallo son los siguientes:

- Lower Sideband Harmonic (LSH):

$$
\begin{gathered}
f_{L S H}=(1-2 s) f_{S}=(1-2 \cdot 0,042) \cdot 50=45,8 \mathrm{~Hz} \\
\circ \quad \text { Upper Sideband Harmonic }(U S H): \\
f_{U S H}=(1+2 s) f_{S}=(1+2 \cdot 0,042) \cdot 50=54,2 \mathrm{~Hz}
\end{gathered}
$$

En el caso del IM sano, estas frecuencias teóricas de fallo corresponderían con los siguientes valores, teniendo en cuenta que el deslizamiento presenta un valor de 0,04 :

○ Lower Sideband Harmonic $(L S H)$ :

$$
\begin{gathered}
f_{L S H}=(1-2 s) f_{S}=(1-2 \cdot 0,04) \cdot 50=46 \mathrm{~Hz} \\
\circ \quad \text { Upper Sideband Harmonic }(U S H): \\
f_{U S H}=(1+2 s) f_{S}=(1+2 \cdot 0,04) \cdot 50=54 \mathrm{~Hz}
\end{gathered}
$$

Los valores teóricos que se obtienen con el motor sano son similares a las frecuencias que se tienen con el motor con fallo, debido a que se han escogido ensayos con un deslizamiento similar.

En la Figura 62, la columna izquierda corresponde a un motor sano, mientras que la columna derecha se trata de un motor con fallo por barra rota. Resultan especialmente interesantes los espectrogramas de la Figura 62 (d). En estas dos Figuras se observa claramente la trayectoria en el plano tiempo-frecuencia del primer armónico. Este es el que tiene más energía en todo instante de tiempo. Realmente estos espectrogramas son representaciones en tres dimensiones, donde en la coordenada $z$ se representa la amplitud de cada componente de frecuencia y en cada instante de tiempo. En estas Figuras se ha proyectado la energía en el planto tiempo-frecuencia y se ha representado mediante una 
escala de colores, donde los más rojos corresponden a una mayor energía y los más próximos al azul, las energías más bajas. El colormap empleado en Matlab es el denominado "jet". En estas Figuras se detecta fácilmente la trayectoria del primer armónico. En la columna de la derecha, en el espectrograma se detectan muy bien las trayectorias de los armónicos de fallo, que no aparecen en el espectrograma de la izquierda (motor sano). Las trayectorias de fallo se detectan durante el transitorio de arranque y también durante el estacionario posterior, además con la misma resolución tiempo-frecuencia. En la transformada Dragón, el átomo se adapta en todo instante a la trayectoria del fallo, y por esta razón, es capaz de detectar el armónico de fallo durante todo el ensayo. En la Figura 62 (f) se muestra un detalle del espectrograma durante el arranque. Aquí se demuestra la capacidad de la transformada para detectar el armónico de fallo. En la Figura 62 (g) se muestra el resultado de la cuantificación del fallo.

En la Figura 62 (d) se observa el resultado de los efectos de borde al aplicar la transformada Dragón al principio y final del ensayo. Este efecto también es visible en el instante donde cambia la trayectoria del armónico principal después del arranque. Sin embargo, esto no afecta a la detección de los armónicos de fallo.
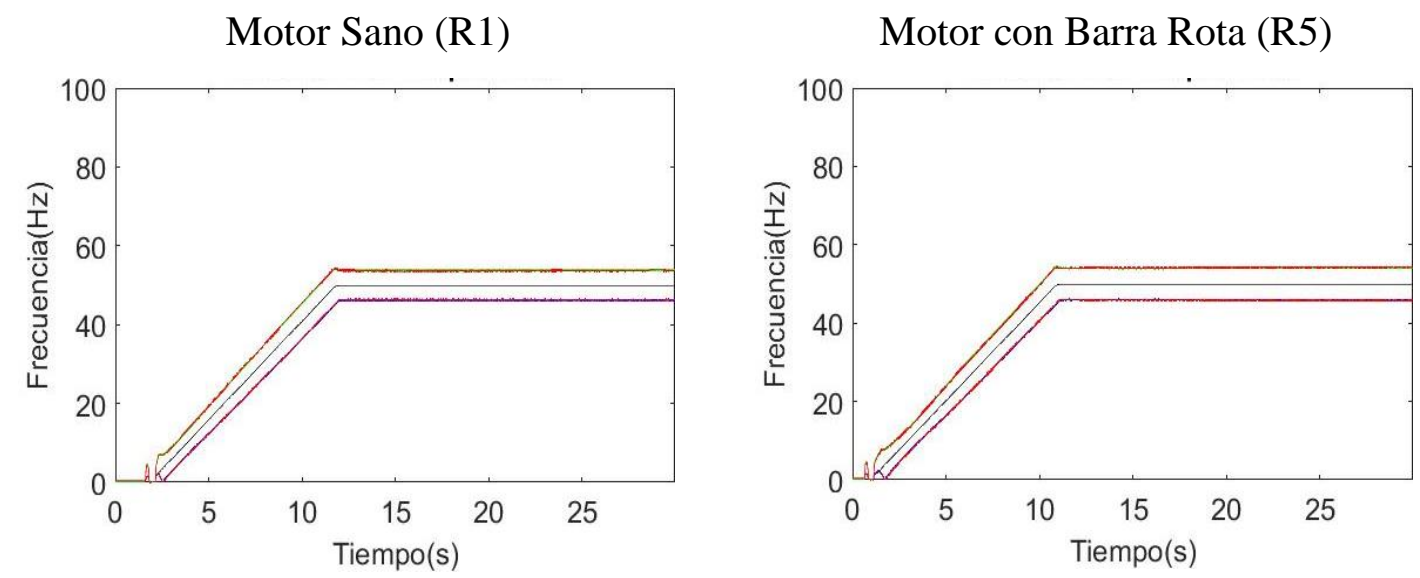

(a) Trayectoria real del primer armónico y teórica de los armónicos de fallo 

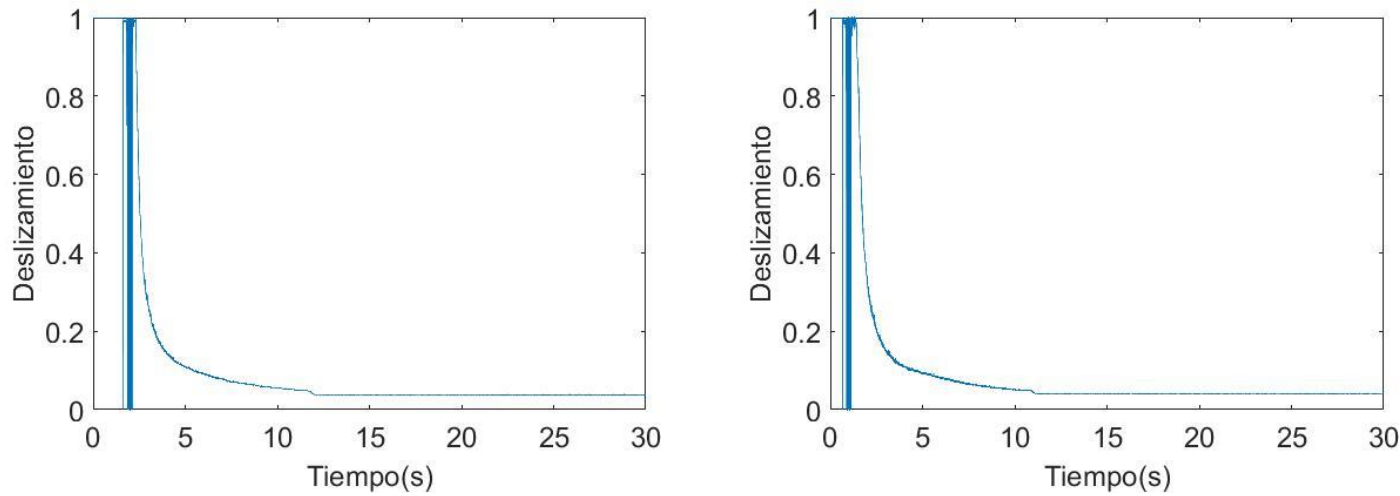

(b) Deslizamiento
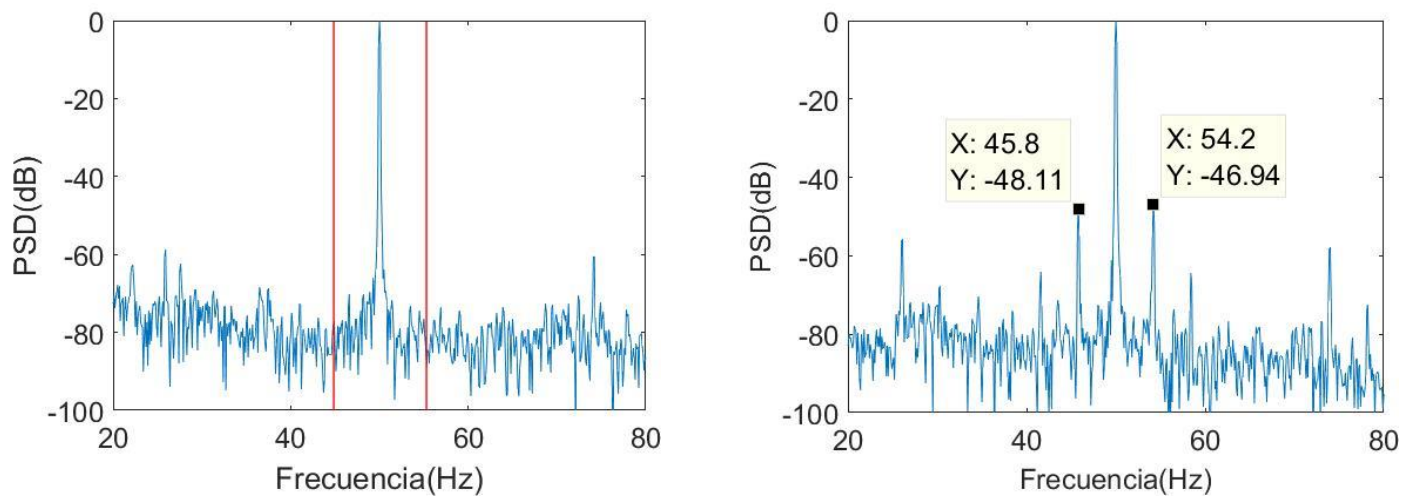

(c) Espectro del estado estacionario
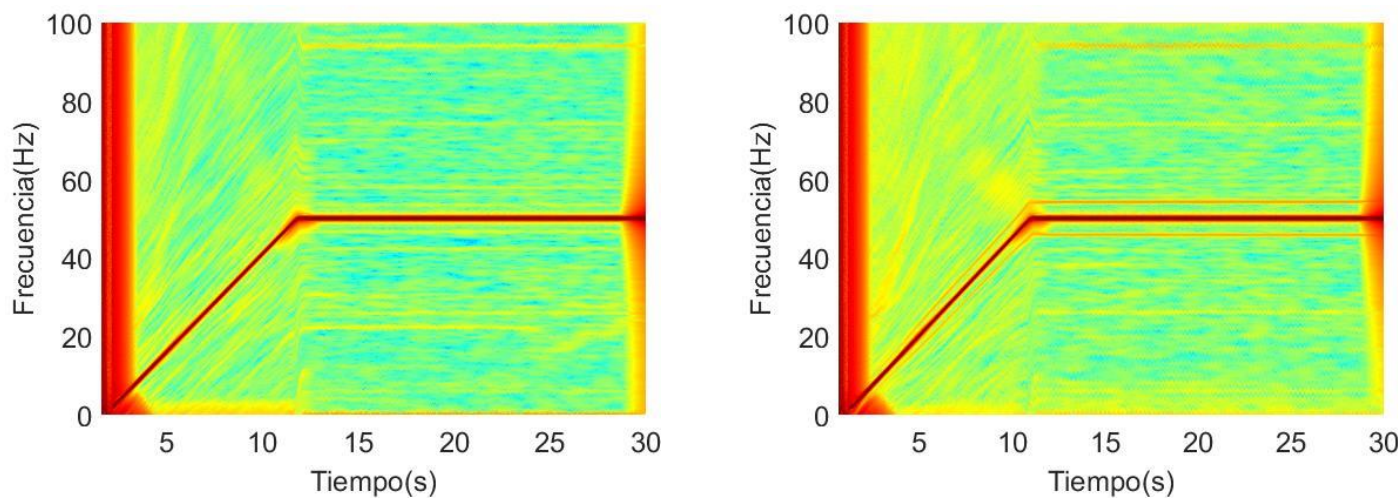

(d) Espectrograma del ensayo
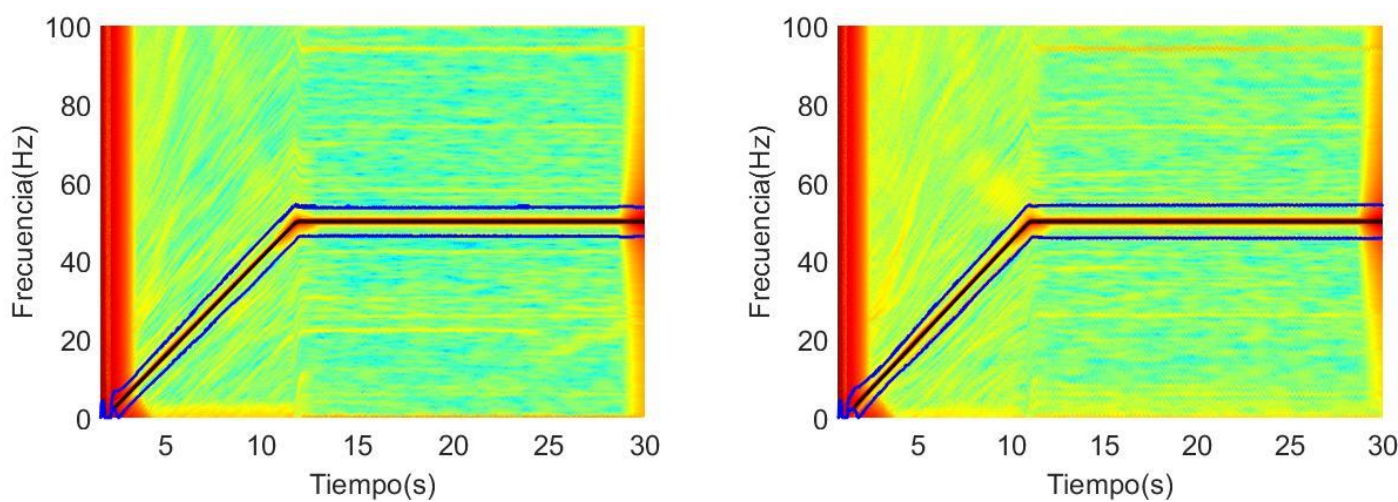

(e) Espectrograma del ensayo y trayectorias de los armónicos de fallo 

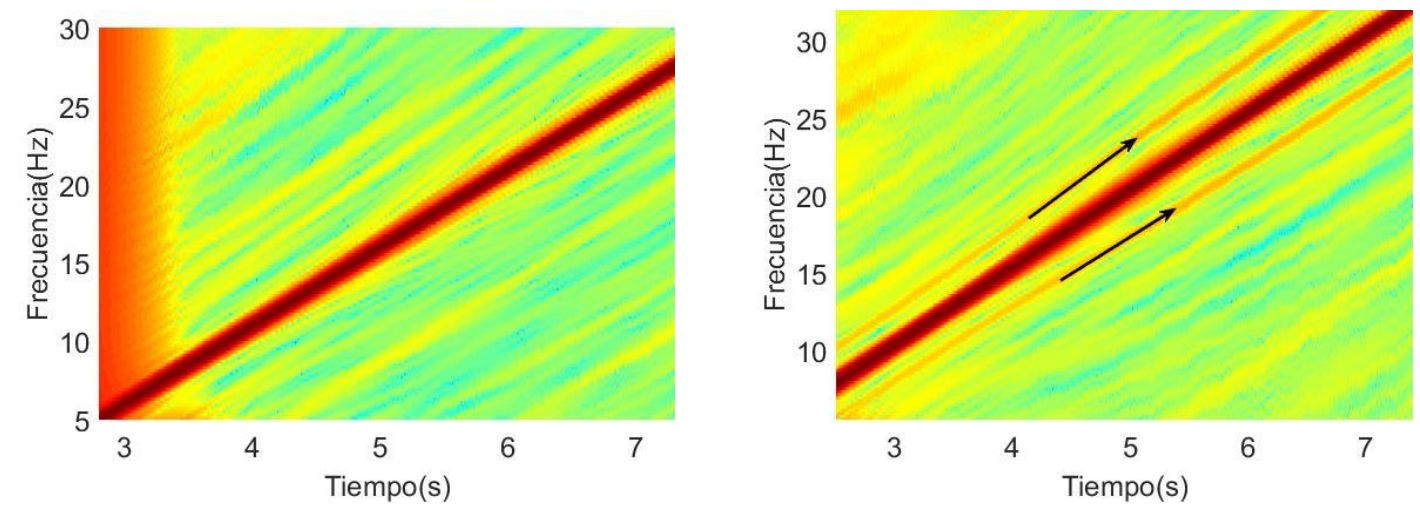

(f) Detalle del espectrograma
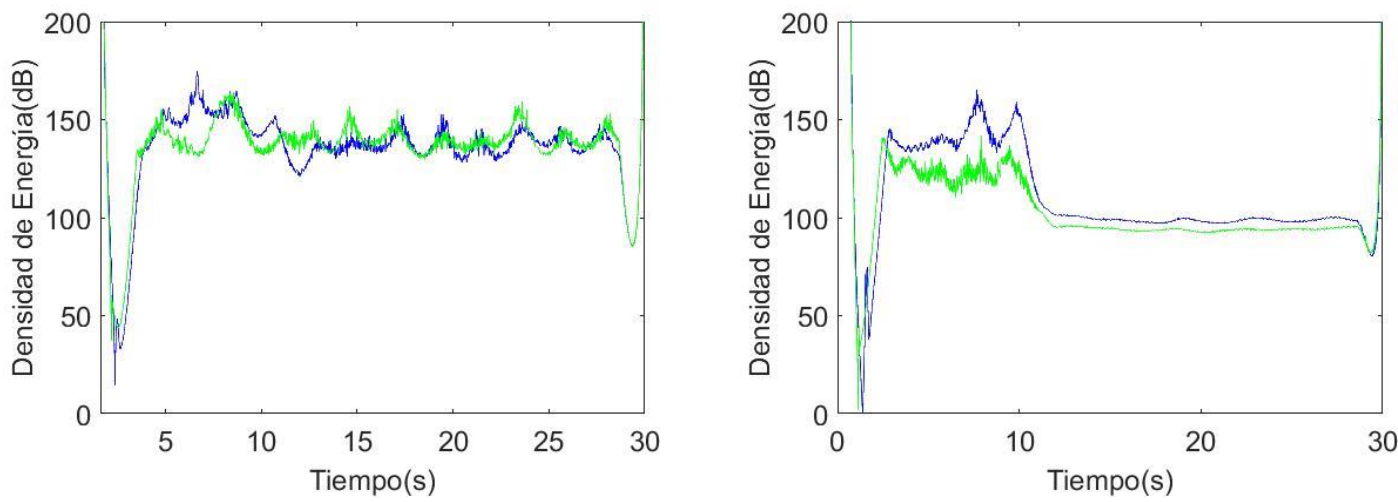

(g) Cuantificación del fallo

Figura 62. Resultados del análisis de la señal de $I M$ alimentado mediante inversor AB con nivel de carga alto.

A continuación, se muestran los resultados relativos al caso de un motor alimentado mediante inversor Allen-Bradley, pero ahora con nivel de carga bajo. Los datos, en este caso, son los siguientes:

- Inversor: Allen-Bradley

- $\quad$ Nivel de carga: Bajo

- Tipo de control: V/f lineal

- $\quad$ Tipo de arranque: lineal

Procediendo análogamente a como se ha hecho en la Figura 62, en la Figura 63 se muestran los resultados del análisis de dos ensayos, solo que ahora el nivel de carga es bajo. En la Figura 63 (c) se muestra el espectro de los dos ensayos en el estado estacionario posterior al arranque. El deslizamiento del motor en el ensayo con una barra 
rota es 0,026. Los valores de las frecuencias de fallo, cuyo cálculo se introdujo en el punto 2 de esta tesis, son los siguientes:

- $\quad$ Lower Sideband Harmonic $(L S H)$ :

$$
f_{L S H}=(1-2 s) f_{S}=(1-2 \cdot 0,026) \cdot 50=47,4 H z
$$

- $\quad$ Upper Sideband Harmonic (USH):

$$
f_{U S H}=(1+2 s) f_{S}=(1+2 \cdot 0,026) \cdot 50=52,6 \mathrm{~Hz}
$$

En el caso del $I M$ sano, estas frecuencias teóricas de fallo corresponderían con los siguientes valores, teniendo en cuenta que el deslizamiento presenta un valor de 0,027:

- Lower Sideband Harmonic (LSH):

$$
f_{L S H}=(1-2 s) f_{S}=(1-2 \cdot 0,027) \cdot 50=47,3 \mathrm{~Hz}
$$

- Upper Sideband Harmonic (USH)

$$
f_{U S H}=(1+2 s) f_{S}=(1+2 \cdot 0,027) \cdot 50=52,7 \mathrm{~Hz}
$$

Así, en la Figura 63, se pueden observar los resultados concernientes al análisis de los dos casos, la columna izquierda corresponde con el motor sano y la columna derecha al motor con una barra rota. En la Figura 63 (c), se demuestra claramente la diferencia de estos dos casos en los que el motor trabajaba en estado estacionario.

La Figura 63 (d) presenta el espectrograma resultante de la aplicación de la técnica desarrollada, en donde resulta sencillo distinguir la componente fundamental. También aquí se puede observar las diferencias entre motor sano y motor con fallo, ya que en el primer caso no aparecen armónicos de fallo, mientras que en el caso del motor con fallo se distinguen los armónicos debidos a fallo de barras, pudiendo distinguirse de una manera nítida y con una fina línea, tanto en el estado estacionario como en el transitorio, por lo que se puede observar su trayectoria a lo largo de todo el plano tiempo-frecuencia. En la Figura 63 (f) se muestra un detalle del espectrograma durante el arranque, donde se puede apreciar con más detalle cómo la técnica es capaz de detectar de manera clara el fallo durante este estado. Finalmente, en la Figura 63 (e) se presentan las curvas de cuantificación de fallo, las cuales se analizan en profundidad en el apartado 6.4. de este punto. 

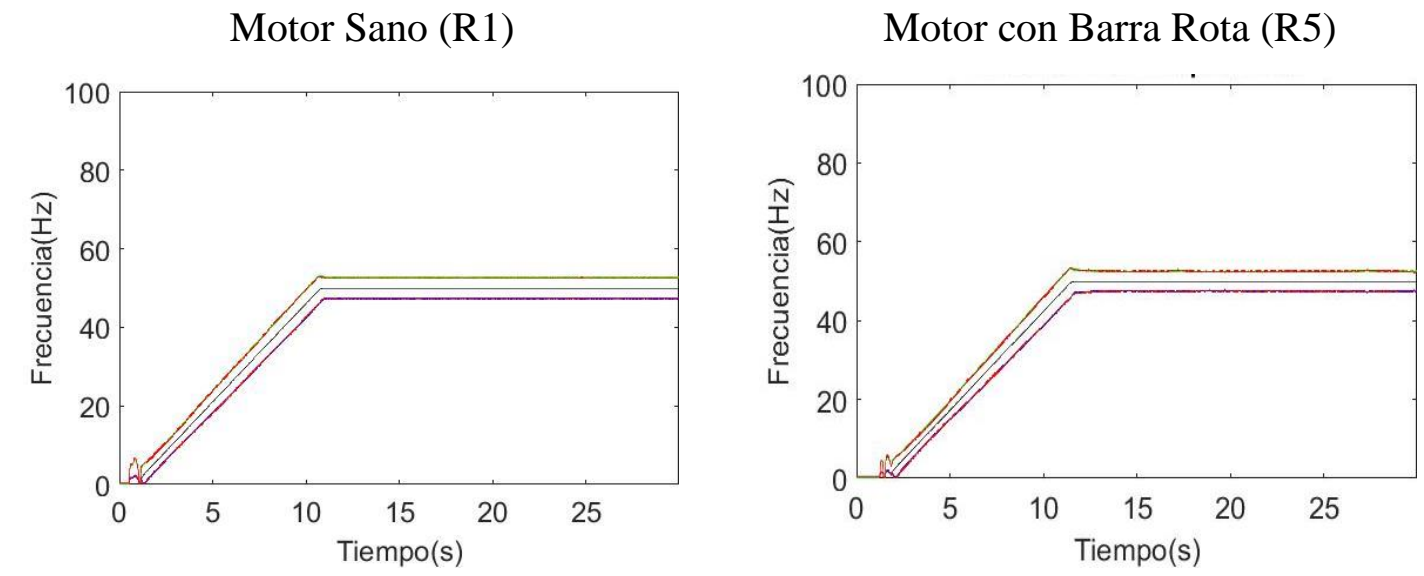

(a) Trayectoria real del primer armónico y teórica de los armónicos de fallo
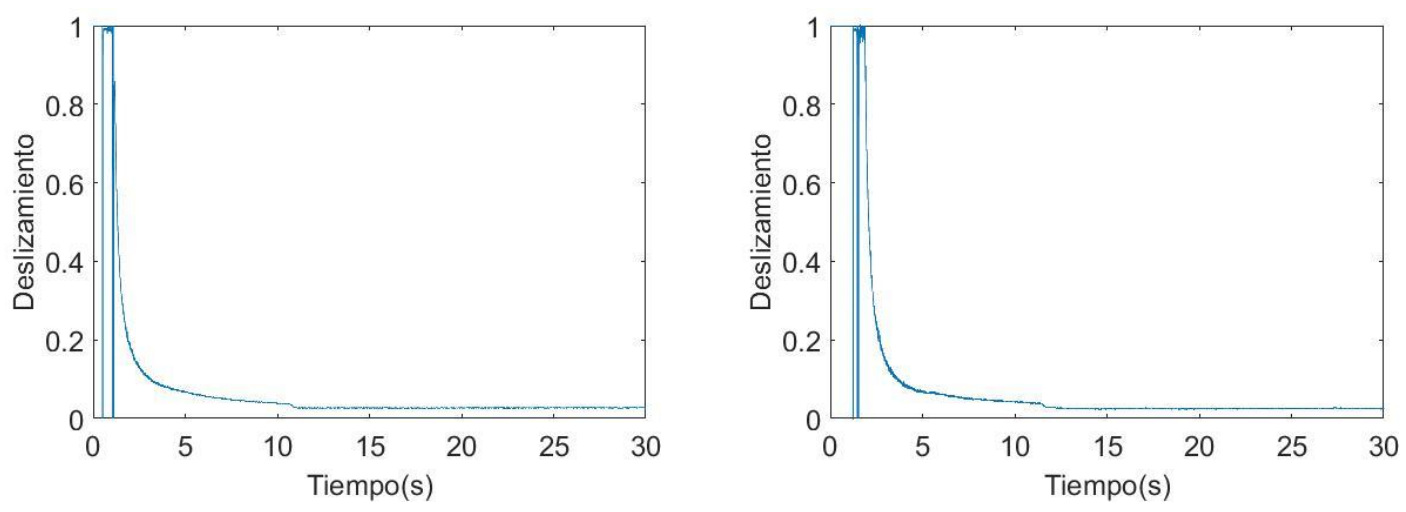

(b) Deslizamiento
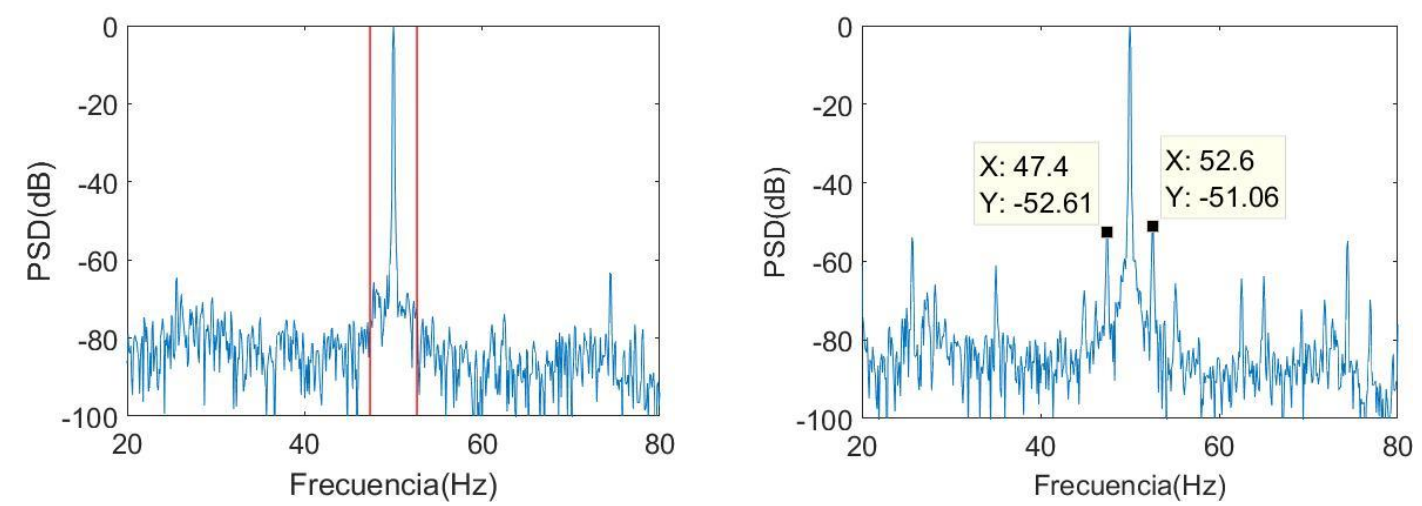

(c) Espectro del estado estacionario
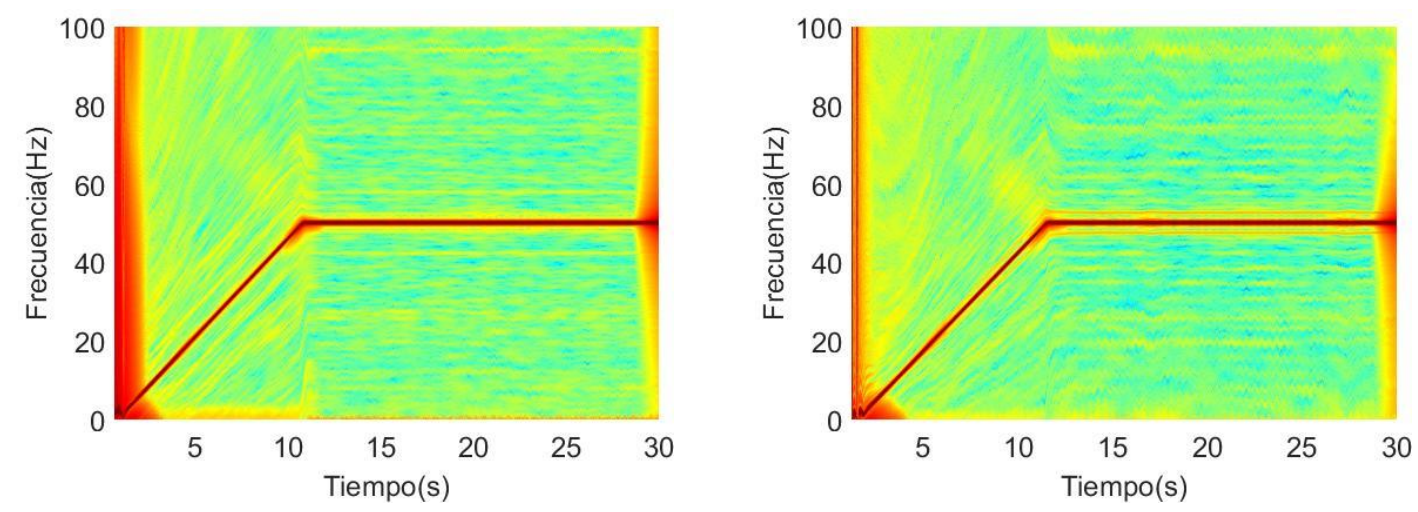

(d) Espectrograma del ensayo 

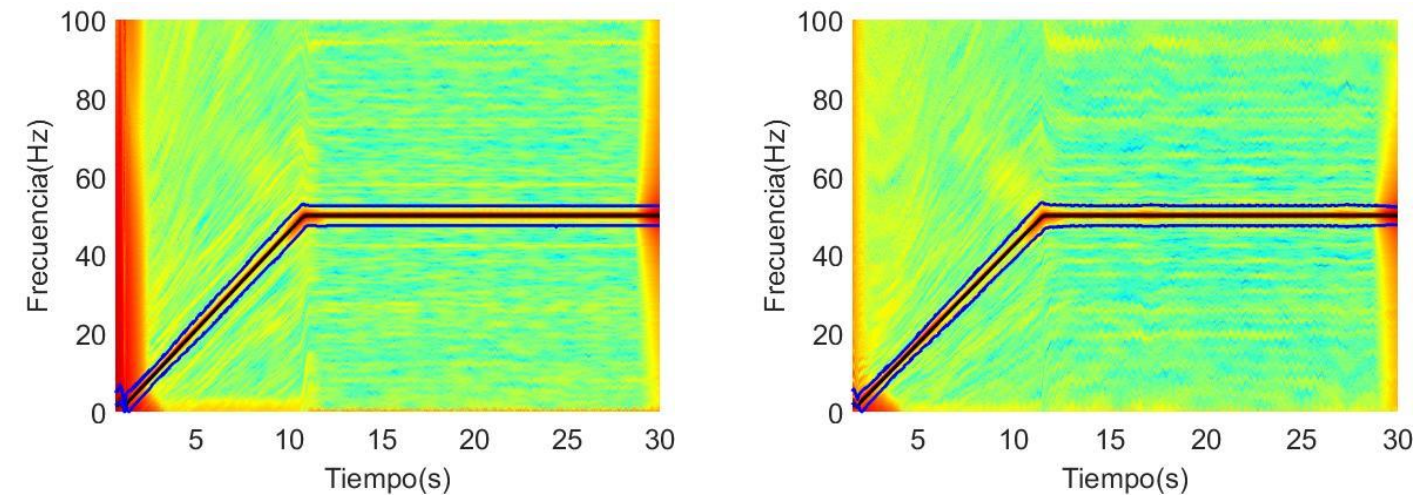

(e) Espectrograma del ensayo y trayectorias de los armónicos de fallo
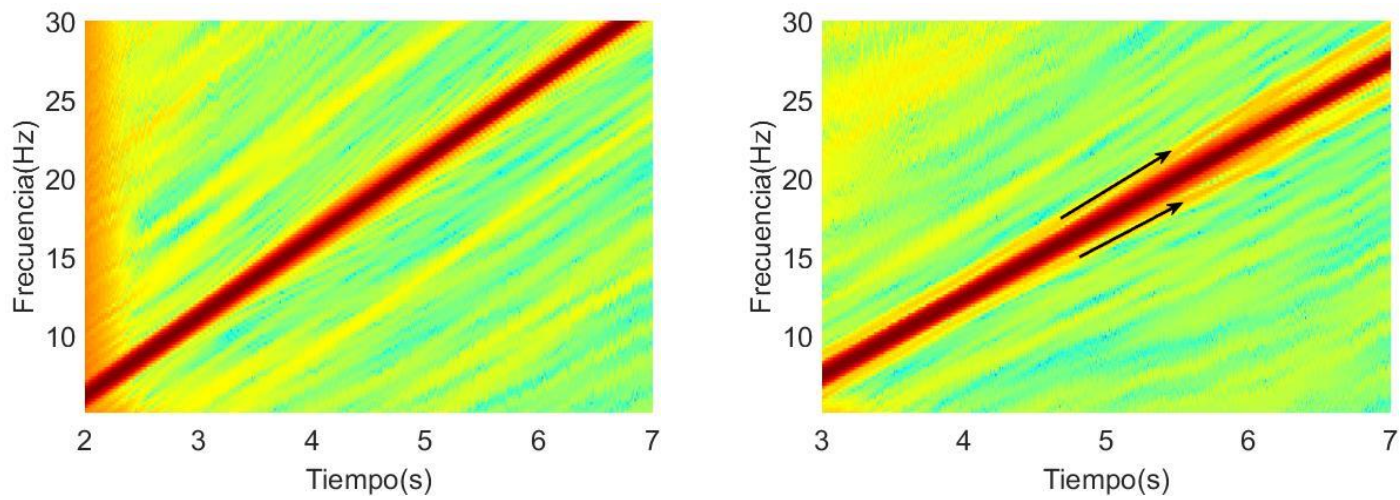

(f) Detalle del espectrograma
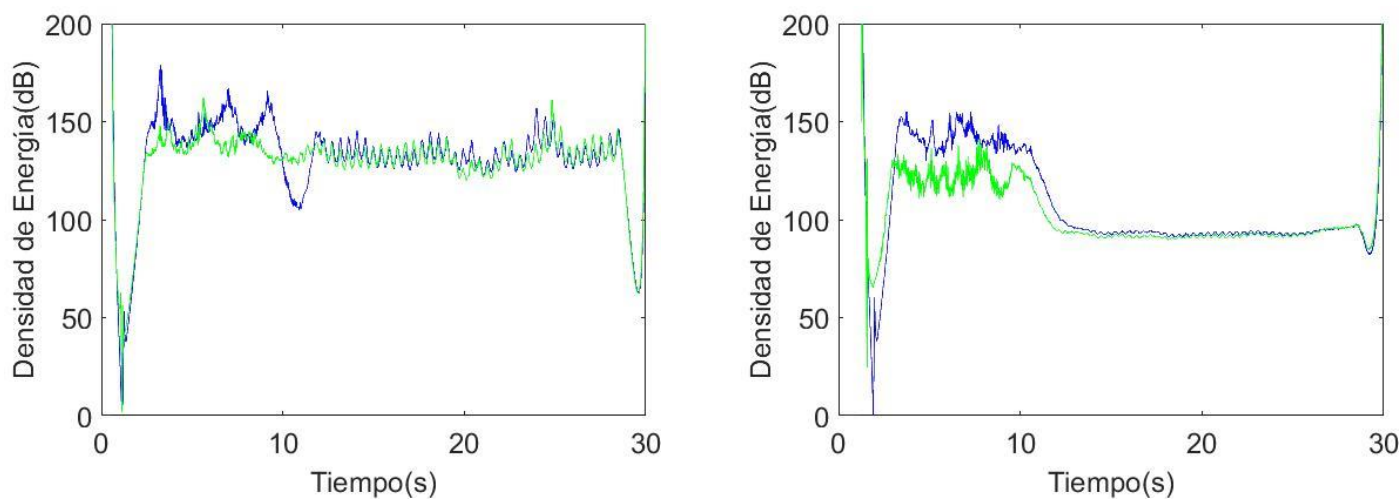

(g) Cuantificación del fallo

Figura 63. Resultados del análisis de la señal de $I M$ alimentado mediante inversor AB con nivel de carga bajo.

Para demostrar la capacidad de detección de la técnica propuesta, se muestran, a continuación, los resultados obtenidos con los diferentes inversores con los dos niveles de carga, tal como pueden verse en las Figuras 64 a 71. Para su análisis se va a proceder de la misma manera que con el inversor de Allen-Bradley. Finalmente, en el apartado 6.2.2. se presentarán las conclusiones concernientes a los mismos. 
A continuación, se muestran los resultados correspondientes al inversor de ABB. Así las características de este ensayo son:

- Inversor: $\mathrm{ABB}$

- Nivel de carga: Alto

- Tipo de control: V/f lineal

- Tipo de arranque: lineal

Como se ha dicho, se analizan los mismos puntos que con los ensayos anteriores, es decir: distribución tiempo-frecuencia (Figura 64 (d)), distribución tiempo-frecuencia con la trayectoria teórica de los armónicos de fallo (Figura 64 (e)), espectro del estado estacionario (Figura 64 (c)) y cuantificación del fallo (Figura 64 (g)).

El deslizamiento del motor en el ensayo con una barra rota es 0,056. Los valores de las frecuencias de fallo son los siguientes:

- $\quad$ Lower Sideband Harmonic $(L S H)$ :

$$
f_{L S H}=(1-2 s) f_{S}=(1-2 \cdot 0,056) \cdot 50=44,4 H z
$$

- Upper Sideband Harmonic (USH):

$$
f_{U S H}=(1+2 s) f_{S}=(1+2 \cdot 0,056) \cdot 50=55,6 \mathrm{~Hz}
$$

En el caso del $I M$ sano, estas frecuencias teóricas de fallo corresponderían con los siguientes valores, teniendo en cuenta que el deslizamiento presenta un valor de 0,05 :

- $\quad$ Lower Sideband Harmonic (LSH):

$$
f_{L S H}=(1-2 s) f_{S}=(1-2 \cdot 0,05) \cdot 50=45 H z
$$

- $\quad$ Upper Sideband Harmonic (USH):

$$
f_{U S H}=(1+2 s) f_{S}=(1+2 \cdot 0,05) \cdot 50=55 \mathrm{~Hz}
$$



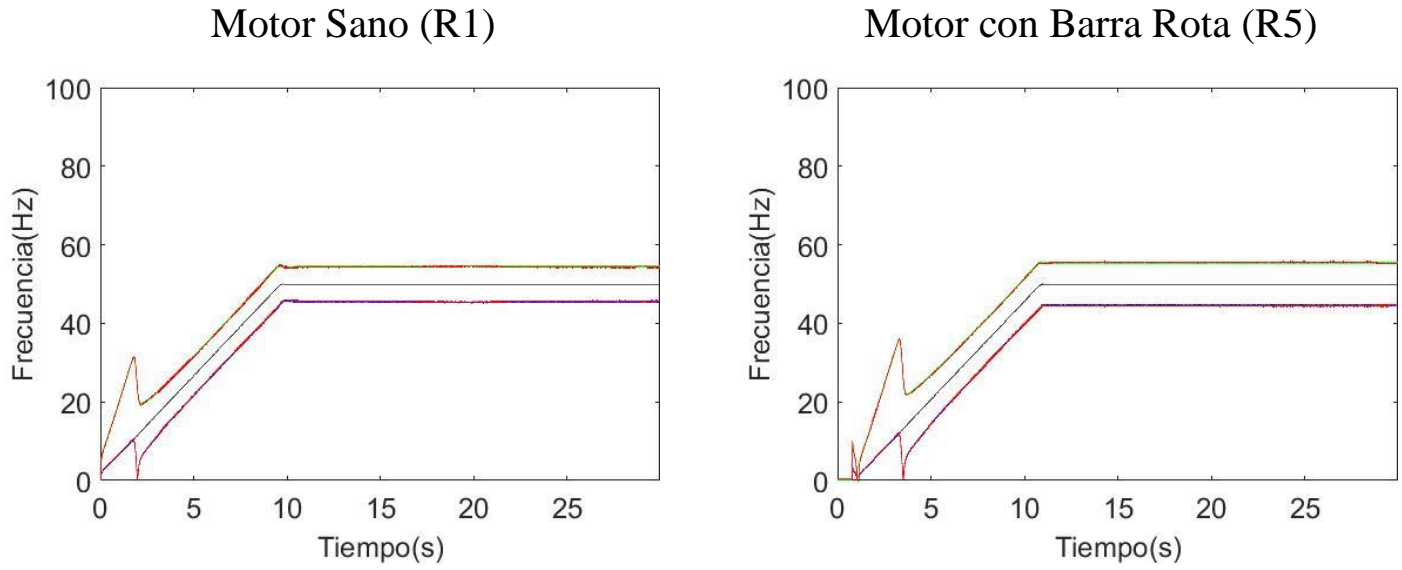

(a) Trayectoria real del primer armónico y teórica de los armónicos de fallo
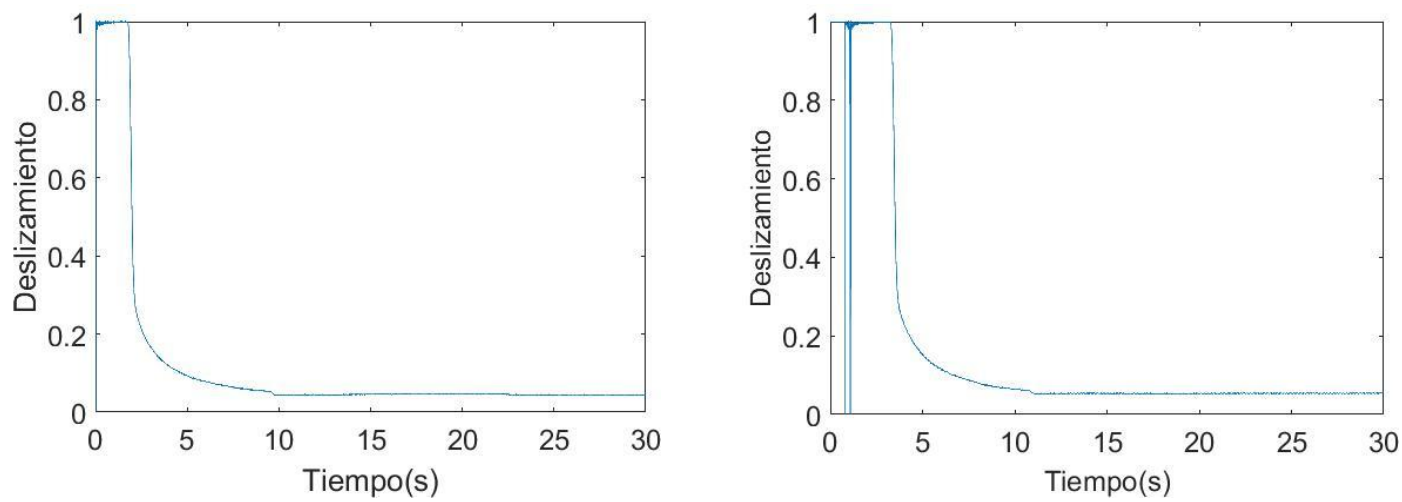

(b) Deslizamiento
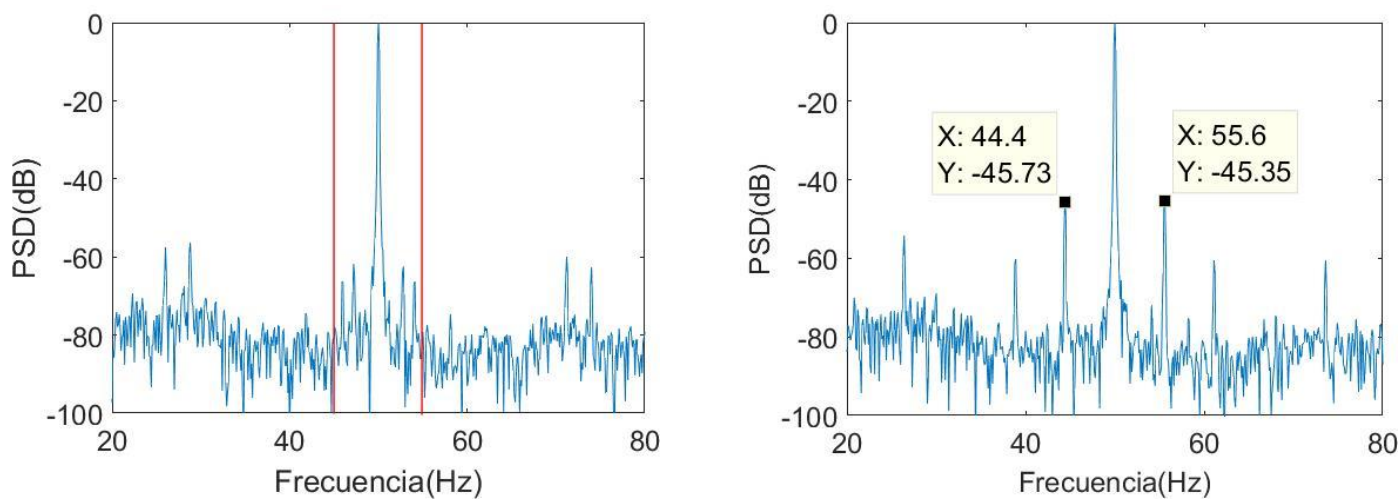

(c) Espectro del estado estacionario
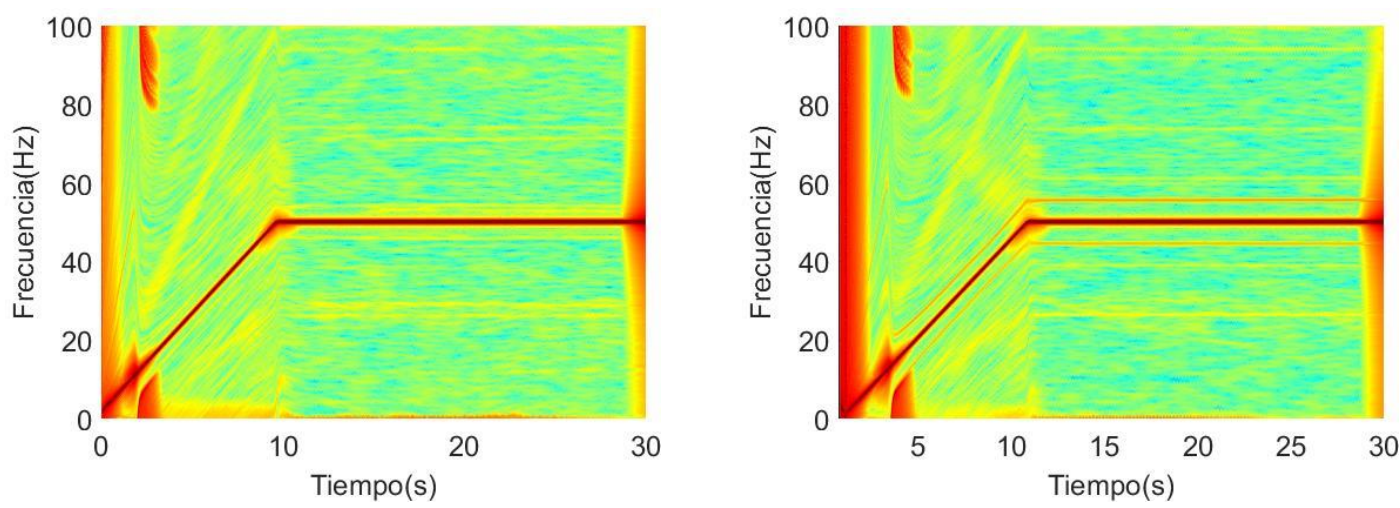

(d) Espectrograma del ensayo 

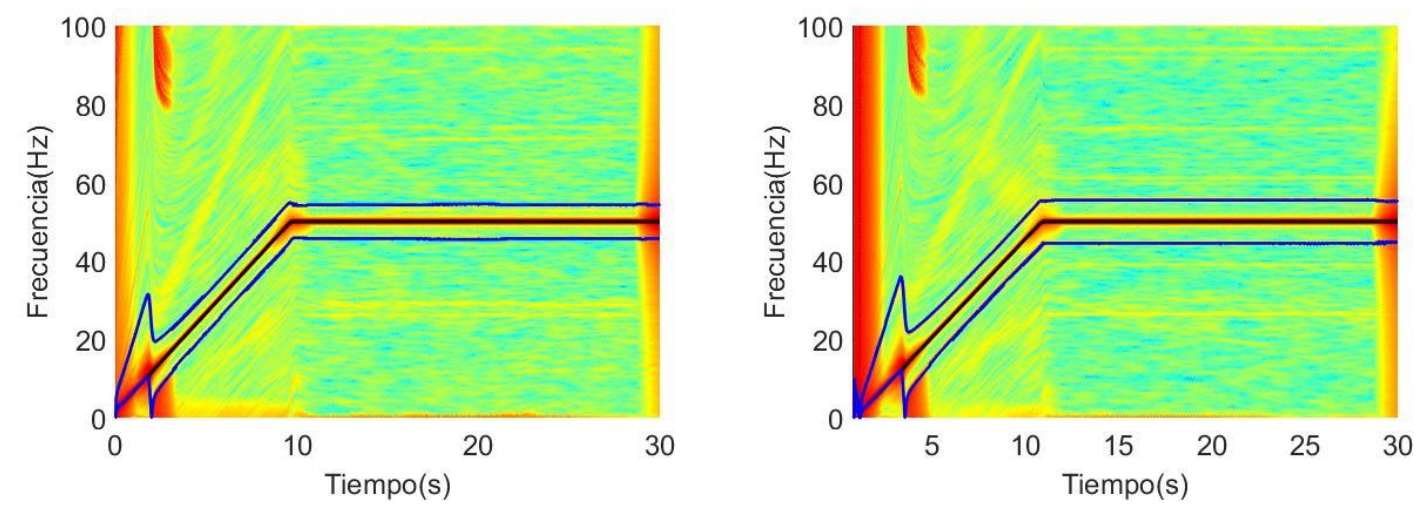

(e) Espectrograma del ensayo y trayectorias de los armónicos de fallo
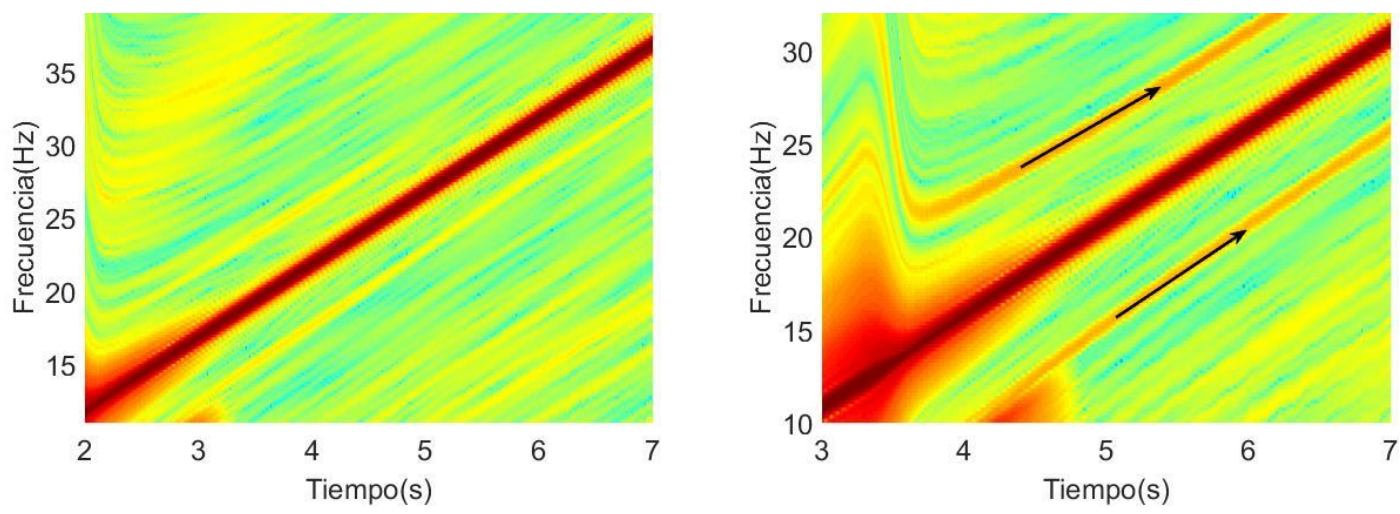

(f) Detalle del espectrograma
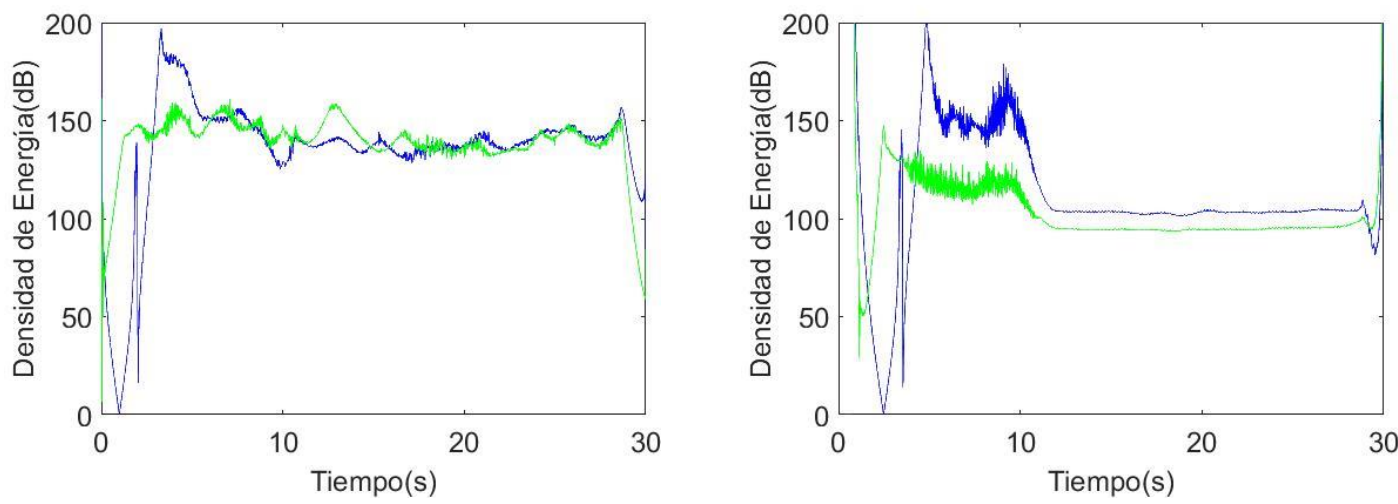

(g) Cuantificación del fallo

Figura 64. Resultados del análisis de la señal de $I M$ alimentado mediante inversor ABB con nivel de carga alto.

La Figura 65 corresponde al mismo inversor (ABB), pero con el motor trabajando con un nivel de carga menor, por tanto, los datos para este ensayo son:

- Inversor: $\mathrm{ABB}$

- Nivel de carga: Bajo

- Tipo de control: V/f lineal

- Tipo de arranque: lineal 
Al ensayar el motor con fallo de barras, el deslizamiento tiene un valor de 0,031 , por lo que los valores de las frecuencias de fallo son:

- Lower Sideband Harmonic $(L S H)$ :

$$
f_{L S H}=(1-2 s) f_{S}=(1-2 \cdot 0,031) \cdot 50=46,9 H z
$$

- Upper Sideband Harmonic (USH):

$$
f_{U S H}=(1+2 s) f_{S}=(1+2 \cdot 0,031) \cdot 50=53,1 \mathrm{~Hz}
$$

En el caso del ensayo del motor en estado sano, el deslizamiento es de 0,03 y las frecuencias teóricas de fallo son las siguientes:

- $\quad$ Lower Sideband Harmonic $(L S H)$ :

$$
f_{L S H}=(1-2 s) f_{S}=(1-2 \cdot 0,03) \cdot 50=47 \mathrm{~Hz}
$$

- Upper Sideband Harmonic (USH):

$$
f_{U S H}=(1+2 s) f_{S}=(1+2 \cdot 0,03) \cdot 50=53 \mathrm{~Hz}
$$

Motor Sano (R1)

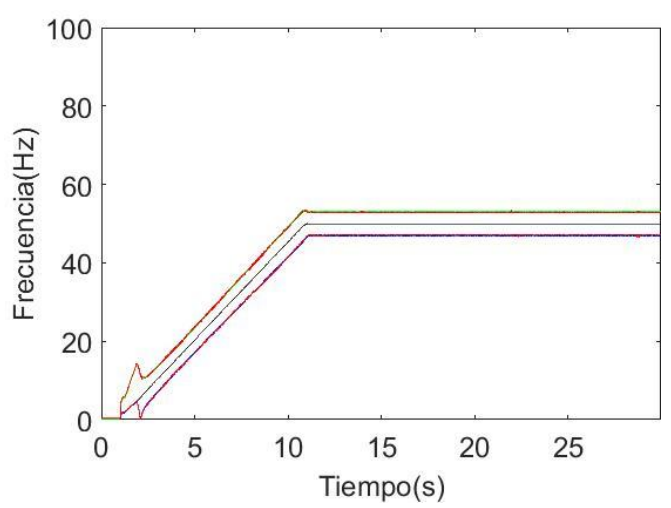

Motor con Barra Rota (R5)

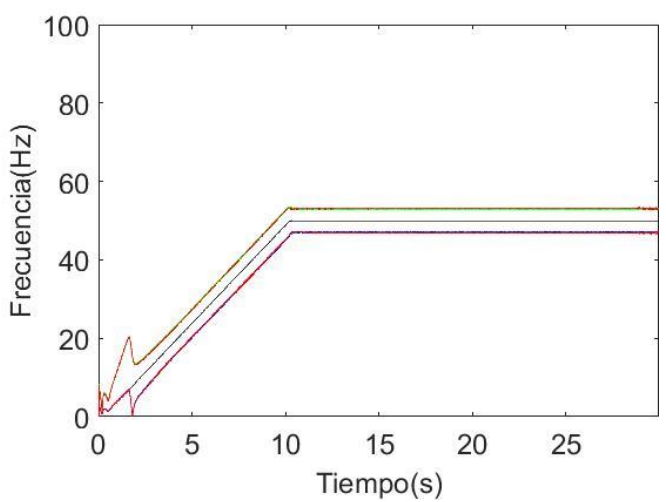

(a) Trayectoria real del primer armónico y teórica de los armónicos de fallo 

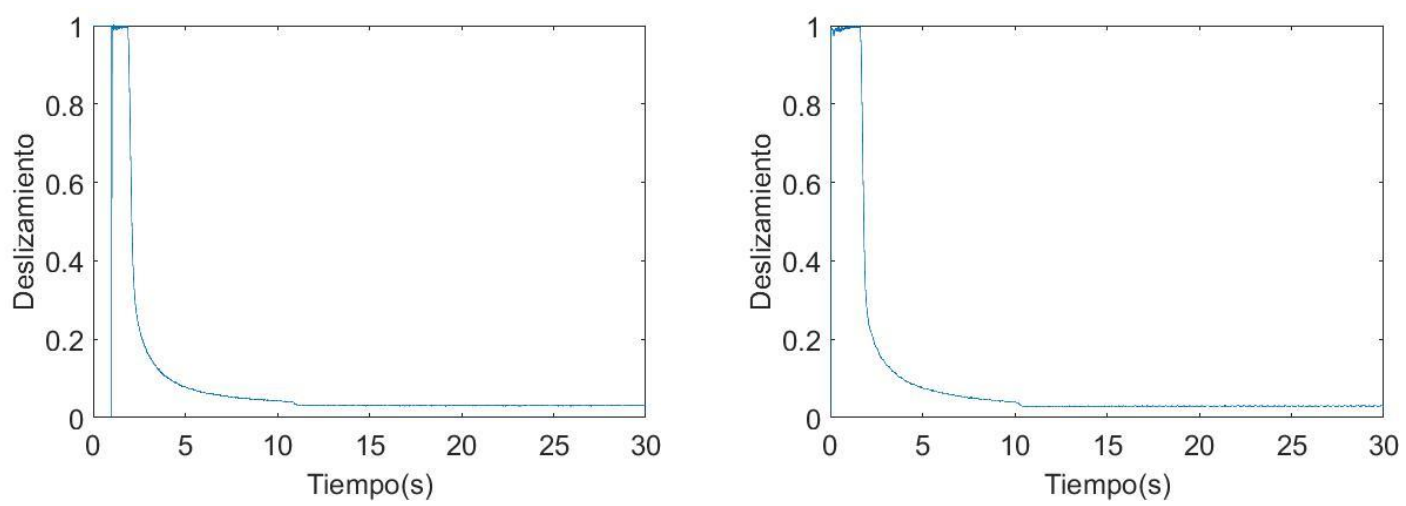

(b) Deslizamiento
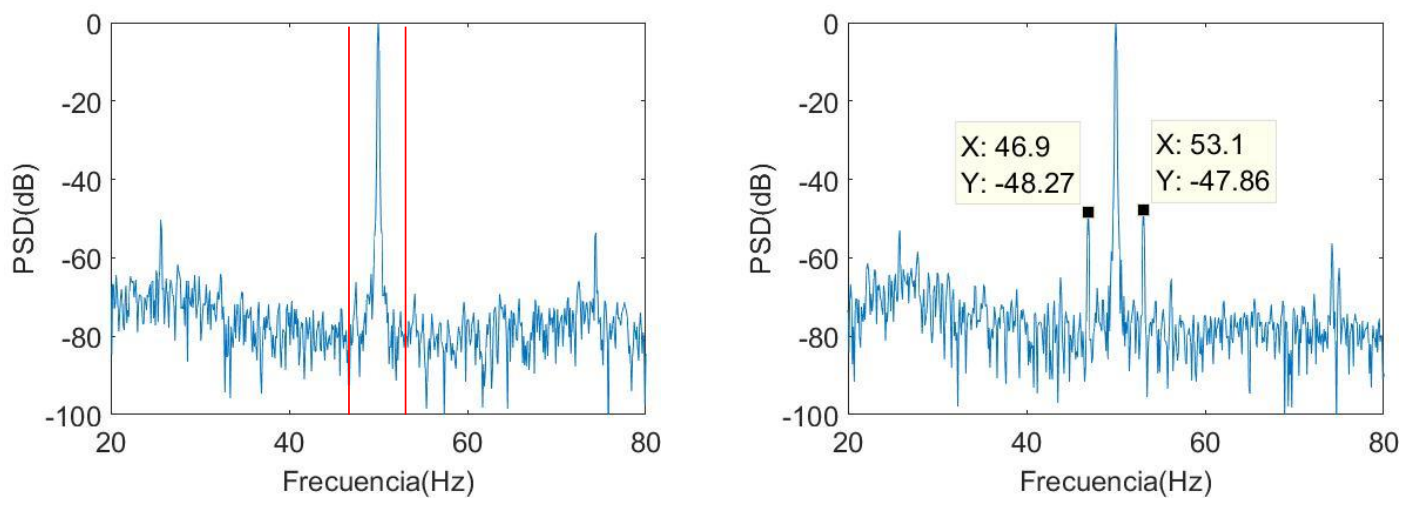

(c) Espectro del estado estacionario
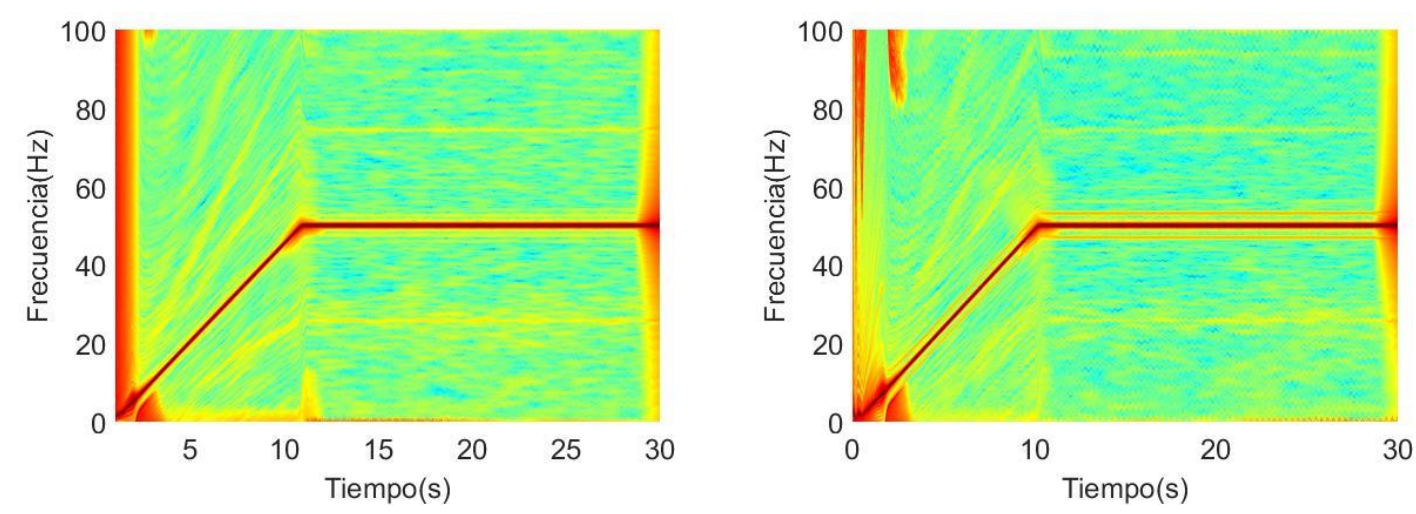

(d) Espectrograma del ensayo
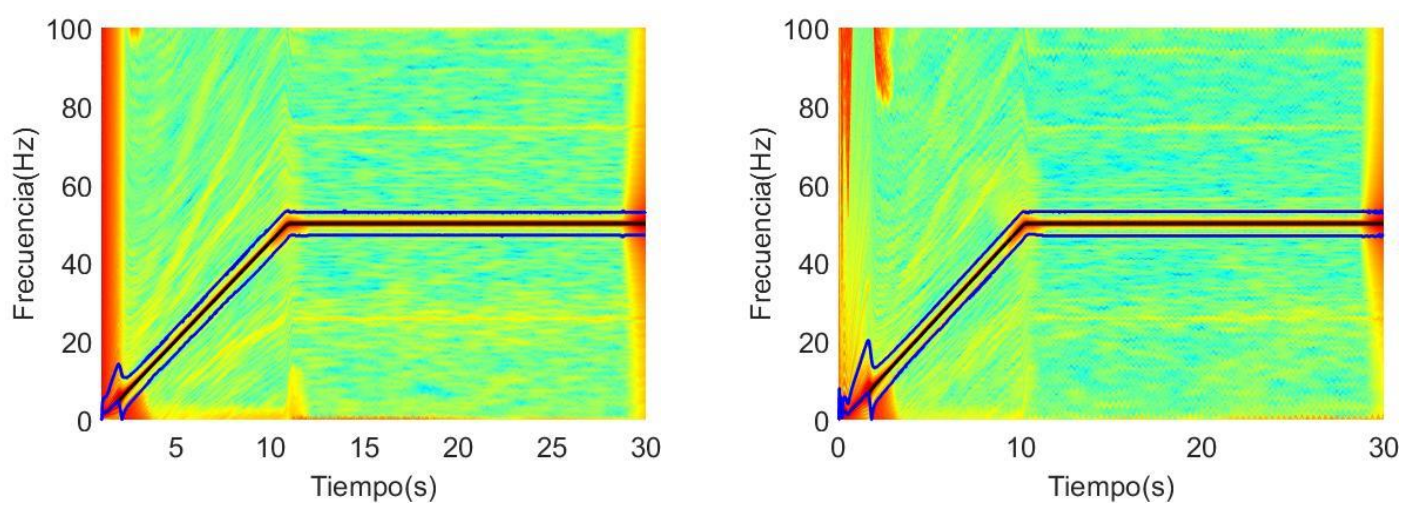

(e) Espectrograma del ensayo y trayectorias de los armónicos de fallo 

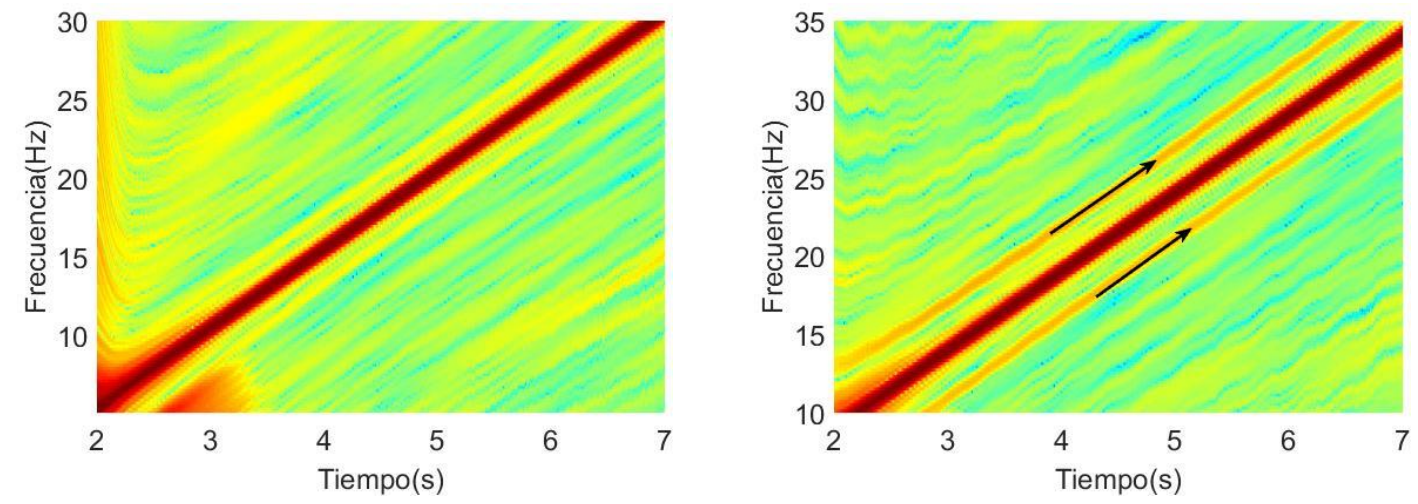

(f) Detalle del espectrograma
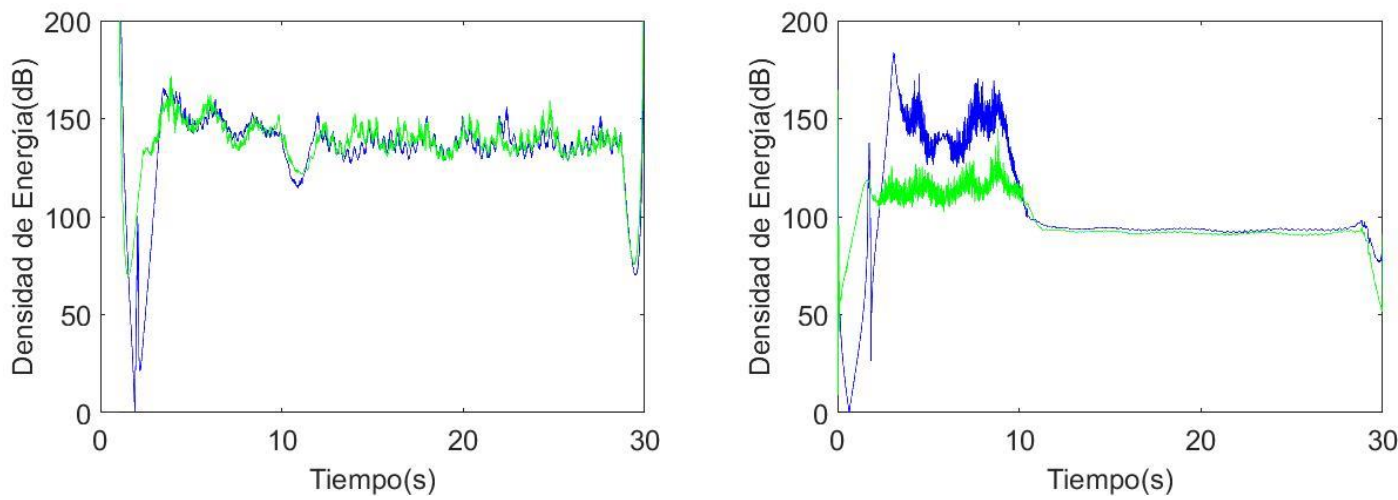

(g) Cuantificación del fallo

Figura 65. Resultados del análisis de la señal de $I M$ alimentado mediante inversor ABB con nivel de carga bajo.

En las Figuras 66 y 67 se ensaya el mismo motor, ahora alimentado mediante el inversor de Siemens, comenzamos con el ensayo con un valor de nivel de carga alto (Figura 66), con lo que los datos de este ensayo son:

- Inversor: SIEMENS

- Nivel de carga: Alto

- Tipo de control: V/f lineal

- $\quad$ Tipo de arranque: lineal

Para proceder del mismo modo que en los casos anteriores, lo primero será obtener los deslizamientos tanto del motor con fallo como del motor en estado sano, así se tiene que para el $I M$ con fallo de barras el deslizamiento es de 0,042 , mientras que para el motor funcionando en estado sano este valor es de 0,051. Por lo que los valores de las frecuencias teóricas de fallo para el motor con fallo son: 
- Lower Sideband Harmonic $(L S H)$ :

$$
f_{L S H}=(1-2 s) f_{s}=(1-2 \cdot 0,042) \cdot 50=45,8 \mathrm{~Hz}
$$

- Upper Sideband Harmonic $(U S H)$ :

$$
f_{U S H}=(1+2 s) f_{S}=(1+2 \cdot 0,042) \cdot 50=54,2 \mathrm{~Hz}
$$

Mientras que para el $I M$ sano serán:

- Lower Sideband Harmonic ( $L S H)$ :

$$
f_{L S H}=(1-2 s) f_{S}=(1-2 \cdot 0,051) \cdot 50=44,9 \mathrm{~Hz}
$$

Upper Sideband Harmonic (USH):

$$
f_{U S H}=(1+2 s) f_{S}=(1+2 \cdot 0,051) \cdot 50=55,1 \mathrm{~Hz}
$$

Motor Sano (R1)

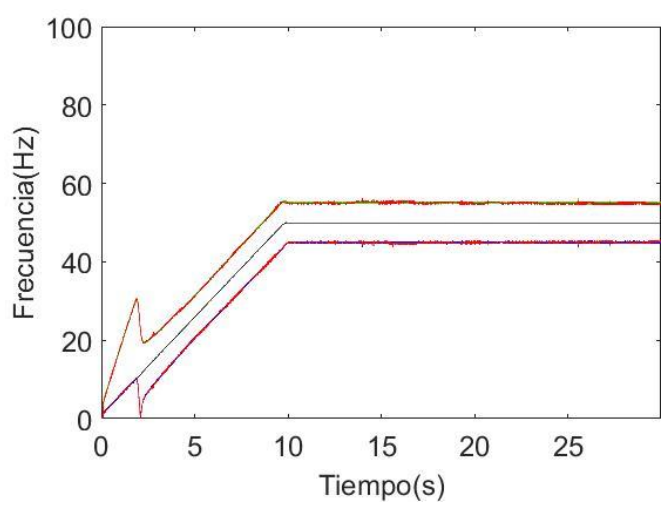

Motor con Barra Rota (R5)

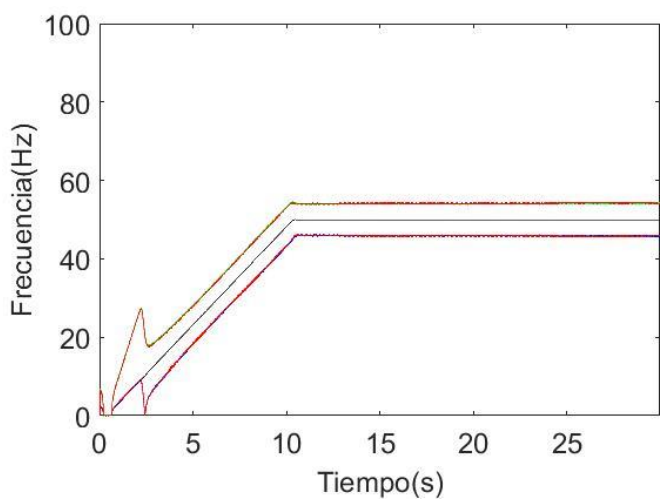

(a) Trayectoria real del primer armónico y teórica de los armónicos de fallo
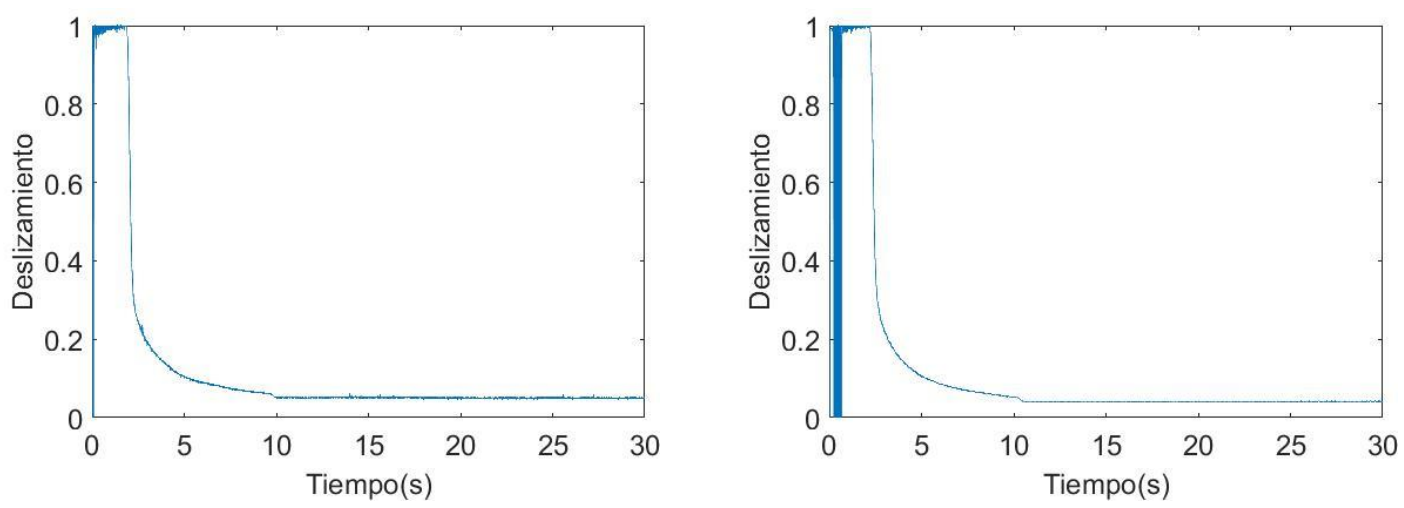

(b) Deslizamiento 

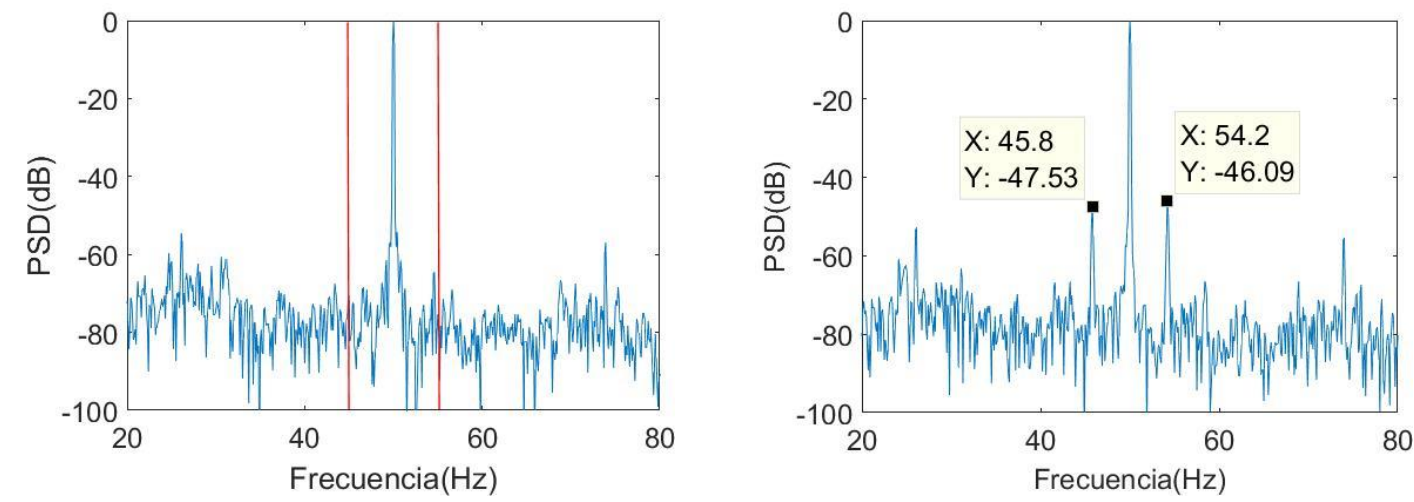

(c) Espectro del estado estacionario
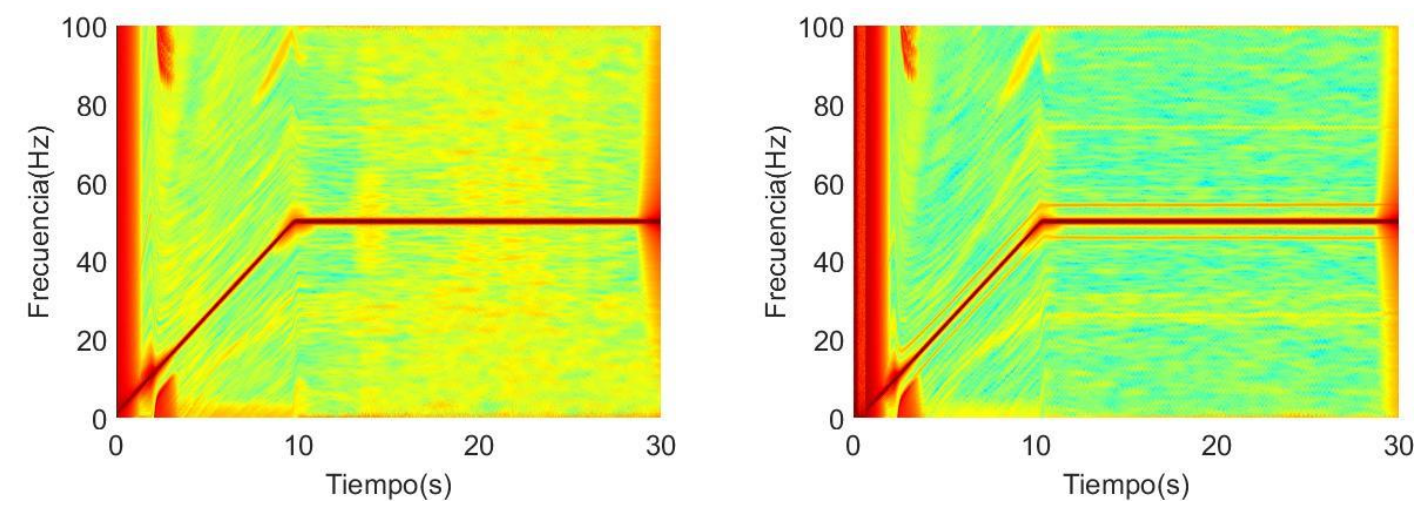

(d) Espectrograma del ensayo
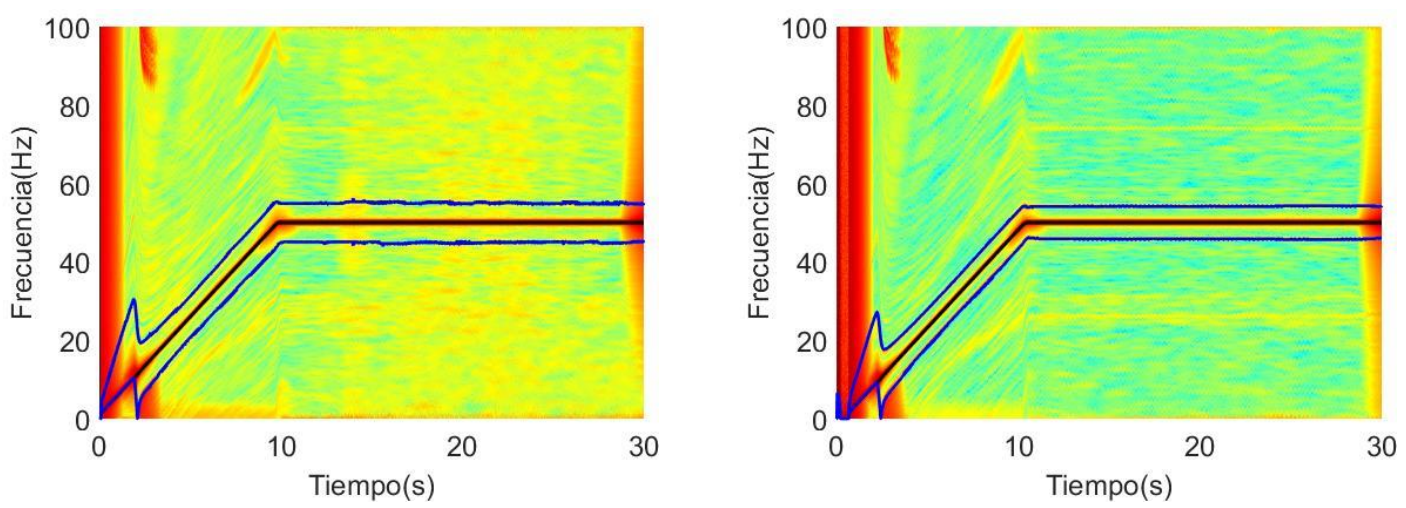

(e) Espectrograma del ensayo y trayectorias de los armónicos de fallo
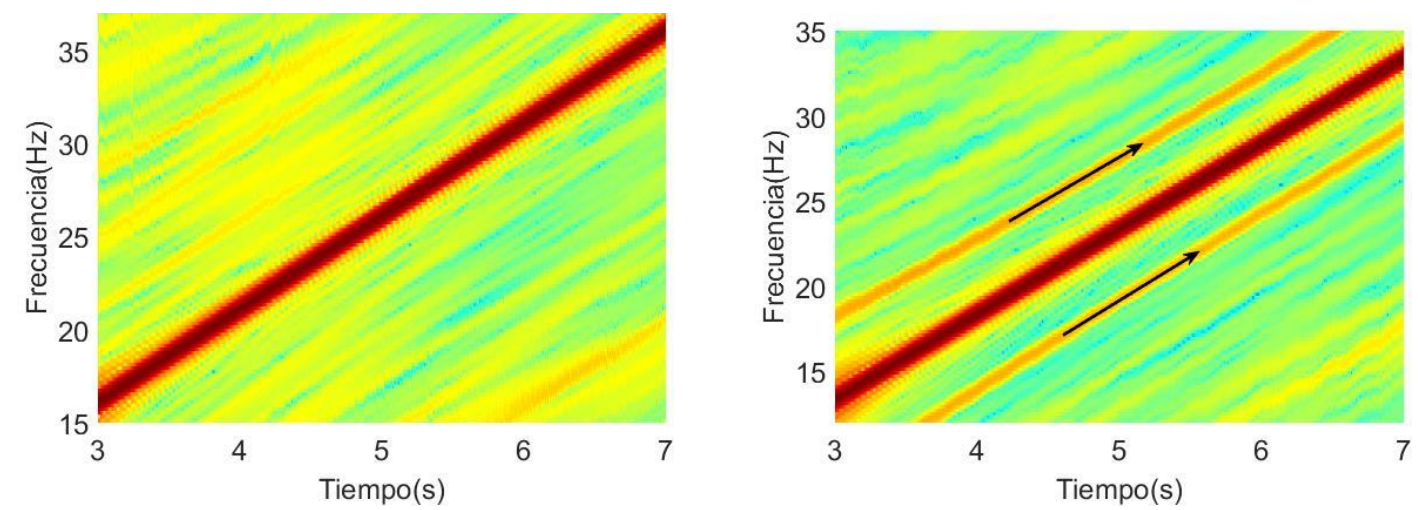

(f) Detalle del espectrograma 

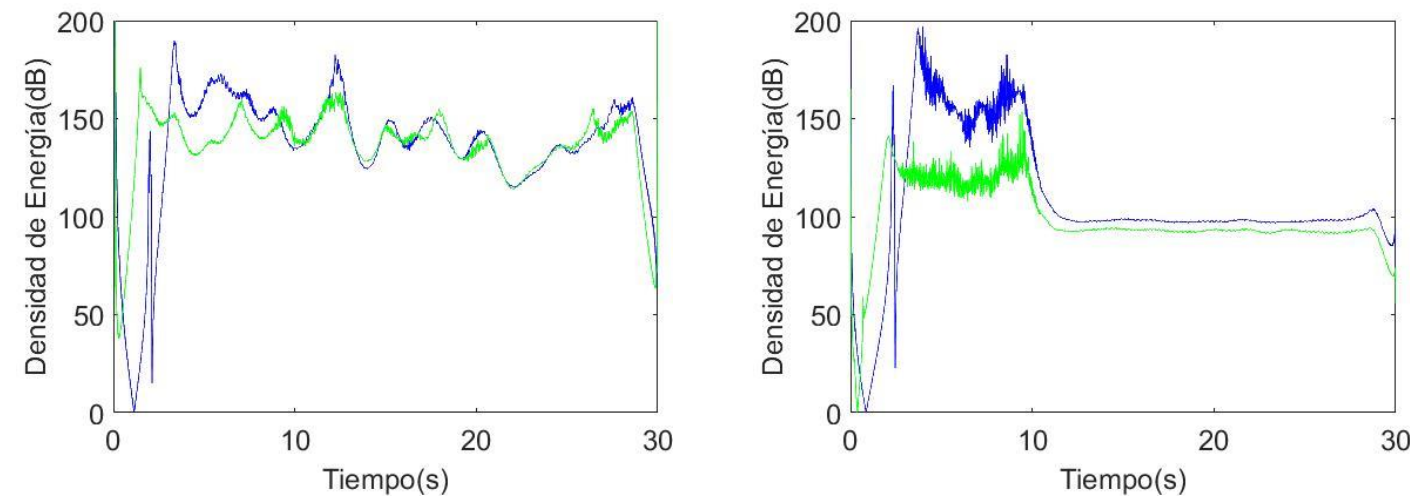

(g) Cuantificación del fallo

Figura 66. Resultados del análisis de la señal de $I M$ alimentado mediante inversor SIEMENS con nivel de carga alto.

El mismo caso anterior, pero ahora se cambia el nivel de carga a bajo, por tanto, en este ensayo se tendrá:

- Inversor: SIEMENS

- $\quad$ Nivel de carga: Bajo

- Tipo de control: V/f lineal

- Tipo de arranque: lineal

Para este ensayo, cuando el motor presenta un fallo de barras, el deslizamiento es de 0,027, siendo así las frecuencias de fallo:

- $\quad$ Lower Sideband Harmonic $(L S H)$ :

$$
f_{L S H}=(1-2 s) f_{S}=(1-2 \cdot 0,027) \cdot 50=47,3 \mathrm{~Hz}
$$

- Upper Sideband Harmonic $(U S H)$ :

$$
f_{U S H}=(1+2 s) f_{s}=(1+2 \cdot 0,027) \cdot 50=52,7 \mathrm{~Hz}
$$

Si el motor está en estado sano presenta un deslizamiento de 0,034 , lo que supone:

- $\quad$ Lower Sideband Harmonic (LSH):

$$
f_{L S H}=(1-2 s) f_{S}=(1-2 \cdot 0,034) \cdot 50=46,6 \mathrm{~Hz}
$$

- Upper Sideband Harmonic (USH):

$$
f_{U S H}=(1+2 s) f_{S}=(1+2 \cdot 0,034) \cdot 50=53,4 H z
$$


La Figura 67 muestra los resultados del análisis de estos dos ensayos.
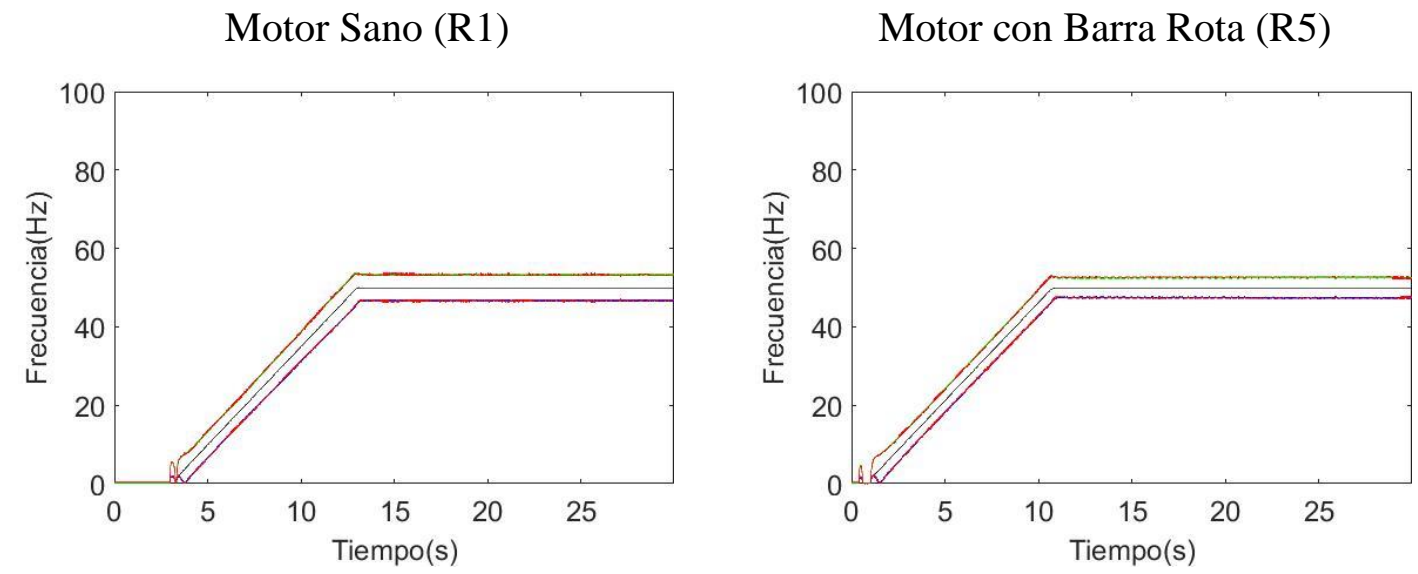

(a) Trayectoria real del primer armónico y teórica de los armónicos de fallo
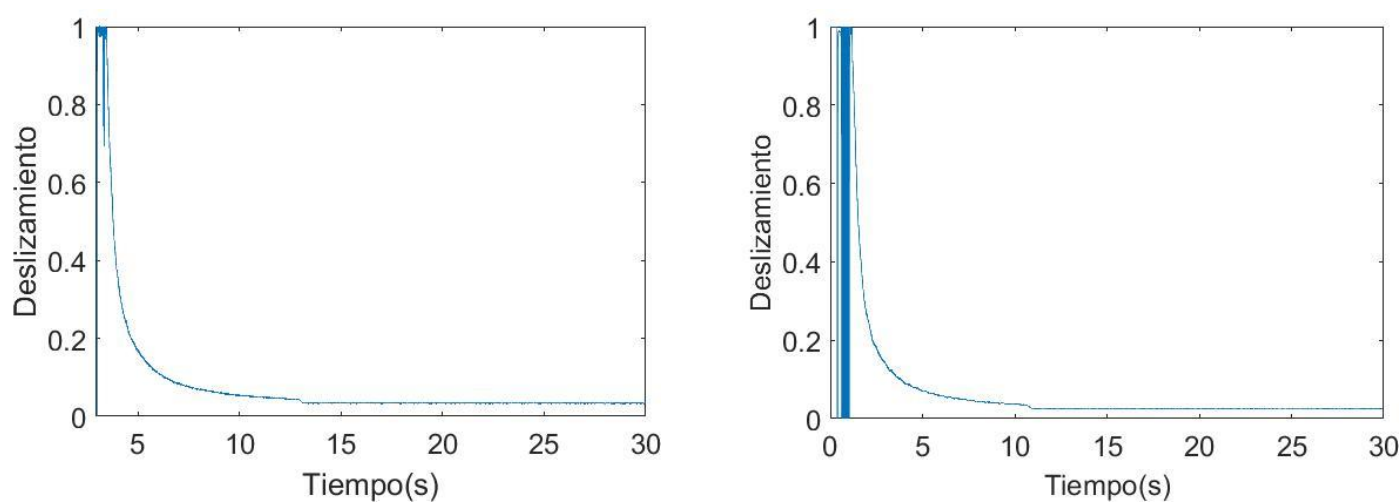

(b) Deslizamiento
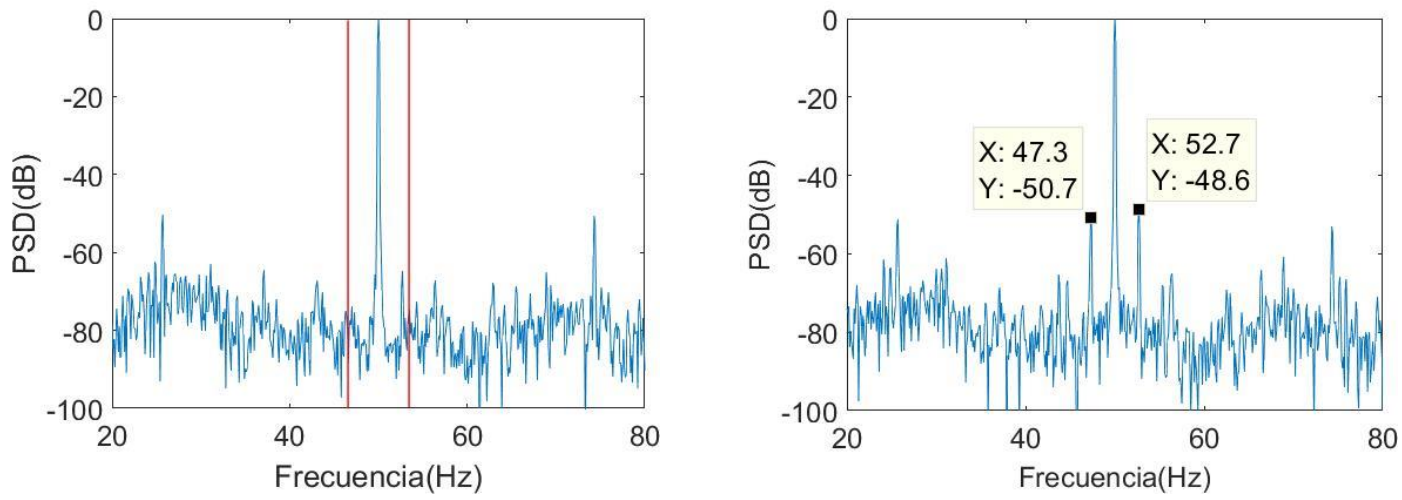

(c) Espectro del estado estacionario 

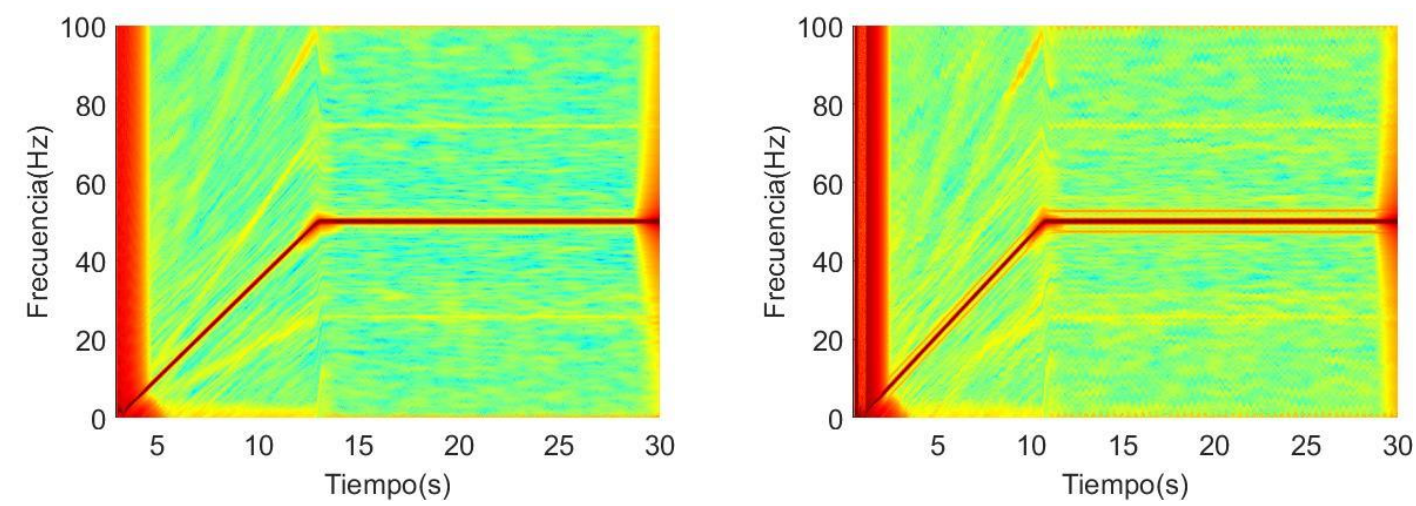

(d) Espectrograma del ensayo
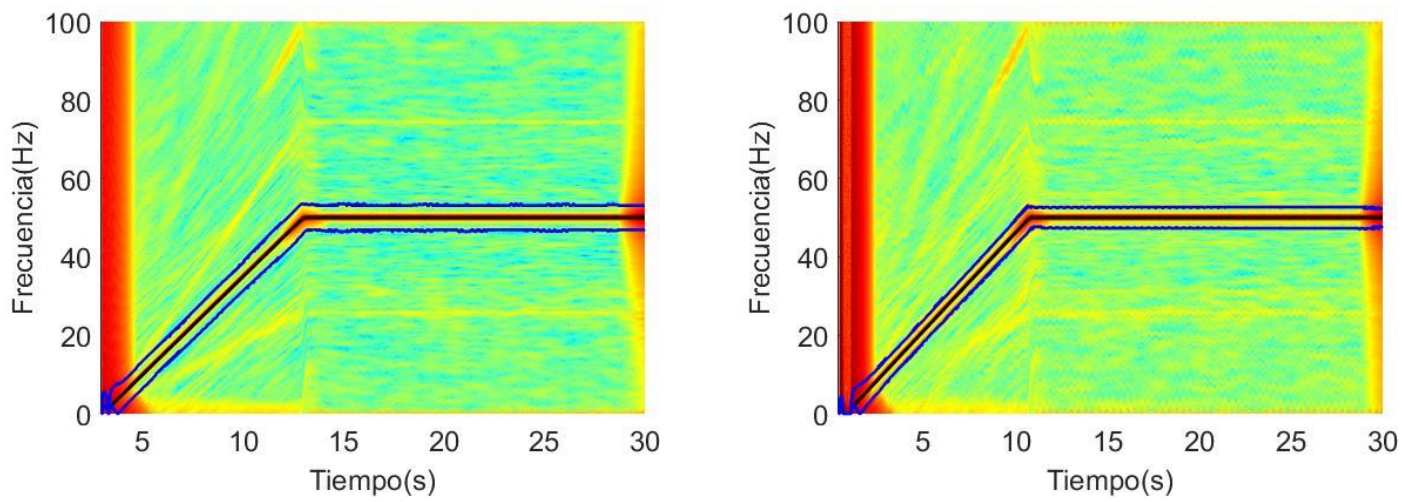

(e) Espectrograma del ensayo y trayectorias de los armónicos de fallo
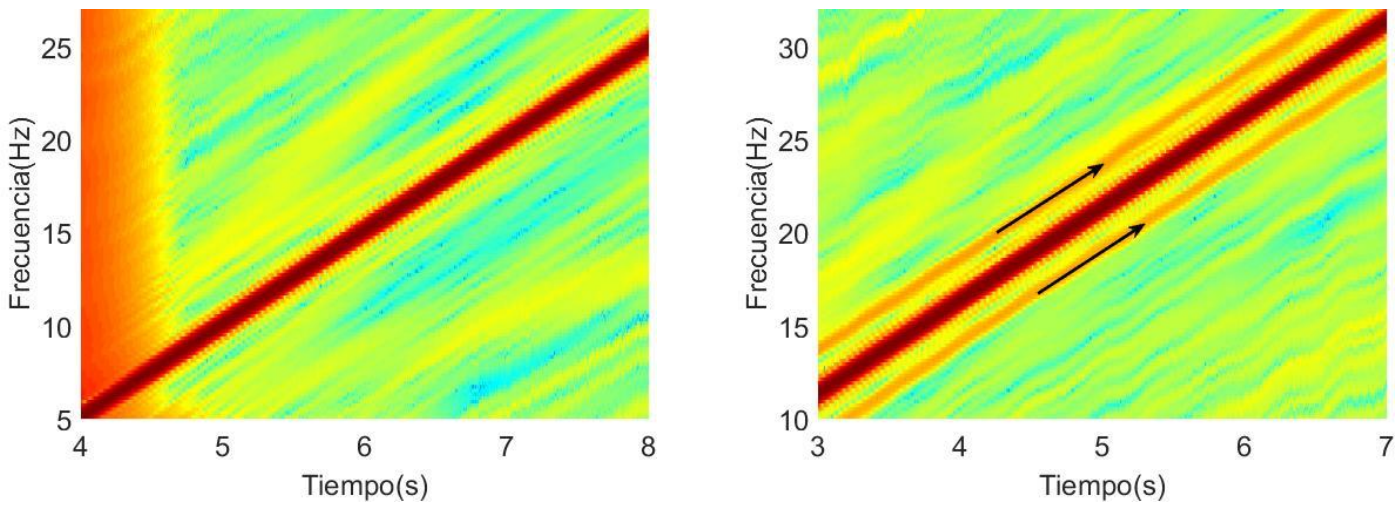

(f) Detalle del espectrograma
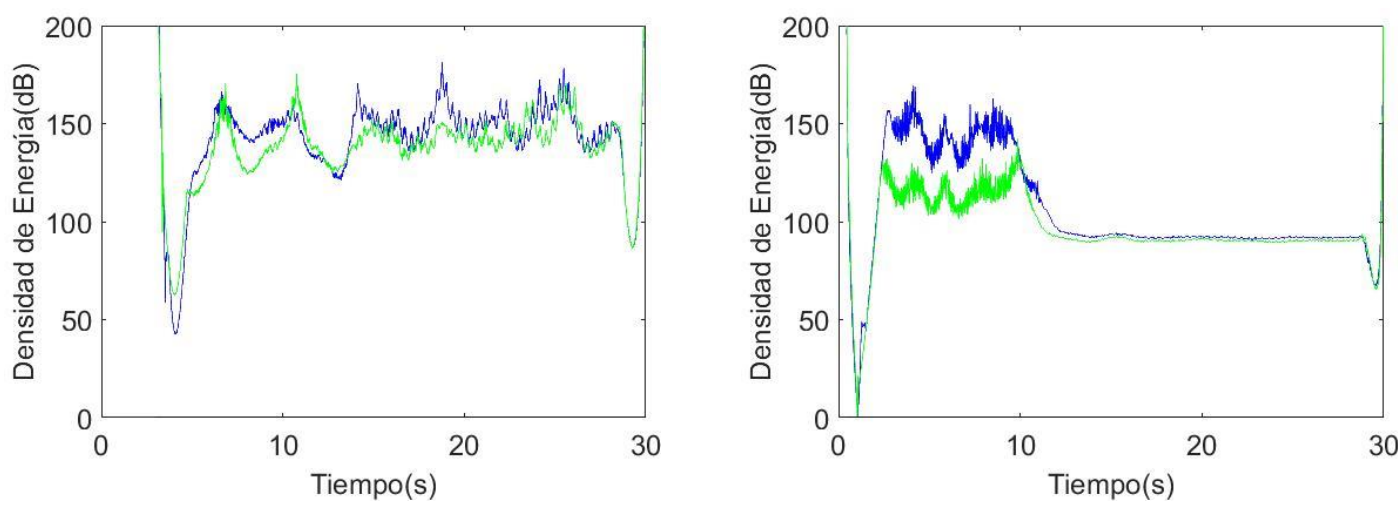

(g) Cuantificación del fallo

Figura 67. Resultados del análisis de la señal de $I M$ alimentado mediante inversor SIEMENS con nivel de carga bajo. 
Para continuar con la validación de la técnica, en las dos siguientes Figuras, 68 y 69, se muestra la detección cuando el motor está alimentado por el inversor de Telemecánica, procediendo de la misma manera que en los casos anteriores.

Se comienza con la Figura 68, en la cual se muestran los resultados del inversor de Telemecánica cuando el nivel de carga es alto, así los datos de estos ensayos son los siguientes:

- Inversor: TELEMECÁNICA

- Nivel de carga: Alto

- Tipo de control: V/f lineal

- Tipo de arranque: lineal

En estas circunstancias el valor del deslizamiento es de 0,045 , por lo que los valores de las frecuencias teóricas de fallo serán:

- Lower Sideband Harmonic (LSH):

$$
f_{L S H}=(1-2 s) f_{S}=(1-2 \cdot 0,045) \cdot 50=45,5 \mathrm{~Hz}
$$

- $\quad$ Upper Sideband Harmonic (USH):

$$
f_{U S H}=(1+2 s) f_{S}=(1+2 \cdot 0,045) \cdot 50=54,5 \mathrm{~Hz}
$$

Y si, en las mismas condiciones, el motor está sano, el deslizamiento que se obtiene es de 0,05 , por tanto:

- $\quad$ Lower Sideband Harmonic $(L S H)$ :

$$
f_{L S H}=(1-2 s) f_{S}=(1-2 \cdot 0,05) \cdot 50=45 \mathrm{~Hz}
$$

- $\quad$ Upper Sideband Harmonic (USH):

$$
f_{U S H}=(1+2 s) f_{S}=(1+2 \cdot 0,05) \cdot 50=55 \mathrm{~Hz}
$$



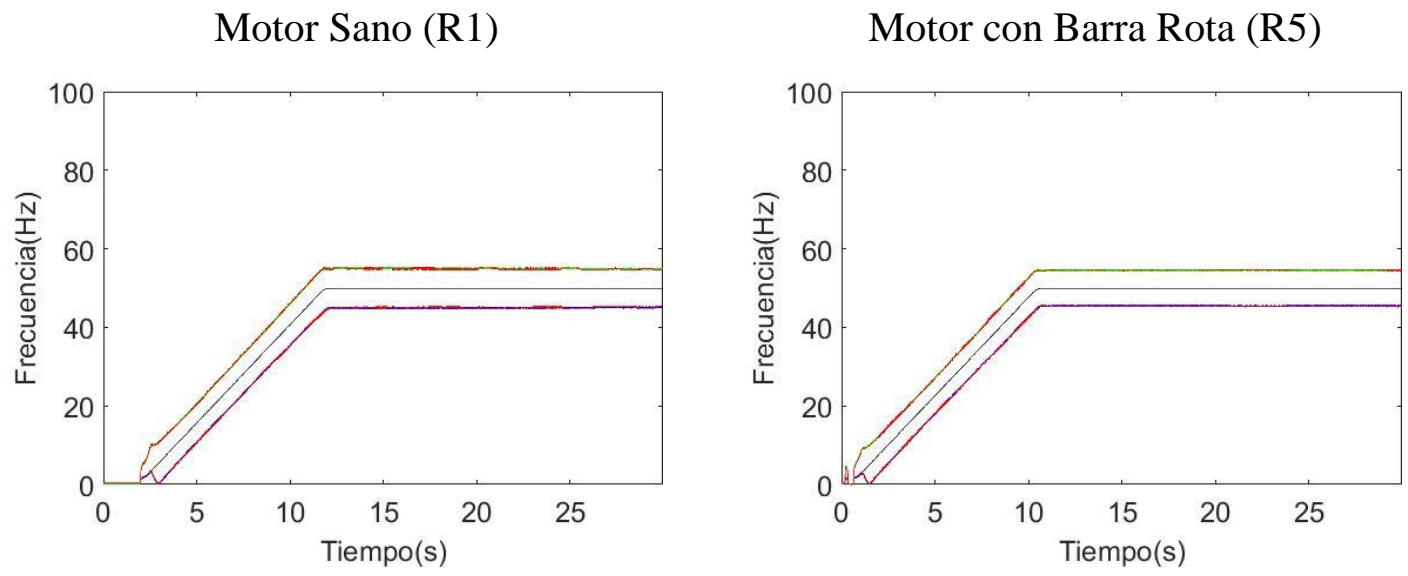

(a) Trayectoria real del primer armónico y teórica de los armónicos de fallo
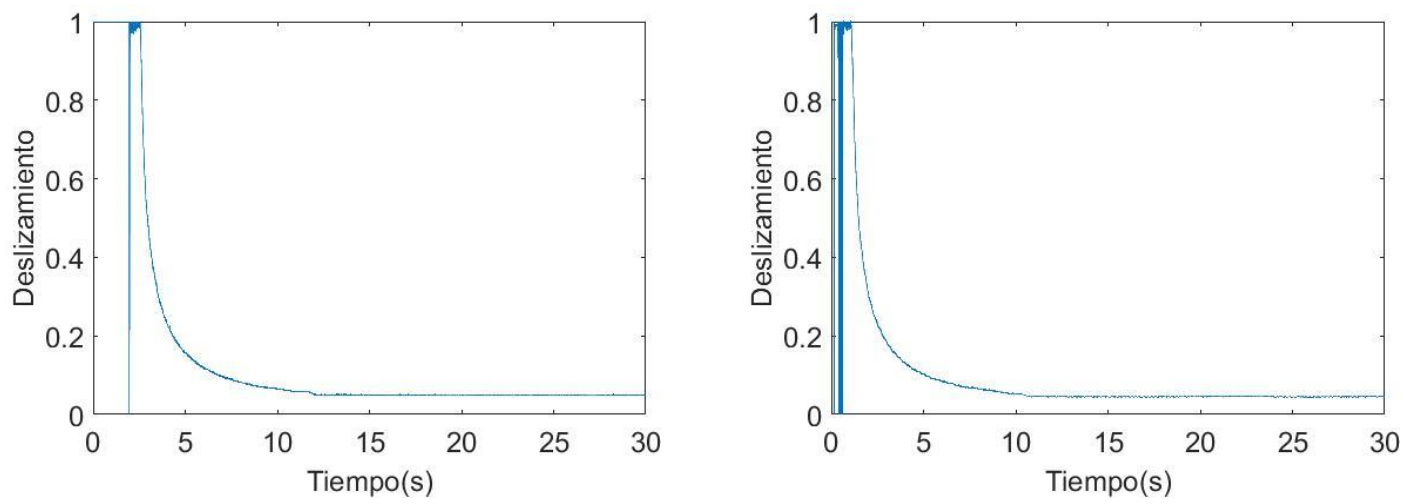

(b) Deslizamiento
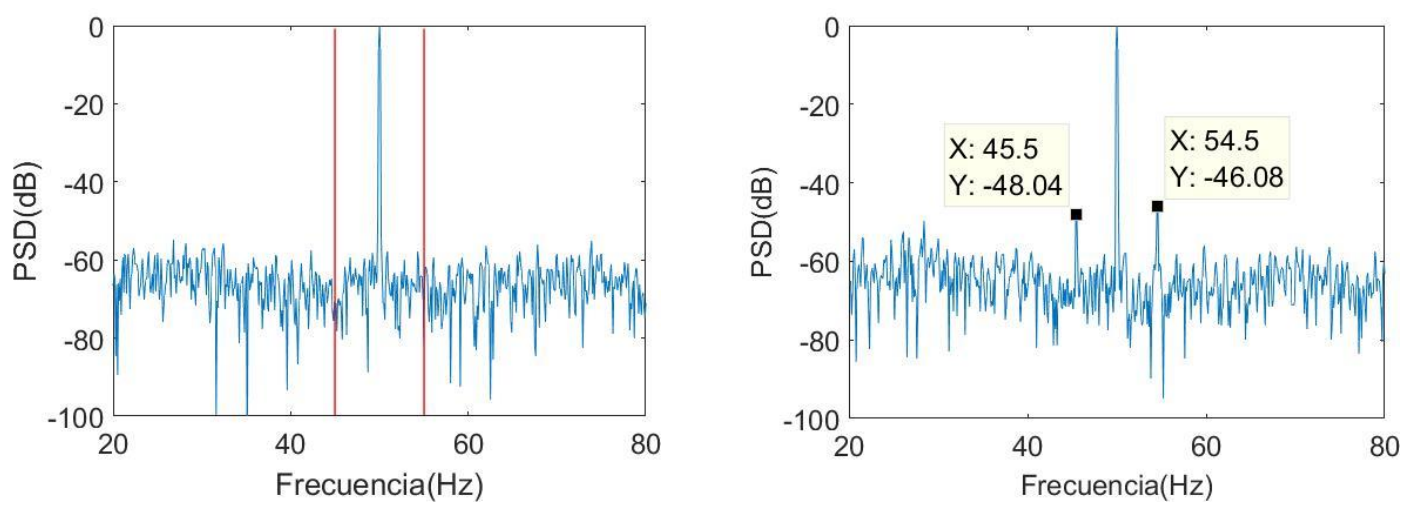

(c) Espectro del estado estacionario
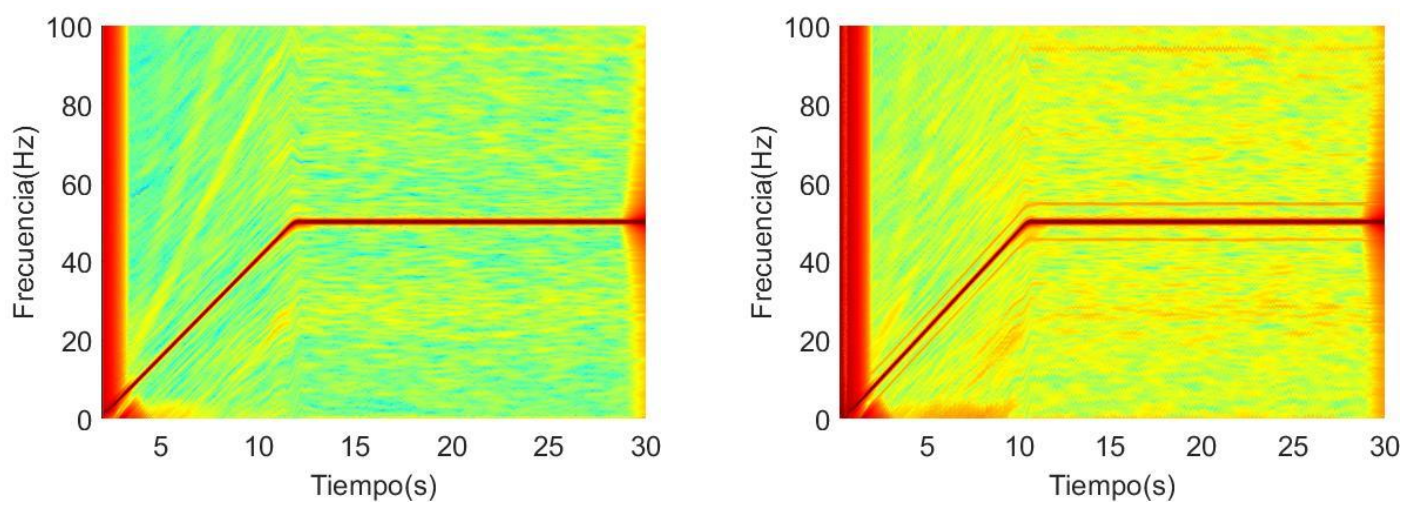

(d) Espectrograma del ensayo 

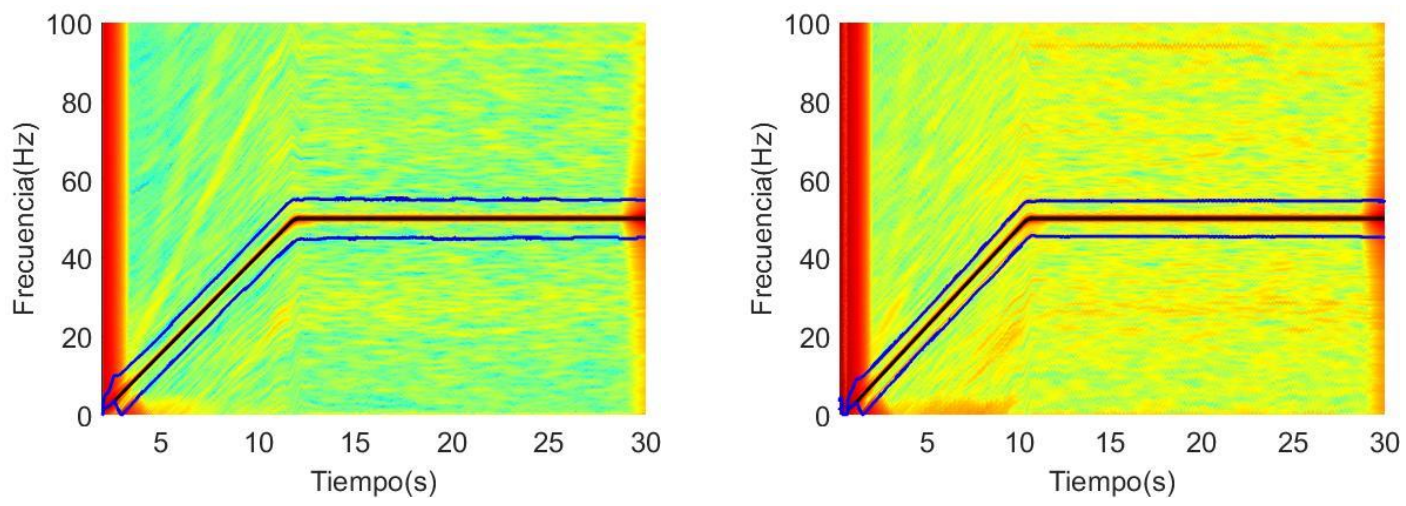

(e) Espectrograma del ensayo y trayectorias de los armónicos de fallo
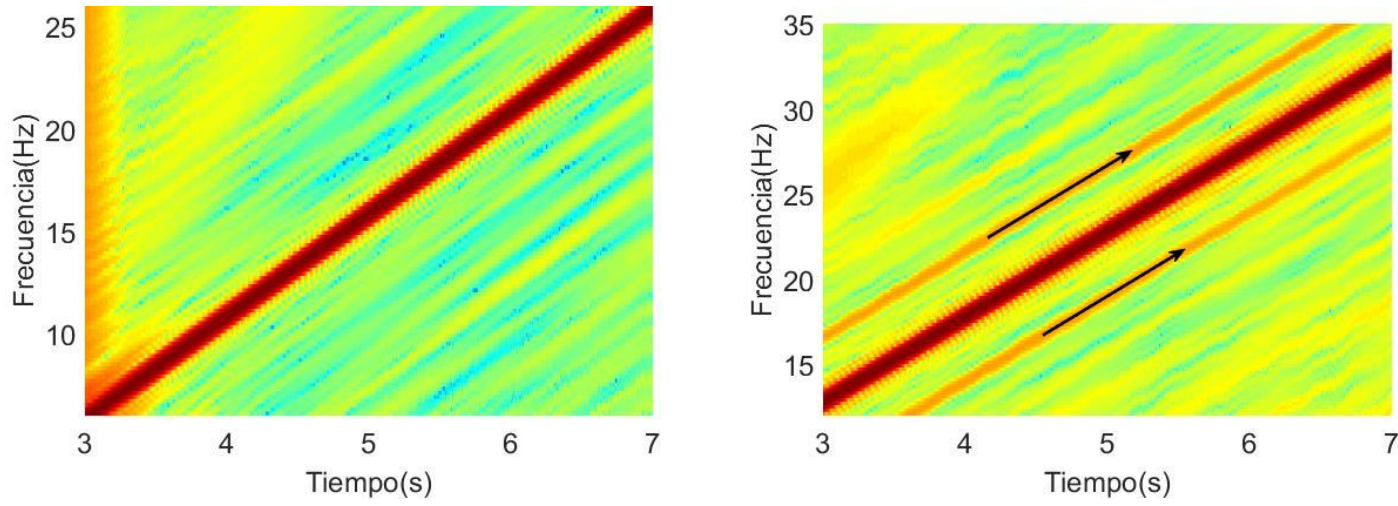

(f) Detalle del espectrograma
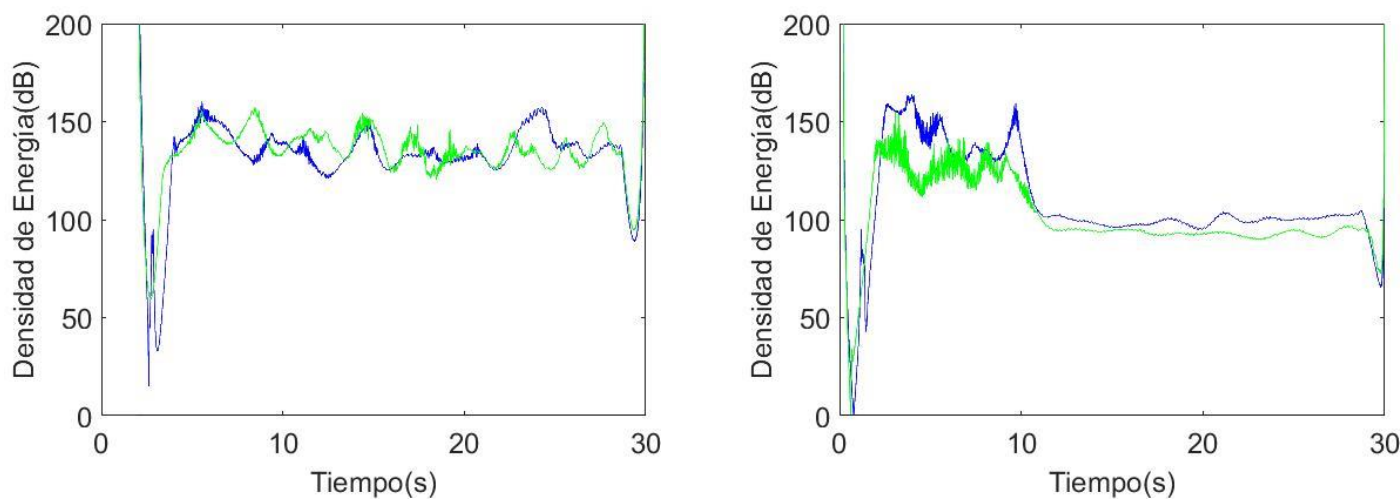

(g) Cuantificación del fallo

Figura 68. Resultados del análisis de la señal de $I M$ alimentado mediante inversor TELEMECANICA con nivel de carga alto.

En la Figura 69 se mantiene el mismo inversor, cambiando ahora el nivel de carga, que pasa a ser de nivel bajo; como resumen los datos de este ensayo son:

- Inversor: TELEMECÁNICA

- Nivel de carga: Bajo

- Tipo de control: V/f lineal

- Tipo de arranque: lineal 
Al medir el deslizamiento del motor cuando presenta barra rota, el valor del mismo es de 0,035 , con lo que las frecuencias teóricas de fallo serán:

- $\quad$ Lower Sideband Harmonic $(L S H)$ :

$$
f_{L S H}=(1-2 s) f_{S}=(1-2 \cdot 0,035) \cdot 50=46,5 H z
$$

- Upper Sideband Harmonic (USH):

$$
f_{U S H}=(1+2 s) f_{S}=(1+2 \cdot 0,035) \cdot 50=53,5 \mathrm{~Hz}
$$

Cuando el motor es sano, y en las mismas condiciones, el deslizamiento presenta un valor de 0,037 , con lo que los valores de las frecuencias son:

- $\quad$ Lower Sideband Harmonic ( $L S H)$ :

$$
f_{L S H}=(1-2 s) f_{S}=(1-2 \cdot 0,037) \cdot 50=46,3 \mathrm{~Hz}
$$

- $\quad$ Upper Sideband Harmonic (USH):

$$
f_{U S H}=(1+2 s) f_{S}=(1+2 \cdot 0,037) \cdot 50=53,7 \mathrm{~Hz}
$$

Motor Sano (R1)

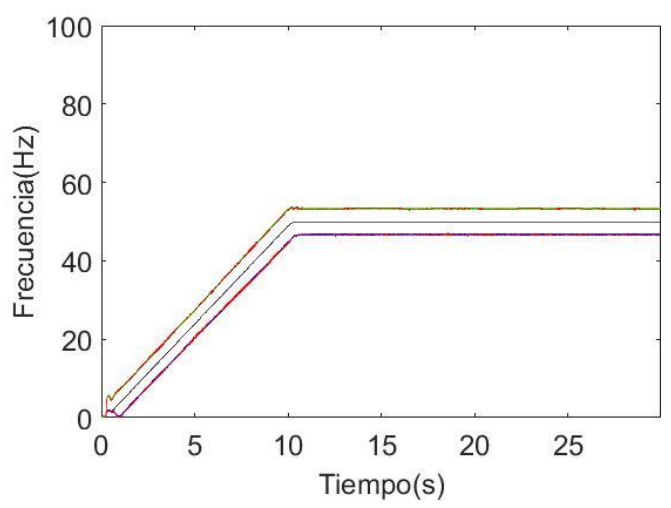

Motor con Barra Rota (R5)

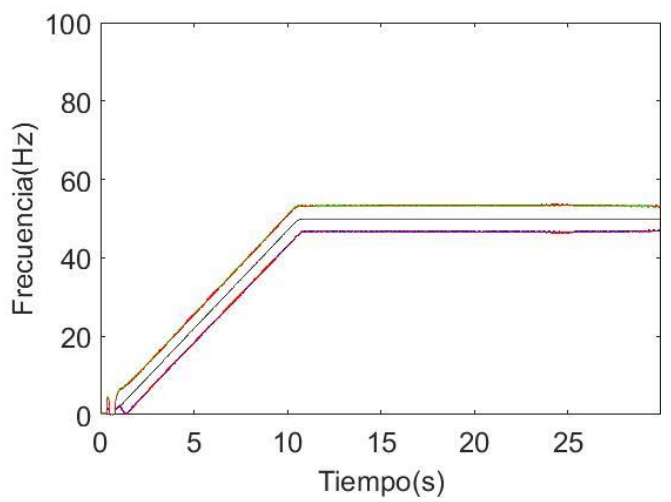

(a) Trayectoria real del primer armónico y teórica de los armónicos de fallo
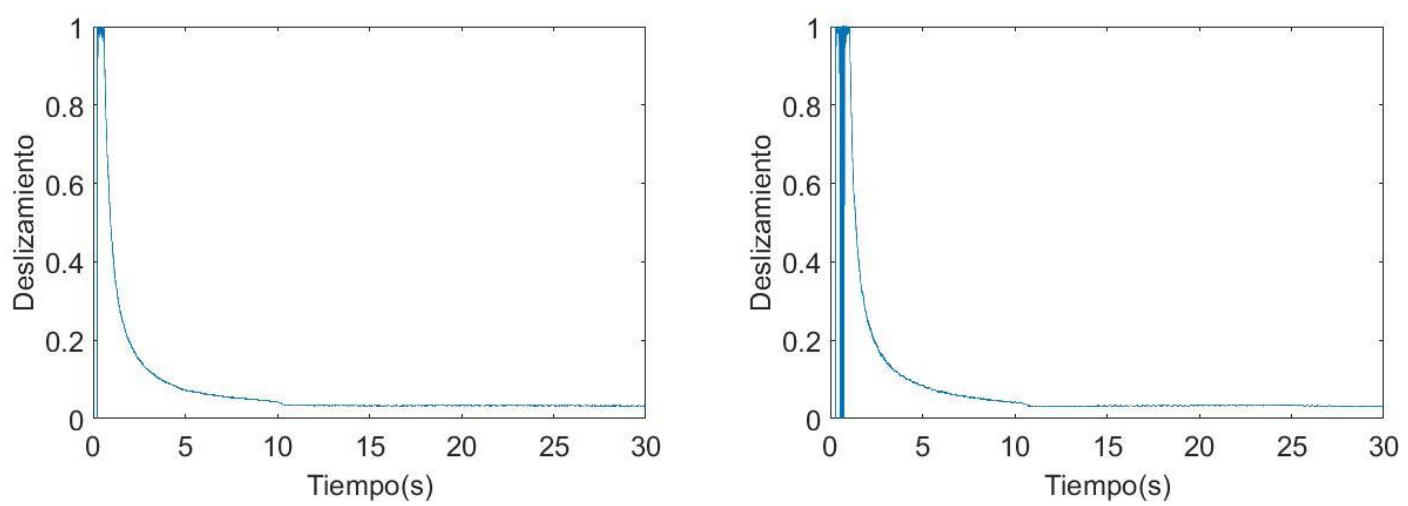

(b) Deslizamiento 

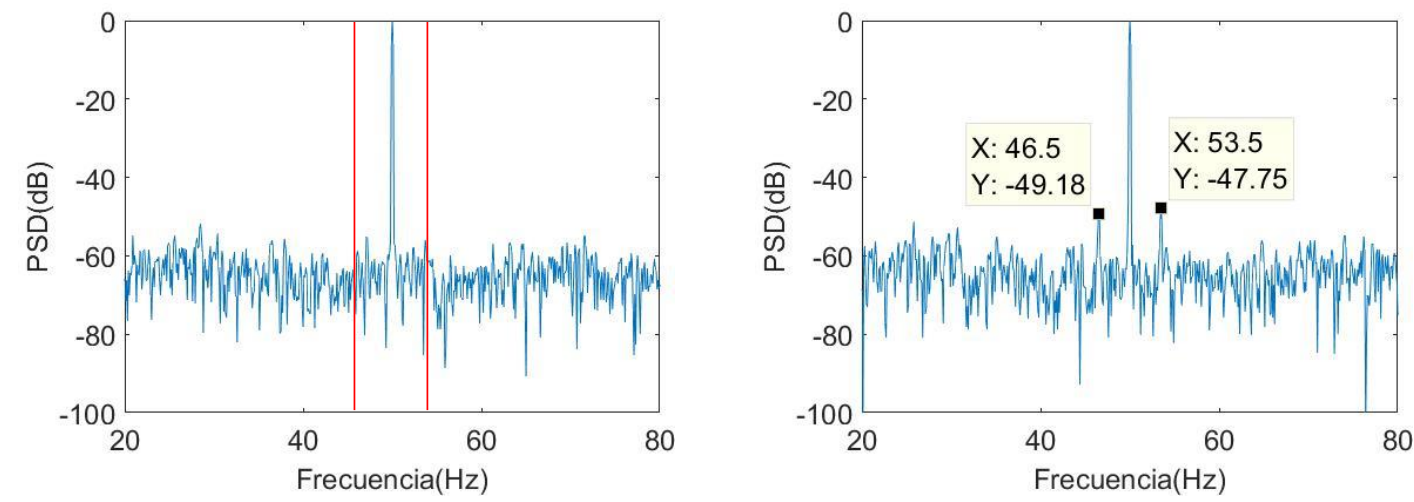

(c) Espectro del estado estacionario
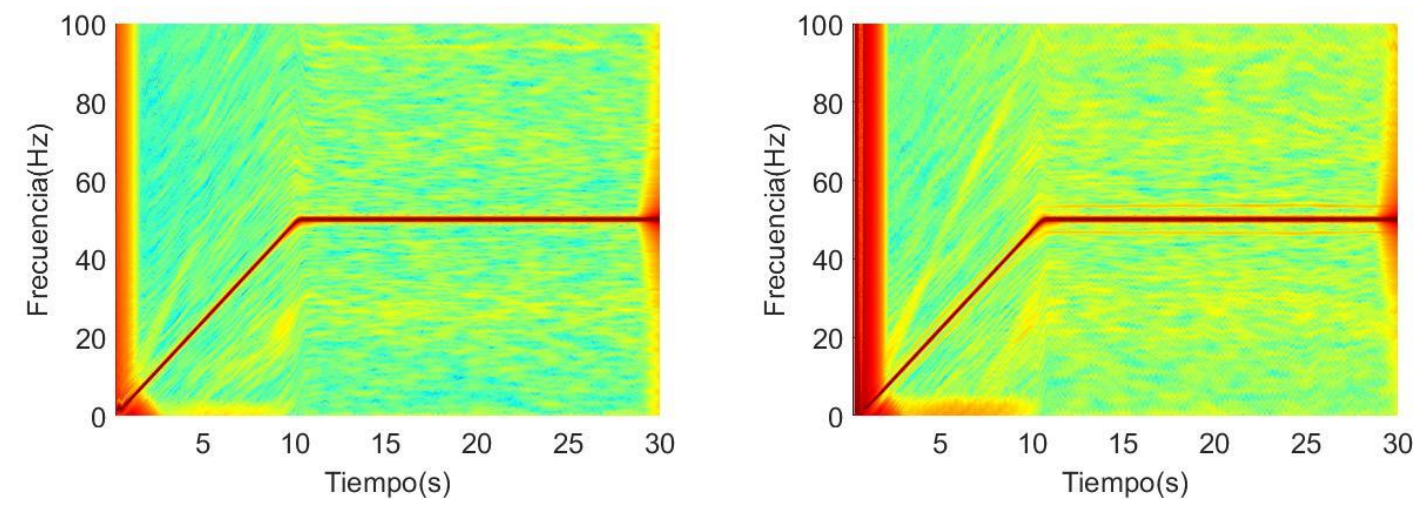

(d) Espectrograma del ensayo
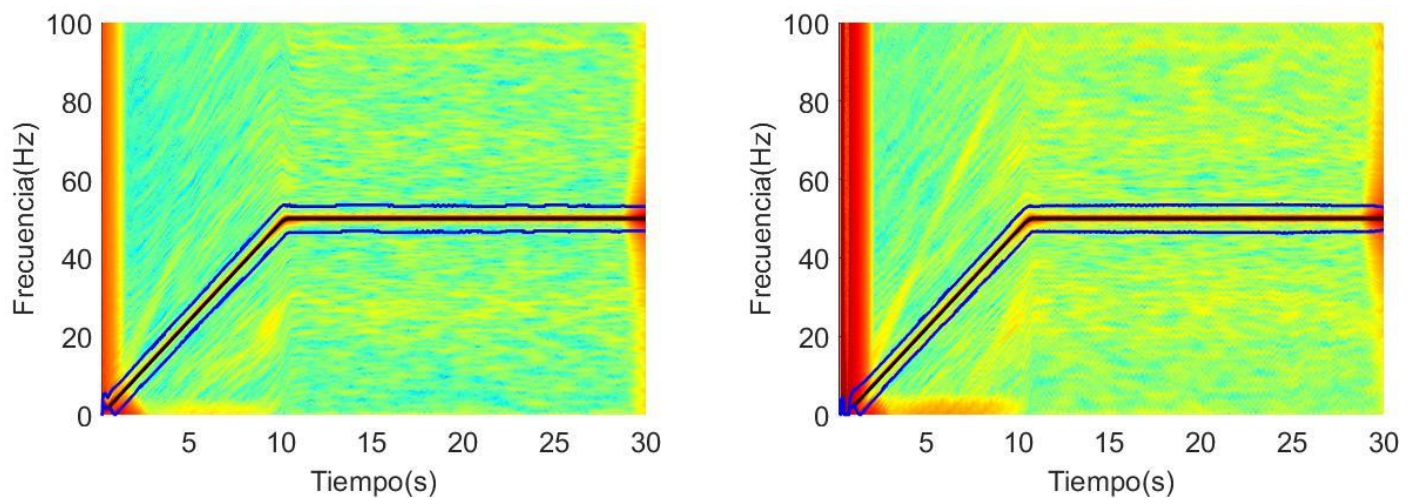

(e) Espectrograma del ensayo y trayectorias de los armónicos de fallo
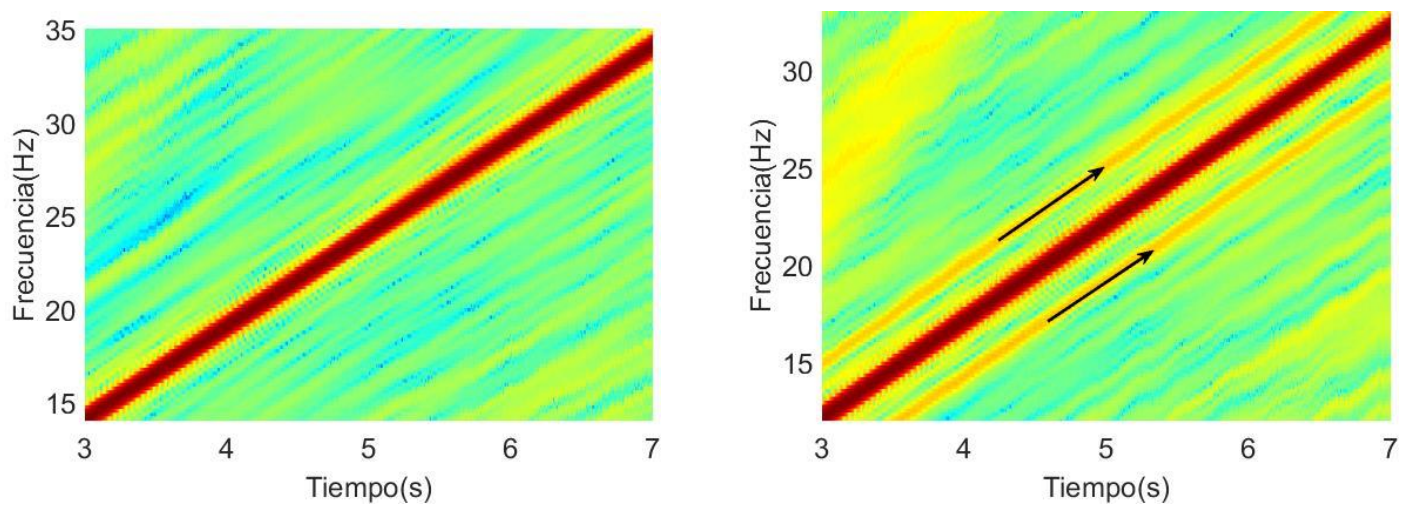

(f) Detalle del espectrograma 

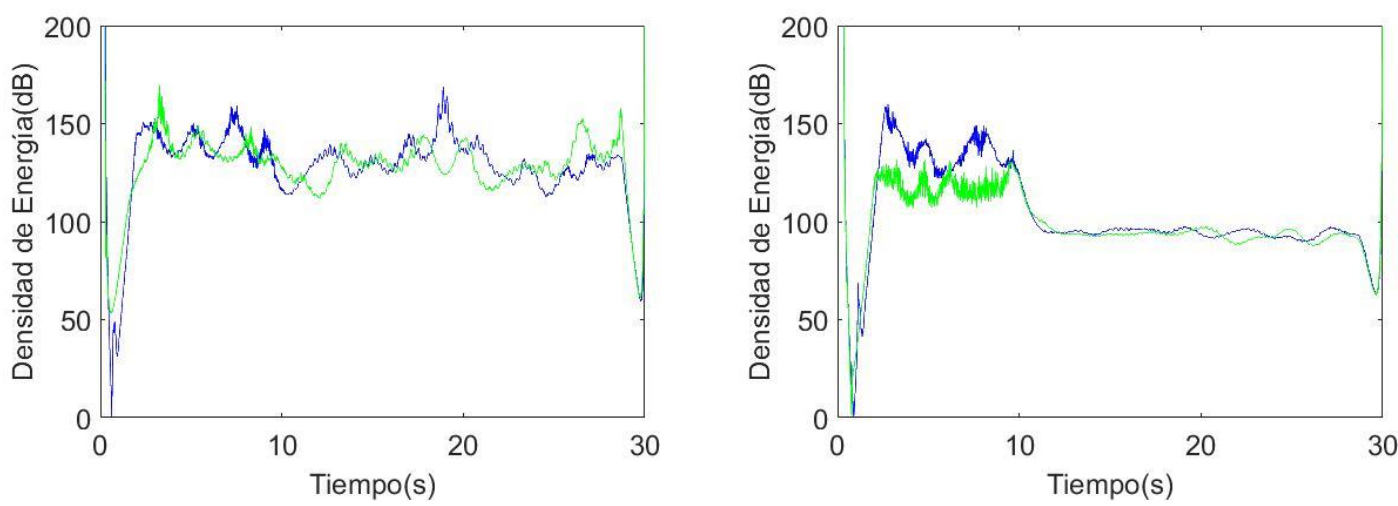

(g) Cuantificación del fallo

Figura 69. Resultados del análisis de la señal de $I M$ alimentado mediante inversor TELEMECANICA con nivel de carga bajo.

Tras el análisis de las Figuras 68 y 69, se comprueba que el inversor Telemecánica introduce una gran cantidad de ruido, que se puede comprobar tanto en el espectrograma resultante como si solo se analiza el estado estacionario. Si bien no es un problema para que la técnica sea capaz de seguir las trayectorias de los componentes de fallo, puede dificultar la apreciación de las mismas.

A continuación, los resultados mostrados en la Figura 70 corresponden al motor con un nivel de carga alto y alimentado con el inversor del fabricante WEG. Los datos de estos ensayos son los siguientes:

- Inversor: WEG

- $\quad$ Nivel de carga: Alto

- Tipo de control: V/f lineal

- $\quad$ Tipo de arranque: lineal

El deslizamiento del motor en el ensayo con una barra rota es 0,049 y los valores de las frecuencias de fallo son los siguientes:

- $\quad$ Lower Sideband Harmonic $(L S H)$ :

$$
f_{L S H}=(1-2 s) f_{S}=(1-2 \cdot 0,049) \cdot 50=45,1 \mathrm{~Hz}
$$

- Upper Sideband Harmonic (USH):

$$
f_{U S H}=(1+2 s) f_{S}=(1+2 \cdot 0,049) \cdot 50=55 H z
$$


En el ensayo con el motor en estado sano, el deslizamiento alcanzado es 0,05 y las frecuencias de fallo teóricas serían las siguientes:

- $\quad$ Lower Sideband Harmonic (LSH):

$$
f_{L S H}=(1-2 s) f_{S}=(1-2 \cdot 0,05) \cdot 50=45 \mathrm{~Hz}
$$

- $\quad$ Upper Sideband Harmonic (USH):

$$
f_{U S H}=(1+2 s) f_{S}=(1+2 \cdot 0,05) \cdot 50=55 \mathrm{~Hz}
$$

\section{Motor Sano (R1)}

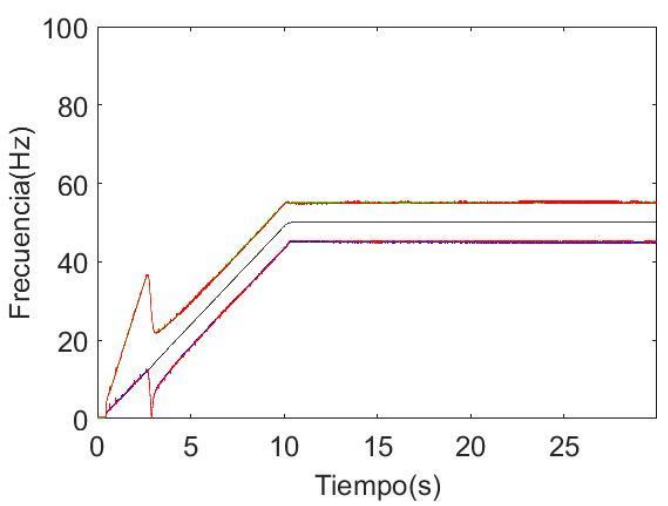

Motor con Barra Rota (R5)

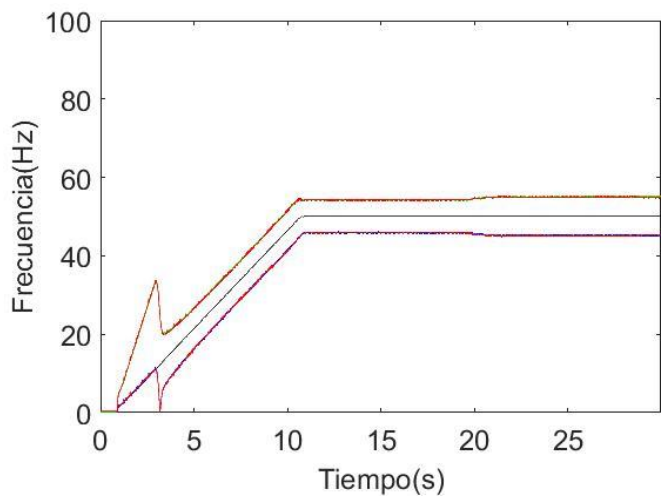

(a) Trayectoria real del primer armónico y teórica de los armónicos de fallo
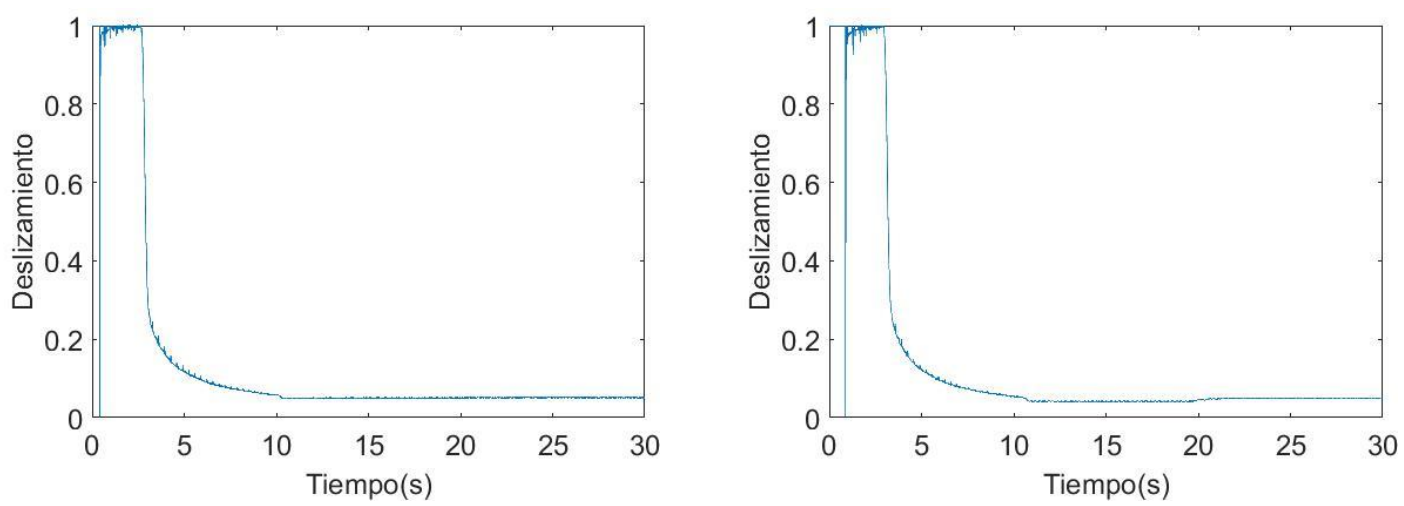

(b) Deslizamiento
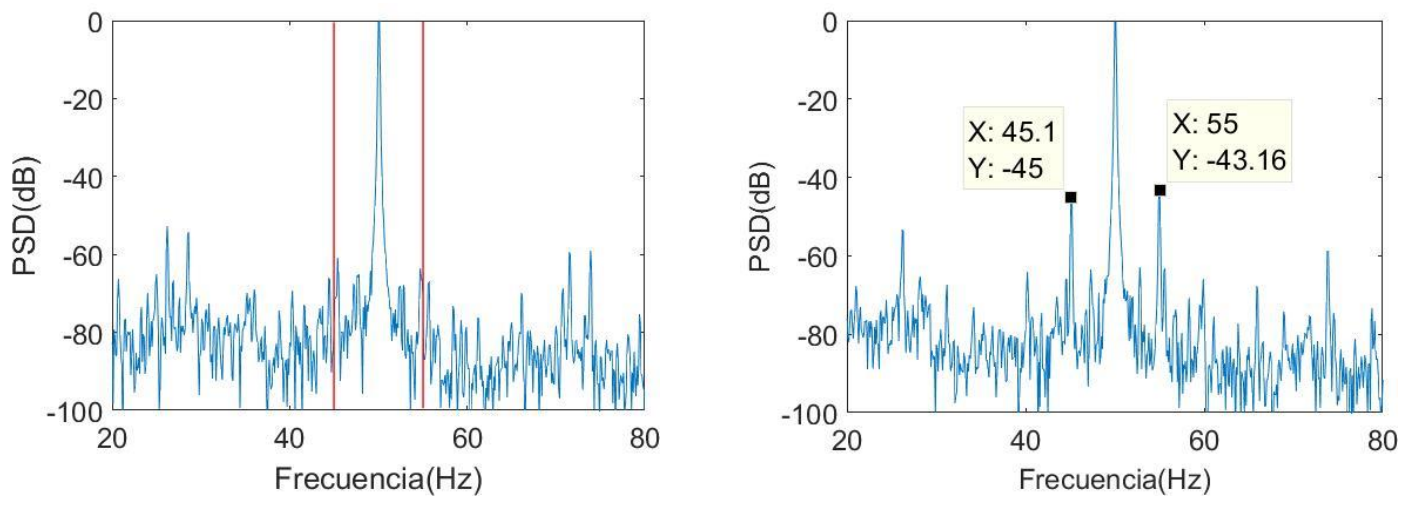

(c) Espectro del estado estacionario 

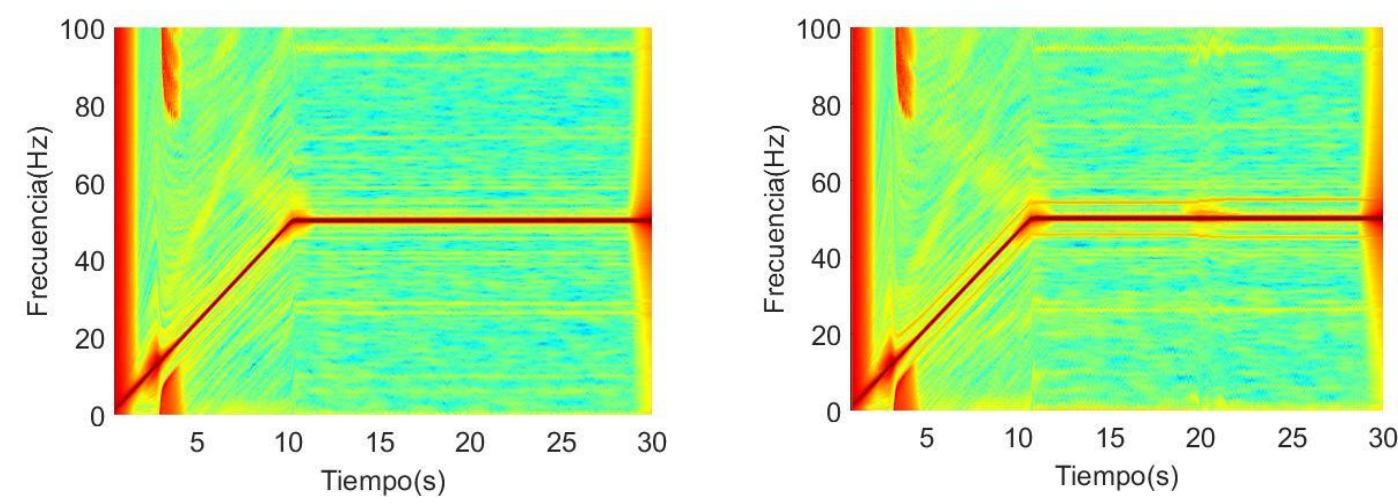

(d) Espectrograma del ensayo
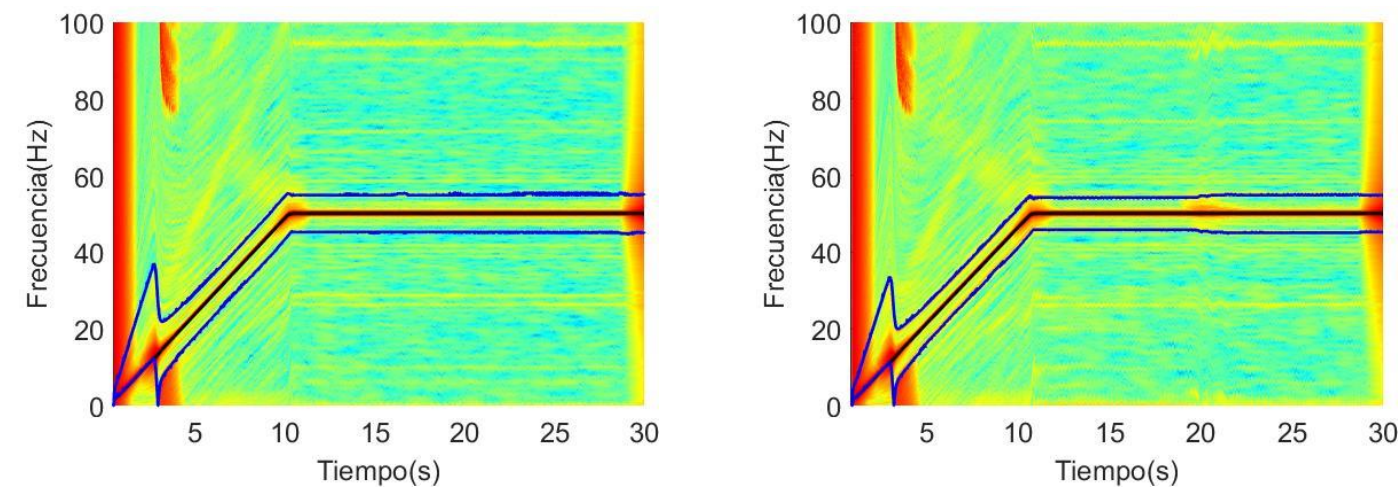

(e) Espectrograma del ensayo y trayectorias de los armónicos de fallo
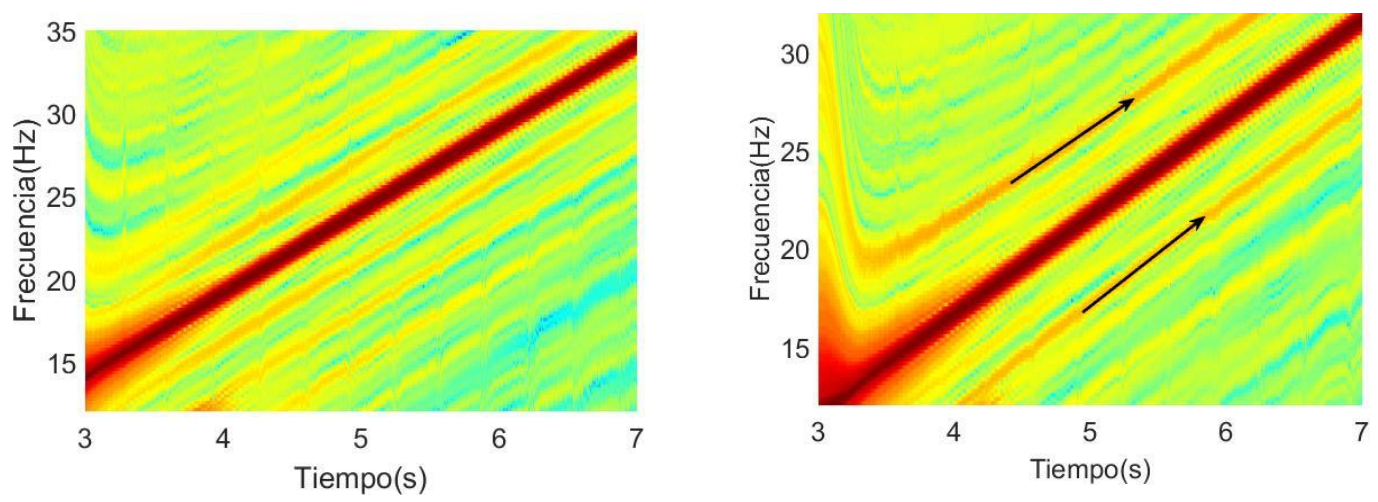

(f) Detalle del espectrograma
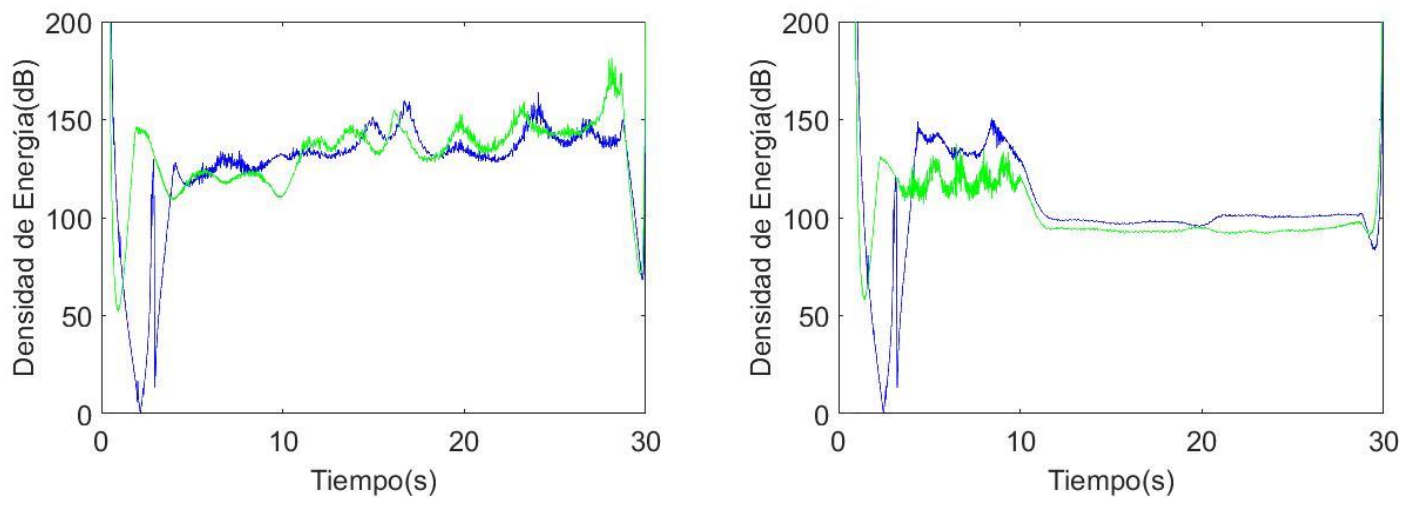

(g) Cuantificación del fallo

Figura 70. Resultados del análisis de la señal de $I M$ alimentado mediante inversor WEG con nivel de carga alto. 
Tras analizar los ensayos concernientes al motor alimentado mediante el inversor de WEG, aparece una nueva circunstancia, que con los anteriores inversores no se había presentado. Esta circunstancia se observa en la Figura 70 (d) y con más detalle en la Figura 70 (f) en donde se ha efectuado un zoom entre los instantes de tiempo 3 y 7 segundos, y es que se encuentran una gran cantidad de armónicos que pueden dar lugar a un error de detección, ya que podrían confundirse con el armónico introducido por el fallo de barras.

Sin embargo, para comprobar que realmente no se trata de un fallo por barras rotas, se hace la comprobación que aparece en la Figura 71, en donde se aprecia el mismo instante de tiempo de la Figura 70 (f), pero con las trayectorias teóricas de fallo superpuestas, con lo que se demuestra que las trayectorias que podían dar lugar a error no son coincidentes con las trayectorias de un posible fallo por barras rotas. Por tanto, se puede concluir que estos armónicos son introducidos por el inversor. Esta circunstancia se va a comprobar mostrando otro ensayo de este mismo variador (Figura 72).

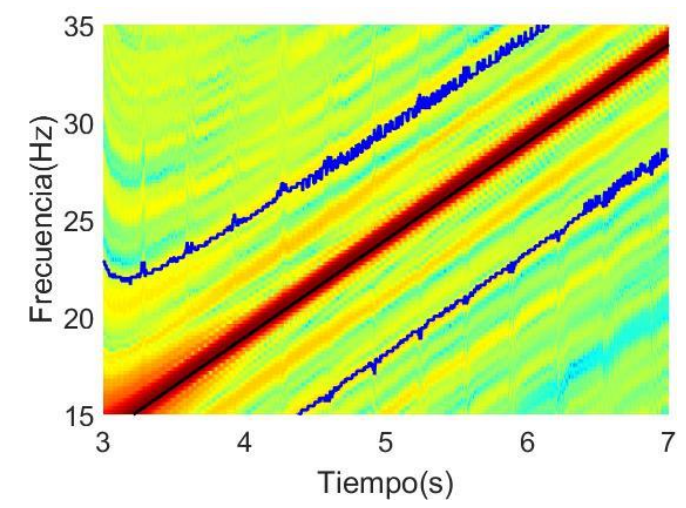

Figura 71. Zoom Figura 70(d) entre los instantes 3 y 7, con las trayectorias teóricas de fallo superpuestas.

En la Figura 72, los resultados corresponden al mismo inversor que en la Figura 66, WEG, pero ahora con un nivel de carga inferior. Los datos de estos ensayos son los siguientes:

- Inversor: WEG

- $\quad$ Nivel de carga: Bajo

- Tipo de control: V/f lineal

- Tipo de arranque: lineal 
El deslizamiento del motor en el ensayo con una barra rota es 0,025. Los valores de las frecuencias de fallo son los siguientes:

- $\quad$ Lower Sideband Harmonic $(L S H)$ :

$$
f_{L S H}=(1-2 s) f_{S}=(1-2 \cdot 0,025) \cdot 50=47,5 \mathrm{~Hz}
$$

- Upper Sideband Harmonic (USH):

$$
f_{U S H}=(1+2 s) f_{S}=(1+2 \cdot 0,025) \cdot 50=52,6 \mathrm{~Hz}
$$

El valor del deslizamiento para el caso de motor en estado sano es de 0,03, por lo, teniendo en cuenta este valor, las frecuencias teóricas de fallo corresponden con:

- $\quad$ Lower Sideband Harmonic ( $L S H)$ :

$$
f_{L S H}=(1-2 s) f_{S}=(1-2 \cdot 0,03) \cdot 50=47 \mathrm{~Hz}
$$

- $\quad$ Upper Sideband Harmonic (USH):

$$
f_{U S H}=(1+2 s) f_{S}=(1+2 \cdot 0,03) \cdot 50=53 \mathrm{~Hz}
$$
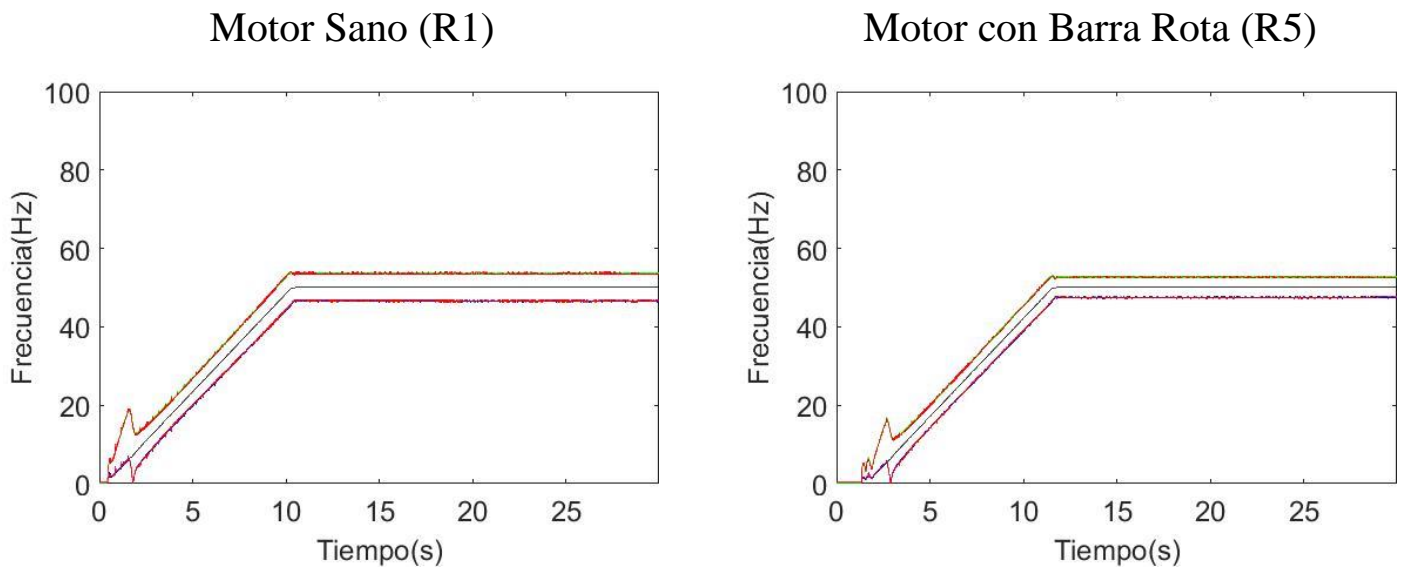

(a) Trayectoria real del primer armónico y teórica de los armónicos de fallo
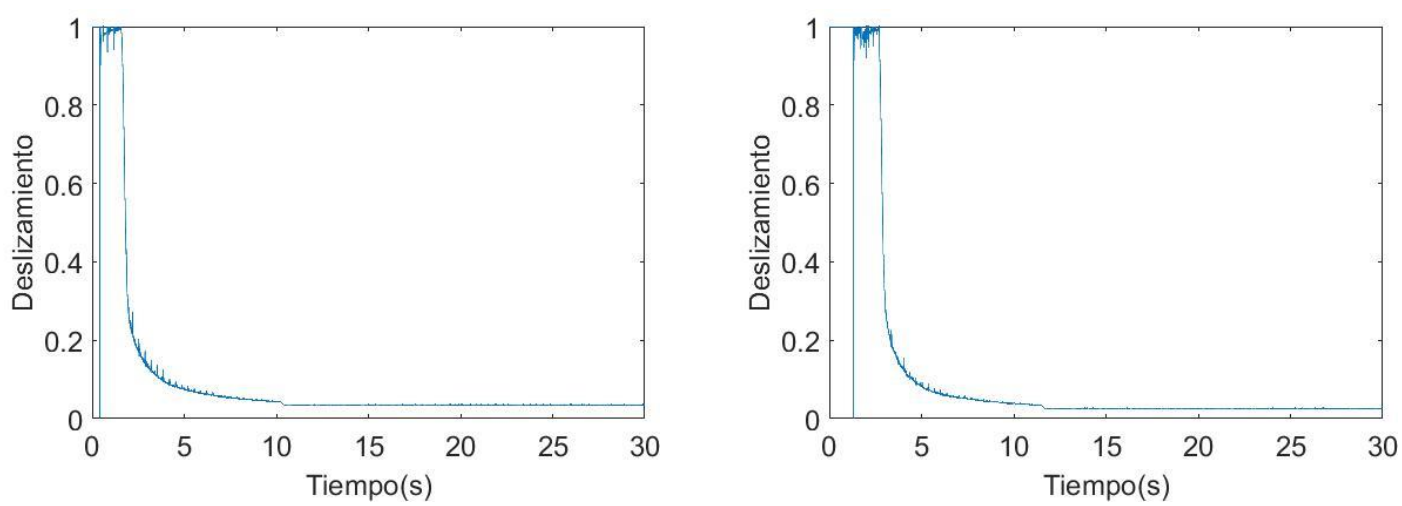

(b) Deslizamiento 

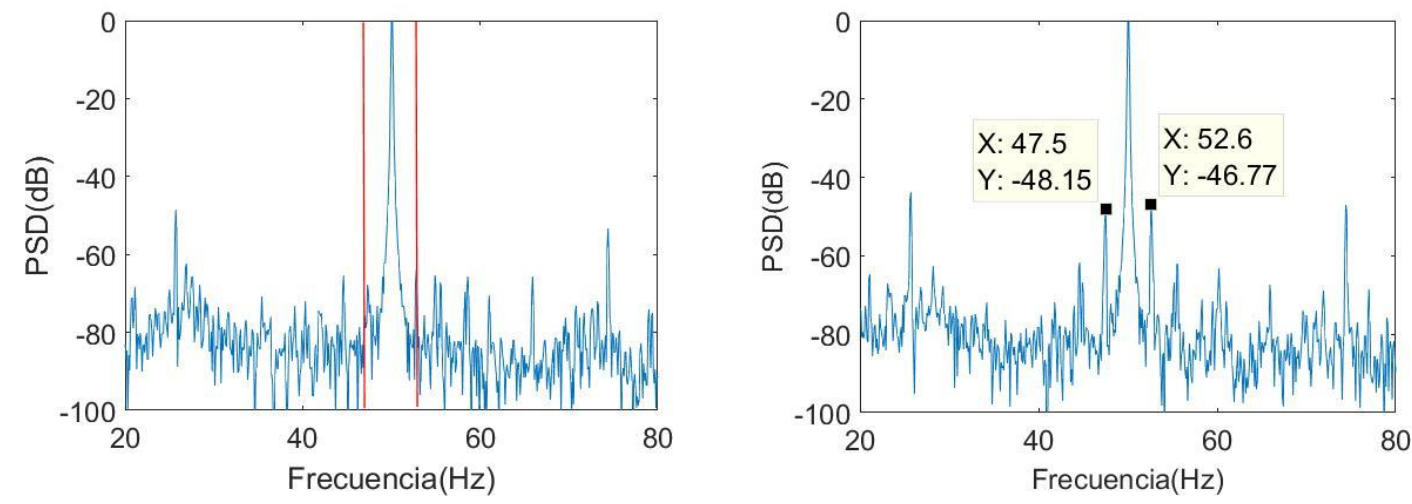

(c) Espectro del estado estacionario
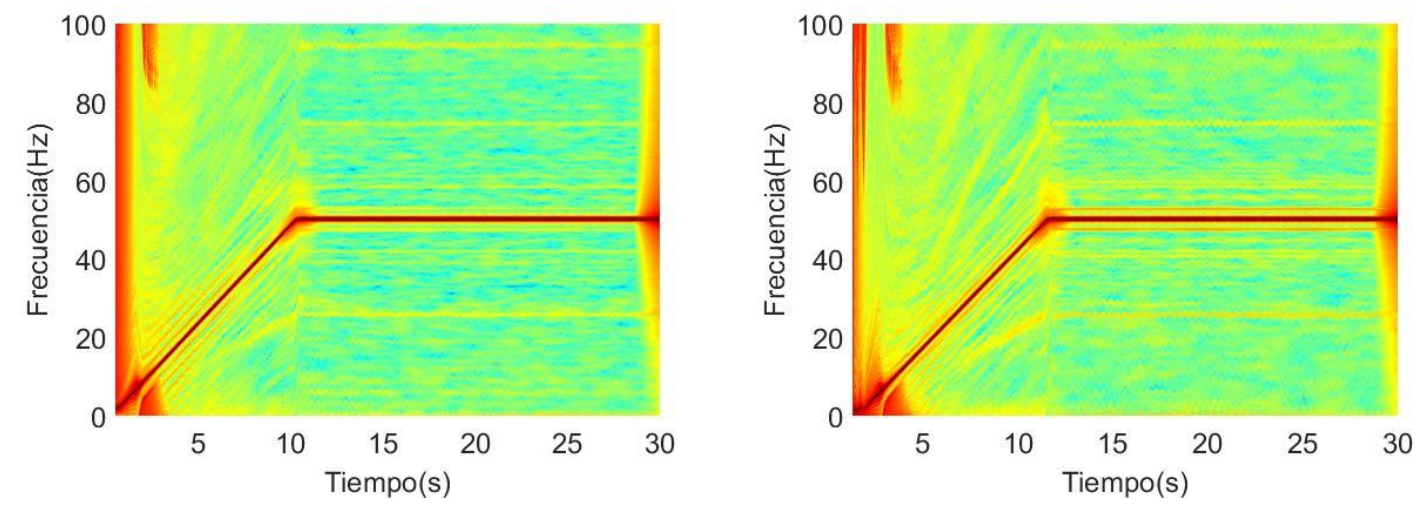

(d) Espectrograma del ensayo
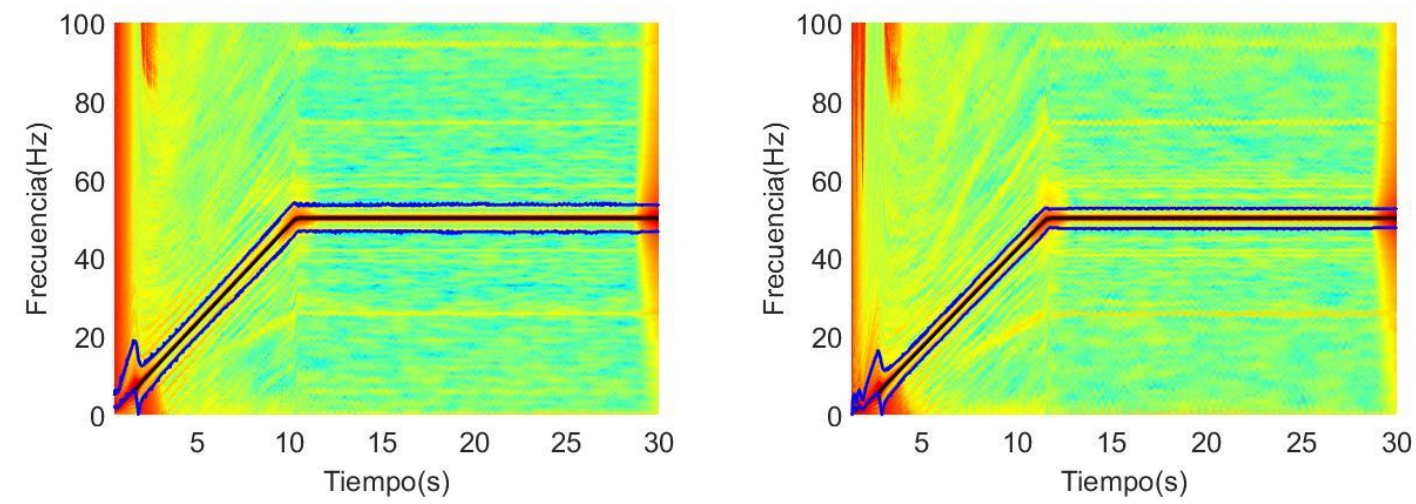

(e) Espectrograma del ensayo y trayectorias de los armónicos de fallo
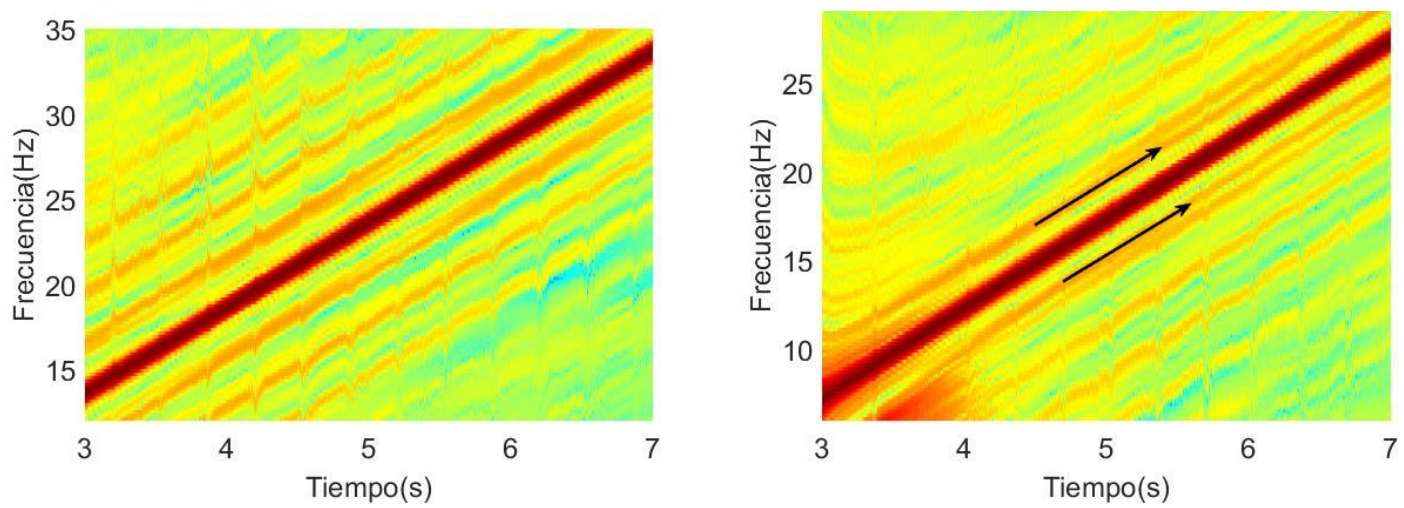

(f) Detalle del espectrograma 

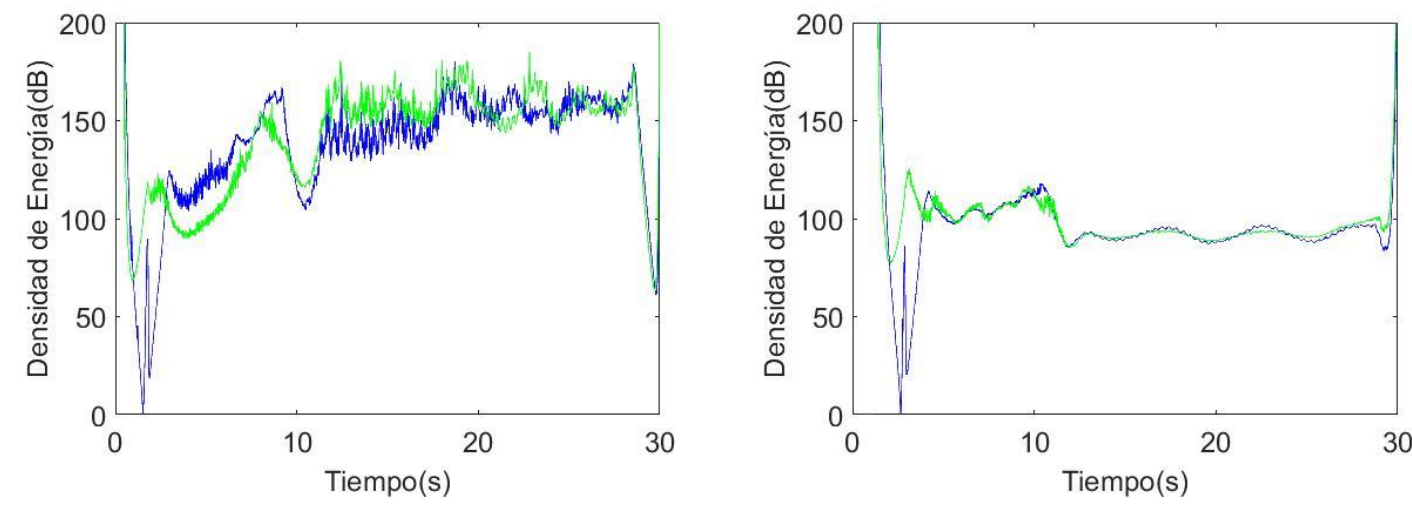

(g) Cuantificación del fallo

Figura 72. Resultados del análisis de la señal de $I M$ alimentado mediante inversor WEG con nivel de carga bajo.

Se corrobora lo que se ha adelantado tras analizar la Figura 70, ya que al analizar con este mismo inversor (WEG), aunque cambiemos de nivel de carga (ahora nivel bajo de carga), se presenta el mismo problema, y es que el inversor introduce una gran cantidad de armónicos, tal como se aprecia en la Figura 72 (d) y que se puede ver con mayor detalle en el zoom de la Figura 72 (f).

Se corrobora, mediante la Figura 73, que realmente no se trata de armónicos debidos a un fallo de barras, ya que al presentar el detalle del espectrograma entre los instantes de tiempo 3 y 7 segundos del arranque del motor con las trayectorias teóricas superpuestas, se observa que estas no son coincidentes.

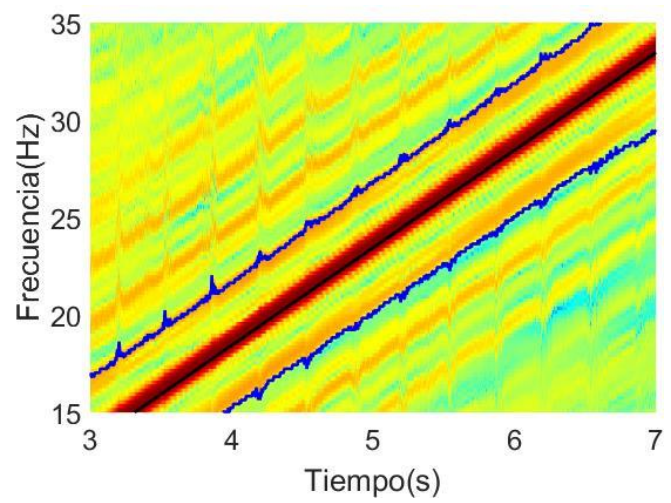

Figura 73. Zoom Figura 68(d) entre los instantes 3 y 7, con las trayectorias teóricas de fallo superpuestas.

Por lo que se puede concluir que, efectivamente, el inversor WEG introduce una gran cantidad de armónicos, que, si bien no se identifican con ningún fallo, pueden dar lugar a error en la interpretación del resultado. Por esta razón, es necesario conocer la 
trayectoria teórica de los armónicos de fallo para interpretar correctamente los resultados en el caso de este inversor.

\subsubsection{Conclusiones parciales}

Una vez que se han analizado los ensayos concernientes a casos en los que el motor presenta fallo por barras rotas, se puede llegar a una serie de conclusiones, que a continuación se exponen.

La primera de ellas es que se ha conseguido desarrollar una técnica que permite seguir las trayectorias de los armónicos presentes en la señal que se analice, independientemente del inversor usado y del nivel de carga.

Se corroboran los resultados mediante la transformada Rápida de Fourier, permite verificar los armónicos de fallo en el estado estacionario coincidente con los valores que se obtienen mediante la transformada Dragón, con la que se obtiene muy buena definición durante este período de funcionamiento.

Sin embargo, cuando se está trabajando con el estado transitorio, la transformada de Fourier no proporciona suficiente información, puesto que en estos casos es importante conocer el contenido de armónicos de la señal, pero también la componente de frecuencia en cada instante de tiempo, y esta información no es suministrada por la $F T$, tal como se explica en el punto 2 de esta memoria, por lo que es necesario recurrir a otras técnicas para poder observar con una buena resolución el arranque del motor. La Transformada desarrollada también es capaz de seguir los armónicos de fallo en este estado transitorio.

Por tanto, la transformado Dragón es capaz de seguir las trayectorias de los armónicos de fallo en todo el plano tiempo-frecuencia, es decir, tanto en estado estacionario como en el transitorio, obteniendo unos transitorios limpios, que permiten una perfecta definición de los armónicos de fallo. Las trayectorias teóricas permiten corroborar los valores reales obtenidos en el espectrograma. 
Por otra parte, en cuanto a los inversores, el inversor de Telemecánica presenta mucho ruido, que, si bien no interfiere a la hora de analizar la señal, puede perturbar los resultados obtenidos, ya que no se obtienen de una forma totalmente nítida.

En cuanto al inversor de WEG, se ha observado que introduce una elevada cantidad de armónicos, lo que pueden dar lugar a un error en la detección del fallo, puesto que estos armónicos se pueden confundir con trayectorias de fallos, por lo que es necesario, en este caso, conocer, previamente, como son estas trayectorias teóricas de fallo (tal como se explican en el punto 2 de esta memoria).

Finalmente, en cuanto a la cuantificación, es observable como existe una diferencia entre las densidades de energía de los armónicos de fallo cuando el motor está sano o presenta un fallo. El estudio de la cuantificación del fallo se desarrolla de manera más exhaustiva en el apartado 6.4. de esta memoria.

\subsubsection{Ensayos especiales}

En este apartado se incluyen una serie de ensayos especiales (Figuras 74 a 76), con el objetivo de ver cómo la transformada desarrollada es capaz de adaptarse a otro tipo de transitorios.

En la Figura 74 se comienza por analizar el caso de un motor alimentado mediante un inversor Allen-Bradley. Este inversor permite un arranque en "S", este tipo de arranque se emplea para aplicaciones en las que se requiera de una transición uniforme de cambio de velocidad, ya que proporciona que este cambio se haga de una manera más suave que cuando el arranque es lineal, (ver descripción inversores, punto 5 de la memoria), con lo que en este ensayo las características son:

- Inversor: Allen-Bradley

- Nivel de carga: Alto

- Tipo de control: V/f lineal

- Tipo de arranque: en forma de S 
Al estar el motor operando en estas condiciones, y cuando éste presenta un fallo por barras rotas, se tiene un valor de deslizamiento de 0,043 , con lo que las frecuencias teóricas de fallo en estado estacionario son las siguientes:

- $\quad$ Lower Sideband Harmonic $(L S H)$ :

$$
f_{L S H}=(1-2 s) f_{S}=(1-2 \cdot 0,043) \cdot 50=45,7 \mathrm{~Hz}
$$

- $\quad$ Upper Sideband Harmonic (USH):

$$
f_{U S H}=(1+2 s) f_{S}=(1+2 \cdot 0,043) \cdot 50=54,3 \mathrm{~Hz}
$$

En el ensayo del motor en estado sano se mide un deslizamiento de 0,05 , por tanto, las frecuencias de fallo son:

- $\quad$ Lower Sideband Harmonic (LSH):

$$
f_{L S H}=(1-2 s) f_{S}=(1-2 \cdot 0,05) \cdot 50=45 \mathrm{~Hz}
$$

- $\quad$ Upper Sideband Harmonic (USH):

$$
f_{U S H}=(1+2 s) f_{S}=(1+2 \cdot 0,05) \cdot 50=55 H z
$$
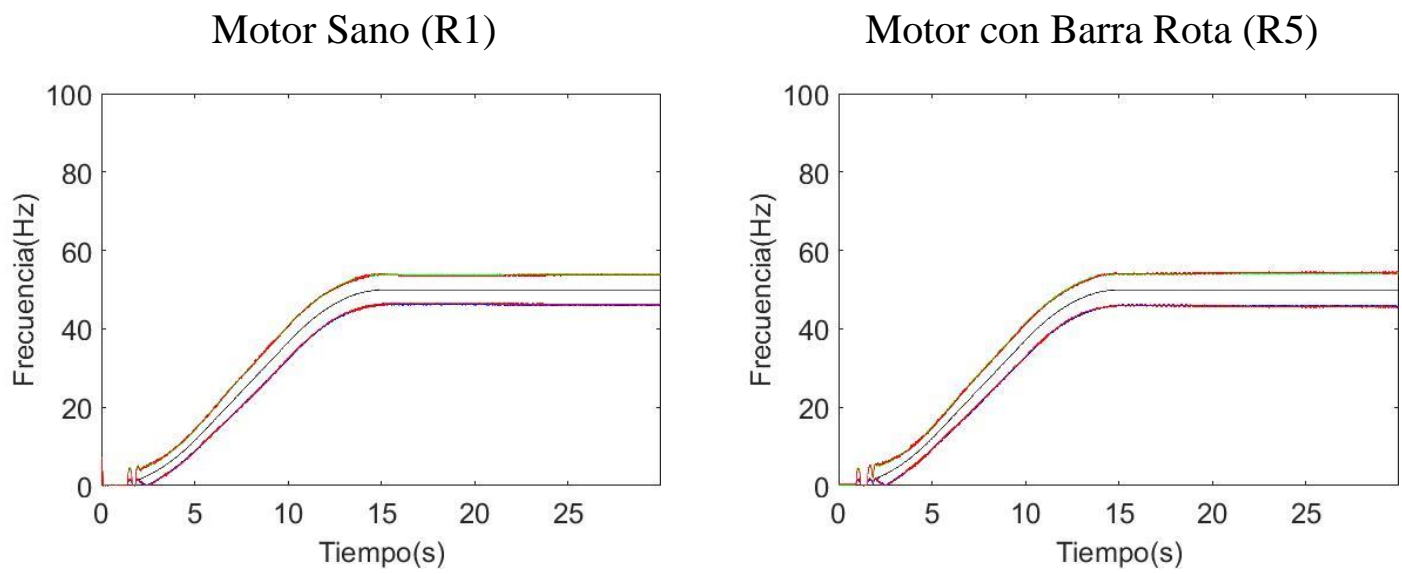

(a) Trayectoria real del primer armónico y teórica de los armónicos de fallo
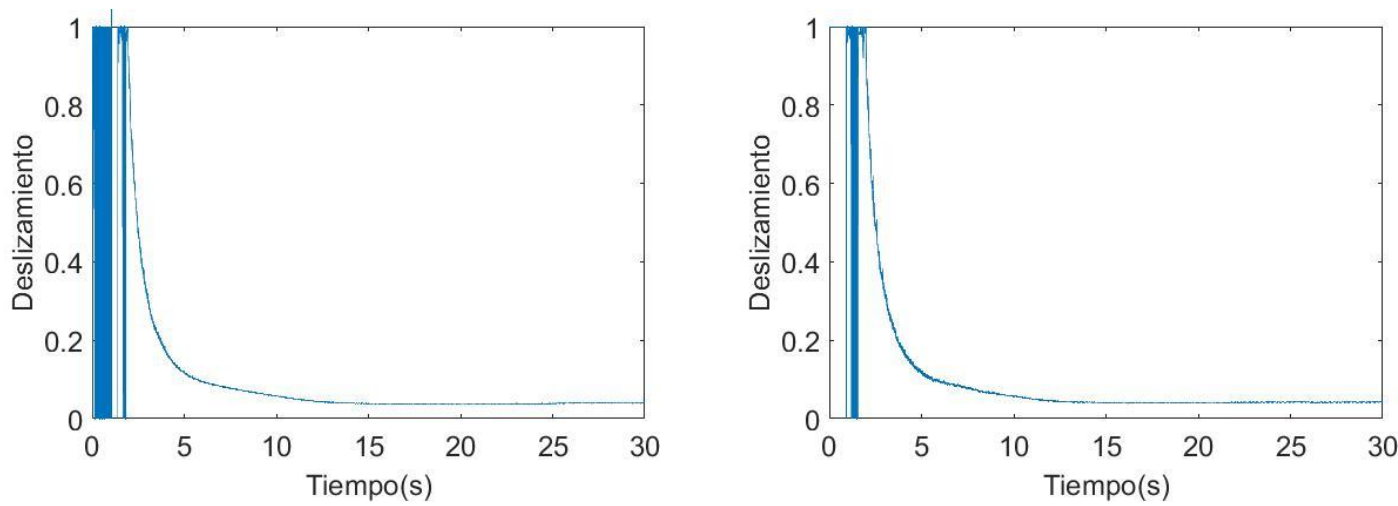

(b) Deslizamiento 

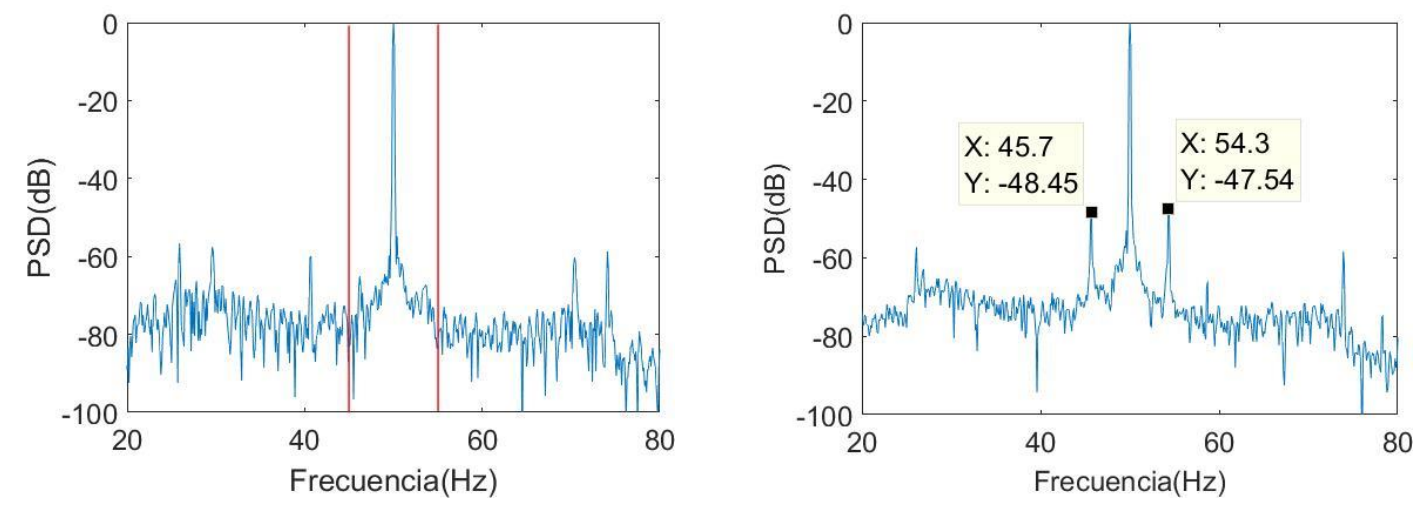

(c) Espectro del estado estacionario
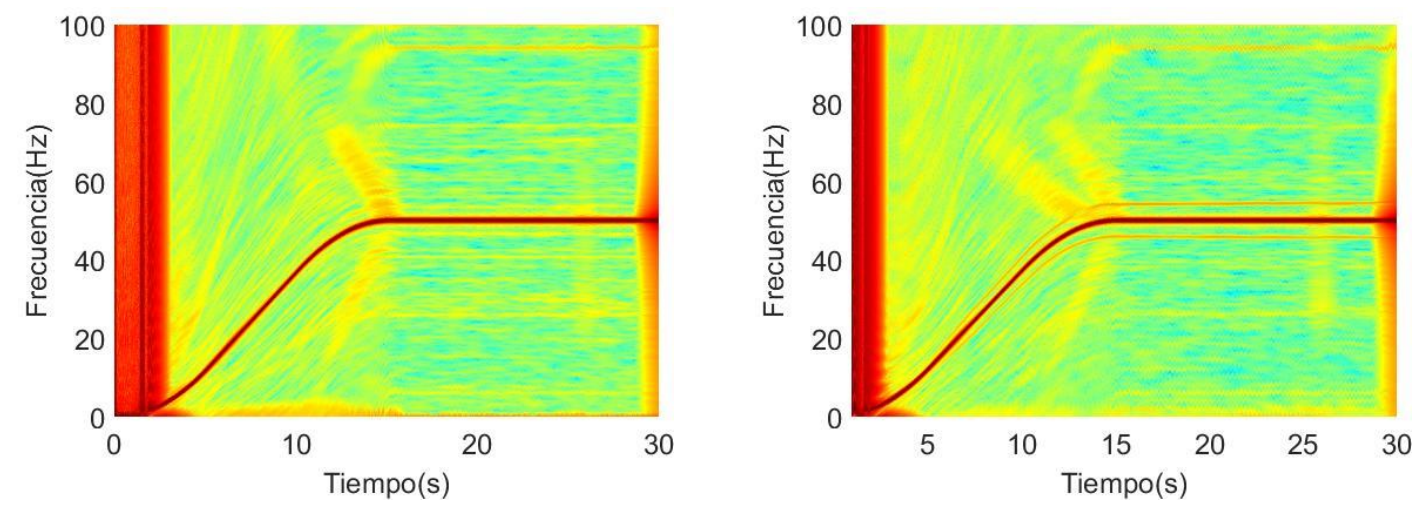

(d) Espectrograma del ensayo
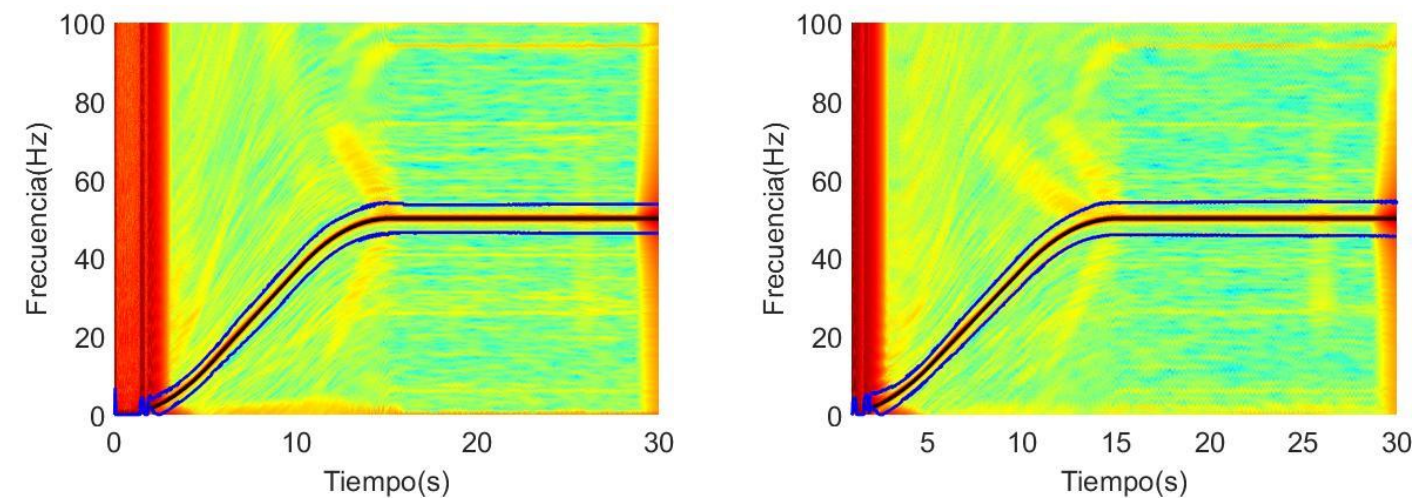

(e) Espectrograma del ensayo y trayectorias de los armónicos de fallo
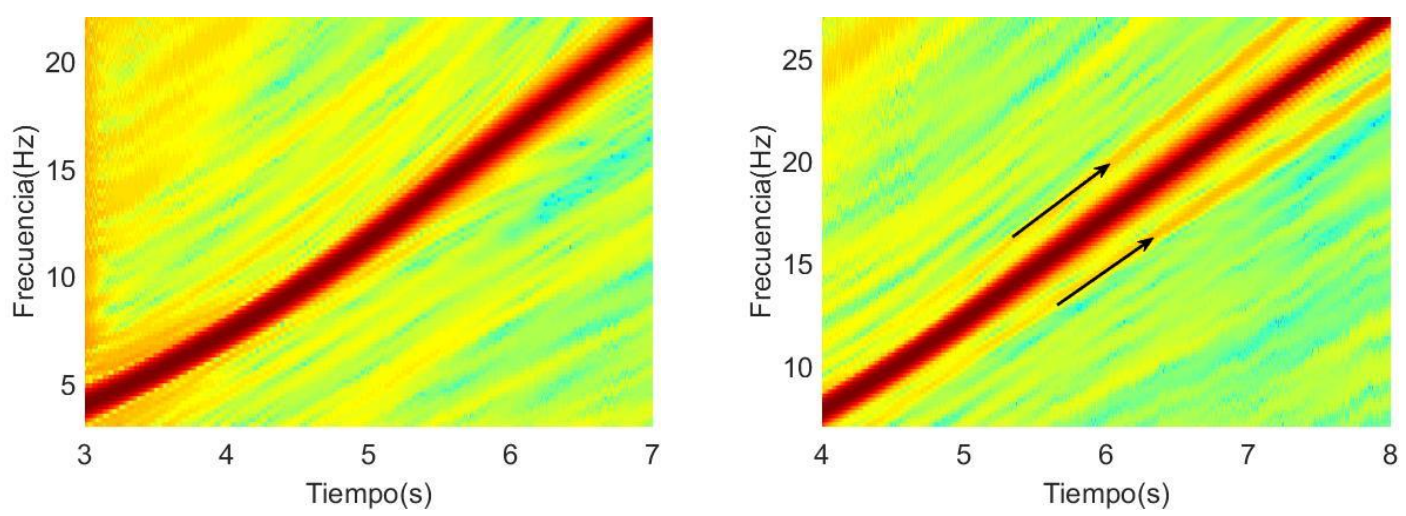

(f) Detalle del espectrograma 

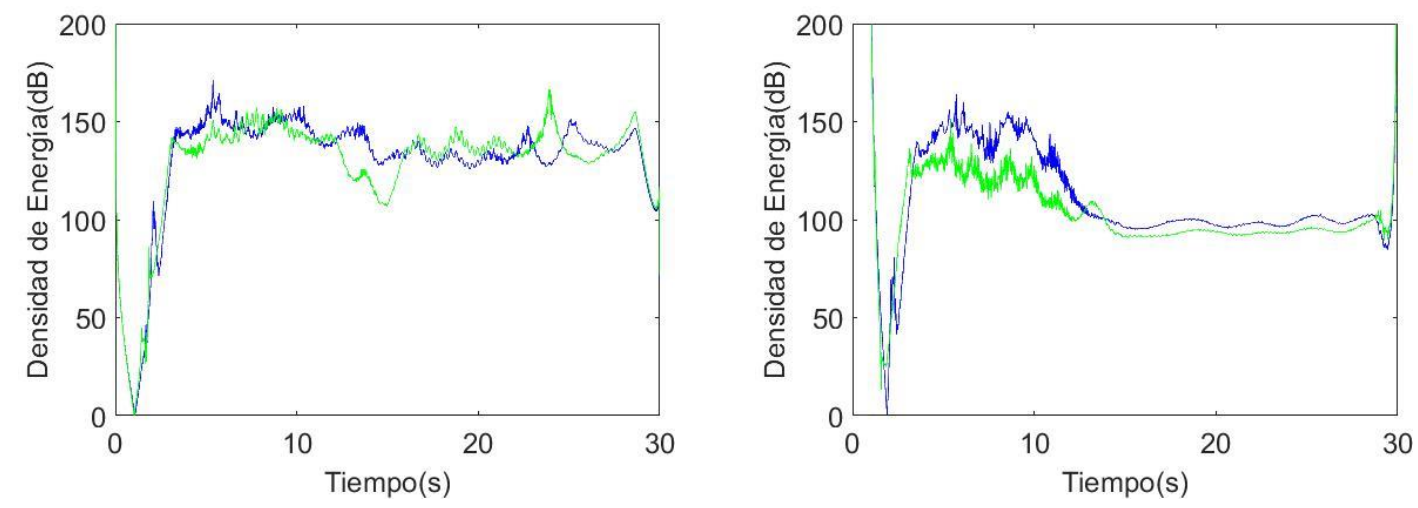

(g) Cuantificación del fallo

Figura 74. Resultados del análisis de la señal de $I M$ alimentado mediante inversor $\mathrm{AB}$ con nivel de carga alto y arranque en $\mathrm{S}$.

Con la Figura 74 (d) se comprueba cómo, efectivamente, la transformada es capaz de seguir un arranque en "S", definiendo, con buena resolución los armónicos de fallo debidos a barra rota, que se corroboran con la Figura 74 (e) y se ve en mayor detalle en la Figura 74 (f), donde se pueden distinguir de manera concisa los armónicos de barras rotas en el estado transitorio.

Para finalizar este apartado, en las Figuras 76 y 77 se presentan dos casos particulares. Estos ensayos analizados tienen por objetivo mostrar las prestaciones de la transformada en otro tipo de transitorios. Quizás no sean representativos de una situación real de funcionamiento, pero sí que imponen unas dificultades especiales para detectar el armónico de fallo. En el primero de ellos (Figura 76), se ha programado un inversor para que produzca una frecuencia de consigna con una oscilación senoidal y manteniendo la carga constante. En el segundo caso, (Figura 77) se consigue una oscilación senoidal tanto de la carga como de la consigna. Con esto se pretende comprobar si la transformada desarrollada es capaz de seguir formas de onda oscilantes.

Para estos ensayos se va a utilizar un variador que no se ha empleado anteriormente. Es un equipo de la marca LS, modelo IG5A (Figura 75), cuyas características se pueden ver en la Tabla 7. 


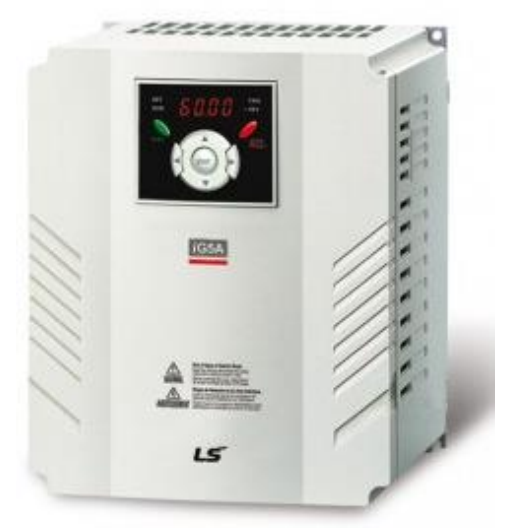

Figura 75. Variador LS, utilizado para ensayos variación carga y consigna.

\section{Variador LS IG5A}

Monofásico 0,4-1,5 kW (200-230 V)

Trifásico 0,4-22 kW (200-230 V)

Trifásico 0,4-22 kW (380-480 V)

Control seleccionable V/f, Vectorial Sensorless.

Algoritmo de control para bombas y ventiladores, (2 motores).

Autoajuste de la frecuencia de corte de los IGBTs.

Selección del tipo de señal de entrada NPN/PNP.

Salida de alimentación a 24 Vcc.

Analógicas de entrada de: $-10+10 ; 0 \ldots 10 \mathrm{Vcc} ; 0$ (4) $20 \mathrm{~mA}$.

Tabla 7. Características variador LS.

Para el primero de los casos (Figura 76), se ha variado la frecuencia de consigna, manteniendo constante la carga. Esto se ha realizado generando una consigna variable con un generador de señales. La frecuencia se varía de $0 \mathrm{~Hz}$ a $5 \mathrm{~Hz}$ con una amplitud que varía, igualmente, de 0 a 5, tal como se recoge en la Tabla 8 . Se han realizado ensayos con el motor sano (R1) y motor con fallo de barras (R5).

\begin{tabular}{|l|c|}
\hline \multicolumn{1}{|c|}{ Frecuencias } & \multicolumn{1}{c|}{ Amplitudes } \\
\hline \hline Frecuencia $0,5 \mathrm{~Hz}$ & 0,$5 ; 2,5 ; 5$ \\
Frecuencia $1 \mathrm{~Hz}$ & 0,$5 ; 2,5 ; 5$ \\
Frecuencia $2 \mathrm{~Hz}$ & 0,$5 ; 2,5 ; 5$ \\
Frecuencia $5 \mathrm{~Hz}$ & 0,$5 ; 2,5 ; 5$ \\
\hline
\end{tabular}

Tabla 8. Ensayos Frecuencia de consigna variable y carga constante.

Para el caso de ensayos a frecuencia de consigna y carga variables, Figura 77, se mantiene la amplitud constante para consigna $+/-1$ y para carga $+/-2$. Quedando las posibles variaciones de la Tabla 9. 


\begin{tabular}{|l|l|}
\hline \multicolumn{1}{|c|}{ Frecuencia Consigna } & Frecuencia carga \\
\hline \hline Frecuencia Consigna 0,5 & 0,$5 ; 1 ; 2$ \\
Frecuencia Consigna 1 & 0,$5 ; 1 ; 2$ \\
Frecuencia Consigna 2 & 0,$5 ; 1 ; 2$ \\
\hline
\end{tabular}

Tabla 9. Valores de frecuencia de consiga y carga variables para los diferentes ensayos.

Para todos los ensayos se ha usado un control V/f lineal, con arranque lineal, llevando a cabo un total de 100 ensayos. Para ilustrar los resultados que se han obtenido con estos ensayos hemos escogido dos casos concretos que a continuación se detallan.

El freno empleado permite introducir una consigna externa para fijar el par. En este caso, también se ha empleado un generador de señales externo para producir una carga oscilante.

En el primer caso (Figura 76), la carga del motor es constante y la consigna de frecuencia es variable. Los datos de estos ensayos son los siguientes (acorde a la Tabla 8):

- Inversor: LS

- Frecuencia: $0,5 \mathrm{~Hz}$

- Amplitud: 0,5

El deslizamiento del motor en la parte estacionaria del ensayo con una barra rota es 0,02. Los valores de las frecuencias de fallo son los siguientes:

- Lower Sideband Harmonic $(L S H)$ :

$$
f_{L S H}=(1-2 s) f_{S}=(1-2 \cdot 0,02) \cdot 50=48 H z
$$

○ Upper Sideband Harmonic (USH):

$$
f_{U S H}=(1+2 s) f_{S}=(1+2 \cdot 0,02) \cdot 50=51,9 H z
$$

Para el caso del motor en estado sano el deslizamiento medido es de 0,021 , con lo que se tiene: 
○ Lower Sideband Harmonic (LSH):

$$
f_{L S H}=(1-2 s) f_{S}=(1-2 \cdot 0,021) \cdot 50=47,9 H z
$$

- Upper Sideband Harmonic (USH):

$$
f_{U S H}=(1+2 s) f_{s}=(1+2 \cdot 0,021) \cdot 50=51,9 \mathrm{~Hz}
$$
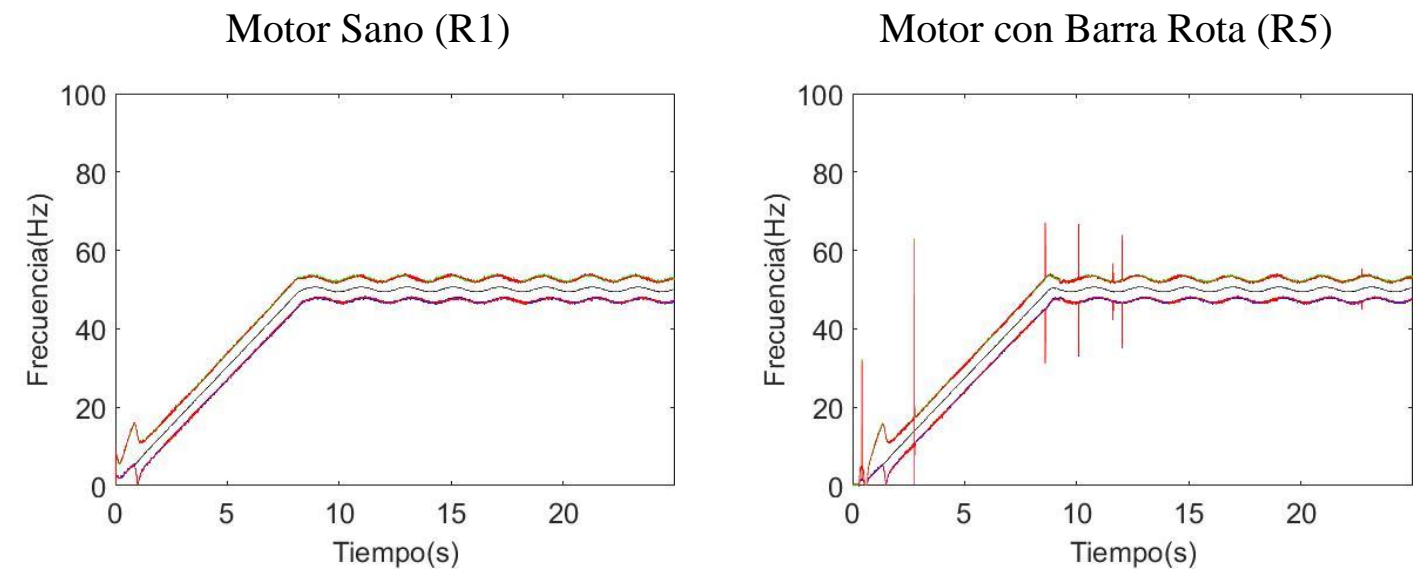

(a) Trayectoria real del primer armónico y teórica de los armónicos de fallo
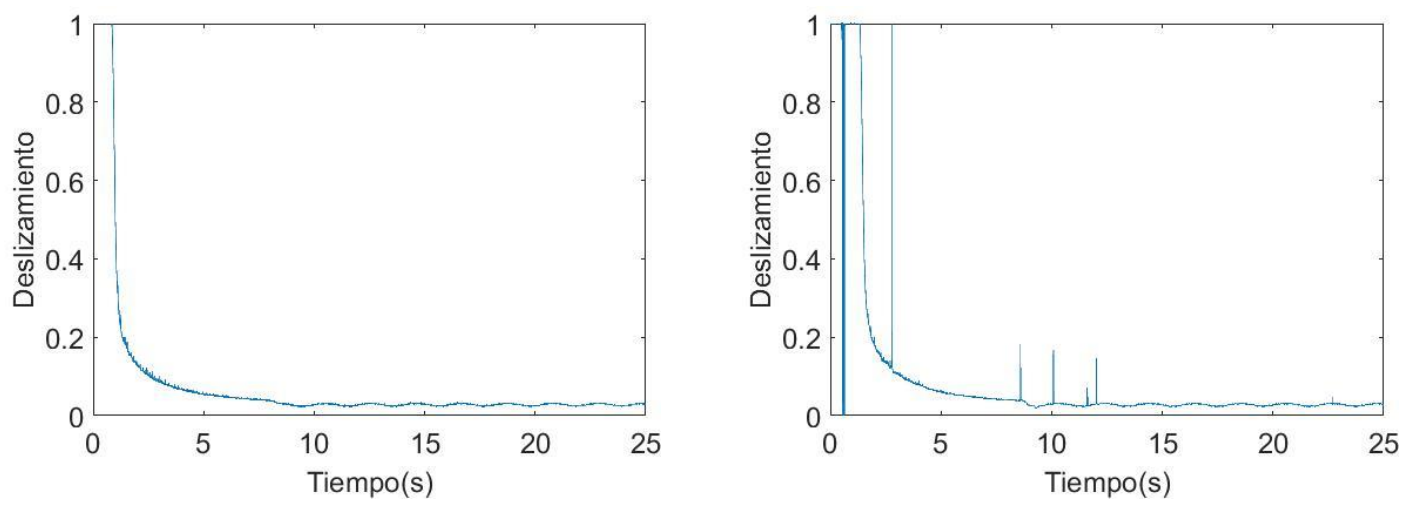

(b) Deslizamiento
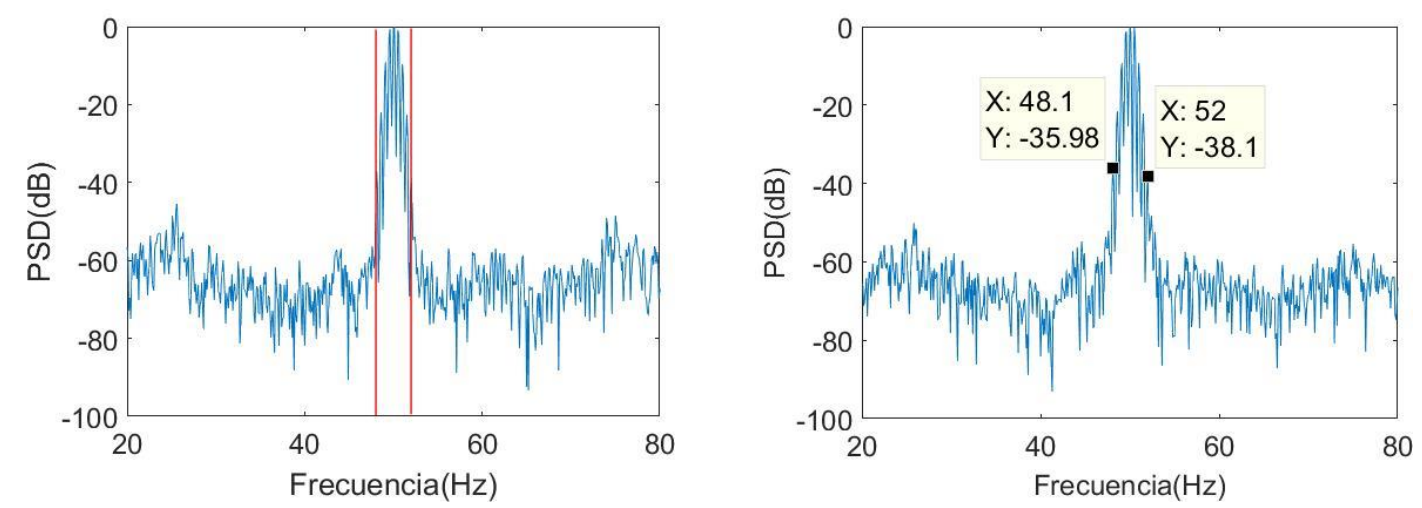

(c) Espectro del estado estacionario 

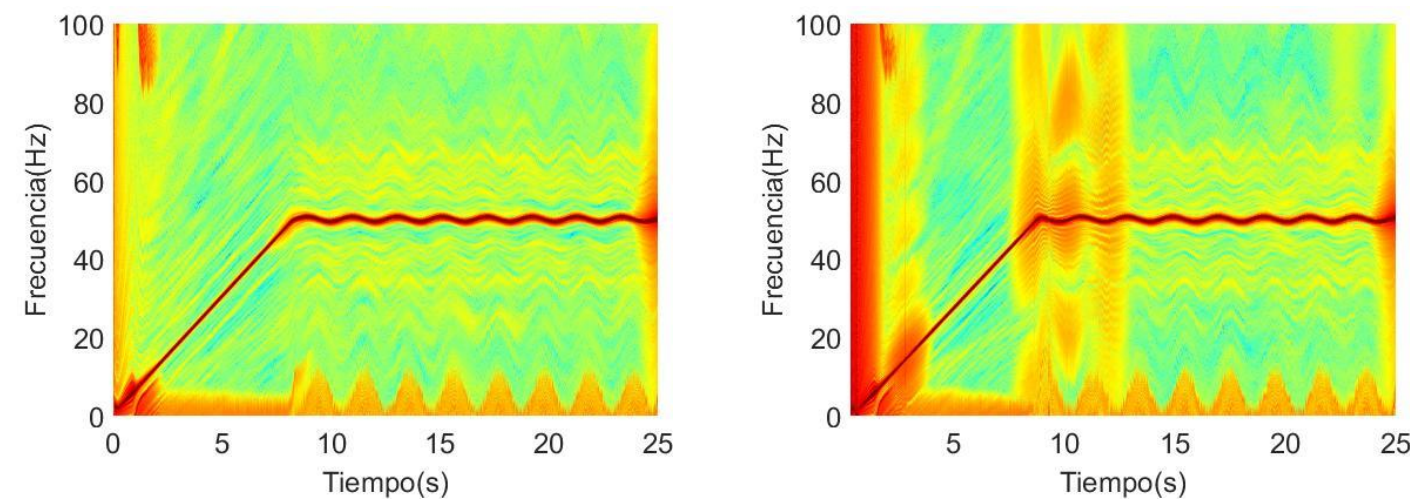

(d) Espectrograma del ensayo
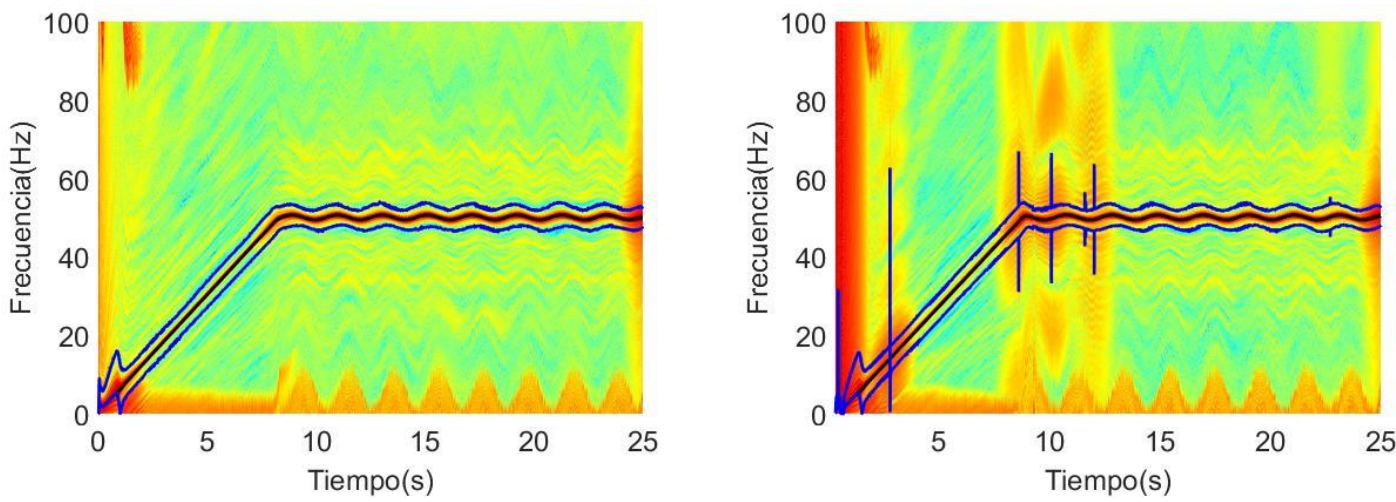

(e) Espectrograma del ensayo y trayectorias de los armónicos de fallo
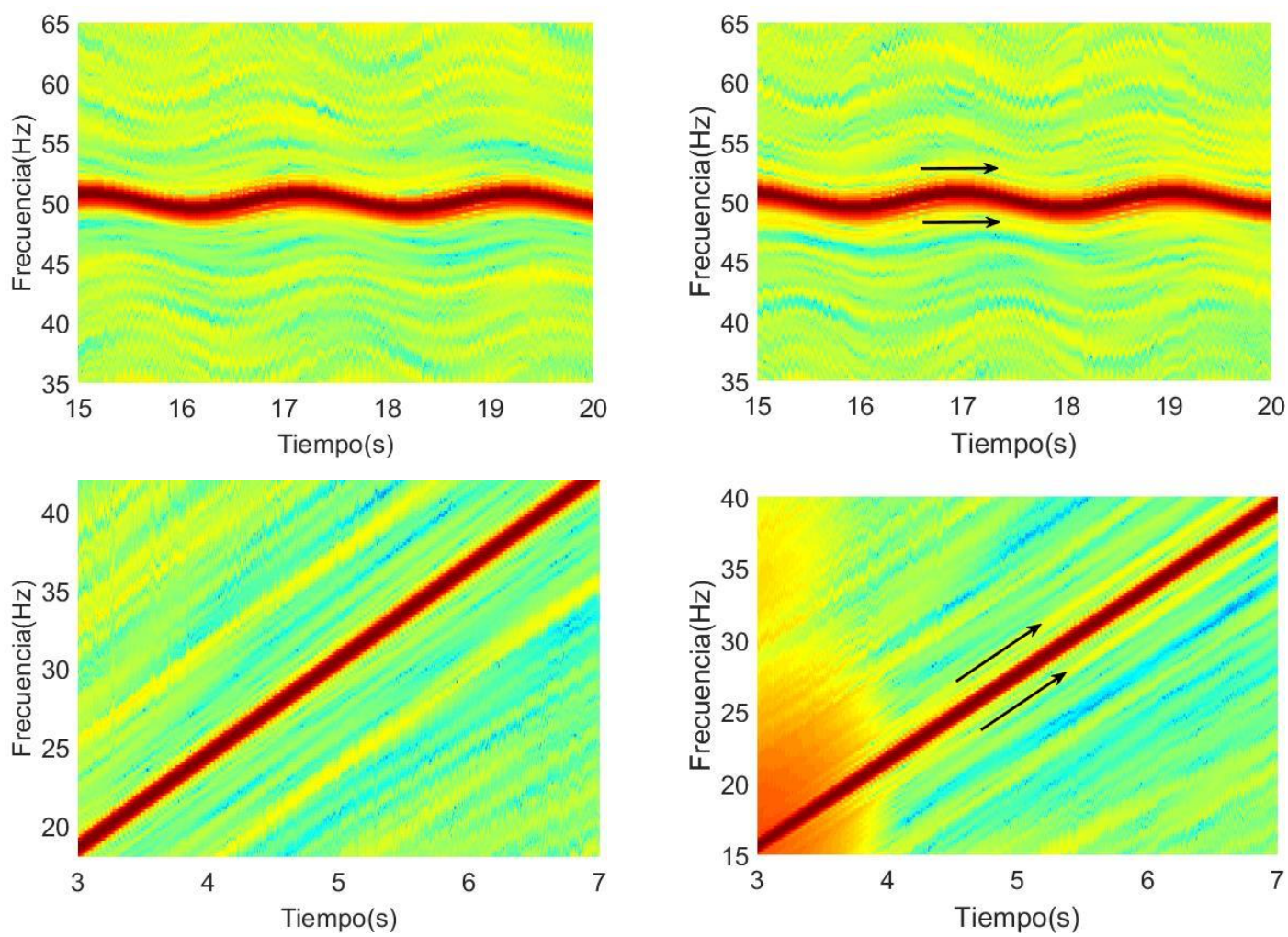

(f) Detalle del espectrograma 

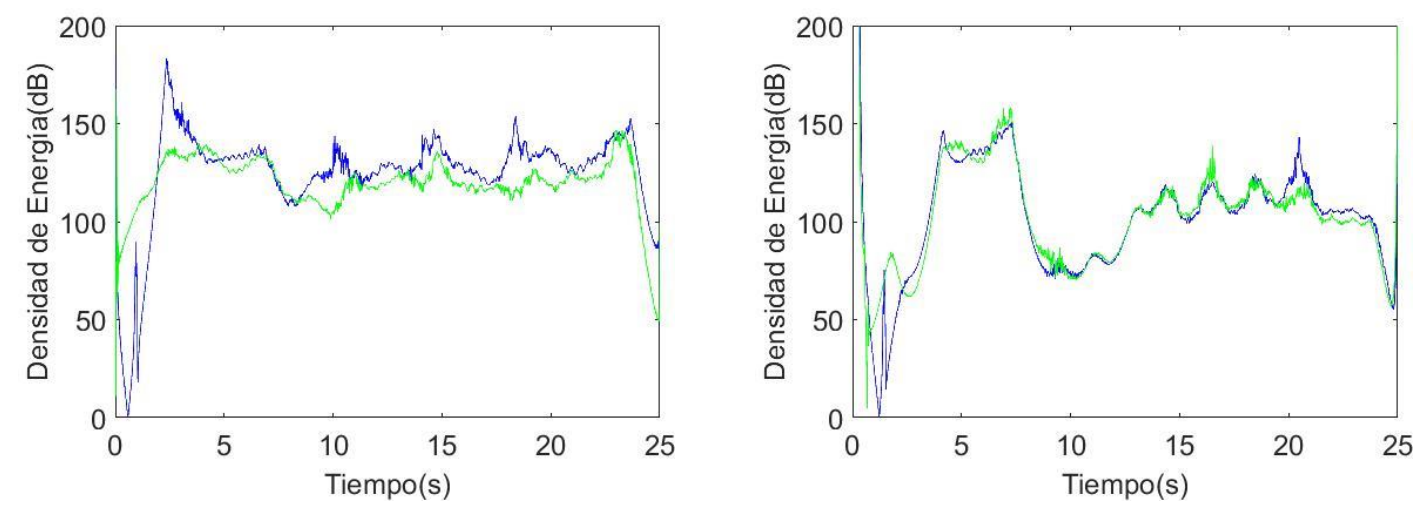

(g) Cuantificación del fallo

Figura 76. Resultados del análisis de la señal de $I M$ alimentado mediante inversor LS con consigna variable.

Por último, la Figura 77, ilustra un ensayo obtenido para frecuencia de consigna y carga variables. Se ilustra con el siguiente caso:

- Inversor: LS

- Frecuencia: $2 \mathrm{~Hz}$

- Amplitud: 2

El deslizamiento del motor en el estado estacionario en el ensayo con fallo por barra rota es 0,02 , siendo así los valores de frecuencias teóricas de fallo:

○ Lower Sideband Harmonic $(L S H)$ :

$$
f_{L S H}=(1-2 s) f_{S}=(1-2 \cdot 0,02) \cdot 50=48 H z
$$

○ Upper Sideband Harmonic (USH):

$$
f_{U S H}=(1+2 s) f_{s}=(1+2 \cdot 0,02) \cdot 50=52 \mathrm{~Hz}
$$

Cuando el motor se encuentra en estado sano, presenta un deslizamiento de 0,02, por tanto, las frecuencias teóricas de fallo tienen los siguientes valores:

○ Lower Sideband Harmonic $(L S H)$ :

$$
f_{L S H}=(1-2 s) f_{S}=(1-2 \cdot 0,022) \cdot 50=48 H z
$$

- Upper Sideband Harmonic $(U S H)$ :

$$
f_{U S H}=(1+2 s) f_{S}=(1+2 \cdot 0,022) \cdot 50=52 H z
$$



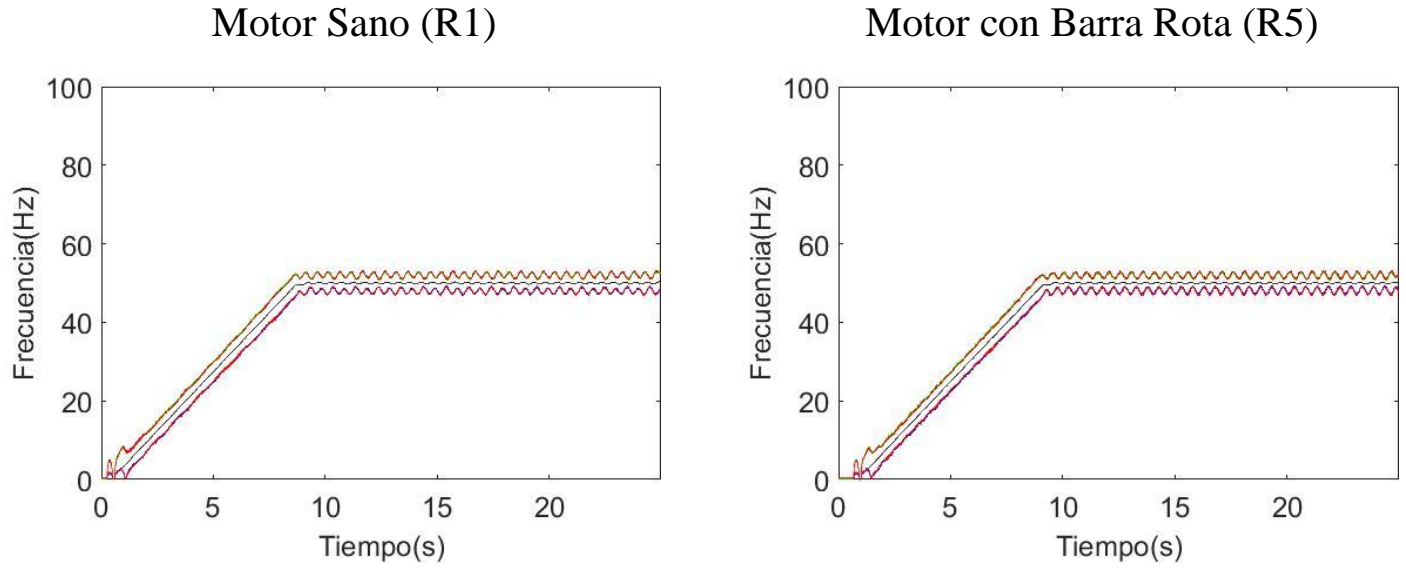

(a) Trayectoria real del primer armónico y teórica de los armónicos de fallo
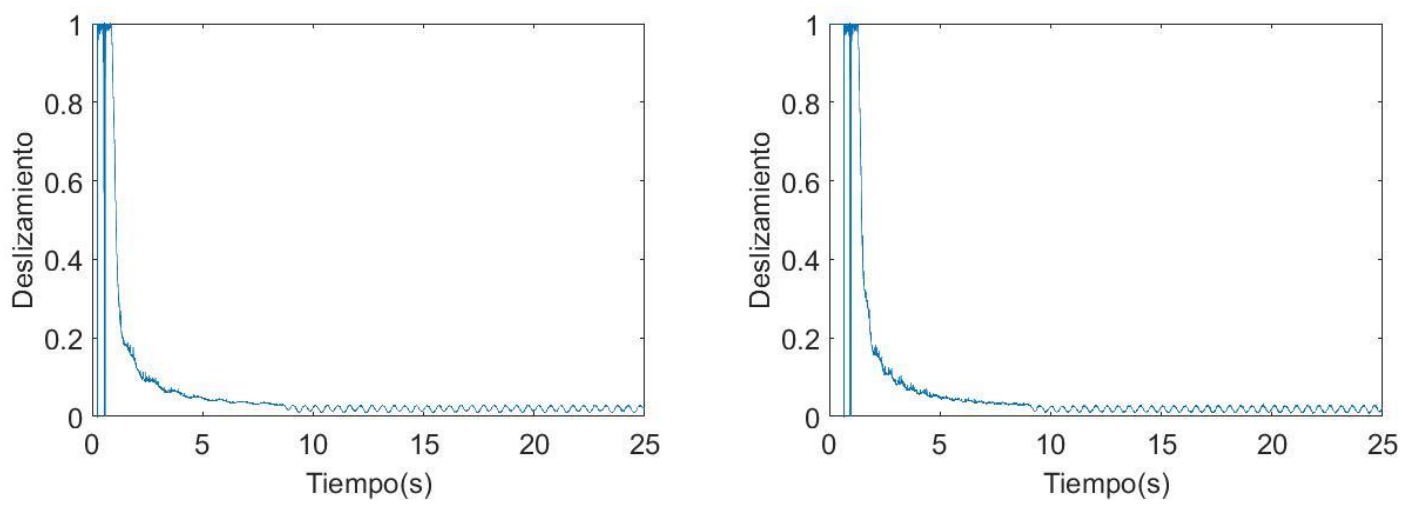

(b) Deslizamiento
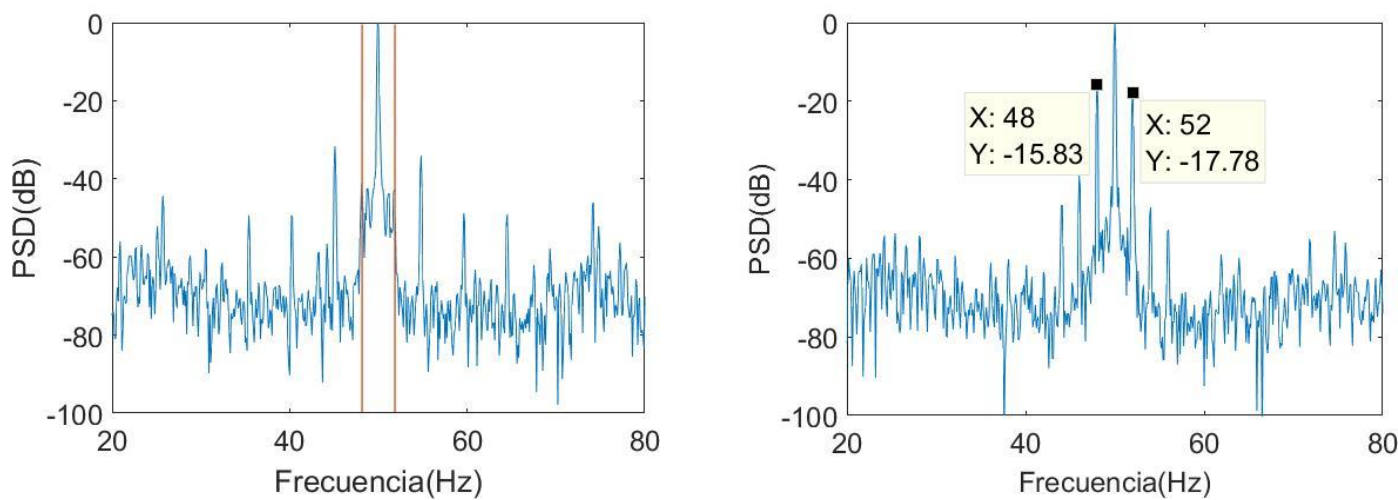

(c) Espectro del estado estacionario
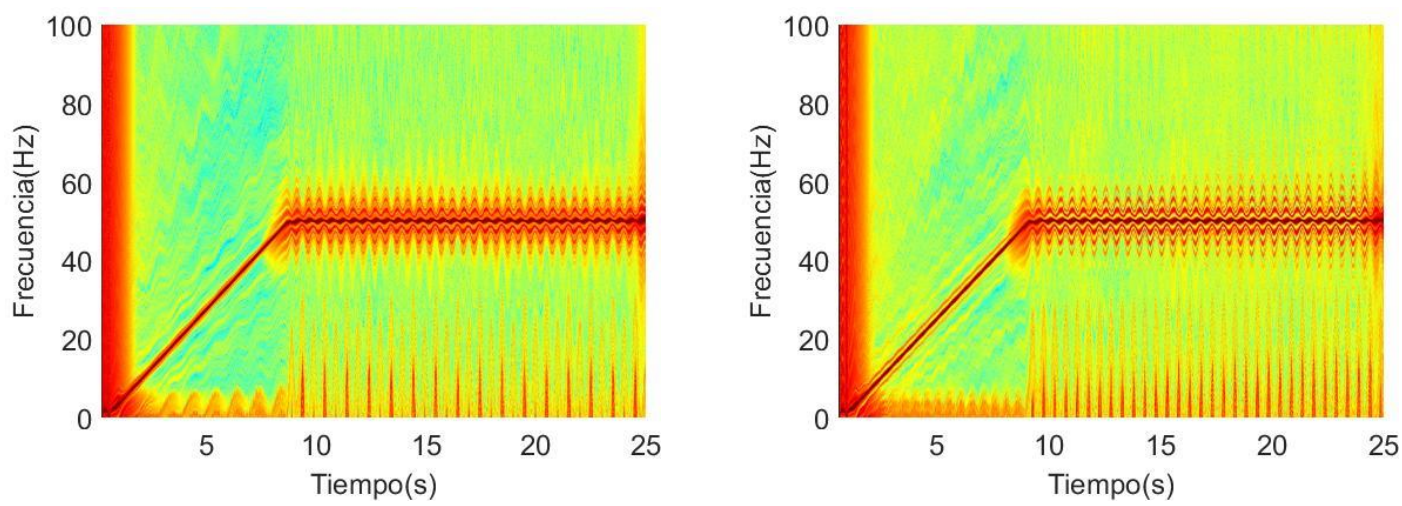

(d) Espectrograma del ensayo 

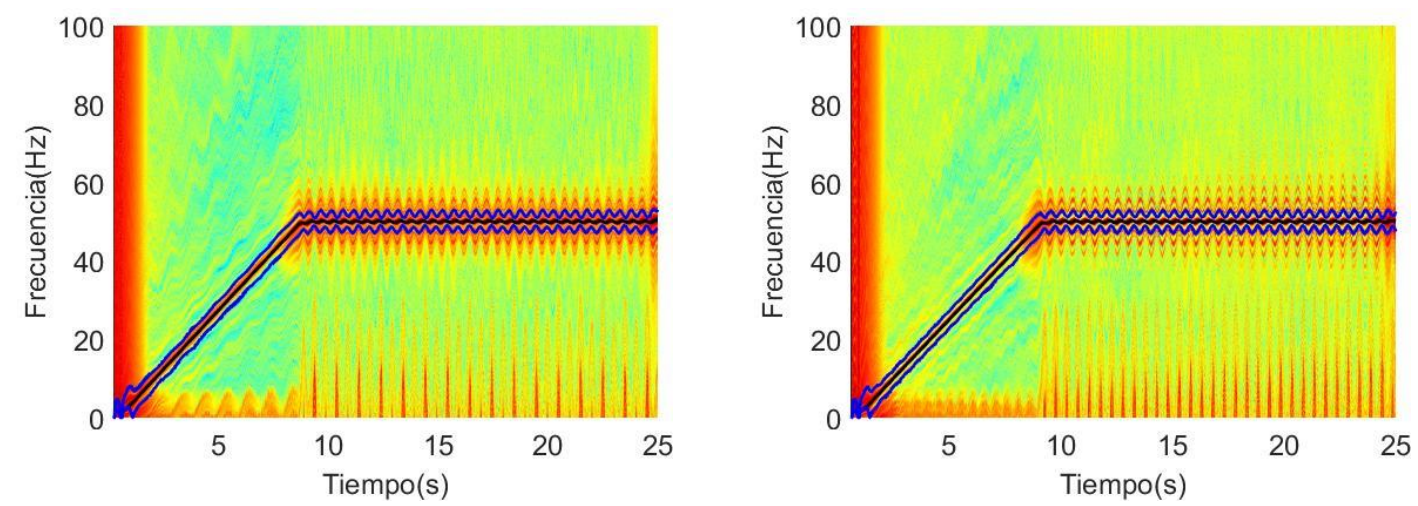

(e) Espectrograma del ensayo y trayectorias de los armónicos de fallo
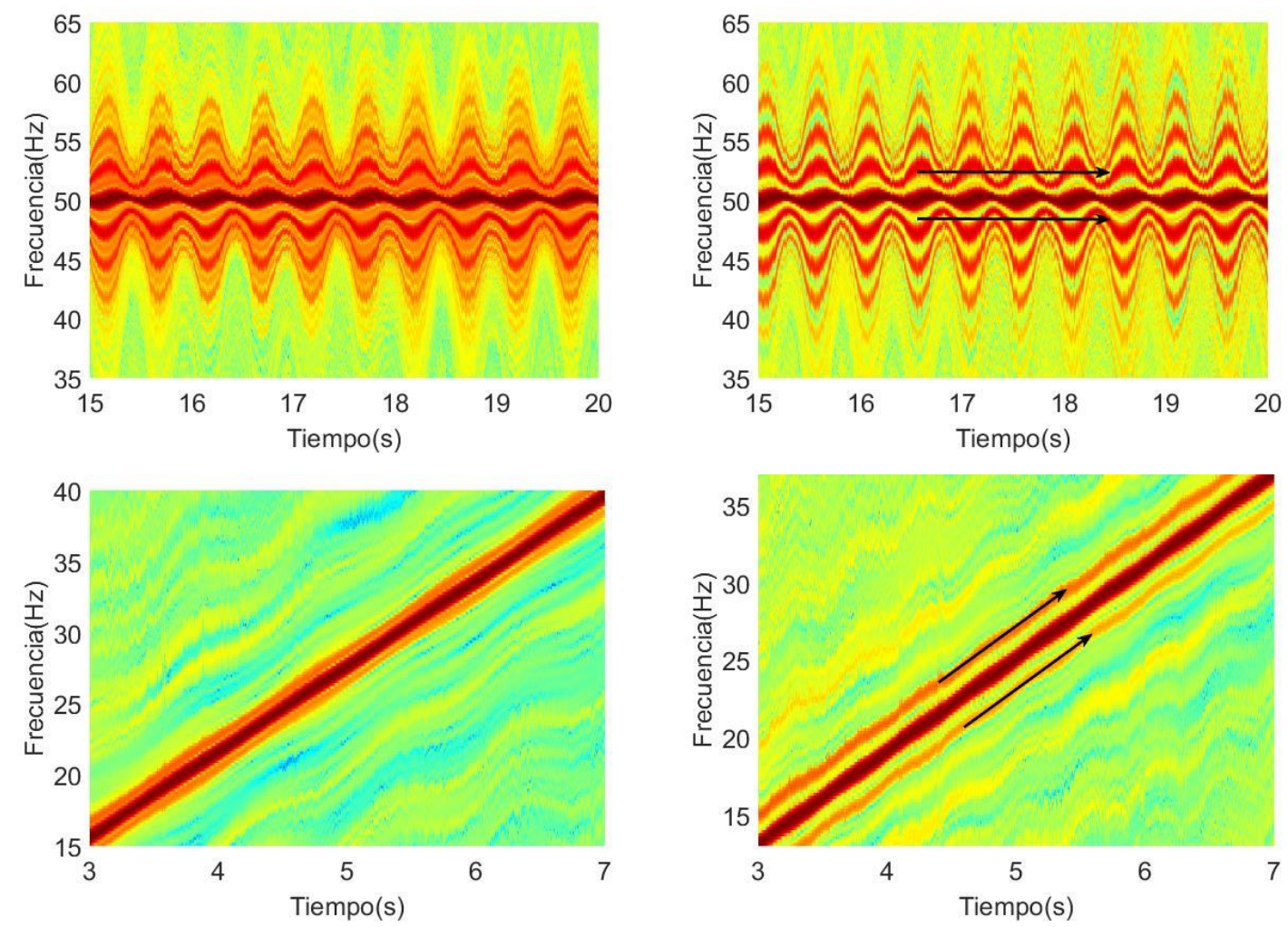

(f) Detalle del espectrograma
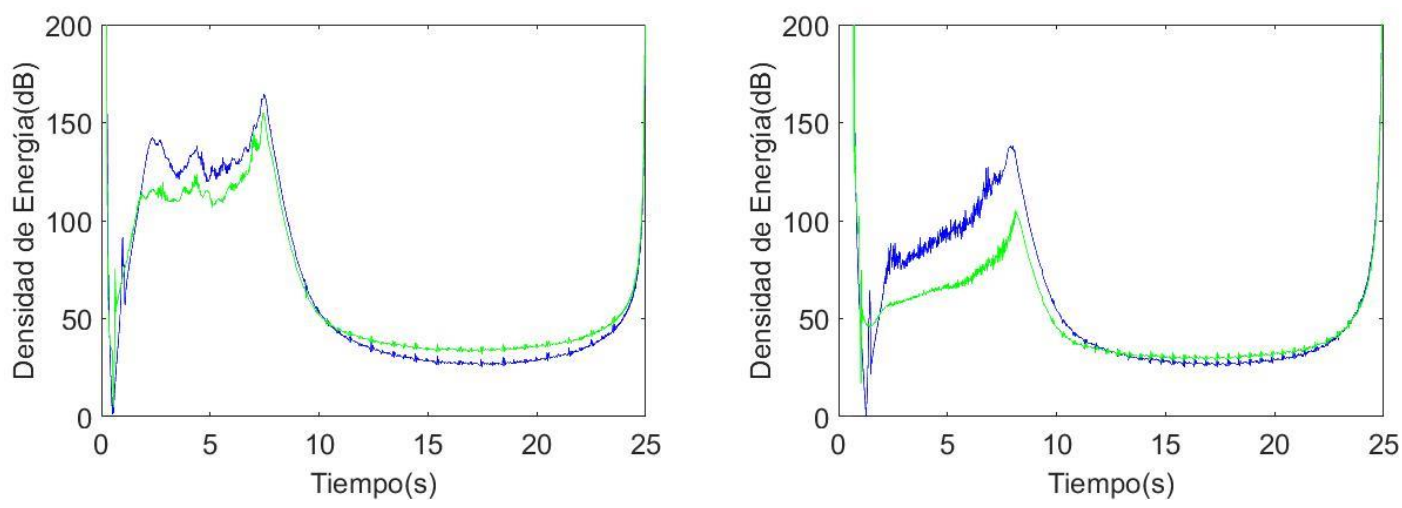

(g) Cuantificación del fallo

Figura 77. Resultados del análisis de la señal de $I M$ alimentado mediante inversor LS con consigna y carga variables. 
Al observar los resultados obtenidos en la Figura 77 (f), se puede apreciar como aparece un nuevo armónico, tanto en el caso del $I M$ sano como en el caso del motor de fallo, dicho armónico es paralelo al fallo por barra rota. Así en este tipo de ensayos en los que no solo es oscilante la consigna, si no que oscila otra variable más, en este caso la carga, se debe de tener especial cuidado al realizar el análisis de los resultados obtenidos. Este análisis puede dar lugar a un error de diagnóstico al confundir este nuevo armónico con un posible fallo de barras, puesto que ambos armónicos son paralelos, aunque presentan distinta amplitud respecto de la componente fundamental. Este nuevo armónico es debido a la oscilación de la frecuencia, que provoca que en el espectrograma resultante se aprecie una nueva evolución que no aparecía cuando esta oscilación no estaba presente.

Con lo cual se puede comprobar la potencialidad de la técnica que nos permite seguir la trayectoria de los armónicos de fallo, con cualquier tipo de señal, incluso si esta es oscilatoria.

\subsection{Detección de excentricidad mixta durante el arranque}

Existen dos tipos de excentricidad, dinámica y estática. En la práctica, estas dos excentricidades aparecen juntas, presentando el motor la denominada excentricidad mixta. Esta excentricidad se da cuando los ejes del motor se encuentran desalineados, sin coincidir ninguno de ellos.

En el desarrollo de la experimentación, para simular el efecto de la rotura de la barra, el motor era desmontado para efectuar la perforación de la jaula. Posteriormente y después de realizar otra vez el ensamblado de la máquina, el motor era montado en el banco de ensayos y acoplado al freno. Este acoplamiento en algunas ocasiones presentaba un cierto desalineamiento entre el eje del motor y del freno que se manifestó en un cierto nivel de excentricidad mixta. Se han aprovechado estos ensayos para probar las prestaciones de la técnica desarrollada en la detección de esta falta. Aquí la dificultad no estriba en la proximidad de los armónicos de fallo a la componente fundamental, como ocurre en el fallo de barras. Como se ha visto en el apartado 2, la trayectoria de los armónicos de excentricidad mixta se aleja del armónico principal, por lo que, en principio, 
no habría problema de resolución en tiempo-frecuencia para distinguirlos. Aquí la dificultad es que la familia de átomos se adapte a las diferentes pendientes de las trayectorias de los armónicos de fallo en cualquier punto del plano.

Para ilustrar los resultados obtenidos del análisis de estos ensayos se procederá de manera análoga a como se ha llevado a cabo en el apartado 6.2. de esta memoria.

Así comenzamos con un ensayo en donde el motor es alimentado mediante inversor Allen-Bradley, con nivel alto de carga, para ilustrar los resultados que se obtienen de aplicar esta técnica en el caso en que el motor presenta excentricidad mixta (Figura 78).

En el primer caso, que se ha introducido, Figura 78, se muestran los resultados del análisis del siguiente ensayo:

- Inversor: Allen-Bradley

- $\quad$ Nivel de carga y deslizamiento: Alto

- Tipo de control: V/f lineal

- Tipo de arranque: lineal

En la Figura 78, se observa como la técnica es capaz de seguir, además de la componente fundamental, las componentes debidas a la excentricidad mixta. El resto de componentes son introducidos por el variador, no correspondiendo con ningún armónico de fallo, tal como se puede ver en la Figura 78 (d), en donde se presenta el espectrograma resultante de aplicar la técnica a esta señal. Esto se puede comprobar de manera más concreta en la Figura 78 (f) en donde se presentan dos zooms, uno de la parte estacionaria y otro de la parte en régimen transitorio.

Para poder calcular las trayectorias teóricas de fallo de los armónicos provocados por excentricidad mixta (Figura 78 (a)) es necesario fijarse en el deslizamiento del motor, que en el estado estacionario presenta un valor de 0,036 (Figura 78 (b)) para el motor que presenta excentricidad y de 0,038 para el motor sano. Al igual que en los casos anteriores, se han escogido ensayos con deslizamiento similar para poder realizar las comparaciones. 
Con este deslizamiento se pueden obtener, de manera teórica, las siguientes frecuencias de fallo para el estado estacionario y para los armónicos asociados a excentricidad mixta, tal como se ha introducido en el punto 2 de esta memoria:

○ $\operatorname{ERH}(-)$

$$
f_{E R H n}=\left(1-\left(\frac{1-s}{p}\right)\right) f_{s}=\left(1-\left(\frac{1-0,036}{2}\right)\right) 50=25,9
$$

○ $\operatorname{ERH}(+)$ :

$$
f_{\text {ERHp }}=\left(1+\left(\frac{1-s}{p}\right)\right) f_{s}=\left(1+\left(\frac{1-0,036}{2}\right)\right) 50=74,1
$$

En el caso del motor sano, las frecuencias teóricas serían:

○ $\operatorname{ERH}(-)$ :

$$
\begin{aligned}
& f_{E R H n}=\left(1-\left(\frac{1-s}{p}\right)\right) f_{s}=\left(1-\left(\frac{1-0,038}{2}\right)\right) 50=25,95 \\
& \quad E R H(+): \\
& f_{E R H p}=\left(1+\left(\frac{1-s}{p}\right)\right) f_{s}=\left(1+\left(\frac{1-0,038}{2}\right)\right) 50=74,05
\end{aligned}
$$

Para corroborar que efectivamente existe esta excentricidad y que la técnica no está dando resultados erróneos, se analiza el estacionario de la señal, mediante la transformada Rápida de Fourier. Así, en la Figura 78 (c) se puede apreciar que efectivamente aparecen armónicos debidos a excentricidad, que corresponden con los calculados de manera teórica. 

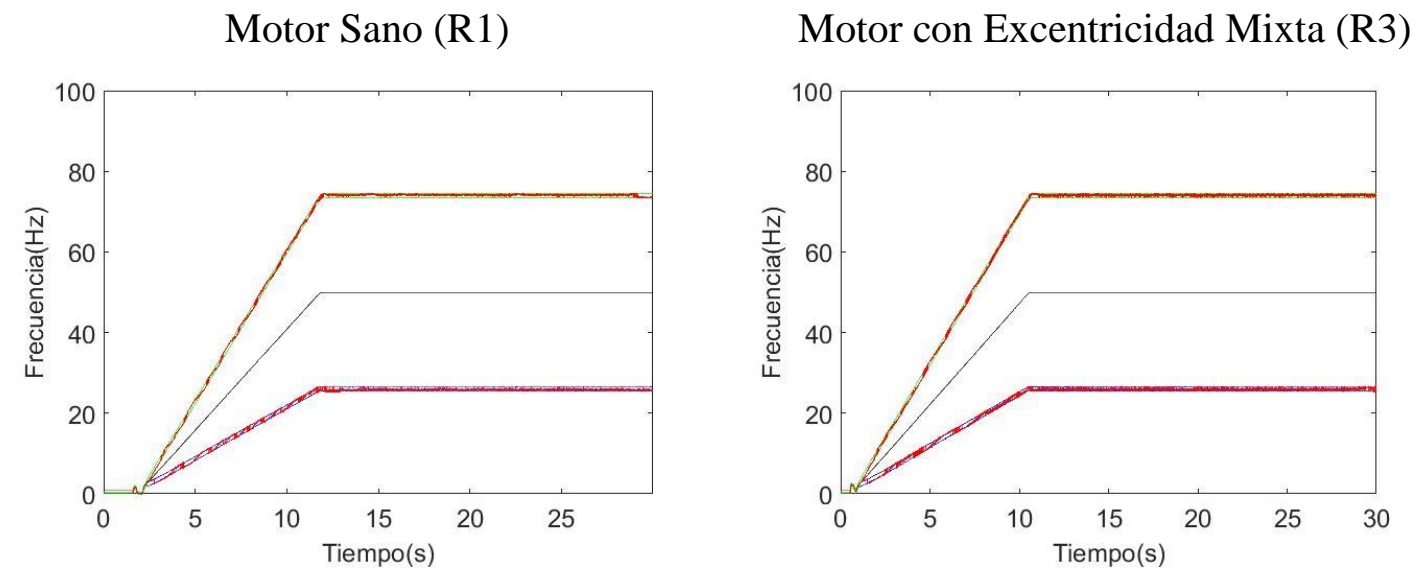

(a) Trayectoria real del primer armónico y teórica de los armónicos de fallo
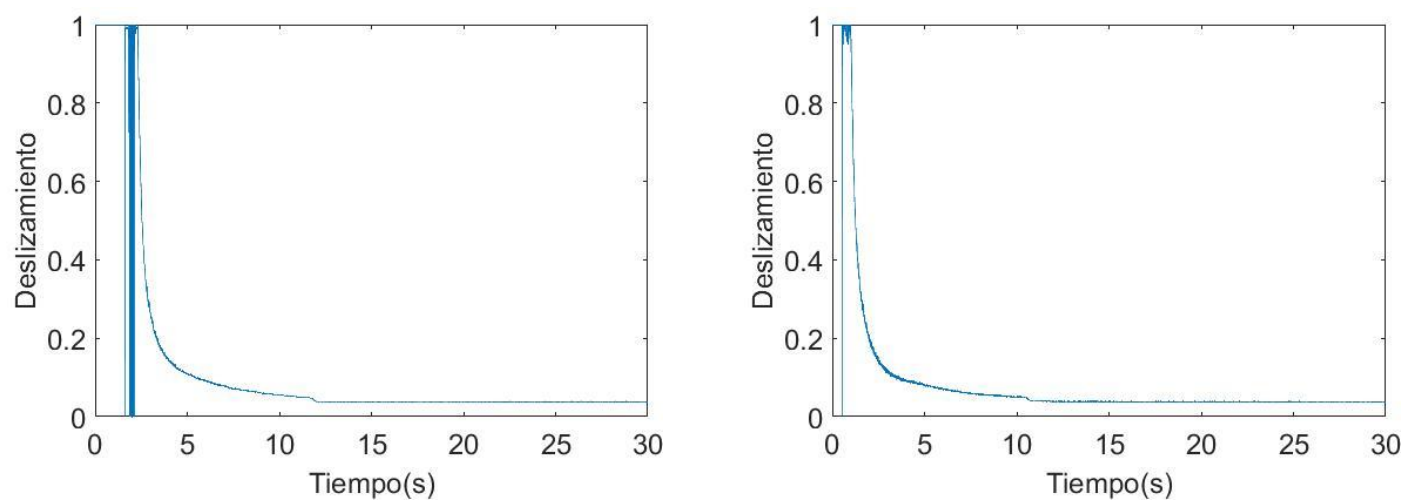

(b) Deslizamiento
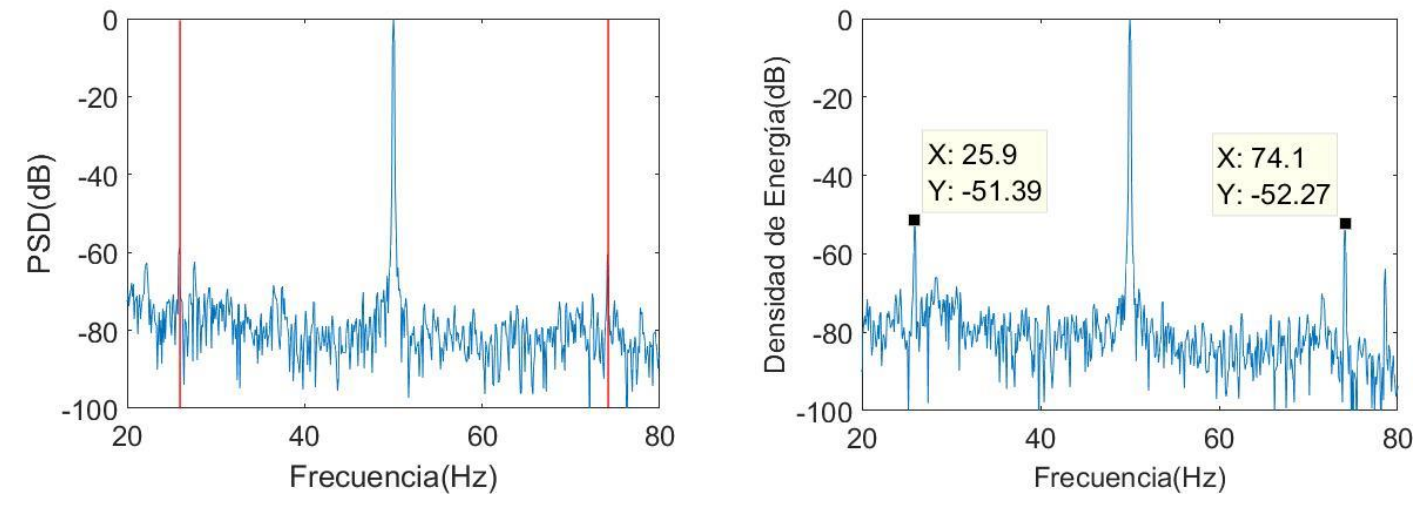

(c) Espectro del estado estacionario 

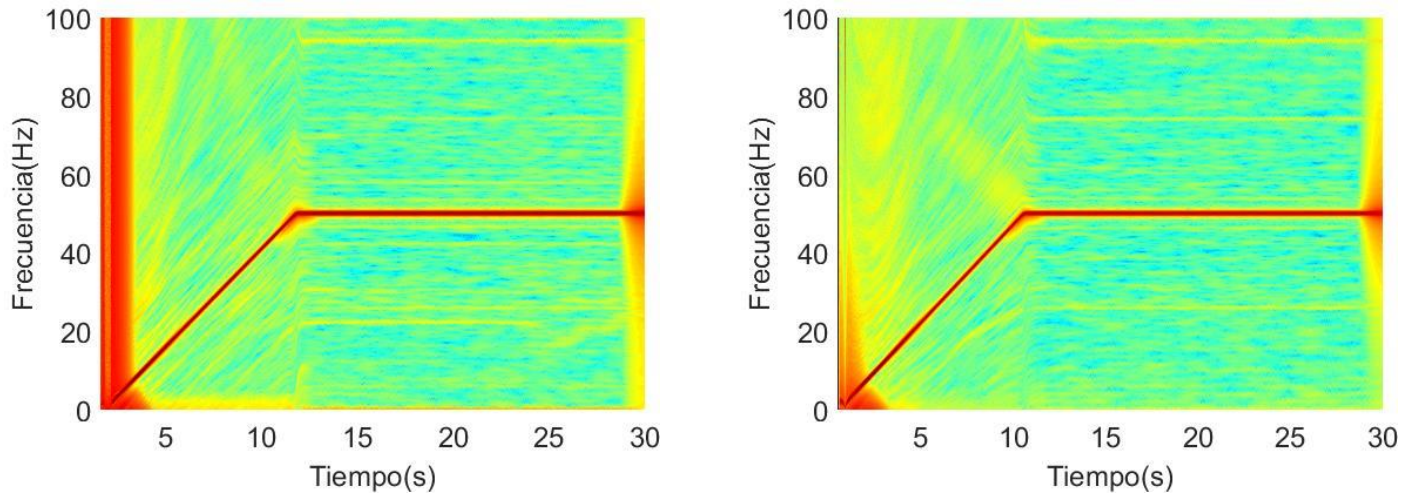

(d) Espectrograma del ensayo
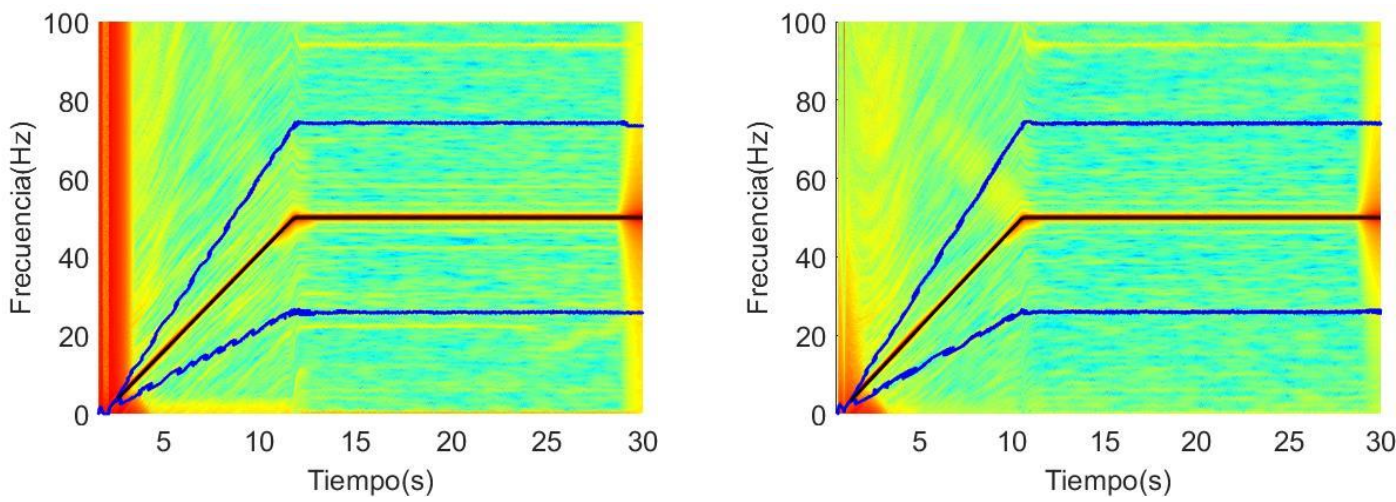

(e) Espectrograma del ensayo y trayectorias de los armónicos de fallo
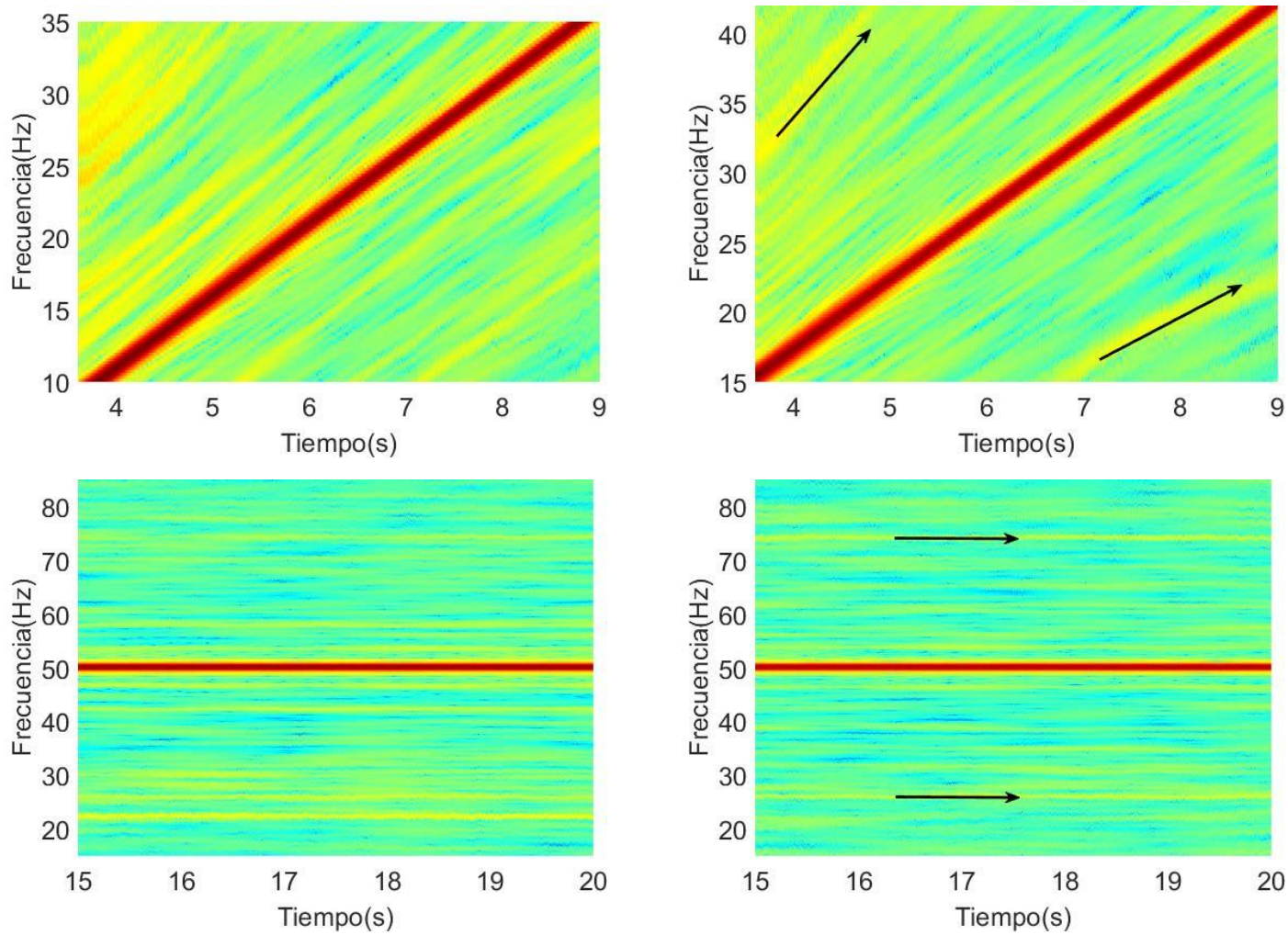

(f) Detalle del espectrograma

Figura 78. Resultados del análisis de la señal de $I M$ alimentado mediante inversor AB con nivel de carga alto. 
En las Figuras 79 y 80 se ilustran dos ejemplos más de ensayos en los que el motor presenta excentricidad mixta, con otros inversores, para así, corroborar que efectivamente la técnica es capaz de detectar este tipo de fallo.

En la Figura 79 se muestran los resultados del análisis del siguiente ensayo:

- Inversor: $\mathrm{ABB}$

- $\quad$ Nivel de carga y deslizamiento: Alto

- $\quad$ Tipo de control: V/f lineal

- $\quad$ Tipo de arranque: lineal

El $I M$ con excentricidad mixta presenta un deslizamiento de 0,04 en el estado estacionario final. Con este deslizamiento se pueden obtener, de manera teórica, las siguientes frecuencias de fallo para los armónicos asociados a excentricidad mixta, que serán:

○ $\operatorname{ERH}(-)$

$$
f_{\text {ERHn }}=\left(1-\left(\frac{1-s}{p}\right)\right) f_{s}=\left(1-\left(\frac{1-0,04}{2}\right)\right) 50=26
$$

○ $E R H(+)$ :

$$
f_{\text {ERHp }}=\left(1+\left(\frac{1-s}{p}\right)\right) f_{s}=\left(1+\left(\frac{1-0,04}{2}\right)\right) 50=74
$$

En el caso del motor sano, el deslizamiento presenta el valor de 0,037 , por tanto, las frecuencias teóricas serían:

○ $\operatorname{ERH}(-)$ :

$$
f_{\text {ERHn }}=\left(1-\left(\frac{1-s}{p}\right)\right) f_{s}=\left(1-\left(\frac{1-0,037}{2}\right)\right) 50=25,925
$$

○ $\operatorname{ERH}(+)$ :

$$
f_{\text {ERHp }}=\left(1+\left(\frac{1-s}{p}\right)\right) f_{s}=\left(1+\left(\frac{1-0,037}{2}\right)\right) 50=74,075
$$



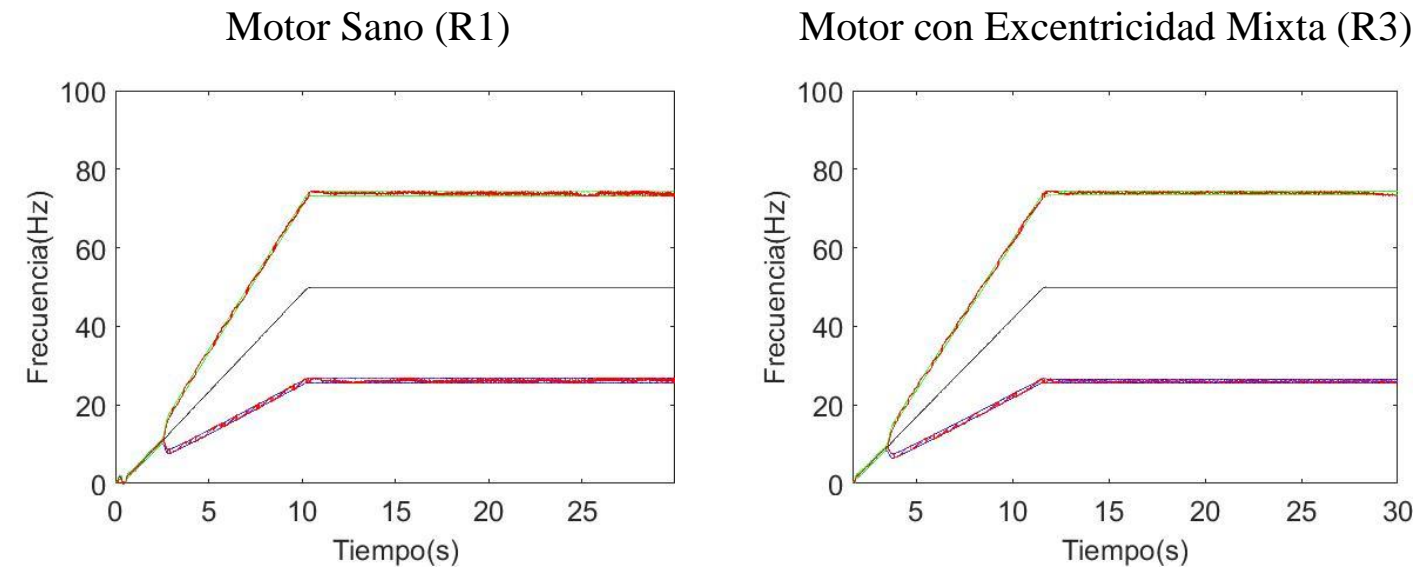

(a) Trayectoria real del primer armónico y teórica de los armónicos de fallo
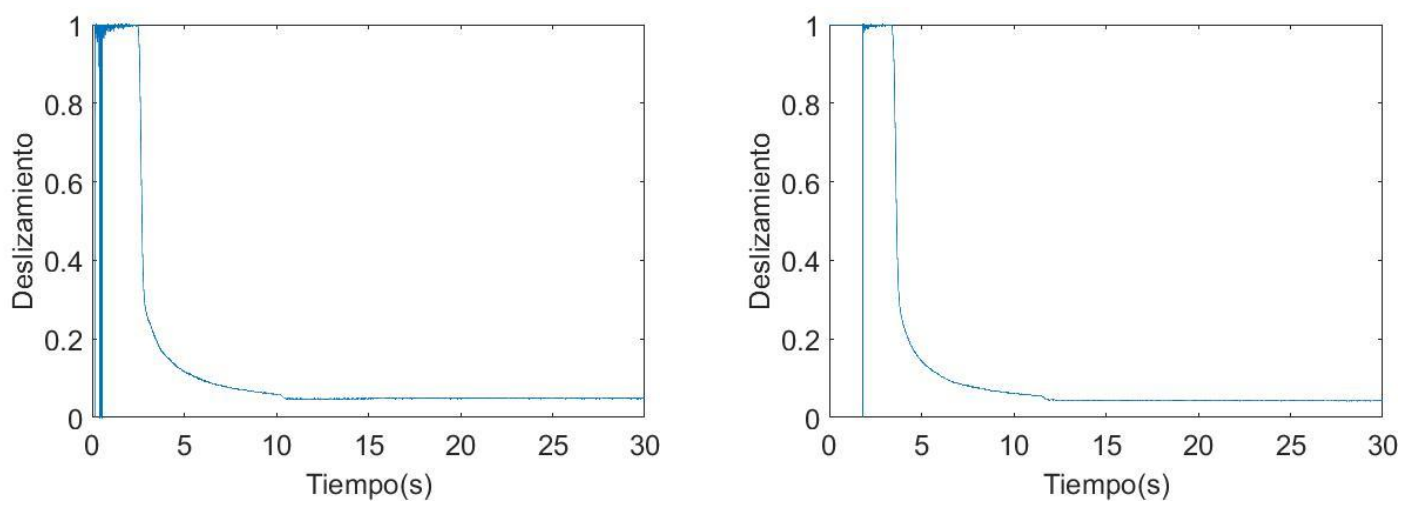

(b) Deslizamiento
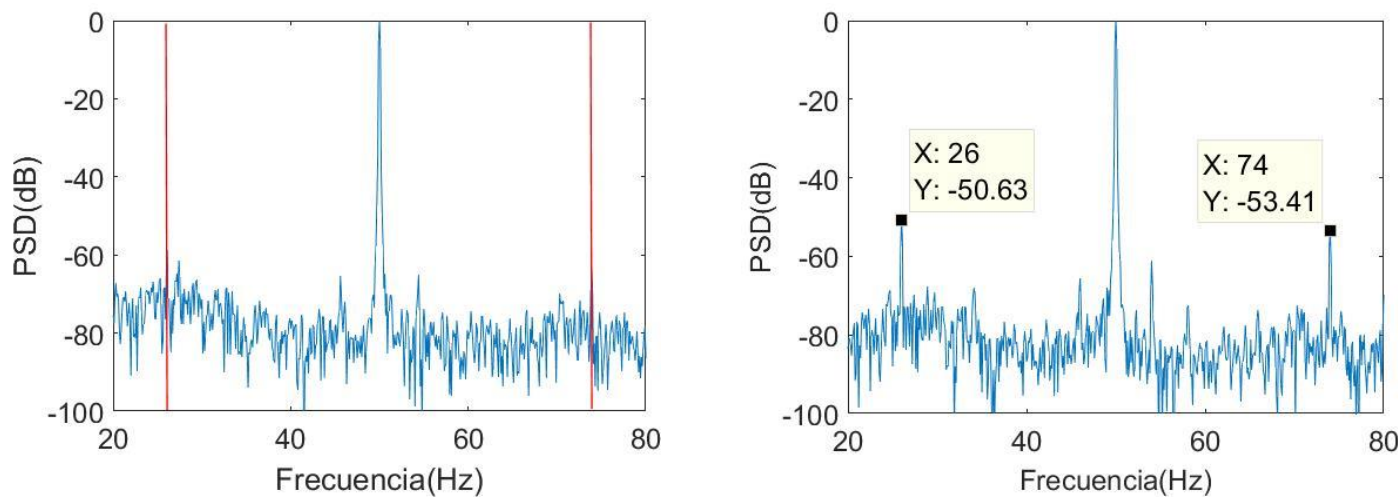

(c) Espectro del estado estacionario
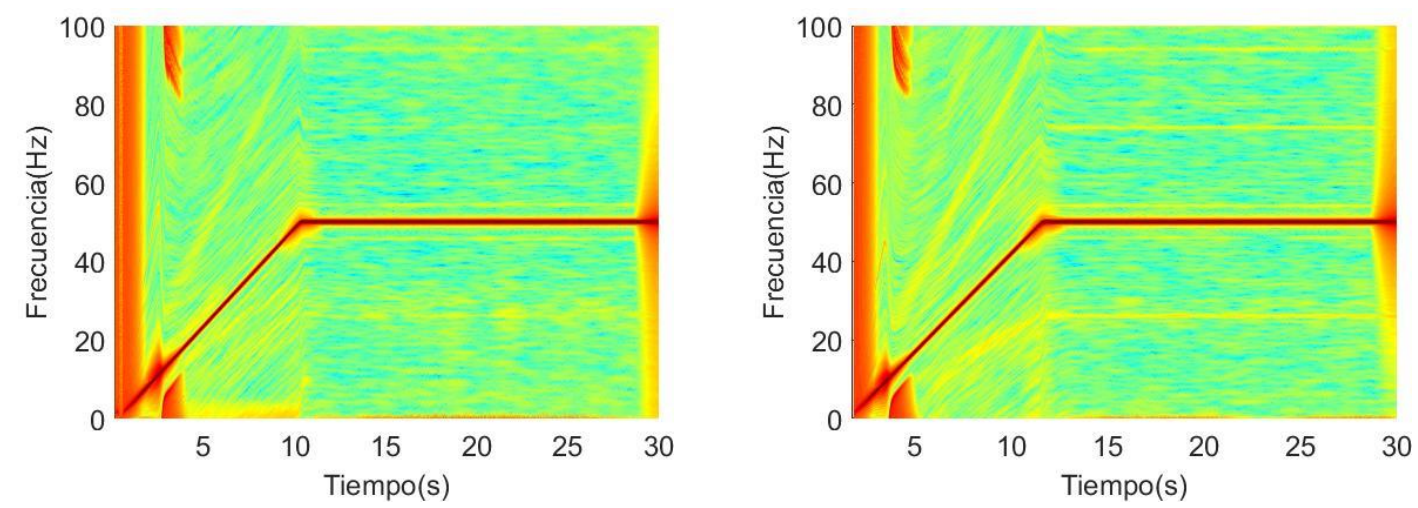

(d) Espectrograma del ensayo 

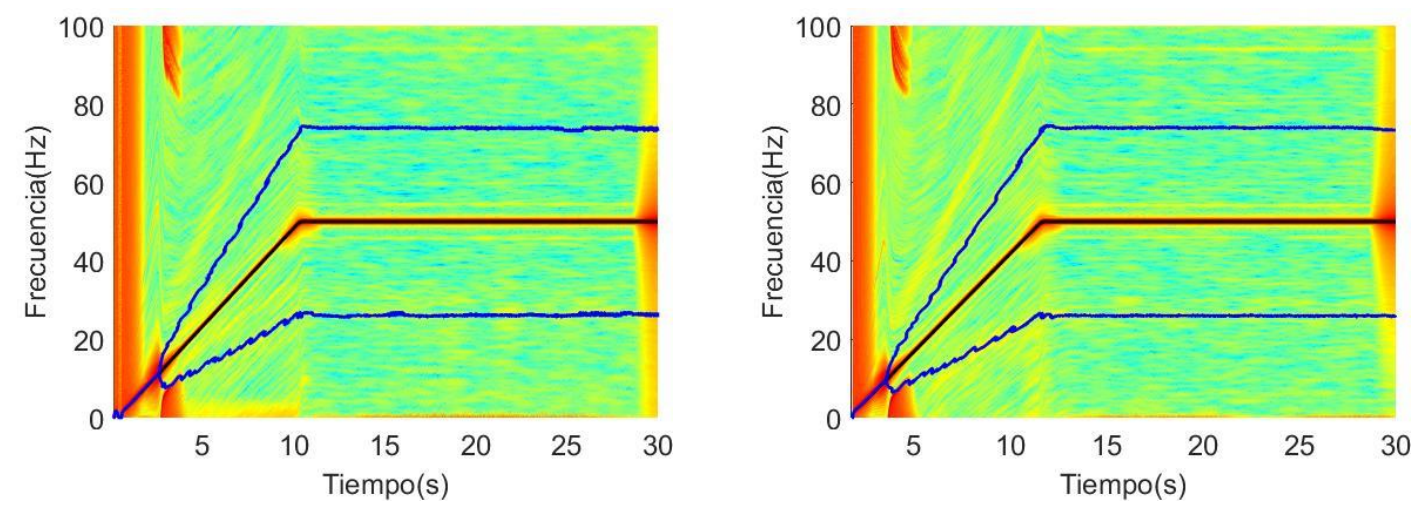

(e) Espectrograma del ensayo y trayectorias de los armónicos de fallo
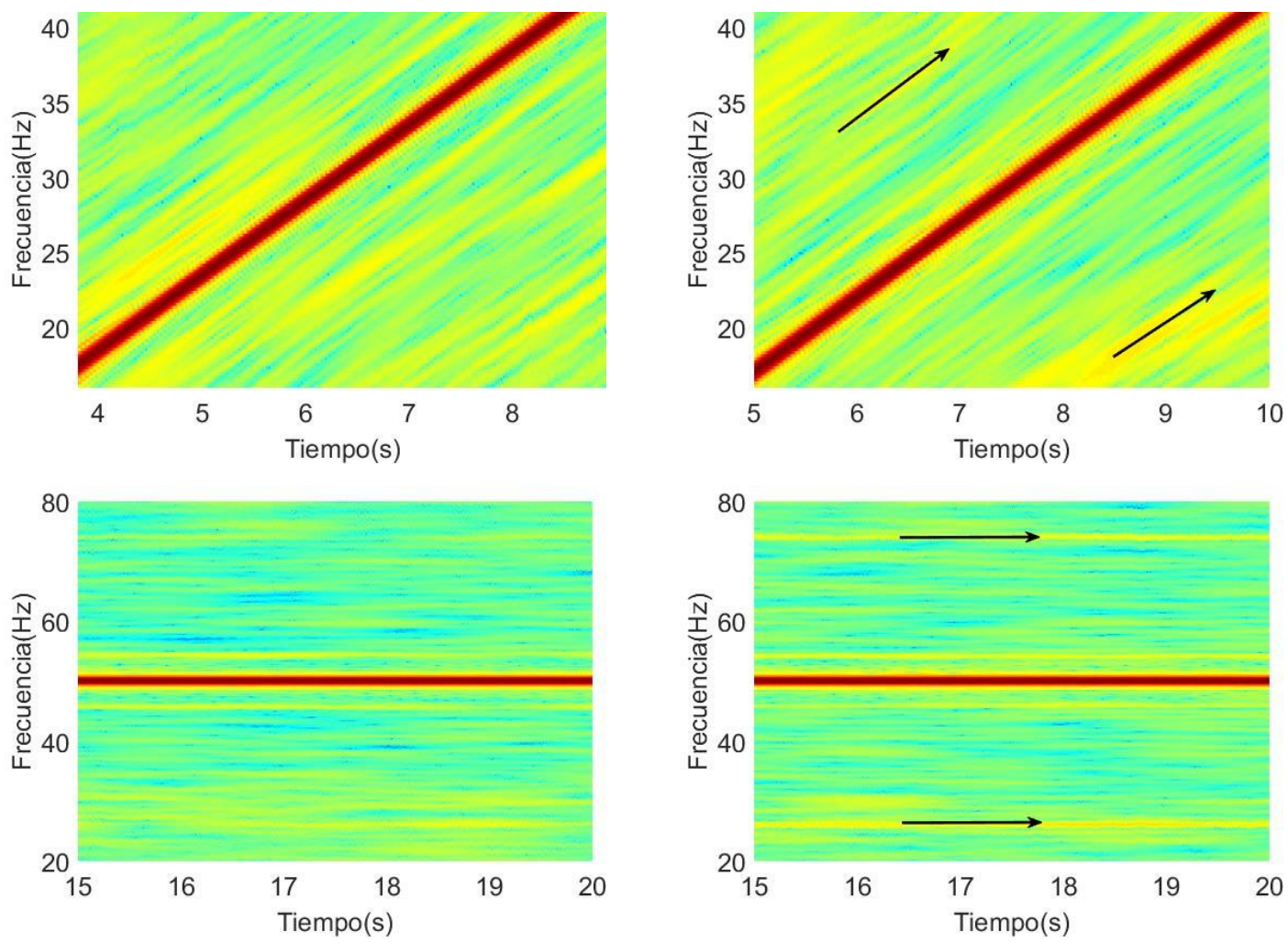

(f) Detalle del espectrograma

Figura 79. Resultados del análisis de la señal de $I M$ alimentado mediante inversor ABB con nivel de carga alto.

La Figura 80 presenta el caso del ensayo realizado con el inversor Siemens, con un nivel de carga bajo. Las características de este ensayo son las siguientes:

- Inversor: Siemens

- Nivel de carga y deslizamiento: Bajo

- Tipo de control: V/f lineal

- Tipo de arranque: lineal 
El motor con fallo en estas condiciones presenta un deslizamiento de 0,028 en el estado estacionario posterior al arranque. Las frecuencias de fallo asociadas a la excentricidad mixta son:

○ $\operatorname{ERH}(-)$

$$
f_{\text {ERHn }}=\left(1-\left(\frac{1-s}{p}\right)\right) f_{s}=\left(1-\left(\frac{1-0,028}{2}\right)\right) 50=25,7
$$

○ $\operatorname{ERH}(+)$ :

$$
f_{\text {ERHp }}=\left(1+\left(\frac{1-s}{p}\right)\right) f_{s}=\left(1+\left(\frac{1-0,028}{2}\right)\right) 50=74,4
$$

En el caso del ensayo con motor sano se obtienen los siguientes valores:

○ $\operatorname{ERH}(-)$ :

$$
f_{\text {ERHn }}=\left(1-\left(\frac{1-s}{p}\right)\right) f_{s}=\left(1-\left(\frac{1-0,028}{2}\right)\right) 50=25,7
$$

○ $\operatorname{ERH}(+)$ :

$$
f_{\text {ERHp }}=\left(1+\left(\frac{1-s}{p}\right)\right) f_{s}=\left(1+\left(\frac{1-0,028}{2}\right)\right) 50=74,3
$$
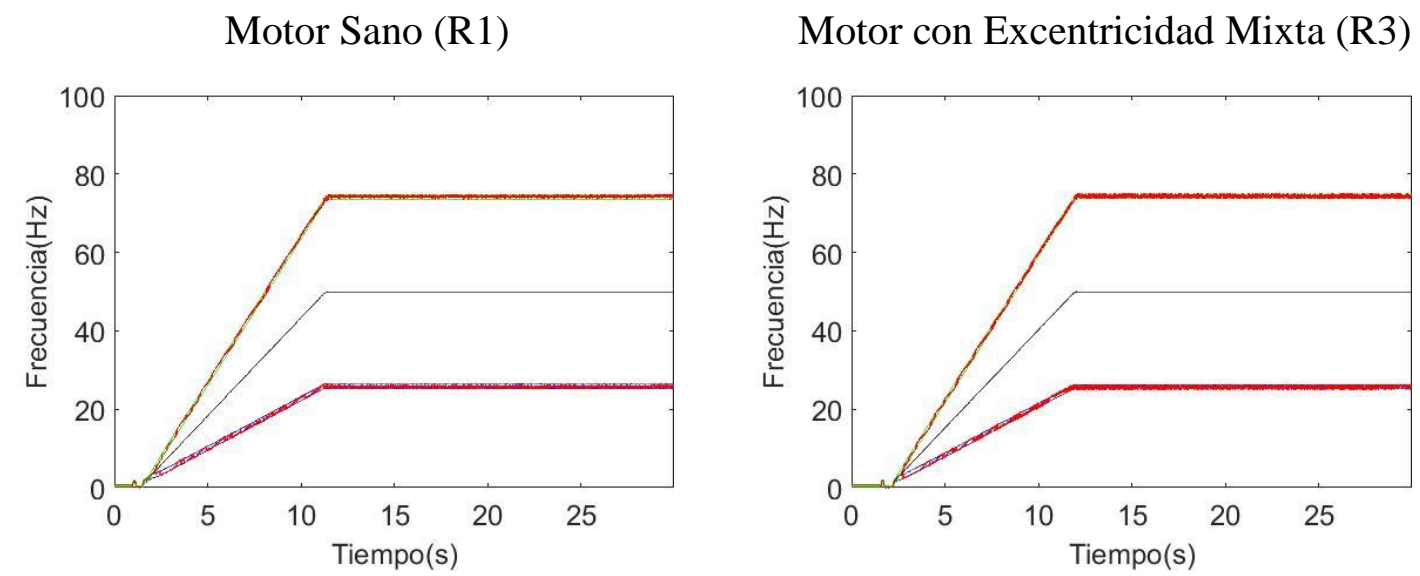

(a) Trayectoria real del primer armónico y teórica de los armónicos de fallo 

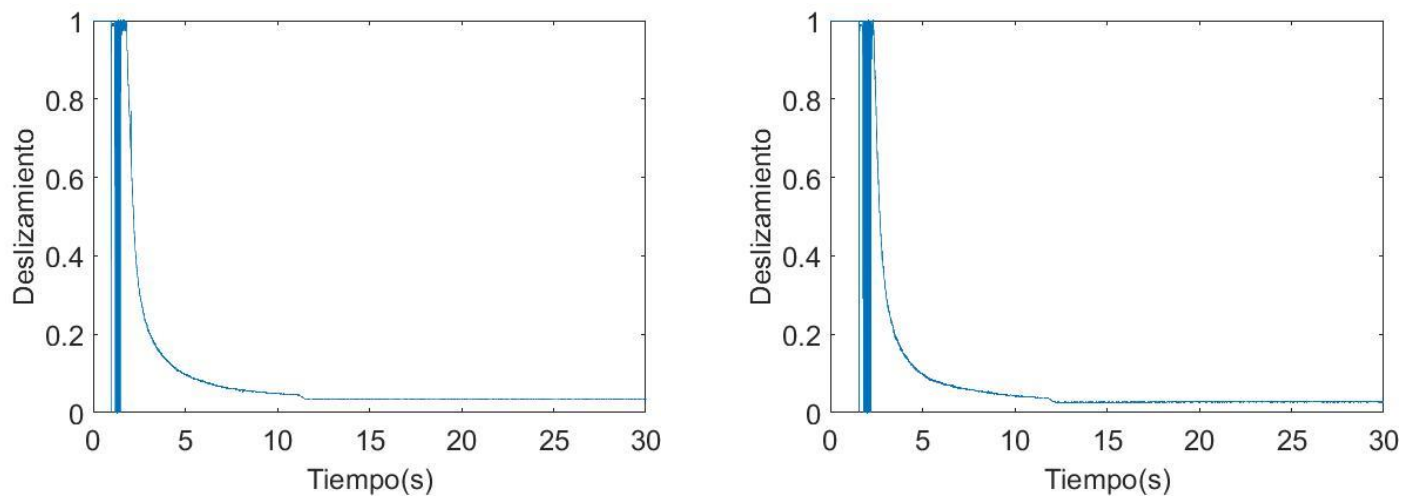

(b) Deslizamiento
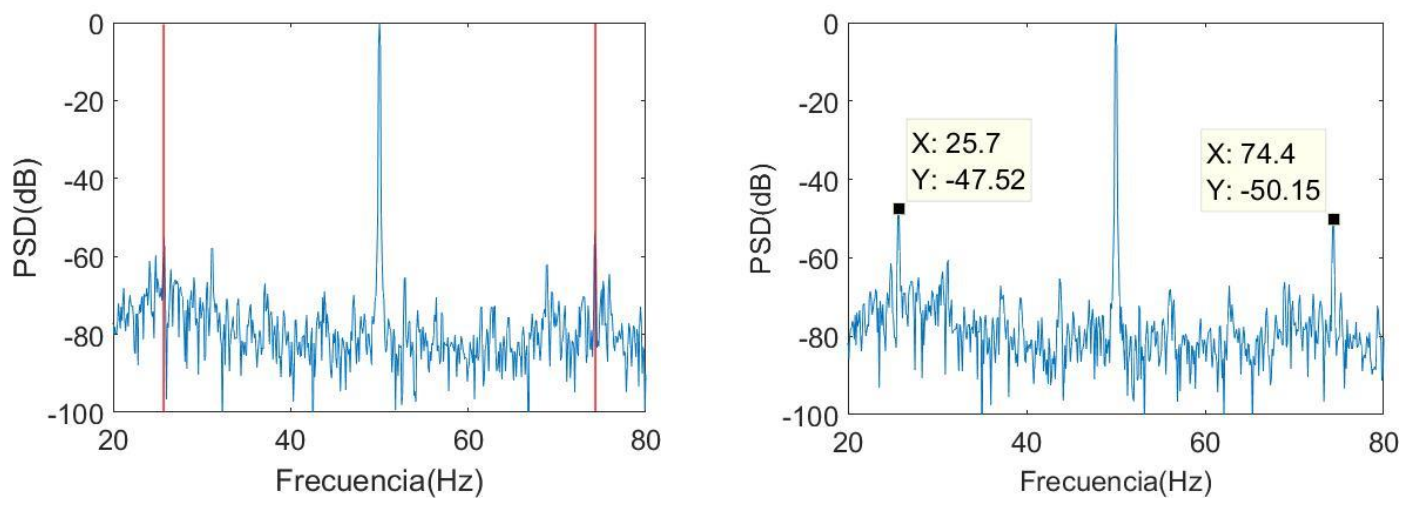

(c) Espectro del estado estacionario
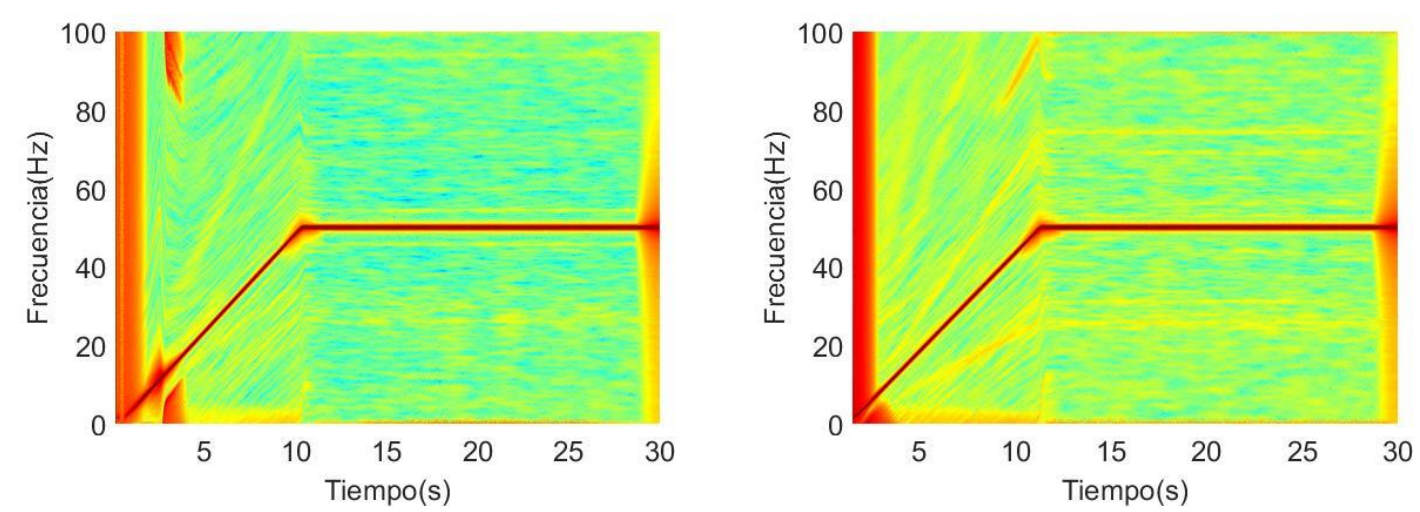

(d) Espectrograma del ensayo
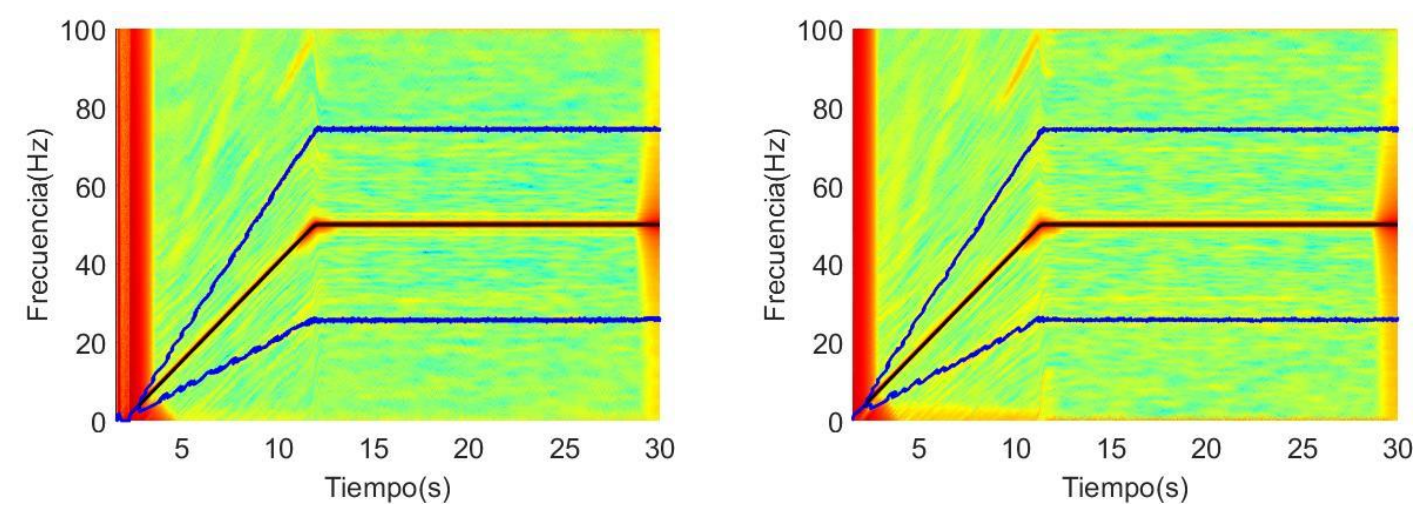

(e) Espectrograma del ensayo y trayectorias de los armónicos de fallo 

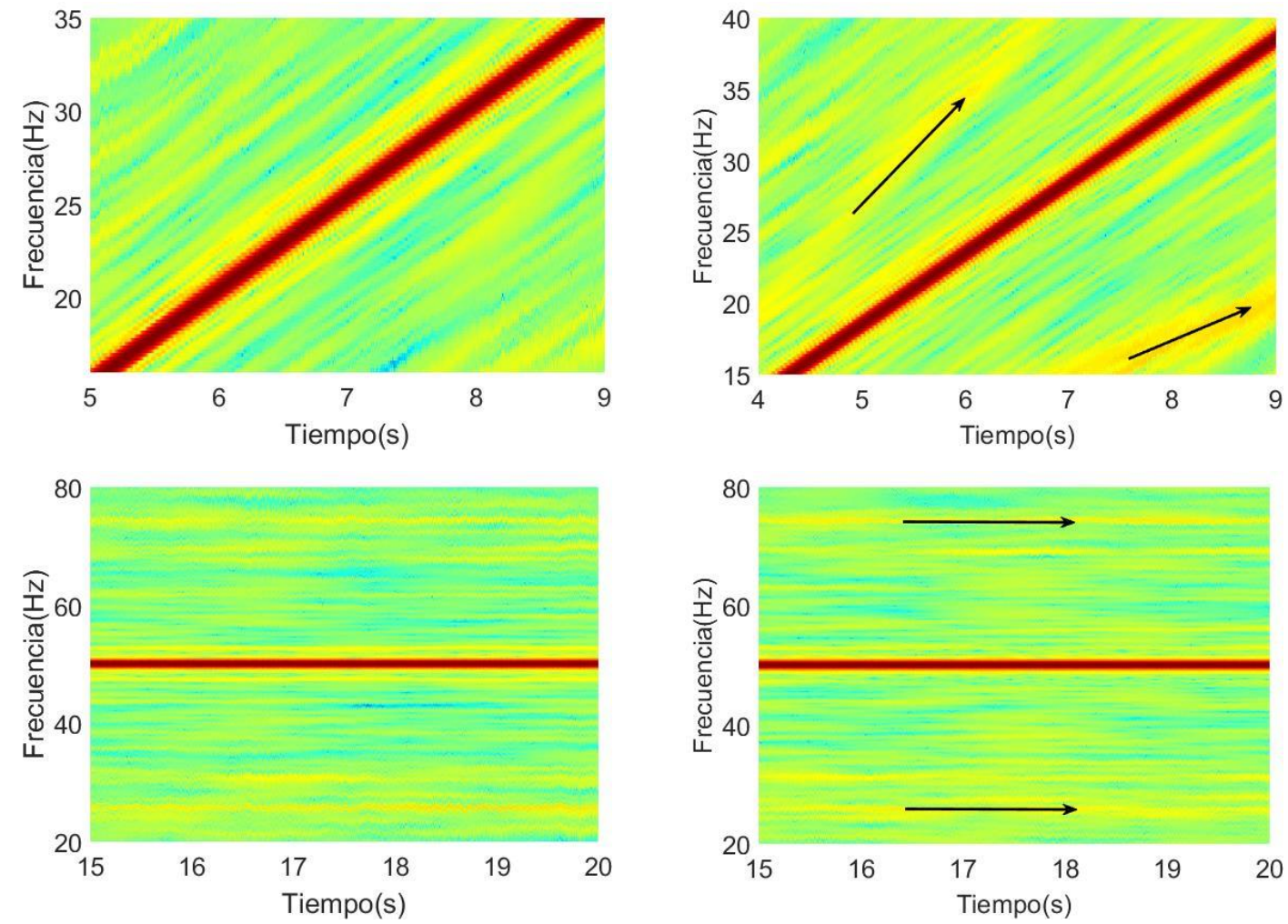

(f) Detalle del espectrograma

Figura 80. Resultados del análisis de la señal de $I M$ alimentado mediante inversor Siemens con nivel de carga bajo.

\subsection{Cuantificación del fallo de barras.}

\subsubsection{Introducción.}

En el apartado 6.2. de este capítulo, al analizar los fallos de barras, uno de los puntos que se expone gráficamente es la cuantificación del fallo. Esta cuantificación merece un apartado aparte para explicar qué es lo que se está obteniendo.

Como ya se ha introducido, se entiende por cuantificación del fallo la amplitud del armónico durante la trayectoria real en el plano tiempo-frecuencia. Es decir, es la energía del armónico de fallo en función del tiempo. Para analizar esta cuantificación, nos centramos en el estado transitorio, que es el que realmente interesa para demostrar la validez de la técnica. Lo primero que se hace para poder calcularla es fijar unas bandas de búsqueda del armónico de fallo. Estas bandas aparecen en la Figura 81(a) y sobre un instante del espectrograma resultante del estado transitorio, en la Figura 81(b). Las dos fronteras que definen la banda de búsqueda están separadas $0,1 \mathrm{~Hz}$. Se ha tomado este 
valor para todos los ensayos. Esta cifra sale de haber probado con diversos espesores hasta comprobar el que ofrece una mejor resolución. Dentro de estas bandas se encuentra el armónico de fallo, tal como puede verse en la Figura 81(b).

Por otra parte, al observar en este estado transitorio, que, como ya se ha mencionado, tiene una duración de 10 segundos, se da la situación que aparece en la Figura 81(c) y (d). Es decir, tanto al inicio de la captura como a los 10 segundos (en el codo), aparecen unos efectos de borde (señalados con círculos negros en dichas Figuras). Al principio del transitorio, se obtiene una banda de mucha energía para todas las frecuencias analizadas. Esto también afecta a la energía de los armónicos de fallo que, durante este período de tiempo corto, son indistinguibles. En la zona del codo, al final del transitorio de arranque y comienzo del funcionamiento estacionario, la energía del armónico principal se dispersa alrededor de su trayectoria. Esto es debido a la forma del átomo empleado. En este caso, esta dispersión de la energía también puede afectar a la energía de los armónicos de fallo, sobre todo si están muy cercanos al fundamental, por ejemplo, con deslizamientos bajos.

Para evitar que estos efectos de borde, que no aportan ninguna información extra, perturben el análisis, se ha reducido el periodo de tiempo donde se cuantifica el fallo a sólo 6 segundos, tal como se muestra en la Figura 81(e). En la Figura 81(f) se muestra la longitud escogida sobre el espectro resultante. De esta forma, se evitan el comienzo y el codo de las componentes. 


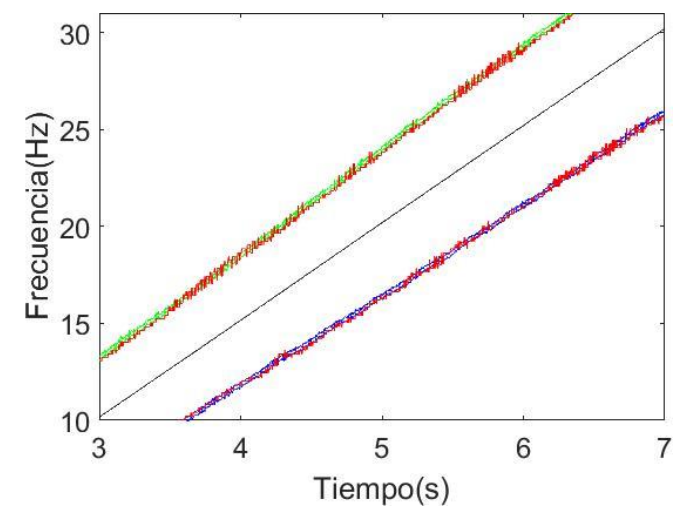

(a) Bandas de búsqueda

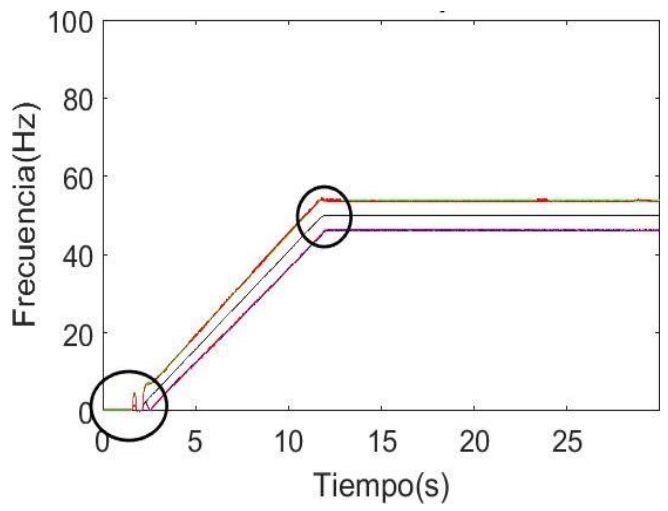

(c) Componente fundamental y trayectorias teóricas.

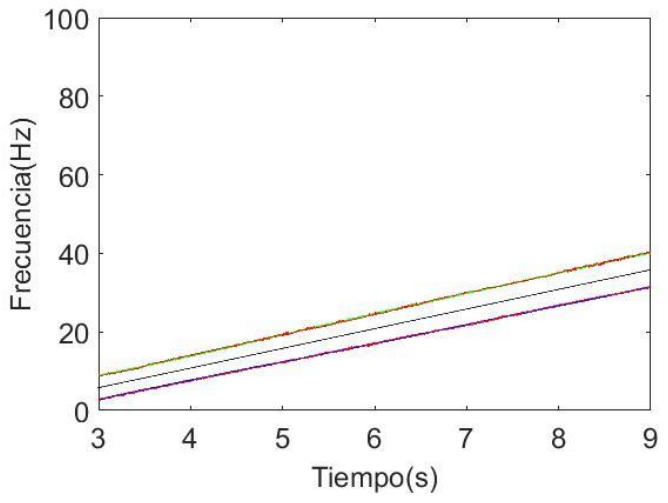

(e) Tramo de 6 s sobre componentes teóricas.

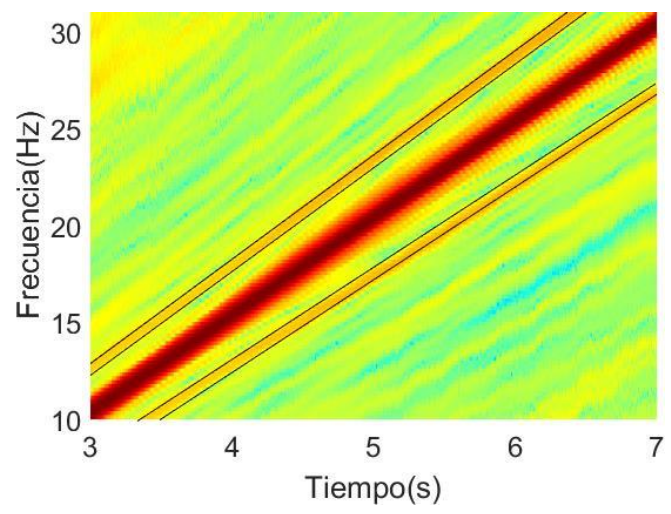

(b) Bandas de búsqueda sobre el espectrograma.

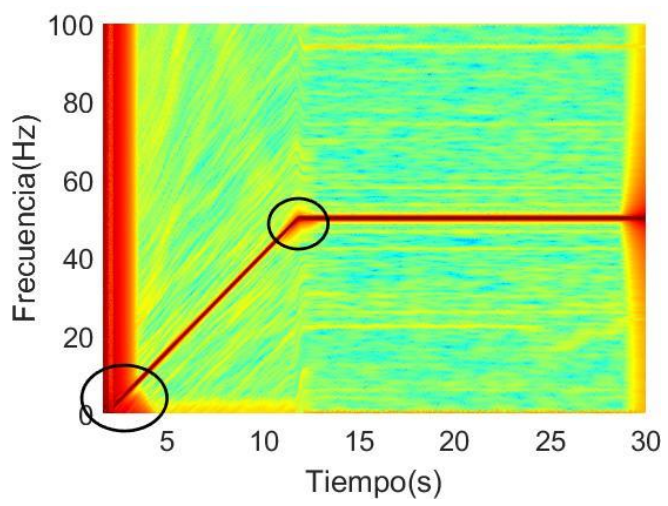

(d) Espectrograma resultante.

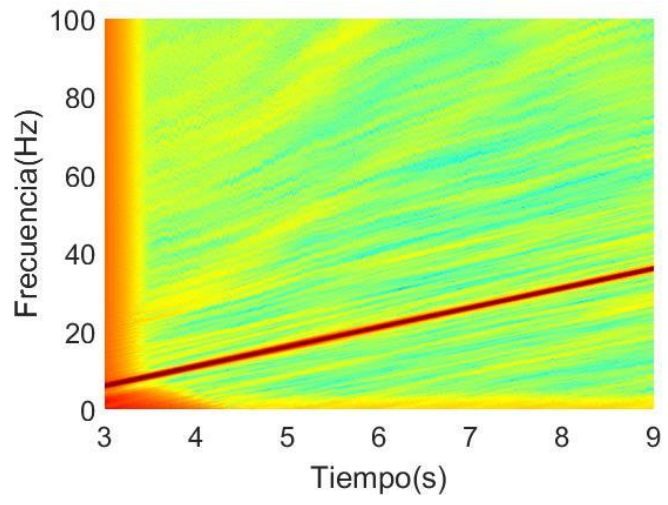

(f) Tramo de 6 s sobre espectrograma resultante.

Figura 81. Explicación gráfica efecto de borde

\subsubsection{Curvas cuantificación.}

A continuación, en las Figuras 85 a 124 se muestran las cuantificaciones obtenidas con los distintos inversores, Allen-Bradley, ABB, Siemens, Telemecánica y WEG. Para el análisis de las curvas resultantes, se van a dividir los resultados en dos: las curvas resultantes del análisis del $L S H$ y las curvas resultantes del análisis del $U S H$. De esta 
forma, se estudia por separado cada componente de los armónicos de fallo de barras. Cada uno de estos apartados se divide en dos subapartados, distinguiendo así entre dos niveles de carga, nivel de carga bajo y nivel de carga alto. A su vez, se distingue entre motor sano y motor con barra rota, por tanto, resumidamente, quedaría de la siguiente manera:

Análisis del LSH:

- Motor sano

○ Nivel de carga bajo

○ Nivel de carga alto

- Motor con BRB

- Nivel de carga bajo

- Nivel de carga alto
Análisis del USH:

- Motor sano

- Nivel de carga bajo

○ Nivel de carga alto

- Motor con BRB

- Nivel de carga bajo

○ Nivel de carga alto

Para el estudio de la cuantificación de las curvas resultantes, se va a utilizar un método estadístico conocido como "Functional Boxplot" [67], ya que resulta ser un buen enfoque para el análisis de datos complejos. Se va a utilizar este método ya que se está trabajando con curvas, y con Functional Boxplot, la unidad básica de información pasa a ser la función a analizar al completo y no una cadena de números. Los autores pretenden desarrollar una herramienta que permita visualizar datos funcionales directamente en el espacio funcional.

Como recordatorio, un boxplot o diagrama de cajas, es una herramienta de la estadística descriptiva que de manera gráfica ilustra cinco parámetros de un conjunto de puntos, que son: la mediana, los cuartiles primeros y terceros, y los máximos y mínimos. Igualmente ilustra los outliers, es decir, los términos atípicos o puntos extraños.

El análisis mediante Functional Boxplot funciona de un modo similar. Con el método de profundidad de banda para datos funcionales se consiguen ordenar todas las curvas de las muestras. Se basa, de igual modo, en la búsqueda de percentiles primeros y terceros, tal y como se procede con el análisis mediante boxplot, determinando la mediana, los máximos y los mínimos y las curvas que serían outliers por salirse de la trayectoria del resto, procediéndose de la manera como se ilustra en la Figura 82. 


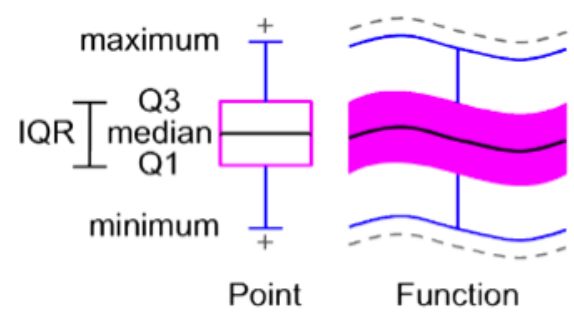

Figura 82. Boxplot y su representación en Functional Boxplot. Fuente: http://cismm.web.unc.edu

Es decir, es como si a cada punto de la curva se le asignara su correspondiente boxplot, buscando en cada uno de estos puntos máximos, mínimos, mediana y los percentiles primero y tercero, tal como se ilustra en la Figura 83.

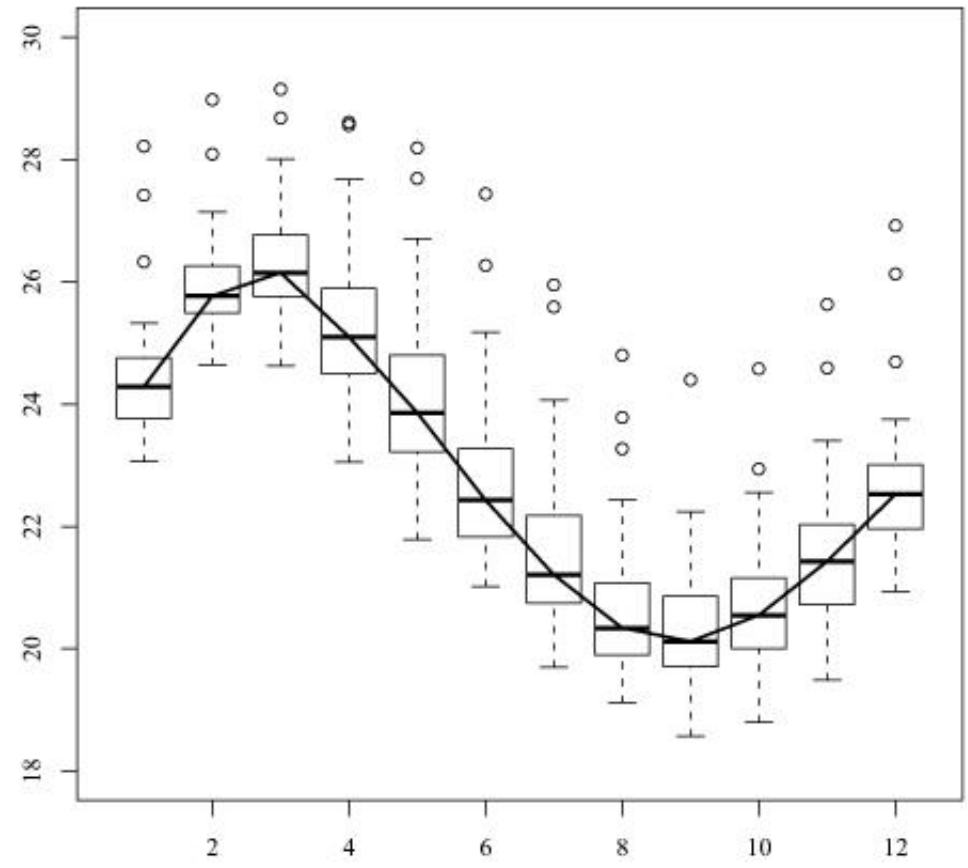

Figura 83. Ejemplo de boxplot asignados a cada punto de la curva. Fuente: http://www.wikiwand.com/en/Functional_boxplot

Por lo que finalmente se obtiene como resultado (Figura 84) una representación de varias curvas donde aparece en negro señalada la mediana, con líneas azules los máximos y los mínimos y la parte de relleno rosa representa la banda de valores comprendida entre los cuartiles primero y tercero. Las líneas discontinuas en rojo serán las curvas. 


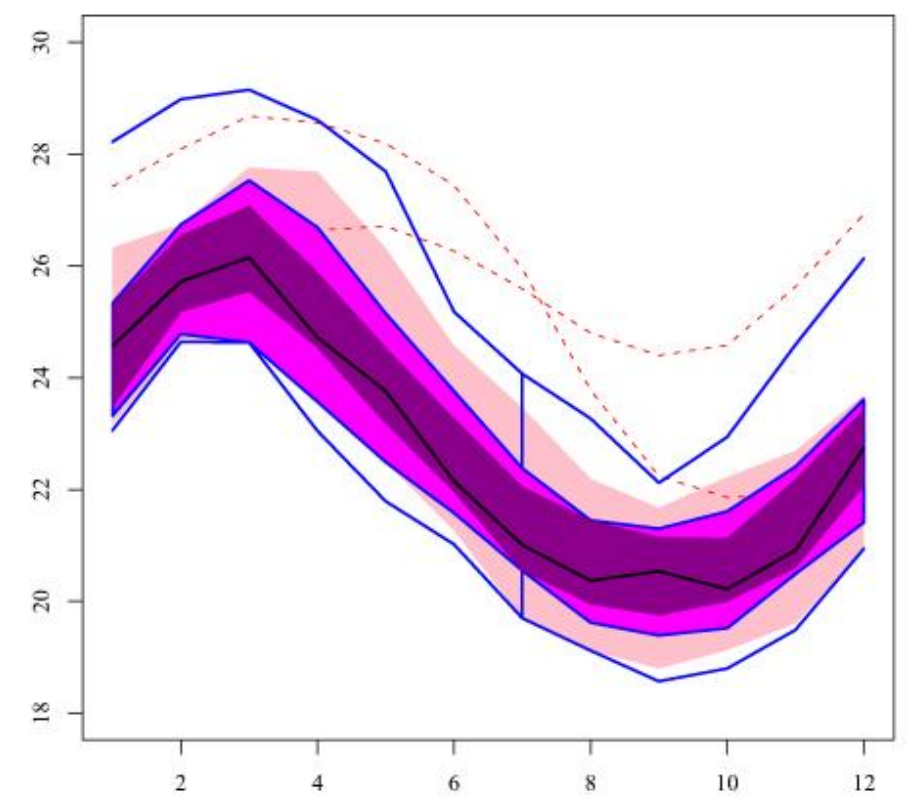

Figura 84. Ejemplo de Functional Boxplot resultante. Fuente: http://www.wikiwand.com/en/Functional_boxplot

El primer análisis se realiza con datos del motor alimentado con el inversor de Allen-Bradley. Primeramente, se presentan las curvas de cuantificación del fallo asociadas al $L S H$ con un nivel de carga bajo (Figura 85 ). Se muestran por separado las curvas correspondientes al motor sano y al motor con la barra rota. Al observar las bandas entre las que se encuentran las curvas se puede observar que en caso del motor sano ésta se encuentra concentrada entre los $140 \mathrm{~dB}$ y $160 \mathrm{~dB}$ (Figura 85(a)), mientras que cuando el motor presenta un fallo esta banda presenta un valor algo inferior, situándose entre los $135 \mathrm{~dB}$ y los $150 \mathrm{~dB}$ (Figura 85(b)). El valor en decibelios de la energía ha disminuido al presentar un fallo el motor.

Cabe aclarar aquí, tanto para este supuesto de la Figura 85 como para los restantes, que realmente la energía aumenta al presentar fallo el motor, ya que el espectro normalmente se expresa en $\mathrm{dB}$ y se normaliza respecto al armónico de mayor amplitud o energía, que suele ser el fundamental.

De esta forma, el resto de componentes del espectro tienen valores negativos, expresados en dB. Lo que ocurre con los supuestos con los que se está trabajando es que se opera con valores absolutos, ya que en esta tesis se decidió representar el valor absoluto del espectro en cada instante de tiempo y no se normalizó la energía respecto a la del 
componente fundamental puesto que su valor va cambiando con el tiempo y se producía una representación errónea del contenido armónico durante todo el transitorio analizado. Por tanto, y al trabajar en valores absolutos, el valor en decibelios de la energía en motor con fallo deberá de disminuir respecto al que presenta cuando el motor está sano. Es decir, en el caso concreto de la Figura 85 se tiene que, al no normalizar y usar valores absolutos, $140 \mathrm{~dB}$ en motor sano frente a $135 \mathrm{~dB}$ en motor con fallo, por lo que, para estos supuestos, con los valores absolutos que se trabaja, el valor en decibelios de la energía en motor con fallo deberá de disminuir respecto al que presenta cuando el motor está sano.

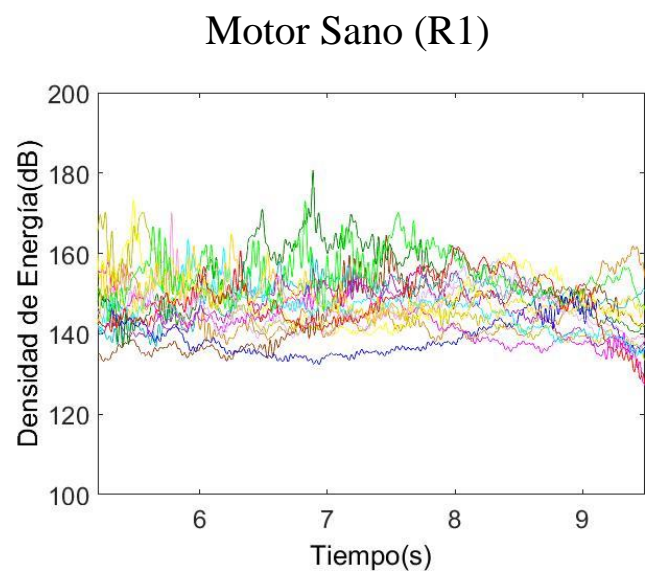

(a) Cuantificación LSH IM sano nivel carga bajo.

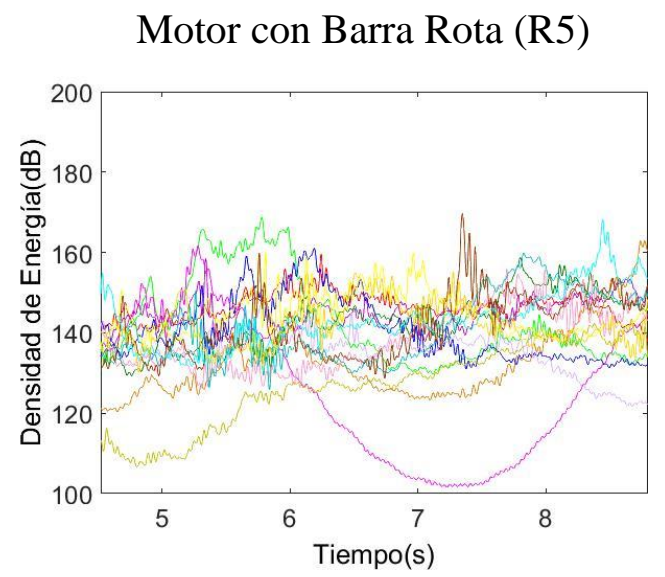

(b) Cuantificación LSH IM BRB nivel carga bajo

Figura 85. Cuantificación del fallo asociado al $L S H$ con nivel de carga bajo, inversor AB.

Motor Sano (R1)

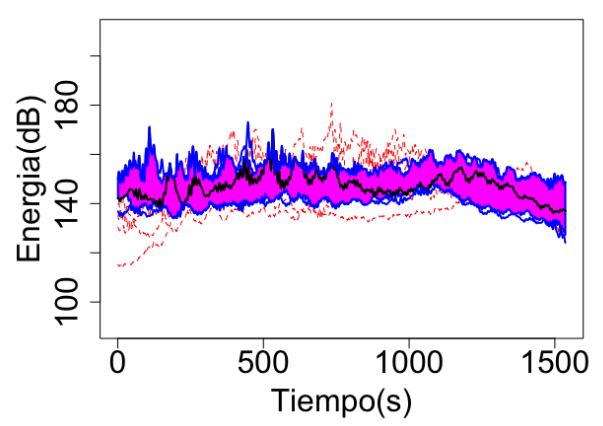

(a) Functional boxplot del LSH IM sano nivel carga bajo.
Motor con Barra Rota (R5)

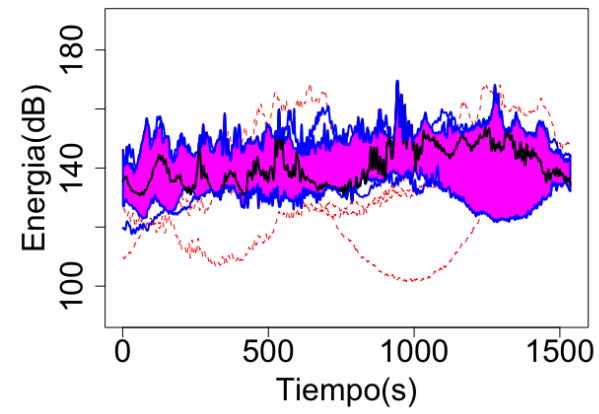

(b) Functional boxplot del LSH IM BRB nivel carga bajo

Figura 86. Functional boxplot del fallo asociado al LSH con nivel de carga bajo, inversor AB.

En la Figura 86 se presenta el análisis mediante Functional Boxplot de las curvas de cuantificación asociadas al $L S H$, cuando el nivel de carga es bajo, es decir, las curvas 
de la Figura 85. Este análisis se ha realizado con el paquete "fda" [67] implementado en la herramienta R [68].

A continuación, se realiza un análisis mediante "Functional Boxplot", que se ha introducido al inicio de este apartado; en cada uno de los casos a estudio ( $L S H$ nivel de carga bajo, LSH nivel de carga alto, USH nivel de carga bajo, USH nivel de carga alto) los elementos a análisis son 15 (15 curvas por cada caso), es decir, en todos los casos que se van a analizar $n=15$. Lo primero que se hace es calcular los percentiles primero y tercero, que definirá la banda entre las que se encuentra el grueso de las curvas analizadas. El siguiente paso es calcular el valor $I Q R$, que se obtiene como la diferencia de ambos percentiles $(\mathrm{IQR}=$ Porcentil3-Percentil1). Finalmente, con este valor se obtienen los valores que delimitarán los outliers. El valor "Value" va a determinar los valores entre los que se moverán las curvas analizadas, es decir, la banda con los valores medios, estos se van a calcular utilizando el criterio del 1,5 [69]. Así se tiene que: Value<Porcentil11,5·IQR y Value> Percentil3+1,5·IQR, por encima de estos valores se van a encontrar las curvas que se salen de rango.

Procediendo de esta manera, se obtiene las Figuras 86, 88, 90, 92, 94, 96, 98, 100, $102,104,106,108,110,112,114,116,118,120,122$ y 124. Este análisis ayudará a verificar si la cuantificación que se ha llevado a cabo es correcta y se está haciendo un análisis correcto de los armónicos de fallo.

Así, al fijarse en la Figura 86, este análisis confirma que cuando el motor presenta fallo el valor promedio de la energía disminuye, por lo que se puede demostrar que se está calculando bien la cuantificación del fallo. Por otra parte, si bien el grueso de las curvas entra entre el percentil primero y tercero, existen algunos outliers o lo que es lo mismo, algunas de las curvas se salen de rango establecido. Estos outliers pueden ser debidos a factores como que el ensayo no se ha llevado a cabo de la manera correcta, o, simplemente, que se ha comenzado a medir más tarde que en el resto de los casos.

Al analizar la Figura 86, en el nivel de carga alto, se ve cómo se eleva el valor de la densidad de energía, pasando, para el motor en estado sano (Figura 86(a)), a presentar un valor medio que se mueve entre la banda $140 \mathrm{~dB}$ y $160 \mathrm{~dB}$ y para el motor con barra 
rota entre $135 \mathrm{~dB}$ y $155 \mathrm{~dB}$ (Figura 86(b)); si bien este valor disminuye con el $I M$ con fallo la diferencia entre ambos no es muy acusada, por lo que se dificulta la detección del fallo al analizarlo mediante éste método.

En la Figura 87 se observa el caso en el que se analiza el $L S H$, pero ahora presentando un nivel de carga alto. Al analizar ambas Figuras (Figura 87(a) y Figura 87(b)) se aprecia que en el caso del $I M$ con fallo parece disminuir el valor promedio de las curvas. Sin embargo, ocurre lo mismo que en el análisis anterior (Figura 85), y es que este valor apenas varía por lo que se hace difícil el comprobar que existe fallo.

Motor Sano (R1)

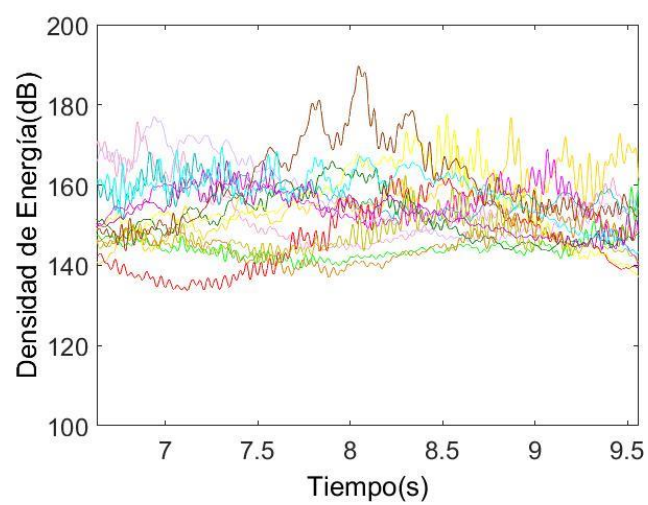

(a) Cuantificación LSH IM sano nivel carga alto.
Motor con Barra Rota (R5)

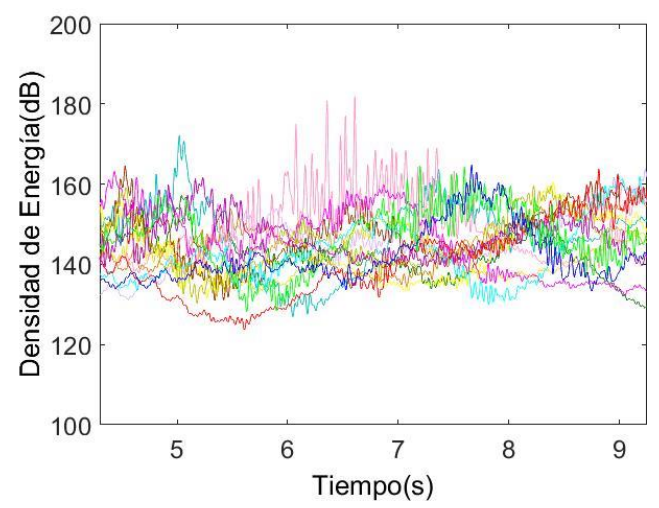

(b) Cuantificación $L S H I M B R B$ nivel carga alto.

Figura 87. Cuantificación del fallo asociado al $L S H$ con nivel de carga alto, inversor AB.

Motor Sano (R1)

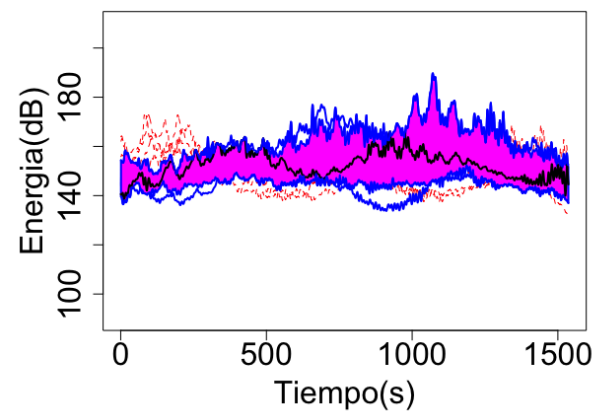

(a) Functional boxplot del LSH IM sano nivel carga alto.
Motor con Barrar Rota (R5)

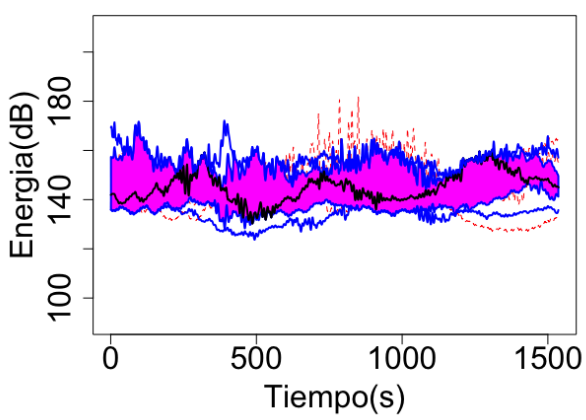

(b) Functional boxplot del LSH IM BRB nivel carga alto.

Figura 88. Functional boxplot del fallo asociado al LSH con nivel de carga alto, inversor AB. 
Esto mismo se demuestra haciendo al análisis de las curvas mediante Functional Boxplot (Figura 88) en donde puede verse como apenas se aprecia una bajada en el valor promedio de las curvas a análisis. Por otra parte, en este caso, apenas existen outliers.

Cuando se analiza el USH (Figuras 89 y 91) para este mismo inversor, AllenBradley, esta diferencia que existe entre motor sano y motor con barra rota, es más acusada, por lo que es más sencillo identificar el fallo. Viendo, primeramente, cómo se comporta cuando se le somete a un nivel de carga bajo (Figura 89), para el caso de un motor sano la media se encuentra en torno a la banda de $130 \mathrm{~dB}$ a $150 \mathrm{~dB}$ (Figura 89(a)), mientras que para el motor con fallo (Figura 89(b)) esta media baja considerablemente, quedándose concentradas las curvas en la banda 120 a 140 dB.

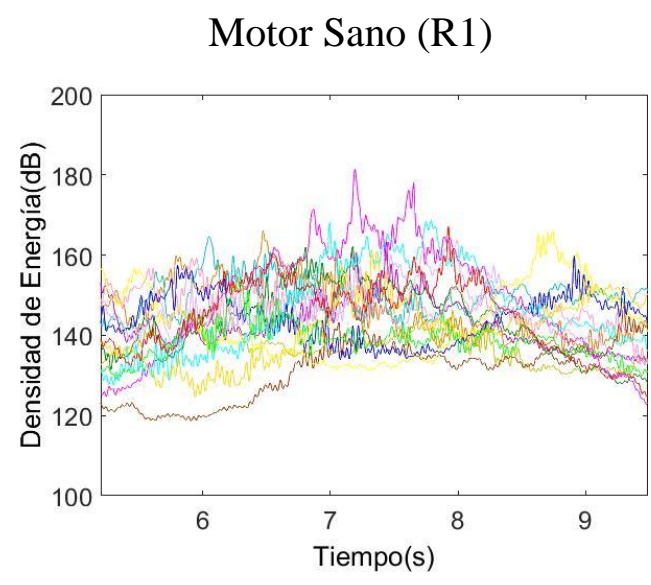

(a) Cuantificación USH IM sano nivel carga bajo.

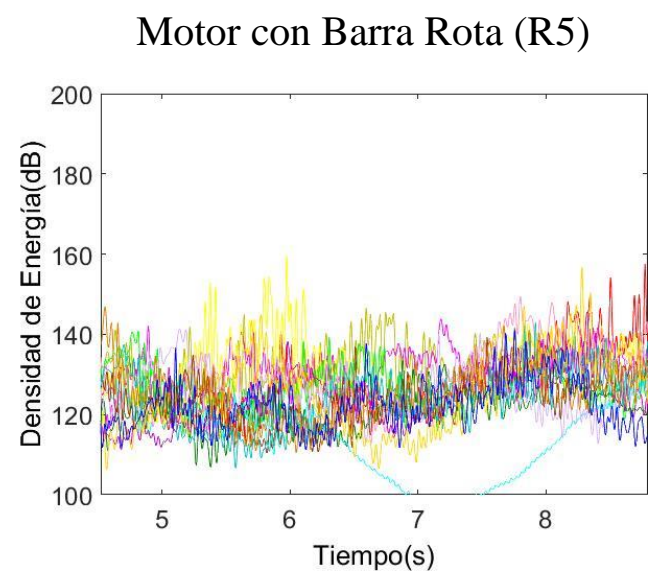

(b) Cuantificación USH IM BRB nivel carga bajo.

Figura 89. Cuantificación del fallo asociado al USH con nivel de carga bajo, inversor AB.

Motor Sano (R1)

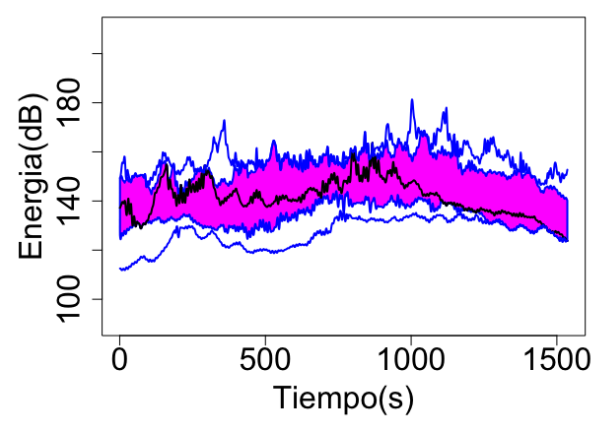

(a) Functional boxplot del USH IM sano nivel carga bajo.
Motor con Barra Rota (R5)

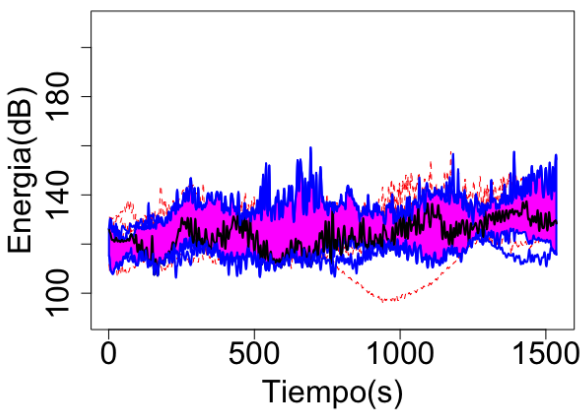

(b) Functional boxplot del USH IM BRB nivel carga bajo.

Figura 90. Functional boxplot del fallo asociado al USH con nivel de carga bajo, inversor AB. 
El estudio mediante el análisis Functional Boxplot (Figura 90) de las curvas de cuantificación de la Figura 89 llevan a comprobar que realmente en el caso del USH, este valor medio es inferior para el caso del motor con fallo. Se comprueba que, además, para el caso del análisis de este armónico, esta bajada en el valor medio es mucho más acusada por lo que resulta más sencillo identificar que realmente existe fallo.

Finalmente, en la Figura 91 se estudia el caso del USH cuando se somete al motor a un nivel de carga alto, siendo el caso en el que mejor se puede observar esta diferencia. Cuando el motor está sano (Figura 91(a)), la media de las curvas se encuentra en la banda 130 dB a 150 dB, mientras que en la Figura 91(b) se comprueba que este valor se sitúa en torno a la banda $125 \mathrm{~dB}$ a $140 \mathrm{~dB}$, luego se identifica mejor la existencia del fallo.

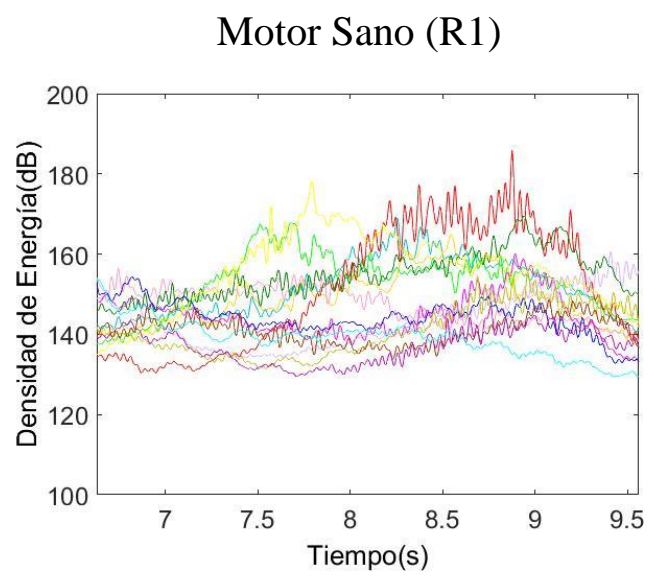

(a) Cuantificación USH IM sano nivel carga alto.

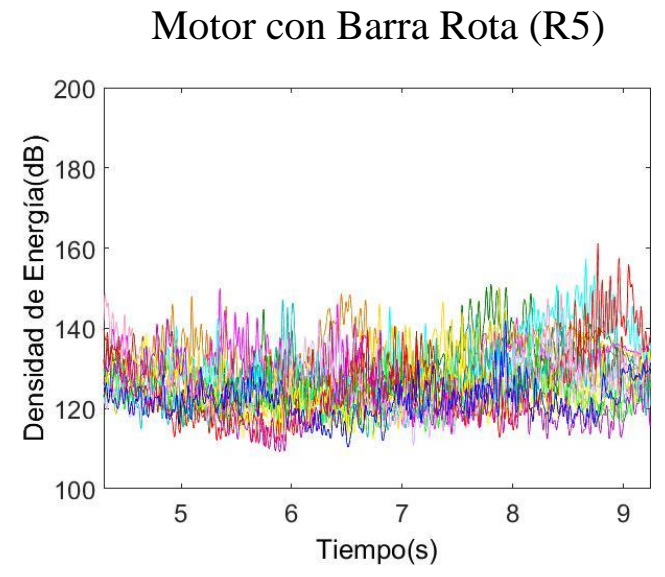

(b) Cuantificación USH IM BRB nivel carga alto.

Figura 91. Cuantificación del fallo asociado al USH con nivel de carga alto, inversor AB.

Motor Sano (R1)

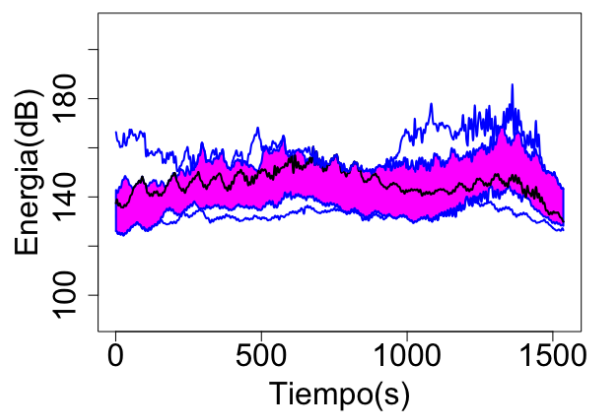

(a) Functional boxplot del USH IM sano nivel carga alto.
Motor con Barra Rota (R5)

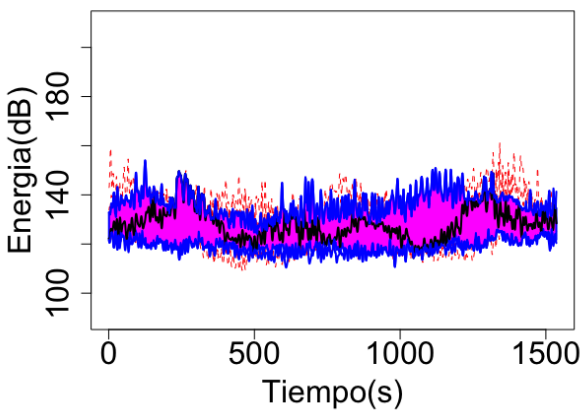

(b) Functional boxplot del USH IM BRB nivel carga alto.

Figura 92. Functional boxplot del fallo asociado al USH con nivel de carga alto, inversor AB. 
Con la Figura 92 queda demostrado como este diferencial entre las bandas que presentan los valores medios de las curvas cuando el motor es sano y el motor que presenta fallo es mucho más acusado cuando lo que se está analizando es el caso del USH. En este caso si bien existen outliers estos no presentan valores tan alejados de las bandas encuadradas dentro del rango $1^{\circ}$-3er percentil.

A continuación, en las Figuras 93 a 124 se procede a realizar un análisis análogo al llevado a cabo con Allen-Bradley, para el resto de los inversores, ABB, Siemens, Telemecánica y WEG.

En las Figuras 93 a 100 se muestran los resultados concernientes al inversor ABB, Así como el análisis mediante Functional Boxplot para cada una de ellas

- $\quad$ Figura 93: curvas de cuantificación: $L S H$ y nivel de carga bajo.

- $\quad$ Figura 94: Functional Boxplot con las curvas de la Figura 117.

- $\quad$ Figura 95: curvas cuantificación: $L S H$ y nivel de carga alto.

- Figura 96: Functional Boxplot de las curvas de la Figura 119.

- Figura 97: curvas cuantificación: USH y nivel de carga bajo.

- $\quad$ Figura 98: Functional Boxplot de las curvas de la Figura 121.

- Figura 99: curvas para el USH con nivel de carga alto.

- Figura 100: Functional Boxplot de las curvas de la Figura 123.

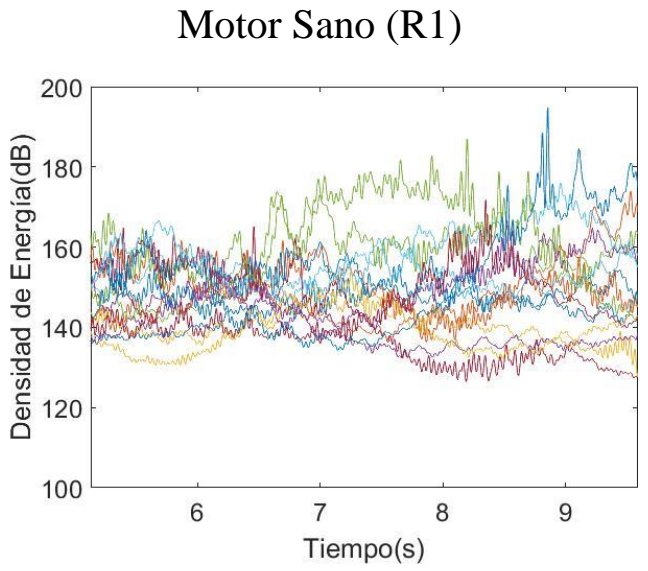

(a) Cuantificación LSH IM sano nivel carga bajo.
Motor con Barra Rota (R5)

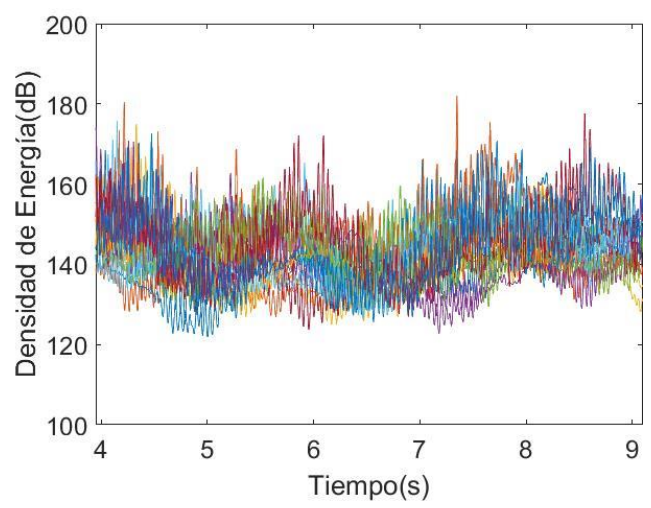

(b) Cuantificación LSH IM BRB nivel carga bajo

Figura 93. Cuantificación del fallo asociado al $L S H$ con nivel de carga bajo, inversor ABB. 


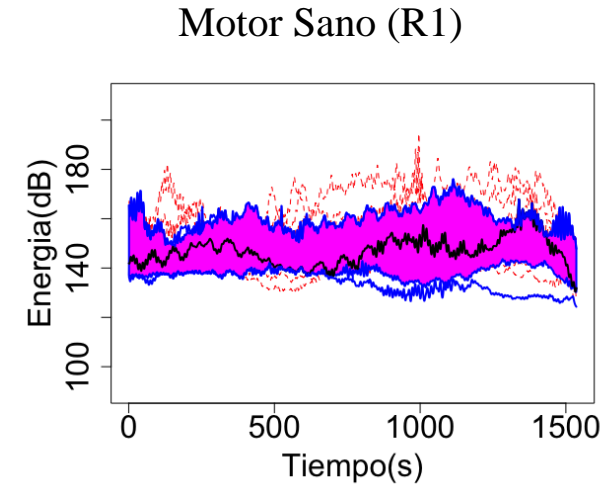

(a) Functional boxplot del LSH IM sano nivel carga bajo.
Motor con Barra Rota (R5)

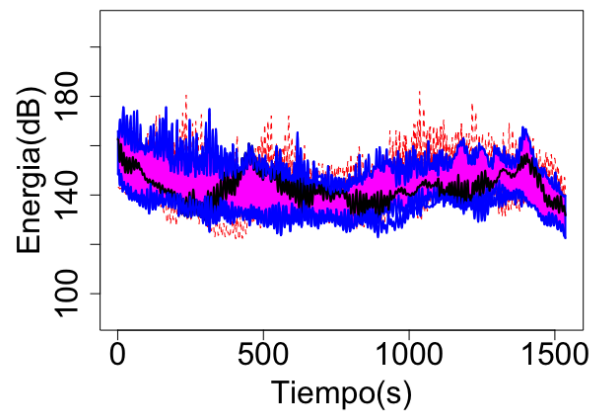

(b) Functional boxplot del LSH IM BRB nivel carga bajo

Figura 94. Functional boxplot del fallo asociado al $L S H$ con nivel de carga bajo, inversor ABB.

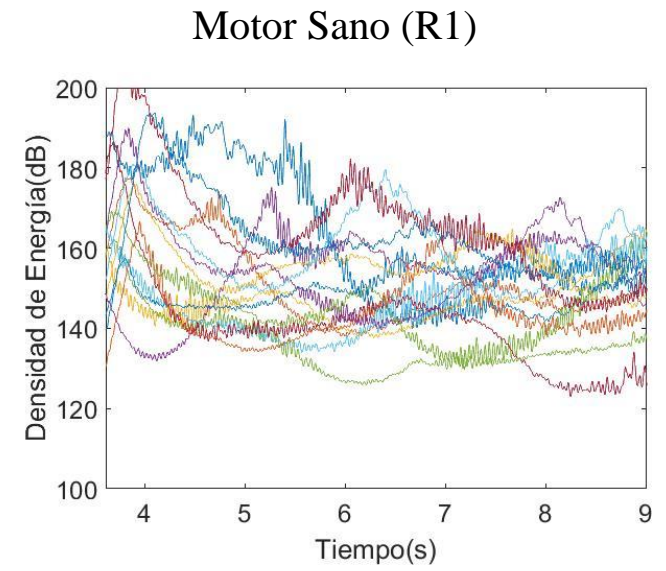

(a) Cuantificación LSH IM sano nivel carga alto.
Motor con Barra Rota (R5)

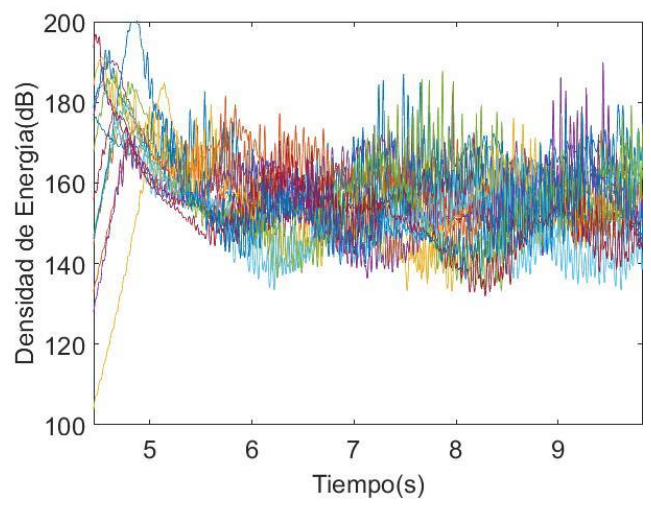

(b) Cuantificación LSH IM BRB nivel carga alto.

Figura 95. Cuantificación del fallo asociado al $\mathrm{LSH}$ con nivel de carga alto, inversor ABB.

Motor Sano (R1)

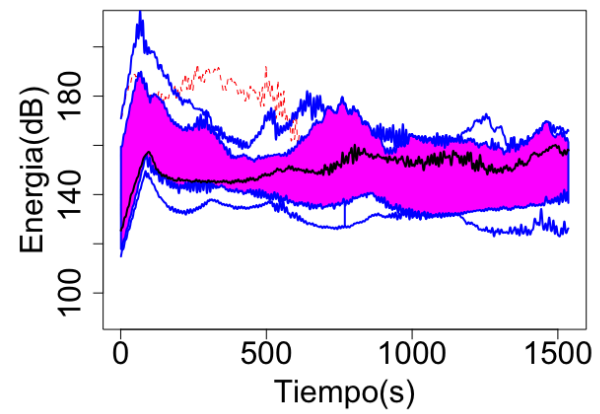

(a) Functional boxplot del LSH IM sano nivel carga alto.
Motor con Barra Rota (R5)

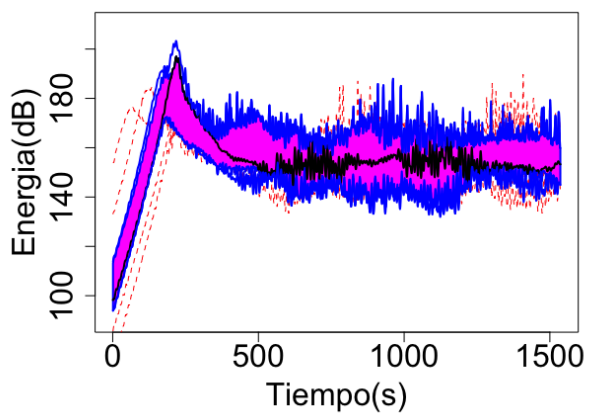

(b) Functional boxplot del LSH IM BRB nivel carga alto.

Figura 96. Functional boxplot del fallo asociado al LSH con nivel de carga alto, inversor ABB. 


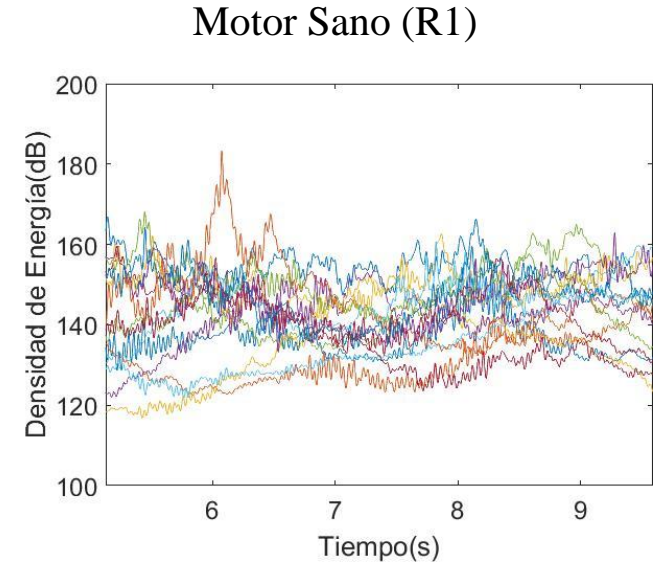

(a) Cuantificación USH IM sano nivel carga bajo.

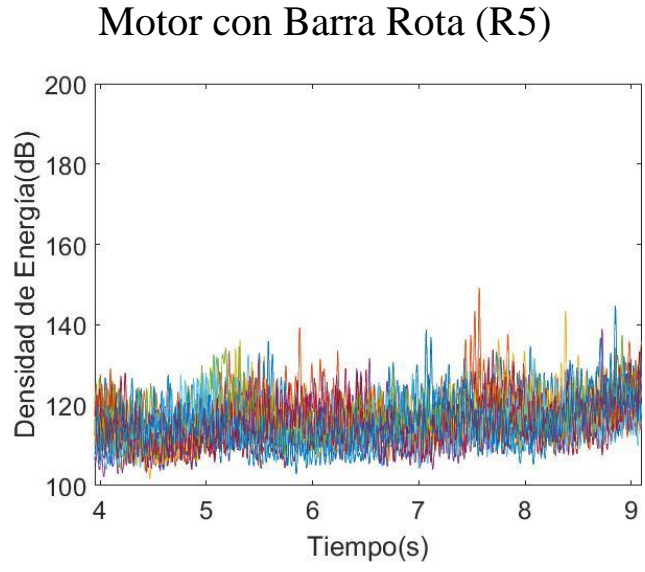

(b) Cuantificación USH IM BRB nivel carga bajo.

Figura 97. Cuantificación del fallo asociado al USH con nivel de carga bajo, inversor ABB.

Motor Sano (R1)

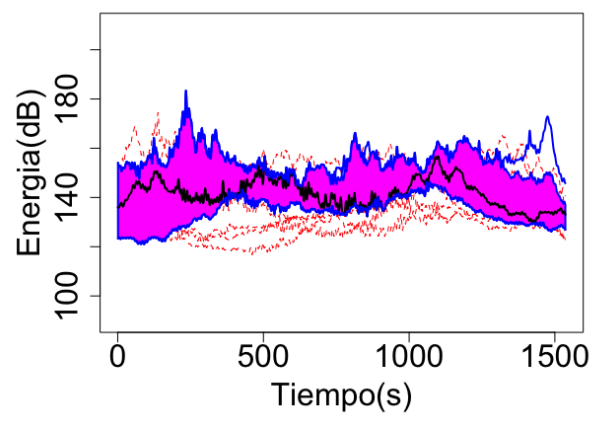

(a) Functional boxplot del USH IM sano nivel carga bajo.
Motor con Barra Rota (R5)

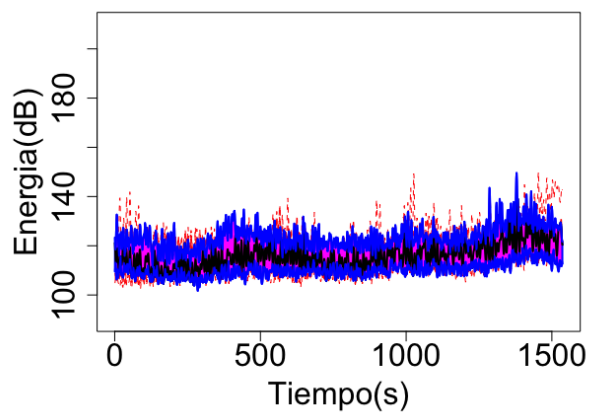

(b) Functional boxplot del USH IM BRB nivel carga bajo.

Figura 98. Functional boxplot del fallo asociado al USH con nivel de carga bajo, inversor ABB.

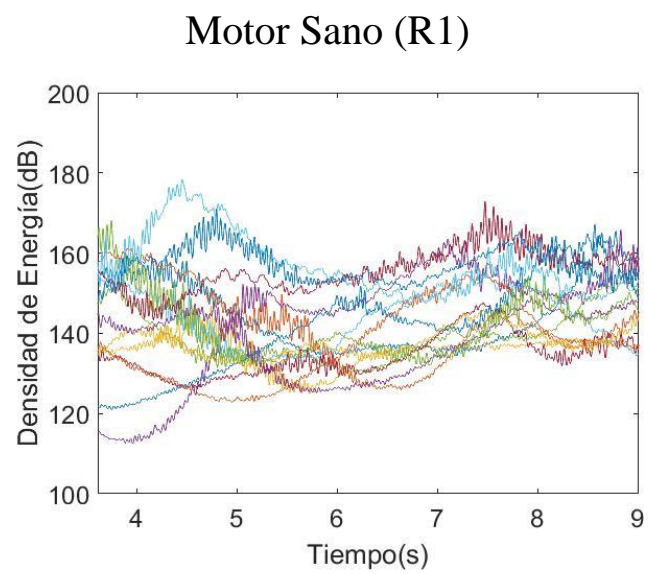

(a) Cuantificación USH IM sano nivel carga alto.
Motor con Barra Rota (R5)

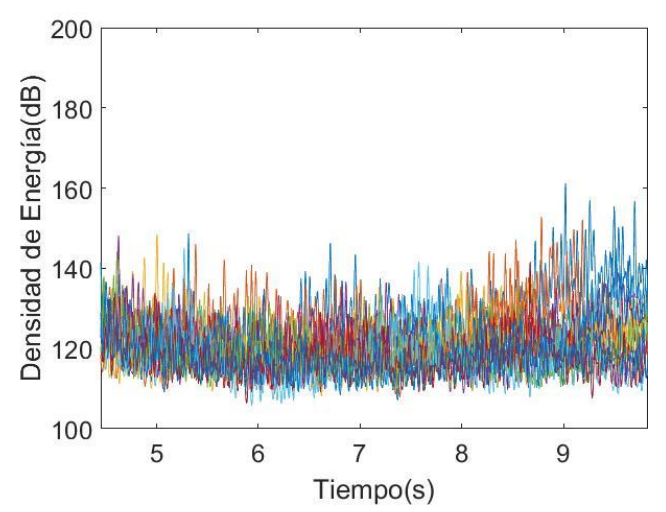

(b) Cuantificación USH IM BRB nivel carga alto.

Figura 99. Cuantificación del fallo asociado al USH con nivel de carga alto, inversor ABB. 


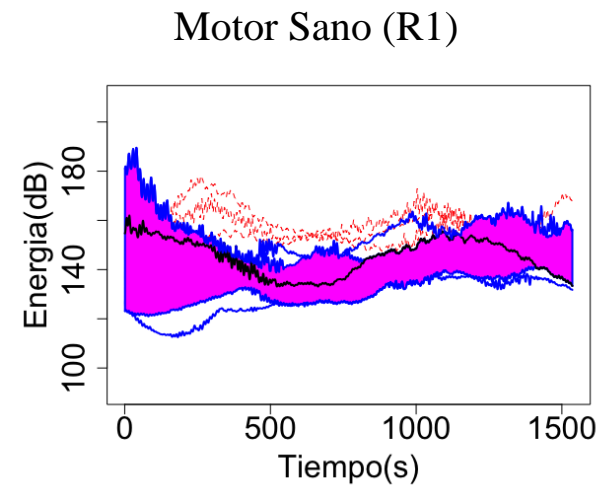

(a) Functional boxplot del USH IM sano nivel carga alto.
Motor con Barra Rota (R5)

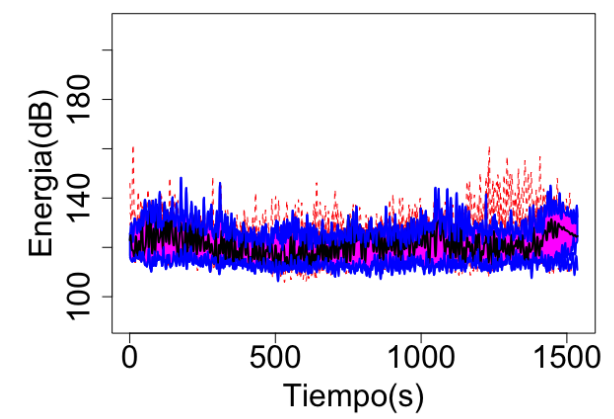

(b) Functional boxplot del USH IM BRB nivel carga alto.

Figura 100. Functional boxplot del fallo asociado al $U S H$ con nivel de carga alto, inversor ABB.

A continuación (Figuras 101 a 108) se analiza el caso de un motor alimentado mediante un inversor Siemens. Procediendo de la misma manera, se obtienen las curvas de cuantificación resultantes para $L S H$ y $U S H$, y se lleva a cabo un análisis mediante Functional Bloxplot de cada una de ellas siendo:

- Figura 101: curvas de cuantificación: $L S H$ y nivel de carga bajo.

- Figura 102: Functional Boxplot de las curvas de la Figura 117.

- Figura 103: curvas cuantificación: $L S H$ y nivel de carga alto.

- Figura 104: Functional Boxplot de las curvas de la Figura 119.

- Figura 105: curvas cuantificación: $U S H$ y nivel de carga bajo.

- Figura 106: Functional Boxplot de las curvas de la Figura 121.

- Figura 107: curvas para el USH con nivel de carga alto.

- Figura 108: Functional Boxplot de las curvas de la Figura 123.

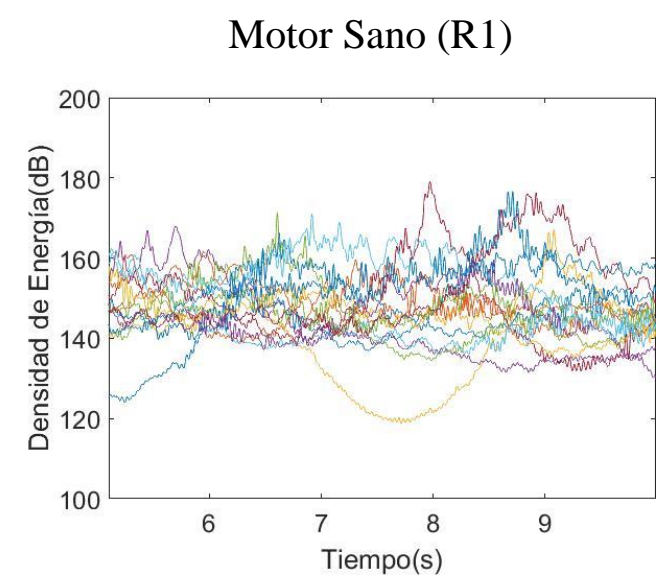

(a) Cuantificación LSH IM sano nivel carga bajo.

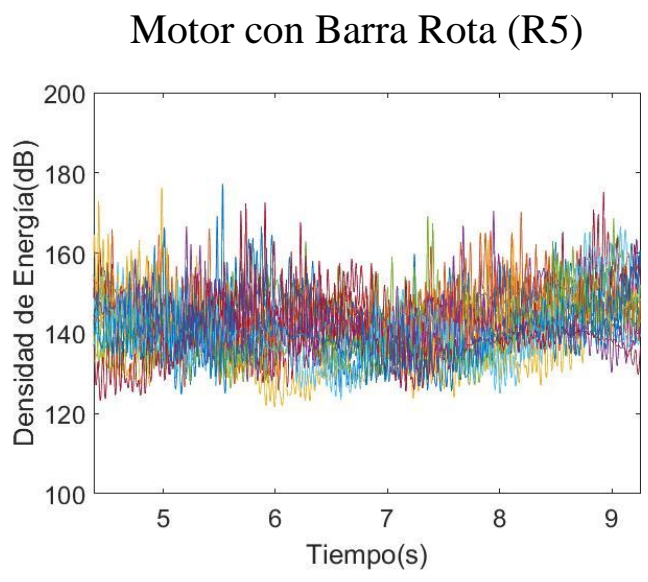

(b) Cuantificación LSH IM BRB nivel carga bajo

Figura 101. Cuantificación del fallo asociado al LSH con nivel de carga bajo, inversor Siemens. 


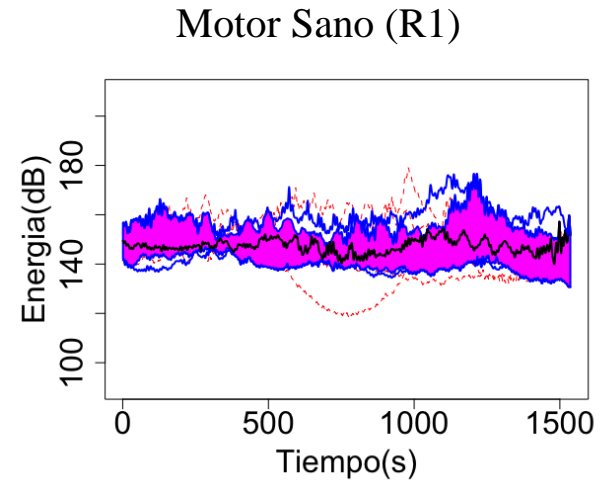

(a) Functional boxplot del LSH IM sano nivel carga bajo.
Motor con Barra Rota (R5)

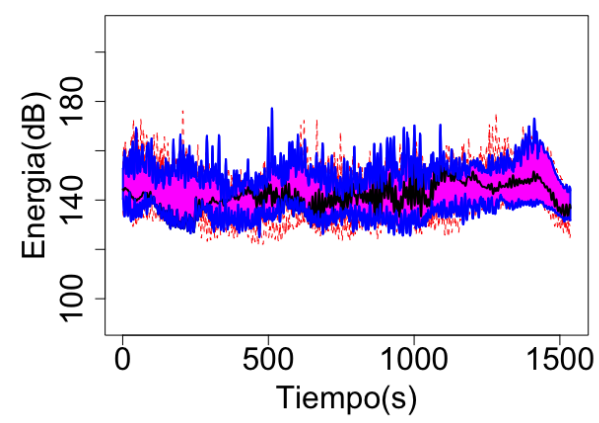

(b) Functional boxplot del LSH IM BRB nivel carga bajo

Figura 102. Functional boxplot del fallo asociado al LSH con nivel de carga bajo, inversor Siemens.

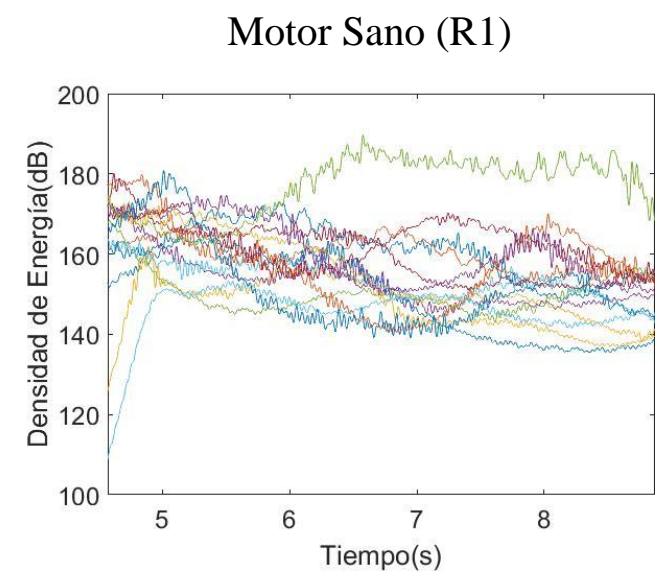

(a) Cuantificación LSH IM sano nivel carga alto.

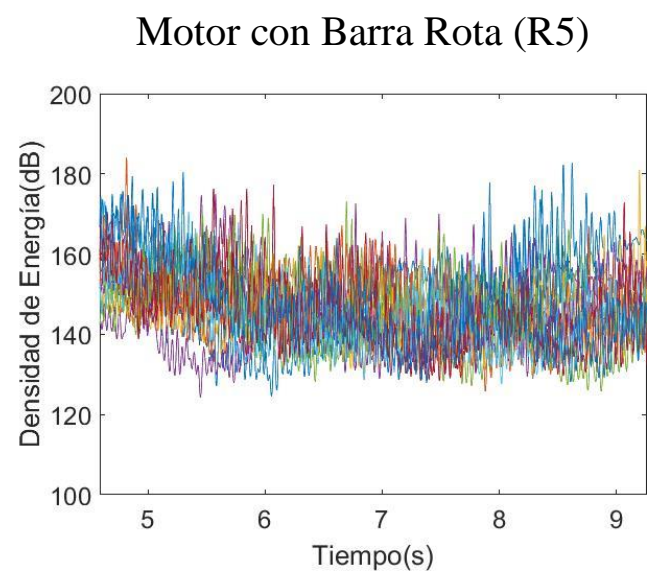

(b) Cuantificación LSH IM BRB nivel carga alto.

Figura 103. Cuantificación del fallo asociado al LSH con nivel de carga alto, inversor Siemens.

Motor Sano (R1)

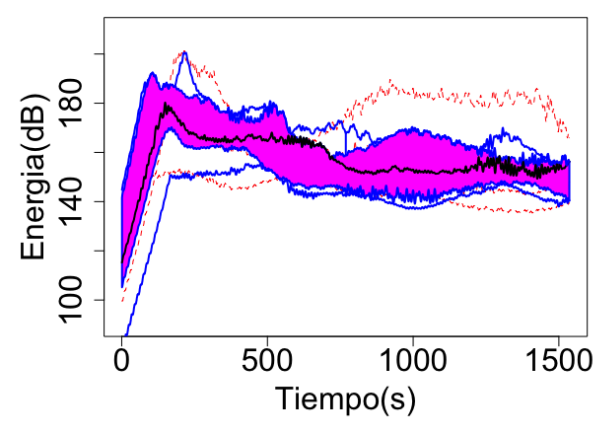

(a) Functional boxplot del LSH IM sano nivel carga alto.
Motor con Barra Rota (R5)

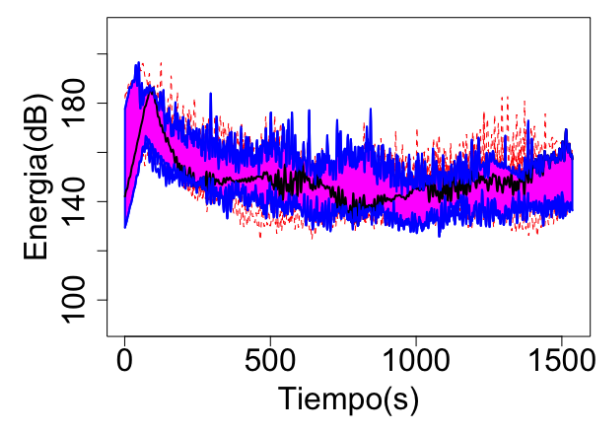

(b) Functional boxplot del LSH IM BRB nivel carga alto.

Figura 104. Functional boxplot del fallo asociado al LSH con nivel de carga alto, inversor Siemens. 


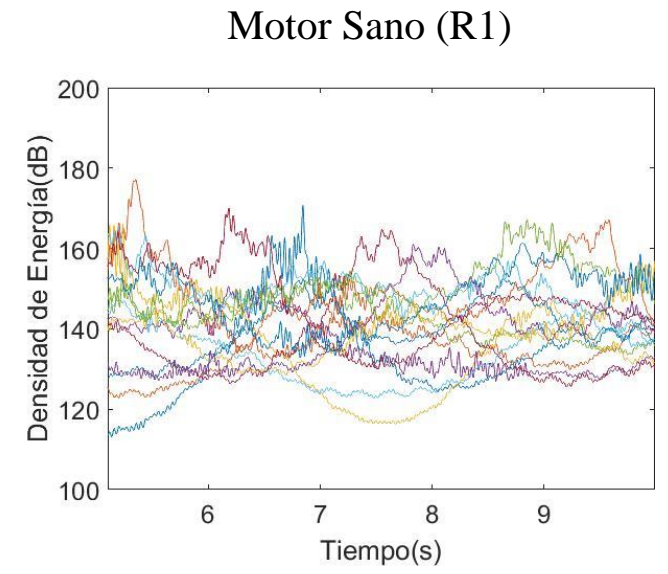

(a) Cuantificación USH IM sano nivel carga bajo.

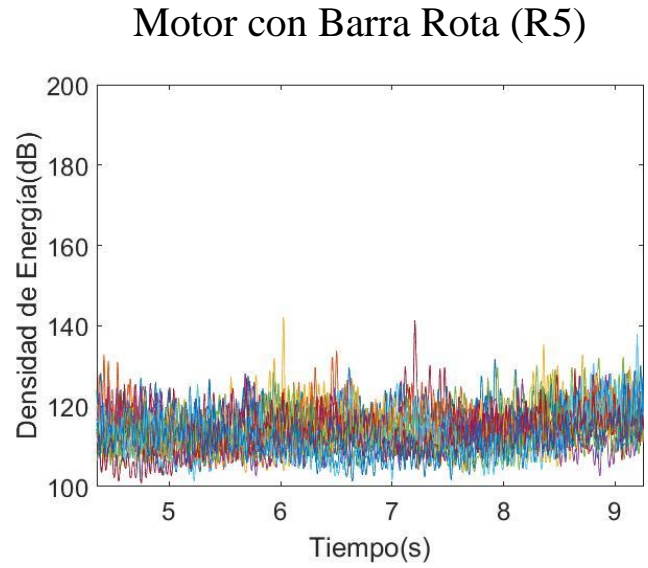

(b) Cuantificación USH IM BRB nivel carga bajo.

Figura 105. Cuantificación del fallo asociado al USH con nivel de carga bajo, inversor Siemens.

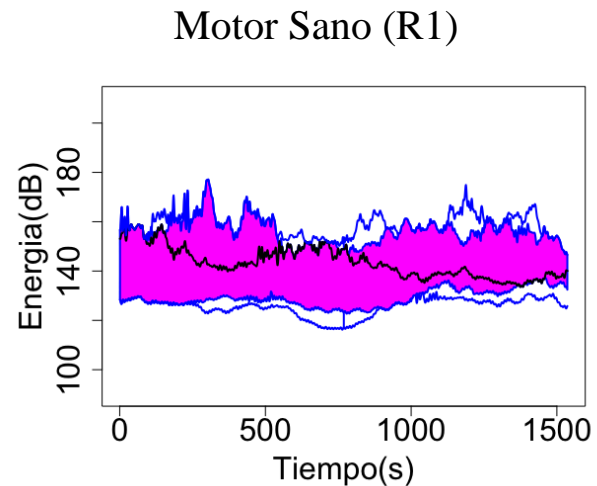

(a) Functional boxplot del USH IM sano nivel carga bajo.
Motor con Barra Rota (R5)

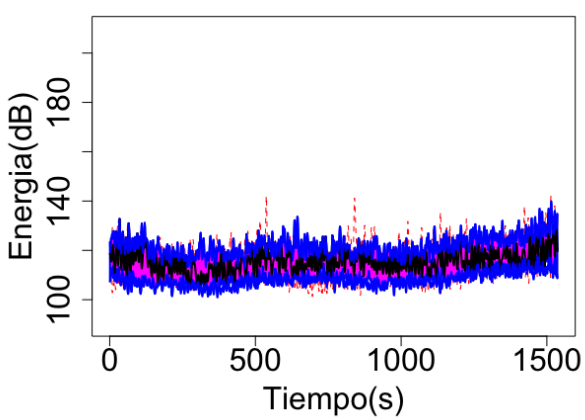

(b) Functional boxplot del USH IM BRB nivel carga bajo.

Figura 106. Functional boxplot del fallo asociado al USH con nivel de carga bajo, inversor Siemens.

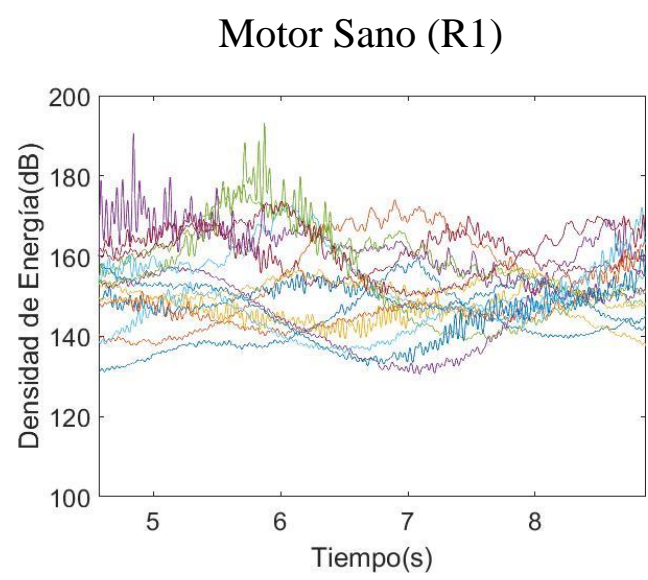

(a) Cuantificación USH IM sano nivel carga alto.
Motor con Barra Rota (R5)

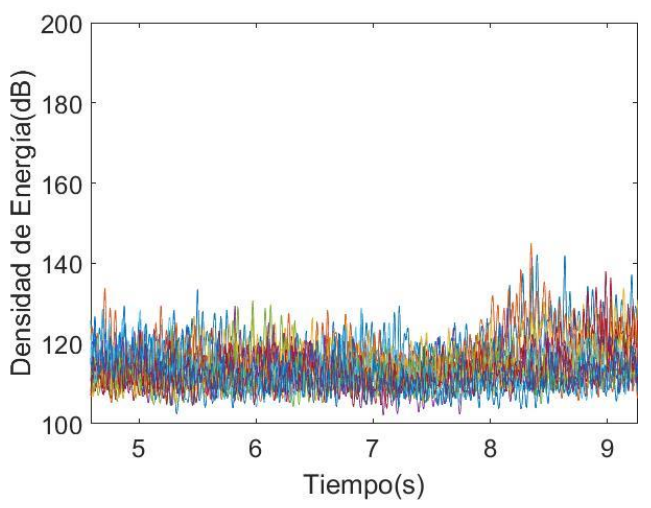

(b) Cuantificación USH IM BRB nivel carga alto.

Figura 107. Cuantificación del fallo asociado al USH con nivel de carga alto, inversor Siemens. 


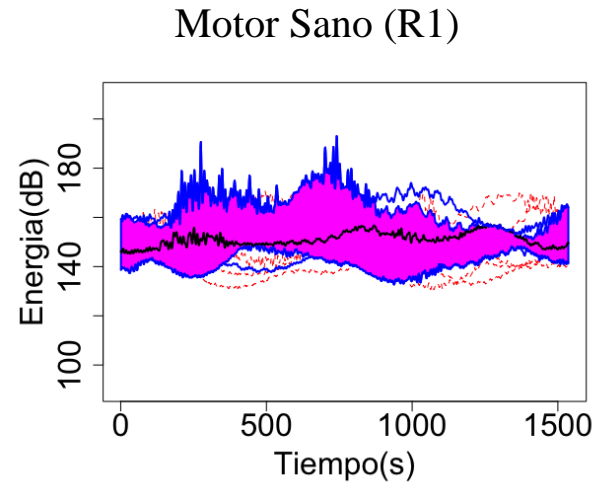

(a) Functional boxplot del USH IM sano nivel carga alto.
Motor con Barra Rota (R5)

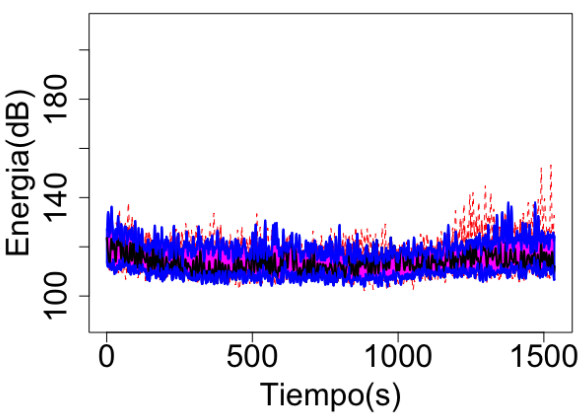

(b) Functional boxplot del USH IM BRB nivel carga alto.

Figura 108. Functional boxplot del fallo asociado al USH con nivel de carga alto, inversor Siemens.

En las Figuras 109 a 116 se analizan las curvas de cuantificación resultantes para el caso de un motor que se alimenta a través de un inversor de Telemecánica. Para el análisis se procede de manera análoga a en el resto de los casos, siendo:

- Figura 109: curvas de cuantificación: $L S H$ y nivel de carga bajo.

- Figura 110: Functional Boxplot de las curvas de la Figura 117.

- Figura 111: curvas cuantificación: LSH y nivel de carga alto.

- Figura 112: Functional Boxplot de las curvas de la Figura 119.

- Figura 113: curvas cuantificación: USH y nivel de carga bajo.

- Figura 114: Functional Boxplot de las curvas de la Figura 121.

- Figura 115: curvas para el USH con nivel de carga alto.

- Figura 116: Functional Boxplot de las curvas de la Figura 123.

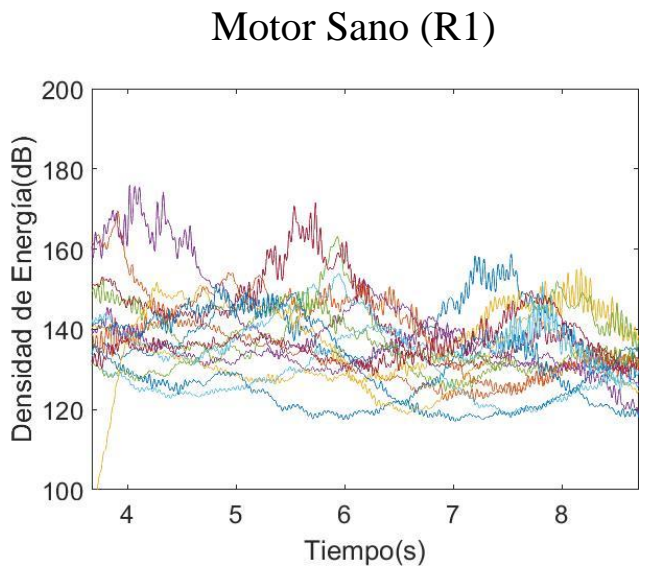

(a) Cuantificación LSH IM sano nivel carga bajo.

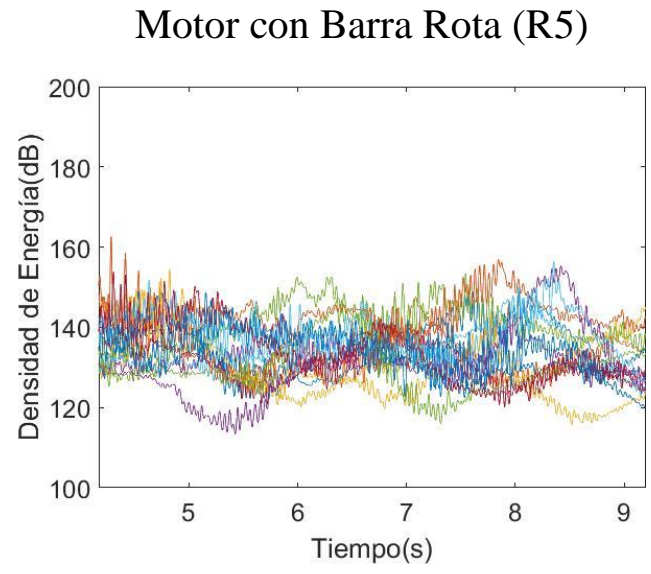

(b) Cuantificación LSH IM BRB nivel carga bajo

Figura 109. Cuantificación del fallo asociado al $L S H$ con nivel de carga bajo, inversor Telemecánica. 


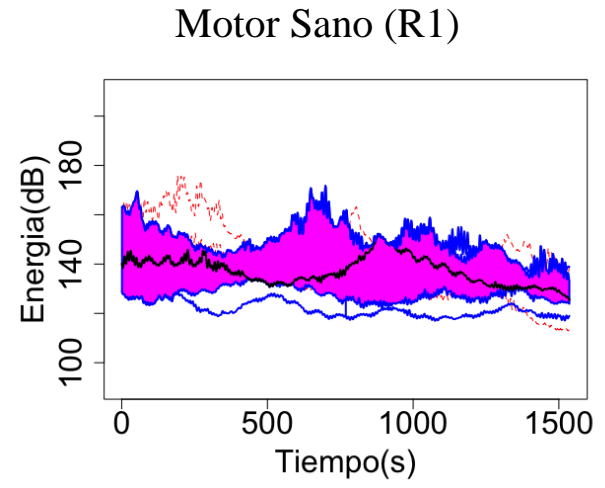

(a) Functional boxplot del LSH IM sano nivel carga bajo.
Motor con Barra Rota (R5)

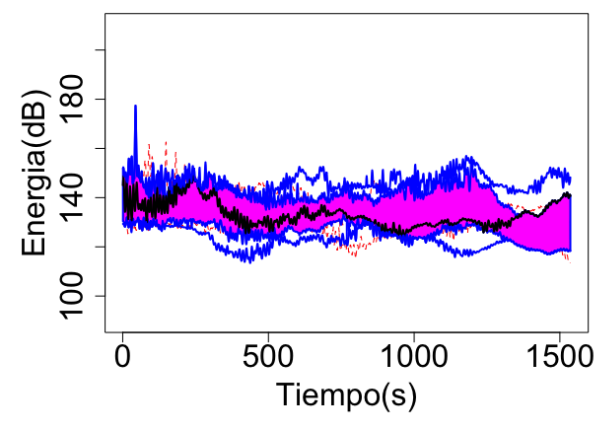

(b) Functional boxplot del LSH IM BRB nivel carga bajo

Figura 110. Functional boxplot del fallo asociado al LSH con nivel de carga bajo, inversor Telemecánica.

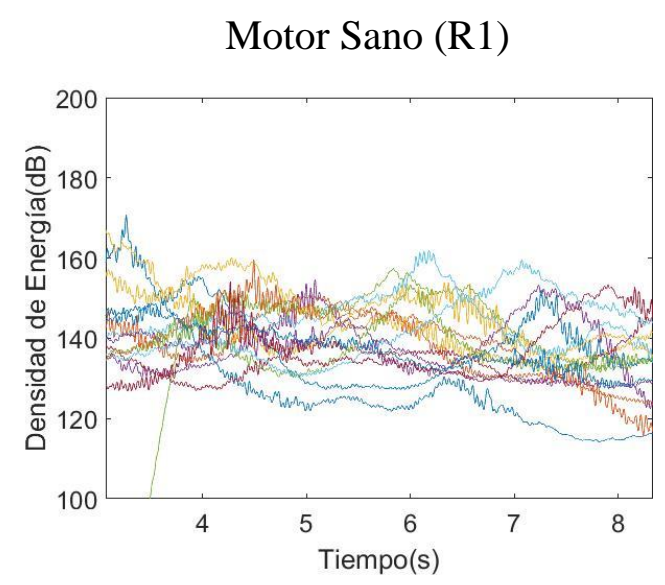

(a) Cuantificación LSH IM sano nivel carga alto.

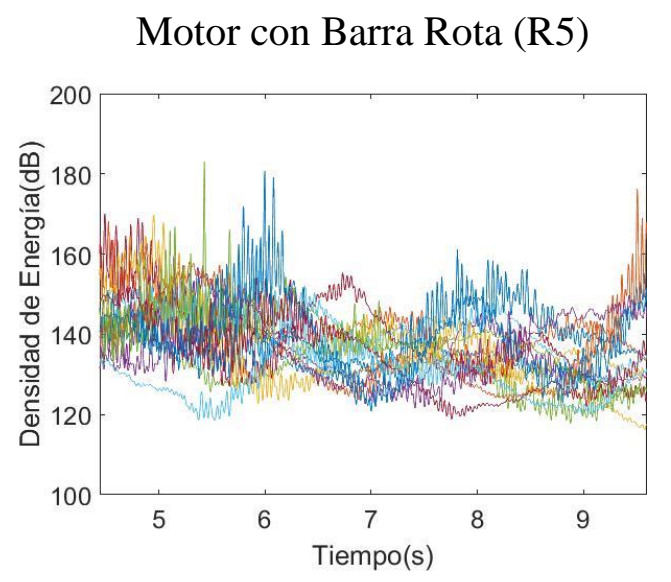

(b) Cuantificación LSH IM BRB nivel carga alto.

Figura 111. Cuantificación del fallo asociado al LSH con nivel de carga alto, inversor Telemecánica.

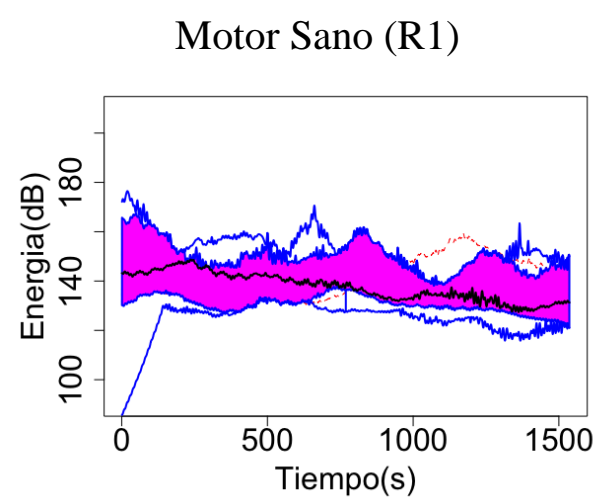

(a) Functional boxplot del LSH IM sano nivel carga alto.
Motor con Barra Rota (R5)

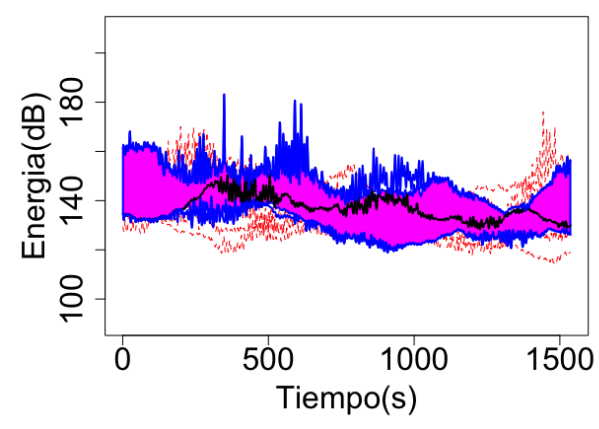

(b) Functional boxplot del LSH IM BRB nivel carga alto.

Figura 112. Functional boxplot del fallo asociado al $L S H$ con nivel de carga alto, inversor Telemecánica. 


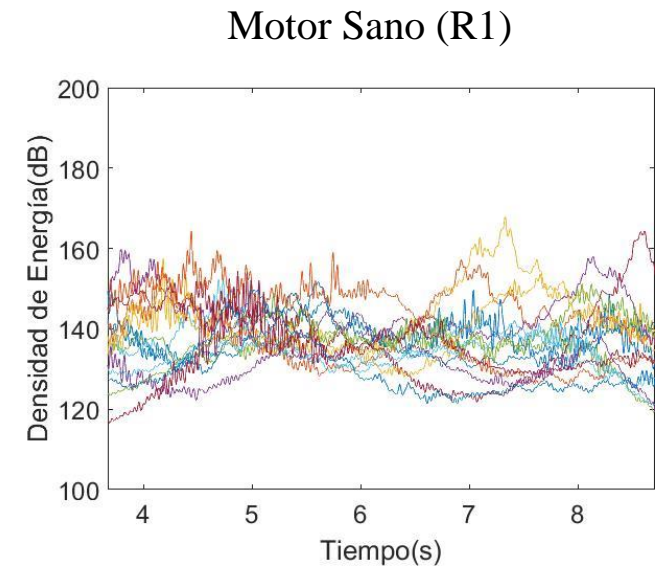

(a) Cuantificación USH IM sano nivel carga bajo.

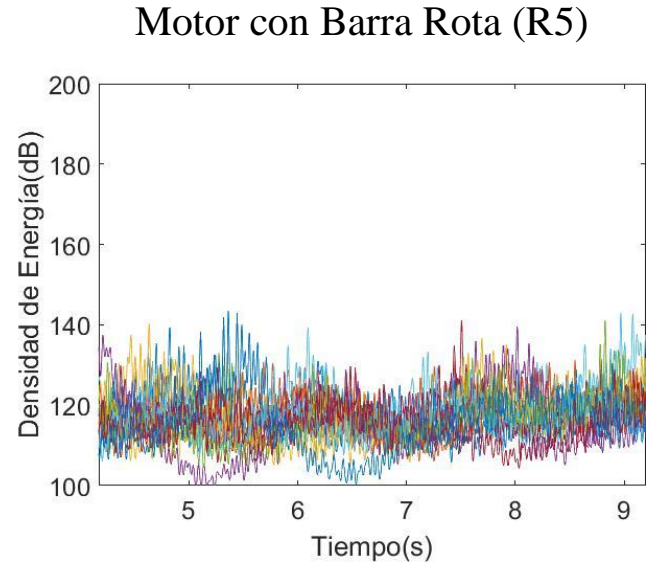

(b) Cuantificación USH IM BRB nivel carga bajo.

Figura 113. Cuantificación del fallo asociado al USH con nivel de carga bajo, inversor Telemecánica.

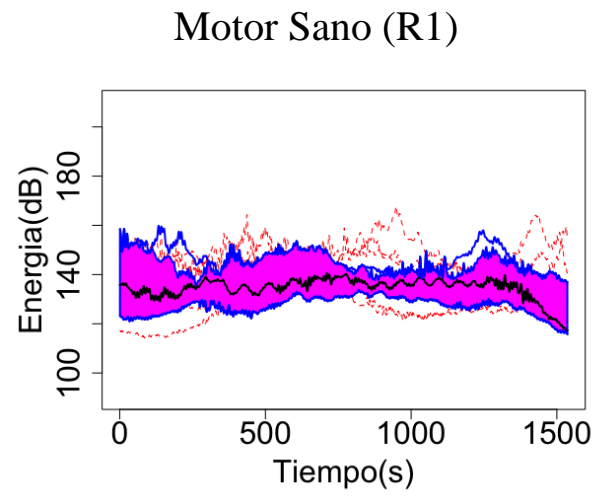

(a) Functional boxplot del USH IM sano nivel carga bajo.
Motor con Barra Rota (R5)

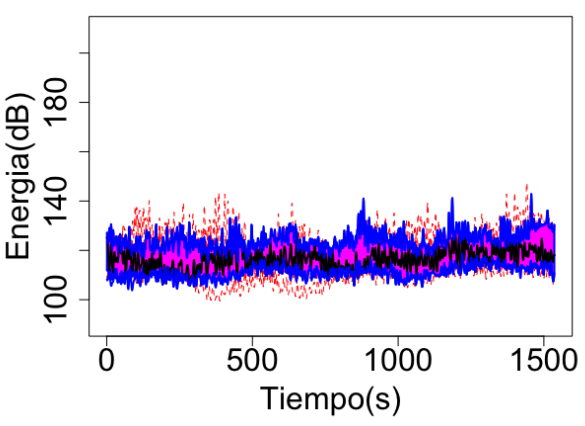

(b) Functional boxplot del USH IM BRB nivel carga bajo.

Figura 114. Functional boxplot del fallo asociado al USH con nivel de carga bajo, inversor Telemecánica.

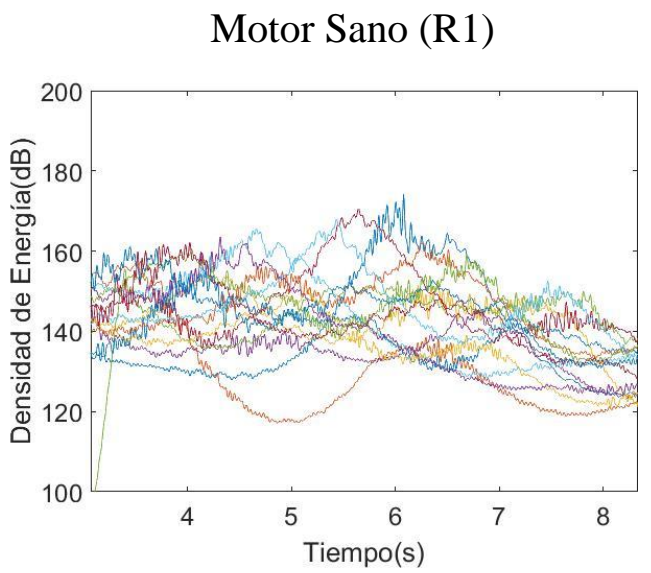

(a) Cuantificación USH IM sano nivel carga alto.

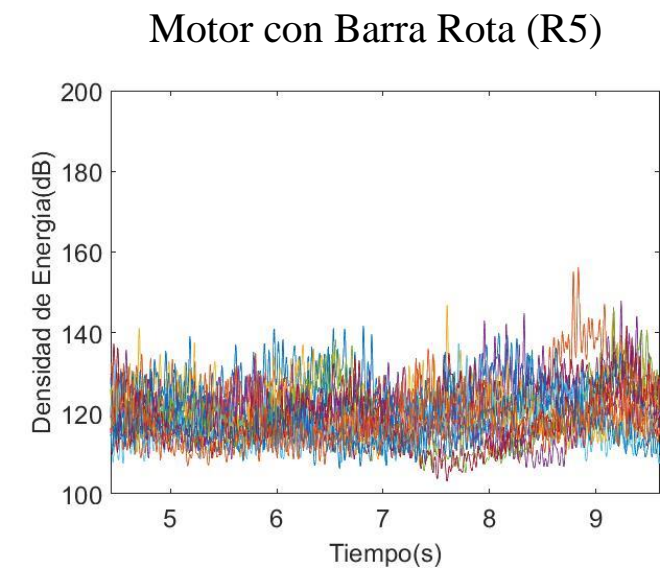

(b) Cuantificación USH IM BRB nivel carga alto.

Figura 115. Cuantificación del fallo asociado al USH con nivel de carga alto, inversor Telemecánica. 


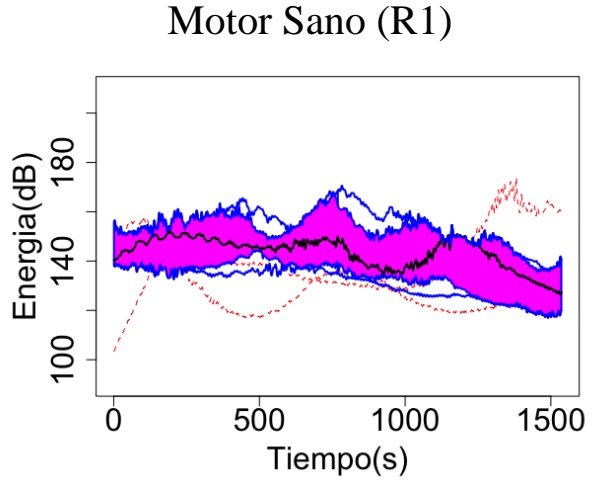

(a) Functional boxplot del USH IM sano nivel carga alto.
Motor con Barra Rota (R5)

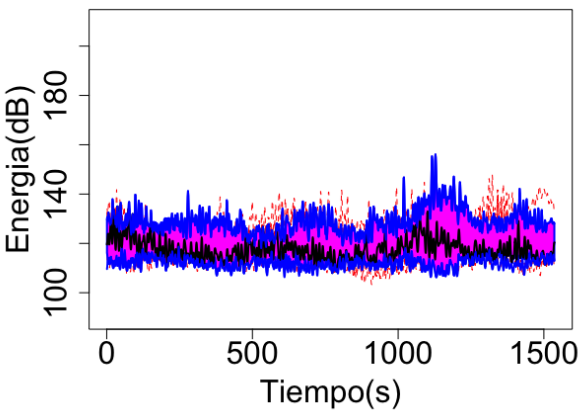

(b) Functional boxplot del USH IM BRB nivel carga alto.

Figura 116. Functional boxplot del fallo asociado al USH con nivel de carga alto, inversor Telemecánica.

Por último, en las Figuras 117 a 124, se analizan las curvas de cuantificación del motor alimentado mediante el inversor WEG, se analiza de la misma manera, por lo que:

- Figura 117: curvas de cuantificación: $L S H$ y nivel de carga bajo.

- Figura 118: Functional Boxplot de las curvas de la Figura 117.

- Figura 119: curvas cuantificación: $L S H$ y nivel de carga alto.

- Figura 120: Functional Boxplot de las curvas de la Figura 119.

- Figura 121: curvas cuantificación: USH y nivel de carga bajo.

- Figura 122: Functional Boxplot de las curvas de la Figura 121.

- Figura 123: curvas para el $U S H$ con nivel de carga alto.

- Figura 124: Functional Boxplot de las curvas de la Figura 123.

Motor Sano (R1)

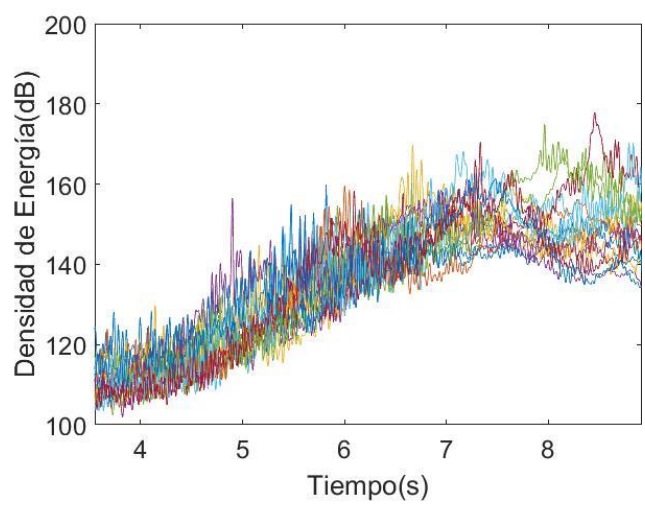

(a) Cuantificación LSH IM sano nivel carga bajo.
Motor con Barra Rota (R5)

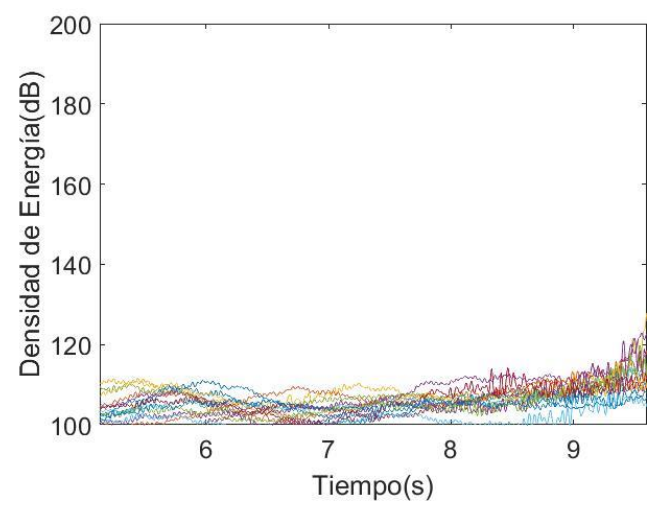

(b) Cuantificación $L S H I M B R B$ nivel carga bajo

Figura 117. Cuantificación del fallo asociado al $L S H$ con nivel de carga bajo, inversor WEG. 


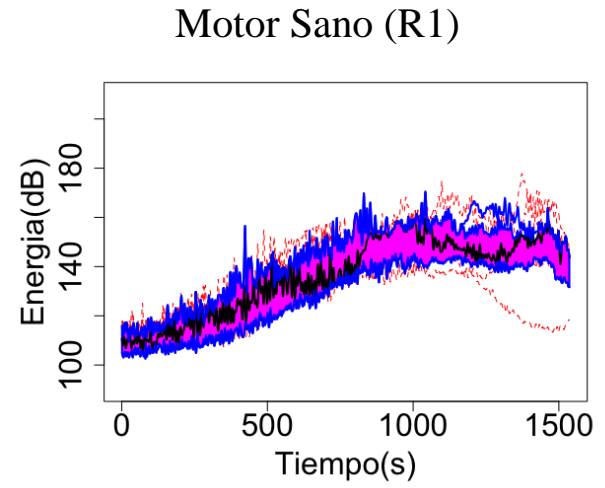

(a) Functional boxplot del LSH IM sano nivel carga bajo.
Motor con Barra Rota (R5)

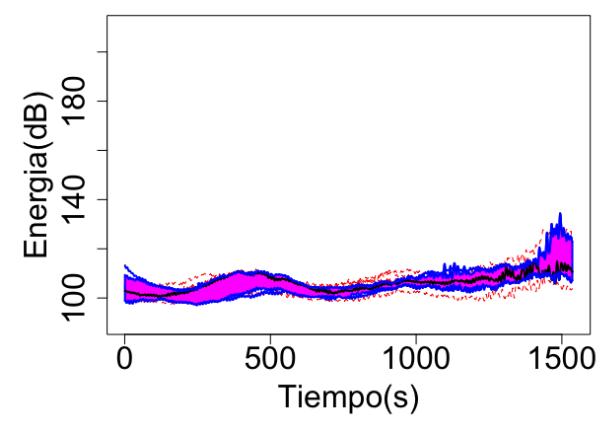

(b) Functional boxplot del LSH IM BRB nivel carga bajo

Figura 118. Functional boxplot del fallo asociado al $L S H$ con nivel de carga bajo, inversor WEG.

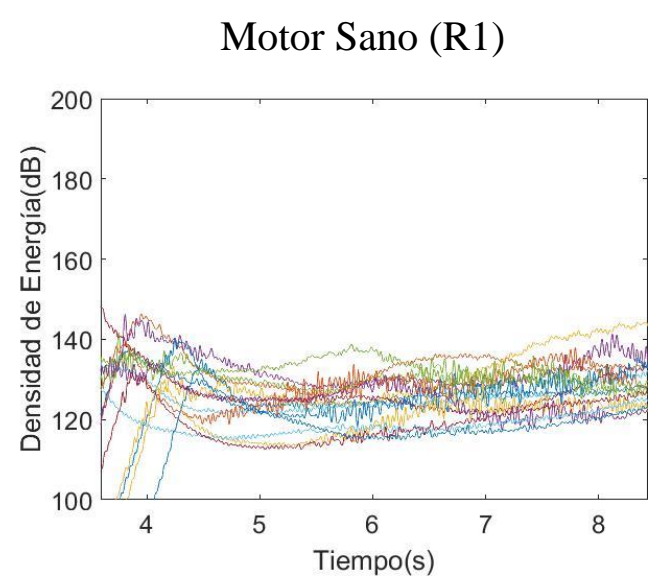

(a) Cuantificación LSH IM sano nivel carga alto.

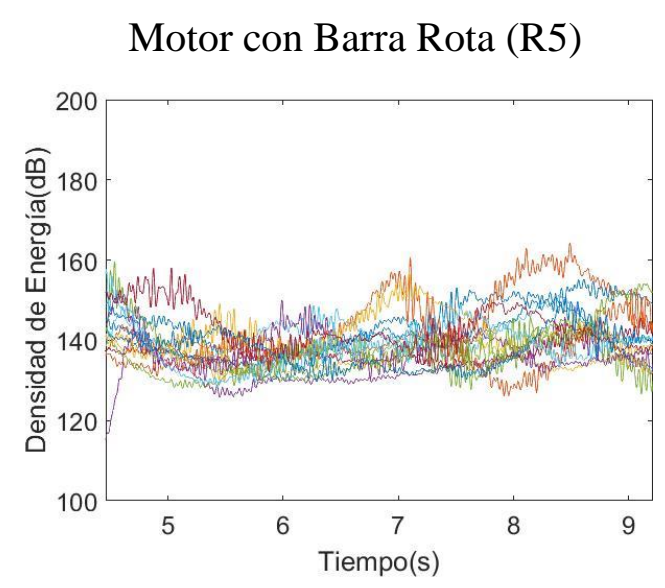

(b) Cuantificación LSH IM BRB nivel carga alto.

Figura 119. Cuantificación del fallo asociado al $L S H$ con nivel de carga alto, inversor WEG.

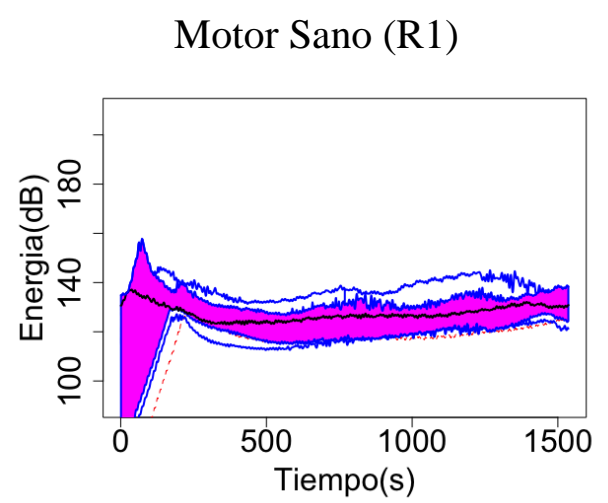

(a) Functional boxplot del LSH IM sano nivel

$$
\text { carga alto. }
$$

\section{Motor con Barra Rota (R5)}

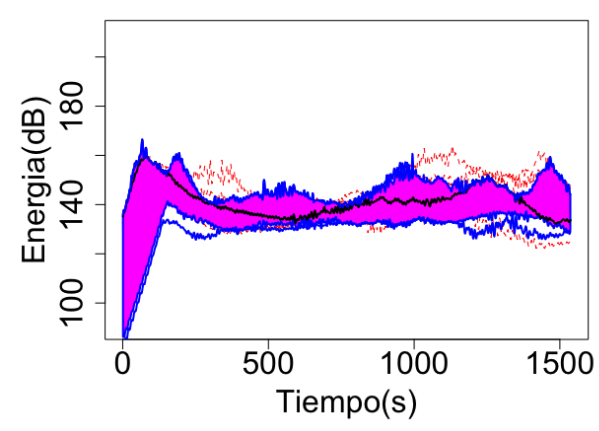

(b) Functional boxplot del LSH IM BRB nivel carga alto.

Figura 120. Functional boxplot del fallo asociado al $L S H$ con nivel de carga alto, inversor WEG. 


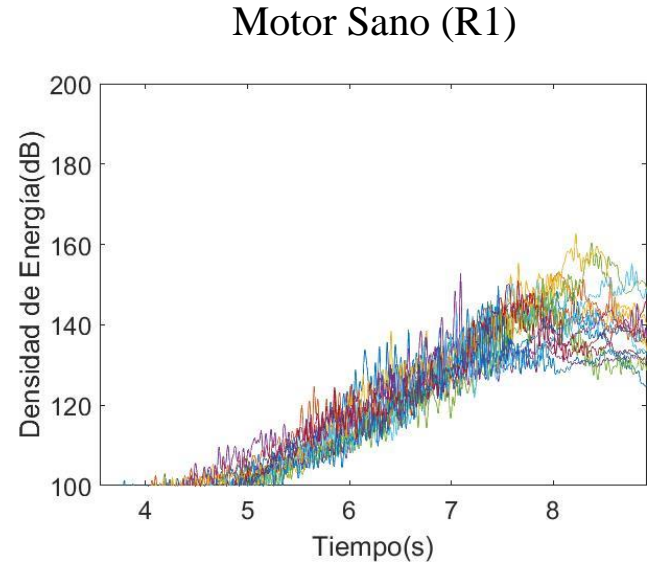

(a) Cuantificación USH IM sano nivel carga bajo.

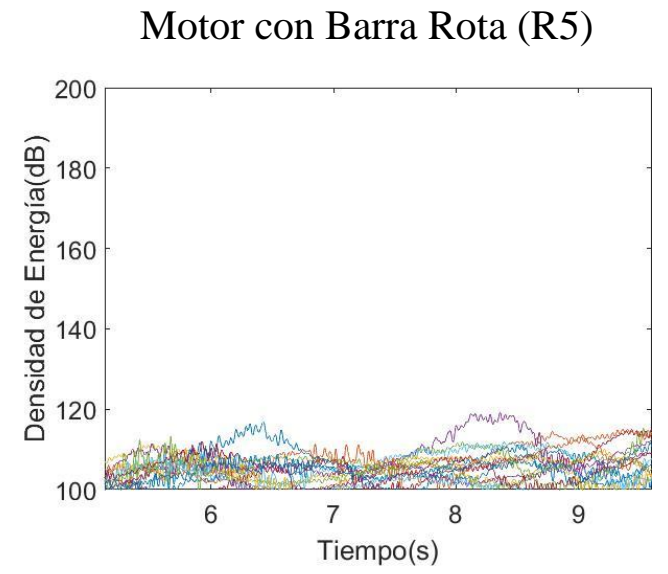

(b) Cuantificación USH IM BRB nivel carga bajo.

Figura 121. Cuantificación del fallo asociado al USH con nivel de carga bajo, inversor WEG.

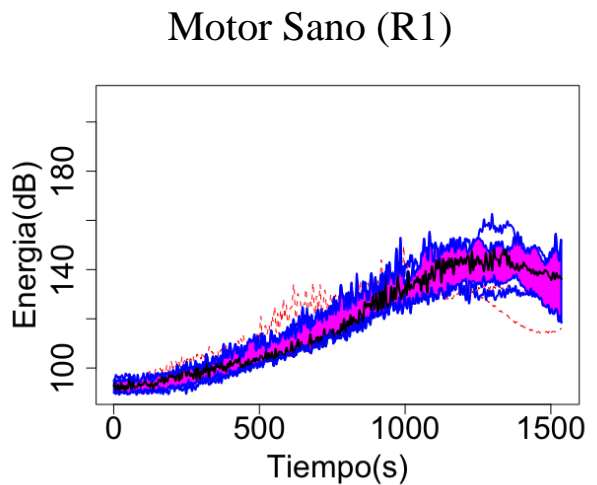

(a) Functional boxplot del USH IM sano nivel carga bajo.
Motor con Barra Rota (R5)

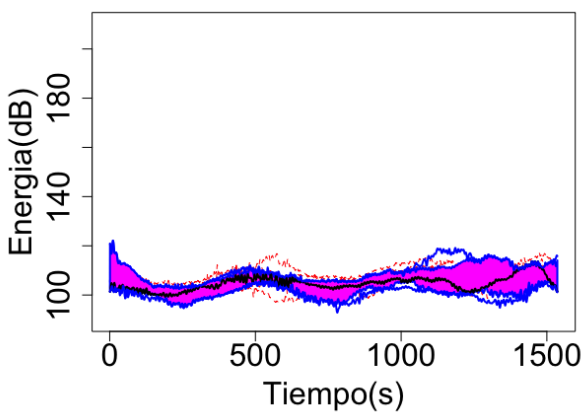

(b) Functional boxplot del USH IM BRB nivel carga bajo.

Figura 122. Functional boxplot del fallo asociado al USH con nivel de carga bajo, inversor WEG.

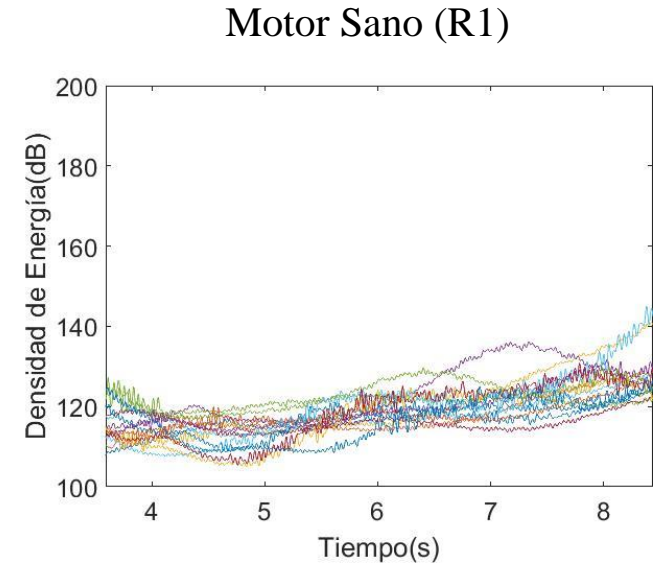

(a) Cuantificación USH IM sano nivel carga alto.

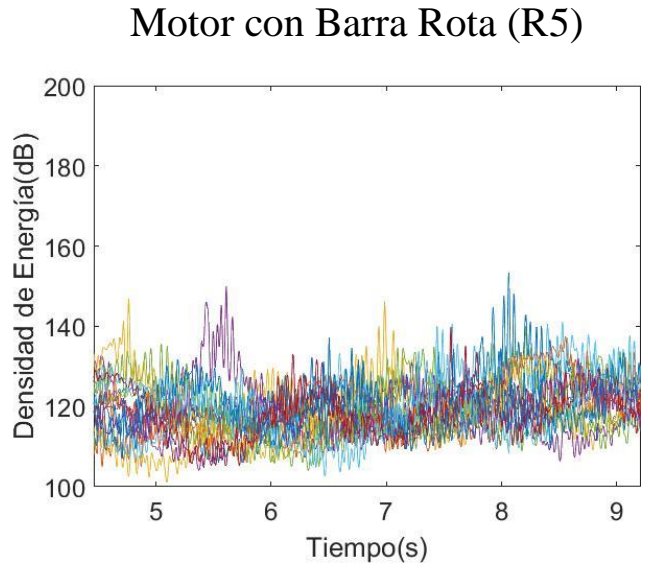

(b) Cuantificación USH IM BRB nivel carga alto.

Figura 123. Cuantificación del fallo asociado al $U S H$ con nivel de carga alto, inversor WEG. 


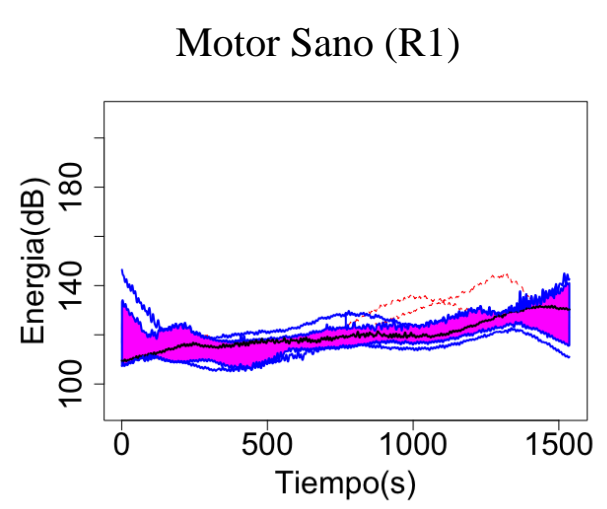

(a) Functional boxplot del USH IM sano nivel carga alto.
Motor con Barra Rota (R5)

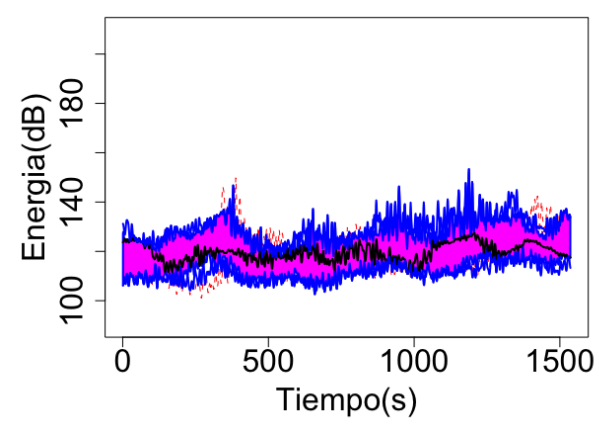

(b) Functional boxplot del USH IM BRB nivel carga alto.

Figura 124. Functional boxplot del fallo asociado al USH con nivel de carga alto, inversor WEG.

En la Tabla 10 se realiza un resumen de las conclusiones sacadas tras analizar las curvas de cuantificación de las Figuras 85 a 124, para cada nivel de carga y clase de armónico. Se especifica si se identifica bien el fallo, si aparecen outliers y otras observaciones. 


\begin{tabular}{|c|c|c|c|c|c|c|}
\hline & Allen-Bradley & ABB & Siemens & Telemecánica & WEG \\
\hline \multirow[t]{2}{*}{$\begin{array}{l}\text { NC1 } \\
\text { (nivel } \\
\text { carga } \\
\text { bajo) }\end{array}$} & LSH & $\begin{array}{l}\text { Se aprecia el } \\
\text { fallo, pero no } \\
\text { hay diferencia } \\
\text { notable de } \\
\text { amplitud con el } \\
\text { sano }\end{array}$ & $\begin{array}{l}\text { Se aprecia el } \\
\text { fallo, pero no } \\
\text { hay diferencia } \\
\text { notable de } \\
\text { amplitud con } \\
\text { el sano }\end{array}$ & $\begin{array}{l}\text { Sí se aprecia } \\
\text { el fallo, pero } \\
\text { no hay } \\
\text { diferencia } \\
\text { notable }\end{array}$ & $\begin{array}{l}\text { No se observa } \\
\text { diferencia de } \\
\text { amplitud con el } \\
\text { sano }\end{array}$ & $\begin{array}{l}\text { Sí se aprecia el fallo, } \\
\text { con una gran } \\
\text { diferencia de } \\
\text { amplitud con el sano }\end{array}$ \\
\hline & $U S H$ & $\begin{array}{l}\text { Se observa muy } \\
\text { bien, diferencia } \\
\text { de amplitud es } \\
\text { notable }\end{array}$ & $\begin{array}{l}\text { Se observa } \\
\text { muy bien, } \\
\text { diferencia de } \\
\text { amplitud es } \\
\text { notable }\end{array}$ & $\begin{array}{l}\text { Se observa } \\
\text { muy bien, } \\
\text { diferencia de } \\
\text { amplitud es } \\
\text { notable }\end{array}$ & $\begin{array}{l}\text { Se observa muy } \\
\text { bien, diferencia } \\
\text { de amplitud es } \\
\text { notable }\end{array}$ & $\begin{array}{l}\text { Sí se aprecia el fallo, } \\
\text { con una gran } \\
\text { diferencia de } \\
\text { amplitud con el sano }\end{array}$ \\
\hline \multirow[t]{2}{*}{$\begin{array}{l}\mathrm{NC} 2 \\
\text { (nivel } \\
\text { carga } \\
\text { alto) }\end{array}$} & $L S H$ & $\begin{array}{l}\text { Se aprecia el } \\
\text { fallo, pero no } \\
\text { hay diferencia } \\
\text { notable de } \\
\text { amplitud con el } \\
\text { sano }\end{array}$ & $\begin{array}{l}\text { Se aprecia el } \\
\text { fallo, pero no } \\
\text { hay diferencia } \\
\text { notable de } \\
\text { amplitud con } \\
\text { el sano } \\
\end{array}$ & $\begin{array}{l}\text { Sí se aprecia } \\
\text { el fallo, pero } \\
\text { no hay } \\
\text { diferencia } \\
\text { notable }\end{array}$ & $\begin{array}{l}\text { No se observa } \\
\text { diferencia de } \\
\text { amplitud con el } \\
\text { sano }\end{array}$ & $\begin{array}{l}\text { No se aprecia el } \\
\text { fallo, (aumenta en } \\
\text { lugar de disminuir) }\end{array}$ \\
\hline & $U S H$ & $\begin{array}{l}\text { Se observa muy } \\
\text { bien, diferencia } \\
\text { de amplitud es } \\
\text { notable }\end{array}$ & $\begin{array}{l}\text { Se observa } \\
\text { muy bien, } \\
\text { diferencia de } \\
\text { amplitud es } \\
\text { notable } \\
\end{array}$ & $\begin{array}{l}\text { Se observa } \\
\text { muy bien, } \\
\text { diferencia de } \\
\text { amplitud es } \\
\text { notable }\end{array}$ & $\begin{array}{l}\text { Se observa muy } \\
\text { bien, diferencia } \\
\text { de amplitud es } \\
\text { notable }\end{array}$ & $\begin{array}{l}\text { No se observa bien el } \\
\text { fallo }\end{array}$ \\
\hline \multicolumn{2}{|c|}{ Outliers } & $\begin{array}{l}\text { Sí, pero no } \\
\text { significativos }\end{array}$ & $\begin{array}{l}\text { Sí, más en el } \\
\text { caso del motor } \\
\text { sano }\end{array}$ & $\begin{array}{l}\text { Sí, más en el } \\
\text { motor sano }\end{array}$ & $\begin{array}{l}\text { Sí, número más } \\
\text { elevado que en } \\
\text { los otros } \\
\text { inversores }\end{array}$ & Apenas presenta \\
\hline \multicolumn{2}{|c|}{ Observaciones } & $\begin{array}{l}\text { Se aprecia } \\
\text { cuando existe } \\
\text { fallo, ya que la } \\
\text { banda con los } \\
\text { valores medios } \\
\text { disminuye, } \\
\text { apreciándose } \\
\text { mejor en el } \\
\text { caso del } U S H \\
\text { (tanto con } \mathrm{NC} 1 \\
\text { con NC2) }\end{array}$ & $\begin{array}{l}\text { Se aprecia } \\
\text { cuando existe } \\
\text { fallo, siendo } \\
\text { más notable en } \\
\text { el caso del } \\
\text { USH (con } \\
\text { ambos niveles } \\
\text { de carga) } \\
\text { Aparecen } \\
\text { perturbaciones } \\
\text { al analizar el } \\
\text { motor con fallo }\end{array}$ & $\begin{array}{l}\text { Se aprecia } \\
\text { cuando existe } \\
\text { el fallo, más } \\
\text { acusadamente } \\
\text { en el caso del } \\
\text { USH }\end{array}$ & $\begin{array}{l}\text { Comportamiento } \\
\text { extraño e } \\
\text { introduce mucha } \\
\text { perturbación }\end{array}$ & $\begin{array}{l}\text { Comportamiento } \\
\text { distinto al resto de } \\
\text { los inversores }\end{array}$ \\
\hline
\end{tabular}

Tabla 10. Tabla resumen respuesta a cuantificación.

\subsubsection{Conclusiones parciales.}

Tras analizar las curvas de cuantificación de fallo de cada uno de los inversores con los que se ha trabajado, se puede llegar a las conclusiones que se exponen a continuación.

La primera conclusión que se saca a primera vista es que al presentar un fallo el motor, el valor en $\mathrm{dB}$ de la media de las curvas baja con respecto al valor que se obtiene cuando el motor está sano. Se puede confirmar tanto al analizar el $L S H$ como al analizar 
el $U S H$. Esta variación en el valor es mucho más acusada en el caso del USH, como puede apreciarse a simple vista con todos los casos de todos los inversores. En el caso del motor alimentado con inversor Siemens, esta diferencia entre el valor medio de las curvas asociadas al USH entre motor con fallo y motor sano es mucho más acusada que en el resto de los inversores.

Por otra parte, el comportamiento de los diversos inversores es análogo, en cuanto que los valores de las curvas se encuentran en el mismo rango. Sin embargo, el caso del inversor WEG es distinto al resto $(\mathrm{AB}, \mathrm{ABB}$, Siemens, Telemecánica), presentando valores diferentes, así como formas de curva distintas al resto de los casos.

En cuanto al análisis mediante Functional Boxplot, éste se ha llevado a cabo con cada uno de los inversores analizados, analizando para cada inversor cada caso posible, es decir: $L S H$ nivel de carga bajo, $L S H$ nivel de carga alto, $U S H$ nivel de carga bajo, $U S H$ nivel de carga alto. Para cada uno de estos casos se han analizado un total de 15 ensayos, es decir, se analizan las 15 curvas de cuantificación obtenidas; buscando con este método los outliers de los ensayos realizados y comprobar así que la cuantificación se ha llevado a cabo de una manera correcta.

Para todos los casos se obtiene que los valores se encuentran mayoritariamente dentro del rango establecido (zona fucsia), presentando algún outlier. Por otra parte, en todos los casos se verifica que motor es el que presenta fallo, ya que el valor promedio (definido mediante una curva negra en las gráficas) de la energía de las curvas analizadas baja en comparación con el valor que tiene cuando el motor está sano. Esta diferencia de valor es más acusada en el caso del análisis del $U S H$, en donde se ve de una manera más sencilla esta diferencia de valor.

Al observar los resultados obtenidos (resumidos en la Tabla 10), se aprecia que para los inversores Allen-Bradley, ABB y Siemens el comportamiento es análogo. El inversor Telemecánica introduce mucha perturbación en el caso del análisis del motor con fallo, salvo en el caso del análisis del $U S H$, aunque permite, igualmente, interpretar los resultados obtenidos. Como cabía esperar, para el inversor WEG se tienen unos resultados que difieren con respecto al resto de inversores, ya que las formas de las curvas 
de las que se parte son diferentes a las de los otros inversores; sin embargo, tras su análisis se puede llegar a las mismas conclusiones.

\subsection{Resultados relativos a la discriminación en motores con compensación por deslizamiento.}

Tras analizar los resultados que se exponen en los epígrafes anteriores (6.2. y 6.3.) se ha descubierto que, al trabajar con inversores, al analizar el estado estacionario, aparece un nuevo componente armónico que no corresponde a ningún tipo de fallo y que puede dar lugar a errores en la detección. Este armónico aparece tanto al trabajar con un motor sano como al trabajar con un motor con fallo.

A continuación, se exponen los resultados experimentales que verifican la aparición de este armónico y que abren una nueva línea de investigación, que se prevé desarrollar más adelante, tal como se explica en el apartado 7 de esta memoria de tesis.

Se comienza el análisis con el inversor Allen-Bradley, tal como se ha hecho en los apartados anteriores, analizando como este parámetro aparece tanto si el motor es sano como si presenta fallo.

\section{Nivel de severidad de fallo R1: Motor sano.}

En el primer caso analizamos un motor en estado sano, alimentado mediante un inversor Allen-Bradley, que, como se explica en el punto 5, presenta un transitorio de 10 segundos, seguido de un estacionario largo, con una duración total de la señal de 30 segundos.

$\mathrm{Al}$ analizar la señal mediante la técnica desarrollada, en la Figura 125 se aprecia como aparece la componente fundamental, y, a su vez, también aparece una componente armónica muy marcada. Esta componente no se visualiza durante el transitorio de arranque, pero sí durante el estacionario, donde se desarrolla paralela al armónico fundamental, como si fuera el LSH. Lo vemos con más detalle en la Figura 125(b), en donde aparece un zoom de este estado estacionario, entre los instantes 15 y 20 segundos. 

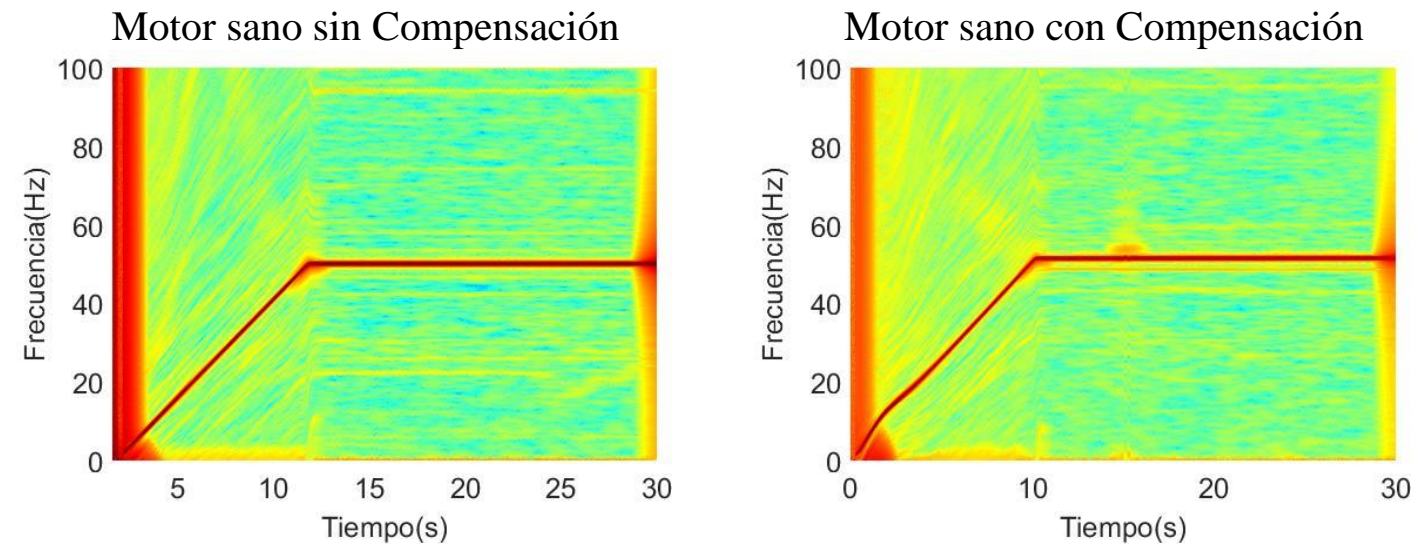

(a) Espectrograma del ensayo
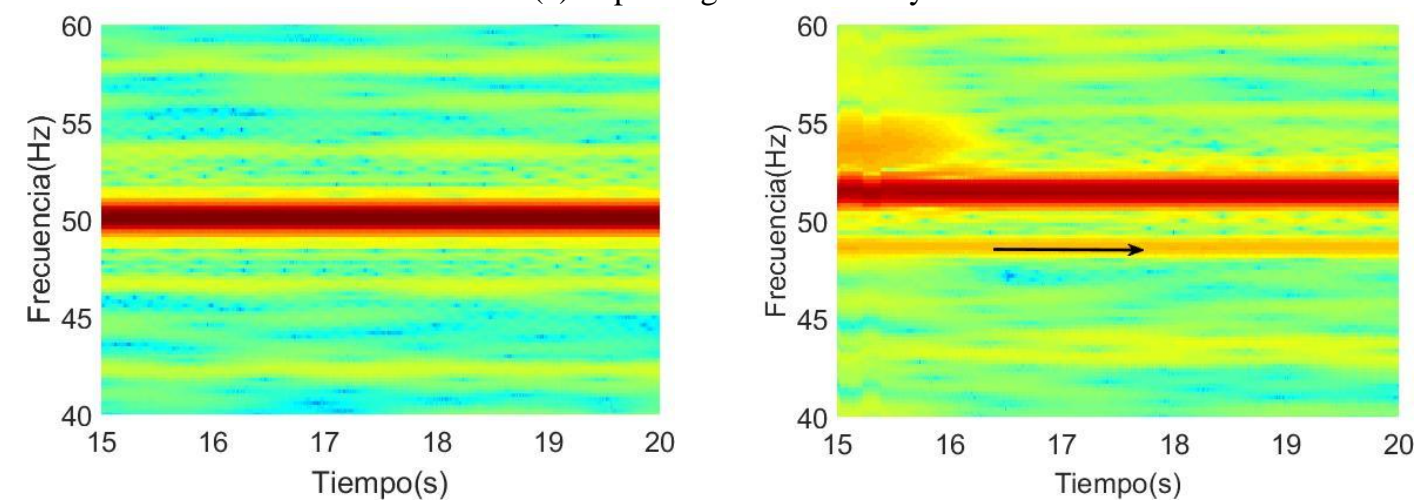

(b) Detalle del espectrograma
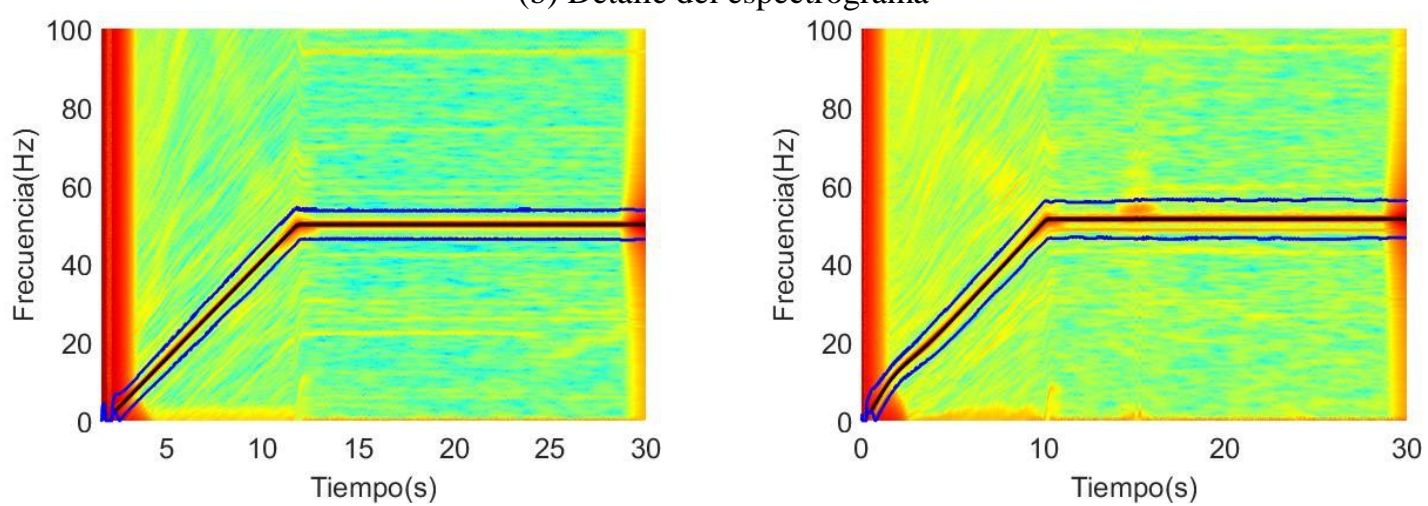

(c) Espectrograma del ensayo y trayectorias de los armónicos de fallo
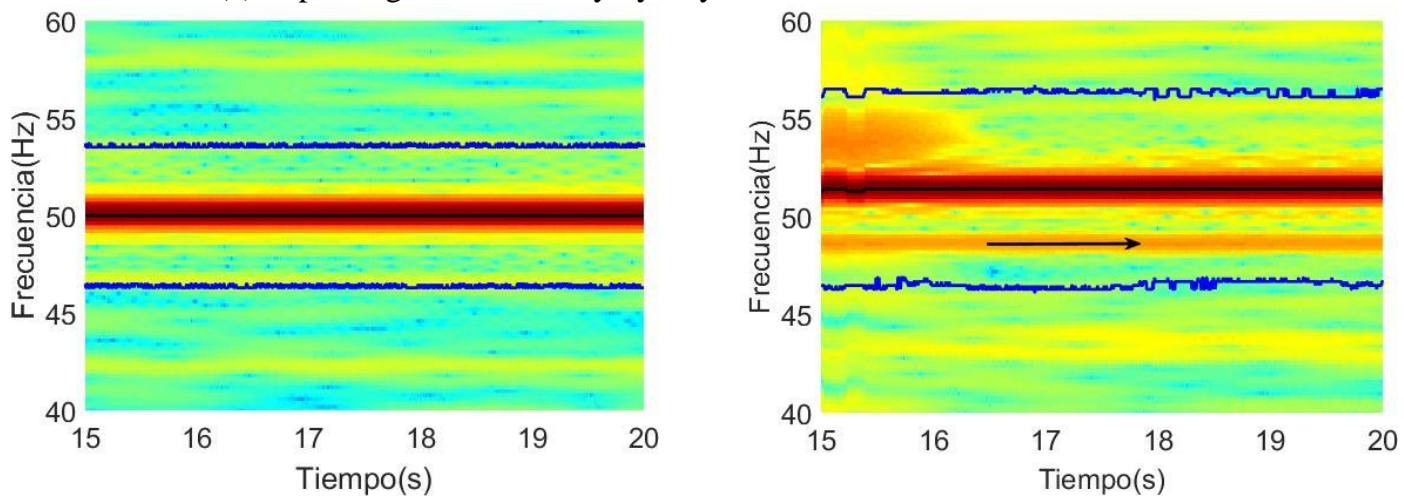

(d) Detalle espectrograma con trayectorias armónicos de fallo 

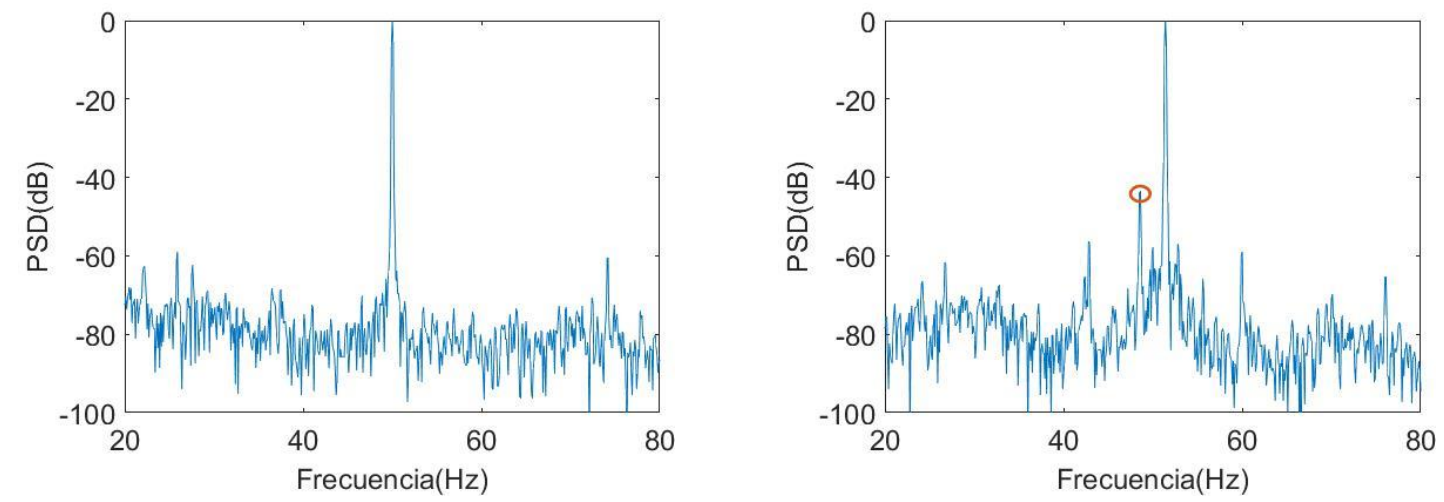

(e) Espectro del estado estacionario

Figura 125. Análisis señal fallo severidad R1, mediante técnica Dragon Transform.

En la Figura 125(c) se ve la trayectoria teórica de los componentes de fallo por barra rota correspondiente al ensayo analizado. Así se puede afirmar que la señal no presenta fallo de este tipo. Hay que tener especial cuidado con no confundir esta componente con la de barra rota, ya que, como se ve en la Figura 125(d), no es coincidente con este armónico. Por tanto, estamos ante un claro ejemplo de falso positivo.

Al observar la Figura 125(d) puede verse como la trayectoria de los componentes de fallo de barras inferior aparece con perturbaciones, esto es debido a la influencia del falso positivo.

Si se analiza la señal mediante la técnica FFT, al presentar falso positivo, debe de aparecer un armónico muy cercano a la componente fundamental, tal y como se puede apreciar en la Figura 125(e). También se puede apreciar como el falso positivo hace que la componente principal ya no esté fija a $50 \mathrm{~Hz}$, si no que se encuentre desplazada hacia la derecha, aumentando su valor.

\section{Nivel de Severidad de fallo R5: Motor con barra completamente rota.}

En segundo lugar se analiza el caso del mismo motor alimentado mediante el mismo inversor (Allen-Bradley), pero ahora presenta fallo por barras rotas.

De la misma manera que con el motor sano, se analiza la batería de ensayos de severidad de fallo R5, con la técnica desarrollada, en busca de un falso positivo. Un ejemplo de lo obtenido se aprecia en la Figura 126. 

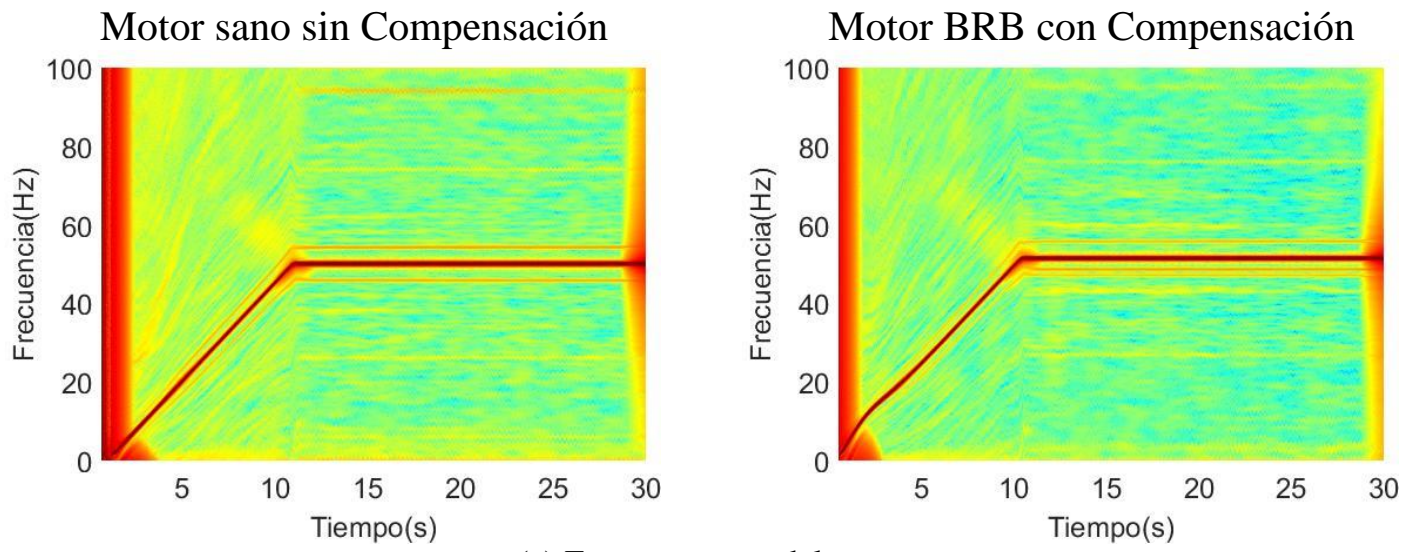

(a) Espectrograma del ensayo
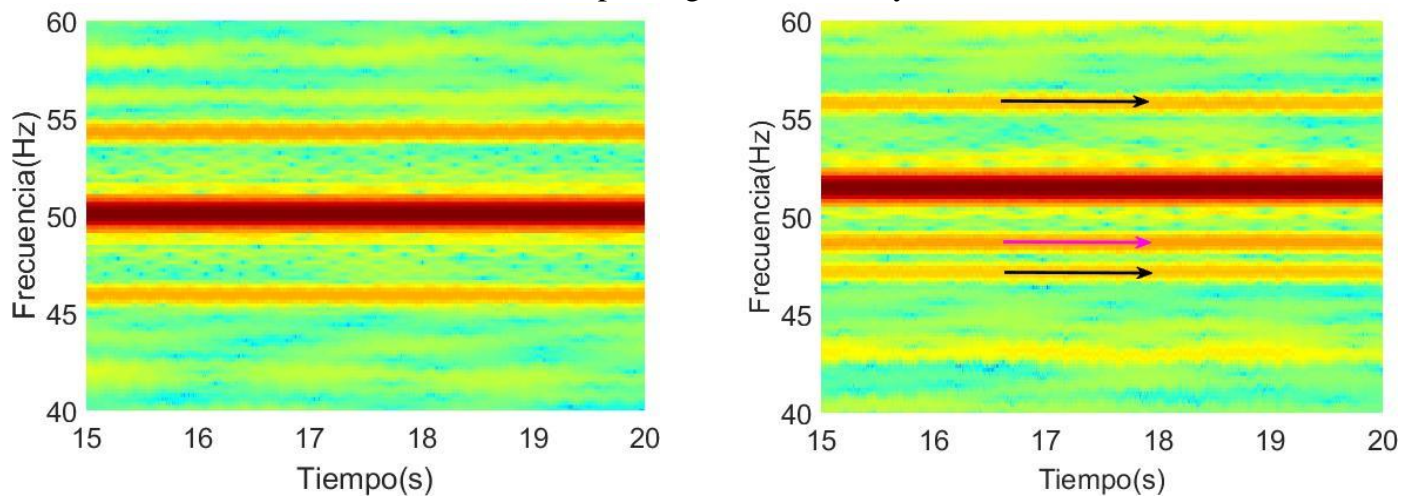

(b) Detalle del espectrograma
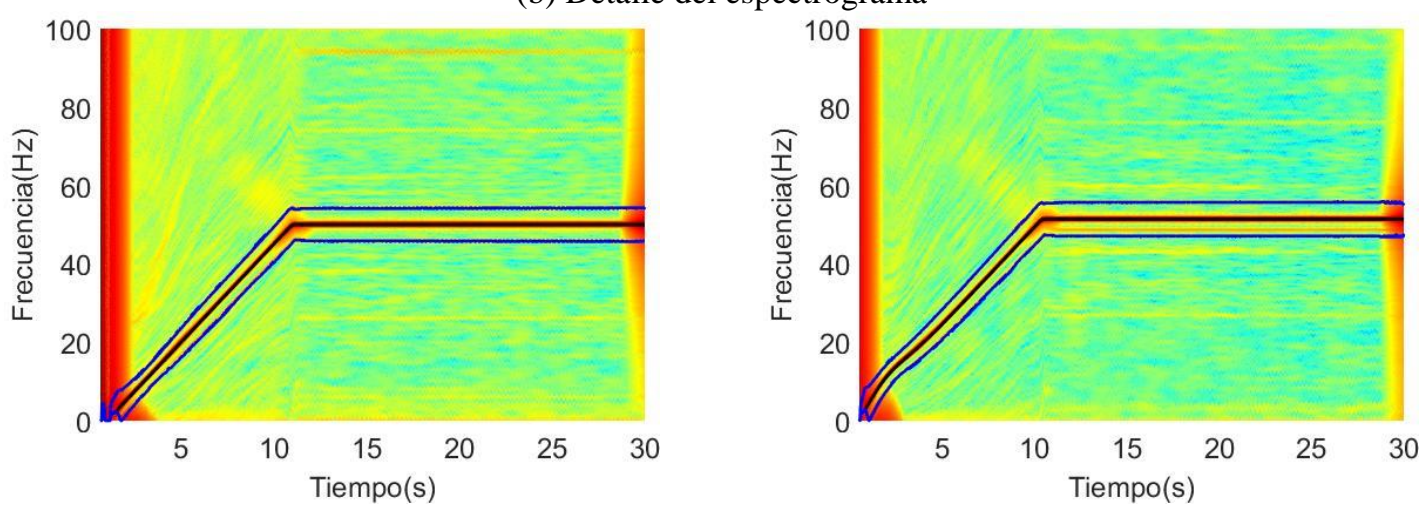

(c) Espectrograma del ensayo y trayectorias de los armónicos de fallo
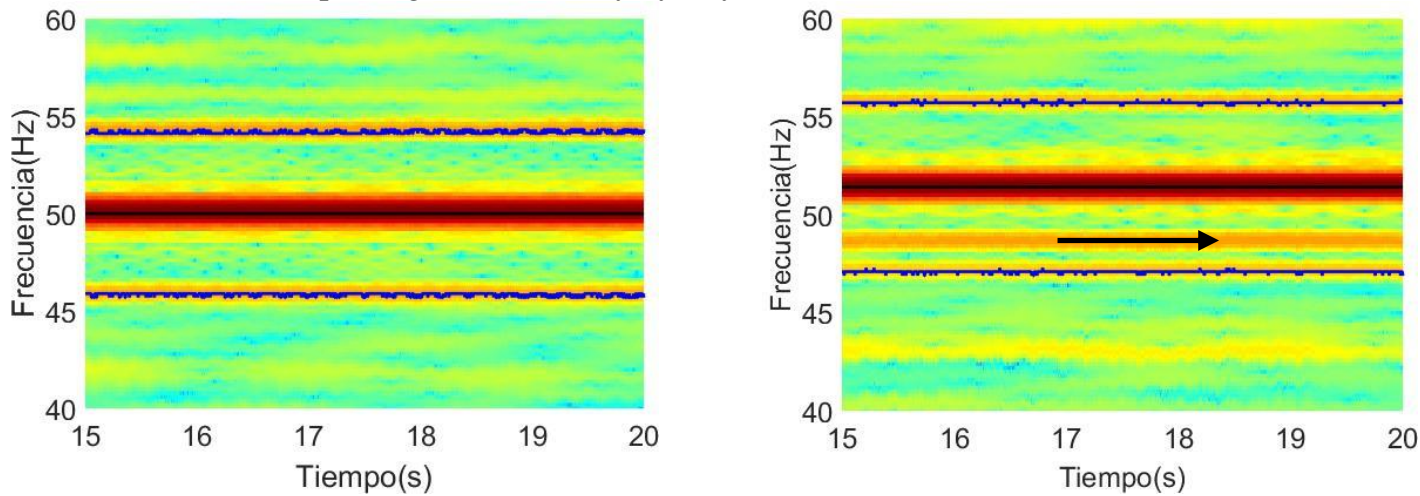

(d) Detalle espectrograma con trayectorias armónicos de fallo 

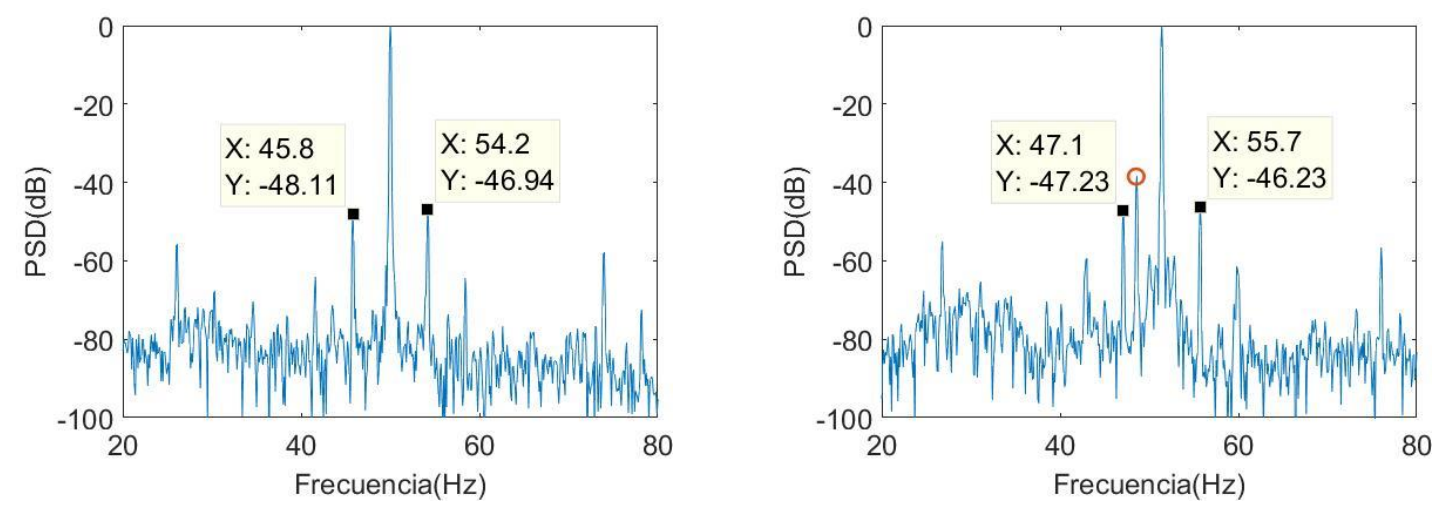

(e) Espectro del estado estacionario

Figura 126. Análisis señal fallo severidad R5, mediante técnica Dragon Transform.

Para verlo en más detalle, vemos en la Figura 126(b), en detalle una parte del estacionario, en donde se puede distinguir fallo de barras $(\rightarrow)$ y el armónico debido al falso positivo $(\rightarrow)$.

En la Figura 126(c) aparecen las trayectorias teóricas de los armónicos debidos al fallo de barras, aquí se puede apreciar mejor la componente debida al falso positivo. En este tipo de caso, donde además del falso positivo se tiene barras rotas, se hace más difícil distinguir qué componente armónica pertenece a cada caso, con lo que se puede cometer un error de diagnóstico.

Viendo la Figura 126(d) se puede comprobar como el efecto del falso positivo hacer que la trayectoria teórica de los armónicos de fallo tienda a irse hacia este falso positivo en varios puntos. Se puede corroborar, realizando un análisis de la señal en estudio, mediante la transformada corta de Fourier. Obteniendo, en la Figura 126(e), cómo se aprecia la componente fundamental y los armónicos debidos a fallo de barras, así como un tercer armónico que se encuentra entre el armónico de barra y la componente fundamental, lo que nos lleva a corroborar que, efectivamente, se da el falso positivo. Así, debido al falso positivo, la componente fundamental se encuentra desplazada de su valor original de $50 \mathrm{~Hz}$, tal y como se ha comentado en el caso anterior, para un motor sano.

Otro caso en el que se puede apreciar claramente el falso positivo es en la Figura 127, donde la señal presenta excentricidad, pero no presenta fallo por barras rotas, sin embargo, el falso positivo podría dar lugar a un error de diagnóstico. 
Motor Excentricidad Sin Compensación

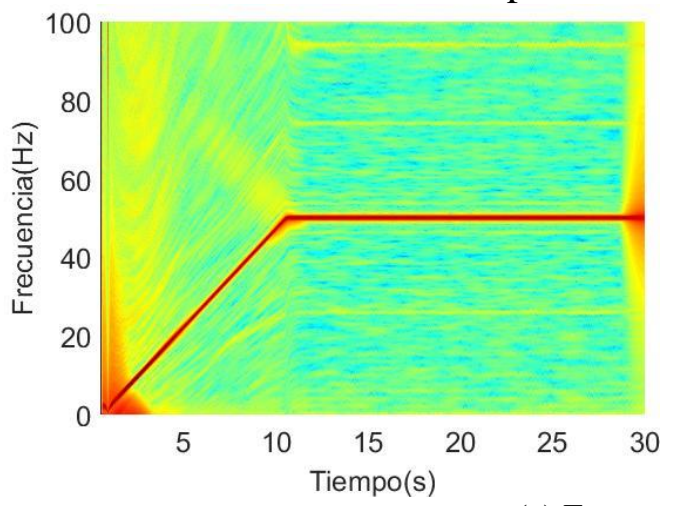

(a) Espectrograma del ensayo

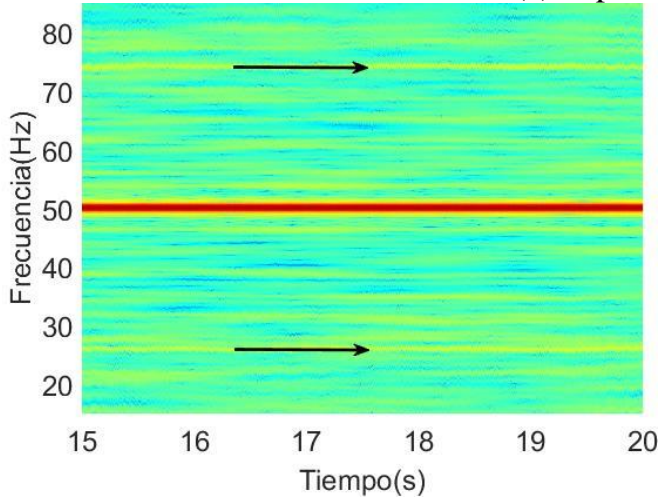

(b) Detalle del espectrograma
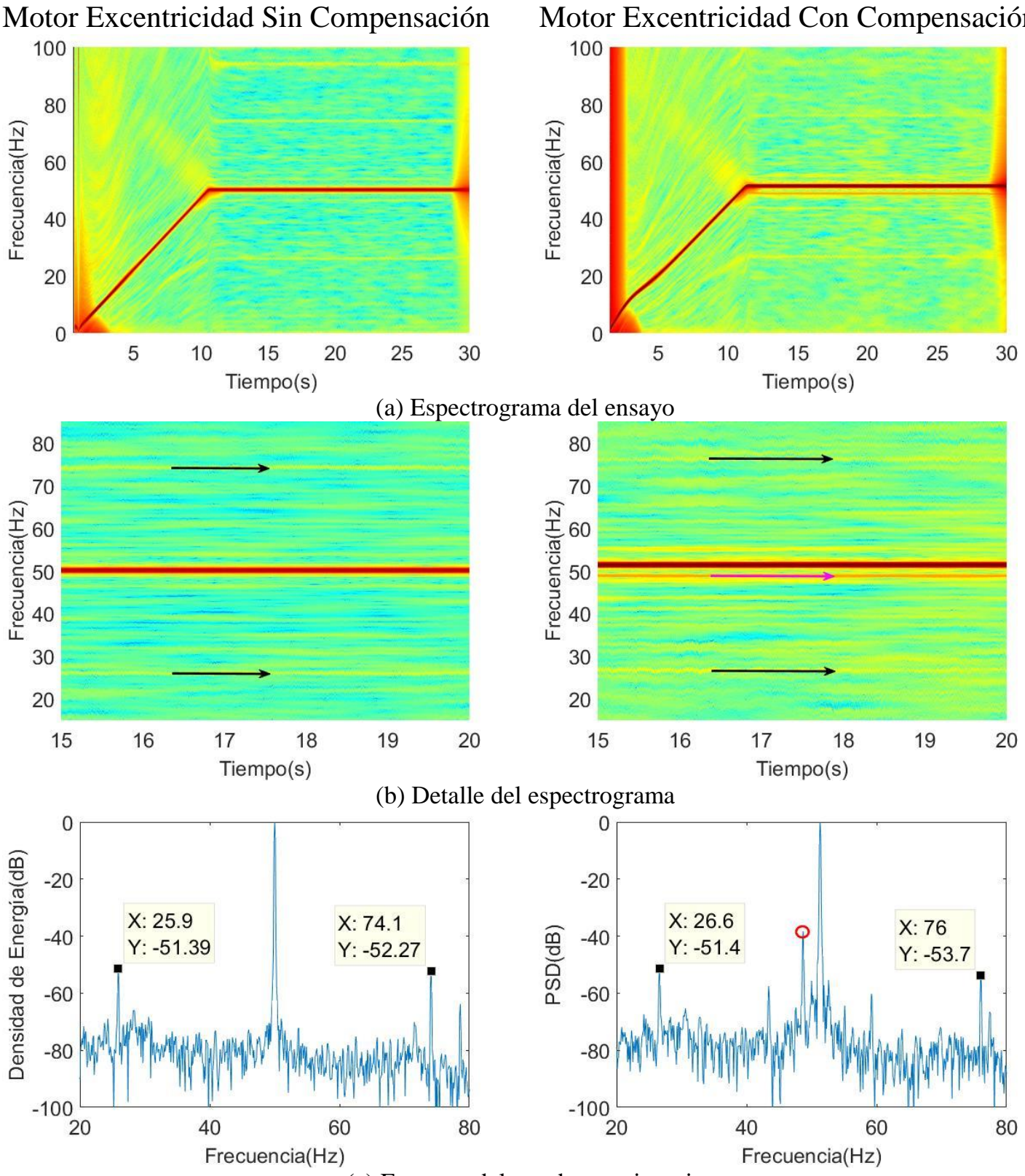

(c) Espectro del estado estacionario

Figura 127. Análisis señal fallo severidad R3, mediante técnica Dragon Transform.

En la Figura 127(b) se puede apreciar el armónico debido a falso positivo $(\rightarrow)$, así como las componentes armónicas causadas por excentricidad $(\rightarrow)$.

Lo vemos aplicando FFT, tal como se muestra en la Figura 127(c). Tal y como se ha explicado en los casos previos, el hecho de tener falso positivo hace que la frecuencia fundamental se vea desplazada de su valor de $50 \mathrm{~Hz}$, tal como se puede apreciar en la citada Figura. 
A continuación, en la Figura 128, analizamos una señal que presenta falso positivo y barras rotas, con los distintos inversores que se han utilizado para ver si existe o no diferencia. Los inversores que se han analizado en este apartado, por poder regular la compensación por deslizamiento son Allen Bradley, Telemecánica y Siemens. Obteniéndose lo siguiente:

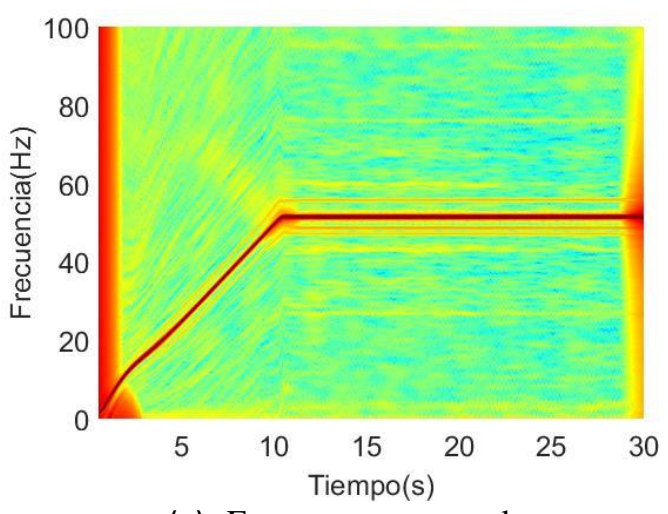

(a) Espectrograma resultante con inversor Allen-Bradley

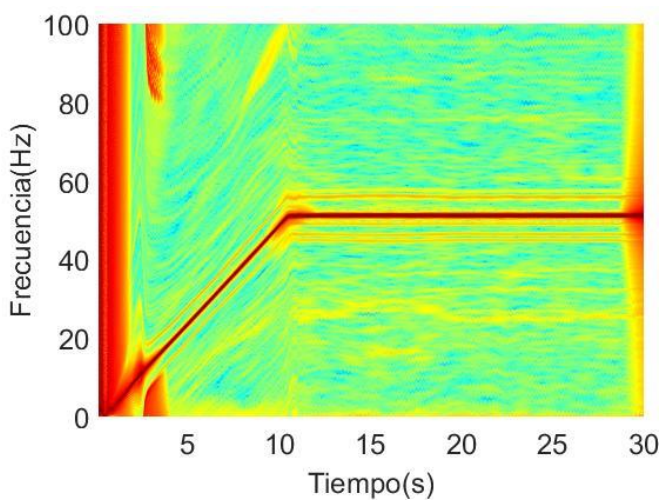

(c) Espectrograma resultante con inversor Siemens

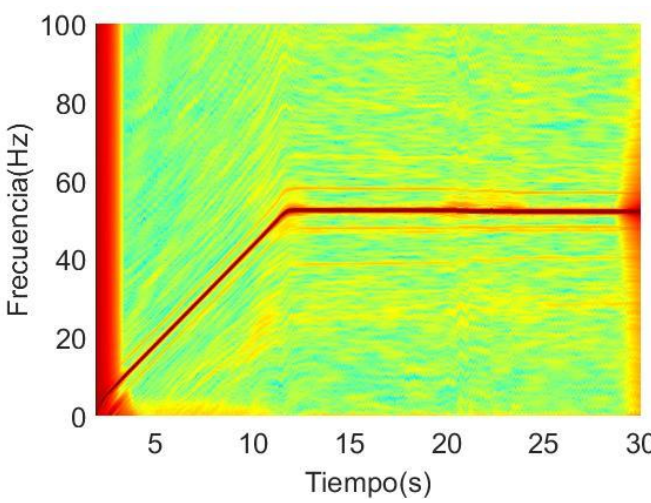

(e) Espectrograma resultante con inversor Telemecánica

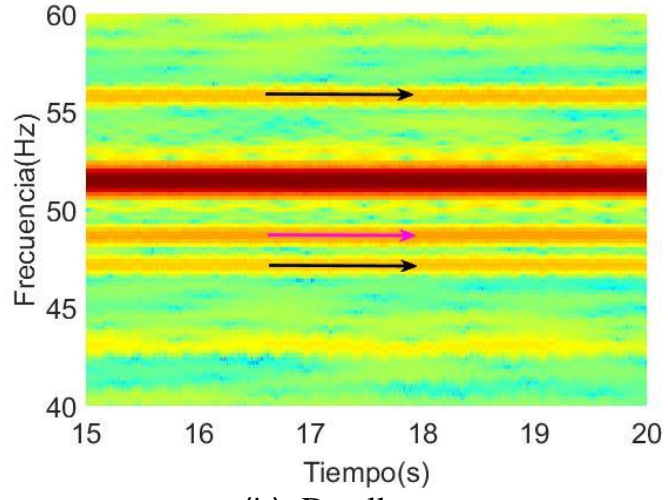

(b) Detalle espectrograma resultante con inversor AllenBradley

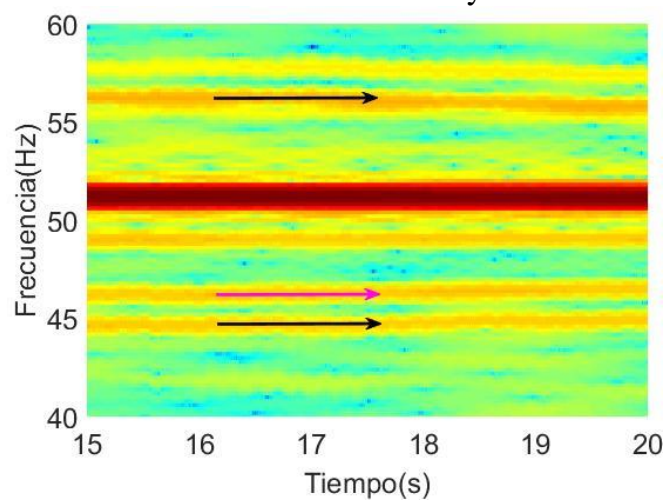

(d) Detalle espectrograma resultante con inversor Siemens

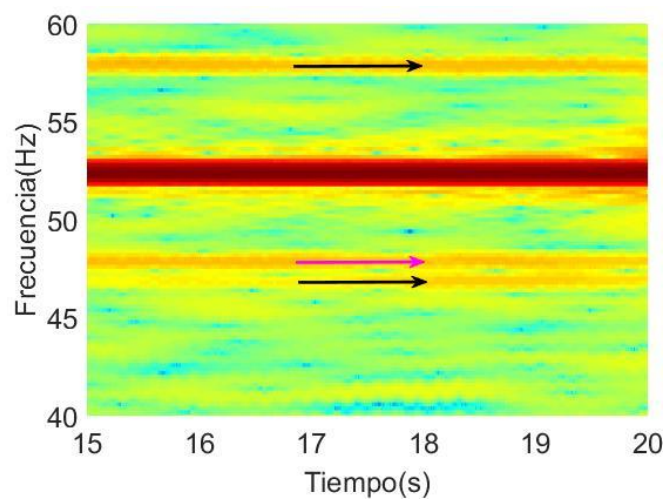

(f) Espectrograma resultante con inversor Telemecánica

Figura 128. Comparativa falso positivo con distintos inversores. 
7. Aportaciones y conclusiones

7.1. Conclusiones

7.2. Aportaciones

7.3. Posibles líneas de investigación a futuro 


\section{Aportaciones y conclusiones (Contributions and conclusions)}

\subsection{Conclusiones}

Una vez analizados todos los puntos anteriores que componen esta memoria de tesis, se presentan a continuación las conclusiones generales que se extraen del estudio realizado, divididas en tres bloques, tal como se ha anticipado en el apartado 1.3.

\section{Estado del arte relativo a la detección de fallos en motores en régimen transitorio}

Con la transformada Rápida de Fourier se pueden apreciar los fallos que el motor de inducción presenta cuando se está analizando el estado estacionario. Sin embargo, esta técnica presenta una serie de limitaciones intrínsecas, que hacen que para la detección de fallos en régimen transitorio sean necesarias otras herramientas matemáticas, distintas de la transformada de Fourier.

Estas transformadas matemáticas pertenecen al campo de las herramientas de descomposición tiempo-frecuencia y, en algunos casos todavía están en desarrollo o proceden de otros campos de la ciencia y la ingeniería. La STFT tiene un compromiso de resolución fijo en todo el plano tiempo-frecuencia, que, si bien es suficiente para obtener la evolución de los componentes de fallo por excentricidad mixta, falla al diagnosticar fallos debidos a rotura de barras. Para solucionar este inconveniente, se propusieron otras técnicas tales como la transformada Wavelet con una resolución mejorada. La CWT permite una resolución variable con la frecuencia analizada. Sin embargo, esta variación no es la adecuada para los tipos de evoluciones de las componentes de fallo en las corrientes de estator de motor de inducción, y, por tanto, se debe evitar. Por otra parte, la DWT descompone la corriente en subseñales relacionadas con diversas bandas de frecuencia, pero no permite obtener las evoluciones de los armónicos de fallo, ya que la presencia de la $F C$ en las subseñales enmascara los componentes. 
WVD elimina el problema de resolución t-f que existe en las anteriores técnicas, pero introduce términos cruzados, impidiendo ver los armónicos de fallo durante el transitorio de arranque. HHT trata de dividir la corriente en sus componentes, para luego obtener su frecuencia instantánea. Sin embargo, sólo tiene éxito para separar la componente de excentricidad mixta inferior de la corriente. MUSIC muestra una resolución uniforme en todo el plano tiempo-frecuencia. La AST ofrece una resolución tf completamente adaptable y esto permite una gran mejora con respecto a la STFT. Sin embargo, se tienen dificultades para observar lo que ocurre en el régimen transitorio. Por su parte, la $C T$ elimina los problemas de resolución de las transformadas anteriores sin introducir términos cruzados. Como consecuencia, se puede distinguir perfectamente lo que ocurre durante el transitorio de arranque.

En conclusión, se puede decir que existe una necesidad actual de técnicas adecuadas para identificar fallos en motores de inducción alimentados por inversor en estados transitorios, lo que supone todo un reto debido a la proximidad de las evoluciones de los armónicos de fallo con la componente fundamental y al cambio continuo de la frecuencia de la componente fundamental durante los estados transitorios.

\section{Transformada Dragón}

Para solventar las limitaciones expuestas en el punto anterior, se ha desarrollado la denominada transformada Dragón. Esta transformada se define en un punto como la correlación entre la señal a analizar y el átomo centrado en ese punto del plano tiempofrecuencia, obteniendo la densidad de energía en el punto. Si se aplica en muchos puntos del plano se pueden observar las evoluciones de los componentes de la señal que se quiere analizar. Con los átomos adaptables desarrollados, “Átomos Dragón”, se puede conseguir seguir la evolución completa de los componentes armónicos de la señal, tanto en el estado estacionario como en el transitorio.

La transformada desarrollada se adecúa a las necesidades de diagnóstico, pues es capaz de seguir las evoluciones de las componentes de fallo que la señal a analizar presente. Las características de la señal a analizar determinan la forma de seleccionar la familia de átomos tiempo-frecuencia a utilizar, que será distinta para cada una de las 
señales a analizar. Así, en comparación con otras transformadas analizadas a lo largo de esta memoria, la mejora que incluye la transformada Dragón es la de poder adaptarse a toda la trayectoria de la señal, tanto en el estado estacionario como transitorio, sin pérdida de resolución.

\section{Resampleado}

La técnica de resampleado planteada permite cambiar la frecuencia de muestreo a un valor adecuado al análisis que se va a realizar posteriormente. Al realizar un remuestreo de la señal, el número de muestras de la señal también cambia, reduciendo el número de muestras de la señal y, por consiguiente, el esfuerzo computacional al aplicar la técnica de análisis tiempo-frecuencia, sin perder calidad o precisión en el resultado.

\section{Resultados obtenidos}

Se ha conseguido desarrollar una técnica que permite seguir las trayectorias de los armónicos presentes en la señal con fallo de barras rotas, independientemente del inversor usado y de la carga a la que esté sometido. Se han corroborado los resultados mediante la $F F T$, que permite verificar los armónicos de fallo en el estado estacionario coincidente con los valores que se obtienen mediante la transformada Dragón, con la que se obtiene muy buena definición de este régimen. Además, la transformada desarrollada también es capaz de seguir los armónicos de fallo en estado transitorio, lo que no se consigue con la FFT.

En el caso de detección de excentricidad, la dificultad no estriba en la proximidad de los armónicos de fallo a la componente fundamental, como ocurre en el fallo de barras. La trayectoria de los armónicos de excentricidad mixta se aleja del armónico principal, por lo que, en principio, no habría problema de resolución en tiempo-frecuencia para distinguirlos. Aquí la dificultad es que la familia de átomos se adapte a las diferentes pendientes de las trayectorias de los armónicos de fallo en cualquier punto del plano.

Se ha realizado también un análisis mediante Functional Boxplot, con el objetivo de cuantificar las curvas resultantes. En este análisis se han apreciado diferencias en el valor en $\mathrm{dB}$ de la media de las curvas con respecto al valor que se obtiene cuando el motor 
está sano. Se puede confirmar tanto al analizar el $L S H$ como al analizar el $U S H$. Esta variación en el valor es mucho más acusada en el caso del USH.

También se han presentado las diferencias de comportamiento entre los diferentes inversores en lo que concierne a la detección del fallo.

\section{English version of the Conclusions Section}

Next, the conclusions extracted from this Thesis are exposed, divided into three sections.

\section{State of the art relating to the fault detection in induction motors in transient regime}

The Fast Fourier transform permits to detect faults in induction motors operating in steady state. However, this technique presents some intrinsic limitations, which make other mathematical tools necessary for the detection of faults in the transient regime.

These mathematical transforms belong to the field of time-frequency decomposition tools and, in some cases, they are still in development or come from other fields of science and engineering. The STFT has a fixed resolution performance in all the time-frequency plane; although it is sufficient to obtain the evolution of the components of failure due to mixed eccentricity, it fails to diagnose failures due to broken rotor bars. To solve this problem other techniques, such as the Wavelet transform, with an improved resolution, were proposed. The $C W T$ allows a variable resolution with the frequency analyzed. However, this variation is not suitable for the evolution of the fault components in the induction motor stator currents, and, therefore, should be avoided. On the other hand, the $D W T$ decomposes the current into sub-signals related to different frequency bands, but it does not allow to obtain the evolutions of the fault harmonics, since the presence of the $F C$ in the sub-signals masks the components.

WVD eliminates the $\mathrm{t}-\mathrm{f}$ resolution problem that exists in the previous techniques, but introduces cross-terms, preventing to see the harmonics of failure during the transient start-up. HHT tries to divide the current into its components, and then obtain its instantaneous frequency. However, it is only successful in separating the lower mixed 
eccentricity components from the current. MUSIC shows an uniform resolution in the whole time-frequency plane. The $A S T$ offers a fully adaptable t-f resolution, and this allows a great improvement over the STFT. However, it is difficult to observe what happens in the transient regime. The $C T$ eliminates the resolution problems of the previous transforms without introducing cross-terms. Therefore, it is possible to distinguish perfectly what happens during the transient start-up.

In conclusion, it can be said that there is a current need for adequate techniques to identify faults in inverter-fed induction motors in transient regimens, which is quite a challenge due to the proximity of the evolutions of the fault harmonics to the fundamental component and the continuous change of the frequency of the fundamental component during the transient regimens.

\section{Dragon Transform}

To solve the limitations exposed in the previous point, the Dragon transform has been developed. This transform is defined in a point as the correlation between the signal to be analyzed and the atom-centered at that point of the time-frequency plane, obtaining the energy density at the point. If it is applied in many points of the plane, the evolutions of the components of the signal to be analyzed can be observed. With the adaptive atoms developed, "Dragon atoms", it is possible to follow the complete evolution of the harmonic components of the signal, both in the steady state and in the transient regime.

The developed transform is adapted to the diagnostic needs. It can follow the evolutions of the fault components that the signal to analyze presents. The characteristics of the signal to be analyzed determine the way to select the family of time-frequency atoms to be used, which will be different for each signal to be analyzed. Thus, in comparison with other transforms analyzed throughout this work, the Dragon transform can adapt to the entire trajectory of the signal, both in the steady state and in the transient regime, without loss of resolution.

\section{Resampling}


The proposed resampling technique allows to change the sampling frequency to a value appropriate to the analysis to be carried out. Reducing the number of samples of the signal, the computational effort is reduced, and it is possible to apply the time-frequency analysis technique, without losing quality or precision in the result.

\section{Results obtained}

It has been possible to develop a technique that allows following the trajectories of the harmonics present in the signal with broken rotor bars faults, independently of the load and the inverter used. The results have been corroborated by the FFT, which allows to verify the harmonics of failure in the steady state coincident with the values obtained by the Dragon transform. In addition, the developed transform is also able to follow the harmonics of failure in the transient regime, which is not achieved with the FFT.

In the case of eccentricity detection, the trajectory of mixed eccentricity harmonics evolves far away from the main harmonic; so, there would be no problem of timefrequency resolution to distinguish them. The difficulty lies in the ability of the family of atoms to be able to adapt to the different slopes of the fault harmonics trajectories at any point in the plane.

An analysis has also been carried out using Functional Boxplot to quantify the resulting curves. In this analysis, the differences in the value, in $\mathrm{dB}$, of the average of the curves have been appreciated with respect to the value obtained when the motor is healthy. It can be confirmed both by analyzing the $L S H$ and the $U S H$. This value variation is more pronounced in the case of the $U S H$.

The differences in performance between the different inverters about the fault detection have also been presented.

\subsection{Aportaciones}


Artículo:

V. Fernandez-Cavero, D. Morinigo-Sotelo, O. Duque-Perez, J. Pons-Llinares, “A Comparison of Techniques for Fault Detection in Inverter-fed Induction Motors in Transient Regime,” IEEE Access, vol. 5, pp. 8048 - 8063, Mayo 2017.

Ponencia en congreso:

V. Fernandez-Cavero, D. Morinigo-Sotelo, O. Duque-Perez, J. Pons-Llinares , "Fault detection in inverter-fed induction motors in transient regime: State of the art," en Proceedings of the IEEE 10th International Symposium on Diagnostics for Electrical Machines, Power Electronics and Drives (SDEMPED), Guarda, Portugal, 1-4 Septiembre 2015.

\subsection{Posibles líneas de investigación a futuro}

Tras la realización de esta tesis, y a tenor de los resultados obtenidos en la misma, cabe la posibilidad de una serie de posibles líneas de investigación a futuro, que aquí se plantean.

Por una parte, sería la mejora de la técnica desarrollada para poder solventar las limitaciones que la misma presenta, haciendo de ésta una técnica más potente de la actualmente conseguida.

Por otra parte, de todos los ensayos que se han venido realizando para la realización de esta tesis, tal como se explica en el punto 5, los resultados se centran en el análisis profundo de los niveles de severidad correspondientes al motor sano y con fallo avanzado, centrándose concretamente en arranque lineal con un tipo de control V/f lineal, tanto con compensación por deslizamiento como sin él. También se ha analizado una severidad de fallo intermedia, para el análisis de la excentricidad mixta.

Por tanto, a futuro queda un trabajo de investigación y profundización de fallos incipientes, tanto con arranques lineales como con arranques en $\mathrm{S}$, así como con distintos tipos de control, V/f lineal, SVC (Vel) y SVC (Par), así mismo con compensación deslizamiento 0 y con compensaciones por deslizamiento distintas de cero. 
Esta tesis ha sido probada en transitorios de arranque, así como en transitorios especiales como el mencionado arranque en S, sin embargo, no ha sido objetivo de esta investigación el estudio de transitorios arbitrarios, lo que puede suponer una nueva línea de trabajo a futuro.

Por último, tal como se ha introducido en el punto 6 de la tesis, concretamente en el epígrafe 6.5., al analizar la respuesta de la transformada se ha llegado a un nuevo descubrimiento, la aparición de un nuevo armónico, que se ha comenzado a analizar, así, se plantea como línea a futuro a desarrollar y verificar el porqué de esta aparición.

\section{English version of possible future lines of research section}

After the achievement of this thesis, and to tenor of the results obtained in it, there fits the possibility of possible future lines of research, which here appear.

On one hand, it would be the progress of the technique developed to be able to settle the limitations that it presents, doing it a more powerful technique.

On the other hand, the results of all the tests that have been carried out for the realization of this thesis, as explained in point 5, are focus on the in-depth analysis of the levels of severity corresponding to a healthy motor and a motor with advanced failure, specifically focusing on linear start-up with a V/f linear control, both with and without slip compensation. An intermediate failure severity has also been analyzed for the mixed eccentricity.

Therefore, in the future there is a work of investigation and deepening of incipient failures, both with linear start-up and with S start-up. As well as with different types of control, linear V/f, SVC (Speed) and SVC (Torque). Also, with slip compensation and without slip compensation. 
This thesis has been tested in start-up transients, as well as in special transients, such as the mentioned start-up in S. However, the study of arbitrary transients has not been the objective of this investigation, which may imply a new line of work in a future.

Finally, as it has been introduced in the point 6 of this thesis memory, specifically in section 6.5., analyzing the response of the transform, a new discovery has been reached. It's the appearance of a new harmonic, which has begun to analyze, thus, it is proposed as a future line to develop and verify the reason for this appearance. 



\section{Abreviaturas}

A:

AST.- Adaptive Slope Transform

AWT.- Analytic Wavelet Transform

B:

BRB.- Broken Rotor Bars

C:

CCWT.- Complex Continous Wavelet Transform

CEEMD.- Complete Ensemble Empirical Mode Decomposition

CT.- Chirplet Transform

CWT.- Continous Wavelet Transform

D:

DWT.- Discrete Wavelet Transform

E:

EEMD.- Ensemble Empirical Mode Decomposition

EMD.- Empirical Mode Decomposition

ERH(-).- Eccentricity Harmonic Negative

ERH(+).- Eccentricity Harmonic Positive

F:

FBS.- Frequency B-Spline

FC.- Fundamental Component

FCC.- Flux Current Control

FFT.- Fast Fourier Transform

FrFT.- Fractional Fourier Transform

FT.- Fourier Transform 
H:

HB.- Heisenberg Box

HHS.- Hilbert Huang Spectrum

HHT.- Hilbert Huang Transform

HT.- Hilbert Transform

I:

IM.- Induction Motor

IMF.- Intrinsic Mode Function

L:

LSH.- Lower Sideband Harmonic

M:

MCSA.- Motor Current Signature Analysis

MUSIC.- Multiple Signal Classification

P:

PSD.- Power Spectral Density

S:

STFT.- Short Time Fourier Transform

SVC.- Sensorless Vector Control

T:

TFA.- Time-Frequency Atom

TFD.- Time-Frequency Decomposition

$\mathbf{U}:$

USH.- Upper Sideband Harmonic 
W:

WD.- Wigner Distribution

WPD.- Wavelet Packets Decomposition

WVD.- Wigner Ville Distribution

Z:

ZSC.- Zero-Sequence Current

Nota. Usamos las abreviaturas en ingles ya que es así como se definen de manera habitual. 



\section{Bibliografía}

[1] J. Tolvanen, "Saving energy with variable speed drives," World Pumps, vol. 501, pp. 32-33, Junio 2008.

[2] C.M.F.S. Reza, M.D. Islam, S. Mekhilef, "A review of reliable and energy efficient direct torque controlled induction motor drives," Renewable and Sustainable Energy Reviews, vol. 37, pp. 919-932, 2014.

[3] J. Pons-Llinares, D. Morinigo-Sotelo, O. Duque-Perez, J. Antonino- Daviu, M. PerezAlonso, "Transient detection of close components through the chirplet transform: Rotor faults in inverter-fed induction motors," in Proc. of 40th Annual Conference of the IEEE Industrial Electronics Society, IECON 2014, pp. 3386-3392, Dallas, TX, USA, 29 Octubre - 1 Noviembre, 2014.

[4] R. Saidur, S. Mekhilef, M.B. Ali, A. Safari, H.A. Mohammed, "Applications of variable speed drive (VSD) in electrical motors energy savings," Renewable and Sustainable Energy Reviews, vol. 16 (1), pp. 543-550, Enero 2012.

[5] V. Ghorbanian, J. Faiz, "A survey on time and frequency characteristics of induction motors with broken rotor bars in line-start and inverter-fed modes," Mechanical Systems and Signal Processing, vol. 54-55, pp. 427-456, Marzo 2015.

[6] Peter Jackson (1998), Introduction to Expert Systems, $3^{\text {a }}$ Ed., Boston, MA, EE.UU. Addison Wesley.

[7] H. Henao, H. Razik, G.A. Capolino, "Analytical approach of the stator current frequency harmonics computation for detection of induction machine rotor faults," IEEE Transactions on Industry Applications, vol. 41 (3), pp. 801-807, Mayo/Junio 2005 .

[8] J. Jee-Hoon, J.J. Lee, K. Bong-Hwan, “Online Diagnosis of Induction Motors Using MCSA," IEEE Transactions on Industrial Electronics, vol. 53 (6), pp. 1842-1852, Diciembre 2006. 
[9] J.P. Amezquita-Sanchez, M. Valtierra-Rodriguez, D. Camarena- Martinez, D. Granados-Lieberman, R., Romero-Troncoso, A. Dominguez-Gonzalez, Aurelio, "Fractal dimension-based approach for detection of multiple combined faults on induction motors," Journal of Vibration and Control, vol. 22 (17), pp. 3638-3648, Enero 2015.

[10] V. Climente-Alarcon, J.A. Antonino-Daviu, F. Vedreno-Santos, R. Puche-Panadero, "Vibration Transient Detection of Broken Rotor Bars by PSH Sidebands," IEEE Transactions on Industry Applications, vol. 49 (6), pp. 2576-2582, Noviembre/Diciembre 2013.

[11] J. Pons-Llinares, J. Antonino-Daviu, J. Roger-Folch, D. Moríñigo- Sotelo, O. DuquePérez, "Mixed eccentricity diagnosis in Inverter- Fed Induction Motors via the Adaptive Slope Transform of transient stator currents," Mechanical Systems and Signal Processing, vol. 48 (1-2), pp. 423-435, Octubre 2014.

[12] D. Morinigo-Sotelo, L.A. Garcia-Escudero, O. Duque-Perez, M. Perez-Alonso, "Practical Aspects of Mixed-Eccentricity Detection in PWM Voltage-Source-InverterFed Induction Motors," IEEE Transactions on Industrial Electronics, vol. 57 (1), pp. 252-262, Enero 2010.

[13] WS. Abu-Elhaija, V. Ghorbanian, J. Faiz, B.M. Ebrahimi, "Impact of closed-loop control on behavior of inverter-fed induction motors with rotor broken-bars fault," in Proc. of 2012 IEEE International Conference on Power Electronics, Drives and Energy Systems (PEDES), Bengaluru, Karnataka, India, 16-19 Diciembre 2012.

[14] J. Faiz, V. Ghorbanian, B.M. Ebrahimi, “A survey on condition monitoring and fault diagnosis in line-start and inverter-fed broken bar induction motors," in Proc. of 2012 IEEE International Conference on Power Electronics, Drives and Energy Systems (PEDES), Bengaluru, Karnataka, India, 16-19 December 2012.

[15] W. Deleroi, "Squirrel cage motor with broken bar in the rotor - Physical phenomena and their experimental assessment," Proc. Int. Conf. Electrical Machines, 1982, pp. 767-770.

[16] J.R. Cameron, W.T. Thomson, A.B. Dow, "Vibration and current monitoring for detecting air gap eccentricity in large induction motors," IEE Proceedings B - Electric Power Applications, vol.133 (3), pp. 155-163, Mayo 1986. 
[17] G.B. Kliman, R.A. Koegl, J. Stein, R.D. Endicott, M.W. Madden, "Noninvasive detection of broken rotor bars in operating induction motors," IEEE Transactions on Energy Conversion, vol.3 (4), pp. 873-879, Diciembre 1988.

[18] J. Fernandez, A. Bediaga, I. Gaston and A. Hernandez, "Evaluation Study on Detection Techniques for Bearing Incipient Faults", The International Conference on Computer as a Tool, vol. 2, pp. 1566-1569, Noviembre 2005.

[19] G.T. Heydt, P.S. Fjeld, C.C. Liu, D. Pierce, L. Tu, G. Hensley, “Applications of the windowed FFT to electric power quality assessment", IEEE Transactions on Power Delivery, vol. 14 (4), Octubre 1999.

[20] Daniel Moríñigo Sotelo, "Introducción a la Máquina de Inducción,” Material del curso celebrado en la Universidad Autónoma de Querétaro San Juan del Río, Querétaro, México, Junio 2014.

[21] R. De Jesús Romero-Troncoso, "Multirate Signal Processing to Improve FFT-based Analysis for Detecting Faults in Induction Motors," IEEE Transactions on Industrial Informatics, vol. 13 (3), pp. 1291-1300, 2016.

[22] F. Hlawatsch y F. Auger (Ed.) (2008), Time-Frequency Analysis. Concepts and Methods, Londres, Reino Unido, ISTE Ltd.

[23] M. Riera-Guasp, et al, "Diagnosis of induction machines under non- stationary conditions: Concepts and tools," in Proc. of 2013 IEEE Workshop on Electrical Machines Design Control and Diagnosis (WEMDCD), pp. 220-231, Paris, France, 1112 Marzo 2013.

[24] J. Pons-Llinares, V. Climente-Alarcon, F. Vedreno-Santos, J. Antonino-Daviu, M. Riera-Guasp, "Electric machines diagnosis techniques via transient current analysis," in Proc. of 38th IEEE Industrial Electronics Annual Conference, pp. 3893-3900, Montreal, QC, Canada, 25-28 Octubre 2012.

[25] J.A. Antonino-Daviu, M. Riera-Guasp, M. Pineda-Sánchez, J. Pons-Llinares, R. Puche-Panadero, J. Pérez-Cruz, "Feature extraction for the prognosis of electromechanical faults in electrical machines through the DWT", International Journal of Computational Intelligence Systems, vol. 2 (2), pp. 158-167, Junio 2009. 
[26] F.J. Vedreno-Santos (2013), Diagnosis of electric induction machines in nonstationary regimes working in randomnly changing conditions, tesis doctoral, Universitat Politècnica de València, Valencia, España.

[27] M. Riera-Guasp, J. Pons-Llinares, V. Climente-Alarcón, F.Vedreño-Santos, M. Pineda-Sánchez, J. Antonino-Daviu, R.Puche-Panadero, J. Perez-Cruz, J. RogerFolch, "Diagnosis of Induction Machines under Nonstationary Conditions: Concepts and Tools," in Proceedings of 2013 IEEE Workshop on Electrical Machines Design, Control and Diagnosis (WEMDCD), pp. 218-229, Paris, Francia, 11-12 Marzo 2013.

[28] F. Vedreño-Santos, M. Riera-Guasp, M. Pineda-Sánchez, "Diagnosis of induction machines under non-stationary conditions by means of the spectral filter," in Proceedings of the 2013 9th IEEE International Symposium on Electric Machines, Power Electronics and Drives (SDEMPED), pp. 91-98, Valencia, España, 27-30 Agosto 2013.

[29] J. Pons-Llinares, M. Riera-Guasp, J. A. Antonino-Daviu, F. Vedreño-Santos, "Transient diagnosis of induction generators via atom-based time-frequency transforms," in Proceedings of the 2014 International Conference on Electrical Machines (ICEM), pp. 1793-1799, Berlin, Alemania, 2-5 Septiembre 2014.

[30] A. Sapena-Baño, M. Riera-Guasp, R. Puche-Panadero, J. Martinez-Roman, J. PerezCruz, J. Roger-Folch, M. Pineda-Sanchez, "Harmonic order tracking analysis: A speed-sensorless method for condition monitoring of wound rotor induction generators in wind turbines," in Proceedings of the IEEE 10th International Symposium on Diagnostics for Electrical Machines, Power Electronics and Drives (SDEMPED), pp. 351-358, Guarda, Portugal, 1-4 Septiembre 2015.

[31] V.T. Tran, F. AlThobiani, A. Ball, B.-K. Choi, “An application to transient current signal based induction motor fault diagnosis of Fourier-Bessel expansion and simplified fuzzy ARTMAP," Expert Systems with Applications, vol. 40 (13), pp. 5372-5384, Octubre 2013.

[32] M. Pineda-Sanchez, M. Riera-Guasp, J.A. Antonino-Daviu, J. Roger- Folch, J. Perez-Cruz, R. Puche-Panadero, "Diagnosis of induction motor faults in the fractional Fourier domain,” IEEE Transactions on Instrumentation and Measurement, vol. 59 (8), pp. 2065-2075, Agosto 2010. 
[33] B. Yazici, G.B. Kliman, “An adaptive statistical time-frequency method for detection of broken bars and bearing faults in motors using stator current," IEEE Transactions on Industry Applications, vol. 35 (2), pp. 442-452, Marzo/Abril 1999.

[34] M. Blödt (2006), Condition Monitoring of Mechanical Faults in Variable Speed Induction Motor Drives - Application of Stator Current Time-Frequency Analysis and Parameter Estimation, tesis doctoral, Institut National Polytechnique de Toulouse (INPT), Toulouse, Francia.

[35] J. Cusidó, L. Romeral, J.A. Ortega, J.A. Rosero, A.G. Espinosa, "Fault detection in induction machines using power spectral density in wavelet decomposition," IEEE Transactions on Industrial Electronics, vol. 55 (2), pp 633-643, Febrero 2008.

[36] J. Antonino-Daviu, M. Riera-Guasp, J. Roger-Folch, F. Martínez-Giménez, A. Peris, "Application and optimization of the discrete wavelet transform for the detection of broken rotor bars in induction machines," Applied and Computational Harmonic Analysis, vol. 21 (2), pp. 268-279, Septiembre 2006.

[37] J.A. Antonino-Daviu, M. Riera-Guasp, M. Pineda-Sanchez, R.B. Pérez, “A critical comparison between DWT and Hilbert-Huang- based methods for the diagnosis of rotor bar failures in induction machines," IEEE Transactions on Industry Applications, vol. 45 (5), pp. 1794-1804, Julio 2009.

[38] J. Pons-Llinares, J. Antonino-Daviu, J. Roger-Folch, D. Morinigo- Sotelo, O. DuquePerez, "Eccentricity diagnosis in Inverter - Fed Induction Motors via the Analytic Wavelet Transform of transient currents," en Proceedings of 2010 XIX International Conference on Electrical Machines (ICEM), Rome, Italia, 6-8 Septiembre 2010.

[39] J. Pons-Llinares, J.A. Antonino-Daviu, M. Riera-Guasp, M. Pineda Sanchez, V. Climente-Alarcon, "Induction motor diagnosis based on a transient current analytic wavelet transform via frequency B- splines," IEEE Transactions on Industrial Electronics, vol. 58 (5), pp. 1530-1554, Mayo 2011.

[40] M. Pineda-Sanchez, M. Riera-Guasp, J. Perez-Cruz, R. Puche- Panadero, "Transient motor current signature analysis via modulus of the continuous complex wavelet: A pattern approach," Energy Conversion and Management, vol. 73, pp. 26-36, Septiembre 2013. 
[41] J.A. Antonino-Daviu, M. Riera-Guasp, M. Pineda-Sánchez, J. Pons- Llinares, R. Puche-Panadero, J. Pérez-Cruz, "Feature extraction for the prognosis of electromechanical faults in electrical machines through the DWT," International Journal of Computational Intelligence Systems, vol. 2 (2), pp. 158-167, Junio 2009.

[42] K. Yahia, A.J.M. Cardoso, A. Ghoggal, S.-E. Zouzou, "Induction Motor Broken Rotor Bars Diagnosis through the Discrete Wavelet Transform of the instantaneous Reactive Power Signal under time- varying load conditions", Electric Power Components and Systems, vol. 42 (7), pp. 682-692, Abril 2014.

[43] K. Yahia, A.J.M. Cardoso, A. Ghoggal, S.E. Zouzou, "Induction motors airgapeccentricity detection through the discrete wavelet transform of the apparent power signal under non-stationary operating conditions," ISA Transactions, vol. 53 (2), pp. 603-611, Marzo 2014.

[44] H.-Y. Zhu, J.-T. Hu, L. Gao, H. Huang, "Fault diagnosis of incipient broken rotor bars for squirrel-cage induction motor under continuous variable load condition," Chinese Journal of Scientific Instrument, vol. 35 (7), pp. 1646-1653, Julio 2014.

[45] K. Teotrakool, M.J. Devaney, L. Eren, “Adjustable-Speed Drive Bearing-Fault Detection Via Wavelet Packet Decomposition,” IEEE Transactions on Instrumentation and Measurement, vol. 58 (8), pp. 2747-2754, Agosto 2009.

[46] A. Yazidi, H. Henao, G.A. Capolino, F. Betin, L. Capocchi, "Experimental inter-turn short circuit fault characterization of wound rotor induction machines," en Proceedings of the 2010 IEEE International Symposium on Industrial Electronics (ISIE), pp. 26152620, Bari, Italia, 4-7 Julio 2010.

[47] A. Yazidi, H. Henao, G.A. Capolino, F. Betin, "Rotor inter-turn short circuit fault detection in wound rotor induction machines," en Proceedings of the 2010 XIX International Conference on Electrical Machines (ICEM), pp. 1-6, Roma, Italia, 6-8 Septiembre 2010.

[48] D. Diaz, M.C. Amaya, A. Paz, "Inter-turn short-circuit analysis in an induction machine by finite elements method and field tests," en Proceedings of the 2012 XXth International Conference on Electrical Machines (ICEM), pp. 1757-1763, Marsella, Francia, 2-5 Septiembre 2012. 
[49] V. Climente-Alarcon, J.A. Antonino-Daviu, M. Riera-Guasp, R. Puche-Panadero, L. Escobar, "Application of the Wigner-Ville distribution for the detection of rotor asymmetries and eccentricity through high-order harmonics," Electric Power Systems Research, vol. 91, pp. 28-36, Octubre 2012.

[50] V. Climente-Alarcon, J. Antonino-Daviu, M. Riera-Guasp, J. Pons- Llinares, J. Roger-Folch, P. Jover-Rodriguez, A. Arkkio, "Transient tracking of low and highorder eccentricity-related components in induction motors via TFD tools", Mechanical Systems and Signal Processing, vol. 25 (2), pp. 667-679, Febrero 2011.

[51] V. Climente-Alarcon, J.A. Antonino-Daviu, A. Haavisto, A. Arkkio, "Particle filterbased estimation of instantaneous frequency for the diagnosis of electrical asymmetries in induction machines," IEEE Transactions on Instrumentation and Measurement, vol. 63 (10), pp. 2454-2463, Octubre 2014.

[52] J. Faiz, V. Ghorbanian, B.M. Ebrahimi, "EMD-Based Analysis of Industrial Induction Motors with Broken Rotor Bars for Identification of Operating Point at Different Supply Modes," IEEE Transactions on Industrial Informatics, vol. 10 (2), pp. 957-966, Mayo 2014.

[53] J. Faiz, V. Ghorbanian, B.M. Ebrahimi, "A new criterion for rotor broken bar fault diagnosis in line-start and inverter-fed induction motors using Hilbert-Huang transform," en Proceedings of the 2012 IEEE International Conference on Power Electronics, Drives and Energy Systems (PEDES), Bengaluru, India, 16-19 Diciembre 2012 .

[54] A.M. da Silva, R.J. Povinelli, N.A.O. Demerdash, "Induction machine broken bar and stator short-circuit fault diagnostics based on three-phase stator current envelopes," IEEE Transactions on Industrial Electronics, vol.55 (3), pp. 1310-1318, Marzo 2008

[55] J. Pons Llinares (2013), Metodología para el diagnóstico de averías en motores de inducción mediante el análisis de corrientes estatóricas transitorias utilizando átomos tiempo-frecuencia, tesis doctoral, Universitat Politècnica de València, Valencia, España. 
[56] R.J. Romero-Troncoso, D. Morinigo-Sotelo, O. Duque-Perez, R.A. Osornio-Rios, M.A. Ibarra-Manzano, A. Garcia-Perez, "Broken rotor bar detection in VSD-fed induction motors at startup by high- resolution spectral analysis," en Proceedings of the 2014 International Conference on Electrical Machines (ICEM), pp. 1848-1854, Berlin, Alemania, 2-5 Septiembre 2014.

[57] D. Morinigo-Sotelo, R.J. Romero-Troncoso, J.A. Antonino-Daviu, K.N. Gyftakis, "Reliable Detection of Broken Rotor Bars in Induction Motors via MUSIC and ZSC Methods", en Proceeding of the XXII International Conference on Electrical Machines (ICEM), pp. 2883-2888, Lausanne, Suiza, 4-7 Septiembre 2016.

[58] J. Pons-Llinares, J. Antonino-Daviu, J. Roger-Folch, D. Morínigo-Sotelo, O. DuquePérez, "Mixed eccentricity diagnosis in Inverter-Fed Induction Motors via the Adaptive Slope Transform of transient stator currents," Mechanical Systems and Signal Processing, vol. 48 (1-2), pp. 423-435, Octubre 2014.

[59] J. Pons-Llinares, M. Riera-Guasp, J. Antonino-Daviu, T. G. Habetler, "Pursuing optimal electric machines transient diagnosis: The adaptive slope transform," Mechanical Systems and Signal Processing, vol. 80, pp. 553-569, Diciembre 2016.

[60] G. Matz, F. Hlawatsch, "Wigner distributions (nearly) everywhere: Time-frequency analysis of signals, systems, random processes, signal spaces, and frames," Signal Processing, vol. 83 (7), pp. 1355-1378, Julio 2003.

[61] A. L. Martinez-Herrera, L. M. Ledesma-Carrillo, M. Lopez-Ramirez, S. SalazarColores, E. Cabal-Yepez, A. Garcia-Perez, "Gabor and the Wigner-Ville transforms for broken rotor bars detection in induction motors," en Proceedings of the 2014 International Conference on Electronics, Communications and Computers (CONIELECOMP), pp. 83-87, Cholula, México, 26-28 Febrero 2014.

[62] D.G. Dorrell, W.T. Thomson, S. Roach, "Analysis of air gap flux, current, and vibration signals as a function of the combination of static and dynamic air gap eccentricity in 3-phase induction motors," IEEE Transactions on Industry Applications, vol. 33 (1), pp. 24-34, Enero/Febrero 1997.

[63] D. Gabor, “Theory of communication," Journal of the IEE - Part III: Radio and Communication Engineering, vol. 93 (26), pp. 429-457, Noviembre 1946. 
[64] J. Pons-Llinares, J. Antonino-Daviu, M. Riera-Guasp, M. Pineda-Sanchez, V. Climente-Alarcon, "Induction motor diagnosis based on a transient current analytic wavelet transform via Frequency B-Splines," IEEE Transactions on Industrial Electronics, vol. 58 (5), pp. 1530-1544, Mayo 2011.

[65] T.A. Garcia-Calva, D. Morinigo-Sotelo, R.J. Romero-Troncoso, "Non-uniform time resampling for diagnosing broken rotor bars in inverter-fed induction motors," IEEE Transactions on Industrial Electronics, vol. 64 (3), pp. 2306-2315, Marzo 2017.

[66] R.J. Romero Troncoso, "Tratamiento Digital de Señales, aspectos teóricos y prácticos," Material del curso celebrado en la Escuela de Ingenierías Industriales, Universidad de Valladolid, Valladolid, España, Febrero 2016.

[67] J. O. Ramsay, Hadley Wickham, Spencer Graves, Giles Hooker (2014). fda: Functional Data Analysis. R package version 2.4.4. URL: https://CRAN.Rproject.org/package $=\mathrm{fda}$

[68] R Development Core Team (2008). R: A language and environment for statistical computing. R Foundation for Statistical Computing, Vienna, Austria. ISBN 3-90005107-0, URL http://www.R-project.org.

[69] Y. Sun, M. G. Genton, "Functional Boxplots," Journal of Computational and Graphical Statistics, vol. 20 (2), pp. 316-334, Enero 2012. 